\title{
Atas do X Simpósio Nacional de Investigação em Psicologia, Universidade da Madeira, 2019
}

\author{
David L. Rodriguesi, Margarida Pocinhoii \& Diniz Lopes ${ }^{i}$ \\ iAssociação Portuguesa de Psicologia / ISCTE-IUL \\ iiUniversidade da Madeira
}

\section{EDITORIAL}

É com grande satisfação que anunciamos a publicação das Atas do X Simpósio Nacional de Investigação em Psicologia (SNIP), realizado entre os dias 30 de Junho e 2 de Julho de 2019 na Universidade da Madeira.

As Atas do X SNIP representam a relevância deste Simpósio Nacional de Investigação em Psicologia na difusão do conhecimento produzido em Portugal nesta área científica. Estas Atas são publicadas, pela segunda vez, na revista PSICOLOGIA, o órgão de difusão científica da Associação Portuguesa de Psicologia. Dado que a revista se encontra indexada em diferentes bases de dados internacionais e nacionais, cremos que estes trabalhos poderão atingir uma comunidade de investigadores bastante alargada, permitindo que a investigação realizada em Portugal desperte o interesse de académicos e profissionais da psicologia em diferentes partes do mundo.

Como os leitores terão oportunidade de verificar, os trabalhos aqui apresentados caracterizam-se pela sua elevada diversidade, quer em termos dos temas abordados, quer das metodologias utilizadas e das instituições científicas de proveniência, refletindo a vitalidade do panorama da investigação em Psicologia realizada no nosso país.

O X SNIP reuniu mais de 250 participantes, com um elevado número de comunicações, quer em formato de simpósio, comunicação oral, ou comunicação em poster, e onde se debateram as questões que preocupam atualmente os investigadores portugueses.

São devidos diferentes agradecimentos a todos os intervenientes, quer na Organização do Simpósio, quer na organização do presente livro de Atas. Em primeiro lugar, queremos expressar o nosso agradecimento a toda a Comissão Organizadora do X SNIP, coordenada pela Prof. Margarida Pocinho, bem como a todos os colegas e estudantes do Departamento de Psicologia da Universidade da Madeira que, de forma incansável, nos ajudaram a realizar mais uma edição deste evento. Uma palavra de agradecimento muito especial é, também, devida à Comissão Científica do X SNIP pela sua inestimável ajuda na revisão do elevado número de submissões que recebemos. Por último, é também devido um agradecimento à Universidade da Madeira, pelo seu caloroso acolhimento do X SNIP.

No que diz respeito às Atas do X SNIP, deixamos o nosso particular agradecimento a todos os investigadores e docentes envolvidos no processo de revisão dos textos que foram submetidos para publicação. Esta tarefa foi longa, mas precisa e aturada. Um agradecimento especial aos autores pelas suas contribuições que excederam, totalmente, as nossas espectativas, quer pela quantidade, quer pela qualidade. Esperamos que os trabalhos agora publicados permitam difundir os vossos contributos de pesquisa por uma comunidade alargada e interessada em diversos temas da Psicologia.

Esperamos contar convosco no XI SNIP, em local a designar em breve, e com os vossos contributos para continuarmos a fazer desta iniciativa a maior e mais completa reunião científica da investigação em Psicologia nacional.

Os organizadores,

David L. Rodrigues

Vice-Presidente da Associação Portuguesa de Psicologia

Margarida Pocinho

Professora Associada da Universidade da Madeira

Diniz Lopes

Presidente da Associação Portuguesa de Psicologia 
Revista PSICOLOGIA, 2020, Vol. 34 (1). doi: 10.17575/psicologia.v34i1.1681

Atas do X Simpósio Nacional de Investigação em Psicologia

\title{
Espiritualidade e religiosidade: Duas medidas diferentes em Psicologia?
}

João Pedro da Silva1i, Anabela M. S. Pereira ${ }^{i} \&$ Sara O. M. Monteiro ${ }^{\mathrm{i}}$

i Universidade de Aveiro - Departamento de Educação e Psicologia

\begin{abstract}
Resumo: 0 problema conceptual acerca da dependência entre religiosidade e espiritualidade tem suscitado inconsistência na investigação psicométrica. Pretende-se com este artigo determinar se uma separação métrica entre constructos é possível e traduz-se numa melhor compreensão destes enquanto objetos de estudo em psicologia. Uma amostra composta por 279 participantes com idades compreendidas entre os 17 e os 69 anos preencheram um questionário com duas escalas em aferição, bem como a versão portuguesa do Questionário de Bem-estar Espiritual usado como medida critério. Concluiu-se que tanto a religiosidade e a espiritualidade são passíveis de serem operacionalizadas independentemente em duas escalas distintas. Ambas as medidas assumem maior significância estatística em avaliar os seus respetivos constructos do que a medida critério. Tanto um constructo como o outro não convergem entre si dada a fraca correlação dos seus índices.
\end{abstract}

\section{Palavras-chave: Espiritualidade; Religiosidade; Escalas; Psicometria; Psicologia.}

Spirituality and Religiosity: Two different measures in Psychology? The conceptual problem upon the unity of religiosity and spirituality led to a systematic inconsistency within psychometric research. With this article it is intended to ascertain a metrical severance between the constructs as also to understand them as research objects in psychology. A sample composed by 279 participants with ages between 17 and 69 filled a questionnaire with two developed scales as also with the Portuguese version of the Spiritual Well-being Questionnaire. Both religiousness and spirituality can be operationalized independently in two distinct scales. The two measures hold more statistical significance in assessing their own constructs than the criterion measure. There is no convergence between each construct since due the weak correlation values.

\section{Keywords: Spirituality; Religiosity; Scales; Psychometrics; Psychology.}

Publicou-se muito sobre espiritualidade em Portugal, desde que o médico e escritor Manuel Laranjeira defendeu a sua tese sobre a nosologia do misticismo na Escola Médico-Cirúrgica do Porto em 1907. Nos últimos 18 anos foram publicadas 37 teses de mestrado, 8 doutoramento e 31 artigos em jornais indexados referentes a estudos portugueses incidentes no tema da vivência espiritual (Romerio, Martins, Pinto \& Caldeira, 2018). A nível internacional também se verifica esta tendência, seja pelo incremento do desenvolvimento de novas medidas de espiritualidade (Hill, 2005, 2015) seja pelo crescente número de publicações que se verifica nas últimas décadas nas áreas disciplinares de medicina, psicologia, enfermagem, terapia ocupacional, sociologia, entre outras (Koenig, 2012) - impulso coincidente com a criação da Divisão 36 da American Psychological Association. 0 incremento do estudo da espiritualidade e da religiosidade foi possível com desenvolvimento de várias medidas métricas sobre estes dois constructos, assentes em propostas teóricas distintas (Hill \& Hood, 1999; Hill \& Edwards, 2013). Porém, ao ler as várias publicações nacionais e internacionais, fica-se com a impressão de que o entendimento dos conceitos de religiosidade e espiritualidade é claro - de facto, não é. Desde o famigerado debate sobre a licitude de uma "psicologia da religião e da espiritualidade" no fim do milénio passado (cf. Emmons \& Crumpler, 1999; Hill, 1999; Pargament, 1999a, 1999b; Reich, 2000; Stifoss-Hanssen, 1999) que se verifica uma dissonância entre modelos teóricos, medidas psicométricas e linhas de investigação que se podem resumir em duas tendências antagónicas entre si: concepção de espiritualidade teísta e concepção de espiritualidade não-teísta. Estas duas tendências ou linhas de investigação afetariam, também, a noção de religiosidade: o teísmo advoga ou uma sobreposição conceptual ou uma sinonímia; o não-teísmo, por seu lado, uma independência conceptual entre espiritualidade e religiosidade. Em função desta dissonância,

${ }^{1}$ Morada para correspondência: João P. Silva, Departamento de Educação e Psicologia, Universidade de Aveiro, Campus de Santiago, 3810193 Aveiro E-mail: jp.dasilva@outlook.com 
estabeleceram-se medidas psicométricas não concordantes com a teoria (Da Silva, Pereira, Monteiro \& Bartolo, 2019) e, com isso, a possibilidade de resultados de investigação discrepantes. Hill (2005) reconheceria o estado periclitante da "psicologia da religião e da espiritualidade" pelas várias direcções teórico-conceptuais existentes e por uma metodologia dúbia, sendo que as diversas medidas de espiritualidade ou de religiosidade não são concordantes entre si e podem não medir aquilo que alegam medir. Este autor estabelece, por este motivo, uma General Rating Criteria para escalas e inventários de espiritualidade e religiosidade, com vista à correção de procedimentos vigentes inadequados (Hill, 2005). Alguns anos depois, Hill (2015) voltaria a reiterar as suas conclusões - nada mudara. A questão é que as linhas de investigação se mantiveram no tempo numa irredutibilidade entre posições teístas e nãoteístas, onde o teísmo espiritual assume preponderância sobre o não teísmo por se valer de um maior suporte psicométrico. De facto, a mensuração da espiritualidade torna-se mais simples quando associada a uma religiosidade (Koenig, 2008), esta última mais 'objectivável', porém, não isenta de críticas e problemas métricos - uma censura já apontada é a de que as escalas disponíveis assentam num logro métrico por incorporarem variáveis que, à partida, não imputam uma mútua associação, advindo daí medidas de "bem-estar espiritual”, "coping religioso", "espiritualidade cristã", entre outras, que nada mais são do que a 'religificação' ou 'espiritualização' semânticas de conceitos psicológicos singulares (Piedmont, 1999; Van Wicklin, 1990). Não há, de momento, em função da listagem de escalas disponíveis (Hill, 2005; 2015), alguma que afira uma religiosidade ou espiritualidade per se. Procurar-se-ia, entretanto, a resolução teórica da questão conceptual para fundamentar a licitude de uma psicologia neste tema (c.f. Oman, 2013; Skrzypinska, 2014). Contudo, reforçando os problemas expostos por Hill $(2005,2015)$ ao invés de superá-los. É o estudo de Handal e colaboradores (2017) que se torna a derradeira tentativa de superar o problema conceptual e métrico. Socorreu-se de uma amostra de 212 participantes, maioritariamente crentes religiosos (92\%), avaliados por três medidas de espiritualidade teísta e duas medidas de religiosidade, cujos indicadores seriam correlacionados com uma identificação auto-referente de "espiritual e religioso", "só religioso", ou "só espiritual". Concluiu-se que não há uma diferenciação entre religiosidade e espiritualidade, ainda que os investigadores considerem que o modelo de espiritualidade não-teísta seja mais consistente, sugerindo que possa haver limitações metodológicas que o seu estudo não conseguiu suprimir (Handal et al., 2017). Este estudo em muitos aspectos seria uma replicação de um outro de Zinnbauer e colaboradores (1997), realizado 20 anos antes e com resultados similares. Será, portanto, uma questão de metodologia que previne o desenvencilhar conceptual e, consequentemente, o métrico?

\section{MÉTODO}

A presente investigação visa o desenvolvimento e a análise das propriedades psicométricas de duas escalas - a Escala do Sentido Religioso e a Escala de Consciência Espiritual - em vista a averiguar a possibilidade de uma separação conceptual entre os constructos de espiritualidade e religiosidade, socorrendo-se de uma amostra heterogénea quanto a atitudes face à religião (i.e. crente praticante, crente não-praticante, céptico e ateu). Este é um aspeto relevante, pois assume-se nesta investigação que uma amostra maioritariamente de crentes praticantes poderá induzir um viés na expressão de resultados tal como terá acontecido em estudos anteriores (e.g. Handal et. al, 2017; Zinnbauer et al., 1997). A construção destas escalas passa por uma operacionalização dos constructos a serem mensurados:

1) Religiosidade - movimento psicológico de natureza teleológica sobre um propósito ético-moral e escatológico da condição humana;

2) Espiritualidade - qualidade da consciência manifesta num sentimento de progressiva humanidade.

Recolheu-se uma amostra de 279 participantes com idades entre 17 e 69 anos $(M=24.42$, $S D=9.463$ ), com colaboração de docentes da Faculdade de Letras da Universidade do Porto e do movimento cívico "Braga Para Todos". Recorreu-se a uma amostragem por conveniência, nomeadamente pelo método bola de neve (Faugier \& Sargeant, 1997), com vista a chegar a grupos de difícil acesso como os ateus e os cépticos, tendo por base os Censos (2011) que revelam que a maioria da população portuguesa professa a crença na Igreja Católica Romana. Foram inicialmente contatados uma diretora de curso de licenciatura na área de sociologia, alguns docentes e a presidente do movimento cívico com vista a auscultar a pertinência da divulgação da presente investigação. A recolha dos dados foi realizada presencialmente pelo investigador responsável, com questionários em formato de papel, após obtido consentimento para divulgação do estudo entre potenciais participantes. Os participantes foram, durante o intervalo das suas atividades académicas/cívicas, introduzidos aos objetivos e âmbito da investigação, sendo garantido o anonimato das respostas, a natureza confidencial da informação prestada e o direito a suspender a participação. Após a obtenção do consentimento informado de cada um dos participantes, 
estes foram convidados a preencher, anonimamente e em grupo, num espaço próprio para o efeito, um questionário com as escalas em aferição e a versão portuguesa do Spiritual Well-being Questionnaire. A recolha de dados decorreu entre Setembro de 2017 e Outubro de 2018. Os critérios de inclusão pressupõem o domínio da língua portuguesa e idade igual ou superior a 17 anos, i.e., na adultez, uma vez que na adolescência e em idades mais precoces a fé ou as crenças teístas assumem um papel mais mítico e convencional do que autorreflexivo (Fowler, 1995). 0 tempo médio de resposta variou entre 10 a 15 minutos. Os dados foram tratados com recurso ao SPSS 25.

\section{Instrumentos}

\section{Processo de Construção da Escala do Sentido Religioso (ESR). A ESR é uma escala do tipo Likert} construída com vista a avaliar a dimensão da religiosidade. Tivemos em linha de conta a literatura sobre psicologia da religiosidade que respeita: a) a separação conceptual entre religiosidade e espiritualidade (Saucier \& Skrzypinska, 2006); e b) a existência de religiosos seculares/ateus, para além dos adeptos das religiões reveladas ou místicas (Pasquale, 2007; Streib \& Hood, 2013; Streib \& Klein, 2013). A respeito dos religiosos seculares, constata-se que também os ateus podem deter crenças sobre o fim último ou o propósito da humanidade que não tem de passar pela admissão da existência do divino ou do sobrenatural (Streib \& Hood, 2013), podendo participar ou agir simbolicamente em ritos tidos como seculares tais como os preconizados pela Igreja Positivista de Auguste Comte (Comte, 2009). Verificou-se que o desenvolvimento psicométrico internacional (Kapuscinski \& Masters, 2010) e nacional (Da Silva, Pereira, Monteiro \& Bartolo, 2019) carece de medidas que atendam aos dois pontos acima mencionados, validando a actualidade do desenvolvimento de uma nova medida de religiosidade. Assim, da literatura encontrada, se decidiu excluir todos os pressupostos ligados a conjecturas para-religiosas, possíveis de encontrar em escalas já existentes (Hill, 2005, 2015; Hill \& Hood, 1999) e que não são concordantes com a nossa definição de constructo, nomeadamente: espiritualidade; orientação teísta; crenças sobrenaturais; satisfação congregacional. Para operacionalização da religiosidade foram tidos oito aspectos que nos pareceram mais apropriados após a revisão da literatura:

1) Crença no Princípio Inteligente ou no Divino - a crença em algo subjacente à prática religiosa é algo tido em consideração na constituição das diversas escalas que aferem uma religiosidade (Hill, 2005, 2015), porém aparece como vinculada à noção de divino (Argyle \& Beit-Hallahmi, 1975; Dollahite, 1998; Fowler, 1995; James, 1919, 2008) que aqui rejeitamos a favor de uma transversalidade a posturas seculares que se enquadram na nossa definição do constructo;

2) Sagrado - livre da conotação das religiões reveladas ou místicas, assume, respeitando a sua raiz etimológica, o sentimento de uma ligação pessoal entre o princípio inteligente ou divino e o próprio sujeito, como parte da formação de um sentido religioso (James, 2008; Koenig, 2009; Zinnbauer \& Pargament, 2005; Pargament, 2013);

3) Fé - de etimologia latina assente no verbo confiar, é tida como um aspecto central na vida do sujeito religioso e diz respeito à confiança que se outorga ao princípio inteligente/divino na mitigação de anseios e necessidades pessoais (Fowler, 1995; James, 1919; Stein; 2011; Reich; 2003). Com cunho teísta e próximo da noção 'acreditar' aparece como dimensão de escalas aferidas em contexto português nomeadamente, a Escala de Avaliação Espiritual e a FACIT-sp - que assumem uma mútua transversalidade entre religiosidade e espiritualidade (Da Silva, Perieira, Monteiro \& Bartolo, 2019);,

4) Fidelidade - a adoção de uma dada religião ou prática religiosa deve ressoar no íntimo do praticante, despoletando o compromisso pessoal para com a prática e os dogmas adotados (James, 1919, 2008);

5) Convicção Religiosa - os preceitos dos dogmas religiosos adotados são entendidos pelo praticante como linhas orientadoras de vida ou conduta pessoal (James, 1919, 2008).

6) Obediência Moral - o praticante adere e obedece ao conjunto de normas expressas pelos dogmas religiosos adotados, essenciais na formação de uma identidade religiosa em particular (James 1919, 2008);

7) Ritualismo - a participação nos ritos tais como instituídos pela religião é um aspeto central na caracterização do religioso, sendo parte integrante da vida religiosa (Argyle \& Beit-Hallahmi, 1975; Comte, 2009; Dollahite, 1998; James, 1919, 2008; Peteet, 1994). Algumas escalas avaliam este aspeto como incluso em medidas multidimensionais que agregam a religiosidade à espiritualidade (Da Silva, Pereira, Monteiro \& Bartolo, 2019; Hill, 2015), sendo a maior referência neste âmbito a Religious Background and Behavior Scale de Connors, Tonigan e Miler (1996) que apresenta algum entrosamento com temas cristãos e com a noção de espiritualidade;

8) Oração - numa aceção ampla e transversal, remete para a comunicação intuitiva, pessoal e autoverbal com o princípio inteligente/divino (James, 2008; Ladd \& Spilka, 2013; Reich, 2003; Zinnbauer \& Pargament, 2005), aparecendo como item teísta em escalas como a versão portuguesa da Spiritual Well- 
being Questionnaire (Gouveia, Marques \& Pais-Ribeiro, 2009; Gouveia, Pais-Ribeiro \& Marques, 2012), em específico o domínio transcendental.

Cada aspeto refere-se a um item em particular cuja formulação atendeu ao sentido etimológico dos conceitos e evitou a 'religificação' de noções não diretamente relacionadas com um sentido religioso. Todos os itens foram adaptados em conformidade com a nossa definição do constructo. Os aspetos 4), 5) e 6) assumem a particularidade de derivarem da desconstrução do enunciado do Religious Commitment Inventory (RCI-10; Worthington et al., 2003) revisto à luz das obras de James $(1919,2008)$. Os itens 5), 4) e 9) do RCI-10 correspondem respetivamente aos itens 4), 5) e 6) do ESR tendo sido adaptados em função da etimologia destes aspetos que não são propriamente identificados ou definidos no RCI-10.

Cada item é de resposta ordinal numa escala que vai de 1 (Discordo) a 5 (Concordo Plenamente).

Processo de Construção da Escala de Consciência Espiritual (ECE). A construção de uma escala que visa avaliar a dimensão da espiritualidade atendeu aos modelos que preconizam uma espiritualidade nãoteísta em psicologia (e.g. Assagioli, 1981; Bucke, 1991; Saucier \& Skrzypinska, 2006). Conceptualizar, em geral, a espiritualidade tem sido uma tarefa pouco consensual (c.f. Oman, 2013; Skrzypinska, 2014), e no que toca à operacionalização da espiritualidade não-teísta, existem propostas psicométricas que não encontram concordância. Duas propostas em psicometria podem encontrar-se nos modelos não-teístas: a) espiritualidade como assente em descritores de bem-estar (Daaleman \& Peyton, 2005); e b) espiritualidade baseada em crenças na vida após a morte e em estados modificados de consciência ou ditos transcendentes (Piedmont, 1999). A espiritualidade como associada ao bem-estar e a variáveis de saúde tem sido alvo de crítica por parte de alguns defensores do modelo teísta, que advogam que uma espiritualidade assim formulada compromete a sua utilização em estudos para com emoções positivas e qualidade de vida por um efeito tautológico (Garssen, Visser, Jager Meezenbroek, 2016; Koenig, 2008; Migdal \& MacDonald, 2013). Já no que respeita à espiritualidade baseada em crenças no além, verifica-se que também os ateus podem ser espirituais (Comte-Sponville, 2008), o que imputa alguma reserva numa espiritualidade assim proposta. A possibilidade da existência de indivíduos "espirituais e não religiosos" (Palmisano, 2010; Saucier \& Skrzypinska, 2006) levou à consideração de que a espiritualidade é um fenómeno subjetivo e individual, com vista a um fim imanente (Skrzypinska, 2014), ou assente no carácter humano, na vivência da própria humanidade (Assagioli, 1981; Bucke, 1991; Hay \& Socha, 2005). 0 intrinsecamente humano será, por seu lado, o produto da evolução da consciência humana em função de uma progressiva humanidade (Hampton, 2010; Miner, Dowson \& Devenish, 2012). 0 desenvolvimento humano, por meio da consciência, tem uma ancestralidade milenar e remete-nos para técnicas hodiernas como o mindfulness, que se desenvolveu a partir dos estudos sobre práticas de base espiritual como a meditação (Assagioli, 1981; Carrington, 1987; Graf Durckheim, 2007; Kabat-Zinn, 2012). A correlação entre mindfulness ou estado mindful e espiritualidade é significativa, podendo encontrar-se neste estado um possível correlato de espiritualidade (Carmody et al., 2008; Cohen, Gruber \& Keltner, 2010; Da Silva \& Pereira, 2017; Greeson et al., 2011). As diversas escalas disponíveis para avaliação da espiritualidade são, por sua vez, lacunares sobre quanto a uma proposta não-teísta (Kapuscinski \& Masters, 2010), e a comparação das suas estruturas fatoriais revela discrepâncias etimológicas e conceptuais que sugerem a possibilidade de a variável latente avaliada não ser a mesma para cada uma das escalas (Da Silva, Pereira, Monteiro \& Bartolo, 2019). Por isso, a análise combinada do constructo teórico e dos itens das dimensões de diferentes escalas ao dispor exigiu o tratamento criterioso e exclusão de todas as noções de paraespiritualidade que possam criar ruído no entendimento de espiritualidade não-teísta: religião; divino; crenças sobrenaturais; bem-estar; e coping A análise desta literatura resultou em 10 aspetos tidos como consensuais:

1) Transcendência - aspeto considerado em escalas de espiritualidade (Piedmont, 1999; Pinto \& Pais-Ribeiro, 2007) é aqui entendido como o sentimento de superação dos próprios limites pessoais ou a sensação de abertura das faculdades latentes do próprio sujeito, habitualmente atribuída à vivência espiritual (Assagioli, 1981; Bucke, 1991; Jung, 1999; Maslow, 1994);

2) Esperança - a esperança é tida como um sentimento positivo perante o futuro que se associa à espiritualidade (Peterson \& Seligman, 2004; Rovers \& Kocum, 2010) e estudos verificaram a existência de uma correlação significativa entre os dois constructos (Da Silva \& Pereira, 2017; Rovers \& Kocum, 2010). Este aspecto encontra-se também presente na Escala de Avaliação de Espiritualidade de Pinto e PaisRibeiro (2007);

3) Capacidade para Amar - o amor altruísta é considerado como um aspecto da vivência espiritual (Comte-Sponville, 2008; Rovers \& Kocum, 2010) podendo assumir uma configuração central no aparecimento de éticas seculares e místicas (Bucke, 1991; Laranjeira, 1907);

4) Contentamento - o contentamento com as pequenas coisas da vida, por oposição à ambição pessoal, é usualmente, tido como afirmação da autossuficiência do sujeito espiritual (Cohen, Gruber \& 
Keltner, 2010; Graf Durckheim, 2007), verificando-se que este aspeto se associa positivamente com a espiritualidade (Da Silva \& Pereira, 2017);

5) Contacto - sentimento de congruência e abertura entre o sujeito, os outros e o mundo, sendo considerado um aspecto da espiritualidade (Bucke, 1991; Cohen, Gruber \& Keltner, 2010). Estudos constataram a associação positiva entre o sentido de contacto e a espiritualidade (Da Silva \& Pereira, 2017; Greeson et al., 2011);

6) Insight - compreensão e entendimento que serve de base ao amor altruísta e contentamento pessoal, é considerado como parte da postura do sujeito espiritual (Assagioli, 1981; Bucke, 1991; Graf Durckheim, 2007), e num estudo realizado por Da Silva e Pereira (2017) verifica-se uma correlação positiva entre o insight de doentes psiquiátricos e a sua espiritualidade;

7) Harmonia - a harmonia entre pensamentos e emoções tem sido considerada como um dos objetivos de práticas espirituais milenares, como a meditação (Assagioli, 1981; Graf Durckheim, 2007), verificando-se a sua associação positiva com a espiritualidade (Da Silva \& Pereira, 2017);

8) Temperança - o controlo dos impulsos é tido como um aspeto da espiritualidade, na medida que se relaciona com a compreensão e o entendimento que conduz à moderação e correção do próprio comportamento (Bucke, 1991; Leuba, 1925);

9) Sublime - o sentimento de autodescoberta nas experiências de vida diária, numa expressão de awe ou contemplação pessoal acerca das próprias qualidades e defeitos, é considerado como um atributo espiritual (Cohen, Gruber \& Keltner, 2010), aparecendo no enunciado de questões do Spiritual Well-being Questionnaire (SWBQ; Gomez \& Fisher, 2003);

10) Sentido Existencial - a atribuição de sentido pessoal é usualmente tida como uma componente da espiritualidade (Frankl, 2006; Gorsuch, 2002; Maslow, 1994; Zinnbauer \& Pargament, 2005), na medida em que o sujeito espiritual é capaz de estabelecer linhas orientadoras da própria vida nas quais se reflete uma ética pessoal (Bucke, 1991). Algumas escalas de espiritualidade arrogam a atribuição de sentido na sua estrutura fatorial (e.g. Jorge, Esgalhado \& Pereira, 2016; Pereira \& Santos, 2011; Gouveia, Marques \& Pais-Ribeiro, 2009).

A ECE é uma escala de tipo Likert cujos itens são de resposta ordinal numa escala que vai de 1 (Discordo) a 5 (Plenamente de Acordo).

Spiritual Well-being Questionnaire (SWBQ). A SWBQ é uma escala que se encontra aferida para a população portuguesa com o nome de Questionário de Bem-estar Espiritual (Gouveia, Marques \& PaisRibeiro, 2009; Gouveia, Pais-Ribeiro \& Marques, 2012). É um questionário do tipo Likert composto por 20 itens, detendo uma ótima consistência interna $(\alpha=.89)$ e possuindo quatro subescalas: Transcendental $(\alpha=.89)$; Ambiental $(\alpha=.84)$; Pessoal $(\alpha=.75)$; e Comunitária $(\alpha=.74)$.

Ainda que a sua estrutura factorial pareça pouco adequada nos estudos de aferição, a escolha desta escala cinge-se ao facto de que possui poucos elementos teístas identificáveis que só são passíveis de serem mensurados pela sub-escala Transcendental, sendo os cinco itens que compõem esta escala os seguintes:1) Relação pessoal com o Divino; 2) Admiração e respeito pela Criação; 3) Sentimento de união com Deus; 4) Sentimento de paz com Deus; e 5) Vida de meditação/oração. Um outro domínio organizado numa concepção similar de espiritualidade com que lidamos é o Pessoal, que possui igualmente cinco itens expressos da seguinte maneira: 1) Sentimento de identidade pessoal; 2) Autoconhecimento; 3) Alegria na vida; 4) Paz interior; e 5) Sentido para a vida. Assim, a sub-escala Transcendental servirá para fazer a validação convergente com a ESR, e a sub-escala Pessoal servirá o mesmo propósito para com a ECE.

\section{RESULTADOS}

Os dados sócio-demográficos revelam que $20.1 \%$ dos participantes $(\mathrm{N}=56)$ é crente praticante, $27.6 \%$ é crente não-praticante $(\mathrm{N}=77), 22.2 \%$ são ateus $(\mathrm{N}=62)$, e $30.1 \%$ são cépticos $(\mathrm{N}=84)$. Quanto à escolaridade $3.2 \%$ são doutorados $(\mathrm{N}=9), 10.8 \%$ são mestres $(\mathrm{N}=30), 30.8 \%$ têm uma licenciatura concluída ( $\mathrm{N}=86), 52.3 \%$ têm o ensino secundário concluído $(\mathrm{N}=146), 2.2 \%$ têm o ensino básico $(\mathrm{N}=6)$ e $0.4 \%$ tem o ensino primário $(\mathrm{N}=1)$. Em relação ao estado civil verifica-se que $85.3 \%$ são solteiros $(\mathrm{N}=238), 6.5 \%$ são casados $(\mathrm{N}=18), 2.5 \%$ são divorciados $(\mathrm{N}=7), 3.9 \%$ estão em união de facto $(\mathrm{N}=11)$, $1.4 \%$ estão separados ( $\mathrm{N}=4)$, havendo apenas uma resposta omissa $(0.4 \%)$.

A respeito da ESR efectuou-se uma análise factorial exploratória com recurso à análise de componentes principais. 0 teste de Barttlet foi significativo, indicando que o modelo de análise factorial para estes dados é apropriado, $X^{2}=2072.56 ; g l=3 ; p \leq .001$. 0 teste de Kaiser-Meyer-Olkin verificou a adequação da correlação entre variáveis $(\mathrm{KMO}=.91)$, permitindo a continuação do tratamento estatístico. Desta análise extraiu-se um único fator que explica $71.62 \%$ da variância. 0 cálculo do alfa de Cronbach revelou uma excelente fiabilidade da escala $(\alpha=.94)$. A análise da validade convergente dada por uma 
correlação de Spearman revelou, por seu lado, uma forte associação positiva e estatisticamente significativa entre o índice geral da ESR e o Domínio Transcendental da SWBQ, $r=.869 ; p \leq .001$. Dado o elevado valor de correlação, realizou-se uma regressão linear simples com vista a determinar a capacidade preditiva da escala em aferição. Verificou-se que o índice da ESR prevê o índice do Domínio Transcendental, $F(1,277)=977.28, p \leq .01$, com um $R^{2}$ de .78 e $\beta=.88$, verificando-se que o índice do Domínio Transcendental previsto é igual a .323 + 1.010 quando o mesmo índice é medido em função das pontuações da ESR. Já no sentido inverso, a regressão linear simples sugeriu que o índice da ESR previsto é de .21 + .77 quando a religiosidade da ESR é medida em função das pontuações do Domínio Transcendental da SWBQ.

No que concerne a ECE, foi empregue a análise fatorial exploratória com método de componentes principais. A significância do teste de Barttlet, $X^{2}=571.961 ; g l=45 ; p \leq .001$, e a adequabilidade do teste de Kaiser-Meyer-Olkin (KMO=.771) permitiu continuar com tratamento dos dados, de onde se extraíram três componentes, em que a primeira explica $32.80 \%$ da variância, a segunda $12.23 \%$ e a terceira $11.37 \%$, para uma fiabilidade aceitável da escala ( $\alpha=.75)$ (ver Tabela 1 e Figura 1$).$

Tabela 1. Matriz Padrão ECE* e Pesos Factoriais

\begin{tabular}{lcccc}
\hline Componentes & $\mathbf{1}$ & $\mathbf{2}$ & $\mathbf{3}$ & Comunalidades \\
\hline Contentamento &. $\mathbf{7 2 5}$ & -.189 & .057 & .520 \\
Capacidade para Amar &. $\mathbf{6 8 1}$ & .260 & .207 & .499 \\
Esperança & .637 & .168 & -.105 & .505 \\
Sentido Existencial & .630 & -.157 & -.150 & .491 \\
Contacto & .590 & -.031 & -.338 & .592 \\
Transcendência & -.052 & .884 & .028 & .777 \\
Sublime & .159 & .431 & -.370 & .411 \\
Temperança & -.057 & -.064 & -.782 & .585 \\
Insight & -.039 & 217 & -.649 & .605 \\
Harmonia & .303 & -.223 & -.639 & .654 \\
\hline Eigenvalues & 3.280 & 1.223 & 1.137 & \\
\hline
\end{tabular}

*Método de Extracção: Análise de Componentes Principais; Método de Rotação Oblimin com Normalização de Kaiser

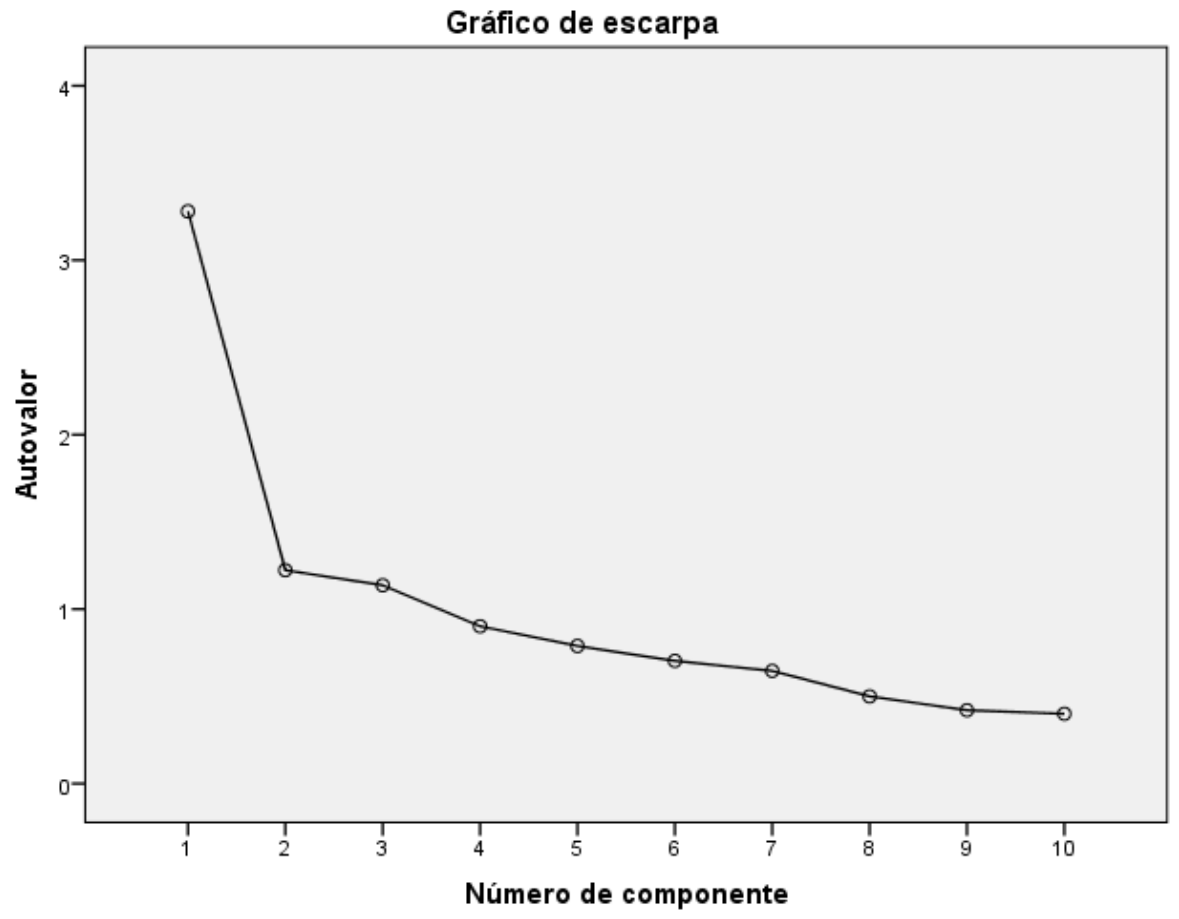

Figura 1. Gráfico de Escarpa. 
As subescalas assumiram, porém, um desempenho factorial pouco adequado, verificando-se que a componente 1 é razoável ( $\alpha=.712)$, a componente 2 não é aceitável $(\alpha=.285)$ e a componente 3 é fraca $(\alpha=.657)$. Constatou-se, a este respeito, que a eliminação do item Transcendência aumentaria o alfa de Chronbach da escala para .78, sendo que a supressão de qualquer um dos outros itens da escala resultaria num alfa inferior ao já encontrado $(\alpha=.75)$. A validade convergente, dada por uma correlação de Spearman, evidenciou uma associação positiva e estatisticamente significativa no limiar do forte entre o índice geral da ECE e o Domínio Pessoal da SWBQ ( $r=.69 ; p \leq .001)$. 0 recurso a uma regressão linear simples revelou que o índice geral da ECE prevê o Domínio Pessoal, $F(1,277)=295.85, p \leq .01$, para um $R^{2}$ de .39 e $\beta$ de .63, em que o índice do Domínio Pessoal previsto é de $.864+720$. Similarmente, procedeu-se à análise inversa procurando determinar se o Domínio Pessoal prevê a espiritualidade tal como ela é dada pela ECE, verificando-se que $\operatorname{sim}, F(1,277)=295.85, R^{2}=.52, p \leq .01$, sendo que o índice de espiritualidade da ECE previsto é de $1.69+54$.

Para verificarmos o grau de associação das variáveis espiritualidade e religiosidade, procedemos a uma nova correlação de Spearman entre os índices da ESR e da ECE. Verificou-se uma correlação positiva e fraca entre o índice da ESR e o índice geral da ECE, $r=.24 ; p \leq .01$.

Pretendemos, também, saber se existem diferenças nas pontuações médias das duas escalas em função de se ser crente praticante, crente não praticante, ateu e cético. Efetuou-se o teste de Shapiro-Wilk para verificar se as pontuações médias da ESR seguem uma distribuição normal. Verificou-se um desvio significativo face à normalidade, $W(279)=.89, p<.001$. Realizou-se, assim, um teste de Kruskal-Wallis para averiguar se existem diferenças significativas entre as pontuações médias da ESR quanto à postura/crenças teístas dos participantes, tendo-se verificado que a religiosidade dada pela ESR varia consideravelmente entre estas posturas, $H(3)=194.99, p<.001$ (ver Tabela 2). Outra análise de diferenças com recurso ao teste de Kruskal-Wallis revelou que o efeito da escolaridade sobre as pontuações médias da ESR não é estatisticamente significativo, $H(2)=.63, p=.73$. No que concerne ao sexo, um teste de MannWhitney permitiu concluir que também não é significativo o efeito do sexo nas pontuações da ESR, $U=7478.00, p=.185$. Em relação à espiritualidade o teste de Shapiro-Wilk revelou que as pontuações médias da ECE seguem uma distribuição normal, $W(279)=.99, p=.336$. Com recurso a uma ANOVA oneway, apurou-se que o efeito das posturas teístas sobre as pontuações médias da ECE não é estatisticamente significativo, $F(3,275)=2.47, p=.062$ (ver Tabela 3).

Tabela 2. Diferenças Médias de Religiosidade por Posturas Teístas

\begin{tabular}{lccccc}
\hline & $\boldsymbol{N}$ & Média & Desvio Padrão & Mínimo & Máximo \\
\hline Crente Praticante & 56 & 3.47 & .84 & 1.63 & 5.00 \\
Crente Não Praticante & 77 & 2.37 & .68 & 1.25 & 4.63 \\
Ateu & 62 & 1.12 & .26 & 1.00 & 2.38 \\
Céptico & 84 & 1.56 & .53 & 1.00 & 3.13 \\
Total & 279 & 2.07 & 1,03 & 1.00 & 5.00 \\
\hline
\end{tabular}

Tabela 3. Diferenças Médias de Espiritualidade por Posturas Teístas

\begin{tabular}{lccccc}
\hline & $\boldsymbol{N}$ & Média & Desvio Padrão & Mínimo & Máximo \\
\hline Crente Praticante & 56 & 3.65 & .50 & 2.50 & 4.80 \\
Crente Não Praticante & 77 & 3.55 & .47 & 2.20 & 4.70 \\
Ateu & 62 & 3.39 & .63 & 2.10 & 5.00 \\
Céptico & 84 & 3.56 & .56 & 1.80 & 5.00 \\
Total & 279 & 3.54 & .55 & 1.80 & 5.00 \\
\hline
\end{tabular}

Uma nova ANOVA one-way evidenciou que o efeito do nível educacional sobre as pontuações médias da ECE não é estatisticamente significativo, $F(2,259)=1.41, p=.25$. 0 uso de um teste $t$ para amostras independentes, revelou, também, que as pontuações médias da ECE não diferem quanto ao sexo masculino $(M=3.50)$ e ao sexo feminino $(M=3.55)$ dos participantes, $t(277)=-.60, p=.549$.

Pretendemos igualmente saber se existe alguma ligação entre o sexo e as atitudes teístas dos participantes. 0 teste de Qui-Quadrado de Pearson revelou que a relação entre estas duas variáveis é significativa, $X^{2}(3)=10.33, p<.05$. 0 sexo feminino é mais propenso a ser crente ou céptico do que o masculino, enquanto este último tende mais para o ateísmo do que o primeiro (ver Tabela 4). 
Tabela 4. Atitudes Teístas por Sexo

\begin{tabular}{lcccc}
\hline & Crente Praticante & Crente Não Praticante & Ateu & Céptico \\
\hline Masculino & $15.1 \%$ & $22.1 \%$ & $33.7 \%$ & $29.1 \%$ \\
Feminino & $22.3 \%$ & $30.1 \%$ & $17.1 \%$ & $30.6 \%$ \\
\hline
\end{tabular}

\section{DISCUSSÃo}

Este trabalho teve como objectivo o desenvolvimento e o estudo preliminar das propriedades psicométricas da Escala de Consciência Espiritual (ECE) e da Escala do Sentido Religioso (ESR).

Os dados permitem constatar que a ESR é uma escala promissora: possui uma excelente fiabilidade e mede um sentido religioso com uma força de previsão do índice do Domínio Transcendental do SWBQ, que não se obtém inversamente. A respeito da ECE, pode concluir-se que esta escala apresenta uma parca adequação psicométrica. A fiabilidade geral é, apenas, aceitável e a sua estrutura factorial é pouco adequada. Dos três componentes extraídos, somente um, constituído pelos aspectos Transcendência e Sublime, não se mostrou aceitável. Verificou-se a este propósito que a eliminação do item Transcendência aumenta a fiabilidade geral da escala. São dois os factores que constituem a escala: factor 1, consciência dos outros $(\alpha=.712)$; e factor 2 , consciência de si $(\alpha=.657)$. Uma possível limitação que se pode outorgar à construção de uma medida de espiritualidade, e que nos permite entender estes resultados, é o facto de que medidas subjectivas, assentes em aspectos idiossincráticos dos participantes, não são estáveis e poderão reduzir a própria fiabilidade da escala que compõem (Hill \& Hill, 2016).

Verificou-se também que, do ponto de vista métrico, espiritualidade e religiosidade não se sobrepõem enquanto constructos. A correlação entre os índices da ESR e da ECE é fraca, o que põe em causa a similitude conceptual entre espiritualidade e religiosidade, tal como preconizada por alguns autores (Koening, 2008; Oman, 2013). A corroborar a independência métrica entre os conceitos, verificase que os índices da ESR e da ECE assumem uma variabilidade discrepante em função da postura teísta dos participantes, sendo que os valores de espiritualidade não acompanham as alterações nos índices de religiosidade em crentes praticantes, crentes não praticantes, ateus e cépticos. Portanto, pode inferir-se que uma convergência entre constructos só poderá ser possível em crentes praticantes e que o uso de amostras maioritariamente constituídas por praticantes de uma religião induz erroneamente a uma sobreposição conceptual; erro cometido por autores passados (Handal et al., 2017; Zinnbauer et al., 1997).

A postura teísta, por sua vez, esteve associada ao sexo dos participantes, constatando-se uma maior predisposição do sexo feminino para crença teísta e do sexo masculino para o ateísmo.

Estes resultados são, contudo, preliminares e a sua extrapolação condicionada às limitações deste estudo. Em primeiro, lugar a ausência de uma análise factorial confirmatória, o que poderia corroborar a necessidade de reformulação do conteúdo das escalas. Nesta fase preliminar, apenas procuramos entender a dimensionalidade das escalas numa perspectiva exploratória, sendo que numa fase posterior, com uma nova amostragem, se testará o ajuste dos modelos encontrados por meio de uma análise factorial confirmatória. Em segundo lugar, a questão da representatividade da presente amostra. A amostra é constituída maioritariamente por jovens adultos e solteiros. Esta é, assim, uma amostra restritiva e que decorre das limitações do uso da técnica de amostragem por bola de neve (Faugier \& Sargeant, 1997): novos participantes são referenciados por indivíduos com os quais partilham um conjunto de características pessoais.

Seja como for, os dados sugerem uma possível via de investigação como alternativa aos estudos que incidem no modelo teísta de espiritualidade, conduzidos em amostras constituídas por uma maioria de religiosos (Kapuscinski \& Masters, 2010). Como principais recomendações para estudos posteriores, recomendamos:

a) Atender à possibilidade da existência de indivíduos "espirituais e religiosos", como "espirituais e não religiosos" e "religiosos e não espirituais" (Palmisano, 2010) na conceção das noções de espiritualidade e religiosidade;

b) Considerar a provável espiritualidade de ateus;

c) Adotar metodologias que atendam à postura teísta/credo pessoal dos participantes como potencial variável parasita;

d) 0 uso criterioso de modelos de espiritualidade teísta em psicologia, em especial, dos trabalhos de Harold Koenig (e.g. Koenig, 2008, 2010), Kenneth Pargament e Brian Zinnbauer (e.g. Pargament, 2013; Zinnbauer \& Pargament, 2005);

e) A realização de estudos comparativos entre espiritualidade e religiosidade. 


\section{REFERÊNCIAS}

Abdel-Khalek, A. M., \& Naceur, F. (2007). Religiosity and its association with positive and negative emotions among college students from Algeria. Mental Health, Religion, and Culture, 10, 159-170. https://doi.org/10.1080/13694670500497197

Argyle, M., \& Beit-Hallahmi, B. (1975). The social psychology of religion. Routledge

Assagioli, R. (1981). Psychosynthesis - A Collection of Basic Writings. Penguin Books Ltd.

Bucke, R. M. (1991). Cosmic Consciousness: A Study in the Evolution of the Human Mind. Penguin Group

Carmody, J., Reed, G., Kristeller, J., \& Merriam, P. (2008). Mindfulness, spirituality, and health-related symptoms. Journal of Psychosomatic Research, 64(4), 393-403. https: // doi.org / 10.1016 / j.jpsychores.2007.06.015

Carrington, P. (1987). Freedom in meditation (3rd ed.). New Jersey: Pace Educational Systems.

Censos (2011). XV Recenseamento Geral da Populacão/V Recenseamento Geral da Habitacão: Resultados Definitivos Portugal. http: // censos.ine.pt / xportal / xmain?xpid=CENSOSandxpgid=ine_censos_publicacao_detandcontexto=puandPUBLICACOESpub_b oui $=73212469$ andPUBLICACOESmodo=2andselTab=tab1andpcensos $=61969554$.

Cohen, A. B., Gruber, J., \& Keltner, D. (2010). Comparing Spiritual Transformations and Experiences of Profound Beauty. Psychology of Religion and Spirituality, 2(3), 127-135. https://doi.org/10.1037/a001912

Comte, A. (2009). The Catechism of Positive Religion - Or Summary Exposition of the Universal Religion in Thirteen Systematic Conversations Between a Woman and a Priest of Humanity. Cambridge University Press

Comte-Sponville, A. (2008). L’Espirit de L'Athéisme: Introduction à Une Spiritualité Sans Dieu. Le Livre de Poche

Connors, G. J., Tonigan, J. S., \& Miler, W. R. (1996). A measure of religious background and behavior for use in behavior change research. Psychology of Addictive Behaviors, 10, 90-96

Daaleman, T. P., \& Frey, B. B. (2004). The Spirituality Index of Well-Being: A New Instrument for HealthRelated Quality-of-Life Research. Annals of Family Medicine, 2(5), 499-503. https://doi.org/10.1370\%2Fafm.89

Da Silva, J. P., \& Pereira, A. M. S. (2017). Perceived Spirituality, Mindfulnes and Quality of Life in Psychiatric Patients. Journal of Religion and Health, 56(1), 130-140. https://doi.org/10.1007/s10943-016-0186-y

Da Silva, J. P., Pereira, A. M. S., Monteiro, S. O. M., \& Bártolo, A. Qualidade Psicométrica de Escalas de Espiritualidade Aferidas para a População Portuguesa: Uma Revisão de Literatura. Psicologia, Saúde \& Doenças, 20(3), 726-739. https://doi.org/10.15309/19psd200314

Dollahite, D. C. (1998). Fathering, faith, and spirituality. Journal of Men's Studies, 7, 3-15. https://doi.org/10.3149/jms.0701.3

Emmons, R. A., \& Crumpler, C. A. (1999). Religion and Spirituality: The Roles of Sanctification and the Concept of God. The International Journal for the Psychology of Religion, 9(1), 17-24

Faugier, J. and Sargeant, M. (1997). Sampling Hard to Reach Populations. Journal of Advanced Nursing, 26, 790-797. https://doi.org/10.1046/j.1365-2648.1997.00371.x

Fowler, J. W. (1995). Stages of Faith: The Psychology of Human Development and the Quest of Meaning. HaperCollins Publishers

Garssen, B., Visser, A., \& de Jager Meezenbroek, E. (2016). Examining whether spirituality predicts subjective well-being: How to avoid tautology. Psychology of Religion and Spirituality, 8(2), 141148. https://doi.org/10.1037/rel0000025

Gomez, R., \& Fisher, J. W. (2003). Domains of spiritual well-being and development and validation of the Spiritual Well-Being Questionnaire. Personality and Individual Differences, 35, 1975-1991 https://doi.org/10.1016/S0191-8869(03)00045-X

Gouveia, M. J., Marques, M., \& Pais-Ribeiro, J. L. (2009). Versão Portuguesa do Questionário de Bem-Estar Espiritual (SWBQ): Análise Confirmatória da Sua Estrutura Factorial. Psicologia, Saúde \& Doenças, 10(2), 285-293

Gouveia, M. J., Pais-Ribeiro, J. L., \& Marques, M. (2012). Estudo da Invariância Factorial do Questionário de Bem-estar Espiritual (SWBQ) em praticantes de Actividades Físicas de Inspiração Oriental. Psychology, Community \& Health, 1(2), 140-150. https://doi.org/10.5964/pch.v1i2.25

Graf Durkheim, K. (2007). The Way of Transformation: Daily Life as a Spiritual Practice. Morning Light Press

Greeson, J. M., Webber, D. M., Smoski, M. J., Brantley, J. G., Ekblad, A. G., Suarez, E. C., \& Wolever, R. Q. (2011). Changes in spirituality partly explains healthrelated quality of life outcomes after mindfulness-based stress reduction. Journal of Behavioral Medicine, 34, 508-518. 
https://doi.org/10.1007/s10865-011-9332-x

Handal, P. J., Creech, C. A., Schwendeman, M. G., Pashak, T. J., Perez, E. J., \& Caver, L. (2017). Distinguishing Between Self-Classified Religious and Spiritual Groups of Emerging Adults: Conceptual and Psychometric Challenges. Journal of Religion \& Health, 56, 1971-1980. https://doi.org/10.1007/s10943-016-0304-x

Hampton, S. (2010). Essential Evolutionary Psychology. Sage Publications, Ltd.

Hay, D., \& Socha, P. M. (2005). Spiritualty as a Natural Phenomenon: Bringing Biological and Psychological Perspectives Together. Zygon, 40, 589-612. https://doi.org/https://doi.org/10.1111/j.14679744.2005.00691.x

Helminiak, D. A. (2008). Confounding the Divine and the Spiritual: Challenges to a Psychology of Spirituality. Pastoral Psychology, 57, 161-182

Hill, P. C. (1999). Giving Religion Away: What the Study of Religion Offers Psychology. The International Journal for the Psychology of Religion, 9(4), 229-249

Hill, P. C. (2005). Measurement in the Psychology of Religion and Spirituality: Current Status and Evaluation. In R. F. Paloutzian \& C. L. Park (Eds.), Handbook of the Psychology of Religion and Spirituality (pp. 21-42). Guilford Press.

Hill, P. C. (2015). Measurement, Assessment and Issues in the Psychology of Religion and Spirituality. In R. F. Paloutzian \& C. L. Park (Eds.), Handbook of the Psychology of Religion and Spirituality (2 ${ }^{\text {nd }}$ Ed., pp. 23-47). Guilford Press

Hill, P. C., \& Edwards, E. (2013). Measurement in the Psychology of Religiousness and Spirituality: Existing Measures and New Frontiers. In K. I., Pargament, J. J. Exline \& J. W. Jones (Eds.), APA handbooks in psychology. APA handbook of psychology, religion, and spirituality (Vol. 1): Context, theory, and research. American Psychological Association.

Hill, P.C. and Hood Jr., R.W. (1999) Measures of Religiosity. Religious Education Press

Hill, M. M., \& Hill, A. (2016). Investigação por Questionário (2ª Edição). Edições Sílabo

James, W. (1919). The Will to Believe and Other Essays in Popular Philosophy. Watchmaker Publishing

James, W. (2008). The Varieties of Religious Experience: A Study in Human Nature. Arc Manor

Jorge, D. F. O., Esgalhado, G., \& Pereira, H. (2016). Inteligência espiritual: validação preliminar da versão portuguesa da escala de inteligência espiritual integrada (EIEI). Análise Psicológica, 3(XXXIV), 325337

Jung (1999). Psicologia e Religião. Vozes

Kabat-Zinn, J. (2012). Mindfulness for Beginners. Sounds True, Inc.

Kapuscinski, A. N., \& Masters, K. S. (2010). The current status of measures of spirituality: A critical review of scale development. Psychology of Religion and Spirituality, 2(4), 191-205. https://doi.org/10.1037/a0020498

Koenig (2008). Concerns about measuring "spirituality" in research. Journal of Nervous and Mental Disease, 196, 349-355. https://doi.org/10.1097/NMD.0b013e31816ff796

Koenig, H. G. (2009). Research on Religion, Spirituality, and Mental Health: A Review. The Canadian Journal of Psychiatry, 54(5), 283-291. https://doi.org/10.1177\%2F070674370905400502

Koenig, H. G. (2012). Religion, Spirituality, and Health: The Research and Clinical Implications. International Scholarly Research Network, 2012, 1-33. https://doi.org/10.5402/2012/278730

Ladd, K. L., \& Spilka, B. (2013). Prayer: A Review of the Empirical Literature. In K. I., Pargament, J. J. Exline \& J. W. Jones (Eds.), APA handbooks in psychology. APA handbook of psychology, religion, and spirituality (Vol. 1): Context, theory, and research. American Psychological Association.

Maslow, A. H. (1994). Religions, Values, and Peak Experiences. Penguin Group

Migdal, L., \& MacDonald, D. A. (2013). Clarifying the relation between spirituality and well-being. Journal $\begin{array}{llll}\text { of Nervous and Mental 201(4), } & \text { 274-280 }\end{array}$ https://doi.org/10.1097/NMD.0b013e318288e26a

Miner, M., Dowson, M. \& Devenish, S. (Eds.) (2012). Beyond Well-being: Spirituality and Human Flourishing. Information Age Publishing, Inc.

Oman, D. (2015). Defining Religion and Spirituality. In R. F. Paloutzian \& C. L. Park (Eds.), Handbook of the Psychology of Religion and Spirituality (pp. 23-47). Guilford Press

Palmisano, S. (2010). Spirituality and Catholicism: The Italian Experience. Journal of Contemporary Religion, 25(2), 221-241. https://doi.org/10.1080/13537901003750910

Pargament, K. I. (1999a). The Psychology of Religion and Spirituality? Yes and No. The International Journal for the Psychology of Religion, 9(1), 3-16

Pargament, K. I. (1999b). The Psychology of Religion and Spirituality? Response to Stifoss-Hanssen, Emmons, and Crumpler. The International Journal for the Psychology of Religion, 9(1), 35-43

Pargament, K. I. (2013). Searching for the Sacred: Toward a Nonreductionist Theory of Spirituality. In K. I., 
Pargament, J. J. Exline \& J. W. Jones (Eds.), APA handbooks in psychology. APA handbook of psychology, religion, and spirituality (Vol. 1): Context, theory, and research. American Psychological Association.

Pasquale, F. L. (2007). The "nonreligious" in the American northwest. In B. A. Kosmin \& A. Keysar (Eds.), Secularism and secularity: Contemporary international perspectives (pp. 41-58). Institute for the Study of Secularism in Society and Culture.

Pereira, F., \& Santos, C. (2011). Adaptação cultural da functional assessment of chronic illness therapyspiritual well-being (FACIT-Sp): estudo de avaliação em doentes oncológicos na fase final de vida. Cadernos de Saúde, 4(2), 37-45

Peteet, J. R. (1994). Approaching spiritual problems in psychotherapy: A conceptual framework. Journal of Psychotherapy Practice and Research, 3, 237-245

Peterson, C., \& Seligman, M. E. P. (2004). Character strengths and virtues: A Handbook and Classification. American Psychological Association and Oxford University Press

Piedmont, R. L. (1999). Does Spirituality Represent the Sixth Factor of Personality? Spiritual Transcendence and the Five-Factor Model. Journal of Personality, 67(6), 985-1013. https://doi.org/https://psycnet.apa.org/doi/10.1111/1467-6494.00080

Pinto, C., \& Pais-Ribeiro, J. L. (2007). Construção de uma Escala de Avaliação da Espiritualidade em Contextos de Saúde. Arquivos de Medicina, 21(2), 47-53

Reich, K. H. (2000). What characterizes Spirituality? A comment on Pargament, Emmons and Crumpler, and Stifoss-Hanssen. International Journal for the Psychology of Religion, 10(2), 125-128 https://doi.org/10.1207/S15327582IJPR1002_05

Reich, K. H. (2003). The Person-God Relationship: A Dynamic Model. International. Journal for the Psychology of Religion, 13(4), 229-247. https://doi.org/10.1207/S15327582IJPR1304_1

Rovers, M., \& Kocum, L. (2010). Development of a holistic model of spirituality. Journal of Spirituality in Mental Health, 12, 2-24. https://doi.org/10.1080/19349630903495475

Saucier, G., \& Skrzypinska, K. (2006). Spiritual But Not Religious? Evidence for Two Independent Dispositions. Journal of Personality, 74(5), 1257-1292. https://doi.org/10.1111/j.14676494.2006.00409.x

Skrzypinska, K. (2014). The Threefold Nature of Spirituality (TNS) in a Psychological Cognitive Framework. Archive for the Psychology of Religion, 36, 277-302. https://doi.org/10.1163/15736121-12341293

Stein, M. (2011). Faith and the practising analyst. Journal of Analytical Psychology, 56(3), 397-406. https://doi.org/10.1111/j.1468-5922.2011.01916.x

Stifoss-Hanssen, A. (1999). Religion and Spirituality: What an European Ear Hears. The International Journal for the Psychology of Religion, 91(1), 25-33. https://psycnet.apa.org/doi/10.1207/s15327582ijpr0901_4

Streib, H., \&, Hood, R. W. (2013). Modeling the Religious Field: Religion, Spirituality, Mysticism, and Related World Views. Implicit Religion, 16(2), 137-155. https://doi.org/10.1558/imre.v16i2.137

Streib, H., \& Klein, C. (2013). Atheists, agnostics, and apostates. In K. I. Pargament, J. J. Exline, \& J. W. Jones (Eds.), APA handbooks in psychology. APA handbook of psychology, religion, and spirituality (Vol. 1): Context, theory, and research (pp. 713-728). American Psychological Association.

Van Wicklin, J. F. (1990). Conceiving and measuring ways of being religious. Journal of Psychology and Christianity, 9(2), 27-40.

Worthington, E. L., Jr., Wade, N. G., Hight, T. L., Ripley, J. S., McCullough, M. E., Berry, J. W., Schmitt, M. M., Berry, J. T., Bursley, K. H., \& O'Connor, L (2003). The Religious Commitment Inventory-10: Development, refinement, and validation of a brief scale for research and counseling. Journal of Counseling Psychology, 50(1), 84-96. https://doi.org/10.1037/0022-0167.50.1.84

Zinnbauer, B. J., Pargament, K. I., Cole, B., Rye, M. S., Butter, E. M., Belavish, T. G., Hipp, K. M., Scott, A. B., \& Kadar, J. L. (1997). Religion and Spirituality: Unfuzzing the Fuzzy. Journal for the Scientific Study of Religion, 36(4), 549-564. https://doi.org/10.2307/1387689

Zinnbauer, B. J., \& Pargament, K. I. (2005). Religiousness and spirituality. In R. F. Paloutzian \& C. L. Park (Eds.), Handbook of the Psychology of Religion and Spirituality (pp. 21-42). Guilford Press

Historial do artigo

Recebido $\quad 06 / 2019$

Aceite $\quad 03 / 2020$

Publicado $\quad 08 / 2020$ 
Revista PSICOLOGIA, 2020, Vol. 34 (1). doi: 10.17575/psicologia.v34i1.1681

Atas do X Simpósio Nacional de Investigação em Psicologia

\title{
Diagnósticos locais de segurança no centro da cidade do Porto - Uma aposta no contexto da Psicologia Policial
}

\author{
Laura M. Nunes $2 \mathrm{i}$ i,iiiii, Ana Sani ${ }^{\mathrm{i}, \mathrm{ii}, \mathrm{iv}}$ \& Maria Alzira Pimenta Dinis $\mathrm{s}^{\mathrm{i}, \mathrm{v}}$ \\ iObservatório Permanente Violência e Crime (OPVC), Universidade Fernando Pessoa, Porto, Portugal \\ ii Centro de Investigação em Ciências Sociais e do Comportamento (FP-2BS), Universidade Fernando Pessoa, \\ Porto, Portugal \\ iii Centro de Investigação em Justiça e Governação (JusGov), Universidade do Minho, Braga, Portugal \\ ${ }^{i v}$ Centro de Investigação em Estudos da Criança, Universidade do Minho, Braga, Portugal \\ ${ }^{v}$ Unidade de Investigação UFP em Energia, Ambiente e Saúde (FP-ENAS), Universidade Fernando Pessoa \\ (UFP), Porto, Portugal
}

\begin{abstract}
Resumo: O Diagnóstico Local de Segurança (DLS) consiste no levantamento de informação que possibilite retratar uma determinada área/comunidade, em termos de criminalidade, vitimação e perceção de segurança, sendo uma avaliação internacionalmente reconhecida como uma mais-valia para as forças policiais. Através do DLS, procurou-se alcançar os objetivos de identificar a perceção das pessoas que residem, trabalham e estudam numa área urbana, sobre segurança e criminalidade, e identificar a satisfação dessa população quanto à atuação policial. Com base no inquérito por questionário sobre uma amostra de 195 participantes com idades entre os 18 e os 87 anos $(M=42.5$; $D P=19.9)$, foram encontradas similaridades aos níveis da perceção dos crimes mais ocorridos, dos mais temidos e da perceção da atuação policial. Os resultados obtidos permitem extrair conclusões que poderão contribuir para um melhor direcionamento nas modalidades de policiamento a implementar.
\end{abstract}

Palavras-chave: Diagnósticos locais de segurança; Criminalidade; Centros urbanos; Ação policial.

Local safety diagnostics in the center of Porto city - An investment in the context of Police Psychology: The Local Safety Diagnostics (DLS) consists in collecting of information that makes it possible to portray a particular area/community in terms of crime, victimization and security perception, an internationally recognized assessment to assist the police forces. Using the DLS, the objective was to identify the perception of people who live, work and study in an urban area, on safety and crime, and to identify the satisfaction of this population with regard to police action. Based on a questionnaire survey of a sample of 195 participants aged 18-87 $(M=42.5 ; S D=19.9)$, similarities were found to the levels of perception of the most frequent crimes and feared crimes, and the perception of the police action. The results obtained allow to draw conclusions that may contribute to a better orientation in the police practices to be implemented.

Keywords: Local safety diagnostics; Crime; Urban centres; Police action.

Considere-se o Diagnóstico Local de Segurança (DLS) como um levantamento de informações que possibilita retratar uma determinada área/comunidade, atendendo à criminalidade, à vitimação, à perceção de segurança e ao sentimento de pertença por parte da população local, não esquecendo a análise à perceção dessa mesma população quanto à atuação policial (Alvarez, 2006; Sani, Nunes, Caridade, \& Azevedo, 2020; Sani, Nunes, Estrada, \& Caridade, 2019). Trata-se de uma avaliação internacionalmente reconhecida como uma mais-valia para as forças policiais, e para as próprias comunidades, tratando-se igualmente de uma prioridade para se conseguir um plano de prevenção criminal realmente adaptado às especificidades locais (Caridade, Sani, Nunes, \& Dinis, 2020; Direcção Geral de Administração Interna, 2009; Nunes \& Sani, 2014; Sani, Nunes, \& Caridade, 2019).

Entenda-se 'crime' como toda a ação que se opõe ao expectável segundo a lei, sendo, portanto, passível de reprovação/condenação (Durkheim, 1895), e impondo-se a participação de um criminoso com um motivo para assim agir, causando dano a uma potencial vítima numa determinada dimensão

\footnotetext{
2 Morada para correspondência: Laura M. Nunes, Universidade Fernando Pessoa, Praça 9 de Abril, 349, 4249-004, Porto, Portugal. E-mail: lnunes@ufp.edu.pt. Este trabalho é financiado por Fundos Nacionais através da FCT - Fundação para a Ciência e a Tecnologia no âmbito do projeto PTDC/DIR-DCP/28120/2017.
} 
espácio-temporal, sem que se interponha algum ator social capaz de impedir a ocorrência que, refira-se, se encontra tipificada no quadro legal/penal (Brookman, Maguire, Pierpoint, \& Bennett, 2010; Dias \& Andrade, 1997). Já o conceito de 'insegurança' pode olhar-se sob dois prismas - o da insegurança real ou objetiva e o da subjetiva -, esta última muito associada ao medo do crime, enquanto a primeira se relaciona, efetiva e realmente, com as ocorrências criminais (Roché, 1996) sendo que, recorde-se, as situações/condições que criam o medo do crime não estão, forçosa e necessariamente, ligadas a reais ocorrências criminais ou riscos de vitimação (Brantingham \& Brantingham, 1995).

Atendendo ao acabado de definir e à essência do designado DLS, ponderou-se sobre as comunidades localizadas nos centros das grandes urbes europeias que apresentam imensas particularidades, incluindo espaços igualmente específicos, pelo que devem ser analisadas sob esta nova perspetiva, a fim de melhor se lidar com o crime e a vitimação que aí possam ocorrer. Foi com base nesta ideia que emergiu o projeto financiado LookCrim, de onde se extraiu este estudo. Assim, neste artigo optou-se por tratar parte da muita informação obtida a partir de um DLS, debruçando-nos sobre as questões da segurança, da criminalidade e da atuação policial, numa área muito caraterística da cidade do Porto - o Centro Histórico - onde está em desenvolvimento o projeto já referido. Genericamente, pretende-se retratar essa região urbana, considerando as perceções de crime, segurança e eficácia policial, por parte da população que ali se move diariamente.

\section{Diagnóstico local de segurança - caraterização e especificidade}

Nunca perdendo de vista o objetivo último de contribuir para uma melhor prevenção criminal, os DLS procuram, de acordo com a Direção Geral de Administração Interna (2009), alcançar o seguinte:

a) Contextualizar a comunidade em estudo, apreendendo os aspetos demográficos e económicos;

b) Analisar o crime, a violência e outros problemas que estejam presentes na comunidade específica em análise;

c) Caraterizar os atores sociais envolvidos na ocorrência criminal, desde as vítimas aos agressores;

d) Caraterizar a comunidade quanto à existência de padrões que possam facilitar ou até potenciar a ocorrência de crime;

e) Identificar os serviços presentes na comunidade e a sua eficácia em termos preventivos;

f) Avaliar o envolvente político e institucional de modo a identificar oportunidades de desenvolvimento no âmbito da prevenção criminal;

g) Identificar as potencialidades existentes na comunidade, de maneira a verificar a potencialidade da estratégia a ser adequadamente adotada.

Em Portugal, para o levantamento de informações que possibilitem a elaboração do DLS, foi publicado um questionário (Sani \& Nunes, 2013), entretanto utilizado em diversos estudos portugueses (e.g., Nunes \& Sani, 2014; Nunes, Sani, Caridade, Sousa, \& Dinis, 2018; Sani \& Nunes, 2012, 2016; Sani, Nunes, Azevedo, \& Sousa, 2019). No estudo de Sani e Nunes (2012) na zona central do Porto, a Sé, com uma amostra de 244 inquiridos, com resultados que apontavam para mais de $30 \%$ de pessoas a sentirem insegurança, muito atribuída à escassez de policiamento e à sensação de presença de perigo/crime, havendo uma perceção algo negativa da atuação policial, com ênfase no défice de policiamento, entre outros motivos. Muitos dos inquiridos referiam que o policiamento era deficitário, já que raramente observavam a presença de agentes nas ruas. Outros DLS foram desenvolvidos em análises internacionais, após adaptação do questionário, nomeadamente, num estudo solicitado às autoras pela Organização das Nações Unidas (Sani \& Nunes, 2014), com resultados de perceção de insegurança por mais de metade dos inquiridos, também fortemente atribuída a défices no policiamento. Junto de comunidades muito específicas, como no Pólo Universitário do Porto (Nunes et al., 2018), foi também realizada uma avaliação por meio do DLS, verificando-se um desfasamento considerável entre crimes temidos e ocorridos, com alguma insatisfação com a polícia. Portanto, e também com base nestas análises, o questionário revelouse excelente para contribuir para uma avaliação ao Centro Histórico do Porto.

Das cinco partes que integram o questionário de DLS - A) dados Sociodemográficos; B) Perceção de Segurança/Insegurança; C) Vitimação; D) Controlo Social; E) Participação Comunitária -, apenas os resultados parciais de três dessas partes, A, B e D, serão aqui apresentados no estudo descrito a seguir.

\section{MÉTODO}

Segundo um plano de estudo descritivo, e através da administração do questionário de DLS, foram recolhidos os dados após ter sido obtida autorização junto da Junta da União de Freguesias do Centro Histórico do Porto, que colaborou também no acesso à amostra, em que apenas participou quem foi previamente informado a respeito da pesquisa, dos seus objetivos e propósitos, e do seu enquadramento 
no âmbito de um projeto de avaliação ao Centro Histórico da cidade, não sendo esquecida a declaração de consentimento informado facultada por cada inquirido.

Recordem-se os objetivos definidos, como o de identificar a perceção das pessoas que residem, trabalham e estudam numa área urbana, sobre segurança e criminalidade, e identificar a satisfação dessa população quanto à atuação policial.

A informação recolhida foi inserida manualmente numa base de dados construída sobre o Statistical Package for Social Sciences (SPSS) - versão 25 -, a partir da qual foi possível obter os resultados decorrentes das análises descritivas dos dados de cariz quantitativo, bem como dos dados de natureza qualitativa, previamente codificados e distribuídos por um sistema de categorias construídas pelo procedimento por Milha, mediante a proposta de Bardin (2004). Uma vez apresentados sumariamente estes procedimentos e algumas decisões metodológicas, passamos à caraterização do conjunto dos inquiridos.

\section{Participantes}

A amostra constituiu-se de 195 participantes com idades entre os 18 e os 87 anos $(M=42.5 ; D P=19.9)$ todos eles a trabalhar, estudar ou a residir naquela área urbana, e apresentando as caraterísticas que se seguem.

Tabela 1. Distribuição dos sujeitos quanto às variáveis sociodemográficas $(N=195)$.

\begin{tabular}{|c|c|c|}
\hline & $n$ & $\%$ \\
\hline \multicolumn{3}{|l|}{ Sexo } \\
\hline Feminino & 112 & 57.4 \\
\hline Masculino & 83 & 42.6 \\
\hline \multicolumn{3}{|l|}{ Idade (anos) } \\
\hline $18-24$ & 43 & 22.1 \\
\hline $25-34$ & 31 & 15.9 \\
\hline $35-44$ & 36 & 18.5 \\
\hline $45-54$ & 36 & 18.5 \\
\hline $55-64$ & 20 & 10.3 \\
\hline 65 ou mais & 27 & 13.8 \\
\hline Omissos & 2 & 1.0 \\
\hline \multicolumn{3}{|l|}{ Escolaridade (anos) } \\
\hline $1^{0}-4^{o}$ & 17 & 8.7 \\
\hline $5^{0}-6^{0}$ & 19 & 9.7 \\
\hline $70-90$ & 14 & 7.2 \\
\hline $10^{\circ}-12^{\circ}$ & 75 & 38.5 \\
\hline Superior & 70 & 35.9 \\
\hline \multicolumn{3}{|l|}{ Situação ocupacional } \\
\hline Trabalhador & 129 & 66.2 \\
\hline Estudante & 31 & 15.9 \\
\hline Reformado & 23 & 11.8 \\
\hline Desempregado & 9 & 4.6 \\
\hline Estudante-Trabalhador & 3 & 1.5 \\
\hline
\end{tabular}

Tal como se verifica na Tabela 1, o sexo feminino apresenta uma ligeira superioridade, com cerca de 57.4\%. Quanto à idade, a faixa etária que se destaca na amostra é o grupo com idades compreendidas entre os 18 e 24 anos, que inclui $22.1 \%$ dos sujeitos, seguindo-se os grupos entre 35 e 44 , e entre 45 e 54 anos, $18.5 \%$ da amostra em cada um destes intervalos. A escolaridade que prevalece é a correspondente aos anos entre $10^{\circ}$ e $12^{\circ}$, em $38.5 \%$ dos participantes, logo seguida do nível superior alcançado por $35.9 \%$ dos inquiridos. Note-se que $66.2 \%$ é trabalhador, havendo depois uma distribuição entre 
estudantes e reformados, com muito poucos sujeitos em situação de desemprego ou de estudantetrabalhador.

\section{RESULTADOS}

Começando pelos resultados referentes às perceções dos inquiridos quanto a questões de segurança e criminalidade naquela região da cidade, pode verificar-se que a maioria referiu sentir segurança, mas ainda assim mais de 1/4 dos participantes (26.2\%) referiu não sentir a área como segura (ver Tabela 2). As categorias de respostas codificadas a respeito da fundamentação que cada participante deu para a sua perceção de segurança foram, no que respeita à perceção de área segura, as seguintes: por experiência/observação (44.1\%), por controlo social formal (9.7\%), segurança limitada apenas aos residentes e conhecidos (5.1\%), por comparação com outras áreas (3.6\%) e por presença de controlo social informal (1\%).

Ainda em relação à perceção de segurança, destaque para as categorias de resposta como fundamento para os que referiram não sentir segurança: para além dos $10.3 \%$ de sujeitos que referiram não saber responder, 9.7\% alegou que a insegurança era predominantemente noturna, seguindo-se a explicação baseada na sensação de presença de crime/perigo (8.7\%), a escassez/limitação de policiamento (4.6\%) e a experiência e observação, bem como a atribuição à presença de tráfico e consumo de drogas, com $1.5 \%$ de respostas em cada uma destas duas últimas categorias.

Tabela 2. Distribuição dos sujeitos quanto à perceção de segurança/insegurança $(N=195)$.

\begin{tabular}{lcc}
\hline Perceção de segurança & $\boldsymbol{n}$ & \% \\
\hline Área segura & 137 & 70.3 \\
Área não segura & 51 & 26.2 \\
Não sabe / Não responde & 7 & 3.6 \\
\hline
\end{tabular}

Analisem-se, agora, as respostas quanto aos crimes mais temidos pelos inquiridos e quanto aos que mais frequentemente ocorrem efetivamente naquela região urbana.

Tabela 3. Distribuição dos sujeitos quanto aos crimes mais temidos e que mais frequentemente ocorrem $(N=195)$.

\begin{tabular}{|c|c|c|c|c|}
\hline \multirow[t]{2}{*}{ Itens } & \multicolumn{2}{|c|}{ Crimes mais temidos } & \multicolumn{2}{|c|}{ Crime mais frequentes } \\
\hline & $n$ & $\%$ & $n$ & $\%$ \\
\hline Roubo & 113 & 68.2 & 87 & 44.6 \\
\hline Furto & 118 & 60.5 & 131 & 67.2 \\
\hline Agressão física & 96 & 49.2 & 64 & 32.8 \\
\hline Assalto residência & 64 & 35.4 & 50 & 25.6 \\
\hline Assalto estabelecimento & 63 & 32.3 & 79 & 40.5 \\
\hline Burla & 60 & 30.8 & 52 & 26.7 \\
\hline Ofensa sexual & 59 & 30.3 & 26 & 13.3 \\
\hline Danos equipamentos & 30 & 15.4 & 69 & 35.4 \\
\hline Tráfico de drogas & 28 & 14.4 & 95 & 48.7 \\
\hline Tráfico de armas & 22 & 11.3 & 4 & 2.1 \\
\hline Crimes rodoviários & 20 & 10.3 & 23 & 11.8 \\
\hline Violência doméstica (cônjuge) & 11 & 5.6 & 10 & 5.1 \\
\hline Violência doméstica (menores) & 11 & 5.6 & - & - \\
\hline Violência doméstica (idosos) & 10 & 5.1 & - & - \\
\hline Nenhum/Desconhece & 13 & 6.7 & 21 & 10.8 \\
\hline
\end{tabular}

Nota: Cada participante pôde optar por mais do que um crime (temido e efetivo)

Conforme pode constatar-se pela Tabela 3, os crimes mais temidos não são coincidentes com os que mais frequentemente ocorrem, havendo até crimes que, sendo temidos, não são apontados como 
ocorrências conhecidas. Tal é o caso da violência doméstica, quer contra idosos, quer contra crianças. 0 furto é o crime percecionado como mais ocorrente e, o mais temido, é o roubo. Já o tráfico de drogas, sendo um crime que acontece frequentemente, nem por isso é temido por muitos inquiridos.

Quanto aos resultados referentes ao grau de satisfação com a atuação policial, vejam-se de seguida (ver Tabela 4).

Tabela 4. Grau de satisfação com a atuação policial e respetiva fundamentação $(N=195)$.

\begin{tabular}{lcc}
\hline & $\boldsymbol{n}$ & $\mathbf{\%}$ \\
\hline Grau de satisfação com a atuação policial & 15 & 7.7 \\
Muito satisfeito & 93 & 47.7 \\
Satisfeito & 52 & 26.7 \\
Pouco satisfeito & 10 & 5.1 \\
Nada satisfeito & 25 & 12.8 \\
$\quad$ Não sabe & & \\
Fundamentação para pouco ou nada satisfeito & 22 & 11.3 \\
Escassez/limitação de policiamento & 17 & 8.7 \\
Inércia/ineficácia na atuação & 8 & 4.1 \\
Atuação reativa/atuação por conveniência & 8 & 4.1 \\
Atuação tardia & 3 & 1.5 \\
Formação/experiência insuficiente & 2 & 1.0 \\
Imagem depreciativa da polícia & & \\
Fundamentação para satisfeito e muito satisfeito & 41 & 21.0 \\
Atuação proativa/disponibilidade & 36 & 18.5 \\
Eficácia/eficiência na atuação & 10 & 5.1 \\
Prontidão de respostas & 1 & 0.5 \\
Formação/experiência & &
\end{tabular}

Muito embora a percentagem prevalecente seja a dos inquiridos que se mostram satisfeitos com a atuação policial, a verdade é que $31.8 \%$ se revelou pouco ou nada satisfeito com essa atuação, apontando, para fundamentar essa classificação negativa, a escassez e limitação de policiamento e, em menor número, outros argumentos como a inércia, a reatividade e a atuação tardia das forças policiais.

\section{DISCUSSÃO}

Muito embora este estudo verse apenas sobre parte dos resultados alcançados com a administração do questionário de DLS, a verdade é que, tal como referido pela Direcção Geral de Administração Interna (2009), esses elementos possibilitam já retratar, pelo menos em parte, aquela comunidade em particular.

Os resultados apontam para uma perceção de insegurança em mais de $26 \%$ dos participantes, numa aproximação aos estudos portugueses referidos (Sani \& Nunes, 2012), embora com uma percentagem consideravelmente inferior à encontrada no estudo realizado a pedido da Organização das Nações Unidas (Sani \& Nunes, 2014). Apesar da diferença ao nível da população inquirida, os resultados aqui encontrados vão ao encontro do verificado na análise realizada no Pólo Universitário do Porto (Nunes et al., 2018), em que se constatou um desfasamento entre crimes ocorridos e crimes temidos pela população. Aliás, esse desfasamento remete para a ideia de insegurança real e subjetiva, apontadas por Roché (1996), estando também ligado ao conceito de medo do crime que nem sempre tem relação com a ocorrência criminal, tal como tão bem referem Brantingham e Brantingham (1995). A atuação policial, avaliada negativamente por um considerável número de sujeitos remete, também, para estudos anteriores (Sani \& Nunes, 2012, 2013) em que essa apreciação negativa era também muito fundada no policiamento deficitário e na postura reativa dos agentes de segurança. 


\section{CONCLUSÃO}

Efetivamente, o objetivo mais genérico de retratar a comunidade retratada foi alcançado, tendo sido identificada a perceção dos inquiridos sobre a segurança e a criminalidade, bem como a respeito da maior ou menor satisfação com a atuação policial.

É importante realçar que poderão notar-se algumas facetas que, nos resultados, poderão vir a justificar uma análise que procure eventuais associações entre diferentes variáveis. Mais especificamente, é de notar que, ao nível das caraterísticas sociodemográficas da amostra, mais de metade dos sujeitos é jovem adulto, com idades entre os 18 e os 44 anos, tendo 10 ou mais anos de escolaridade e sendo predominantemente trabalhadores no ativo ou estudantes. Seria, portanto, de ponderar a existência de uma relação entre tais caraterísticas e os resultados referentes à perceção de segurança maioritariamente apontada, havendo uma razoável percentagem de indivíduos satisfeitos com a atuação policial e, salientese, remetendo para alguma familiaridade com o crime de tráfico de drogas que, estando entre os que mais ocorrem não é dos mais temidos. A verdade é que, ainda assim, mais de $26 \%$ dos sujeitos não sente segurança, e mais de 30\% não está satisfeito com a atuação da polícia, pelo que há necessidade de trabalhar estas questões aos níveis das prevenções primária, secundária e terciária.

É certo que a amostra não permite generalizações, mas também é correto que alcançado um retrato da comunidade, se torna possível apoiar as autoridades no sentido de um maior conhecimento sobre aquela, facilitando a maior adequação das estratégias de vigilância e policiamento e, consequentemente, da prevenção criminal naquela área específica. Tal prevenção será também potenciada pelo maior conhecimento da população local, o que por sua vez conduz à maior aproximação polícia/população.

Conhecida a perceção de policiamento deficitário, será possível repensá-lo e torná-lo mais visível o que, por vezes, já leva à dissuasão do criminoso, ao aumento da satisfação da população, e, portanto, à maior prevenção com aproximação e cooperação da população relativamente aos agentes de segurança. Estas e outras medidas que se esperam encontrar com o desenvolvimento do projeto, são de extrema importância para o bem-estar sentido nos grandes centros urbanos.

\section{REFERÊNCIAS}

Alvarez, J. (2006). Réalisation d'un diagnostic de sécurité. Trousse à l'intention des collectivités locales. Centre international pour la prévention de la criminalité.

Bardin, L. (2004). Análise de conteúdo (3ª ed.; L. Reto \& A. Pinheiro, Trad.). Edições 70. (Original publicado em 1977).

Brantingham, P., \& Brantingham, P. (1995). Criminality of place: crime generators and crime attractors. European Journal on Criminal Policy and Research, 3(3), 5-26. https://doi.org/10.1007/BF02242925

Brookman, F., Maguire, M., Pierpoint, H., \& Bennett, T. (2010). Handbook on Crime. Willian Publishing. https://doi.org/10.4324/9781843929680

Caridade, S., Sani, A. I., Nunes, L., \& Dinis, A. (2020). Youth victims of community violence: developmental outcomes and prevention challenges. In M. L. Knudsen (Eds.), Victims of Violence: Support, Challenges and Outcomes (pp. 279-290). Nova Science Publishers, Inc.

Dias, J., \& Andrade, M. (1997). Criminologia: o homem delinquente e a sociedade criminógena. Editora.

Direcção Geral de Administração Interna (2009). Manual de diagnósticos locais de segurança. Uma compilação de normas e práticas internacionais (M. Correia, Trad.). Ministério da Administração Interna.

https://efus.eu/files/fileadmin/efus/Publications/Manual_Seguranca_miolo_166pp_23_12_09.pdf

Durkheim, E. (1895). Les Règles de la méthode sociologique. Presses Universitaires de France.

Nunes, L. \& Sani, A. (2014). Avaliação de uma comunidade urbana - diagnóstico local de segurança e soluções de policiamento. Revista Direito e Democracia, 15(1), 4-17. http://www.ulbra.br/upload/ce0573673bdb2fbcdb11420bcb21dcb6.pdf

Nunes, L., Sani, A., Caridade, S., Sousa, H., \& Dinis, M. (2018). Segurança e vitimação entre estudantes universitários na cidade do Porto. Análise Psicológica, 36(2), 169-183. http://dx.doi.org/10.14417/ap.1395

Roché, S. (1996). La société incivile. Qu'est-ce que l'insécurité ? Seuil.

Sani, A., \& Nunes, L. (2012). Relatório do inquérito Diagnóstico Local de Segurança na Sé (Porto). Edições UFP. http://hdl.handle.net/10284/3313

Sani, A., \& Nunes, L. (2013). Questionário de diagnóstico local de segurança: Estudo numa comunidade urbana. Análise Psicológica, 31(2), 185-195. http://dx.doi.org/10.14417/ap.609

Sani, A., \& Nunes, L. (2014). Relatório Diagnóstico Local de Segurança Guiné Bissau. Porto: Edições UFP. http://hdl.handle.net/10284/4536 
Sani, A. I., \& Nunes, L. M. (2016). Diagnóstico de seguridad/inseguridad. Un estudio exploratorio en na comunidad urbana. Anuario de Psicología Jurídica, 26, 102-106. http://dx.doi.org/10.1016/j.apj.2015.07.001

Sani, A., Nunes, L., Azevedo, V., \& Sousa, H. (2020). Campus criminal victimization among higher education students: A diagnosis of local security in Porto. Journal of Criminal Justice Education, 31(2), 250266. http://dx.doi.org/10.1080/10511253.2019.1702219

Sani, A., Nunes, L., \& Caridade, S. (2019). Comunidades urbanas, prevenção criminal e diagnóstico local de segurança. In J. Trindade, \& F. Molinari (Org.), Temas de Psicologia Forense: um olhar para o futuro (pp. 335-349). Editora Imprensa Livre.

Sani, A. I., Nunes, L., Caridade, S., \& Azevedo, V. (2020). Violence in urban community: diagnosis of local security and victimization. In M. L. Knudsen (Eds.), Victims of Violence: Support, Challenges and Outcomes (pp. 291-314). Nova Science Publishers, Inc.

Sani, A., Nunes, L., Estrada, R., \& Caridade, S. (2019). Avaliação-intervenção comunitária Diagnóstico Local de Segurança - Perceção de violência e crime. In A. Neves, F. Veiga, I. Machado, R. Miranda, S. Almeida, \& T. Riboira (Org.), III Congresso Ibero-americano de Intervenção Social - Direitos Humanos e Mediação (pp. 265-277). Lema d' Origem, Editora Lda.

Historial do artigo

Recebido 06/2019

Aceite $\quad 03 / 2020$

Publicado $\quad 08 / 2020$ 
Revista PSICOLOGIA, 2020, Vol. 34 (1). doi: 10.17575/psicologia.v34i1.1681

Atas do X Simpósio Nacional de Investigação em Psicologia

\title{
Criminalidade no centro histórico do Porto e distribuição de espaços físicos
}

\author{
Maria Alzira Pimenta Dinis ${ }^{3 i, i i}$, Laura M. Nunes ${ }^{i, i i i, i v}$ \& Ana Sani i,iii,v \\ i Observatório Permanente Violência e Crime (OPVC), Universidade Fernando Pessoa, Porto, Portugal \\ ii Unidade de Investigação UFP em Energia, Ambiente e Saúde (FP-ENAS), Universidade Fernando Pessoa \\ (UFP), Porto, Portugal \\ iii Centro de Investigação em Ciências Sociais e do Comportamento (FP-2BS), Universidade Fernando Pessoa, \\ Porto, Portugal \\ ${ }^{i v}$ Centro de Investigação em Justiça e Governação (JusGov), Universidade do Minho, Braga, Portugal \\ ${ }^{v}$ Centro de Investigação em Estudos da Criança (CIEC), Universidade do Minho, Braga, Portugal
}

\begin{abstract}
Resumo: Os programas Crime Prevention Through Environmental Design (CPTED) procuram analisar o impacto dos espaços sobre os comportamentos das pessoas, designadamente sobre as condutas criminais. Apresenta-se um estudo exploratório e observacional, explorando uma das vertentes do projeto financiado LookCrim, com uma amostra de 195 participantes entre os 18 e os 87 anos $(M=42.5$; $D P=19.9$ ), e com registos de observações nas seis freguesias que integram o Centro Histórico do Porto. Procurou-se cruzar a perceção de criminalidade da população local com as caraterísticas espaciais. Tratase de uma análise comunitária que permitiu constatar o imperativo de mudança ao nível da reabilitação urbana, nos espaços privados e nos públicos e comuns à comunidade. Entre as conclusões são apontadas medidas que, não sendo dispendiosas nem complicadas, podem vir a prevenir a ocorrência criminal.
\end{abstract}

Palavras-chave: Crime prevention through environmental design; Prevenção criminal; Insegurança; Espaços físicos.

Crime in the historic centre of Porto and distribution of physical spaces: The Crime Prevention Through Environmental Design (CPTED) programs aim to analyse the impact of spaces on people's behaviour, which includes criminal conduct. This is an exploratory and observational study, exploring one of the aspects focused on the LookCrim funded project. The study encompasses a sample of 195 participants aged 18-87 years $(M=42.5, S D=19.9)$, and comprises observation records in the six parishes of the Historic Centre of Porto, intending to cross the perception of crime of the local population with the characteristics of physical spaces in that urban area. This is a community analysis allowing to verify the imperative of change in urban rehabilitation, either in private spaces as in public spaces and in common community spaces. The conclusions point out strategies that, being neither expensive nor complicated, can contribute to prevent the criminal occurrence.

Keywords: Crime prevention through environmental design; Criminal prevention; Insecurity; Physical spaces.

Entendam-se os designados Crime Prevention Through Environmental Design (CPTED), enquanto ancorados na ideia inicialmente apresentada por Jeffery (1971; 1999), como programas de análise refletida e focada nos espaços físicos, atendendo a princípios e fatores estabelecidos, de forma a reconhecer nesses espaços as caraterísticas que podem estar a potenciar o crime, visando a introdução de alterações que possam prevenir a ocorrência criminal (Lab, 2014). Estes esforços, programados no sentido de manusear o espaço para prevenir o crime, genericamente designados por CPTED, incluem uma abordagem arquitetónica, territorial e ambiental, a que se junta o reconhecimento dos utilizadores/frequentadores dos espaços como tendo legitimidade de intervenção naquela área, sendo esta uma ideia que emergiu do conceito de espaço defensível apresentado por Newman (1972). Segundo o autor, a ideia é a de que as caraterísticas espaciais podem afetar os comportamentos das pessoas, quer dos residentes nesses locais, quer dos potenciais ofensores. Nos primeiros, a aparência e o estado dos

${ }^{3}$ Morada para correspondência: Laura M. Nunes, Universidade Fernando Pessoa, Praça 9 de Abril, 349, 4249-004, Porto, Portugal. E-mail: lnunes@ufp.edu.pt. Este trabalho é financiado por Fundos Nacionais através da FCT - Fundação para a Ciência e a Tecnologia no âmbito do projeto PTDC/DIR-DCP/28120/2017. 
espaços podem induzir um maior envolvimento e cuidado com o local, numa atmosfera de contacto e empenho que poderá afastar e melhor controlar o crime, enquanto os potenciais ofensores se aperceberão, pelo estado e cuidado dos espaços, que os residentes estão atentos e vigilantes a ponto de um crime ter mais probabilidades de ser observado por alguém presente e atento.

Acrescente-se a consideração do Diagnóstico Local de Segurança (DLS) como um levantamento de informações conducentes ao traçado de um retrato de determinada área/comunidade, em que são consideradas as dimensões de criminalidade e da vitimação, entre outras, sendo uma avaliação internacionalmente reconhecida como uma fonte útil de informações e conclusões, para que as forças policiais e as próprias populações adaptem medidas preventivas do crime (Direcção Geral de Administração Interna, 2009).

Partindo destas duas vertentes de análise comunitária, e tendo por base o projeto financiado LookCrim em desenvolvimento no Centro Histórico do Porto, este estudo apresenta os resultados parcelares que, entretanto, foram já recolhidos no âmbito da criminalidade e da análise das condições físico-espaciais registadas naquela área urbana. Genericamente, os objetivos traçados passaram por i) analisar a perceção da população a respeito da (in)segurança sentida na área em estudo; ii) avaliar/identificar as caraterísticas dos espaços físicos que eventualmente possam estar ligadas à perceção de insegurança. A delimitação do projeto e, portanto, deste estudo, ao Centro Histórico da cidade do Porto, fundamenta-se em aspetos que se relacionam com o aumento da circulação de pessoas, nomeadamente de turistas, naquela área que se revela por isso de extrema importância para a cidade e, por outro lado, de acordo com o Ministério da Administração Interna (2019), pelo facto de o Porto, em 2018, ter estado entre os distritos de maior índice de criminalidade, revelando-se pertinente e útil circunscrever o desenvolvimento dos estudos deste projeto àquela região.

\section{Crime, insegurança e espaços físicos}

De acordo com o Relatório Anual de Segurança Interna de 2017 (Ministério da Administração Interna, 2018), entre 2008 e 2017, temos vindo a assistir a um decréscimo da criminalidade geral, embora a mesma fonte em 2019 revele que o Porto apresenta um dos maiores índices de criminalidade. Não obstante, apesar dos números nos indicarem este decréscimo, a verdade é que segundo Fernandes e Rêgo (2011), o sentimento de insegurança tem vindo a crescer e, note-se, de acordo com o Ministério da Administração Interna (2018), as cidades maiores, como é o caso do Porto e Lisboa, revelam o aumento de alguma criminalidade. Se atendermos ao último relatório publicado por aquele ministério, importa reforçar e potenciar a prevenção criminal apostando em: renovação/formação das forças policiais e nos dispositivos de vigilância dos espaços; reabilitação de estruturas e modernização de equipamentos; reforço do pilar da prevenção, vigilância e proteção. Portanto, medidas que remetem para a ideia dos espaços e do seu impacto sobre o comportamento, designadamente, sobre a conduta criminosa.

$\mathrm{Na}$ verdade, pode aqui fazer-se uma estreita ligação à Teoria dos Vidros Partidos - Broken Windows Theory - de Wilson e Kelling (1982). Segundo os autores, pode considerar-se que a desordem e as incivilidades estariam estreitamente ligadas ao desenvolvimento de ações cada vez mais desordeiras e incívicas, chegando a potenciar o crime. De acordo com os autores da teoria, a difusão de sinais de abandono, descuido e vandalismo, não tendo sido atalhados por algum tipo de intervenção, funcionam como sinal de indiferença social, num espaço que se revelará como desprotegido e, portanto, inseguro, e interpretado pelo potencial delinquente como um espaço de oportunidade para o crime. Está-se, claramente, na orla dos espaços e das suas caraterísticas que podem funcionar como potenciadores do crime.

Dentro desta lógica de pensamento, e atendendo aos programas CPTED, deve atender-se aos seus princípios básicos para avaliar um determinado espaço, tendo em vista identificar o que há a alterar para dele se fazer um local mais seguro. Esses princípios podem apresentar-se como 4 aspetos focais (National Crime Prevention Council, 2003):

a) Vigilância Natural, que remete para o objetivo de controlar visualmente os espaços de maneira a identificar rápida e claramente qualquer intrusão;

b) Controlo Natural, que se refere ao controlo do acesso aos espaços, implicando a adaptação e adequação de medidas que incluem a inserção de barreiras físicas que limitem o acesso a estranhos sem o dificultar àqueles que legitimamente devem aceder-lhe;

c) Reforço Territorial, com inevitável necessidade de desenvolvimento de sentimentos de pertença por parte daqueles que ali residem ou trabalham, estimulando a proteção e o cuidado com tais espaços;

d) Manutenção e Gestão, diretamente relacionado com o princípio anterior, remetendo para a atitude de cuidar, melhorar e proteger por parte dos residentes e de todos quantos frequentem diariamente essa área. 
Estes pilares devem ser os sustentáculos deste tipo de programas, que se desenvolvem de forma adaptada a cada área específica, devendo também ancorar-se na Three D Approach (Crowe, 2000). Segundo o mesmo autor, esta abordagem integra os designados três "D":

a) 0 primeiro é o da Designação, mediante o qual se tenta retratar o local/espaço identificando os fins do seu uso, os objetivos do mesmo e se foi pensado de raiz tendo em vista esses mesmos objetivos, ou se, pelo contrário, reúne caraterísticas que estejam a chocar com tais metas e propósitos;

b) 0 segundo é o da Definição, com busca ativa de dados que permitam aceder à forma como está definido o espaço, a quem pertence e para que fins se destina, o que regulamenta os comportamentos e os usos de tal espaço, quais os limites e se a sua utilização é a mais adequada;

c) 0 último dos três " $\mathrm{D}$ " remete para o Design, como ponto mais focado na forma, procurando apurar se o espaço foi desenhado para os fins a que se destina, se o seu projeto arquitetónico favorece a regulação comportamental e se reúne caraterísticas que possam atrair/potenciar o desenvolvimento de ações que colidam com o que se considera ser o adequado uso daquele espaço.

Assim, e estando na posse desta informação que procura contextualizar teoricamente o estudo do crime e a distribuição/caraterização espacial, passamos à apresentação da investigação propriamente dita.

\section{MÉTODO}

Segundo um plano de estudo descritivo e observacional, foi usado o questionário de DLS (Sani \& Nunes, 2013), do qual se extraíram os dados referentes a uma das suas partes analisadas e que remete para a criminalidade percebida pelos inquiridos. De salientar que o questionário de DLS, publicado em 2013 na sua versão portuguesa, foi pré-testado, tendo dado provas de que é claramente compreendido pela população e de que obtém as informações sugeridas internacionalmente para os chamados DLS (cf. European Forum for Urban Safety, 2007). Este questionário foi, entretanto, alvo de adaptações por forma a realizar diagnósticos no Brasil, em curso, e na Guiné, por solicitação da Organização das Nações Unidas (cf. Sani \& Nunes, 2017). É constituído por 5 partes, das quais apenas as partes dos dados sociodemográficos e da parte referente à perceção de crime foram analisadas neste estudo.

Os dados foram recolhidos após ter sido obtida autorização junto da Junta da União de Freguesias do Centro Histórico do Porto, que tem colaborado, tal como a Câmara Municipal do Porto, para o desenvolvimento do projeto. Apenas integraram a amostra os sujeitos previamente informados a respeito da pesquisa, dos seus objetivos e propósitos, e do seu enquadramento no âmbito de um projeto de avaliação ao Centro Histórico da cidade, não sendo esquecida a declaração de consentimento informado facultada por cada inquirido.

Os objetivos traçados foram de i) analisar a perceção da população a respeito da (in)segurança sentida na área em estudo - Centro Histórico do Porto, e ii) avaliar/identificar as caraterísticas dos espaços físicos que, naquela área urbana, eventualmente, possam estar ligadas à perceção de insegurança.

A informação referente aos espaços foi obtida por observação de 18 pontos distribuídos pelas 6 antigas freguesias que atualmente integram o Centro Histórico da cidade do Porto, com registo das respetivas caraterísticas numa grelha previamente concebida para o efeito e testada por diferentes observadores - Prevenção do Crime pela Gestão de Espaços (PCGE) (Nunes \& Caridade, s/d). A construção do instrumento de registo de observações espaciais seguiu rigorosamente os indicadores que as análises internacionais no âmbito dos CPTED têm proporcionado a respeito das caraterísticas espaciais consideradas potenciadoras do crime, como passa a listar-se:

a) Esquinas e locais designados como 'cegos' por contribuírem para redução de visibilidade;

b) Espaços não vigiados e espaços pouco movimentados;

c) Espaços de elevado risco (com becos sem saída, degradação/deterioração, vegetação descontrolada, ...);

d) Acessos deficitários/difíceis

e) Iluminação reduzida ou mal distribuída;

f) Fraca delimitação de espaços públicos e privados;

g) Degradação habitacional, das áreas comuns, dos pavimentos, ...;

h) Práticas incívicas (e respetivos sinais visíveis);

i) Ambiente/Clima social instalado, entre outras.

A informação recolhida, quer a partir do questionário, quer com base nos registos observacionais em grelha, foi inserida numa base de dados construída sobre o Statistical Package for Social Sciences (SPSS) - versão 25 -, a partir da qual foi possível obter os resultados decorrentes das análises descritivas dos dados de cariz quantitativo, bem como dos dados de natureza qualitativa, previamente codificados e distribuídos por um sistema de categorias construídas pelo procedimento por Milha, mediante a proposta 
de Bardin (2004), tendo sido também inseridos os dados obtidos pela técnica da observação. Após esta apresentação dos procedimentos, a par da exposição das decisões metodológicas, passamos à caraterização do conjunto dos inquiridos que integrou a amostra.

\section{Participantes}

A amostra contou com um total de 195 indivíduos cujas idades oscilaram entre os 18 e os 87 anos $(M=42.5$; $D P=19.9)$. Este constituiu o número de inquiridos que, até ao momento, tinha sido abordado pela equipa anteriormente treinada para recolher dados com o questionário de DLS, estando ainda a decorrer a recolha de dados para o projeto LookCrim, antes de se fechar a amostra final. A totalidade dos participantes trabalhava, estudava ou residia naquela área urbana, conhecendo-a bem, e a sua seleção constou na abordagem às pessoas nas áreas apontadas pela Junta da União de Freguesias do Centro Histórico do Porto. Apresentam-se na Tabela 1 as caraterísticas sociodemográficas da amostra.

Tabela 1. Distribuição dos sujeitos quanto às variáveis sociodemográficas $(N=195)$.

\begin{tabular}{|c|c|c|}
\hline & $\bar{n}$ & $\%$ \\
\hline \multicolumn{3}{|l|}{ Sexo } \\
\hline Feminino & 112 & 57.4 \\
\hline Masculino & 83 & 42.6 \\
\hline \multicolumn{3}{|l|}{ Idade (anos) } \\
\hline $18-24$ & 43 & 22.1 \\
\hline $25-34$ & 31 & 15.9 \\
\hline $35-44$ & 36 & 18.5 \\
\hline $45-54$ & 36 & 18.5 \\
\hline $55-64$ & 20 & 10.3 \\
\hline 65 ou mais & 27 & 13.8 \\
\hline Omissos & 2 & 1.0 \\
\hline \multicolumn{3}{|l|}{ Escolaridade (anos) } \\
\hline $1^{0}-4^{\circ}$ & 17 & 8.7 \\
\hline $5^{0}-6^{0}$ & 19 & 9.7 \\
\hline $7 \underline{0}-9 \underline{0}$ & 14 & 7.2 \\
\hline $10^{\mathrm{o}}-12^{\mathrm{o}}$ & 75 & 38.5 \\
\hline Superior & 70 & 35.9 \\
\hline \multicolumn{3}{|l|}{ Situação Ocupacional } \\
\hline Trabalhador & 129 & 66.2 \\
\hline Estudante & 31 & 15.9 \\
\hline Reformado & 23 & 11.8 \\
\hline Desempregado & 9 & 4.6 \\
\hline Estudante-Trabalhador & 3 & 1.5 \\
\hline
\end{tabular}

De acordo com a Tabela 1, a amostra inclui mais de metade de sujeitos do sexo feminino (57.4\%), sendo que as idades que mais se destacam estão entre os 18 e 24 (22.1\%), seguindo-se os grupos etários entre 35 e 44, e entre 45 e 54 anos, com 18.5\% da amostra em cada uma daquelas faixas etárias. No que à escolaridade diz respeito, destaquem-se os $38.5 \%$ de indivíduos entre os $10^{\circ}$ e $12^{\circ}$ anos, sendo ainda de referir que 35.9\% dos inquiridos apresentou formação superior. A situação laboral remete para uma maioria (66.2\%) em situação de emprego, havendo muito poucos desempregados (4.6\%). 


\section{RESULTADOS}

\section{Perceção dos participantes}

Atenda-se aos resultados referentes à criminalidade percebida pelos participantes neste estudo. Estes resultados decorreram da administração individual do questionário DLS em papel, por uma equipa treinada nesse sentido.

Excluindo os 7 indivíduos que preferiram não se manifestar quanto à perceção de segurança/insegurança, pode constatar-se que uma maioria de sujeitos, mais precisamente 137 (70.3\%), aponta o Centro Histórico do Porto como seguro. Não obstante, não podemos ignorar que mais de $1 / 4 \mathrm{da}$ amostra (26.1\%) sente aquela região como insegura. Trata-se de uma percentagem algo significativa de pessoas que percecionam insegurança na área urbana em que circulam diariamente (ver Tabela 2).

Tabela 2. Distribuição dos sujeitos quanto à perceção de segurança/insegurança $(N=195)$.

\begin{tabular}{lcc}
\hline Perceção de Segurança & $\boldsymbol{n}$ & \% \\
\hline Área segura & 137 & 70.3 \\
Área não segura & 51 & 26.2 \\
Não sabe/Não responde & 7 & 3.6 \\
\hline
\end{tabular}

Perante tais respostas, e uma vez inquiridos sobre como fundamentariam a sua perceção de segurança/insegurança, os participantes foram explicando essa perceção através de respostas que foram codificadas nas seguintes categorias: perceção de segurança dada a experiência/observação (44.1\%), ou por atribuição à ação dos meios de controlo social formal (9.7\%), havendo inquiridos que referiram que a segurança era limitada apenas aos residentes e conhecidos (5.1\%), enquanto outros sujeitos preferiram fundamentar a sua perceção de segurança por comparação estabelecida com outras áreas (3.6\%).

A perceção de insegurança foi justificada por alguns (9.7\%) que alegaram que tal insegurança era sentida essencialmente durante o período noturno, havendo inquiridos que explicaram a perceção de insegurança dada a sensação de presença de crime/perigo (8.7\%). Outros houve que apontaram a escassez/limitação de policiamento (4.6\%), a experiência e observação, bem como a presença de tráfico e consumo de drogas como razões que os levavam a sentir-se pouco seguros.

Quanto aos crimes efetivamente ocorridos e observados naquela área, o furto (67.2\%), o tráfico de drogas (48.7\%) e o roubo (44.6\%) foram os que mais frequentemente foram apontados (cf. Tabela 3).

Tabela 3. Distribuição dos sujeitos quanto aos crimes que mais frequentemente ocorrem $(N=195)$.

\begin{tabular}{lcc}
\hline Crime mais frequentes: & $\boldsymbol{n}$ & $\mathbf{\%}$ \\
\hline Furto & 131 & 67.2 \\
Tráfico drogas & 95 & 48.7 \\
Roubo & 87 & 44.6 \\
Assalto estabelecimento & 79 & 40.5 \\
Danos equipamentos & 69 & 35.4 \\
Agressão física & 64 & 32.8 \\
Burla & 52 & 26.7 \\
Assalto residência & 50 & 25.6 \\
Ofensa sexual & 26 & 13.3 \\
Crimes rodoviários & 23 & 11.8 \\
Violência conjugal & 10 & 5.1 \\
Tráfico armas & 4 & 2.1 \\
Desconhece & 21 & 10.8 \\
\hline
\end{tabular}

Nota: Cada participante pôde optar por mais do que um crime

Vejam-se, agora, os resultados referentes aos registos das observações dos espaços. 


\section{Registo das observações aos espaços físicos}

No que se refere aos espaços físicos, as observações foram realizadas e registadas conforme se passa a apresentar na Tabela 4.

Tabela 4. Indicadores espaciais registados, definidos como eventuais potenciadores do crime.

\begin{tabular}{|c|c|c|c|}
\hline $\begin{array}{l}\text { Designação do } \\
\text { Elemento/ } \\
\text { Indicador }\end{array}$ & $\begin{array}{c}\text { Descrição do } \\
\text { Elemento/Indicador ( } N=18 \text { observações/registos) }\end{array}$ & $\begin{array}{c}\text { Presente } \\
\quad N_{p}\end{array}$ & $\begin{array}{c}\text { Ausente } \\
\qquad N_{a}\end{array}$ \\
\hline Esquinas Cegas & Que impedem visibilidade & 18 & 0 \\
\hline Locais Cegos & Locais de visibilidade reduzida & 18 & 0 \\
\hline $\begin{array}{l}\text { Espaços não } \\
\text { vigiados }\end{array}$ & Zonas de risco sem vigilantes / dispositivos de vigilância & 15 & 3 \\
\hline $\begin{array}{l}\text { Espaços pouco } \\
\text { movimentados }\end{array}$ & $\begin{array}{l}\text { Constituindo locais de oportunidade para ações sem qualquer } \\
\text { testemunha }\end{array}$ & 18 & 0 \\
\hline \multirow{6}{*}{$\begin{array}{l}\text { Espaços de elevado } \\
\text { risco }\end{array}$} & Túneis ou pontes pedonais & 15 & 3 \\
\hline & Becos sem saída & 15 & 3 \\
\hline & Parques de estacionamento e outras áreas de confinamento & 18 & 0 \\
\hline & Terrenos abandonados e com vegetação sem controlo & 3 & 15 \\
\hline & Casas abandonadas e com possibilidade de abrigar/esconder ... & 18 & 0 \\
\hline & Áreas ou arruamentos sem vigilância formal e informal & 18 & 0 \\
\hline \multirow[t]{2}{*}{ Acessos } & Ruas estreitas com esquinas cegas e/ou barreiras à visibilidade & 18 & 0 \\
\hline & Escadas, clareiras ou rampas sem visibilidade & 6 & 12 \\
\hline \multirow[t]{2}{*}{ Iluminação } & Escassa ou mal distribuída & 12 & 6 \\
\hline & Fraca iluminação dos caminhos pedonais & 12 & 6 \\
\hline \multirow[t]{3}{*}{ Funcionalidades } & Zona mista & 18 & 0 \\
\hline & Não delimitação de espaços públicos e privados & 12 & 6 \\
\hline & Ausência de áreas lúdicas/desportivas & 18 & 0 \\
\hline Condições & Degradação habitacional & 18 & 0 \\
\hline
\end{tabular}


Degradação áreas comuns

Degradação pavimentos

Não delimitação de caminhos pedonais

Sinalética pouco visível ou em falta

Incivilidades conducentes a degradação

Vizinhança antissocial

Presença de consumo e venda de drogas

Flutuação populacional notória
18

15

12

12

18

Frequência

18

0

18

369

0

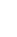

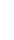

TOTAL Presença / Ausência de caraterísticas espaciais no total de observações

Nota: $\mathrm{N}_{\mathrm{p}}=$ Número de observações de presença do indicador; $\mathrm{N}_{\mathrm{a}}=$ Número de observações de ausência do indicador.

Dos resultados decorrentes da observação, e dos respetivos registos obtidos nos dezoito espaços distribuídos pelas seis regiões anteriormente consideradas como freguesias e atualmente como parte integrante do Centro Histórico do Porto, pode verificar-se (ver Tabela 4) que apenas em 69 do total das observações se encontrou ausência de alguns dos indicadores espaciais que concorrem para potenciar a ocorrência criminal (15.8\%). Foi ainda observável que um grande número desses indicadores se encontra bem presente nas seis regiões que constituem o Centro Histórico do Porto. Nomeadamente, certos elementos são recorrentemente assinalados, como é o caso de: esquinas e locais cegos e, portanto, dificultadores de visibilidade; áreas com reduzido movimento de pessoas, reduzindo-se assim o controlo social informal; áreas de confinamento, casas abandonadas e abertas para abrigar estranhos, e locais sem suficiente controlo social formal e informal; ruas estreitas e que apresentam oposição à boa visibilidade; ausência de espaços para atividades lúdicas/desportivas; degradação habitacional e decorrente de prática de incivilidades; flutuação/inconstância populacional, vizinhança antissocial e franca presença de tráfico/consumo de drogas.

Então, estão reunidas naquela área algumas das condições que, sendo potenciadoras do crime, podem e devem ser alvo de intervenção. Neste momento, importa discutir estes resultados, à luz dos pressupostos teóricos antes apresentados.

\section{DISCUSSÃo}

Comecemos, nesta discussão, pela amostra, com sujeitos de idades num intervalo de grande amplitude, entre os 18 e os 87 anos e uma média de cerca de 40 anos, espelhando opiniões, pareceres e perceções de díspares gerações, predominantemente pertencentes à população ativa.

Os resultados remetem para uma perceção de segurança predominante, muito embora haja uma percentagem considerável que aponte para uma perceção de insegurança num contexto em que os espaços, conforme ficou claro, evidenciam uma série de caraterísticas que são consideradas potenciadoras do crime. De facto, existem resultados algo contrastantes que denunciam algum desfasamento entre a perceção dos participantes, o que nos pode levar à necessidade de alguma aposta no empowerment da população relativamente a algumas noções referentes a segurança / criminalidade / perceção de crime. Por outro lado, os espaços parecem ter tido aqui um importante papel a salientar. Estes resultados parecem ir ao encontro do referido por vários autores (e.g., Jeffery, 1971; 1999; Newman, 1972), a respeito da relação estreita entre o estado e as caraterísticas dos espaços e os comportamentos manifestados, designadamente os delituosos.

Sendo certo que este estudo traduz apenas parte dos resultados a obter ao longo do projeto de onde foi retirado, não é menos correto que, numa perspetiva exploratória, nos permite traçar um retrato 
sobre aquela região - vejam-se a Direcção Geral de Administração Interna (2009) e Sani e Nunes (2013) quer pelos dados obtidos pelo DLS, com referência a uma perceção considerável de insegurança, identificação de crimes frequentes e alegadamente observados, como furto, tráfico de drogas, roubo e assalto a estabelecimento, quer ainda pelas observações feitas aos espaços que ficaram identificados com caraterísticas muito marcantes sobre os comportamentos. Pode ainda afirmar-se que o retrato assim traçado daquela área tem espelhada a Teoria dos Vidros Partidos (Wilson \& Kelling, 1982), dada a degradação e o mau estado geral denunciado pelas caraterísticas espaciais observadas.

Efetivamente, é observável o comprometimento dos quatro pilares apontados e expostos pelo National Crime Prevention Council (2003), o que remete para a ausência destes princípios relativamente à abordagem do três " $\mathrm{D}$ ".

Assim, estes resultados possibilitam já uma análise conclusiva que proporcionará orientação para o prosseguimento do projeto.

\section{CONCLUSÃO}

Efetivamente, os objetivos da investigação foram plenamente alcançados, tendo sido possível fazer uma análise conducente à identificação da perceção da população local em termos de segurança/insegurança, obtendo-se, também, o rol de crimes que os participantes consideraram objetivamente mais observados/percebidos naquela região. 0 outro objetivo, referente à avaliação/identificação das caraterísticas espaciais, foi igualmente conseguido, sendo até possível elencar as caraterísticas que, no Centro Histórico do Porto, se revelam menos adequadas, devendo, portanto, ser alvo de intervenção.

$\mathrm{Na}$ verdade, o estudo procurou tais caraterísticas para, conjuntamente com os poderes locais, ser possível perceber exatamente o que deve ser alterado e como devem tais alterações ser feitas. Algumas sugestões passariam pela reabilitação habitacional, num gesto de melhoria direta do Centro e que, simultaneamente, teria um efeito indireto, mas também positivo, que se traduziria na mobilização das pessoas que residam nessas habitações, envolvendo-as entusiasticamente no cuidado e na melhoria dos próprios espaços públicos adjacentes às suas casas. Ainda decorrente da reabilitação (habitacional e dos espaços públicos), haveria uma maior fixação da população residente, evitando-se a rotatividade sentida e, consequentemente, aumentando a ligação das pessoas ao local, com desenvolvimento de sentimentos de pertença que levariam a maiores cuidados no tratamento e na proteção do local. 0 aumento de iluminação e a sua adequada distribuição seriam também importantes, tal como o uso dos espaços menos movimentados para o desenvolvimento de atividades lúdicas/desportivas que atraíssem as pessoas.

Uma articulação com as polícias, quer a de segurança pública, quer a municipal, seria uma ideia para se definir conjuntamente e apelando a todos os recursos um plano estratégico de maior controlo social formal, prevenindo-se a prática de incivilidades e de crimes que ocorrem naquela área, como o tráfico de drogas. Por outro lado, esta união estratégica de forças transmitiria às populações uma maior perceção de segurança.

Entre outras medidas, o traçado claro, bem iluminado e com fronteiras delineadas por sebes ou vegetação bem controlada, das passagens para pedestres seria excelente. Além disso, com estas medidas, esses caminhos tornar-se-iam mais apelativos, chamando mais turistas e mais transeuntes, o que aumentaria o controlo social informal.

Todas estas medidas aumentariam a possibilidade de vigilância, a ligação dos residentes e comerciantes ao local que, consequentemente, passaria a ser mais cuidado e protegido, facilitariam o policiamento e seriam inibidoras de determinadas condutas como as práticas incívicas, antissociais e criminosas que os inquiridos tão bem identificaram. Tornar melhor ainda o Centro Histórico do Porto é, real e efetivamente, a grande meta destes estudos que integram o projeto LookCrim.

\section{REFERÊNCIAS}

Bardin, L. (2004). Análise de conteúdo (3ª ed.; L. Reto \& A. Pinheiro, Trad.). Edições 70. (Original publicado em 1977).

Crowe, T. (2000). Crime Prevention Through Environmental Design: Applications of architectural design and space management concepts. Butterworth-Heinemann.

Direcção Geral de Administração Interna (2009). Manual de diagnósticos locais de segurança. Uma compilação de normas e práticas internacionais (M. Correia, Trad.). Ministério da Administração Interna. https://efus.eu/files/fileadmin/efus/Publications/Manual_Seguranca_miolo_166pp_23_12_09.pdf

European Forum for Urban Safety (2007). Guidance on Local Safety Audits: A compendium of international practice. European for Urban Safety. https://www.saferspaces.org.za/uploads/files/EFUS_Safety_Audit_e_WEB.pdf 
Fernandes, L., \& Rêgo, X. (2011). Por onde anda o sentimento de insegurança? Problematizações sociais e científicas do medo à cidade. Etnográfica Revista do Centro em Rede de Investigação em Antropologia, 15(1), 167-181. https://doi.org/10.4000/etnografica.869

Jeffery, C. (1971). Crime Prevention Through Environmental Design. Sage Publications. https://doi.org/10.1177/000276427101400409

Jeffery, C. (1999). CPTED: Past, present, and future. 4th Annual International CPTED Association Conference, Ontario.

Lab, S. (2014). Crime prevention. Aproaches, practices, and evaluations (8 $8^{\mathrm{a}}$ ed). Anderson Publishing. https://doi.org/10.4324/9781315721927

Ministério da Administração Interna. (2018). Relatório Anual de Segurança Interna 2017. https://www.portugal.gov.pt/download-ficheiros/ficheiro.aspx?v=9f0d7743-7d45-40f3-8cf2e448600f3af6

Ministério da Administração Interna. (2019). Relatório Anual de Segurança Interna - 2018. https://www.portugal.gov.pt/download-ficheiros/ficheiro.aspx?v=ad5cfe37-0d52-412e-83fb7f098448dba7

National Crime Prevention Council (2003). Crime Prevention Through Environmental Design Guidebook. National Crime Prevention Council.

Newman, O. (1972). Defensible space: People and design in the violent city. Macmillan.

Nunes, L., \& Caridade, S. (s/d). Grelha de Registo de Observações Prevenção do Crime pela Gestão de Espaços (PCGE). Instrumento não publicado.

Sani, A., \& Nunes, L. (2013). Questionário de diagnóstico local de segurança: Estudo numa comunidade urbana. Análise Psicológica, 2(31), 185-195. https://doi.org/10.14417/ap.609

Sani, A., \& Nunes, L. (2017). Diagnóstico Local de Segurança no bairro militar da Guiné-Bissau - Em busca de esquadras adequadas. In L. Nunes, A. Sani, R. Estrada, F. Viana, S. Caridade, \& R. Maia (Coords.). Crime e segurança nas cidades contemporâneas (pp. 51-72). Fronteira do Caos.

Wilson, J., \& Kelling, G. (1982). Broken windows. The police and neighborhood safety. The Atlantic Online. https://www.ncjrs.gov/App/Publications/abstract.aspx?ID=185996

$\begin{array}{lr}\text { Historial do } & \text { artigo } \\ \text { Recebido } & 06 / 2019 \\ \text { Aceite } & 03 / 2020 \\ \text { Publicado } & 08 / 2020\end{array}$


Revista PSICOLOGIA, 2020, Vol. 34 (1). doi: 10.17575/psicologia.v34i1.1681

Atas do X Simpósio Nacional de Investigação em Psicologia

\title{
Teaching styles and burnout: A short-term longitudinal study
}

\author{
Lénia Carvalhais ${ }^{4 i}$, Carolina Carlos ${ }^{\text {ii }}$ \& Paula Vagos ${ }^{\mathrm{i}}$ \\ ${ }^{i}$ Departamento de Psicologia e Educação, Universidade Portucalense Infante D. Henrique \\ ii Departamento de Educação e Psicologia, Universidade de Aveiro
}

\begin{abstract}
The aim of this short-term longitudinal study was to investigate the associations between teaching styles and burnout. The participants, 73 teachers, were asked to answer the Teaching Styles Questionnaire and the Cuestionario de Burnout del Professorado twice, with a three-months gap; both instruments were presented in their Portuguese version. Results point to the stability of teaching styles and lowered personal accomplishment, but not of emotional exhaustion. Also, we found negative associations between authoritative teaching and burnout and positive associations between permissive teaching and burnout. Only the lowered personal accomplishment facet of burnout was significantly predicted by authoritative and permissive teaching. The implications of this study suggest the importance of preventing burnout, namely by aiding teachers in developing more efficacious teaching styles (e.g., authoritative). The authoritative style is considered as ideal, having in mind its correlation with higher levels of work accomplishment and lower levels of emotional exhaustion.
\end{abstract}

\section{Keywords: Teaching styles; Burnout; Emotional exhaustion; Personal accomplishment.}

Estilos de ensino e burnout: Um estudo longitudinal de curta duração: 0 objetivo deste estudo longitudinal de curto prazo foi investigar as associações entre estilos de ensino e burnout. Aos participantes, 73 professores, foi solicitado que respondessem ao Questionário de Estilos de Ensino e ao Cuestionario de Burnout do Professorado duas vezes, com um intervalo de 3 meses; ambos os instrumentos foram apresentados na sua versão portuguesa. Os resultados apontam para a estabilidade dos estilos de ensino e da dimensão 'falta de realização pessoal' do burnout, mas não do desgaste emocional. Além disso, encontrámos associações negativas entre ensino autoritativo e burnout e associações positivas entre ensino permissivo e burnout. Apenas a faceta do burnout respeitante a falta de realização pessoal foi significativamente prevista pelo ensino autoritário e permissivo. As implicações deste estudo sugerem a importância de prevenir o burnout, nomeadamente auxiliando os professores no desenvolvimento de estilos de ensino mais eficazes (por exemplo, autoritativos). 0 estilo autoritativo é considerado ideal, tendo em vista sua correlação com níveis mais elevados de realização profissional e menores níveis de exaustão emocional.

Palavras-chave: Estilos de ensino; Burnout; Exaustão emocional; Realização pessoal.

Over the last decades, there has been an increasing research focus on teachers' burnout (i.e., teachers' experience of emotional exhaustion, depersonalization, and perceived lowered personal accomplishment), which impacts on their professional activity (Aloe, Amo, \& Shanahan, 2014; Butler, 2007; Evers, Tomic, \& Brouwers, 2004; Lauermann \& König, 2016; Maslach, 2003). Teachers tend to have higher levels of burnout than other workers in the field of human services (Maslach, Jackson, \& Leiter, 1996), which consists in one of the reasons for leaving the profession (Aloe et al., 2013). Estimates in the Europe are that 25\%-30\% of teachers experience burnout (Tschiesner, Tauber, Martina, \& Farneti, 2014). The increasing diversity in school context, the decreasing levels of parental involvement, and major changes in school policies are potential factors affecting teacher burnout (Aloe et al., 2014). It may also be the case that teachers feel unprepared to deal with classroom management (Melnick \& Meister, 2008). Results from a multivariate meta-analysis on sixteen studies showed that there is a significant relation between classroom management self-efficacy and burnout; alternatively, when teachers have a greater sense of classroom management self-efficacy, they also feel more accomplished (Aloe et al., 2014).

Classroom management may be practiced through different teaching styles, which may be conceptualized based on the three parental styles. The parents' practices are classified as permissive, authoritarian, and authoritative and the differences between categories are based on how parents control children's behavior (Baumrind, 1966). Little control is applied by permissive parents in opposition to

${ }^{4}$ Correspondence address: Lénia Carvalhais, R. Dr. António Bernardino de Almeida 541, 4200-072 Porto. E-mail: leniac@upt.pt 
authoritarian parents who apply rigid rules and expect children to obey them without questioning or else they will get punished. Authoritative parents implement rules but explain to their children the basis for these rules and expect children to internalize the standards of behavior instead of acting based on fear of punishment. Recent studies propose that teaching styles may be considered based on the same typology either when considering the perspective of college students (Bassett, Snyder, Rogers, \& Collins, 2013) or the perspective of teachers (Barni, Russo, \& Danioni, 2018; Carlos, Carvalhais, \& Vagos, 2020 ).

The association between diverse ways of managing classroom, based on parenting styles (Baumrind, 1966), and burnout has not, however, to our knowledge, been previously investigated. By applying the framework of parenting styles to classroom management (Bassett et al., 2013), three teaching styles may be contrasted: authoritative, authoritarian, and permissive. Authoritative teachers show interest and affection for their students, and place limits while encouraging the students' independence. The authoritarian teacher expects that students pay attention at classes as the way to acquire knowledge and act according to a restrictive discipline. The permissive teacher disconnects from students, their needs and classroom management (Barni et al., 2018). The authoritative teaching style has been associated with better outcomes among elementary and middle school students (Walker, 2009; Wentzel, 2002) and university students (Bassett et al., 2013). Still, little is known about how specific classroom management styles may associate with burnout among teachers over time.

The present study explored the relationship between teacher's classroom management styles and teachers' burnout using a short-term (i.e., three months) longitudinal study. We expected teaching styles to be stable over this period. In addition, we expected that teaching styles predicted burnout over time. Specifically, we expected that an authoritative style would predict less burnout, whereas an authoritarian or permissive style would predict higher burnout.

\section{METHOD}

\section{Participants}

Participants were 73 Portuguese teachers, mostly female $(n=66,90.4 \%)$ and married $(n=58,79.5 \%)$. They taught compulsory grades in public schools in the north and center regions of Portugal for an average of 24.28 years $(S D=6.66)$; men and women had, on average, similar years of past teaching experience. Participants' age ranged from 34 to 61 years old $(M=24.29, S D=6.64)$; men were significantly, $t(71)=53,407, p<.001$, older $(M=52.43, D P=6.87)$ then women $(M=48.18, D P=6.87)$. Most of these teachers $(n=69,94.5 \%)$ referred they were not at the time of the study or had in the past received psychological counselling. All participants filled in the research instruments in two time-points (cf. Instruments and Procedure section respectively).

\section{Measurements}

Teaching Styles Questionnaire (TSQ). The TSQ intends to address three teaching styles (i.e., authoritarian, authoritative, and permissive) and results from adapting the Parenting Styles and Dimensions Questionnaire (Robinson, Mandleco, Olsen, \& Hart, 2001; Portuguese version Pedro, Carapito, \& Ribeiro, 2015) to a school context. Preliminary analyses on its internal structure provides evidence on a 21-item three-style factor structure and on the reliability and construct validity of those factors (Carlos et al., 2020 ). At time 1 and 2 of data collection, respectively, the internal consistency of those teaching styles were: $\alpha=.85$ and .86 for authoritative, $\alpha=.55$ and .16 for authoritarian, and $\alpha=.57$ and .67 for permissive; due to its unacceptable internal consistency value, data for time 2 for the authoritarian style was not considered in the current work.

Questionário de Burnout para Professores (CBP-R). The CBP-R (Moreno-Jiménez, Hernández \& Gutiérrez, 2000; Portuguese version by Patrão, Rita \& Maroco, 2012) intends to assess stress and burnout in teachers. It consists of 66 items that are organized into 6 dimensions, only one of which (i.e., burnout) was psychometrically validated by Patrão, Rita, and Maroco (2012) with a Portuguese sample. This work confirmed a three-factor structure for burnout, namely emotional exhaustion, lowered personal accomplishment and depersonalization, and adequate reliability of those three-factors; the burnout dimension and its factors were the ones used in the current work. Emotional exhaustion represents a personal level of burnout and comprises feelings of being emotionally and physically overwhelmed, unable to use other resources to cope with demanding tasks and time pressure. Depersonalization refers to a social level of burnout and is characterized by intentional detachment from others, namely colleagues or students. The lowered personal accomplishment withstands a professional level of burnout and consists of a sense of inefficacy and a perceived lowered achievement of professional goals (Aloe et al., 2014; Maslach et al., 2001). The emotional exhaustion ( $\alpha=.81$ at Time $1 ; \alpha=.79$ at Time 2$)$ and lowered 
personal accomplishment ( $\alpha=.77$ at Time $1 ; \alpha=.79$ at Time 2$)$ were considered for the present study. The depersonalization factor was not considered given that it presented internal consistency values lower than .50 .

\section{Procedure}

The sample was recruited based on geographic convenience. After the school board had approved the study, participants were approached individually in order to explain the main goals and procedures of the study and ask for informed consent and voluntary and confidential participation. Using a short-term longitudinal study, teachers filled in the questionnaire at times 1 and 2, between which occurred a threemonth gap. One hundred and twenty-five questionnaires were distributed in the first data collection moment (i.e., time 1), of which 115 were returned. In the second moment of data collection (i.e., time 2), 115 questionnaires were distributed but only 73 were returned; participants who are engaged in both data collection moments comprised the sample for the current work.

Non-parametric analyses were used via the IBM SPSS 24. Specifically, we conducted mean level comparisons between times 1 and 2, correlated data collected from times 1 and 2, and performed hierarchical regression analyses. For these last analyses, the dependent variables were each burnout dimension taken from time 2 , whereas the independent variables were the teaching styles taken from time 1.

\section{RESULTS}

\section{Stability of teaching styles and burnout over time}

No significant differences were found between measures of teaching styles over time (i.e., $z=-.09, p=.92$ for authoritarian and $z=-.51, p=.61$ for permissive ${ }^{5}$ ). No differences were likewise found for lowered personal accomplishment, $z=-1.03, p=.30$, but emotional exhaustion seems to significantly decrease over time, $z=2.28, p=.02(M=20.49, S D=6.74$ at time 1 and $M=19.38, S D=5.81$ at Time 2$)$.

\section{Associations between teaching styles and burnout over time}

Teaching styles taken from time 1 showed large correlation to themselves as taken from time 2 (i.e., $r_{s}=$ $.64, p<.001$ for authoritarian and $r_{s}=.63, p<.001$ for permissive); the same was found for emotional exhaustion, $r_{s}=.86, p<.001$, and lowered personal accomplishment, $r_{s}=.81, p<.001$. Furthermore, the authoritative teaching style at time 1 correlated negatively with emotional exhaustion, $r_{s}=-.26, p=.027$, and lowered personal accomplishment, $r_{s}=-.26, p=.028$, at time 2 , whereas the permissive teaching style at time 1 correlated positively with emotional exhaustion, $r_{s}=.27, p=.023$, and lowered personal accomplishment at time $2, r_{s}=.37, p=.001$.

Emotional exhaustion at time 2 was not significantly predicted by teaching styles at time $1, r^{2}=.10$, $F(3,72)=2.65, p=.055$. Lowered personal accomplishment at time 2 was predicted by teaching styles at time $1, r^{2}=.17, F(3,72)=4.79, p=.004$; the authoritative, $\beta=-.25, p=.027$, and permissive, $\beta=.34, p=$ .006 , styles were significant predictors, whereas the authoritarian style was not, $\beta=-.01, p=.91$. Multiple regression equation is 'Lowered personal accomplishment at time $2{ }_{i}^{\prime}=21.195+(-0.163$ 'authoritative teaching style $\left.{ }_{i}^{\prime}\right)+\left(1.033\right.$ 'permissive teaching style $\left.{ }_{i}^{\prime}\right)+\left(-0.038{ }^{\prime}\right.$ authoritarian teaching style $\left.{ }_{i}^{\prime}\right)+4.319$.

\section{DISCUSSION}

The current work intended to address the stability of teaching styles and burnout over a three-month period, and to investigate the impact that different teaching styles might have on experiencing burnout over time. Though burnout has been associated with several professional risk factors, its association with classroom management practices thought of as mirroring the styles of parent's interactions with children (Baumrind, 1966) in particular has not, to our knowledge, been previously addressed.

Our findings point to teaching styles being stable over time; in other words, teachers seem to employ similar classroom management behaviors throughout the school year. Even if teachers' preference for management practices may vary according to cultural and social contexts or students' age and grade level (cf. Uibu \& Kikas, 2014), management styles as a pattern of behavior seem to be relatively stable. The manner of teaching is expressed by a style and could be considered as 'the image of character' of each teacher (Jarvis, 2002).

Similarly, teachers seem to experience similar levels of lowered personal accomplishment, though they seem less emotionally exhausted as the end of the school year approaches. Though burnout seems to be relatively stable over time (Peiró, González-Romá, Tordera, \& Mañas, 2001), its emotional exhaustion facet may be more dependent on contextual changes, namely, in the current case, the moment of data

\footnotetext{
${ }^{5}$ Similarly, no significant differences emerged for the authoritarian style $(z=-1.28, p=.20)$.
} 
collection. In other words, teacher may feel more exhausted from the beginning of the school year, which requires adaptations to new students and, in some cases, to new contents, to the middle of the school year, when those adaptations may be well underway and thus, be less demanding and provoke less stress. The assumption that emotional exhaustion may be more circumstance dependent (e.g., quantity of work experienced at a given time and/or time pressure) (Gomes \& Quintão, 2011; Maslach, Schaufeli, \& Leiter, 2001) then lowered personal accomplishment may also underlie it not been significantly predicted by teaching styles.

Our results indicate a negative association between being an authoritative teacher and experiencing burnout; being such a teacher significantly predicted experiencing less lowered personal accomplishment. So, it may be the case that the benefits of this teaching style are not reflected only on better academic and personal outcomes among students from elementary to university grades (Bassett et al., 2013; Ertesvåg, 2011; Walker, 2009), but also on the mental health of teachers. Particularly, because an authoritative teacher is invested in managing its class, it may be the case that when teachers feel that they can manage the classroom, they also feel more accomplished (Aloe et al., 2014). Alternatively, we found that being a permissive teacher associated with higher levels of burnout, particularly in what concerns lowered personal accomplishment. The permissive teacher is not engaged with the classroom environment nor with her/his students' learning (Barni et al., 2018); in a sense, he/she may have given up on managing his/her class, due to low self-efficacy beliefs, which in turn can lead to higher levels of burnout (Aloe et al., 2014). It is also worth mentioning that teachers who experience burnout are more likely to create negative environments for their students (Lambert, McCarthy, O'Donnell, \& Wang, 2009).

There were some limitations to this study that should be noticed. First, larger samples than in the present study may be necessary to add statistical power to the current analyses. The sample could also be more heterogeneous, namely concerning gender, as almost all our participants are women. Furthermore, relying on methods other than self-report may be useful, namely for understanding the associations between teaching styles (as they are manifested and observed by others, and not only self-perceived), teachers' mental health (namely but not restricted to burnout), and students' outcomes.

Nevertheless, the present short-term longitudinal study extends previous research addressing teaching styles and burnout, suggesting that more positive (i.e., authoritative) and less negative (i.e., permissive) styles may contribute to experiencing less burnout. Professional burnout may result from a mismatch between job demands and available resources to cope with these demands (Lauermann \& König, 2016; Maslach et al., 2001), and so may be responsive to individual, group and/or organizational interventions (Gil-Monte, 2003). Though our findings support the relevance of personal interventions, peer support and a shared and participative environment should not be overlooked, as they could also be important to lighten the burden usually described by teachers.

\section{REFERENCES}

Aloe, A. M., Amo, L. C., \& Shanahan, M. E. (2014). Classroom management self-efficacy and burnout: a multivariate meta-analysis. Educational Psychology Review, 26, 101-126. https://doi.org/10.1007/s106448-013-9244-0

Barni, D., Russo, C., \& Danioni, F. (2018). Teachers' values as predictors of classroom management styles: a relative weight analysis. Frontiers in Psychology, 9. https://doi.org/ 10.3389/fpsyg.2018.01970

Bassett, J., Snyder, T., Rogers, D., \& Collins, C. L. (2013). Permissive, authoritarian, and authoritative instructors: applying the concept of parenting styles to the college classroom. Individual Differences Research, 11(1), 1-11.

Baumrind, D. (1966). Effects of authoritative parental control on child behavior. Child Development, 37, 887-907. https://doi.org/10.2307/1126611

Butler, R. (2007). Teachers' achievement goal orientations and associations with teachers' help seeking: Examination of a novel approach to teacher motivation. Journal of Educational Psychology, 99(2), 241-252. https://doi.org/ 10.1037/0022-0663.99.2.241

Carlos, C., Carvalhais, L., \& Vagos, P. (in press). Development and preliminary psychometric study of the Teaching Styles Questionnaire.

Ertesvåg, S. K. (2011). Measuring authoritative teaching. Teaching and Teacher Education, 27, 51-61. https://doi.org/ 10.1016/j.tate.2010.07.002

Evers, W. J. G., Tomic, W., \& Brouwers, A. (2004). Burnout among teachers-students' and teachers' perceptions compared. School Psychology International, 25, 131-148. https://doi.org/ $10.1177 / 0143034304043670$

Gomes, A., \& Quintão, S. (2011). Burnout, satisfação com a vida, depressão e carga horária em professores. Análise Psicológica, 2(XXIX), 335-344. https://doi.org/10.14417/ap.56 
Jarvis, P. (2002). Teaching styles and teaching methods. In The theory and practice of teaching, ed. P. Jarvis, 22-38. Kogan Page.

Lambert, R. G., McCarthy, C., O’Donnell, M., \& Wang, C. (2009). Measuring elementary teacher stress and coping in the classroom: validity evidence for the Classroom Appraisal of Resources and Demands. Psychology in the Schools, 46, 973-988. https://doi.org/ 10.1002/pits.20438

Lauermann, F., \& König, J. (2016). Teachers' professional competence and wellbeing: understanding the links between general pedagogical knowledge, self-efficacy and burnout. Learning and Instruction, 45, 9-19. https://doi.org/ 10.1016/j.learninstruc.2016.06.006

Maslach, C., Jackson, S. E., \& Leiter, M. P. (1996). Maslach Burnout Inventory (3 ${ }^{\text {rd }}$ ed.). Consulting Psychologists Press.

Maslach, C., Schaufeli, W. B., \& Leiter, M.P. (2001). Job burnout. Annual Review of Psychology, 53, $397-$ 422. https://doi.org/ 10.1146/annurev.psych.52.1.397

Maslach, C. (2003). Job burnout: new directions in research and intervention. Current Directions in Psychological Science, 12, 189-192. https://doi.org/ 10.1111/1467-8721.01258

Melnick, S. A., \& Meister, D. G. (2008). A comparison of beginning and experienced teachers' concerns. Educational Research Quarterly, 31(3), 39-56.

Moreno-Jiménez, B., Hernández, E. G., \& Gutiérrez, J. L. G. (2000). La evaluación del estrés y el burnout del profesorado: el CBP-R. Revista de Psicología del Trabajo y las Organizaciones, 16(1), 331349.

Patrão, I., Rita, J., \& Maroco, J. (2012). Avaliação do burnout em professores: Contributo para o estudo de adaptação do CBP-R. Psychology, Community \& Health, 1(2), 179-188. https://doi.org/10.5964/pch.v1i2.29

Pedro, M., Carapito, E., \& Ribeiro, T. (2015). Parenting Styles and Dimensions Questionnaire - Versão portuguesa de autorrelato. Psicologia: Reflexão e Crítica, 28(2), 302-312. https://doi.org/ 10.1590/1678-7153.201528210

Peiró, J. M., González-Romá, V., Tordera, N., \& Mañas, M. A. (2001). Does role stress predict burnout over time among health care professionals? Psychology \& Health, 16(5), 511-525. https://doi.org/ 10.1080/08870440108405524

Robinson, C., Mandleco, B., Olsen, S., \& Hart, C. (2001) The Parenting Styles and Dimensions Questionnaire (PSDQ). In B. F. Perlmutter, J. Touliatos \& G. W. Holden (Eds.), Handbook of family measurement techniques (Vol. 3. Instruments and index, pp. 319-321). Sage.

Tschiesner, R., Tauber, S., Martina, P., \& Farneti, A. (2014). Pupils' Interpersonal Problems and Occupational Stress in Teacher. Preliminary Results. Procedia - Social and Behavioral Sciences, 140, 197-199. https://doi.org/ 10.1016/j.sbspro.2014.04.409

Uibu, K., \& Kikas, E. (2014). Authoritative and authoritarian-inconsistent teachers' preferences for teaching methods and instructional goals. Education 3-13, 42(1), 5-22. https://doi.org/ 10.1080/03004279.2011.618808

Walker, J. M. T. (2009). Authoritative classroom management: how control and nurturance work together. Theory into Practice, 48, 122-129. https://doi.org/ 10.1080/00405840902776392

Wentzel, K. R. (2002). Are effective teachers like good parents? Teaching styles and student adjustment in early adolescence. Child Development, 73, 287-301. https://doi.org/ $10.1111 / 1467-8624.00406$

Historial do artigo

Recebido $\quad 07 / 2019$

Aceite $\quad 04 / 2020$

Publicado 08/2020 
Revista PSICOLOGIA, 2020, Vol. 34 (1). doi: 10.17575/psicologia.v34i1.1681

Atas do X Simpósio Nacional de Investigação em Psicologia

\title{
Development and preliminary psychometric study of the student version of the Teacher-Student Relationship Scale
}

\author{
Rafaela Maia6i, Lénia Carvalhaisi ${ }^{\mathrm{i}}, \&$ Paula Vagos ${ }^{\mathrm{i}}$ \\ iDepartamento de Psicologia e Educação, Universidade Portucalense Infante D. Henrique
}

\begin{abstract}
This study developed a version for students of the Teacher-Student Relationship Scale and evaluated its psychometric characteristics. In line with its teacher version, this student version intends to evaluate conflict and closeness as dimensions characterizing teacher-student interactions. Qualitative evaluation (i.e. via thinking aloud) of the instrument with a group of eight 7 th grade students showed that the instrument had good facial validity. The instrument was then applied to 297 students, boys and girls, attending the 7th trough 9th grade. The two-factor internal structure of the instrument was confirmed, and both factors had good internal consistency values; furthermore, this measurement model proved invariant by sex. This instrument will allow (re)addressing the student's perspective on his / her relationship with the teacher and may allow further work into how this perception can impact on several aspects of the intra and interpersonal functioning of both student and teacher.
\end{abstract}

\section{Keywords: Teacher; Student; Relationship; Validation; Psychometrics.}

Desenvolvimento e estudo psicométrico preliminar da versão do aluno da Escala Relação Professor-Aluno: Este estudo desenvolveu uma versão para alunos da Escala de Relação Professor Aluno e avaliou as suas características psicométricas. Coincidente com a versão do instrumento dirigida ao professor, a versão para o aluno pretende avaliar as dimensões do conflito e proximidade presentes nas interações entre o professor e o aluno. A versão do aluno foi qualitativamente avaliada (i.e., via reflexão falada) e apresentou boa validade facial. 0 instrumento foi, então, aplicado a 297 alunos do sétimo ao nono ano de escolaridade, de ambos os sexos. Confirmou-se uma estrutura interna do instrumento em dois fatores, ambos com boa consistência interna; este modelo de medida mostrou também ser invariante por sexo. Este instrumento permitirá (re)conhecer a perspetiva do aluno sobre a sua relação com o professor, bem como explorar a forma como esta perceção pode ter impacto em diversos aspetos do funcionamento intra e interpessoal do aluno e do professor.

Palavras-chave: Professor; Aluno; Relação; Validação; Psicometria.

The teacher-student relationship plays an essential role in the achievement of academic success and the development of social and behavioral skills (Patrício, Barata, Calheiros, \& Graça, 2015). Still, this may depend on the quality of the established relationship, which in turn depends on what both teacher and student bring into the day-to-day interactions (i.e. teacher's support and concern, students respect; Hughes, 2012). Besides transmitting knowledge, teachers must also invest in building empathic relationships with students that may contribute to students' holistic development and success (Brait, Macedo, Silva, Silva, \& Souza, 2010). Such relationship is associated with several academic success factors (e.g., academic motivation, involvement in the school, diminished behavioral problems, development of positive relationships and of socio-emotional competencies; Gomes, 2012), and with positive peer relationships (Hughes, 2012).

The quality of the teacher-student relationship has been conceptualized as organized into two dimensions, namely conflict and closeness, which may have an impact on internalizing or externalizing behavior trajectories of students (Hughes, 2012). Conflict, as negative, unpredictable and unpleasant interactions, relates to a problematic relationship process between the teacher and the student, which tends to exacerbate over time. Closeness refers to positive affect and communication between teacher and student; the greater the communication of positive affection, the greater the closeness between teacher and student (Tsigilis, Gregoriadis, \& Grammatikopoulos, 2017).

The conflict and closeness dimensions are evaluated by the Teacher-Student Relationship Scale (Pianta, 1992), which intends to consider the perspective of teachers about the quality of their

${ }^{6}$ Correspondence address: Paula Vagos, R. Dr. António Bernardino de Almeida, 541, 4200-072, Porto. E-mail: pvagos@upt.pt 
relationship with each student. This instrument has been used extensively and in diverse cultural contexts, namely Portugal and Brazil. Psychometric investigation into its Portuguese version concurs with a two-factor (i.e., conflict and closeness) structure, with both constructs presenting good internal consistency values (Patrício, Barata, Calheiros, \& Graça, 2015). This study points to the fact that teachers evaluated their relationships with their students as being based on more closeness then conflict. Similar psychometric results were found for the Brazilian version concerning its internal structure and internal consistency indicators (Petrucci, Borsa, Barbosa, \& Koller, 2014). Furthermore, Petrucci and collaborators (2014) considered students' sex differences and reported that teachers tend to evaluate their relationships with female students as closer than their relationship with male students.

The literature give greater emphasis to the teacher's perspective on his/her relationship with the students (e.g., Barbosa, Campos, \& Valentim, 2011; Baker, Grant, \& Morlock, 2008; Runions\& Shaw, 2013; Tsigilis, Gregoriadis, \& Grammatikopoulos, 2017) than to the students' perspective of this relationship. The perspective of students, especially the older ones (i.e., adolescents), may be particularly relevant, because it is their perception on what the teacher expects from them. The way teachers relate to students them may also have an increased impact on their behavior and academic investment (Martinelli, Schiavoni, \& Bartholomeu, 2009). Moreover, being able to assess the teachers and the students perspectives on that relationship using similar constructs might prove useful for unequivocally investigating the mutual impact they have on each other's behavioral patterns, which has been point out, particularly for disruptive behavior (Maia, 2009; Nelson \& Roberts, 2000)

Thus, the aim of this study was to develop and psychometrically evaluate a student version of the Teacher-Student Relationship Scale. Alike the original teachers' version, we expect to find confirmation on the instrument evaluating the dimensions of conflict (unpleasant, negative and unpredictable interactions with the teacher) and closeness (open communication and affection). Both factors are expected to present at least good internal consistency indicators. Furthermore, we tested for measurement invariance of the two-factor internal structure, which has not, to our knowledge been previously ascertained even in the case of the original teachers' version of the instrument.

\section{METHOD}

\section{Participants}

The participants of this study constitute a non-probabilistic sample of 297 students, 166 girls (55.9\%) and 131 boys (44.1\%), who attended the $7^{\text {th }}$ grade $(n=90,35.7 \%)$, 8th grade $(n=101,30.3 \%)$ and $9^{\text {th }}$ school grade (34\%). Upon data collection, participants were aged between 12 and 15 years old $(M=$ $13.47, S D=0.97)$. Most of these participants had never been retained in the same school year before $(n=$ $26,87.87 \%$ ) and a little over half of them had not been subjected to a disciplinary measure due to inappropriate behavior $(n=157,52.9 \%)$.

\section{Measurements \\ Student Version of the Teacher-Student Relationship Scale -short form [SV-TSQ-SF; Versão Aluno da Escala de Relação Professor-Aluno - Versão Aluno]. The Student Version of Teacher-Student Relationship Scale - short form is composed of 16 items and was developed to evaluate the student's perspective on the quality of the relationship with his/her teachers, thus resulting from the adaptation of the Teacher-Student Relationship Scale (Pianta, 1992; cf. Results section), which as shown to be psychometrically sound (cf. Introduction section). It contains nine items related to conflict dimension (i.e. unpleasant, negative and unpredictable student-teacher interactions) and seven items relating to closeness dimension (i.e. open communication and affection between student and teacher). Items are rated using a five-point scale, ranging from 1 (i.e., has nothing to do with what happens to me) to 5 (i.e., is exactly what happens to me).}

\section{Procedure}

Participants were recruited based on geographic convenience from two public schools in the northern region of Portugal. Schools were contacted after the General Directorate for Education approved the study; in turn, students were contacted only after the schools' boards gave permission and their legal guards gave informed consent for their participation. Students were also informed on the goals of the study and on the confidentiality, anonymity and voluntary nature of their participation; finally, they were asked verbal assent. Inclusion criteria were being between 12 and 15 years old at the time of data collection and exclusion criteria were presence of specific educational needs that might question the way the self-report instruments were understood and answered. Participants who assented and were eligible 
to the study were asked to fill in the questionnaire within their classrooms, using time made available by their teachers; participation took about 10 to 15 minutes.

A two-step approach for data analyses was taken. First, the instrument was developed, and its facial validity and understandability was investigated based on a thinking aloud strategy. Then, the psychometric properties of the instrument, namely its factorial validity and internal consistency were assessed. A confirmatory factor analyses approach was adopted for factorial validity, given that the instrument was developed to mirror closely its two-factor teacher version, which has been previously ascertain; a one-factor model was also tested for comparison purposes. Then, the measurement invariance of the best fitting model by sex was investigated; we sought for strong measurement invariance (i.e., metric and scalar invariance) as it is necessary for conducting reliable group comparisons (Dimitrov, 2010). The scores of boys and girls were then compared via latent mean comparison. Finally, the internal consistency of the instrument was also reported, based on the Cronbach alpha values. ${ }^{7}$

\section{RESULTS}

\section{Development of the Student Version of the Teacher-Student Relationship Scale - Short form}

All items were adapted to refer to the students' perspective instead of the teachers' point of view. To guarantee that the student version of the item related as closely as possible to its original intended content, we considered both the original English version of the items (Pianta, 1992), its Portuguese version (Patrício et al., 2015) and its Brazilian version (Petrucci et al.,2014). Items 4, 7, 10, 12 and 14 required the greatest attention to guarantee the content to be appropriate as considered by students ${ }^{8}$. Table 1 shows the process of adapting one item pertaining to conflict (i.e., item 14) and one item pertaining to closeness (i.e., item 7), contrasting their original English, Portuguese and Brazilian versions into an adapted Portuguese version that was afterwards subjected to a thinking aloud procedure. It should also be noted that the original items referred to a specific student, whereas we proposed a general approach to teachers (and not to any one specific teacher); this option intended answers not to be biased by students choosing to reply based on one specific teacher they particularly liked or disliked.

Table 1. Adaption of item from a teachers' perspective to a students' point of view

\begin{tabular}{ll}
\hline $\begin{array}{l}\text { Item 7 } \\
\text { English version }\end{array}$ & This student spontaneously, shares information about himself/herself. \\
Brazilian version & Essa criança compartilha espontaneamente informações sobre si mesma. \\
$\begin{array}{l}\text { Portuguese version } \\
\text { Adapted version }\end{array}$ & $\begin{array}{l}\text { Este/a aluno/a partilha espontaneamente informação acerca de si próprio/a. } \\
\text { Final version }\end{array}$ \\
$\begin{array}{l}\text { Item 14 } \\
\text { English version }\end{array}$ & Partilho informações sobre mim com os/as professores/as por vontade própria. \\
Brazilian version & Essa criança é dissimulada ou manipuladora comigo. \\
Portuguese version & Este/a aluno/a é sorrateiro/a ou manipulador/a comigo. \\
Adapted version & Os/as professores/as são enganadores ou falsos comigo. \\
Final version & Os/as professores/as mentem-me ou enganam-me. \\
\hline
\end{tabular}

The thinking aloud procedure took place in a public school in the northern region of Portugal and intended to ascertain if students understood the items as addressing their intended content. Eight 7 th grade students, four boys and four girls, were randomly selected and asked to read the instrument in its entirety and then discuss it together. After reading and exchanging ideas, students themselves suggested some changes orally so that the intended contents could be more easily understood. After this procedure, a final version of the items was achieved (cf., Table 1).

\footnotetext{
${ }^{7}$ Confirmatory factor analyses and measurement invariance analyses were conducted using the Mplus v7.4 software (Muthén \&Muthén, 2012). The Cronbach alpha values were computed using the IBM SPSS v. 24 software.

${ }^{8}$ Further information on the adaptation process for all those five items may be requested from the corresponding author.
} 


\section{Psychometric analyses on the Student Version of the Teacher-Student Relationship Scale - Short form}

Preliminary data analyses showed between 1 to 5 missing values for 20 participants, which were not missing completely at random (MCAR $\left.\chi^{2}{ }_{(193)}=296.66, p<.001\right)$ and so were dealt with via multiple imputation. Data was also found not to be multivariate normal (Mardias' $\chi^{2}{ }^{(55.79)}=2575.74$ for skewness and $\chi^{2}(341.71)=18.63$ for kurtosis with $p<.001$ ) and so the Maximum Likelihood Robust estimator was used for CFA and measurement invariance analyses.

The two-factor model portraying closeness and conflict was found to be a better fit for the data then the one-factor $\operatorname{model}^{9}$. However, for its fit to be acceptable for boys and girls considered separately and thus configural invariance be found, it was necessary to allow for three residual correlations ${ }^{10}$ (cf. Table 2).

Table 2. Fit indices for confirmatory factor analyses and measurement invariance analyses

\begin{tabular}{lccccc}
\hline & $\chi^{2}$ & df & RMSEA (90\% CI) & CFI & SRMR \\
\hline One-factor model & 540.84 & 104 & $.119(.109 ; .129)$ & .40 & .131 \\
Two-factor model & 267.46 & 103 & $.073(.063 ; .084)$ & .78 & .074 \\
Two-factor model modified & 198.87 & 100 & $.065(.046 ; .069)$ & .87 & .068 \\
Male participants & 150.36 & 100 & $.062(.040 ; .082)$ & .86 & .083 \\
Female participants & 194.28 & 100 & $.075(.059 ; .091)$ & .80 & .081 \\
Baseline model & 339.08 & 198 & $.069(.057 ; .082)$ & .83 & .082 \\
Full metric invariance & 357.83 & 214 & $.067(.055 ; .079)$ & .83 & .087 \\
Full scalar invariance & 395.08 & 228 & $.070(.058 ; .082)$ & .80 & .090 \\
Partial scalar invariance & 378.19 & 226 & $.067(.055 ; .079)$ & .82 & .087 \\
\hline
\end{tabular}

Note: All $\chi^{2}$ values were significant at $p<.001 . \mathrm{df}=$ degree of freedom, RMSEA $=$ Root Mean Square Error of Approximation; $\mathrm{CI}=$ confidence interval; $\mathrm{CFI}=$ Comparative Fit Index; SRMR = Standardized Root Mean Square Residual

For this modified model, loading values ranged from .380 for item 6 and .671 for item 5 for the closeness factor and from .308 for item 15 and .654 for item 8 for the conflict factor in the complete sample. For boys, loading values varied between .397 for item 7 and .681 for item 9 and between .335 for item 4 and .607 for item 8 for the closeness and conflict factors respectively. For girls, loading values varied between .356 for item 6 and .720 for item 5 and between .115 for item 15 and .713 for item 8 for the closeness and conflict factors respectively; in this case, exclusion of item 15 worsened the fit of the model.

Using the complete sample, both factors achieved good internal consistency values $(\alpha=.77$ for closeness and $\alpha=.72$ for conflict) and they were not significantly correlated $\left(r_{s}=-.02, p=.75\right)$. Similar results were found by gender: $\alpha=.78$ and $\alpha=.74$ for closeness and conflict respectively and $r_{s}=.10, p=$ .28 for boys and $\alpha=.76$ and $\alpha=.72$ for closeness and conflict respectively and $r_{s}=-.04, p=.61$, for girls.

Following configural invariance, full metric invariance was further achieved $(\triangle \mathrm{RMSEA}=.002, \Delta \mathrm{CFI}$ $=.003, \Delta \mathrm{SRMR}=.005)$; in turn, only partial scalar invariance was achieved $(\triangle \mathrm{RMSEA}=.000, \Delta \mathrm{CFI}=-.01$, $\triangle \mathrm{SRMR}=.000)^{11}$ after allowing the intercepts of items 1 and 6 to vary between male and female participants. Latent mean comparisons indicate that there are no significant differences between boys and girls on the closeness and conflict reported $(p>.05)$. Table 3 presents the descriptive values for those measures.

\footnotetext{
9 The models' fit was considered acceptable if at least two of the following adjustment indices abided by the necessary thresholds: Root Mean Square Error of Approximation (RMSEA) $\leq .08$, Comparative Fit Index (CFI) > .09, or Standardized Root Mean Square Residual (SRMR) <.10. Furthermore, lower values of RMSEA and/or SRMR and higher values of CFI were considered indicative of better fit (Schermelleh-Engel, Moosbrugger, \& Müller, 2003).

10 Residual correlations were added between items 7 and 16, both portraying conflicts, and between items 11 and 13 and items 12 and 15 , all relating to closeness. As only two items were associated at a time, the hypothesis of an underlying construct addressing the specific variance of those items was not considered plausible.

11 Criteria for deciding on invariance at the metric and scalar level were taken from the guidelines provided by Chen (2007): for metric invariance, $\Delta \mathrm{CFI}<-.01$, combined with $\triangle \mathrm{SRMR}<.03$ or with $\triangle \mathrm{RMSEA}<.03$; for scalar invariance, $\Delta \mathrm{CFI}<-.01$, combined with $\Delta \mathrm{SRMR}<.03$ or with $\triangle$ RMSEA $<.01$.
} 
Table 3. Descriptive values for the closeness and conflict measures

\begin{tabular}{lccc}
\hline & $\begin{array}{c}\text { Complete sample } \\
(\boldsymbol{N}=\mathbf{2 9 7})\end{array}$ & $\begin{array}{c}\text { Male participants } \\
(\boldsymbol{n}=\mathbf{1 3 1})\end{array}$ & $\begin{array}{c}\text { Female participants } \\
(\boldsymbol{n}=\mathbf{1 6 6})\end{array}$ \\
\hline Closeness & $20.46(5.33)$ & $20.80(5.62)$ & $20.20(5.11)$ \\
Conflict & $16.12(5.33)$ & $16.36(5.25)$ & $15.93(5.40)$ \\
\hline
\end{tabular}

Note: Results are presented as $M(S D)$

\section{DISCUSSION}

Teacher-student relationship may have a noteworthy impact on the psychosocial and academic performance of students, particularly in its conflict or closeness dimensions (Patrício at al., 2015). Still, to our knowledge, only the teachers' point of view has been considered, leaving out the students' perspective on how this interaction unfolds. Because human beings react to the way they perceive their world (more than to its objective characteristics) it may be the case that students' perspective impact more strongly on their behavior then the teachers' point of view (Martinelli et al., 2009). The current work sought to develop and psychometrically evaluate an instrument to assess students' perspective, based on an instrument concerning the teachers' point of view that had been proven sound (i.e., StudentTeacher Relationship Questionnaire; e.g., Patrício et al., 2015, Petrucci et al., 2014; Pianta, 1992; Tsigilis et al., 2017).

In line with the version of the instrument intended for teachers, the student version of the Teacher-Student Relationship Scale was found to be organized into two dimensions (i.e., conflict and closeness) as main descriptors of the teacher-student relationship; both achieved good internal consistency values. Those dimensions were not significantly associated, though its correlation value was negative; previous works with teachers' samples show not only a negative but also a significant correlation (Patrício et al., 2015; Petrucci et al., 2014; Pianta, 1992). These findings seem to point to some specific perspectives, depending on the respondent, which should be further analyzed. This may be particularly relevant in the case of boys, given that, even if non-significant, the correlation between conflict and closeness was positive.

Gender-based measurement invariance of the two-factor structure had not been previously addressed in relation to teacher-student relationships. We found evidence on such invariance, which allows greater confidence in gender-based comparisons (Chen, 2007). Our findings indicate that boys and girls report similar levels of conflict and closeness to their teachers. This is not in line with previous works based on the teachers' perspective that show that teachers perceive to relate differently to their male and female students (Patrício et al., 2015; Petrucci et al., 2014; Pianta, 1992). These previous studies did not consider the teachers' gender as in our study, which may sustain future works. The current work used a relatively small sample size, which was restricted to the $7^{\text {th }}$ to $9^{\text {th }}$ grade students, and did not gather data on which to further evaluate the construct validity of the instrument. Future works may consider using larger and more diverse samples. Other ways of assessing student-teacher relationships (e.g., observation) may also be useful for establishing the construct validity of the student (and teacher) versions of the instrument.

This study provides preliminary evidence on an appropriate tool to evaluate the student's perspective, as he/she may be the most credible rapporteur of his/her behavior. Specifically, the student version of the Student-Teacher Relationships Scale was found to have a theoretically relevant and consistent internal structure that is applied to both boys and girls. Such an instrument was not previously available and may prove useful in further exploring several aspects relating to teacher-student relationship, namely how it affects the psychosocial development, social behavior, or academic performance of students. Promoting higher quality teacher-student relationships may come to be conceptualized as a way of improving all of those indicators, aiming for the holistic development of students.

\section{REFERENCES}

Barbosa, A. J. G., Campos, R. A., \& Valentim, T. A. (2011). A diversidade em sala de aula e relação professoraluno [Diversity in the classroom and the teacher-student relationship]. Estudos de Psicologia, 28, 453-461. https://doi.org/10.1590/S0103-166X2011000400006

Baker, J. A., Grant, S., \& Morlock, L. (2008). The teacher-student relationship as a developmental context for children with internalizing or externalizing behavior problems. School Psychology Quarterly, 23, 3-15. https://doi.org/10.1037/1045-3830.23.1.3 
Brait, L. F. R., Macedo, K. M. F., Silva, F. B., Silva, M. R., \& Souza, A. L. R, (2010). A relação professor/aluno no processo de ensino e aprendizagem. Revista Eletrónica do Curso de Pedagogia do Campus Jataí UFG, 8.

Chen, F. F. (2007). Sensitivity of goodness of fit indexes to lack of measurement invariance. Structural Equation Modeling: A Multidisciplinary Journal, 14, 464-504. https://doi.org/10.1080/10705510701301834.

Dimitrov, D. M. (2010). Testing for Factorial Invariance in the Context of Construct Validation. Measurement and Evaluation in Counseling and Development, 43, 121-149. https://doi.org/ 10.1177/0748175610373459.

Gomes, A. F. C. (2012). Perceção da interação professor-aluno como fator motivacional [Perception of the teacher-student as a motivational factor]. [Unpublished master thesis, Universidade Católica Portuguesa]

Hughes, J. N. (2012). Teacher-student relationships and school adjustment: Progress and remaining challenges. National Institutes of Health, $14, \quad 319-327$. https://doi.org/10.1080/14616734.2012.672288.

Maia, R. (2019). Comportamento agressivo e relação professor-aluno: A perspetiva do aluno [Agressive behavior and student-teacher relation: The students' perspective]. [Unpublished master thesis, Universidade Portucalense Infante D. Henrique].

Martinelli, S. C., Schiavoni, A., \& Bartholomeu, D. (2009). Propriedades psicométricas de uma escala de percepção de alunos sobre as expectativas do professor [Psychometric properties of a students' perception about the teachers' expectations scale]. Avaliação Psicológica, 8, 119-129.

Muthén, L. K., \& Muthén, B. O. (2012). Mplus User's Guide. Muthén \& Muthén.

Patrício, J. N., Barata, M. C., Calheiros, M. M., \& Graça, J. (2015). A Portuguese version of the studentteacher relationship scale - short form. Spanish Journal of Psychology, 18, 1-12. https://doi.org/ 10.1017/sjp.2015.29

Petrucci, G. W., Borsa, J. C., Barbosa, A. J. G., \& Koller, S. H. (2014). Adaptação cultural e evidências de validade da escala de relacionamento professor-aluno [Cultural adaptation and evidences of validity of the Student-Teacher Relationship Scale]. Avaliação Psicológica, 13, 133-142.

Pianta, R. C. (1992). The student-teacher relationship scale. Charlottesville.

Nelson, J. R., \& Roberts, M. L. (2000). Ongoing reciprocal teacher-student interactions involving disruptive behaviors in general education classrooms. Journal of Emotional and Behavioral Disorders, 8(1), 2737. https://doi.org/10.1177/106342660000800104

Runions, K. C., \& Shaw, T. (2013). Teacher-child relationship, child withdrawal and aggression in the development of peer victimization. Journal of Applied Developmental Psychology, 34, 319-327. https://doi.org/10.1016/j.appdev.2013.09.002

Schermelleh-Engel, K., Moosbrugger, H., \& Müller, H. (2003). Evaluating the fit of structural equation models: tests of significance and descriptive goodness-of-fit measures. Methods Psychol. Res. Online, 8(2), 23-74.

Tsigilis, N., Gregoriadis, A., \& Grammatikopoulos, V. (2017). Evaluating the student-teacher relationship scale in the Greek educational setting: an item parceling perspective. Research Papers in Education, 33, 414-426. https://doi.org/10.1080/14616734.2012.672.

Van de Schoot, R., Lugtig, P., \& Hox, J. J. (2012). A checklist for testing measurement invariance. European $\begin{array}{lllll}\text { Journal of } & \text { Developmental }\end{array}$ https://doi.org/10.1080/17405629.2012.686740

Historial do artigo

Recebido $\quad 07 / 2019$

Aceite $\quad 01 / 2020$

Publicado 08/2020 
Revista PSICOLOGIA, 2020, Vol. 34 (1). doi: 10.17575/psicologia.v34i1.1681

Atas do X Simpósio Nacional de Investigação em Psicologia

\title{
Symptomatology and social support of family of young people with nonfatal suicidal behaviours
}

\author{
Marta Sofia Ventosa Brás ${ }^{12 i}$, Tina Jardimii, Margarida Henriques ${ }^{i i i}$ \& Inês Rothes ${ }^{\text {iv }}$ \\ ${ }^{i}$ Centro de Investigação em Psicologia (CIP/UAL); Universidade do Algarve, Algarve, Portugal \\ ii Universidade do Algarve, Algarve Portugal \\ iii Universidade do Porto, Porto, Portugal \\ iv Centro de Psicologia da Universidade do Porto, CPUP, Porto, Portugal
}

\begin{abstract}
Suicidal behaviors are a serious public health problem, affecting individual, family and society. The aims of this study are to identify the effects of suicidal behaviors on different dimensions of family life and to explore differences in depressive, anxiogenic symptomatology, and levels of stress and satisfaction with social support among family members of young people with and without suicidal behavior. Participants were 107 relatives of young people with and without suicidal behavior. Self-report questionnaires were used. Descriptive analyzes and nonparametric tests of comparison of means were used. The components of life most affected by youth suicidal behaviors were family dynamics, considering positive effects and effects in health. There were no differences between family members of young people with and without suicidal behaviors in symptomatology and satisfaction with social support. Results have implications for research in suicidology and for practices and policies to prevent suicidal behaviors of young people, namely in supporting parenting.
\end{abstract}

Keywords: Suicidal behaviors; Effects on family members; Youth.

Sintomatologia e suporte social dos familiares de jovens com comportamentos suicidários nãofatais: Os comportamentos suicidários são um grave problema de saúde pública afetando o indivíduo, família e comunidade. 0 presente estudo tem como objetivos identificar os efeitos dos comportamentos suicidários na vida de familiares, e explorar a existência de diferenças na sintomatologia e satisfação com o suporte social entre familiares de jovens com e sem comportamentos suicidários. Participaram 107 familiares de jovens com e sem comportamentos suicidários. Usaram-se questionários de autorresposta. Análises descritivas e testes não paramétricos de comparação de médias foram as análises usadas. As componentes mais afetadas pelos comportamentos suicidários foram: as dinâmicas familiares - efeitos positivos e efeitos na saúde. Não se verificaram diferenças entre familiares de jovens com e sem comportamentos suicidários na sintomatologia e na satisfação com o suporte social. Os resultados têm implicações para a investigação em suicidologia e para práticas e políticas de prevenção dos comportamentos suicidários de jovens, nomeadamente na fundamentação do suporte aos pais.

Palavras-chave: Comportamentos suicidários; Efeitos nos familiares, Jovens.

Suicidal behaviours are a serious public health problem. Data provided by the World Health Organization indicate that around 800000 people die by suicide every year and nonfatal suicidal behaviours are thought to be 20 to 30 times more frequent than suicides (WHO, 2018). In young people this ratio is even higher and nonfatal suicidal behaviours are estimated to be 100 to 200 times more frequent than suicide (Bertolote \& Fleischmann, 2009).

Suicidal behaviours rate among the leading positions of the ranking of health problems when nonfatal attempts are considered. For 15 to 24 years old suicide is the second cause of years of life lost (YLL). There is an existing consensus in literature indicating that young people find it difficult to ask for help. Peers are usually referred to as the first people they ask for help, but parents and close friends are potential protective factors and mediators in seeking help (e.g., Berger, Hasking, \& Martin, 2013; Fortune, Sinclair, \& Hawton, 2008). Acknowledging that family's participation can be an important preventive

\footnotetext{
${ }^{12}$ Correspondence address: Marta Brás. Universidade do Algarve, Faculdade de Ciências Humanas e Sociais. Departamento de Psicologia e Ciências da Educação. Campus de Gambelas. 8005-139 Faro, Algarve. E-mail: mbras@ualg.pt. This work was funded by national funds through FCT - Fundação para a Ciência e a Tecnologia - as part the project CIP/UAL - Ref FEDER / COMPETE2020 POCI-01-0145-FEDER-007294 and by Research Center in Psychology(CIP/UAL).
} 
factor when it comes to the repetition of suicidal behaviours, some studies try to understand the dimensions of family experience in these situations (Curtis, Thorn, McRoberts, Hetrick, Rice, \& Robinson, 2018). This new line of investigation has been focusing on the experience of these families. These studies have mainly adopted qualitative approaches and seek to understand how parents respond and deal with the situation (Buus, Caspersen, Hansen, Stenager, \& Fleischer, 2014; Byrne et al., 2008; Ferrey et al., 2016; Greene-Palmer et al., 2015; Kelada et al., 2016; Lindgren, Aström, \& Graneheim, 2010; Oldershaw et al., 2008; Stewart, Hughes, Simkin, Locock, Ferrey, Kapur et al., 2016; Trinco, Santos, \& Barbosa, 2017).

These investigations consider these behaviours as a traumatic and stigmatising event (Buus et al., 2014; Byrne et al., 2008). They have also identified the presence of depressive symptomatology, anxiety symptoms and sleep problems in the parents (Ferrey et al., 2016; Lindgren et al., 2010). Feelings of guilt, shame, anguish and loneliness (Lindgren et al., 2010), of rage against oneself (Greene-Palmer, 2015) and failure as a parent (Daly, 2005; McDonald, O'Brien, \& Jackson, 2007) stand out. These feelings along with the fear of stigma lead to social isolation of the parents and to feelings of loss of social support (Byrne et al., 2008; Lindgren et al. 2010; McDonald et al., 2007). Most of these studies suggest that suicidal behaviours negatively affect several areas of family life (e.g. the relationship with the parents gets more distant and there is a breach in trust after the suicidal behaviour - Kelada et al., 2016; Whitlock et al., 2018). Nevertheless, some - even though fewer - studies suggest that suicidal behaviours may also have positive outcomes in family life (e.g., Kelada et al., 2016; Oldershaw et al., 2008). For example, some parents were able to develop a better relationship with their child (Kelada et al., 2016; Oldershaw et al., 2008; Whitlock et al., 2018).

When a young person has engaged in suicidal behaviours, the process of asking others (family members, friends, health professionals) for help and receiving it can be hard and difficult. Some studies indicate that the parents felt judged and criticized by their relatives (Ferrey et al., 2016). Other investigations reported similar findings concerning friends and neighbours (Asare-Doku et al., 2017; Lindgren et al., 2010; Morgan et al., 2013). This acquires special relevance as it is known that friends and family can represent a fundamental support, especially those who have already experienced similar situations (Ferrey et al., 2016). Some research reports that some parents also felt being blamed by health professionals (e.g., Trinco et al., 2017).

In short, suicidal behaviours of young people can have an important impact (both negative and positive) in several dimensions of family life. Yet, in Portugal there are few studies about these families. There is a need to learn more about these families in order to improve their protective and supportive role.

\section{OBJECTIVES}

To identify the effects of suicidal or self harm behaviours in different dimensions of family life (mothers, fathers and other close relatives).

To explore the differences in depressive symptomatology, anxiety symptoms and stress levels in relatives of young people with suicidal behaviours.

To explore the existence of differences in the satisfaction with social support in relatives of young people with and without suicidal behaviours.

\section{METHOD}

\section{Participants}

A process of convenience sampling has been adopted, and two samples have been used: relatives of young people with suicidal behaviours and relatives of young people without suicidal behaviours. To define suicidal or self harming behaviours the definition of self-harm has been adopted: intentional self poisoning or self harming, regardless of the motive and/or the level of suicidal intent (Hawton, Saunders, \& O'Connor, 2012). One hundred and seven relatives participated in the study. The sample comprising the young people with suicidal behaviours was composed of 27 participants reaching from 18 to 80 years $(M=47.26 ; S D=15.25), 18$ female (66.7\%). Nine of the relatives are mothers $(33.3 \%)$, six are fathers (22.2\%) and 12 are other close relatives (44.4\%).

The remaining 80 participants are relatives of young people with no suicidal behaviours reaching from 19 to 63 years of age. Sixty-four of them (80.0\%) are female.

\section{Measurements}

Scale of Effects of Suicidal Behaviours in Relatives (SESB-R-26). The SESB-R-26 was built in the scope of a broader investigation, comprising this study. The development of the SESB-R-26 was carried out according to the proceedings established in literature (Almeida \& Freire, 2008; Gutierrez \& Osman, 2008; 
Hill \& Hill, 2008; Moreira, 2004; Ribeiro, 1999). The items were designed and organized into six theoretical dimensions, based on the results obtained in qualitative studies with samples displaying similar characteristics (e.g., Buus et al., 2014; Ferrey et al., 2016; Greene-Palmer et al., 2015; Kelada et al., 2016). After creating the first version a panel of five judges specialised in Suicidology was consulted. According to the instructions pointed out by Gutierrez and Osman (2008) about the conception of instruments in the field of Suicidology, each jury was asked to rate the items in a 5- point Likert scale, based on the following criteria: relevance, representativeness, specificity, clarity, language and extension. In a second phase, the judges were asked to pair each item with one of the six previously designed theoretical dimensions. In a third phase, the judges were asked to give a technical opinion about the clarity of the instructions, the adjustment of the measuring scale, the global extent and suggestions. The technical opinion was favorable. Some items were altered according to suggestions. The first three times the SESB-R-26 was implemented, the participants were questioned about understanding the items and encouraged to suggest changes, but no suggestions were made. Thus, a final version of the SESB-R-26 was assembled considering 26 items rated on a Likert scale from 1 (Hasn't affected at all) to 5 (Affected very much). The six dimensions of the effects consisted in (a) life in general, (b) negative effects on family, positive effects on family, (d) social life, (e) professional life and (f) health. In 5 components, Cronbach's alpha was between .75 and .88 and in the component displaying Cronbach's alpha below .70, the mean inter-item-correlation (MIC) was .35, thus revealing a good internal consistency. SESB-R-26 revealed a satisfactory sensitivity, answers reached from the lowest to the highest level (1 to 5) in 12 items, from 1 to 4 in another 12 items and from 1 to 3 in only 2 items.

Depression Anxiety Stress Scales (DASS-21; Lovibond \& Lovibond, 1995; Pais-Ribeiro, Honrado, \& Leal, 2004). DASS- 21 is a self-administration scale aimed at assessing anxiety, depression and stress. This scale contains 21 items. In this study it obtained a high total internal consistency $(\alpha=.91)$, as well as its three sub-scales - Anxiety ( $\alpha=.83)$, Depression $(\alpha=.79)$ and Stress $(\alpha=.81)$.

Scale of Satisfaction with Social Support (ESSS; Pais-Ribeiro, 1999). It consists of a selfadministration scale with the aim of measuring satisfaction with social support. The ESSS contains 15 items. In this study it obtained a good total internal consistency $(\alpha=.80)$ and also in its four sub-scales: Satisfaction with friends $(\alpha=.74)$, Satisfaction with intimacy ( $\alpha=.57)$, Satisfaction with family $(\alpha=.85)$ and Satisfaction with social activities $(\alpha=.74)$.

A questionnaire for gathering sociodemographic data was also used.

\section{Data collection and ethical considerations}

The study protocol was approved by the Ethics Committee of the Faculty of Psychology and Educational Sciences of the University of Porto, the Hospital Magalhães Lemos, the University Hospital Center of Coimbra and the Regional Board of Health of the Algarve. All the participants signed an informed consent document after the study was explained to them. Anonymity and confidentiality of data were guaranteed.

\section{Data analysis}

Statistical analyses were carried out using the Statistical Package for the Social Sciences (IBM SPSS Statistics - Version 24). To calculate the internal consistency of the scales Cronbach's Alpha and the Mean inter-item-correlation (MIC) was used. To explore the existence of differences in the symptomatology and in satisfaction with social support the Mann-Whitney non-parametric test was used.

\section{RESULTS}

Effects of young people's suicidal behaviours in the life of relatives.

As displayed in Table 1, the component with more effects as a consequence of suicidal behaviours is positive effects on the family, followed by the dimension health, where the increase of family anxiety and changes in sleep stand out. 
Table 1. Effects of young people's suicidal behaviours in the life of relatives

\begin{tabular}{|c|c|c|c|c|c|}
\hline $\begin{array}{c}\text { Subcategories } \\
\text { (Cronbach's alfa } \\
\text { and/or MIC; M; SD) }\end{array}$ & Items & $\begin{array}{c}\text { (1) It } \\
\text { hasn't } \\
\text { affected at } \\
\text { all } \\
(N ; \%)\end{array}$ & $\begin{array}{l}\text { (2) Affected } \\
\text { slightly and } \\
\text { (3) Affected } \\
\text { somewhat } \\
(N ; \%)\end{array}$ & $\begin{array}{l}\text { (4) Affected } \\
\text { and (5) } \\
\text { Affected a } \\
\text { lot }(N ; \%)\end{array}$ & $M(S D)$ \\
\hline \multirow{3}{*}{$\begin{array}{l}\text { Life in general } \\
(\alpha=.75 ; M=2.29 \\
S D=.88)\end{array}$} & 1. It affected my life in general. & $4(15.4)$ & $15(57,7)$ & 7 (26.9) & $\begin{array}{c}2.85 \\
(1.16)\end{array}$ \\
\hline & 11. It affected my daily life. & $8(30.8)$ & $14(53.8)$ & $4(15.3)$ & $\begin{array}{c}2.38 \\
(1.17)\end{array}$ \\
\hline & $\begin{array}{l}\text { 15. It harmed my relationships } \\
\text { in general. }\end{array}$ & $15(55.6)$ & $11(40.7)$ & $1(3.7)$ & $\begin{array}{l}1.70 \\
(.91)\end{array}$ \\
\hline \multirow{11}{*}{$\begin{array}{l}\text { Negative effects on } \\
\text { family } \\
(\alpha=.83 ; M=1.86 ; \\
S D=.65)\end{array}$} & 2. It affected my family life. & $6(23.1)$ & $12(46.1)$ & $8(30.8)$ & $\begin{array}{c}2.92 \\
(1.41)\end{array}$ \\
\hline & $\begin{array}{l}\text { 4. It affected my relationship } \\
\text { with my partner. }\end{array}$ & $16(61.5)$ & $8(30.8)$ & $2(7.7)$ & $\begin{array}{l}1.62 \\
(.94)\end{array}$ \\
\hline & $\begin{array}{l}\text { 5. It affected my relationship } \\
\text { with my relative. }\end{array}$ & $12(44.4)$ & $12(44.4)$ & $3(11.1)$ & $\begin{array}{l}1.85 \\
(.99)\end{array}$ \\
\hline & $\begin{array}{l}\text { 6. It affected my relationship } \\
\text { with my child(ren)/parents. }\end{array}$ & $19(73.1)$ & $4(15.4)$ & $3(11.5)$ & $\begin{array}{c}1.58 \\
(1.07)\end{array}$ \\
\hline & $\begin{array}{l}\text { 7. It affected my relationship } \\
\text { with close relatives. }\end{array}$ & $19(73.1)$ & $5(19.2)$ & $2(7.7)$ & $\begin{array}{l}1.50 \\
(.95)\end{array}$ \\
\hline & $\begin{array}{l}\text { 12. It affected my family's } \\
\text { finances (e.g. paying } \\
\text { appointments) }\end{array}$ & $12(46.2)$ & $10(38.5)$ & $4(15.4)$ & $\begin{array}{c}2.08 \\
(1.29)\end{array}$ \\
\hline & 13. It affected the siblings. & $11(40.7)$ & $11(40.7)$ & $5(18.5)$ & $\begin{array}{c}2.30 \\
(1.33)\end{array}$ \\
\hline & $\begin{array}{l}\text { 16. It harmed my family } \\
\text { relationships. }\end{array}$ & $16(59.3)$ & $10(37.0)$ & $1(3.7)$ & $\begin{array}{l}1.67 \\
(.92)\end{array}$ \\
\hline & $\begin{array}{l}20 . \text { It made my life as a couple } \\
\text { worse. }\end{array}$ & $22(84.6)$ & $2(7.7)$ & $2(7.7)$ & $\begin{array}{l}1.69 \\
(.93)\end{array}$ \\
\hline & $\begin{array}{l}\text { 24. It put pressure/ discomfort } \\
\text { in my life as a couple. }\end{array}$ & $19(73.0)$ & 5 (19.2) & $2(7.7)$ & $\begin{array}{c}1.81 \\
(1.02)\end{array}$ \\
\hline & $\begin{array}{l}25 \text {. The relationship with my } \\
\text { relative became worse. }\end{array}$ & $22(84.6)$ & $4(15.4)$ & 0 & $\begin{array}{l}1.58 \\
(.76)\end{array}$ \\
\hline \multirow{3}{*}{$\begin{array}{l}\text { Positive effects on } \\
\text { the family } \\
(\alpha=.84 ; M=2.56 ; \\
S D=.97)\end{array}$} & $\begin{array}{l}\text { 21. It strengthened } \\
\text { relationships within the family. }\end{array}$ & $14(53.8)$ & $8(30.8)$ & $4(15.4)$ & $\begin{array}{c}2.31 \\
(1.19)\end{array}$ \\
\hline & $\begin{array}{l}22 . \text { It made my relationship } \\
\text { with my relative become } \\
\text { closer. }\end{array}$ & $13(50.0)$ & $6(23.1)$ & 7 (26.9) & $\begin{array}{c}2.65 \\
(1.02)\end{array}$ \\
\hline & $\begin{array}{l}\text { 23. It made my family more } \\
\text { united. }\end{array}$ & $12(46.2)$ & $6(23.1)$ & $8(30.7)$ & $\begin{array}{c}2.73 \\
(1.15)\end{array}$ \\
\hline \multirow{2}{*}{$\begin{array}{l}\text { Social life } \\
(\alpha=.88 ; M=1.56 ; \\
S D=.93)\end{array}$} & 3. It affected my social life. & $15(57.7)$ & $9(34.6)$ & $1(7.6)$ & $\begin{array}{c}1.77 \\
(1.11)\end{array}$ \\
\hline & $\begin{array}{l}\text { 9. It affected my relationships } \\
\text { with my friends. }\end{array}$ & $21(80.8)$ & $3(11.5)$ & $2(7.6)$ & $\begin{array}{l}1.35 \\
(.85)\end{array}$ \\
\hline $\begin{array}{l}\text { Professional life } \\
(\alpha=.43 ; M I C=.35 ; \\
M=1.47 ; S D=.53)\end{array}$ & $\begin{array}{l}\text { 8. It affected my relationships } \\
\text { with my colleagues from } \\
\text { work/school. }\end{array}$ & $22(84.6)$ & $3(11.5)$ & $1(3.8)$ & $\begin{array}{l}1.23 \\
(.65)\end{array}$ \\
\hline
\end{tabular}




\begin{tabular}{|c|c|c|c|c|c|}
\hline & $\begin{array}{l}\text { 10. It affected my relationships } \\
\text { with my superiors at work. }\end{array}$ & $22(85.6)$ & 3 (11.5) & $1(3.8)$ & $\begin{array}{l}1.19 \\
(.49)\end{array}$ \\
\hline & $\begin{array}{l}\text { 14. It negatively reflected on } \\
\text { my work/school. }\end{array}$ & $12(44.4)$ & $12(44.4)$ & 3 (11.1) & $\begin{array}{c}2.07 \\
(1.11)\end{array}$ \\
\hline \multirow{4}{*}{$\begin{array}{l}\text { Health } \\
(\alpha=.88 ; M=2.43 ; \\
S D=.96)\end{array}$} & $\begin{array}{l}\text { 17. It harmed my mental } \\
\text { health. }\end{array}$ & $8(30.8)$ & $16(61.6)$ & $2(7.6)$ & $\begin{array}{c}2.12 \\
(1.03)\end{array}$ \\
\hline & $\begin{array}{l}\text { 18. It harmed my physical } \\
\text { health. }\end{array}$ & $14(53.8)$ & $10(38.4)$ & $2(7.6)$ & $\begin{array}{c}1.77 \\
(1.07)\end{array}$ \\
\hline & $\begin{array}{l}\text { 19. It changed my sleep routine } \\
\text { (e.g. not being able to fall } \\
\text { asleep or waking up in the } \\
\text { middle of the night) }\end{array}$ & $10(38.4)$ & $10(38.4)$ & $6(23.1)$ & $\begin{array}{c}2.62 \\
(1.20)\end{array}$ \\
\hline & $\begin{array}{l}\text { 26. It made the levels of anxiety } \\
\text { in the family rise. }\end{array}$ & 5 (19.2) & $10(38.4)$ & $11(42.3)$ & $\begin{array}{c}3.23 \\
(1.14)\end{array}$ \\
\hline
\end{tabular}

Note: MIC = Mean-inter-item correlation; \% = percentage; $M=$ Mean; SD = Standard Deviation

Depressive symptomatology, anxiety symptoms and stress levels in relatives of young people with and without suicidal behaviour

As displayed in table 2, no significant statistical differences were found between family/relatives of young people with and without suicidal behaviours.

Table 2. Depressive symptomatology, anxiety symptoms and stress levels in relatives of young people with and without suicidal behaviours

\begin{tabular}{lcccccc}
\hline & $\begin{array}{c}\text { Relatives of young } \\
\text { people with suicidal } \\
\text { behaviours }(\boldsymbol{N = 2 7 )}\end{array}$ & $\begin{array}{c}\text { Relatives of young } \\
\text { people without suicidal } \\
\text { behaviours }(\boldsymbol{N}=\mathbf{8 0})\end{array}$ & $\boldsymbol{U}$ & $\boldsymbol{p}$ \\
\cline { 2 - 7 } & $M$ & $S D$ & $M$ & $S D$ & & \\
\hline Anxiety & 2.30 & 2.54 & 3.21 & 3.47 & 940.50 & .308 \\
Depression & 3.48 & 3.49 & 3.55 & 3.42 & 106300 & .902 \\
Stress & 5.52 & 3.73 & 5.25 & 3.49 & 1031.50 & .727 \\
\hline
\end{tabular}

Note. M=Mean; SD=Standard Deviation; U=Mann-Whitney Test.

Satisfaction with social support in relatives of young people with and without suicidal behaviours As displayed in table 3, no significant statistical differences were found in the satisfaction with social support in relatives of young people with and without suicidal behaviours.

\section{DISCUSSION}

This study is the first to investigate the effects of non-suicidal behaviours in Portuguese families. The only existing study to this day analysing the Portuguese context and in line with this investigation excluded self-harm behaviours with suicidal intent (Trinco, Santos, \& Barbosa, 2017). Internationally, there are few quantitative investigations that evaluate the effects of nonfatal suicidal behaviours in relatives. The existing studies do not use specific instruments to assess this event (Morgan et al. 2013).

Results from this study highlight that the dimensions which were most affected were family dynamics in their positive effects and health, namely changes in sleep and increased anxiety. No differences were found between relatives from young people with and without suicidal behaviours concerning depressive symptomatology, anxiety symptoms and stress levels, nor concerning satisfaction with social support. It was also established that the questionnaire SESB-R-26 presented satisfactory psychometric properties, although preliminary, thus encouraging further research. 
Table 3. Satisfaction with social support in relatives of young people with and without suicidal behaviours

\begin{tabular}{|c|c|c|c|c|c|c|c|}
\hline & & \multicolumn{2}{|c|}{$\begin{array}{c}\text { Relatives of young } \\
\text { people with suicidal } \\
\text { behaviours } \\
(N=27)\end{array}$} & \multicolumn{2}{|c|}{$\begin{array}{c}\text { Relatives of young } \\
\text { people without suicidal } \\
\text { behaviours } \\
(N=80)\end{array}$} & \multirow[t]{2}{*}{$\boldsymbol{U}$} & \multirow[t]{2}{*}{$p$} \\
\hline & & $M$ & $S D$ & $M$ & $S D$ & & \\
\hline \multirow[t]{5}{*}{ Total } & & 2.73 & .58 & 2.72 & .65 & 1044.00 & .796 \\
\hline & $\begin{array}{l}\text { Satisfaction with } \\
\text { friends. }\end{array}$ & 2.82 & .70 & 3.03 & .79 & 882.00 & .153 \\
\hline & $\begin{array}{l}\text { Satisfaction with } \\
\text { intimacy. }\end{array}$ & 2.72 & .88 & 2.62 & .87 & 1006.00 & .594 \\
\hline & $\begin{array}{l}\text { Satisfaction with } \\
\text { family. }\end{array}$ & 2.94 & .81 & 3.09 & 1.01 & 909.00 & .211 \\
\hline & $\begin{array}{l}\text { Satisfaction with social } \\
\text { activities. }\end{array}$ & 2.38 & 1.02 & 1.97 & 1.22 & 847.50 & .094 \\
\hline
\end{tabular}

Note. M=Mean; $S D=$ Standard Deviation; U=Mann-Whitney Test

The results of the effects of young people's suicidal behaviours on their relatives in the Portuguese context are in line with international research (e.g., Buus et al., 2014; Byrne et al., 2008; Ferrey, et al., 2016) showing that an attempted suicide is a disturbing emotional experience and that it can endanger the health and well-being and family relationships (Curtis et al., 2018; Ferrey et al., 2016; Kelada et al., 2016). About $30 \%$ of the relatives consider that family life was affected by the young person's suicidal behaviour. Over $40 \%$ report an increase in anxiety levels in the family and $23 \%$ state that their sleep routine has changed.

Nevertheless, when symptomatology is analysed, there are no differences between relatives of young people with and without suicidal behaviours, and the means obtained are lower than those from previous studies (Lovibond \& Lovibond, 1995). These findings seem to indicate that, albeit most relatives experience the suicidal behaviour as an emotionally distressing event, they find resources to adjust and deal with the situation. A different explanation may relate to the psychological support some parents were receiving, enabling the development of skills to deal with the event and to lower psychopathology. These results are encouraging, as parents are identified by young people as potential facilitators of seeking help (e.g., Berger et al., 2013; Fortune et al., 2008). As such, intervention politics should include measures directed at the parents and other potential 'protectors' and informal resources (e.g., friends, social groups). Public health measures promoting an increasing literacy in mental health, including suicidal behaviours, are recommended. Previous studies refer that the parents experience doubts about the best way to help their child and usually do not seek professional help at the proper moment (Berger et al., 2017). Despite the parent's insecurity, if appropriately informed and supported, they can play an essential role in starting treatment and in providing support to young people (Glenn et al., 2015). Results from this research are in line with previous studies that mention positive effects on family dynamics after the suicidal behaviour (Ferrey et al., 2016; Kelada et al., 2016; Oldershaw et al., 2008). Over 30\% of the participants in the present investigation remark that the family got more united and $27 \%$ that the relationships became closer.

Additionally, results seem to indicate less disturbance in social and professional life, when compared to the data obtained by international investigations (e.g., Byrne et al., 2008; Daly, 2005; McDonald, 2007). One possible explanation for this seemingly lower disturbance level can be the fact that most international studies include mothers and fathers and the present study also includes other relatives. Another explanation might be related to cultural differences of the social support network that overcome the social stigma of this issue. These possible explanations are apparently sustained by the non-existence of differences in the satisfaction with the social support experienced by relatives of young people with and without suicidal behaviours. This result suggests the protective role played by satisfaction with social support that may reduce the negative impact of suicidal behaviours in family dynamics and health. The association between the variables - suicidal behaviour, social support and experience/ impact - and the mechanism responsible for how this association seems to occur, can also help to interpret the absence of differences in psychopathological symptoms. The few existing studies 
that include satisfaction with social support (e.g., Asare-Dokar et al., 2017; Byrne et al., 2008; Ferrey et al., 2016; Lindgren et al., 2010; McDonald et al., 2007), suggest that suicidal behaviour may have negative impacts on this variable leading to isolation and making the impact more negative. Other results (AsareDokar et al., 2017; Ferrey et al., 2016) suggest that satisfaction with social support rather works as a mediator, increasing or reducing the negative impact. The results gathered by the present investigation provide further arguments consolidating the second hypothesis, thus pointing out the role of satisfaction with social support as a moderating variable and recommending further investigations on this moderating role.

Notwithstanding the limitations of this study, which recommend a broader sample and the use of factor analysis, the results express clues for clinical and psychoeducational practices in order to foster the protective role played by families. Results also suggest that the dimensions of modifications in family dynamics, anxiety and changes in sleep should be addressed in order to prevent health problems and to promote adjustments. Finally, including parents in support groups moderated by health professionals is suggested.

\section{REFERENCES}

Almeida, L. S., \& Freire, T. (2008). Metodologia da investigação em psicologia e educação. Psiquilíbrios.

Asare-Doku, W., Osafo, J., \& Akotia, C. S. (2017). The experiences of attempt survivor families and how they cope after a suicide attempt in Ghana: a qualitative study. BMC Psychiatry, 17(1), 178. https://doi.org/10.1186/s12888-017-1336-9.

Buus, N., Caspersen, J., Hansen, R., Stenager, E., \& Fleischer, E. (2014). Experiences of parents whose sons or daughters have (had) attempted suicide. Journal of Advanced Nursing, 70(4), 823-832. https://doi.org/10.1111/jan.12243.

Berger, E., Hasking, P., \& Martin, G. (2013). 'Listen to them': Adolescents' views on helping young people $\begin{array}{llll}\text { who self-injure. Journal of } & \text { Adolescence, }\end{array}$ https://doi.org/10.1016/j.adolescence.2013.07.011.

Bertolote, J. M., \& Fleischmann, A. (2009). A global perspective on the magnitude of suicide mortality. In D. Wasserman \& C. Wasserman (Eds.), Suicidology and suicide prevention: A global perspective (pp. 9198). Oxford University Press.

Byrne, S., Morgan, S., Fitzpatrick, C., Boylan, C., Crowley, S., Gahan, H., Howley, J., Staunton, D., \& Guerin, S. (2008). Deliberate self-harm in children and adolescents: a qualitative study exploring the needs of parents and careers. Clinical Child Psychology and Psychiatry, 13(4), 493-504. https://doi.org/10.1177/1359104508096765.

Curtis, S., Thorn, P., McRoberts, A., Hetrick, S., Rice, S., \& Robinson, J. (2018). Caring for Young People Who Self-Harm: A Review of Perspectives from Families and Young People. International Journal of Environmental Research and Public Health, 15(5), 950. https://doi.org/10.3390/ijerph15050950

Daly, P. (2005). Mothers living with suicidal adolescents: a phenomenological study of their experiences. Journal of Psychosocial Nursing, 43(3), 22-29.

Doessel, D.P., Williams, R. \& Whiteford H. (2009). Policy-appropriate measurement of suicide: headcount vs. potential years of life lost, Australia, 1907-2005. Archives of Suicide Research, 13(1), 87-99. https://doi.org/10.1080/13811110802572197.

Ferrey, A. E., Hughes, N. D., Simkin, S., Locock, L., Stewart, A., Kapur, N., Gunnell, D., \& Hawton, K. (2016). The impact of self-harm by young people on parents and families: a qualitative study. BMJ Open, 6(1). https://doi.org/10.1136/bmjopen-2015-009631.

Fortune, S., Sinclair, J., \& Hawton, K. (2008). Help-seeking before and after episodes of self-harm: A descriptive study in school pupils in England. Bmc Public Health, 8. https://doi.org/10.1186/14712458-8-369.

Greene-Palmer, F. N., Wagner, B. M., Neely, L. L., Cox, D. W., Kochanski, K. M., \& Perera, K. U., \& Ghahramanlou-Holloway, M. (2015). How parental reactions change in response to adolescent suicide attempt. Archives of Suicide Research, 19, 414-421. https://doi.org/10.1080/13811118.2015.1094367.

Gutierrez, P. M. \& Osman, A. (2008). Adolescent suicide: an integrated approach to the assessment risk and protective factors. Northern Illinois University Press.

Hawton, K., Saunders, K. E. A., \& O'Connor, R. C. (2012). Self-harm and suicide in adolescents. The Lancet, $379,2373-2382$.

Hill, M. M., \& Hill, A. (2008). Investigação por questionário. Edições Sílabo.

Hughes, N. D., Ferrey, A. E., Locock, L., Simkin, S., Stewart, A., Gunnell, D., Kapur, N., \& Hawton, K. (2015). Making sense of an unknown terrain: how parents understand self-harm in young people. Qualitative Health Research, 27(2), 215-225. https://doi.org/10.1177/1049732315603032 
Kelada, L., Whitlock, J., Hasking, P., \& Melvin, G. (2016). Parents' experiences of nonsuicidal self-injury among adolescents and young adults. Journal of Child and Family Studies, 25(11), 3403-3416. https://doi.org/10.1007/s10826-016-0496-4.

Lindgren, B. M., Åström, S., \& Graneheim, U. H. (2010). Held to ransom: parents of self-harming adults describe their lived experience of professional care and caregivers. International Journal of Qualitative Studies on Health and Well-Being, 5(5482), 1-11. https://doi.org/10.3402/qhw.v5i3.5482.

Lovibond, P. \& Lovibond, S. (1995). The structure of negative emotional states: Comparison of the depression anxiety stress scales (DASS) with the Beck Depression and Anxiety Inventories. Behaviour Research and Therapy, 33(3), 335-343. https://doi.org/10.1016/0005-7967(94)00075u.

Moreira, J. M. (2004). Questionários: Teoria e prática. Livraria Almedina.

Morgan, S., Rickard, E., Noone, M., Boylan, C., \& Carthy, A., Crowley, S., Butler, J., Geurin, S., \& Fitzpatrick, C. (2013). Parents of young people with self-harm or suicidal behaviour who seek help - a psychosocial profile. Child and Adolescent Psychiatry and Mental Health, 7(13), 1-10. https://doi.org/10.1186/1753-2000-7-13.

Oldershaw, A., Richards, C., Simic, M., \& Schmidt, U. (2008). Parents' perspectives on adolescent self-harm: qualitative study. British Journal of Psychiatry, 193(2), 140-144. https://doi.org/10.1192/bjp.bp.107.045930.

Pais-Ribeiro, J. L. (1999). Escala de satisfação com o suporte social (ESSS). Análise Psicológica, 3(17), 547558.

Pais-Ribeiro, J. L., Honrado, A., \& Leal, I. (2004). Contribuição para o estudo da adaptação portuguesa das escalas de ansiedade, depressão e stress (EADS) de 21 itens de Lovibond e Lovibond. Psicologia, Saúde \& Doenças, 5(2), 229-239.

Raphael, H., Clarke, G., \& Kumar, S. (2006). Exploring parents' responses to their child's deliberate selfharm. Health Education, 106(1), 9-20. https://doi.org/10.1108/09654280610637166.

Ribeiro, J. L. P. (1999). Investigação e avaliação em psicologia e saúde. Climepsi editores.

Stewart, A., Hughes, N. D., Simkin, S., Locock, L., Ferrey, A., Kapur, N., ... Hawton, K. (2016). Navigating an unfamiliar world: how parents of young people who self-harm experience support and treatment. Child and Adolescent Mental Health. https://doi.org/10.1111/camh.12205.

Trinco, M. E., Santos, J. C., \& Barbosa, A. (2017). Vivências e necessidades dos pais no internamento do filho adolescente com comportamento autolesivo. Revista de Enfermagem Referência, 4 (13), 115124. http://dx.doi.org/10.12707/RIV17008.

Whitlock, J., Lloyd-Richardson, E., Fisseha, F., \& Bates, T. (2018). Parental secondary stress: the often hidden consequences of nonsuicidal self-injury in youth. Journal of Clinical Psychology, 74(1), 178196. https://doi.org/10.1002/jclp.22488.

World Health Organization (2014). Preventing suicide: a global imperative. Luxembourg: WHO. http://www.who.int/mental_health/suicide-prevention/world_report_2014/en/

World Health Organization (2018). Latest data on suicide. https://www.who.int/mental_health/suicideprevention/en/

Historial do artigo

Recebido $\quad 07 / 2019$

Aceite $\quad 03 / 2020$

Publicado $\quad 08 / 2020$ 
Revista PSICOLOGIA, 2020, Vol. 34 (1). doi: 10.17575/psicologia.v34i1.1681

Atas do X Simpósio Nacional de Investigação em Psicologia

\title{
Development and preliminary psychometric study of the Teaching Styles Questionnaire
}

\author{
Carolina Carlos ${ }^{13 i}$, Paula Vagosii \& Lénia Carvalhaisii \\ i Departamento de Educação e Psicologia, Universidade de Aveiro \\ ii Departamento de Psicologia e Educação, Universidade Portucalense Infante D. Henrique
}

\begin{abstract}
The aim of this study was to develop and preliminary evaluate a teacher's version of the Parenting Styles and Dimensions Questionnaire. The Teaching Styles Questionnaire intends to explore the three parental styles as they may be applied to the teacher-student relationship (i.e., authoritative, authoritarian and permissive); items were reworded accordingly. Participants were 128 teachers $(93.8 \%$ female), 73 of which answered the instrument twice, with a 3-month gap, to ascertain for test-retest reliability, whereas the remaining 51 in addition filled in the Student-Teacher Relationship Questionnaire for validity evidence. Preliminary psychometric analyses confirm that the instrument addresses three teaching styles, which presented with reasonable reliability and validity indicators. The way teachers relate with their students has a significant impact not only on their students' academic success, but also on their intraclass behavior. The instrument under analyses may prove to be an important tool to better understand this impact, and how it relates to better holistic school outcomes.
\end{abstract}

Keywords: Teaching styles, Psychometrics, Validation, Teachers.

Desenvolvimento e estudo psicométrico preliminar do Questionário de Estilos de Ensino: 0 objetivo deste estudo foi desenvolver e avaliar preliminarmente a versão para professores do Questionário de Estilos e Dimensões Parentais. O Questionário de Estilos de Ensino pretende explorar os três estilos parentais enquanto aplicados à relação professor-aluno (ou seja, autoritativo, autoritário e permissivo); os itens foram reformulados nesse sentido. Os participantes foram 128 professores $(93,8 \%$ do sexo feminino), 73 dos quais preencheram o instrumento duas vezes, com um intervalo de 3 meses, para verificar a fidelidade teste-reteste, enquanto os 51 restantes preencheram o Questionário de Relação Estudante-Professor para análise de validade de constructo. Análises psicométricas preliminares confirmam que o instrumento considera três estilos de ensino, que apresentaram níveis razoáveis de fidelidade e validade. A maneira como os professores se relacionam com seus alunos tem um impacto considerável não apenas no sucesso académico dos seus alunos, mas também no comportamento dos mesmos. 0 instrumento em análise pode revelar-se uma ferramenta importante para entender melhor este impacto e como se relaciona com melhores resultados escolares holísticos.

\section{Palavras-chave: Estilos de ensino; Psicometria; Validação; Professores.}

Teachers are important agents for promoting a healthy social and emotional development on the part of their students (Barni, Russo, \& Danioni, 2018). A closer teacher-student relationship can lead to students' higher academic performance and lower externalizing behaviors (Pianta \& Stuhlman, 2004; Sabol \& Pianta, 2012). The interactions that take place within the classroom are a key element of reciprocity between students and teachers (Catalán, Serrano, Lucas, Clemente, \& García-González, 2018), and teachers may have an active role in the way these interactions unfold, namely by the teaching styles they enact.

Based on the similarity between parent-child and teacher-student relationships, teacher-student relationships may serve a regulatory function in child's social and emotional development (Pianta \& Stuhlman, 2004; Tsigilis \& Gregoriadis, 2008). This regulatory function can have positive or negative effects on the child's school adjustment; also, relations between children and teachers can determine school success (Pianta, Steinberg, \& Rollins, 1995). Bassett and colleagues (2011) developed a study to examined how teachers interact with their college students, categorizing this interaction in the same way that developmental psychologist Baumrind categorized parent's interactions with their children (1966, 1971). In Bassett and colleagues' study (2011), college students described their instructor according to

${ }^{13}$ Correspondence address: Paula Vagos, Universidade Portucalense Infante D. Henrique, R. Dr. António Bernardino de Almeida 541, 4200072 Porto. E-mail: pvagos@upt.pt 
three teacher's practices, namely permissive, authoritarian, or authoritative. The authoritative teaching style is a student-oriented style in which the teacher is concerned with what could be important for his/her students and interacts with them using both control and warmth (Barni et al., 2018; Bassett et al., 2013). The authoritative teaching style was associated in previous studies with better academic outcomes among elementary and middle school students (Walker, 2009; Wentzel, 2002). The authoritarian teacher expects students to pay attention at classes as a way to acquire knowledge, and to act according to a restrictive discipline. The permissive teacher is disconnected from classroom management and is not in synchrony with students and their needs (Barni et al., 2018). Teacher-student relationships may be based on different teaching styles, meaning the ways in which teachers interact with their students and how they perceive those interactions.

To our knowledge there is currently no measure to address teaching styles as reported by the teacher. The closest alternative is Pianta and collaborators' (Pianta \& Nimetz, 1991; Pianta, 1992) Student-Teacher Relationship Scale (in its complete and short form). This instrument intends to measure teacher-student relationship from the teacher's perspective, namely referring to a conflict (i.e., if the student-teacher relationships are negative, unpredictable or unpleasant) and a closeness dimension (i.e., if the teacher feels that his/her relationship with the students is warm, affective and if the communication is open; Pianta, 2001). Though the relevance of these dimensions has been established (Tsigilis \& Gregoriadis, 2008), they do not consider the specific interpersonal behaviors that may characterize different teaching styles.

\section{Present study}

The purpose of the present study was to develop a Portuguese version of the Parenting Styles and Dimensions Questionnaire (Pedro, Carapito, \& Ribeiro, 2015) as applied to a teaching context, which resulted in the Teaching Styles Questionnaire (TSQ). We also purpose to preliminarily examine the psychometric characteristics of the TSQ as applied to the Portuguese school setting, namely its internal structure, reliability, and associations with teacher-student relationship. We expected to confirm the presence of three teaching styles (i.e., authoritative, authoritarian, and permissive); the authoritative style was further expected to associate with closeness in teacher-student relationship, whereas the authoritarian and permissive styles should appear related to conflict in such relationship.

\section{METHOD}

\section{Participants}

Participants were 128 teachers recruited from the north region of Portugal, mostly female $(\mathrm{n}=120$, $93.8 \%$ ) and married ( $\mathrm{N}=92,71.9 \%)$, who taught compulsory teaching grades in Portuguese schools for a mean of 22.85 years $(S D=8.66)$. Their ages varied between 26 and 64 years old $(M=47.51, S D=7.91)$. There were no significant statistical differences in the mean years of teaching experience and age between male and female participants. Participants were asked to contribute to the study under one of two conditions: 1) by filling in the Teaching Styles Questionnaire along with the Student-Teacher Relationship Scale - Short Form only once (i.e., validity sample) or 2) by filling in only the Teaching Styles Questionnaire twice with a 3-month gap (i.e., reliability sample). Teachers were thus included in one of two subsamples according to their stated convenience. The validity sample included 51 participants and the reliability sample included 73 participants. See table 1 for further information on the composition of these subsamples.

Table 1. Composition of the subsamples

\begin{tabular}{lcc}
\hline & Reliability subsample & Validity subsample \\
\hline Sex & $7(9.1 \%)$ & $1(2 \%)$ \\
$\quad$ Male & $70(90.9 \%)$ & $50(98 \%)$ \\
Female & $48.87(6.72)$ & $45.45(9.13)$ \\
Mean age $(S D)$ & $24.61(6.65)$ & $20.26(10.52)$ \\
\hline
\end{tabular}

Note: Participants from the reliability sample had a significantly higher mean of years of teaching experience when compared to participants from the validity sample $(t(75.35)=2.59, p=.01)$; the same was found regarding mean age $(t(85.07)=2.29, p=.02)$. 


\section{Measurements}

Teaching Styles Questionnaire. The Teaching Styles Questionnaire (i.e., TSQ) resulted from adapting the Portuguese version of the Parenting Styles and Dimensions Questionnaire (Pedro, Carapito, \& Ribeiro, $2015)$ that intends and has shown evidence via confirmatory factor analyses of assessing three parenting styles with appropriate internal consistency values: authoritative (15 items; $\alpha=.88$ ), authoritarian (12 items; ; $\alpha=.73$ ) and permissive (5 items; ; $\alpha=.62$ ). Each scale is composed by the sum of the answers that each participant gives to the items composing it. The current work intended to ascertain if the same interaction styles could characterize the way teachers relate with their students. The 32 items that compose the Parenting Styles and Dimensions Questionnaire were adapted to include the word 'students' in replacement of the word 'child'; the plural was used so that no particular student was targeted when answering the instrument (cf. Appendix 1 for examples of how items were adapted). The same scoring scale was used ranged from 1 (i.e., never) to 5 (i.e., always).

Student-Teacher Relationship Scale - Short form (STRS-SF). The Portuguese version of the STRS-SF (Patrício, Barata, Calheiros, \& Graça, 2015) was applied as a way of gathering construct validity evidence on the TSQ. This instrument intends to assess two dimensions of student-teacher relationship, namely closeness and conflict, using a five-point Likert type scale ranging from 1 (i.e., definitely does not apply) to 5 (i.e., definitely applies). Alike previous works (e.g., Tsigilis \& Gregoriadis, 2008), both dimensions achieved good internal consistency values within the current subsample (i.e., $\alpha=.82$ for closeness and $\alpha=$ .85 for conflict).

\section{Procedure}

A confirmatory factor analysis (CFA) approach was used to test for the factorial validity of the TSQ. Specifically, we mirrored the analytic strategy used for testing the Portuguese version of the parenting version of the instrument and tested four consecutive models (cf. Pedro et al., 2015 and Table 2): Model 1 represents a second-order model, posing three dimensions within the authoritative style (i.e., connection, regulation, and autonomy granting), two dimensions within the authoritarian style (i.e., verbal hostility and non-reasoning/punition), and the permissive style. Model 2 portrays three factors representing the authoritative, authoritarian and permissive styles. Model 3 refers to two factors conveying positive parenting (i.e., authoritative) and negative parenting (i.e., authoritarian and permissive). Model 4 includes a single parenting factor. Acceptable fit was considered to be achieved based on a Root Mean Square Error Of Approximation value <.10, combined with the a Comparative Fit Value $>.90$ (Hu \& Bentler, 1999); lower values of the Weighted Root Mean Square Residual were considered as indicative of better fit, as it refers to the existence of residual variance within the model, not accounted for the proposed measurement model. Descriptive values for the measures composing the best fitting model are presented and pairwise comparisons were carried out to characterize the teaching style mostly used in our sample. The reliability of the TSQ was investigated via internal consistency values and test-retest analyses; evidence was also sought for the TSQ in relation to external variables.

\section{RESULTS}

Preliminary analyses showed that seven participants had between 1 to 5 missing values on the TSQ, which were missing completely at random $\left(\chi^{2} \operatorname{MCAR}(183)=183.30, p=.48\right)$; so, they were dealt with via multiple imputation in Mplus. Also, items 6 and 32 showed minimal variance ${ }^{14}$, which questioned their sensitivity; so, they were excluded from the remaining analyses. Finally, all items showed strong deviations from the normal distribution ${ }^{15}$, which lead to considering data as categorical and using the Weighted Least Square Mean and Variance Adjusted estimator for CFA.

None of the four CFA models presented a good fit to the data (cf. Table 2). The two best fitting models were model 1 and 2; model 1 presented with better comparative fit (i.e., CFI) and less residual variance (i.e., WRMR) whereas model 2 presented with the bestr absolute fit (i.e., RMSEA). We opted for trying to improve the fit of the more parsimonious model (i.e., model 2) by eliminating items with loading values lower then .32 (Fabrigar, Wegner, MacCallum, \& Strahan, 1999; i.e., items 13, 22, 23, 24 and 30) or that seemed to be representative of multiple latent variables, as suggested by the modification indices

\footnotetext{
${ }^{14}$ For item 6 all but one participant scored 1; for item 32 all participants scored 1. Both proposed to assess physical coercion, within an authoritarian style.

${ }^{15}$ Items 19 and 28 are especially noteworthy as participants scored only in two of the five response options. Both proposed to portray the authoritarian style, namely physical coercion and non-reasoning/ punition. ${ }^{16}$ Morada para correspondência: Cristina Queirós, Faculdade de Psicologia e de Ciências da Educação da Universidade do Porto, Rua Alfredo Allen, s/n, 4200-135, Porto, Portugal. E-mail: cqueiros@fpce.up.pt. Este estudo foi financiado pela Fundação para a Ciência e Tecnologia através da bolsa de doutoramento SFRH/BD/135619/2018, atribuída à primeira autora.
} 
(i.e., items 25 and 28). This resulted in a 21-item 3-first order factors that adjusted reasonably to the data (cf. Table 2); the authoritative scale included 13 items (i.e., items 1, 3, 5, 7, 9, 11, 12, 14, 18, 21, 27, 29, 31), the authoritarian scale included 5 items (i.e., items $2,10,16,19,26$ ), and the permissive scale included 3 items (i.e., items $15,17,20)$. It is worth mentioning that the same improvement strategies as applied to model 1 resulted in an adjusted model presenting the following fit indicators: $\chi^{2}(200)=423.76, p<.001$, RMSEA $=.093(.081 ; .106), \mathrm{CFI}=.890, \mathrm{WRMR}=1.184$.

Table 2. Adjustment indicators for models of the internal structure of the Teaching Styles Questionnaire

\begin{tabular}{lcccccc}
\hline & $\chi^{2}$ & df & RMSEA & $\mathbf{9 0 \%}$ CI for RMSEA & CFI & WRMR \\
\hline Model 1 & $818.91^{*}$ & 342 & 0.104 & $0.095 ; 0.114$ & 0.767 & 1.559 \\
Model 2 & $919.16^{*}$ & 402 & 0.100 & $0.092 ; 0.109$ & 0.742 & 1.638 \\
Model 2 adjusted & $344.89^{*}$ & 186 & 0.082 & $0.068 ; 0.095$ & 0.910 & 1.153 \\
Model 3 & $978.67^{*}$ & 404 & 0.105 & $0.097 ; 0.114$ & 0.713 & 1.638 \\
Model 4 & $1009.08^{*}$ & 405 & 0.108 & $0.100 ; 0.116$ & 0.698 & 1.651 \\
\hline
\end{tabular}

$\mathrm{df}=$ degrees of freedom, RMSEA $=$ Root Mean Square Error of Approximation, $\mathrm{CI}=$ confidence interval, $\mathrm{CFI}=$ Comparative Fit Index, WRMR = Weighted Root Mean Square Residual; ${ }^{*} p<.001$

The authoritative measure achieved good reliability indicators and related significantly with closeness. The authoritarian and permissive measures achieved only acceptable internal consistency values and were significantly and positively correlated with each other. The authoritarian measure was not related to either closeness or conflict whereas the permissive measure related significantly and positively with both closeness and conflict (cf. Table 3).

Table 3. Loading, descriptive, reliability and validity results for the three measures of the Teaching Styles Questionnaire

\begin{tabular}{|c|c|c|c|c|c|c|c|c|}
\hline & $\begin{array}{c}\text { Range of } \\
\text { loading } \\
\text { values }\end{array}$ & $\begin{array}{c}M \\
(S D)\end{array}$ & $\alpha$ & $\begin{array}{l}\text { Test- } \\
\text { retest }\end{array}$ & Authoritative & Authoritarian & Closeness & Conflict \\
\hline $\begin{array}{l}\text { Authoritative } \\
\text { (13 items) }\end{array}$ & $.46-.83$ & $\begin{array}{l}51.81 \\
(7.36)\end{array}$ & .86 & $.64^{* * *}$ & - & - & $.51^{* * *}$ & -.08 \\
\hline $\begin{array}{l}\text { Authoritarian } \\
\text { (5 items) }\end{array}$ & $.43-.93$ & $\begin{array}{c}6.34 \\
(1.65)\end{array}$ & .62 & $.49^{* * *}$ & -.09 & - & .04 & .04 \\
\hline $\begin{array}{l}\text { Permissive } \\
\text { (3 items) }\end{array}$ & $.59-.86$ & $\begin{array}{c}5.31 \\
(1.86)\end{array}$ & .66 & $.63^{* * *}$ & .16 & $.31^{* * *}$ & $.41^{* *}$ & $.32^{*}$ \\
\hline
\end{tabular}

Note: All loading values were significant at $p<.001{ }^{* * *} p<.001,{ }^{* *} p<.01,{ }^{*} p<.05$

Pairwise comparisons using the weighted mean for each measure further show that teachers in the current study resort more significantly to the authoritative than to the authoritarian teaching style, $z=-$ 9.62, $p<.001$, or to the permissive teaching style, $z=-9.71, p<.001$; they also scored higher on the permissive style in comparison with the authoritarian one, $z=-7.98, p<.001$.

\section{DISCUSSION}

The current work set out to develop and investigate the psychometric properties of the Teaching Style Questionnaire (TSQ), which resulted from an adaptation of the Parenting Styles and Dimensions Questionnaire (Robinson, Madleco, Olsen, \& Harte, 2001). Teaching styles arise from applying a parenting style typology to the teacher-student relationship, given that the child's interactions with both parents and teachers may, in either case, serve a regulatory function (Pianta \& Stuhlman, 2004; Tsigilis \& Gregoriadis, 2008), albeit applied to different contexts.

Analyses into the internal structure of the TSQ points to the suitability of a three-factor measure assessing authoritative, authoritarian, and permissive teaching styles, though several items needed to be eliminated because they were either not representative of any construct or unable to distinguish between them. The typology for parenting styles was replicated when applied to a teaching context, which is in line with most studies on management styles similarly focusing on these three main parenting styles 
(Baumrind, 1966, 1971; Bassett et al., 2013; Walker, 2008). The authoritative measure proved to be a reliable and valid measure, associating, as expected, with higher levels of closeness in student-teacher relationship. This finding is in line with that reported relying on the student's perspectives (Bassett et al., 2013). The authoritative style was also, as expected, the most frequently used by our participants. Though social desirability issues may be in place when responding to the TSQ, in addition to legal impositions regarding the behavior that teachers may or may not practice towards their students, this finding is encouraging regarding the social climate that Portuguese students may be experiencing within their classrooms.

Alternatively, the authoritarian measure showed the poorest reliability indicators and did not correlate to either closeness or conflict in student-teacher relationship. One would expect that more authoritarian teachers would have less close and more conflicting relationships with their students, but rather it may be case that conflict (and closeness) are not given space in this kind of teaching style. According to previous research (Bassett et al., 2013), authoritarian teachers are perceived by their students as uncaring and not engaged with students' motivations. This teaching style is also characterized by self-focused attitudes as the authoritarian teachers are concentrated with what is important for themselves, while holding high standards and expectations of obedience on the part of their students (Barni et al., 2018).

The permissive measure seems to be stable over time, albeit marginally internally consistent. It seems to associate with both negative (i.e., authoritarian style and student-teacher conflict) and positive aspects of teaching (i.e., closeness in student-teacher relationships). Permissive teachers may be less consistent in the way they interact with their students, focusing on external and transient circumstances, and thus be at times more or less strict and more or less close or in conflict with their students. This inconsistency seems in line with this style being characterized as a form of disengagement from the situation as teachers do not care much about what students are doing (Barni et al., 2018).

Results presented in this work should be seen as restricted and preliminary, due to some limitations worth mentioning. Most importantly, data was gathered from a small and non-representative sample, which may have resulted in the limited variability of responses that participants gave, particularly for items intending to evaluate authoritarian behaviors in the classroom; such limited variability had a strong impact on the results that were achieved. Even if it is expected that teachers express that they more rarely resort to authoritarian teaching attitudes, a larger and more diverse sample might, nevertheless, provide more varied and representative results. Also, only the perspective of teachers was considered. Additionally, considering the perspective of students might be elucidative; for example, even if authoritarian teachers perceive not to be in conflict with their students, students may have a different perception.

Understanding and characterizing how teachers relate with their students may have important implications for teacher training, given that the students' intra and interpersonal development, as well as academic achievement, may be dependent not only on the contents to be taught but also on the interpersonal context in which they are taught. The current work provided preliminary evidence on the TSQ being a potentially important tool to better describe teaching styles and how they impact on the teaching-learning experiences, namely how they may relate to better holistic school outcomes.

\section{REFERENCES}

Barni, D., Russo, C., \& Danioni, F. (2018). Teachers' values as predictors of classroom management styles: a relative weight analysis. Frontiers in Psychology, 9: 1970. https://doi.org/ 10.3389/fpsyg.2018.01970

Bassett, J., Snyder, T., Rogers, D., \& Collins, C. L. (2013). Permissive, authoritarian, and authoritative instructors: applying the concept of parenting styles to the college classroom. Individual Differences Research, 11 (1), 1-11.

Baumrind, D. (1966). Effects of authoritative parental control on child behavior. Child Development, 37, 887-907.

Baumrind, D. (1971). Current patterns of parental authority. Developmental Psychology Monograph, 4, (1, Pt. 2), 1-103.

Catalán, A. A., Serrano, J. S., Lucas, J., Clemente, J., \& García-González, L. (2018). An integrative framework to validate the Need-Supportive Teaching Style Scale (NSTSS) in secondary teachers through exploratory structural equation modelling, Contemporary Educational Psychology, 52, 48-60. http://doi.org/10.1016/j.cedpsych.2018.01.001.

Fabrigar, L., Wegener, D., MacCallum, R., \& Strahan, E. (1999). Evaluating the use of exploratory factor analysis in psychological research. Psychological Methods, 4 (3), 272-299 
Hu, L. \& Bentler, P. (1999). Cutoff criteria for fit indexes in covariance structure analysis: Conventional criteria versus new alternatives. Structural Equation Modeling: A Multidisciplinary Journal, 6(1), 1-55.

Patrício, J. N., Barata, M. C., Calheiros, M. M., \& Graça, J. (2015). A Portuguese version of the StudentTeacher Relationship Scale - Short Form. The Spanish Journal of Psychology, 18, E30. https://doi.org/10.1017/sjp.2015.29

Pedro, M., Carapito, E., \& Ribeiro, T. (2015). Parenting Styles and Dimensions Questionnaire - Versão portuguesa de autorrelato. Psicologia: Reflexão e Crítica, 28(2), 302-312. https://doi.org/ 10.1590/1678-7153.201528210

Pianta, R. C., \& Nimetz, S. L. (1991). Relationships between children and teachers: Associations with classroom and home behavior. Journal of Applied Developmental Psychology, 12(3), 379-393. https://doi.org/10.1016/0193-3973(91)90007-Q

Pianta, R. C. (1992). The Student Teacher Relationship Scale. University of Virginia.

Pianta, R. C., Steinberg, M. S., \& Rollins, L. B. (1995). The first two years of school: Teacher-child relationships and deflections in children's classroom adjustment. Development and Psychopathology, 7, 295-312. https://doi.org/10.1017/S0954579400006519

Pianta, R. C. (2001). STRS Student-Teacher Relationship Scale. Professional manual. Psychological Assessment Resources.

Pianta, R. C., \& Stuhlman, M.W. (2004). Teacher-child relationships and children's success in the first years of school. School Psychology Review, 33(3), 444-458.

Robinson, C. C., Mandleco, B., Olsen, S.F., \& Hart, C.H. (2001). The parenting Styles and Dimensions (PSDQ). In B. F. Perlmutter, J. Touliatos, \& G. W. Holden (Eds.), Handbook of family measurement techniques: Vol. 3. Instruments \& index (pp. 319 - 321). Sage Publications.

Sabol, T. J., \& Pianta, R.C. (2012) Recent trends in research on teacher-child relationships. Attachment \& Human Development, 14(3), 213-231. https://doi.org/10.1080/14616734.2012.672262

Tsigilis, N., \& Gregoriadis, A. (2008). Measuring teacher-child relationships in the Greek kindergarten setting: A validity study of the Student-Teacher Relationship Scale - Short Form. Early Education and Development, 19, 816-835. https://doi.org/10.1080/10409280801975826

Walker, J. M. T. (2008). Looking at teacher practices through the lens of parenting style. The Journal of Experimental Education, 76(2), 218-240. https://doi.org/10.3200/ JEXE.76.2.218-240

Walker, J. M. T. (2009). Authoritative classroom management: how control and nurturance work together. Theory into Practice, 48, 122-129. https://doi.org/10.1080/00405840902776392

Wentzel, K. R. (2002). Are effective teachers like good parents? Teaching styles and student adjustment in early adolescence. Child Development, 73, 287-301. https://doi.org/10.1111/1467-8624.00406

Historial do artigo

Recebido 07/2019

Aceite $\quad 03 / 2020$

Publicado $\quad 08 / 2020$ 
Revista PSICOLOGIA, 2020, Vol. 34 (1). doi: 10.17575/psicologia.v34i1.1681

Atas do X Simpósio Nacional de Investigação em Psicologia

\title{
Trauma em técnicos de emergência pré-hospitalar: Contributo da ansiedade, depressão e stress
}

\author{
Sílvia Monteiro Fonseca16i, Sónia Cunhaii, Rui Camposii \& Cristina Queirós ${ }^{\mathrm{i}}$ \\ i Faculdade de Psicologia e de Ciências da Educação da Universidade do Porto \\ ii Instituto Nacional de Emergência Médica, Instituto Público
}

\begin{abstract}
Resumo: Os técnicos de emergência pré-hospitalar (TEPH) estão mais propensos ao trauma e ansiedade/depressão/stress (ADS). Estudos recentes apontam para o impacto de ADS no trauma, mas não analisam em profundidade estas relações nestes profissionais. Pretende-se conhecer os níveis de trauma e ADS, a influência de características sociodemográficas e profissionais, e o contributo da ADS no trauma. Através da DAAS e IES-R inquiriram-se 593 TEPH, com 34.83 anos $(D P=5.29,20-56)$ e 7.64 anos de experiência profissional ( $D P=3.86,1-28)$. Encontraram-se níveis baixos de ADS e trauma, superiores nos profissionais do sexo feminino, com filhos e mais experiência. A ADS prediz a severidade de trauma (29\%), pensamentos intrusivos (23\%), evitamento (23\%) e hiperativação $(28 \%)$. 0 stress teve maior peso para severidade de trauma e pensamentos intrusivos, a depressão para evitamento, e a ansiedade para hiperativação. Os resultados permitiram aprofundar estas interações e compreender a importância de prevenir a sintomatologia de ADS, pois pode potenciar o trauma.
\end{abstract}

\section{Palavras-chave: Trauma; Ansiedade; Depressão; Stress; Técnicos de emergência pré-hospitalar.}

Trauma in pre-hospital emergency medicine: Contribution of anxiety, depression and stress: PreHospital Emergency Technicians (TEPH) are more prone to trauma and anxiety/depression/stress (ADS). Recent studies point to the impact of ADS on trauma but are scarce the ones that analyze these relationships in depth, within this population. This study aims to identify trauma and ADS levels, the influence of sociodemographic and professional characteristics, and the contribution of ADS on trauma. Through DAAS and IES-R, we inquired 593 TEPH with 34.83 years $(S D=5.29,20-56)$ and 7.64 years of experience $(S D=3.86,1-28)$. Low levels of ADS and trauma were found, which increased in female TEPH and TEPH with sons/daughters and more professional experience. ADS explained trauma (29\%), intrusive thoughts (23\%), avoidance (23\%) and hyperactivation (28\%). Stress had the highest contribution for trauma and intrusive thoughts, depression for avoidance, and anxiety for hyperactivation. Results allowed to deepen the knowledge on these interactions and to understand the need to prevent ADS, which can promote trauma.

Keywords: Trauma; Anxiety; Depression; Stress; Pre-hospital emergency technicians.

Os profissionais de emergência médica são expostos a estímulos potencialmente traumáticos, entre os quais se salientam cenários de violência, acidente/trauma, morte, entre outras (e.g., Carleton et al., 2019; Declercq, Meganck, Deheegher, \& Van Hoorde, 2011; Halpern, Maunder, Schwartz, \& Gurevich, 2012). Várias investigações apontam para o impacto destes incidentes na saúde psicológica/ocupacional dos profissionais (e.g., Berger et al., 2012; Carleton et al., 2019; Donnelly \& Bennett, 2014; Knowles, Sripada, Defever, \& Rauch, 2019; Petrie et al., 2018), em especial para os técnicos de emergência pré-hospitalar (TEPH; Johnson et al., 2005).

A perturbação de stress pós-traumática (PTSD), psicopatologia mais estudada nesta área, apresenta uma prevalência entre 10-19\% nos profissionais de emergência (Berger et al., 2012; Cunha, Queirós, Fonseca, \& Campos, 2017; Davis, MacBeth, Warwick, \& Chan, 2019; Marcelino, Figueiras, \& Claudino, 2012; Petrie et al., 2018). Os seus sintomas podem ser agrupados em (APA, 2014; Pereira \& MonteiroFerreira, 2003): pensamentos intrusivos - revisitação do acontecimento involuntariamente; evitamento de memórias/pensamentos/sentimentos relacionados com o incidente; hiperativação - reações intensas que resultam num aumento do estado de vigília; e pensamentos e humor negativos - expetativas negativas que podem resultar em auto-culpabilização e estados emocionais negativos. Variáveis

${ }^{16}$ Morada para correspondência: Cristina Queirós, Faculdade de Psicologia e de Ciências da Educação da Universidade do Porto, Rua Alfredo Allen, s/n, 4200-135, Porto, Portugal. E-mail: cqueiros@fpce.up.pt. Este estudo foi financiado pela Fundação para a Ciência e Tecnologia através da bolsa de doutoramento SFRH/BD/135619/2018, atribuída à primeira autora. 
sociodemográficas (como idade, sexo, rede de suporte familiar) e profissionais (como anos de experiência profissional) demostraram contribuir para PTSD, ainda que os resultados sejam incongruentes (Cunha et al., 2017; Donnelly \& Bennett, 2014; Greinacher, Derezza-Greeven, Herzog, \& Nikendei, 2019; Marcelino et al., 2012; Oginska-Bulik, 2015; Oravecz, Penko, Suklan, \& Krivec, 2018; Scully, 2011; ShakespeareFinch, Rees, \& Armstrong, 2014; Skeffington, Rees, \& Mazzucchelli, 2017).

Para além da PTSD, outras perturbações são frequentemente associadas à vivência do préhospitalar, como a ansiedade, depressão e stress (ADS), apresentando até maior prevalência (Petrie et al., 2018). A literatura aponta para interações entre estas variáveis, porém carece de estudos que analisem em profundidade estas relações, especialmente neste grupo profissional.

A ansiedade constitui-se como uma resposta normativa face a estímulos stressantes, que resulta em alterações cognitivas, afetivas, fisiológicas e comportamentais (Baptista, Carvalho, \& Lory, 2005; Beck \& Clark, 1997). A depressão pode também comprometer várias áreas do funcionamento (APA, 2014), e caracteriza-se por cognições e sentimentos negativos, acompanhados de alterações fisiológicas e comportamentais, que persistem por pelo menos duas semanas (Beck, 1963). Por sua vez, o stress surge após a exposição a stressores que podem originar estados positivos - eustress - ou negativos - distress (Selye, 1980). 0 distress surge como resultado de um desequilíbrio entre os recursos do indivíduo e as exigências da situação (Everly \& Mitchell, 1997). Apesar da idiossincrasia que caracterizam as reações individuais face aos incidentes críticos, são normativas reações agudas de stress (RAS), com sintomatologia cognitiva, emocional, física e interpessoal associada (APA, 2014; Cooper, Dewe, \& O' Driscoll, 2001; Ehrenreich \& McQuaide, 2001; Seynaeve, 2001). Estas reações podem persistir no tempo e originar PTSD (APA, 2014; Berger et al., 2012; Declerq et al., 2011).

Vários estudos apontam para níveis mais elevados de sintomatologia de ADS nos profissionais de emergência (Mao, Fung, Hu, \& Loke, 2018; Santos et al., 2019; Ward, Lombard, \& Gwebushe, 2006). Petrie e colaboradores (2018), identificaram a prevalência de $27 \%$ para distress e $15 \%$ para ansiedade e depressão, em profissionais do pré-hospitalar. Outros estudos identificaram correlações positivas entre estas sintomatologias (e.g., Courtney, Francis, \& Paxton, 2010; Donnelly, 2012; Kerai et al., 2017; Straud, Henderson, Vega, Black, \& Van Hasselt, 2018), bem como a influência de características sociodemográficas e profissionais, entre os quais se destaca a idade, sexo e experiência profissional (Alharthy, Alrajeh, Almutairi, \& Alhajri, 2017; Donnelly \& Bennett, 2014; Kerai et al., 2017; Lee \& Lee, 2019; Yahaya, Wahab, Yusoff, Yasin, \& Rahman, 2018).

Contudo, ainda não é muito clara a direccionalidade das interações entre ADS e PTSD, pois apesar de alguns estudos identificaram interações, bem como o impacto da ADS no trauma (Donnelly, 2012; Kerai et al., 2017; Straud et al., 2018), outros identificaram efeitos diretos e indiretos de PTSD na ADS (Ginzburg, Ein-Dor, \& Solomon, 2010; Kim, Park, \& Kim, 2018), ainda que com outras populações (bombeiros e veteranos de guerra). Além disso, os investigadores não se têm focado de igual forma na ADS, nem têm estudado aprofundadamente a contribuição de cada uma destas sintomatologias na experiência de trauma, especialmente nestes profissionais.

Assim, este estudo pretende analisar o papel preditor da ADS no trauma, bem como conhecer os níveis destas sintomatologias e a variação em função de variáveis sociodemográficas e profissionais nos TEPH. Serão expetáveis níveis moderados a elevados de trauma e ADS que variem em função de variáveis sociodemográficas e profissionais, bem como a contribuição positiva da ADS para a variabilidade do trauma.

\section{MÉTODO}

\section{Participantes}

Participaram 593 TEPH, sendo 203 mulheres (35\%) e 382 homens (65\%), com idades entre os 20-56 anos $(M=34.83, D P=5.29)$, estando $47 \%$ solteiros, divorciados ou viúvos e $53 \%$ casados ou em união de facto. A maioria não estava deslocada do local de residência (69\%) e trabalhava na delegação sul (41\%), seguida da delegação norte (34\%) e delegação centro (25\%). Os participantes trabalhavam entre 8-86 horas por semana $(M=41.49 ; D P=6.06)$, tendo em média 7.64 anos $(D P=3.86,1-28)$ de experiência profissional no INEM.

\section{Instrumentos}

O trauma foi avaliado através da Impact of Event Scale - Revised (IES-R, de Weiss \& Marmar, 1997; Matos, Pinto-Gouveia, \& Martins, 2011), composta por 22 itens avaliados numa escala de tipo Likert de 5 pontos $(0=n a d a$ a $4=$ muitíssimo) e agrupados em três dimensões: pensamentos intrusivos ( 8 itens), evitamento (8 itens) e hiperativação (6 itens). De uma escala total com valores entre $0-88$, valores superiores a 33 são 
indicativos da presença e severidade dos sintomas de trauma (quantitativa e qualitativamente). A consistência interna variou entre 0.86 e 0.96 (Tabela 1).

A ansiedade, depressão e stress foram avaliadas através da Anxiety Depresssion Stress Scales (ADS de Lovibond \& Lovibond, 1995; Pais-Ribeiro, Honrado, \& Leal, 2004), que contém 21 itens avaliados

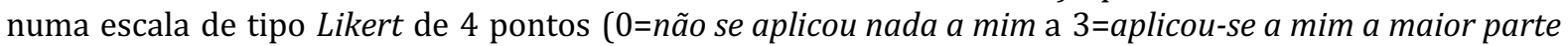
das vezes). A cada uma das variáveis correspondem 7 itens, podendo apresentar valores entre 0-21, e valores mais elevados correspondem a níveis mais elevados da sintomatologia avaliada. A consistência interna variou entre 0.84 e 0.88 (ver Tabela 1 ).

\section{Procedimento}

Após a aprovação do Conselho Diretivo do INEM, todos os TEPH com pelo menos 1 ano de experiência foram contactados. A recolha de dados envolveu a distribuição, pelas delegações do INEM, de consentimentos informados e questionários. Estes foram preenchidos em reuniões de equipa ou formações contínuas, sem a presença dos colegas/chefias, não existindo qualquer contacto entre investigadores e participantes. Todos os procedimentos éticos foram respeitados e assegurados. Os participantes no estudo representaram $62 \%$ dos TEPH a nível nacional $(N=958)$.

A análise de dados, através do programa IBM SPSS (v.25), assegurou a normalidade univariada das variáveis (através do teste Kolmogorov-Smirnov e dos critérios de assimetria e curtose, conforme Kline, 2011). Conforme Field (2009), foram realizadas estatísticas descritivas, testes $t$ de Student para amostras independentes, análise de variância a um fator (através do teste post-hoc GT2 de Hochberg), correlações através do coeficiente $r$ de Pearson e regressões múltiplas (método Enter). Nas regressões foram assegurados os pressupostos de homoscedasticidade, linearidade, normalidade da distribuição dos erros, multicolinearidade e independência dos erros (Durbin-Watson com valores entre 1.99-2.08).

\section{RESULTADOS}

Relativamente às estatísticas descritivas de ADS e trauma (ver Tabela 1), verificou-se baixa manifestação de sintomatologia em todas as dimensões, considerando os valores médios obtidos, em comparação com os extremos possíveis. Apenas $20 \%$ da amostra reuniu critérios para a presença de trauma $(n=102)$, verificando-se menor severidade da sintomatologia traumática nos participantes.

Tabela 1. ADS e Trauma: Consistência Interna, Mínimo, Máximo, Média e Desvio-Padrão

\begin{tabular}{lccccc}
\hline Dimensões & $\boldsymbol{\alpha}$ & Min. & Máx. & $\boldsymbol{M}$ & $\boldsymbol{D P}$ \\
\hline Ansiedade (0-3) & .84 & 0 & 2.57 & 0.30 & 0.41 \\
Depressão (0-3) & .87 & 0 & 2.86 & 0.39 & 0.46 \\
Stress (0-3) & .88 & 0 & 2.71 & 0.72 & 0.52 \\
Pensamentos Intrusivos (0-4) & .93 & 0 & 4.00 & 1.01 & 0.87 \\
Evitamento (0-4) & .91 & 0 & 3.50 & 0.93 & 0.76 \\
Hiperativação (0-4) & .86 & 0 & 4.00 & 0.71 & 0.84 \\
Trauma (0-88) & .96 & 0 & 84 & 19.58 & 16.73 \\
\hline
\end{tabular}

Foram analisadas as variações de ADS e trauma em função das variáveis sociodemográficas (sexo, estado civil, existência de filhos e deslocado do local de residência) e profissionais (anos de experiência profissional, média de horas semanais e região de trabalho), encontrando-se apenas diferenças para o sexo dos TEPH e existência de filhos (ver Tabela 2). Os profissionais do sexo feminino apresentaram níveis mais elevados de stress, e os TEPH com filhos apresentaram níveis mais elevados de sintomatologia de evitamento. Nas variáveis profissionais, apenas os anos de experiência se mostraram correlacionados positivamente com depressão, $r(592)=.10, p=.020$, stress, $r(592)=.09, p=.022$, e pensamentos intrusivos, $r(511)=.10, p=.019$, verificando-se que quanto maior era a experiência profissional, mais elevados eram os níveis de depressão, stress e pensamentos intrusivos. 
Tabela 2. Análise Comparativa em Função do Sexo e da Existência de Filhos

\begin{tabular}{lcccccc}
\hline Dimensões & Variáveis & Categorias & $\boldsymbol{M ( D P )}$ & $\boldsymbol{t}(\boldsymbol{d} \boldsymbol{f})$ & IC a 95\% & $\boldsymbol{d}$ \\
\hline \multirow{2}{*}{ Stress } & \multirow{2}{*}{ Sexo } & Feminino & $0.81(0.51)$ & & & 0.27 \\
& & Masculino & $0.67(0.52)$ & $-3.06(582)^{* *}$ & {$[-0.23,-0.05]$} & \\
\multirow{2}{*}{ Evitamento } & \multirow{2}{*}{ Filhos } & Sim & $1.01(0.76)$ & & & 0.18 \\
\hline
\end{tabular}

Notas. Tamanho de efeito (Cohen, 1988): $d \leq 0.2$ - pequeno; $d=$ ]0.2, 0.5] - moderado; $d=$ ]0.5, 1.0] - elevado; $d>1.0$ muito elevado; ${ }^{*} p<.050 .{ }^{* *} p<.010$.

Encontraram-se correlações positivas entre as dimensões de ADS e as dimensões de trauma (ver Tabela 3), e quanto mais elevados foram os níveis de ADS, mais elevados se mostraram os níveis de trauma e sintomatologia associada.

Tabela 3. Correlações entre ADS e Trauma

\begin{tabular}{|c|c|c|c|c|c|c|}
\hline Dimensões & 1 & 2 & 3 & 4 & 5 & 6 \\
\hline 1. Ansiedade & - & & & & & \\
\hline 2. Depressão & $.70^{* * *}$ & & & & & \\
\hline 3. Stress & $.72^{* * *}$ & $.74^{* * *}$ & & & & \\
\hline 4. Pensamentos Intrusivos & $.44^{* * *}$ & $.42^{* * *}$ & $.45^{* * *}$ & & & \\
\hline 5. Evitamento & $.42^{* * *}$ & $.45^{* * *}$ & $.45^{* * *}$ & $.79^{* * *}$ & & \\
\hline 6. Hiperativação & $.49^{* * *}$ & $.48^{* * *}$ & $.47^{* * *}$ & $.88^{* * *}$ & $.76^{* * *}$ & \\
\hline 7. Trauma & $.48^{* * *}$ & $.48^{* * *}$ & $.49^{* * *}$ & $.95^{* * *}$ & $.91^{* * *}$ & $.93^{* * *}$ \\
\hline
\end{tabular}

Nota. ${ }^{* * *} p<.001$.

Através de regressões múltiplas procurou-se compreender o papel preditor da ADS (variáveis independentes) na sintomatologia traumática (variáveis dependentes). Verificou-se que a ADS explicou $28.6 \%$ da variância da severidade de trauma, 23.2\% dos pensamentos intrusivos, $23.2 \%$ do evitamento e $28.2 \%$ da hiperativação. A sintomatologia de ADS explicou positivamente todas as dimensões do trauma (ver Tabela 4).

Tabela 4. Modelos de Regressão Múltipla do Papel Preditor da ADS no Trauma

\begin{tabular}{|c|c|c|c|c|c|c|c|c|}
\hline & \multicolumn{2}{|c|}{ Trauma } & \multicolumn{2}{|c|}{$\begin{array}{c}\text { Pensamentos } \\
\text { Intrusivos }\end{array}$} & \multicolumn{2}{|c|}{ Evitamento } & \multicolumn{2}{|c|}{ Hiperativação } \\
\hline & $\boldsymbol{\beta}$ & $p$ & $\bar{\beta}$ & $p$ & $\beta$ & $p$ & $\bar{\beta}$ & $p$ \\
\hline Ansiedade & .202 & .001 & .200 & .001 & .132 & .028 & .242 & $<.001$ \\
\hline Depressão & .184 & .002 & .116 & .060 & .213 & .001 & .209 & $<.001$ \\
\hline Stress & .212 & .001 & .221 & .001 & .193 & .003 & .142 & .022 \\
\hline$F(3,506)$ & \multicolumn{2}{|c|}{$68.89^{* * *}$} & \multicolumn{2}{|c|}{$52.13^{* * *}$} & \multicolumn{2}{|c|}{$52.12^{* * *}$} & \multicolumn{2}{|c|}{$67.51^{* * *}$} \\
\hline$R^{2} \mathrm{a}$ & \multicolumn{2}{|c|}{.286} & \multicolumn{2}{|c|}{.232} & \multicolumn{2}{|c|}{.232} & \multicolumn{2}{|c|}{.282} \\
\hline
\end{tabular}

Nota. $^{* * *} p<.001$.

Para analisar em maior profundidade o papel preditor de ADS no trauma, analisaram-se os valores de $\beta$ (ver Tabela 4 e Figura 1), tendo-se verificado que para a severidade de trauma e pensamentos intrusivos o stress teve maior peso, seguido da ansiedade e depressão. No caso dos pensamentos intrusivos a depressão não se constituiu como preditor significativo, enquanto para o evitamento a depressão teve maior peso, seguida do stress e ansiedade. Já a ansiedade teve maior peso na variabilidade da sintomatologia hiperativa, seguida da depressão e stress. 


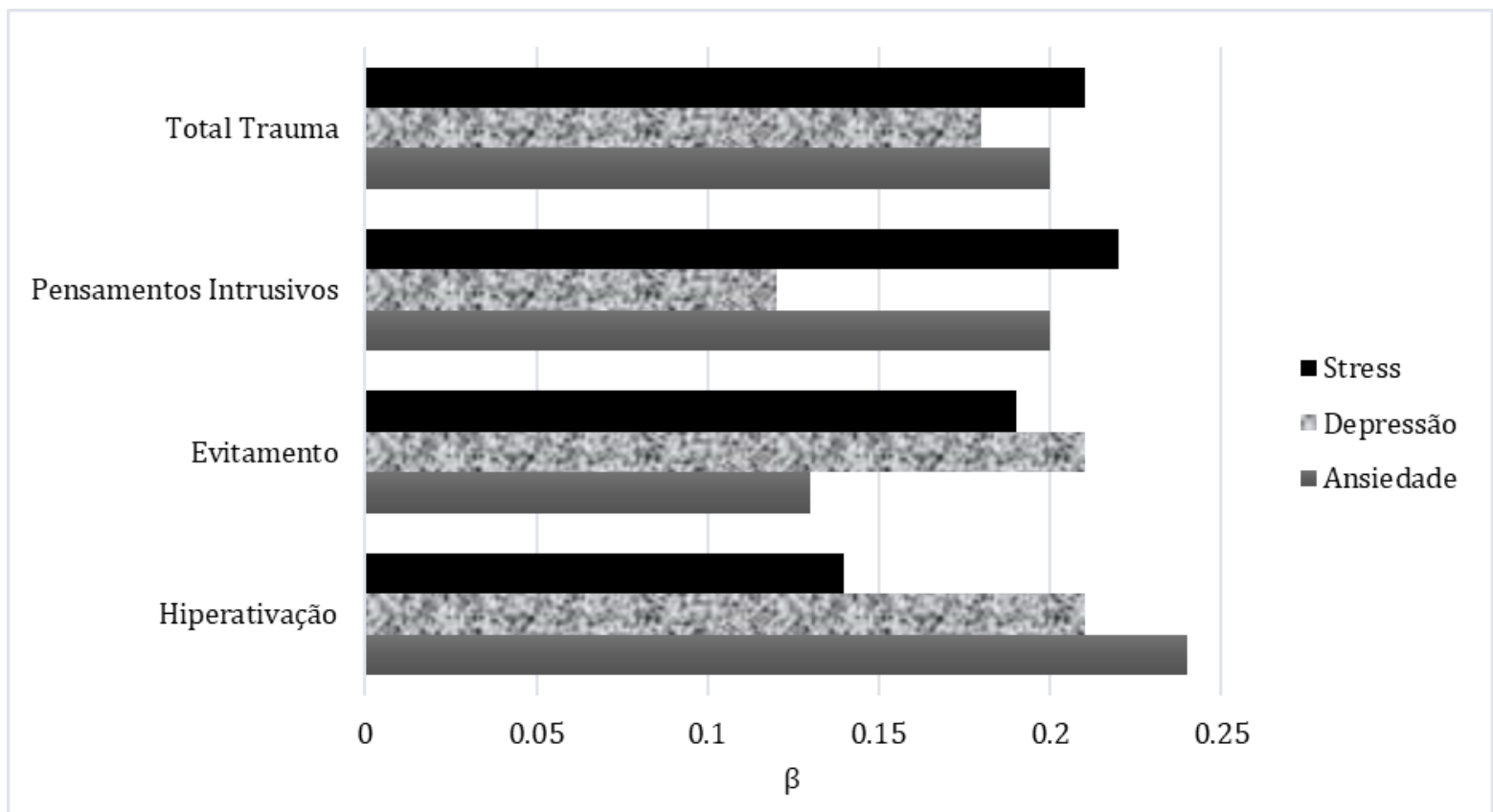

Figura 1. Valores de $\beta$ do Papel Preditor da ADS no Trauma

\section{DISCUSSÃo}

Neste estudo encontraram-se níveis reduzidos de ADS e trauma nos TEPH, o que sugere a positiva resolução das ocorrências e capacidade de resposta das políticas institucionais do INEM às necessidades dos colaboradores, no que respeita à saúde psicológica e ocupacional. Apesar da maioria dos inquiridos (80\%) não reunir critérios para PTSD, em consonância com a literatura (Berger et al., 2012; Cunha et al., 2017; Marcelino et al., 2012; Petrie et al., 2018), realça-se que os profissionais com maior severidade de trauma poderão não estar no ativo e, por isso, não estarem representados neste estudo, expressando o mito do trabalhador saudável (Shah, 2009).

Relativamente às variáveis sociodemográficas e profissionais, apenas se verificaram diferenças significativas em função do sexo, existência de filhos e anos de experiência. As TEPH mulheres apresentaram níveis mais elevados de stress, e os TEPH com filhos níveis mais elevados de evitamento. Ser do sexo feminino surge como fator de risco para o stress, sugerindo-se a necessidade de desenvolver estratégias dirigidas para esta população, ainda que alguns estudos tenham identificado níveis mais elevados em profissionais do sexo masculino (Donnelly \& Bennett, 2014; Greinacher et al., 2019; Yahaya et al., 2018). A existência de filhos constitui-se como uma característica pouca estudada, embora se possa enquadrar na rede de suporte familiar/social, enquanto fator protetor (Greinacher et al., 2019; OginskaBulik, 2015; Shakespeare-Finch et al., 2014). Contudo, neste estudo, os filhos constituem-se como fator de risco, sugerindo-se que exigem maior cuidado e que, portanto, as estratégias de evitamento são necessárias para que os estímulos traumáticos não impactem o exercício da parentalidade.

Verificou-se, ainda, que quanto maior era a experiência profissional, mais elevados eram os níveis de depressão, stress e pensamentos intrusivos, em consonância com estudos que concluíram acerca do risco acrescido para o trauma (Carleton et al., 2019; Cunha et al., 2017; Donnelly \& Bennett, 2014; Oravecz et al., 2018). 0 mesmo aconteceu para ADS, mas a literatura é escassa, demonstrando a necessidade de desenvolver mais estudos para compreender estas interações, pois alguns investigadores verificaram que os anos de experiência se constituíam como fator de risco para ADS (Donnelly \& Bennett, 2014), outros como protetor (Kerai et al., 2017) e outros não verificaram qualquer interação (Yahaya et al., 2018).

Encontraram-se correlações positivas entre a ADS e a sintomatologia traumática, permitindo compreender a comorbidade entre estes sintomas (Donnelly, 2012; Kerai et al., 2017; Straud et al., 2018). Nas regressões, verificou-se uma contribuição significativa e positiva da ADS no trauma, em consonância com alguns estudos (Donnelly, 2012; Kerai et al., 2017; Knowles et al., 2019; Straud et al., 2018), sugerindo que a ADS poderá potenciar a sintomatologia traumática. Contudo, isto não se aplicou à depressão na explicação da variabilidade dos pensamentos intrusivos. Coloca-se como hipótese que os TEPH com sintomatologia depressiva se foquem mais no self e nos sentimentos de desvalorização pessoal e autoculpabilização (APA, 2014; Beck, 1963), ao invés de se focarem nos incidentes propriamente ditos. Além disso, verificou-se que a sintomatologia de ADS contribuiu especialmente para variância da 
severidade do trauma e da hiperativação (28.6\% e 28.2\%, respetivamente), em comparação com pensamentos intrusivos e evitamento (23.2\% para ambos). Apesar de não ser objetivo deste estudo e da literatura ainda se mostrar inconsistente, foi também explorado o valor preditivo do trauma na variância explicada da ADS, mas os resultados não se mostraram significativos, com exceção da predição do stress pelo total de trauma e pensamentos intrusivos (24.5\%). Note-se que a sintomatologia de trauma e pensamentos intrusivos, ao implicarem uma ativação cognitiva pela revisitação do acontecimento involuntariamente (APA, 2014), parecem contribuir para uma ativação psicofisiológica e ao nível do stress experienciado (e.g., Kim et al., 2018).

Esta comorbilidade entre ADS e trauma salienta a necessidade de estudos longitudinais que permitam clarificar a direccionalidade destas relações, dado que a natureza transversal deste estudo poderá limitar as conclusões obtidas (e.g., Taris \& Kompier, 2003, 2014). De facto, no seu estudo longitudinal, Ginzburg e colaboradores (2010) com 664 veteranos, ao longo de 20 anos, constataram que PTSD predizia a ansiedade e depressão, mas estas sintomatologias não alteravam a probabilidade de manifestação de PTSD ao longo do tempo. Ora, no presente estudo pretendeu-se compreender como poderiam ser identificados perfis de profissionais mais vulneráveis ao desenvolvimento de trauma, através da ADS, numa lógica de desenvolvimento de estratégias preventivas. Assim, o impacto do trauma na ADS parece surgir como consequência natural, dado o seu impacto na saúde mental e a comorbidade com diversas sintomatologias (e.g., Ginzburg et al., 2010; Terhaag et al., 2019) e, como tal, as estratégias preventivas já não são adequadas.

Na severidade do trauma e pensamentos intrusivos, o stress apresentou maior peso e a depressão menor (não significativa para pensamentos intrusivos), sendo esta contribuição do stress para o trauma congruente com a literatura, uma vez que as RAS, normativas face aos incidentes críticos, podem originar PTSD (APA, 2014; Berger et al., 2012; Declercq et al., 2011). Além disso, o stress parece contribuir especialmente para os pensamentos intrusivos, demonstrando a sua contribuição para as cognições associadas ao trauma. A menor contribuição da depressão para ambos, sugere novamente o maior foco nas cognições e sentimentos depreciativos direcionados para o self, ao invés dos estímulos traumáticos.

No que diz respeito ao evitamento, apresentou maior e menor peso, respetivamente, a depressão e ansiedade. Novamente, estes dados reforçam o predomínio de cognições e sentimentos depreciativos, em TEPH depressivos, que privilegiam o evitamento do incidente. A sintomatologia ansiosa contribuiu com baixa expressão para o evitamento destes estímulos, possivelmente devido à consciencialização dos stressores e estado de vigília em indivíduos ansiosos (e.g., Beck \& Clark, 1997).

Por fim, a hiperativação recebeu a maior contribuição da ansiedade, sugerindo-se a existência de processos semelhantes em ambas as sintomatologias, que resultam num aumento do estado de vigília (APA, 2014; Beck \& Clark, 1997; Pereira \& Monteiro-Ferreira, 2003). 0 stress apresentou menor peso para a hiperativação, o que poderá sugerir que a hiperativação não resulta do desequilíbrio entre os recursos disponíveis e as exigências da situação, tal como se sucede no stress (Everly \& Mitchell, 1997; Selye, 1980), mas receberá maior influência de componentes mais cognitivas tais como a memória de incidentes passados, característico de quadros ansiosos (Baptista et al., 2005; Beck \& Clark, 1997).

\section{CONCLUSÃo}

Os dados permitem compreender a necessidade de prevenir a sintomatologia de ADS, dado que poderá potenciar de forma distinta a sintomatologia traumática, bem como a necessidade de atentar a TEPH do sexo feminino, com filhos e mais experiência. Estes resultados levantam também importantes pistas teóricas e conceptuais para melhor compreensão dos construtos avaliados, e para melhor compreensão da sintomatologia de trauma a partir da ADS. Contudo, deverá considerar-se a existência de limitações neste estudo, nomeadamente ser um estudo transversal, retrospetivo e de autorrelato. Os dados poderão também estar enviesados por apenas se ter acedido a profissionais no ativo, possivelmente mais saudáveis. Finalmente, salienta-se a necessidade de ampliar este estudo a outros profissionais/instituições de urgência/emergência, assim como se salienta que a realização de estudos longitudinais poderá ser determinante para clarificar relações pouco claras entre as variáveis consideradas neste estudo, assim como para melhor compreender a direccionalidade da relação entre trauma e ADS.

\section{REFERÊNCIAS}

Alharthy, N., Alrajeh, O., Almutairi, M., \& Alhajri, A. (2017). Assessment of anxiety level of emergency health-care workers by generalized anxiety disorder-7 tool. International Journal of Applied and Basic Medical Research, 7, 150-155. https://doi.org/10.4103/2229-516x.212963

American Psychiatric Association (2014). DSM-V: Manual de diagnóstico e estatístico de transtornos mentais (5 $\underline{a}$ ed.). Climepsi Editores. 
Baptista, A., Carvalho, M., \& Lory, F., (2005). 0 medo, a ansiedade e suas perturbações. Psicologia, 19, 267278. https://doi.org/10.17575/rpsicol.v19i1/2.407

Beck, A. T. (1963). Thinking and depression. Archives of General Psychiatry, 9, 324-333. https://doi.org/10.1001/archpsyc.1963.01720160014002

Beck, A. T., \& Clark, D. A. (1997). An information processing model of anxiety: Automatic and strategic processes. Behavior Research and Therapy, 35, 49-58. https://doi.org/10.1016/s00057967(96)00069-1

Berger, W., Coutinho, E., Figueira, I., Marques-Portella, C., Luz, M., Neylan, T., ... Mendlowicz, M. (2012). Rescuers at risk: A systematic review and meta-regression analysis of the worldwide current prevalence and correlates of PTSD in rescue workers. Social Psychiatry and Psychiatric Epidemiology, 47, 1001-1011. https://doi.org/10.1007/s00127-011-0408-2

Carleton, R. N., Afifi, T. O., Taillieu, T., Turner, S., Krakauer, R., Anderson, G. S., ... McCreary, D. R. (2019). Exposures to potentially traumatic events among public safety personnel in Canada. Canadian Journal of Behavioural Science, 51, 37-52. https://doi.org/10.1037/cbs0000115

Cohen, J. (1988). Statistical power analysis for the behavioral sciences. Lawrence Erlbaum Associates.

Cooper, C. L., Dewe, P., \& O'Driscoll, M. (2001). Organizational stress: A review and critique of theory, research, and applications. Sage.

Courtney, J. A., Francis, A. J. P., \& Paxton, S. J. (2010). Caring for the carers: Fatigue, sleep, and mental health in Australian paramedic shiftworkers. The Australian and New Zealand Journal of Organisational Psychology, 3, 32-41. https://doi.org/10.1375/ajop.3.1.32

Cunha, S., Queirós, C., Fonseca, S., \& Campos, R. (2017). Resiliência como preditor do impacto traumático em técnicos de emergência pré-hospitalar. International Journal on Working Conditions, 13, 51-67.

Davis, K., MacBeth, A., Warwick, R., \& Chan, S.W.Y. (2019). Posttraumatic stress symptom severity, prevalence and impact in ambulance clinicians: The hidden extent of distress in the emergency services. Traumatology, 25, 282-288. https://doi.org/10.1037/trm0000191

Declercq, F., Meganck, R., Deheegher, J., \& Van Hoorde, H. (2011). Frequency of and subjective response to critical incidents in the prediction of PTSD in emergency personnel. Journal of Traumatic Stress, 24, 133-136. https://doi.org/10.1002/jts.20609

Donnelly, E. A. (2012). Work-related stress and posttraumatic stress in emergency medical services. Prehospital Emergency Care, 16, 76-85. https://doi.org/10.3109/10903127.2011.621044

Donnelly, E. A., \& Bennett, M. (2014). Development of a critical incident stress inventory for the emergency medical services. Traumatology: An International Journal, 20(1), 1-8. https://doi.org/10.1177/1534765613496646

Ehrenreich, J. H., \& McQuaide, S. (2001). Coping with disaster: A guidebook to psychological intervention. Center for Psychology and Society.

Everly, G. S., \& Mitchell, J. T. (1997). Critical Incident Stress Management (CISM): A new era and standard of care in crisis intervention. Chevron Publishing.

Field, A. (2009). Discovering statistics using SPSS ( $2^{\mathrm{a}}$ ed.). Artmed Editora.

Ginzburg, K., Ein-Dor, T., \& Solomon, Z. (2010). Comorbidity of posttraumatic stress disorder, anxiety and depression: A 20-year longitudinal study of war veterans. Journal of Affective Disorders, 123, 249257. https://doi.org/10.1016/j.jad.2009.08.006

Greinacher, A., Derezza-Greeven, C., Herzog, W., \& Nikendei, C. (2019). Secondary traumatization in first responders: A systematic review. European Journal of Psychotraumatology, 10(1), 1-21. https://doi.org/10.1080/20008198.2018.1562840

Halpern, J., Maunder, R. G., Schwartz, B., \& Gurevich, M. (2012). The critical incident inventory: Characteristics of incidents which affect emergency medical technicians and paramedics. $B M C$ Emergency Medicine, 12(1), 1-10. https://doi.org/10.1186/1471-227X-12-10

Johnson, S., Cooper, C., Cartwright, S., Donald, I., Taylor, P., \& Millet, C. (2005). The experience of workrelated stress across occupations. Journal of Managerial Psychology, 20, 178-187. https://doi.org/10.1108/02683940510579803

Kerai, S. M., Khan, U. R., Islam, M., Asad, N., Razzak, J., \& Pasha, O. (2017). Post-traumatic stress disorder and its predictors in emergency medical service personnel: A cross-sectional study from Karachi, Pakistan. BMC Emergency Medicine, 17, 26-33. https://doi.org/10.1186/s12873-017-0140-7

Kim, J. I., Park, H., \& Kim, J. H. (2018). The mediation effect of PTSD, perceived job stress and resilience on the relationship between trauma exposure and the development of depression and alcohol use problems in Korean firefighters: A cross-sectional study. Journal of Affective Disorders, 229, 450455. https://doi.org/10.1016/j.jad.2017.12.055

Kline, B. (2011). Principles and practice of structural equation modeling (2 $2^{\mathrm{a}}$ ed.). The Guilford Press. 
Knowles, K. A., Sripada, R. K., Defever, M., \& Rauch, S. A. M. (2019). Comorbid mood and anxiety disorders and severity of posttraumatic stress disorder symptoms in treatment-seeking veterans. Psychological Trauma: Theory, Research, Practice, and Policy, 11, 451-458. https://doi.org/10.1037/tra0000383

Lee, E., \& Lee, H. (2019). Disaster awareness and coping: Impact on stress, anxiety, and depression. Perspectives in Psychiatric Care, 55, 311-318. https://doi.org/10.1111/ppc.12351.

Lovibond, P., \& Lovibond, S. (1995). The structure of negative emotional states: Comparison of the Depression Anxiety Stress Scales (DASS) with the Beck Depression and Anxiety Inventories. Behaviour Research and Therapy, 33, 335-343. https://doi.org/10.1016/0005-7967(94)00075-U

Mao, X., Fung, O. M., Hu, X., \& Loke, A. Y. (2018). Psychological impacts of disaster on rescue workers: A review of the literature. International Journal of Disaster Risk Reduction, 27, 602-617. https://doi.org/10.1016/j.ijdrr.2017.10.020

Marcelino, D., Figueiras, M. J., \& Claudino, A. (2012). Impacto da exposição a incidentes críticos na saúde e bem-estar psicológico dos tripulantes de ambulância. Psicologia, Saúde e Doenças, 13, 110-116.

Matos, M., Pinto-Gouveia, J., \& Martins, S. (2011). 0 impacto traumático de experiências de vergonha: Estudo das propriedades psicométricas da versão portuguesa da Impact of Event Scale - Revised. Psychologica, 54, 413-438. https://doi.org/10.14195/1647-8606_54_16

Ogińska-Bulik, N. (2015). Social support and negative and positive outcomes of experienced traumatic events in a group of male emergency service workers. International Journal of Occupational Safety and Ergonomics, 21, 119-127. https://doi.org/10.1080/10803548.2015.1028232

Oravecz, R., Penko, J., Suklan, J., \& Krivec, J. (2018). Prevalence of post-traumatic stress disorder, symptomatology and coping strategies among Slovene medical emergency professionals. Sigurnost, 60,117-127. https://doi.org/10.31306/s.60.2.2

Pais-Ribeiro, J. L., Honrado, A., \& Leal, I. (2004). Contribuição para o estudo da adaptação portuguesa das Escalas de Ansiedade, Depressão e Stress (EADS) de 21 itens de Lovibond e Lovibond. Psicologia Saúde \& Doenças, 5, 229-239.

Pereira, M. G., \& Monteiro-Ferreira, J. (2003). Stress traumático: Aspectos teóricos e intervenção. Climepsi.

Petrie, K., Milligan-Saville, J., Gayed, A., Deady, M., Phelps, A., Dell, L., ... Harvey, S. B. (2018). Prevalence of PTSD and common mental disorders amongst ambulance personnel: A systematic review and meta-analysis. Social Psychiatry and Psychiatric Epidemiology, 53, 897-909. https://doi.org/10.1007/s00127-018-1539-5

Santos, J., Longuiniere, A., Vieira, S., Amaral, A., Sanches, G., \& Vilela, A. (2019). Occupational stress: The exposure of an emergency unit nursing team. Journal of Clinical Nursing, 11, 455-463. https://doi.org/10.9789/2175- 5361.2019.v11i2.455-463

Scully, P. J. (2011). Taking care of staff: A comprehensive model of support for paramedics and emergency medical dispatchers. Traumatology, 17, 35-42. https://doi.org/10.1177/1534765611430129

Selye, H. (1980). Selye's guide to stress research. Van Nostrand Reinhold.

Seynaeve, G. J. R. (2001). Psycho-social support in situations of mass emergency. European policy paper concerning different aspects of psycho-social support for people in major accidents and disasters. Ministry of Public Health.

Shah, D. (2009). Healthy worker effect phenomenon. Indian Journal of Occupational and Environmental Medicine, 13, 77-79. https://doi.org/10.4103/0019-5278.55123

Shakespeare-Finch, J., Rees, A., \& Armstrong, D. (2014). Social support, self-efficacy, trauma and wellbeing in emergency medical dispatchers. Social Indicators Research, 123, 549-565. https://doi.org/10.1007/s11205-014-0749-9

Skeffington, P. M., Rees, C. S., \& Mazzucchelli, T. (2017). Trauma exposure and post-traumatic stress disorder within fire and emergency services in Western Australia. Australian Journal of Psychology, 69, 20-28. https://doi.org/10.1111/ajpy.12120

Straud, C., Henderson, S. N., Vega, L., Black, R., \& Van Hasselt, V. (2018). Resiliency and posttraumatic stress symptoms in firefighter paramedics: The mediating role of depression, anxiety, and sleep. Traumatology, 24, 140-147. https://doi.org/10.1037/trm0000142

Taris, T. W., \& Kompier, M. (Eds.). (2003). Challenges in longitudinal designs in occupational health psychology [Special issue]. Scandinavian Journal of Work, Environment and Health, 29(1), 1-4. https://doi.org/10.5271/sjweh.697

Taris, T. W., \& Kompier, M. (Eds.). (2014). Cause and effect: Optimizing the designs of longitudinal studies in occupational health psychology [Special issue]. Work and Stress, 28(1), 1-8. https://doi.org/10.1080/02678373.2014.878494

Terhaag, S., Cowlishaw, S., Steel, Z., Brewer, D., Howard, A., Armstrong, R., ... Phelps, A. (2019). Psychiatric comorbidity for veterans with posttraumatic stress disorder (PTSD): A latent profile analysis and 
implications for treatment. Psychological Trauma: Theory, Research, Practice, and Policy. Advance online publication. https://doi.org/10.1037/tra0000520

Ward, C. L., Lombard, C. J., \& Gwebushe, N. (2006). Critical incident exposure in South African emergency services personnel: Prevalence and associated mental health issues. Emergency Medical Journal, 23, 226-231. https://doi.org/0.1136/emj.2005.025908

Weiss, D. S., \& Marmar, C. R. (1997). The Impact of Event Scale - Revised. In J. P. Wilson, \& T. M. Keane (Eds.), Assessing psychological trauma and ptsd (pp. 399-411). Guilford Press.

Yahaya, S. N., Wahab, S. F. A., Yusoff, M. S. B., Yasin, M. A. M., \& Rahman, M. A. A. (2018). Prevalence and associated factors of stress, anxiety and depression among emergency medical officers in Malaysian hospitals. World Journal of Emergency Medicine, 9, 178-187. https://doi.org/10.5847/wjem.j.1920-8642.2018.03.003

\author{
Historial do artigo \\ Recebido $\quad 07 / 2019$ \\ Aceite $\quad 03 / 2020$ \\ Publicado $\quad 08 / 2020$
}


Revista PSICOLOGIA, 2020, Vol. 34 (1). doi: 10.17575/psicologia.v34i1.1681

Atas do X Simpósio Nacional de Investigação em Psicologia

\title{
Os consumos de álcool e tabaco da rede social nos comportamentos de policonsumos dos estudantes do ensino superior
}

\author{
António Ramalho Mostardinha ${ }^{17 i}$ \& Anabela Pereira ${ }^{i}$ \\ iUniversidade de Aveiro
}

\begin{abstract}
Resumo: 0 presente estudo pretende avaliar a relação entre os consumos (álcool e tabaco) dos progenitores e dos amigos com comportamentos de policonsumo dos estudantes do ensino superior. Foi desenvolvido um estudo transversal cuja amostra foi constituída por 338 estudantes (51.8\% homens), com uma idade média de 20.6 \pm 3.4 anos. Modelos de regressão logística multivariada foram aplicados, avaliando a significância do modelo e o seu ajustamento, bem como as variáveis com um efeito nos comportamentos dos participantes. Observou-se que os estudantes que apresentam amigos (OR=3.298; IC95\% 1.266-8.592) e progenitores fumadores (OR=1.831; IC95\% 1.154-2.906) são mais prováveis recorrerem a policonsumos do tipo A (consumir álcool e tabaco). Ainda, para policonsumos do tipo B (consumir álcool e/ou tabaco mais cannabis), ser do sexo feminino apresenta-se como fator protetor (OR=0.508; IC95\% 0.302-0.854) e a idade como fator de risco (OR=1.849; IC95\% 1.084-3.153). Desta forma, observou-se que consumo de substâncias é influenciado pela idade, sexo e pelos consumos de substâncias, por parte de amigos e progenitores. São sugeridas algumas implicações, tais como a necessidade de intervir nesta população sem desconsiderar o contexto social em que o estudante se insere.
\end{abstract}

Palavras-chave: Determinantes sociais de saúde; Consumo de substâncias; Policonsumos; Estudantes do ensino superior.

Alcohol and tobacco consumption of the social network on university students' polydrug consumption behaviors: The present study aims to evaluate the association between parents and friends' consumption behaviours (alcohol and tobacco) with polydrug consumption behaviour of university students. A Cross-sectional study was developed, whose sample was composed by 338 students $(51.8 \%$ men $)$ with a mean age of $20.6 \pm 3.4$ years old. Multivariate logistic regression models were performed, as well as the assessment of the model significance and its adjustment and the variables with an effect on the participants' behaviours. It was observed that the students who present friends $(\mathrm{OR}=3.298 ; 95 \% \mathrm{CI} 1.266-8.592)$ and smoking parents (OR=1.831; 95\%CI 1.154-2.906) are more likely to present type A polydrug consumption (alcohol and tobacco consumption). Also, for type B polydrug consumption (to consume alcohol and/or tobacco plus cannabis), being female showed to be a protective factor (OR=.508; 95\%CI .302-.854) and age as a risk factor (OR=1.849; 95\%ci 1.084-3.153). Thereby, it was observed that substance consumption is influenced by age, sex, and substance consumption of friends and parents. Some implications are suggested such as the need to intervene in this population without disregarding the students' social context.

\section{Keywords: Health social determinants; Substance consumption; Polydrug; University students.}

O consumo recreacional de álcool, tabaco e substâncias ilícitas (e.g. cannabis) (Rehm et al., 2017), representa um risco para o desenvolvimento de diferentes morbilidades (Peacock et al., 2018). Neste sentido, estima-se que $4,9 \%$ da população mundial adulta sofra de transtornos advenientes de consumo de bebidas alcoólicas, 22,5\% apresentam consumos de produtos de tabaco (e.g., cigarros) e 3,5\% de cannabis (Gowing et al., 2015), sendo os Anos de Vida Perdidos Ajustados por Incapacidade (sigla em inglês DALYs), a uma escala global, mais elevados para o tabaco (170,9 milhões DALYs), seguindo-se o álcool (85,0 milhões DALYs) e as substâncias ilícitas (27,8 milhões DALYs) (Peacock et al., 2018). Neste contexto, as taxas de mortalidade atribuídas a consumos de substâncias são mais elevadas para tabaco (110,7/100 000 indivíduos), seguindo-se bebidas alcoólicas (33,0/100 000 indivíduos) e substâncias ilícitas (6,9/100 000 indivíduos) (Peacock et al., 2018).

${ }^{17}$ Morada para correspondência: António Ramalho Mostardinha, Centro de Investigação em Didática e Tecnologia na Formação de Formadores , Campus Universitário de Santiago, 3810-193, Aveiro, Portugal. E-mail: antonio.mostardinha@ua.pt 
Uma vez que o consumo de substâncias atinge o seu pico na faixa etária entre os 18 e os 25 anos de idade, os estudantes universitários constituem-se como uma população de risco (Pilatti, Read, \& Pautassi, 2017), apesar de diversos fatores contribuírem para o consumo nestes jovens adultos (Ansari \& Stock, 2012; Dhanookdhary et al., 2010).

Focando nos fatores socioambientais, os consumos por parte dos grupos de referência (e.g. pais e amigos) apresentam uma associação com os consumos dos estudantes (Mak, Ho, \& Day, 2012; Schultz, Nowatzki, \& Ronson, 2013), devido a processos de aprendizagem social e auto-regulação (Bandura, 1991, 1999). Paralelamente aos processos de aprendizagem social (Petraitis, Flay, \& Miller, 1995), o consumo de substâncias por parte dos estudantes poderá ser explicado pelas normas sociais (Perkins \& Berkowitz, 1986), em que os estudantes tendem a sobrestimar o consumo (normas descritivas) e a aprovação desse comportamento por parte dos seus pares (normas injuntivas), influenciando no seu próprio consumo (Perkins, 2002).

A universidade representa um período de transição, onde ocorrem diversos processos de mudança (Baer, Kivlahan, Blume, McKnight, \& Marlatt, 2001), tais como alterações da composição da rede social, (Meisel \& Barnett, 2017), fomentando a necessidade do sentimento de pertença e do desenvolvimento da identidade social (Rimal \& Real, 2005). Neste ambiente académico, onde o consumo de substâncias é comum (Lipari \& Jean-Francois, 2016), os fatores sociais e ambientais, bem como as normas culturais (moldadas por crenças, atitudes e comportamentos), poderão influenciar os comportamentos e intenções de consumo (Grønkjær, Curtis, De Crespigny, \& Delmar, 2011; McGloin, Sullivan, \& Thomas, 2014). Desta forma, será premente o aprofundamento do entendimento da relação entre os consumos da rede social, isto é, dos pais e amigos, com os consumos dos estudantes do ensino superior.

Por conseguinte, objetiva-se com o presente trabalho, avaliar a relação entre os consumos (álcool e tabaco) da rede social com os comportamentos de policonsumo dos estudantes do ensino superior.

\section{MÉTODO}

\section{Participantes}

Estudo transversal a uma população de estudantes universitários inscritos no ano letivo de 2016/2017. 0 processo de amostragem decorreu entre fevereiro e maio de 2017, numa universidade da região centro de Portugal, e os dados foram recolhidos através de um questionário constituído por questões sociodemográficas e questões para avaliar o consumo de substâncias. Na Tabela 1, pode ser observada a caracterização da amostra. A amostra por conveniência foi constituída por 338 estudantes, dos quais $51.8 \%$ eram do sexo masculino, com uma média de idades de 20.6 anos (DP = 3.4). Ainda, 40.3\% apresentam consumos tabágicos (definido neste estudo como consumo diário) e $94.6 \%$ consumos etílicos (definido como consumo pelo menos uma vez por semana). Para ambos os consumos, na análise de dados, não foram analisados dados referentes a estudantes que consumiram ou experimentaram, ao longo da sua vida, estas substâncias.

O presente estudo foi aprovado pela Comissão Científica do Programa Doutoral em Educação do Departamento de Educação e Psicologia da Universidade de Aveiro, e todos os participantes incluídos assinaram um consentimento conformado. Foram, ainda, garantidos os direitos dos indivíduos de acordo com a Declaração de Helsínquia (World Medical Association, 2008).

\section{Instrumentos}

0 instrumento de recolha de dados foi constituído por variáveis sociodemográficas (sexo e idade), variáveis relativas a consumos de álcool e tabaco dos progenitores e amigos (álcool e tabaco) e variáveis relativas a consumos de substâncias - policonsumo do Tipo A (consumir álcool e tabaco) e policonsumo do Tipo B (consumir álcool e/ou tabaco mais cannabis) (European Monitoring Centre for Drugs and Drug Addiction, 2009).

\section{Análise de dados}

Por forma a avaliar a relação entre os consumos (álcool e tabaco) dos progenitores e dos amigos com comportamentos de policonsumo dos estudantes do ensino superior, calcularam-se modelos de regressão logística multivariada. Teve-se em consideração a significância (estatística de teste $\mathrm{G}^{2}$ ) e qualidade de ajustamento do modelo obtido pelo cálculo da estatística $\chi^{2} \mathrm{HL}$ e pelo cálculo dos seguintes Pseudo- $\mathrm{R}^{2}: \mathrm{R}^{2}$ de Cox \& Snell ( $\mathrm{R}^{2} \mathrm{CS}$ ), $\mathrm{R}^{2}$ de Nagelkerke $\left(\mathrm{R}^{2} \mathrm{~N}\right)$ e $\mathrm{R}^{2}$ de MacFadden $\left(\mathrm{R}^{2} \mathrm{MF}\right.$ ) (Marôco, 2018). Para a análise de dados utilizou-se um nível de significância $(\alpha)$ de 0.05 . A análise de dados foi realizada através do IBM SPSS Statistics, versão 23. 
Tabela 1. Caracterização da amostra quanto a variáveis sociodemográficas, variáveis relativas a consumo de progenitores e amigos e variáveis relativas a policonsumos

\begin{tabular}{lc}
\hline Variável & $\mathbf{n}(\%)$ \\
\hline Sexo & $175(51.8)$ \\
Masculino & $163(48.2)$ \\
Feminino & \\
Idade & $140(41.7)$ \\
$<20$ anos & $196(58.3)$ \\
$\geq 20$ anos & \\
Ter um pai progenitor que consome bebidas alcoólicas & $38(11.3)$ \\
Não & $297(88.7)$ \\
Sim & \\
Ter amigo(s) que consome(m) bebidas alcoólicas & $6(1.8)$ \\
Não & $332(98.2)$ \\
Sim & \\
Ter um progenitor que fuma ou alguma vez fumou & $132(39.5)$ \\
Não & $202(60.5)$ \\
Sim & \\
Ter amigo(s) fumador(es) & $21(6.3)$ \\
Não & $315(93.8)$ \\
\hline Sim
\end{tabular}

\section{RESULTADOS}

De uma forma geral, observaram-se percentagens de $59.6 \%$ e de $29.0 \%$, respetivamente para policonsumos do Tipo A e B.

No âmbito dos policonsumos do Tipo A, a regressão logística, com todos os preditores, revelou que ter amigo(s) fumador(es) (OR 3,298; IC 95\% - 1.266; 8.592) e ter um pai fumador (OR 1.831; IC 95\% $1.154 ; 2.906$ ) apresentaram um efeito estatisticamente significativo (ver Tabela 2 ).

Tabela 2. Preditores de policonsumos do Tipo A $\left[G^{2 *}(4)=16.776 ; p=.002 ; R^{2} C S=.050 ; R^{2} N=.067 ; R^{2} M F\right.$ $\left.=.038 ; \chi^{2} H L(7)=0.652 ; p=.999\right]$

\begin{tabular}{|c|c|c|c|c|c|c|}
\hline Variável & $B$ & S.E. & Wald & $g I$ & $p$ & OR (IC95\%) \\
\hline Sexo & 0.165 & 0.239 & 0.478 & 1 & .489 & \\
\hline \multicolumn{7}{|l|}{ Masculino } \\
\hline Feminino & & & & & & $1.179(0.739 ; 1.884)$ \\
\hline Idade & 0.415 & 0.238 & 3.037 & 1 & .081 & \\
\hline \multicolumn{7}{|l|}{$<20$ anos } \\
\hline$\geq 20$ anos & & & & & & $1.515(0.950 ; 2.416)$ \\
\hline Ter amigos fumadores & 1.193 & 0.489 & 5.965 & 1 & .015 & \\
\hline \multicolumn{7}{|l|}{ Não } \\
\hline Sim & & & & & & $3.298(1.266 ; 8.592)$ \\
\hline $\begin{array}{l}\text { Ter um pai progenitor que fuma ou alguma } \\
\text { vez fumou }\end{array}$ & 0.605 & 0.236 & 6.592 & 1 & .010 & \\
\hline Não & & & & & & $1.831(1.154 ; 2.906)$ \\
\hline Sim & & & & & & \\
\hline
\end{tabular}

*G2: estatística de teste à significância do modelo 
No que diz respeito aos policonsumos do tipo B (ver Tabela 3), observou-se que ser estudante do sexo feminino (OR 0.508; IC95\% - 0.302; 0.854) representa um fator protetor para apresentar policonsumos. Ainda, estudantes com idades iguais ou superiores a 20 anos apresentam um maior risco de apresentarem estes consumos (OR 1.849; IC95\% 1.084;3.153).

Tabela 3. Preditores de policonsumos do Tipo B $\left[G^{2}(4)^{*}=20.891 ; p<0.001 ; R^{2} C S=0.063 ; R^{2} N=0.090\right.$; $\left.R^{2} M F=0.054 ; X 2 H L(6)=0.9,731 ; p=0.136\right]$

\begin{tabular}{|c|c|c|c|c|c|c|}
\hline Variável & $\boldsymbol{B}$ & S.E. & Wald & $g I$ & $\bar{p}$ & OR (IC95\%) \\
\hline Sexo & -0.678 & 0.265 & 6.538 & 1 & 0.011 & \\
\hline \multicolumn{7}{|l|}{ Masculino } \\
\hline Feminino & & & & & & $0.508(0.302 ; 0.854)$ \\
\hline Idade & 0.614 & 0.272 & 5.088 & 1 & 0.024 & \\
\hline \multicolumn{7}{|l|}{$<20$ anos } \\
\hline$\geq 20$ anos & & & & & & 1.849 (1.084; 3.153) \\
\hline Ter amigos fumadores & 0.791 & 0.652 & 1.473 & 1 & 0.225 & \\
\hline \multicolumn{7}{|l|}{ Não } \\
\hline Sim & & & & & & $2.206(0.615 ; 7.914)$ \\
\hline $\begin{array}{l}\text { Ter um pai que fuma ou alguma vez } \\
\text { fumou }\end{array}$ & 0.518 & 0.269 & 3.699 & 1 & 0.054 & \\
\hline Não & & & & & & $1.678(0.990 ; 2.844)$ \\
\hline Sim & & & & & & \\
\hline
\end{tabular}

* $\mathrm{G}^{2}$ : estatística de teste à significância do modelo

\section{DISCUSSÃo}

De uma forma geral verificou-se que ter amigo(s) fumador(es) e um progenitor fumador são preditores de policonsumos do Tipo A, e ser do sexo masculino e idade são preditores para policonsumos do Tipo B.

No âmbito da influência das variáveis sociodemográficas (sexo e idade) nos policonsumos do tipo $\mathrm{B}$, diferentes estudos obtiveram resultados semelhantes, em que estudantes do sexo masculino são mais prováveis de experimentar e/ou consumir substâncias (King \& Chassin, 2007), e a idade atua como um preditor nestes consumos (Lamont, Woodlief, \& Malone, 2014).

Estes resultados poderão ser explicados pela Teoria do Comportamento Social Normativo (Rimal \& Real, 2005), pois esta teoria propõe que a relação entre um determinado comportamento e as percepções sobre a prevalência desse comportamento (ex. normas descritivas) são moderadas pela proximidade dos grupos de referência (neste estudo, pelos progenitores e amigos). Desta forma, a proximidade dos grupos de referência (e.g., progenitores e amigos) apresenta um efeito moderador na relação entre as normas descritivas e as intenções de consumo dos estudantes, isto é, quanto maior a perceção da prevalência de consumo (normas descritivas), maior as intenções de consumo (Rimal, 2008). Ademais, tendo em consideração a relação explanada anteriormente, os estudantes tendem a sobrestimar o consumo de álcool dos pares (normas descritivas) e, consequentemente, aumentam o seu próprio consumo (Borsari \& Carey, 2003).

0 presente trabalho de investigação, além das limitações inerentes ao tipo de estudo (transversal), à amostragem (por conveniência) e à operacionalização das variáveis de consumo de tabaco e de álcool (como explanado nas metodologias), apresenta como principal limitação a natureza sensível das questões, que poderá conduzir a um viés da desejabilidade social (Bryman, 2012), que não foi possível avaliar por não incluir escalas que o detetem (van de Mortel, 2008).

Futuros trabalhos poderão focar-se nos efeitos de moderação e mediação que os grupos de referência apresentam nos policonsumos dos estudantes do ensino superior, fornecendo evidência que suporte o desenvolvimento de estratégias a serem implementadas em projetos de educação para a saúde, dado a prevalência de consumos nocivos que os estudantes universitários apresentam (O'Grady, Arria, Fitzelle, \& Wish, 2008), e os problemas de saúde associados (Degenhardt et al., 2018). 


\section{CONCLUSÃO}

O consumo de tabaco por parte dos grupos de referência (e.g., progenitores e amigos), apresenta-se como preditor dos policonsumos do tipo A em estudantes do ensino superior. Por outro lado, o sexo e a idade constituem preditores para os policonsumos do tipo B. Tais conclusões alertam para a necessidade de intervenções não só ao nível do jovem adulto inserido no ensino superior, bem como nos seus contextos familiares e sociais. Estas intervenções multidisciplinares deverão ser planeadas e executadas, dando enfoque à prevenção dos comportamentos de risco, visando a saúde e bem-estar do indivíduo.

\section{REFERÊNCIAS}

Ansari, W. El, \& Stock, C. (2012). Factors Associated With Smoking, Quit Attempts and Attitudes towards Total Smoking Bans at University: A Survey of Seven Universities in England, Wales and Northern ireland. Asian Pacific Journal of Cancer Prevention, 13(2), 705-714. https://doi.org/10.7314/APJCP.2012.13.2.705

Baer, J. S., Kivlahan, D. R., Blume, A. W., McKnight, P., \& Marlatt, G. A. (2001). Brief intervention for heavydrinking college students: 4-year follow-up and natural history. American Journal of Public Health, 91(8), 1310-1316. https://doi.org/10.2105/AJPH.91.8.1310

Bandura, A. (1991). Social cognitive theory of self-regulation. Organizational Behavior and Human Decision Processes, 50(2), 248-287. https://doi.org/10.1016/0749-5978(91)90022-L

Bandura, A. (1999). Social Cognitive Theory: An Agentic Perspective. Asian Journal of Social Psychology, 2(1), 21-41. https://doi.org/10.1111/1467-839X.00024

Borsari, B., \& Carey, K. B. (2003). Descriptive and injunctive norms in college drinking: a meta-analytic integration. Journal of Studies on Alcohol, 64(3), 331-341. https://doi.org/10.15288/jsa.2003.64.331

Bryman, A. (2012). Social Research Methods (Fourth Edi). New York: Oxford University Press.

Degenhardt, L., Charlson, F., Ferrari, A., Santomauro, D., Erskine, H., Mantilla-Herrara, A., ... Vos, T. (2018). The global burden of disease attributable to alcohol and drug use in 195 countries and territories, 1990-2016: a systematic analysis for the Global Burden of Disease Study 2016. The Lancet Psychiatry, 5(12), 987-1012. https://doi.org/10.1016/S2215-0366(18)30337-7

Dhanookdhary, A. M., Gomez, A. M., Khan, R., Lall, A., Murray, D., Prabhu, D., ... Youssef, F. F. (2010). Substance use among university students at the St augustine campus of the University of the West Indies. West Indian Medical Journal, 59(6), 641-649.

European Monitoring Centre for Drugs and Drug Addiction. (2009). Polydrug use: Patterns and responses. https://doi.org/10.2810/26783

Gowing, L. R., Ali, R. L., Allsop, S., Marsden, J., Turf, E. E., West, R., \& Witton, J. (2015). Global statistics on addictive behaviours: 2014 status report. Addiction, 110(6), 904-919. https://doi.org/10.1111/add.12899

Grønkjær, M., Curtis, T., De Crespigny, C., \& Delmar, C. (2011). Acceptance and expectance: Cultural norms for alcohol use in Denmark. International Journal of Qualitative Studies on Health and Well-Being, 6(4), 8461. https://doi.org/10.3402/qhw.v6i4.8461

King, K. M., \& Chassin, L. (2007). A prospective study of the effects of age of initiation of alcohol and drug use on young adult substance dependence. Journal of Studies on Alcohol and Drugs, 68(2), 256-265. https://doi.org/ 10.15288/jsad.2007.68.256

Lamont, A. E., Woodlief, D., \& Malone, P. S. (2014). Predicting high-risk versus higher-risk substance use during late adolescence from early adolescent risk factors using Latent Class Analysis. Addiction Research \& Theory, 22(1), 78-89. https://doi.org/10.3109/16066359.2013.772587

Lipari, R. N., \& Jean-Francois, B. (2016). A Day in the Life of College Students Aged 18 to 22: Substance Use Facts. Retrieved May 7, 2018, from https://www.samhsa.gov/data/sites/default/files/report_2361/ShortReport-2361.html

Mak, K.-K., Ho, S.-Y., \& Day, J. R. (2012). Smoking of Parents and Best Friend-Independent and Combined Effects on Adolescent Smoking and Intention to Initiate and Quit Smoking. Nicotine \& Tobacco Research, 14(9), 1057-1064. https://doi.org/10.1093/ntr/nts008

Marôco, J. (2018). Análise Estatística com o SPSS Statistics (7a ed.). Pêro Pinheiro: Report Number.

McGloin, J. M., Sullivan, C. J., \& Thomas, K. J. (2014). Peer Influence and Context: The Interdependence of Friendship Groups, Schoolmates and Network Density in Predicting Substance Use. Journal of Youth and Adolescence, 43(9), 1436-1452. https://doi.org/10.1007/s10964-014-0126-7

Meisel, M. K., \& Barnett, N. P. (2017). Protective and Risky Social Network Factors for Drinking During the Transition From High School to College. Journal of Studies on Alcohol and Drugs, 78(6), 922-929. https://doi.org/10.15288/jsad.2017.78.922

O'Grady, K. E., Arria, A. M., Fitzelle, D. M. B., \& Wish, E. D. (2008). Heavy Drinking and Polydrug Use among 
College Students. Journal of Drug Issues, 38(2), 445-465.

https://doi.org/10.1177/002204260803800204

Peacock, A., Leung, J., Larney, S., Colledge, S., Hickman, M., Rehm, J., ... Degenhardt, L. (2018). Global statistics on alcohol, tobacco and illicit drug use: 2017 status report. Addiction. https://doi.org/10.1111/add.14234

Perkins, H W, \& Berkowitz, A. D. (1986). Perceiving the community norms of alcohol use among students: some research implications for campus alcohol education programming. The International Journal of the Addictions, 21(9-10), 961-976. https://doi.org/10.3109/10826088609077249

Perkins, H Wesley. (2002). Social norms and the prevention of alcohol misuse in collegiate contexts. Journal of Studies on Alcohol, Supplement, (s14), 164-172. https://doi.org/10.15288/jsas.2002.s14.164

Petraitis, J., Flay, B. R., \& Miller, T. Q. (1995). Reviewing theories of adolescent substance use: Organizing pieces in the puzzle. Psychological Bulletin, 117(1), 67-86. https://doi.org/10.1037/00332909.117.1.67

Pilatti, A., Read, J. P., \& Pautassi, R. M. (2017). ELSA 2016 Cohort: Alcohol, Tobacco, and Marijuana Use and Their Association with Age of Drug Use Onset, Risk Perception, and Social Norms in Argentinean College Freshmen. Frontiers in Psychology, 8. https://doi.org/10.3389/fpsyg.2017.01452

Rehm, J., Gmel, G. E., Gmel, G., Hasan, O. S. M., Imtiaz, S., Popova, S., ... Shuper, P. A. (2017). The relationship between different dimensions of alcohol use and the burden of disease-an update. Addiction, 112(6), 968-1001. https://doi.org/10.1111/add.13757

Rimal, R. N. (2008). Modeling the Relationship Between Descriptive Norms and Behaviors: A Test and Extension of the Theory of Normative Social Behavior (TNSB). Health Communication, 23(2), 103116. https://doi.org/10.1080/10410230801967791

Rimal, R. N., \& Real, K. (2005). How Behaviors are Influenced by Perceived Norms. Communication Research, 32(3), 389-414. https://doi.org/10.1177/0093650205275385

Schuckit, M. A., Smith, T. L., Clausen, P., Skidmore, J., Shafir, A., \& Kalmijn, J. (2016). Drinking Patterns Across Spring, Summer, and Fall in 462 University Students. Alcoholism: Clinical and Experimental Research, 40(4), 889-896. https://doi.org/10.1111/acer.13019

Schultz, A. S. H., Nowatzki, J., \& Ronson, G. (2013). Effects of Household Socialization on Youth Susceptibility to Smoke: Differences Between Youth Age Groups and Trends Over Time. American Journal of Public Health, 103(7), e39-e42. https://doi.org/10.2105/AJPH.2013.301344

van de Mortel, T. F. (2008). Faking it: social desirability response bias in self-report research. Australian Journal of Advanced Nursing, 25(4), 40-48.

World Medical Association. (2008). World Medical Association Declaration os Helsinki: Ethical Principles for Medical Research Involving Human Subjects. Retrieved March 21, 2016, from http://www.wma.net/en/30publications/10policies/b3/17c.pdf

$\begin{array}{ll}\text { Historial do } & \text { artigo } \\ \text { Recebido } & 07 / 2019 \\ \text { Aceite } & 11 / 2019 \\ \text { Publicado } & 08 / 2020\end{array}$


Revista PSICOLOGIA, 2020, Vol. 34 (1). doi: 10.17575/psicologia.v34i1.1681

Atas do X Simpósio Nacional de Investigação em Psicologia

\title{
A análise dos projetos pessoais em psicoterapia: As potencialidades de uma abordagem idiográfica
}

\author{
Isabel Albuquerque ${ }^{18 i}$, António J. Gonzalez ${ }^{\mathrm{ii}}$, Paulo Martins ${ }^{\mathrm{iii}} \&$ Margarida P. Lima ${ }^{\mathrm{iv}}$ \\ ${ }^{i}$ CINEICC - FPCE UC \\ ii APPsyCI, Applied Psychology Research Center, Capabilities and Inclusion, ISPA - Instituto Universitário, \\ Lisboa, Portugal \\ iii Laboratory of Sport and Exercise Psychology, Faculty of Human Kinetics, University of Lisbon, Portugal \\ iv Clinical Psychology, Faculdade de Psicologia e de Ciências da Educação da Universidade de Coimbra, \\ Portugal
}

\begin{abstract}
Resumo: A investigação tem mostrado que a monitorização do progresso do cliente em processo terapêutico mediante uma abordagem idiográfica que contemple a sua individualidade, pode trazer benefícios substantivos na superação de dificuldades e de problemas específicos. A utilização de metas pessoais é uma das formas propostas para a concretização desta abordagem. A metodologia de Análise de Projetos Pessoais proporciona meios para reformular o sistema de projetos, encorajar o indivíduo a investir em novos projetos, observar o modo como estes se promovem entre si ou criam conflitos ou resistências, reconhecer e trabalhar as estruturas ecológicas que os impedem de ser sustentáveis, realizar uma hierarquização que ajude o indivíduo a organizar o seu sistema de projetos, de modo a que ele seja funcional, exequível e tenha coerência e significado. Todas estas características dotam esta metodologia de potencialidades para ser implementada na psicoterapia e aconselhamento, como forma de ultrapassar as fragilidades de uma abordagem apenas nomotética.
\end{abstract}

Palavras-chave: Metas; Projetos pessoais; Psicoterapia; Abordagem idiográfica.

The analysis of personal projects in psychotherapy: The potential of an idiographic approach: Research has shown that monitoring client's progress in the therapeutic process through an idiographic approach that addresses individuality can bring substantive benefits in overcoming specific difficulties and problems. The use of personal goals is one of the proposed ways to achieve this approach. The Personal Project Analysis methodology provides means to restructure the project system, encourage individuals to invest in new projects, observe how projects promote themselves, or otherwise create conflict or resistance, recognize and work on the ecological structures that prevent projects from being sustainable, carry out a project hierarchy that helps the individual to organize their project system so that it is functional, achievable and coherent and meaningful. All these features give this methodology the potential to be implemented in psychotherapy and counseling to overcome the weaknesses of a nomothetic approach only.

Keywords: Goals; Personal projects; Psychotherapy; Idiographic approach.

\section{A importância da utilização de metas em psicoterapia}

A monitorização do progresso do cliente em processo terapêutico tem-se socorrido, sobretudo, de medidas nomotéticas, nas quais os itens estão predefinidos e predeterminados (Sales \& Alves, 2016). No entanto, este tipo de abordagem não contempla aspetos capazes de refletir a individualidade de cada cliente, negligenciando, nomeadamente, o progresso na superação de dificuldades e problemas específicos (Lloyd, Duncan, \& Cooper, 2019). A utilização de medidas idiográficas para este fim atingiu alguma popularidade nas últimas décadas (Elliott et al., 2016, p. 263). As mesmas possibilitam aceder ao progresso do cliente, e a processos complexos de mudança que têm relevância para a própria pessoa, e que são mais consistentes com o próprio trabalho psicoterapêutico (Edbrooke-Childs, Jacob, Law, Deighton, \& Wolpert, 2015).

As metas pessoais são uma das formas para a concretização desta abordagem idiográfica em contexto de aconselhamento (Cooper, 2018; Tryron, 2018), e recentes meta-análises apontam para um

\footnotetext{
${ }^{18}$ Morada para correspondência: Isabel Albuquerque. CINEICC - Faculdade de Psicologia e de Ciências da Educação da Universidade de Coimbra, Rua do Colégio Novo, 3000-115 Coimbra. E-mail: ialbuquerque.ialbuquerque@gmail.com
} 
efeito positivo da definição e da sua monitorização numa diversidade de aspetos comportamentais (Epton, Currie, \& Armitage, 2017; Harkin et al., 2016). Desde a década de oitenta que a investigação psicológica tem mostrado interesse pela psicologia conativa, enquanto análise e explicação dos conteúdos, da estrutura e das dinâmicas da atividade pessoal direcionada a metas (Albuquerque, 2015). Os resultados que emergem desta área de investigação ligada à ação intencional oriundos sobretudo da psicologia da personalidade, como os empenhamentos pessoais (Emmons, 1986), as preocupações atuais (Klinger, 1977), as tarefas de vida (Cantor \& Zirkel, 1990) ou os projetos pessoais (Little, 1983), podem constituir-se como uma base de apoio importante para a prática psicoterapêutica (Cooper \& Joseph, 2016) e podem ajudar a alicerçar a mesma em modelos válidos e defensáveis ao nível do funcionamento humano e da mudança (Cooper, 2018, 2019; Cooper \& Joseph, 2016). Um aspeto importante que ressalta de um vasto conjunto de estudos é o facto de as metas pessoais, e os múltiplos processos que lhe são inerentes, serem preditores robustos do bem-estar subjetivo, sobretudo das suas duas componentes afetivas, afeto negativo e afeto positivo (Cooper, 2018; Epton, Currie, \& Armitage, 2017; Harkin et al., 2016; Kaftan \& Freund, 2018; Klug \& Maier, 2015; Milyavskaya \& Werner, 2018).

A metodologia de Análise dos Projetos Pessoais - APP (Little, 1983) - integra-se nesta abordagem cognitivo-motivacional e permite aceder, por um lado, às metas pessoais que as pessoas formulam e tentam atingir e, por outro, providencia uma via de acesso às avaliações cognitivas e afetivas que cada sujeito faz sobre os seus projetos pessoais (Albuquerque, 2015). Em Portugal, esta metodologia tem sido utilizada maioritariamente no âmbito de estudos da relação entre a personalidade e o bem-estar psicológico e subjetivo (Albuquerque, 2006, 2015; Albuquerque \& Lima, 2007; Albuquerque, Lima, Matos \& Figueiredo, 2013, 2014). No entanto, desde a sua formulação, têm sido inventariadas inúmeras potencialidades do seu uso em contexto de aconselhamento e psicoterapia (Little, 2011, Little \& Coulombe, 2015). Numa revisão recente relativa à utilização das metas pessoais em psicoterapia, Lloyd, Duncan e Cooper (2018) apresentam a Análise dos Projetos Pessoais como uma das metodologias capazes de responder a esse propósito.

\section{Projetos pessoais: 0 conceito}

Os projetos pessoais são entendidos como conjuntos de ações diferidas, pessoalmente relevantes, e em contexto (Little, 2011, 2014). Little (2007, p. 26) clarifica cada um destes aspetos: "conjuntos" porque os projetos pessoais raramente são uma única ação, mas antes um conjunto de ações geralmente interrelacionadas; "ações" porque os projetos envolvem transações entre o indivíduo e os contextos que o cercam; "diferidas" porque os projetos pessoais não são comportamentos momentâneos; "pessoalmente relevantes" porque se referem às ações idiossincráticas que têm relevância para os indivíduos; "em contexto" uma vez que os projetos pessoais acontecem numa ecologia social que integra contextos físicos, sociais, culturais e históricos. Enquanto unidades interacionais de análise, apreendem do comportamento humano os aspetos da ação estimulada pela intenção interna, mas embebida nos contextos reais. Segundo o modelo de ecologia social do desenvolvimento humano (Little 1999; Little \& Coulombe, 2015), os projetos pessoais são essenciais ao ajustamento do indivíduo aos diferentes contextos em que se move, uma vez que a sua implementação possibilita aos indivíduos integrar fontes de influência estáveis, quer de natureza biológica (e.g. Traços de personalidade), quer de natureza contextual (e.g. Normas culturais), promovendo maior bem-estar psicológico e físico.

\section{Análise dos Projetos Pessoais: pressupostos teóricos}

Este enquadramento concetual reclama uma abordagem metodológica específica, capaz de captar unidades de análise relativamente complexas pelo que foram formuladas algumas proposições relativas à natureza das pessoas, à natureza dos contextos e às características das transações entre as pessoas e os seus contextos, que estabelecem uma ligação entre as linhas teóricas e a metodologia de avaliação dos projetos pessoais. A Análise dos Projetos Pessoais (APP) assenta em quatro pressupostos fundamentais (ver Tabela 1) necessários à compreensão da mesma (Little \& Chambers, 2004; Little \& Balsari-Palsule, 2019). 
Tabela 1. Síntese dos pressupostos teórico-metodológicos da abordagem dos projetos pessoais (Little, 2005; Little \& Coulumbe, 2015)

Pressupostos Implicações Metodológicas na Avaliação dos Projetos Pessoais
Teóricos

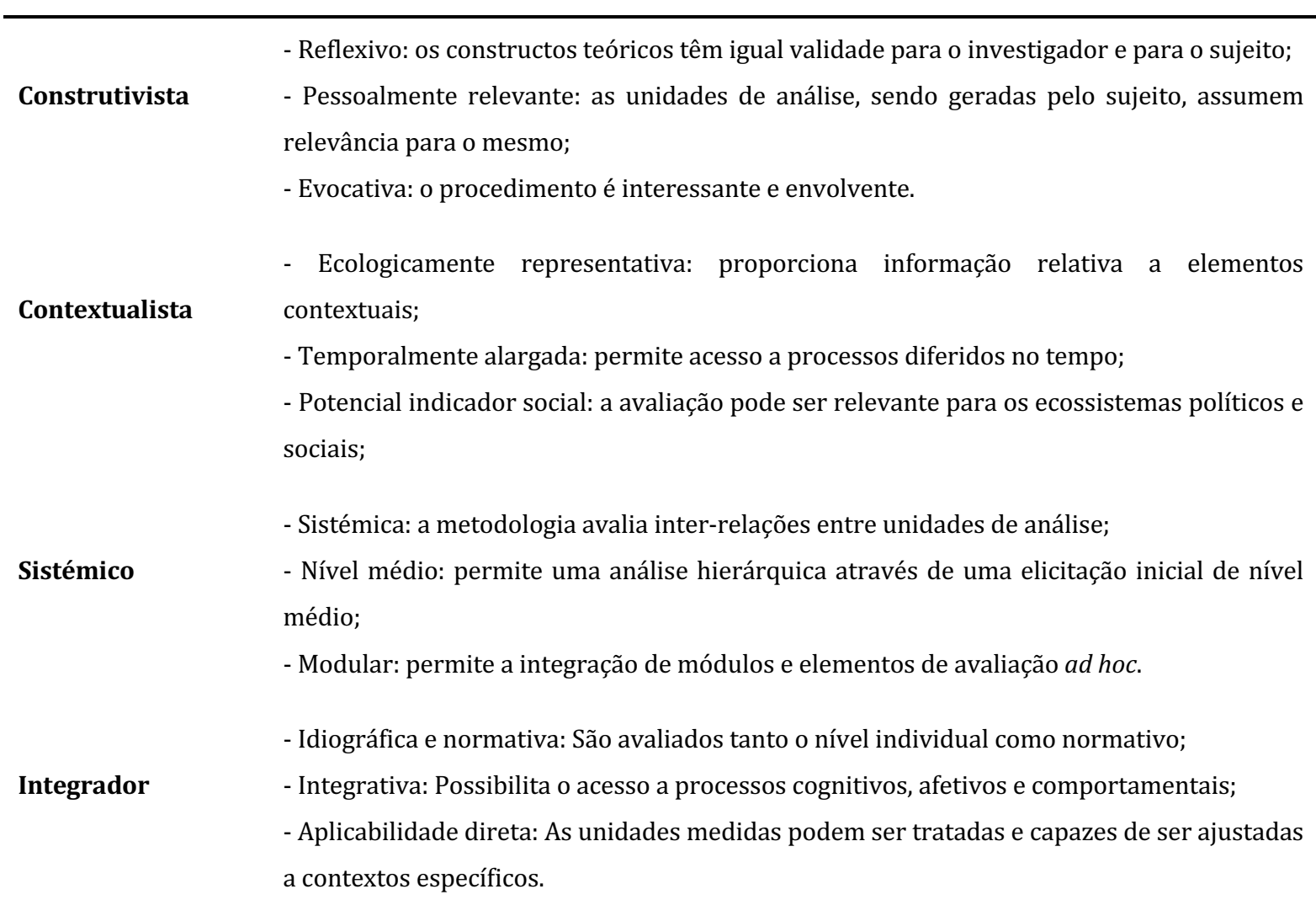

\section{Os diversos módulos de análise da metodologia}

A Análise dos Projetos Pessoais - APP (Little, 1983) - é flexível, funcionando como um sistema de avaliação abrangente concebido para ser aplicado a um amplo conjunto de questões teóricas e práticas, e tem sido utilizada em diferentes programas de investigação e também em contexto laboral.

No âmbito da terapia e do aconselhamento, a APP, ao centrar-se no modo como os indivíduos formulam e avaliam cognitiva e afetivamente os seus projetos pessoais, ao analisar o impacto entre esses mesmos projetos, ao permitir construir uma rede hierarquizada de projetos pessoais, bem como identificar projetos fundamentais que dão coerência e significado à vida, pode contribuir para detetar problemas ou questões que na vida de cada indivíduo são capazes de frustrar ou facilitar a implementação do programa terapêutico (Little \& Chambers, 2004). A adoção da APP na psicoterapia e aconselhamento proporciona meios para reformular o sistema de projetos, encorajar o cliente a investir em novos projetos, observar o modo como os projetos se promovem entre si ou se, por outro lado, criam conflitos ou resistências, reconhecer e trabalhar as estruturas ecológicas que impedem que os projetos sejam sustentáveis, realizar uma hierarquização de projetos que ajude o indivíduo a organizar o seu sistema de projetos de modo a que ele seja funcional, exequível e tenha coerência e significado.

A APP integra diversos módulos e consente uma utilização parcial por módulo, mas a sua utilização completa é particularmente importante em terapia e aconselhamento pois permite trabalhar variáveis importantes, específicas de cada um dos módulos, que têm implicações no bem-estar dos indivíduos (ver Tabela 2). 
Tabela 2. Módulos de avaliação que integram a APP e variáveis de análise que emergem de cada um deles

Módulos de avaliação $\quad$ Variáveis de análise que emergem do módulo

1 Explicitação de projetos $\quad \begin{aligned} & \text { Peso dos projetos, frequência das categorias, análise linguística e } \\ & \text { sintática, categorias e subcategorias, categorias problemáticas, } \\ & \text { equilíbrio entre categorias. }\end{aligned}$

2 Classificação de projetos

3 Matriz de impacto cruzado individual

4 Matriz de impacto cruzado conjunta

5 Hierarquização de projetos

6 Avaliação dos projetos fundamentais
Dimensões de classificação/itens e fatores emergentes dessa

classificação (significado, manejabilidade, conexão, afeto

positivo e afeto negativo)

Conflito ou promoção entre os projetos que integram o sistema a um nível individual.

Conflito ou promoção entre os projetos ao nível das pessoas que partilham a mesma ecologia social.

Nível de molaridade dos projetos e relação entre os mesmos.

Qual ou quais os projetos que dão coerência ao sistema de projetos pessoais, redes do sistema de projetos.

Módulo 1 - explicitação dos projetos pessoais. A explicitação de projetos pessoais, primeiro módulo da APP, inicia-se com uma explicação sobre o que são os projetos pessoais, sendo apresentados exemplos representativos de diversos níveis e conteúdos de projetos, e da sua ecologia social. Embora Little (1989) admita a possibilidade de limitar a listagem de projetos a uma determinada área de interesse (e.g., projetos da vida social), quando se trabalha no contexto do aconselhamento clínico pensamos que dar liberdade aos sujeitos para gerarem os projetos sem limitações permite que estes sejam representativos do seu sistema de projetos, e das relações entre os mesmos. Little e Chambers (2000) referem que só muito raramente surgem dificuldades por parte dos sujeitos em compreender o conceito de projeto pessoal, e em gerarem uma listagem de projetos. 0 resultado obtido no módulo explicitação de projetos concorre para vários tipos de análise.

Módulo 2 - a Classificação dos projetos. Neste módulo, são selecionados entre sete e dez projetos que são transcritos para a matriz de dimensões cognitivas e para a matriz de dimensões afetivas, sendo classificados entre 0 e 10 ao longo dessas dimensões.

0 conjunto destas dimensões cognitivas fazem emergir três fatores cognitivamente orientados significado, conexão e manejabilidade - e as dimensões-padrão afetivas dão origem a dois fatores - afeto positivo e afeto negativo (Little, 2011). É possível realizar análises normativas obtendo médias de cada uma das dimensões, examinando a relação entre as dimensões dos projetos e medidas do bem-estar. Num estudo realizado em Portugal, a consistência interna remeteu para um alfa de Cronbach de .95 para as dimensões cognitivas e de .90 para as dimensões afetivas (Albuquerque, 2006). Ao nível idiográfico, poder-se-ão estabelecer relações entre as diversas dimensões dos projetos relativamente a um só sujeito (Little \& Chambers, 2004). A investigação tem mostrado que cada uma das variáveis possibilitadas por este módulo tem ligações independentes com diversos índices do bem-estar e da qualidade de vida (Albuquerque, 2006, 2015; Little, 2008).

Módulos 3 e 4 - as matrizes de impacto cruzado. As matrizes de impacto cruzado integram aspetos tanto de natureza intrapessoal como interpessoal, e ajudam a perceber as dinâmicas presentes da vida de cada pessoa.

Na matriz de impacto cruzado do módulo 3, os indivíduos avaliam o impacto de cada um dos seus projetos face aos restantes que integram o sistema (Little \& Chambers, 2004). Riediger e Freund (2004) encontraram resultados que sugerem que o impacto entre as nossas perseguições pessoais se caracteriza 
pela facilitação e pela interferência, e que a interferência exerce uma influência negativa em diversas medidas do bem-estar.

Embora os respondentes refiram que este é o módulo mais informativo da APP, o contributo empírico das pontuações do impacto cruzado na predição do bem-estar é menor do que os que resultam das pontuações de dimensões como a eficácia ou o stresse (Little, 1998; Little \& Chambers, 2004).

No módulo 4, uma matriz de impacto cruzado a nível interpessoal permite examinar o impacto dos projetos pessoais entre pessoas que pertencem à mesma ecologia social, e apreciar até que ponto esses projetos se promovem mutuamente ou se, por outro lado, se embaraçam (Little, 2000; Little \& Chambers, 2004). No entanto, existem ainda poucos dados empíricos que permitam sustentar uma conclusão acerca da sua utilização (Little \& Chambers, 2000, 2004).

Módulo 5 - Hierarquização de projetos dos pessoais. A hierarquização permite ter uma visão sistémica inter-relações de projetos que pertencem a um mesmo sistema, mas que assumem molaridades e importâncias diferentes. Atos, projetos e valores integrados no sistema de projetos influenciam-se mutuamente, podendo o mesmo ato ser subordinado a mais de um projeto e um mesmo projeto servir mais do que um valor (Little, 2011; Little \& Chambers, 2000). A ação de hierarquizar os próprios projetos exige um questionamento específico para cada projeto que permite traçar a relação de cada projeto com outros de nível mais elevado, e traçar a relação do mesmo projeto com outros de nível hierárquico inferior (Little, 2011; Little \& Coulumbe, 2015). Quanto mais próximos os projetos estão de um valor final, mais significado têm para os indivíduos e quanto mais próximos os projetos estão dos atos, maior é a sua manejabilidade, ou seja, mais fácil é a sua concretização (Little \& Gee, 2007). A investigação tem mostrado que não basta os projetos terem significado para o indivíduo para que promovam o seu bemestar psicológico e subjetivo, sendo também necessário que, em conjunto com o significado, haja eficácia e progresso (manejabilidade) e, para isso, os projetos necessitam de serem desdobrados em atos concretizáveis (Albuquerque, 2006, 2015).

Para identificar as ligações entre estes níveis, adota-se a adaptação de uma técnica utilizada na investigação no âmbito dos construtos pessoais (Kelly, 1955). Essa técnica é constituída por dois processos que permitem, através de uma sequência contínua de perguntas e respostas, desdobrar os projetos, por um lado, em sentido ascendente conduzindo o indivíduo ao(s) projeto(s) que se encontram no topo e que, geralmente, têm mais significado dando sentido e propósito à vida e, por outro lado, permite, igualmente, desdobrar os projetos em sentido descendente conduzindo o indivíduo aos atos realizáveis, sem os quais o projeto em questão não poderia ser concretizado (Little \& Coulombe, 2015). Concretizar esta técnica implica utilizar apenas adequadamente duas questões: 1) "porquê?" e este porquê interrogativo sequencial permite ascender a níveis mais elevados da estrutura de projetos; e 2) "como?" e este como interrogativo sequencial conduz ao desdobrar de projetos em sentido descendente. No primeiro processo, é perguntado ao indivíduo "Porque é que está envolvido no projeto x?", enquanto que no segundo o indivíduo é questionado "Como é que o projeto x está ou pode ser levado a cabo?".

Módulo 6 - Avaliação de Projetos Fundamentais. Little (2007, 2011) defende que o florescimento humano é contingente à persecução sustentável de projetos fundamentais que são essenciais para o sistema de projetos de cada indivíduo, pois possibilitam a ligação dos projetos do sistema e lhe conferem coerência e operacionalidade. A incapacidade de reconhecer quais são esses projetos, a sua ausência ou a impossibilidade de os cumprir por dificuldades várias, nomeadamente por inexistência de projetos pessoais de nível mais molecular que os suportem, podem ter impacto muito negativo no bem-estar da pessoa. São esses projetos fundamentais que permitem chegar ao reconhecimento ou estabelecimento de um propósito e um significado de vida enquadrado por valores.

A noção de projetos pessoais fundamentais, apesar de ter grande relevância nesta abordagem, tem recebido pouca atenção por parte dos investigadores (Little, 2011). Este aspeto da avaliação dos projetos pessoais permite observar o nível na hierarquia de cada projeto e a existência de projetos moleculares, ou não, que possibilitam a sua concretização. A investigação mostrou que esses projetos são os que mais estão interconectados com os restantes (McDiarmid, 1990). Quando um projeto desses é retirado, isso pode comprometer todo o sistema.

No contexto da psicoterapia, um estudo recente de Di Malta, Oddli e Cooper (2019) aponta para o facto de as metas pessoais, em clínica, levarem a uma consciência das aspirações, ao envolvimento e à motivação, e suportarem a manutenção das ações, sendo igualmente úteis para desdobrar as metas de ordem superior em tarefas concretizáveis.

A Análise dos Projetos Pessoais é suscetível de ser utilizada em diversos contextos psicoterapêuticos e clínicos. De facto, o estabelecimento de metas é uma das técnicas mais utilizadas no contexto da mudança comportamental (Epton, Currie, \& Armitage, 2017). Grey e colegas (2018) 
consideram que a adoção de metas pessoais tem um papel central em diversas terapias, nomeadamente na terapia cognitivo-comportamental, terapia familiar e sistémica, psicanálise, psicoterapia interpessoal e psicoterapia existencial humanista. Cox e Klinger (2011) referem que o seu uso é fundamental nas terapias ligadas ao aconselhamento motivacional sistemático. Por sua vez, Grawe (2004) considera o seu uso relevante no âmbito da psicoterapia compreensiva.

\section{CONCLUSÃo}

Nas últimas duas décadas a adoção de metodologias no âmbito das metas pessoais em psicoterapia temse generalizado como uma forma de monitorizar o progresso do cliente, com a vantagem de respeitar a sua individualidade (Cooper \& Law, 2018).

A Análise dos Projetos Pessoais (Little, 1983) tem sido usada, em Portugal, sobretudo em investigação na população geral como forma de estudar a personalidade, tendo mostrado boa qualidade preditiva, nomeadamente a capacidade de as variáveis delas emergentes serem preditoras do bem-estar psicológico e subjetivo (Albuquerque, 2006, 2015), e de se constituírem como variáveis mediadoras (Albuquerque, Lima, Matos, \& Figueiredo, 2013) e moderadoras na relação dos traços de personalidade com esses dois tipos de bem-estar (Albuquerque, Lima, Matos, \& Figueiredo, 2014).

No entanto, o seu autor sempre argumentou em favor da sua utilidade no processo psicoterapêutico e de aconselhamento (Little \& Chambers, 2000, 2004; Little, 2011; Little \& Coulumbe, 2015), defendendo que pode levar à superação de diversos problemas presentes quando usamos outro tipo de metodologias.

De um ponto de vista teórico, parece-nos que esta metodologia responde às diversas demandas que são apresentadas pelos investigadores que procuram explorar a implementação das metas pessoais em psicoterapia e aconselhamento (Cooper, 2018, 2019; Cooper \& Law, 2018). A investigação futura na área dos projetos pessoais deverá explorar as potencialidades da APP em psicoterapia e aconselhamento, tanto mais que a definição de metas e a sua monitorização têm mostrado, nas últimas duas décadas, resultados positivos nesse contexto.

\section{REFERÊNCIAS}

Albuquerque, I. (2006). O florescimento dos professores: projectos pessoais, bem-estar subjectivo e satisfação profissional. [Dissertação de mestrado, Faculdade de Psicologia e de Ciências da Educação da Universidade de Coimbra].

Albuquerque, I. (2015). Traços disposicionais e projectos pessoais. O diálogo entre o "ter" e o "fazer" da personalidade na produção do bem-estar dos professores. [Tese de doutoramento, Faculdade de Psicologia e de Ciências da Educação da Universidade de Coimbra].

Albuquerque, I. \& Lima, M. P. (2007). Personalidade e bem-estar subjectivo: Uma abordagem com os projectos pessoais. Psicologia.com. http://www.psicologia.com.pt/artigos/textos/A0373.pdf

Albuquerque, I., Lima, M. P., Matos, M. \& Figueiredo, C. (2014). Work matters. Work personal projects and the idiosyncratic linkages between traits, eudaimonic and hedonic well-being. Social Indicators Research, 115, 855-906. https://doi.org/10.1007/s11205-013-0238-6

Albuquerque, I., Lima, M. P., Matos, M. \& Figueiredo, C. (2013). The interplay among levels of personality: The mediator effect of personal projects between the big five and subjective well-being. The Journal of Happiness Studies, 14, 235-250. https://doi.org/10.1007/s10902-012-9326-6

Ashworth, M., Guerra, D. \& Kordowicz M. (2019). Individualised or Standardised Outcome Measures: A Co-habitation? Administration and Policy in Mental Health and Mental Health Services Research, 46, 425-428. https://doi.org/10.1007/s10488-019-00928-z

Cooper, M. (2018). The psychology of goals: A practice-friendly review. In M. Cooper \& D. Law (Eds.), Working with goals in counselling and psychotherapy (pp. 35-71). Oxford University.

Cooper, M. (2019). Integrating counselling and psychotherapy: Directionality, synergy, and social change. Sage.

Cooper, M., \& Joseph, S. (2016). Psychological foundations for humanistic psychotherapeutic practice. In D. Cain, K. Keenan \& S. Rubin (Eds.), Humanistic psychotherapies (2nd ed., pp. 11-46). APA.

Cooper, M., \& Law, D. (2018). Introduction. In M. Cooper, \& D. Law (Eds.), Working with goals in counselling and psychotherapy (pp. 1-13). Oxford University.

Cooper, M., \& Joseph, S. (2016). Psychological foundations for humanistic psychotherapeutic practice. In D. Cain, K. Keenan \& S. Rubin (Eds.), Humanistic psychotherapies (2nd ed., pp. 11-46). APA.

Cantor, N., \& Zirkel, S. (1990). Personality, cognition, and purposive behavior. In L. Pervin (Ed.), Handbook of Personality Psychology: Theory and Research (pp. 135-164). Guilford Press. 
Klinger, E., Cox, W.M. (2011). Motivation and the goal theory of current concerns. In W.M. Cox \& E. Klinger, (Eds.), Handbook of Motivational Counseling: Goal-Based Approaches to Assessment and Intervention with Addiction and Other Problems (pp.1-47). Wiley-Blackwell.

Di Malta, G., Oddli, H. W., \& Cooper, M. (2019). From intention to action: A mixed methods study of clients' experiences of goal-oriented practices. Journal of Clinical Psychology. Advanced on-line publication. https://doi.org/10.1002/jclp.22821

Edbrooke-Childs, J., Jacob, J., Law, D., Deighton, J., \& Wolpert, M. (2015). Interpreting standardized and idiographic outcome measures in CAMHS: What does change mean and how does it relate to functioning and experience? Child and Adolescent Mental Health, 20, 142-148.

Elliott, R., Wagner, J., Sales, C., Rodgers, B., Alves, P., \& Café, M. J. (2016). Psychometrics of the Personal Questionnaire: A client generated outcome measure. Psychological Assessment, 28, 263-278. https://doi.org/10.1037/pas0000174

Emmons, R. A. (1986). Personal strivings: An approach to personality and subjective well-being. Journal of Personality and Social Psychology, 51, 1058 - 1068.

Epton, T., Currie, S., \& Armitage, C. J. (2017). Unique effects of setting goals on behavior change: Systematic review and meta-analysis. Journal of Consulting and Clinical Psychology, 85, 1182-1198. https://doi.org/10.1037/ccp0000260

Grawe, K. (2004). Psychological therapy. Hogrefe \& Huber Publishers.

Grey, N., Byrne, S., Taylor, T., Shmueli, A., Troupp, C., Stratton, P., Sefi, A., Law, R., \& Cooper, M. (2018). Goal-oriented practice across therapies. In M. Cooper \& D. Law (Eds.), Working with goals in psychotherapy and counselling (p. 181-203). Oxford University Press.

Harkin, B., Webb, T. L., Chang, B. P. I., Prestwich, A., Conner, M., Kellar, I., ... Sheeran, P. (2016). Does monitoring goal progress promote goal attainment? A meta-analysis of the experimental evidence. Psychological Bulletin, 142, 198-229. https://doi.org/10.1037/bul0000025

Kaftan, O. J., \& Freund, A. M. (2018). The way is the goal: The role of goal focus for successful goal pursuit and subjective well-being. In E. Diener, S. Oishi, \& L. Tay (Eds.), Handbook of well-being. DEF Publishers.

Kelly, G. A. (1955). The psychology of personal constructs. Norton.

Klinger, E. (1977). Meaning and void: Inner experience and the incentives in people's lives. University of Minnesota Press.

Klug, H. J. P., \& Maier, G. W. (2015). Linking goal progress and subjective well-being: A meta-analysis. Journal of Happiness Studies, 16, 37-65. https://doi.org/10.1007/s10902-013-9493-0

Lima, M. P. (2002). Personal Projects Analysis (Portuguese version for research). Unpublished manuscript.

Little, B. R. (1983). Personal projects: A rationale and method for investigation. Environment and Behavior, 15, 173-309.

Little, B. R. (1998). Personal project pursuit: Dimensions and dynamics of personal meaning. In P. T. P. Wong, \& P. S. Fry (Eds.), The human question for meaning: A handbook of psychological research and clinical applications (pp. 197-221). Sage

Little, B. R. (2000). Persons, contexts, and personal projects: Assumptive themes of a methodological transnationalism. In S. Wapner, J. Demick, T. Yamamoto, \& H Minami (Eds.), Theoretical perspectives environment-behavior research (pp. 79 - 88). Plenum.

Little, B. R. (2005). Personality science and personal projects: Six impossible things before breakfast. Journal of Research in Personality, 39, 4-21. https://doi.org/10.1016/j.jrp.2004.09.003

Little, B. R. (2007). Little, B. R. (2007). Prompt and circumstance: Generative contexts of personal projects. In B. R. Little, K. Salmela-Aro, \& S. D Phillips, Personal projects pursuit: Goals, action, and human flourishing (pp. 3-51). Lawrence Erlbaum Associates Publishers.

Little, B. R. (2008). Personal projects and free traits: Personality and motivation reconsidered. Social and Personality Compass, 2, 1235 - 1254. https://doi.org/10.1111/j.1751-9004.2008.00106.x

Little, B. R (2011). Personal projects and motivational counseling: The quality of lives reconsidered. In W. M. Cox \& E. Klinger (Eds.), Handbook of Motivational Counseling: Goal-Based Approaches to Assessment and Intervention with Addiction and Other Problems (pp. 73-88). John Wiley \& Sons.

Little, B. R. (2014). Well-doing: Personal projects and the quality of lives. Theory and Research in Education, 12, 329-346. https://doi.org/10.1177/1477878514545847

Little, B. R., \& Balsari-Palsule, S. (2019). Fates Beyond Traits: The Dynamics and Impacts of Personal Project Pursuit. Manuscript in preparation.

Little, B. R., \& Chambers, N. C. (2000). Personal Projects Analysis: An integrative framework for clinical and counselling psychology. Revue Québecoise de Psychologie, 21, 153-190.

Little, B. R., \& Chambers, N. C. (2004). Personal project pursuit: On human doings and well-beings. In W. Miles Cox \& E. Klinger (Eds.), Handbook of motivational counseling (pp. 65 - 82). John Wiley \& Sons. 
Little, B. R., \& Gee, T. L. (2007). The methodology of personal projects analysis: Four modules and a funnel. In B. R. Little, K. Salmela-Aro \& S. D. Phillips (Eds.), Personal project pursuit: Goals, action and human flourishing (pp. 51-93). Lawrence Erlbaum Associates.

Little, B. R., \& Coulombe, S. (2015). Personal projects analysis. In International Encyclopedia of the Social and Behavioral Sciences (2nd edition). Elsevier.

Lloyd, C. E. M., Duncan, C., \& Cooper, M. (2019). Goal measures for psychotherapy: A systematic review of self-report, idiographic instruments. Clinical Psychology: Science and Practice. Advanced on-line publication. https://doi.org/10.1111/cpsp.12281|

McDiarmid, E. (1990). Level of molarity, project cross-impact and resistance to change in personal project systems. [Unpublished master's thesis, Carleton University]

Milyavskaya, M., \& Werner, K. M. (2018). Goal pursuit: Current state of affairs and directions for future

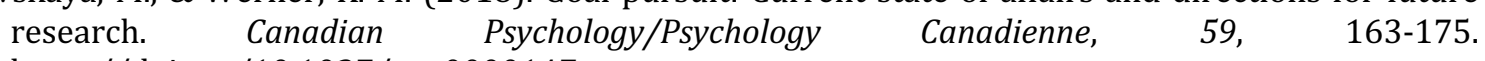
https://doi.org/10.1037/cap0000147

Riediger, M., \& Freund, A. M. (2004). Interference and facilitation among personal goals: Differential associations with subjective well-being and persistent goal pursuit. Personality and Social Psychology Bulletin, 30, 1511-1523. https://doi.org/10.1177/0146167204271184

Sales, C., \& Alves, P. C. (2016). Patient-centered assessment in psychotherapy: A review of individualized tools. Clinical Psychology: Science and Practice, 23, 265-283. https://doi.org/10.1111/cpsp.12162

Tryon, G. S. (2018). Goals and psychotherapy research. In M. Cooper \& D. Law (Eds.), Working with goals in counselling and psychotherapy (pp. 87-111). Oxford University.

Historial do artigo

Recebido $\quad 07 / 2019$

Aceite $\quad 03 / 2020$

Publicado $\quad 08 / 2020$ 


\title{
Preparando-se para a Copa do mundo: 0 que leva os brasileiros a comprar impulsivamente produtos para apoiar o seu país?
}

\author{
Samuel Lins ${ }^{19 \mathrm{i}}$ \\ i Universidade do Porto, Portugal
}

\begin{abstract}
Resumo: Os megaeventos esportivos são momentos em que o espírito de nacionalismo costuma ser elevado, fazendo com que as pessoas adquiram produtos para demonstrar apoio ao seu país na competição. 0 objetivo deste estudo correlacional é o de verificar quais as variáveis que influenciam o comportamento de compra por impulso de acessórios de torcida. Participaram do estudo 441 brasileiros (264 mulheres e 177 homens, $M_{\text {idade }}=34.56, D P=15.89$ anos). A recolha de dados foi realizada através de um questionário online (plataforma SurveyMonkey), que foi divulgado através do Facebook, do WhatsApp, e listas de e-mails durante a Copa do mundo de Futebol FIFA 2018. Os resultados mostraram que os homens são mais fanáticos pela copa e tendem a se envolver mais no evento. Um modelo de mediação sequencial, aplicado a ambos os sexos, revelou que quanto mais forte é a identidade nacional, maior é o fanatismo, que leva a um maior envolvimento, resultando na compra por impulso de acessórios de torcida.
\end{abstract}

Palavras-chave: Compra por impulso; Identidade nacional; Fanatismo; Emoções; Futebol.

Getting ready for the World Cup: What drives Brazilians to buy products impulsively to support their country? Sports mega-events are periods when the spirit of nationalism is high, leading people to buy products to express support for their country in the competition. The purpose of this correlational study is to verify which variables influence the impulse buying behavior of merchandise. 441 Brazilians participated in the study (264 women and $177 \mathrm{men}, M_{\mathrm{age}}=34.56 . S D=15.89$ years). Data collection was carried out through an online questionnaire (SurveyMonkey platform), which was shared through Facebook, WhatsApp, and email lists during the FIFA World Cup 2018. The results showed that men are more fanatic about the cup and tend to become more involved in the event. A sequential mediation model, applied to both sexes, revealed that the stronger the national identity, the greater the fanaticism, which leads to greater involvement, resulting in impulse buying of merchandise.

Keywords: Impulse buying; National identity; Fanaticism; Emotions; Soccer.

A Copa do mundo de futebol FIFA é o maior evento desportivo do planeta, e tem uma grande importância para os torcedores dos países que participam da competição. Durante a sua realização, o patriotismo emerge com mais força, levando as pessoas a se envolverem e a expressarem apoio ao seu país. Particularmente, no Brasil, a Copa pode ser considerada um fenómeno social relevante, pois tem uma grande repercussão e mobilização entre os brasileiros, fomentando um sentimento de orgulho nacional. Inclusive, é muito comum ouvir entre os brasileiros que eles são patriotas apenas de quatro em quatro anos, quando ocorre o evento.

Neste sentido, durante o evento, muito brasileiros passam a acompanhar de forma mais frequente os jogos da seleção brasileira de futebol, como também começam a procurar mais informações sobre o evento, e a se envolverem em atividade sociais, como se encontrar com amigos e familiares para assistir aos jogos. Outas atividades comuns nesta época são a compra de coisas para apoiar o país na competição (e.g., bandeiras, chapéus, camisas, etc.), e a manifestação da identificação com o país (e.g., vestir a camisa e decorar a casa e a vizinhança com o verde-amarelo). Assim, surgem as questões: em quais atividades os brasileiros mais se envolvem durante a Copa do mundo? E que variáveis estão relacionadas com a compra de produtos para demonstrar apoio ao seu país na competição?

\footnotetext{
${ }^{19}$ Morada para correspondência: Samuel Lins, Faculdade de Psicologia e de Ciências da Educação da Universidade do Porto, Rua Alfredo Allen, 4200-135, Porto, Portugal. E-mail: samuellins@fpce.up.pt. 0 autor agradece a Ana Eberhardt pelos comentários e pela revisão de versões do artigo
} 


\section{O Brasil e a Copa}

O futebol e a Copa do mundo estão diretamente relacionados com a identidade nacional do Brasil. Um estudo realizado por Lima e Santos (2016), solicitou a brasileiros que referissem imagens, pensamentos ou sentimentos que lhes ocorriam primeiro quando ouviam a expressão "Brasil". As expressões "futebol, "copa" e "seleção" foram muito evocadas. Por outro lado, um estudo realizado por Wachelke (2008), solicitou a uma amostra de brasileiros que indicassem palavras que lhes vinham à mente quando pensavam em "Futebol", e expressões como "Brasil" e "copa" também emergiram de forma frequente.

Estes estudos revelam como os dois termos, "Futebol" e "Brasil", estão fortemente associados. Portanto, é inegável o papel central que o futebol tem desempenhado na unidade nacional do país ao longo da história, e que o futebol tenha se tornado um símbolo de brasilidade (Penfold, 2018). Por isso, é de se esperar que quanto maior a identificação com o ser brasileiro, mais as pessoas terão uma forte atitude a favor da Copa do mundo (fanatismo), e uma maior probabilidade de se envolverem com o evento.

\section{Fanatismo}

O fanatismo pode ser definido como uma forma extrema de entusiasmo e devoção, resultando em elevados níveis de envolvimento, comprometimento e ligação emocional (Chung, Beverland, Farrelly, \& Quester, 2009). 0 objeto de devoção pode ser uma marca, uma celebridade ou um desporto, por exemplo (Thorne \& Bruner, 2006).

Segundo Wachelke, de Andrade, Tavares e Neves (2008), o fanatismo expressa níveis mais elevados de identificação grupal, ou seja, o fanatismo diz respeito a comportamentos, situações, crenças e atitudes extremas. Por exemplo, o fanatismo por um clube de futebol é evidenciado quando um adepto fica muito ansioso nos dias anteriores aos jogos e sente muita angústia durante os jogos, como também pensa que o sucesso do seu clube é uma das coisas mais importantes da sua vida, e pensa que o futebol não é diversão, mas um assunto sério (Wachelke et al., 2008). Neste sentido, espera-se que quanto maior o fanatismo pela Copa do mundo, maior será o envolvimento com o evento.

Para além da tradição histórica - o Brasil é o único país que participou de todas as edições do evento, como também é o país que mais venceu a competição, tendo ganho cinco copas - e do impacto social, a Copa também tem um impacto económico, principalmente no comércio. As lojas ficam cheias de produtos, dos mais variados tipos (e.g., camisas, chapéus, acessórios e bandeiras). Assim, é habitual que durante o evento, as pessoas comprem produtos para apoiar seu país, vestindo-se com as cores nacionais, pendurando bandeiras nas casas, e decorando os mais diversos lugares (e.g., ruas, escolas, centros comerciais e locais de trabalho). Tudo se preparando para o evento.

Durante a Copa, é muito comum ver bandeiras brasileiras expostas em muitos locais de forma mais frequente do que o habitual, aumentando ainda mais o sentimento de patriotismo. Neste sentido, alguns estudos revelam que a exposição frequente da bandeira nacional resulta num maior sentimento de nacionalismo, influenciando diversos tipos comportamentos (ex. julgamentos, comportamentos altruístas e comportamento de compra) (Guéguen, Martin, \& Stefan 2017).

Num estudo realizado no contexto esportivo, verificou-se que quanto mais forte as pessoas se identificavam com uma equipe maior era a tendência de comprar produtos de merchandise (e.g., bonés, camisas, canecas) (Fisher \& Wakefield, 1998). Importa destacar que quando as pessoas compram produtos que representam seu grupo desportivo, têm como objetivo vivenciar estados emocionais positivos, como a excitação e o orgulho, expressar sua identidade, como também socializar-se com outros (Kwon \& Kwak, 2014).

\section{Compra por impulso, emoções e o sexo}

Neste estudo, será abordado um tipo de compra específico - o comportamento de compra impulsiva. Segundo Rook e Fisher (1995), a compra impulsiva pode ser definida como uma "tendência do consumidor para comprar espontaneamente, sem reflexão, de forma imediata, estimulado pela proximidade física do objeto desejado, dominado pela atração emocional e absorvido pela promessa de gratificação imediata" (p. 306). A compra por impulso é um comportamento de compra complexo e hedónico, caracterizado por desconsiderar as consequências e a análise de alternativas (Sharma, Sivakumaran, \& Marshall, 2010), sendo acompanhada por um desejo poderoso e persistente de adquirir um produto (Xiao \& Nicholson, 2013).

Muitas vezes, a compra impulsiva é utilizada como sinónimo de compra compulsiva, por isso é importante fazer a diferenciação entre os dois conceitos. A compra por impulso pode ser realizada de forma habitual por qualquer pessoa. Já a compra compulsiva é patológica e crónica, sendo caracterizada por um desejo repetitivo e incontrolável de comprar, tendo como consequência o surgimento de sentimentos negativos, como o arrependimento e a culpa. Portanto, enquanto na compra por impulso o 
foco é o produto (o desejo repentino adquirir algo), na compra por compulsão o foco é o comportamento (o desejo de comprar por comprar).

Alguns estudos apontam que quanto maior a identificação com um grupo, mais as pessoas tendem a comprar impulsivamente produtos que representem este grupo (Kwon \& Armstrong, 2002). Assim, espera-se que quanto mais forte for a identidade nacional, maior será a tendência de comprar impulsivamente acessórios de torcida. Entretanto, apesar de pesquisas mostrarem que existe uma relação entre a identidade social e a compra por impulso, são escassos os estudos que aprofundam esta relação. Nesse sentido, torna-se importante identificar quais fatores poderiam mediar ou moderar a relação entre estas duas variáveis.

Adicionalmente, a realização de megaeventos desportivos tem uma forte simbologia, proporcionando diferentes maneiras de as pessoas se interligarem, levando-as a vivenciar um sentimento de pertença compartilhado por todo um país (Heere et al., 2013). Grandes eventos desportivos aumentam o envolvimento e o entusiasmo, como também provocam uma maior emotividade (Sullivan, 2009), o que pode levar as pessoas a vivenciarem tanto emoções positivas como negativas (Lage et al., 2014).

Como a compra por impulso está fortemente relacionada com os estados emocionais do indivíduo (Flight, Rountree, \& Beatty, 2012), também podemos esperar que as emoções vivenciadas (positivas e negativas), quando se pensa na Copa do mundo, influenciam o comportamento de compra impulsiva. Contudo, a literatura não é consensual sobre a relação entre as emoções e a compra por impulso, pois tanto estados emocionais positivos como negativos podem levar as pessoas a comprarem impulsivamente (Iyer, Blut, Xiao, \& Grewal, 2020). Por sua vez, neste estudo, espera-se que quanto mais as pessoas sentirem emoções positivas ao pensar no evento, mais propensas estarão a comprar coisas para apoiar o Brasil. Por outro lado, também se espera que quanto mais emoções negativas as pessoas vivenciarem ao pensar na Copa do mundo, menos comprarão acessórios de torcida impulsivamente.

Por fim, é possível que o sexo do participante também exerça uma influência nas variáveis em estudo. Relativamente a compra por impulso, estudos apontam que as mulheres tendem a comprar mais por impulso do que os homens (Fenton-0'Creevy \& Furnham, 2019; Santini, Ladeira, Vieira, Araújo, \& Sampaio, 2019). Entretanto, importa destacar que o tipo de produto também pode influenciar de forma distinta o comportamento de compra de ambos os sexos. Enquanto os homens tendem a comprar mais impulsivamente produtos de lazer e que refletem a sua independência, as mulheres tendem a comprar por impulso produtos cujas características estão mais relacionadas com a aparência e aspectos emocionais (Dittmar, Beattie, \& Friese, 1995).

No que diz respeito às variáveis relacionadas com a Copa do mundo (Fanatismo, Envolvimento e Emoções), estudos revelam que os homens são mais fanáticos por futebol do que as mulheres (Wachelke et al., 2008). Adicionalmente, homens e mulheres fazem julgamentos e vivenciam emoções de formas diferentes quando pensam no evento. Um estudo realizado por Lins e Borsa (2014), revelou que os homens, em comparação com as mulheres, sentem mais emoções positivas e menos emoções negativas quando pensam na Copa do mundo.

Assim, este estudo correlacional teve como objetivo: (1) Verificar quais as atividades que os brasileiros mais se envolvem durante a Copa do mundo; (2) Comparar as variáveis em estudo (Compra por impulso, Identidade nacional, Fanatismo e Envolvimento com a Copa, e Emoções positivas e negativas), em função do sexo; (3) Identificar quais variáveis estão relacionadas com a compra por impulso de acessórios de torcida; e, (4) Testar um modelo de mediação sequencial para verificar se o fanatismo e o envolvimento com a Copa medeiam a relação entre a identidade nacional e a compra por impulso.

\section{MÉTODO}

\section{Participantes}

Participaram do estudo 441 brasileiros (264 mulheres e 177 homens com idade média de 34.56 anos (DP $=15.89, \min =18$, máx $=87)$, de todos os estados da federação, sendo a maioria do estado do Rio de Janeiro $(n=115)$, São Paulo $(n=64)$, Paraíba $(n=62)$ e Minas Gerais $(n=33)$. Relativamente a classe social, ao comparar-se com a população da cidade onde morava, a maioria afirmou pertencer a classe média (40.6\%), seguido das classes média alta (23.4\%) e média baixa (22.4\%), alta ou muito alta $(7.2 \%)$, e baixa ou muito baixa (6.3\%).

Adicionalmente, $38.8 \%$ da amostra se consideram fãs de futebol, $27.4 \%$ costumam frequentar eventos desportivos (e.g., jogos de futebol, tênis, lutas, maratonas, etc.), apenas $10.2 \%$ foram assistir a algum jogo no estádio na Copa do mundo anterior no Brasil, em 2014, e 44.7\% residiam em alguma cidade-sede na última Copa. 


\section{Instrumentos}

\section{Compra por impulso}

Para avaliar a compra por impulso de acessórios de torcida, adaptou-se a escala de Rook e Fisher (1995), validada para o contexto brasileiro por Aquino, Natividade e Lins (2020). Todos os itens da escala foram modificados, incluindo a expressão "acessórios de torcida". A escala é unidimensional e composta por nove itens ( 1 = discordo totalmente, 5 = concordo totalmente), e apresentou propriedades psicométricas satisfatórias (ver Tabela 1) ${ }^{20}$.

Tabela 1. Análise fatorial da escala de Compra por Impulso de Acessórios de Torcida (Método: fatorização em eixos principais; rotação: oblimin direto)

\begin{tabular}{lc}
\hline Itens & $\begin{array}{c}\text { Peso } \\
\text { fatorial }\end{array}$ \\
\hline 1. "Eu vejo, eu compro acessórios de torcida": esta afirmação me descreve & .86 \\
2. Eu costumo comprar "acessórios de torcida" sem pensar & .81 \\
3. Eu frequentemente compro "acessórios de torcida" de forma espontânea & .81 \\
4. "Simplesmente compro": isto descreve a maneira como eu compro "acessórios de torcida" & .80 \\
5. "Compro acessórios de torcida agora e penso sobre isto mais tarde": esta afirmação me & .77 \\
descreve & .73 \\
6. Eu compro "acessórios de torcida" de acordo com o que sinto no momento & .65 \\
7. Às vezes eu fico com vontade de comprar "acessórios de torcida" no impulso do momento & .65 \\
8. Às vezes eu sou um pouco imprudente com os "acessórios de torcida" que eu compro & .39 \\
9. Eu planejo cuidadosamente a maioria dos "acessórios de torcida" que eu compro &
\end{tabular}

Nota: KMO Kaiser-Meyer-Olkin = .91; Teste de esfericidade de Bartlett $=X^{2}(36)=2316.24, p<.001 ;$ Eigenvalue $=5.22$; Variância explicada $=57.99 \% ; \alpha=.89$

\section{Identidade nacional}

A identificação com os(as) brasileiros(as) foi medida através de uma escala de cinco pontos $(1=$ discordo completamente; 5 = concordo completamente) de Lins e Borsa (2014), composta por 5 itens (Ser brasileiro(a) é importante pra mim, Gosto de ser brasileiro(a), Me identifico com os(as) brasileiros(as) em geral, Me orgulho de ser brasileiro(a), Sinto satisfação em pensar que sou brasileiro(a), $\alpha=.91$ ).

\section{Emoções}

Relativamente às emoções, o respondente foi solicitado a indicar a intensidade $(1=$ nada; $10=$ muitíssimo) da emoção que ele(a) sentia quando pensava na Copa do mundo FIFA 2018. Foram avaliadas 9 emoções, 5 positivas (alegria, satisfação, entusiasmo, admiração e orgulho, $\alpha=.95$ ) e 4 negativas (raiva, vergonha, tristeza e deceção, $\alpha=.87$ ).

\section{Fanatismo pela Copa}

O fanatismo pela Copa do mundo foi avaliado através da adaptação da Escala de Fanatismo em Torcedores de Futebol (EFTF) de Wachelke e colaboradores (2008). Para além dos onze itens da EFTF (e.g., Durante a Copa, eu fico muito ansioso nos dias anteriores aos jogos da seleção; Quando a seleção perde um jogo importante na Copa, eu fico perturbado, e isso afeta o andamento de outras tarefas do meu dia-adia; O sucesso da seleção na Copa é uma das coisas mais importantes da minha vida), também foi adicionado o item "Eu sou fanático(a) pela Copa do mundo", totalizando 12 itens $(1=$ discordo completamente; 5 = concordo completamente, $\alpha=.91$ ).

\section{Envolvimento com a Copa}

Os participantes foram solicitados a indicar qual era a probabilidade de se envolverem (1= nada provável a 5 = muito provável), em onze atividades relacionadas com a Copa do mundo (e.g., Acompanhar os jogos

\footnotetext{
${ }^{20}$ Antes de responderem a esta escala, foi apresentada a seguinte instrução: Durante a Copa do mundo é comum as pessoas comprarem coisas (ex. bandeiras, chapéus, camisas, etc.) para apoiar o Brasil. Vamos chamar estas coisas de "acessórios de torcida". A seguir temos algumas afirmações sobre este tipo de acessórios. Por favor, responda sendo (a) mais sincero(a) possível, indicando o seu grau de concordância.
} 
da seleção brasileira; Encontrar com outras pessoas (e.g., amigos, familiares, etc.) para assistir os jogos; Vestir a camisa com as cores do Brasil mais frequentemente; $\alpha=.91$ ).

\section{Procedimento}

A recolha de dados foi realizada através de um questionário online (plataforma SurveyMonkey), que foi divulgado através do Facebook, do WhatsApp, e listas de e-mails. Todos os participantes participaram de forma voluntária, e foram informados dos objetivos e procedimentos da pesquisa. Os respondentes apenas tinham acesso ao questionário, após concordarem com o termo de consentimento informado. A recolha de dados começou uma semana antes (6 de Junho de 2018) do início do evento (14 de Junho de 2018), e foi finalizada antes do jogo que o Brasil foi eliminado da Copa, jogando contra a Bélgica nas quartas de final, perdendo por 2 x 1 (6 de Julho de 2018).

\section{RESULTADOS}

Relativamente às atividades relacionadas com a Copa, verificou-se que as atividades com maior probabilidade de envolvimento eram "Acompanhar os jogos da seleção brasileira" e "Encontrar com outras pessoas (e.g., amigos, familiares, etc.) para assistir os jogos", e as de menor probabilidade eram "Colecionar álbum de figurinhas da Copa" e "Ajudar os vizinhos a decorar locais (e.g., rua, prédio, salão da comunidade, etc.) com as cores do Brasil" (ver Tabela 2).

Tabela 2. Média da probabilidade de se envolver em atividades relacionadas com a copa (1=nada provável; $5=$ muito provável)

\begin{tabular}{llcc}
\hline & Envolvimento na Copa & $M$ & $D P$ \\
\hline 1. & Acompanhar os jogos da seleção brasileira & 3.53 & 1.51 \\
2. & Encontrar com outras pessoas (e.g., amigos, familiares, etc.) para assistir os jogos & 3.27 & 1.58 \\
3. & Procurar informações sobre a Copa (e.g., em jornais, revistas, internet ou programas de & 2.61 & 1.57 \\
& TV) & 2.56 & 1.60 \\
4. & Acompanhar o máximo de jogos possível, não apenas os da seleção brasileira & 2.52 & 1.56 \\
5. & Procurar informações sobre a seleção brasileira de futebol (e.g., em jornais, revistas, & & \\
& internet ou programas de TV) & 2.46 & 1.55 \\
6. & Vestir a camisa com as cores do Brasil mais frequentemente & 2.27 & 1.63 \\
7. & Ter uma tabela ou um aplicativo no celular para acompanhar os resultados dos jogos & 1.81 & 1.27 \\
8. & Participar de apostas (e.g., bolões) com amigos, família, ou colegas de trabalho & 1.64 & 1.19 \\
9. & Decorar a casa com as cores do Brasil (e.g., pendurar a bandeira do Brasil na janela) & 1.59 & 1.17 \\
10. & Ajudar os vizinhos a decorar locais (e.g., rua, prédio, salão da comunidade, etc.) com as & 1.59 & 1.28 \\
\hline
\end{tabular}

Ao comparar as variáveis em estudo em função do sexo, verificou-se que os homens são mais fanáticos pela Copa, $t(439)=3.08, p=.002, d=0.30,95 \%$ IC $[0.11,0.49]$, e tem maior probabilidade de se envolverem em atividades relacionadas ao evento, $t(439)=2.83, p=.005, d=0.27,95 \%$ IC $[0.08,0.47]$, (ver Tabela 3).

As análises de correlações $r$ de Pearson mostraram que quanto mais forte for a identidade nacional, $r=.24 ; p<.001$, quanto mais emoções positivas forem vivenciadas ao pensar na Copa do mundo, $r=.50 ; p$ $<.001$, quanto maior for o fanatismo, $r=.58$; $p<.001$, e o envolvimento com o evento, $r=.62 ; p<.001$, maior é a tendência de comprar acessórios de torcida por impulso. Por outro lado, quanto mais emoções negativas são vivenciadas ao pensar na Copa, $r=-.18$; $p<.001$, menor é a tendência de comprar acessórios de torcida impulsivamente (ver Tabela 4). 
Tabela 3. Diferenças de Média das Variáveis em Estudo em Função do Sexo, segundo teste t de Student

\begin{tabular}{|c|c|c|c|c|c|c|c|}
\hline & \multicolumn{2}{|c|}{ Sexo } & \multirow[b]{2}{*}{$t(439)$} & \multirow[b]{2}{*}{$p$} & \multirow[b]{2}{*}{$\begin{array}{c}d \text { de } \\
\text { Cohen }\end{array}$} & \multicolumn{2}{|c|}{$95 \%$ IC } \\
\hline & $\begin{array}{l}\text { Feminino } \\
(n=264)\end{array}$ & $\begin{array}{c}\text { Masculino } \\
(n=177)\end{array}$ & & & & Inf. & Sup. \\
\hline Compra por impulso & 1.53 & 1.44 & 1.10 & .272 & - & - & - \\
\hline Identidade nacional & 3.56 & 3.46 & 0.92 & .359 & - & - & - \\
\hline Emoções positivas & 4.63 & 5.20 & 1.78 & .075 & - & - & - \\
\hline Emoções negativas & 3.61 & 3.35 & 0.94 & .346 & - & - & - \\
\hline Fanatismo pela Copa & 1.71 & 1.97 & 3.08 & .002 & 0.30 & 0.11 & 0.49 \\
\hline Envolvimento com a Copa & 2.24 & 2.53 & 2.83 & .005 & 0.27 & 0.08 & 0.47 \\
\hline
\end{tabular}

Nota. Os valores em negrito indicam a maior média entre os sexos

Tabela 4. Correlação das Variáveis em Estudo

\begin{tabular}{|c|c|c|c|c|c|c|c|}
\hline & $M(D P)$ & 1 & 2 & 3 & 4 & 5 & 6 \\
\hline 1. Compra por impulso & $1.49(0.84)$ & 1 & & & & & \\
\hline 2. Identidade nacional & $3.52(1.07)$ & $.24^{*}$ & 1 & & & & \\
\hline 3. Emoções positivas & $4.86(3.27)$ & $.50^{*}$ & $.39^{*}$ & 1 & & & \\
\hline 4. Emoções negativas & $3.51(2.79)$ & $-.18^{*}$ & $-.18^{*}$ & $-.29 *$ & 1 & & \\
\hline 5. Fanatismo pela Copa & $1.82(0.86)$ & $.58^{*}$ & $.32^{*}$ & $.72^{*}$ & $-.23^{*}$ & 1 & \\
\hline 6. Envolvimento com a Copa & $2.35(1.07)$ & $.62^{*}$ & $.31^{*}$ & $.74^{*}$ & $-.32 *$ & $.81^{*}$ & 1 \\
\hline
\end{tabular}

Nota. $^{*} p<.001$

Por fim, como o objetivo de compreender melhor a relação entre a identidade nacional e a compra por impulso de acessórios de torcida, foi testado se o fanatismo e envolvimento com a Copa mediavam de forma sequencial a relação entre estas duas variáveis. Para isto, foi realizada uma análise de mediação no macro PROCESS do SPSS (Modelo 6, Hayes, 2017), com método Bootstrap de 10000 amostras.

Como podemos verificar na Figura 1, o efeito total da identidade nacional na compra por impulso foi significativo, mas o efeito direto sem o efeito dos mediadores não foi significativo, indicando uma mediação total, $b=0.09,95 \%$ IC [0.06, 0.13]. Este modelo de mediação foi observado tanto na amostra geral, como em ambos os sexos separadamente (Mulheres: $b=0.11,95 \%$ IC [0.06, 0.17]; Homens: $b=0.06$, $95 \%$ IC $[0.03,0.11]$. Entretanto, é no sexo masculino que apresenta uma maior variância explicada (Mulheres: 36\%; Homens: 56\%).

Devido à forte relação entre Fanatismo e Envolvimento, surgiu a suspeita de que poderíamos estar diante de um caso de multicolinariedade. Contudo o teste de colinearidade não apresentou valores de VIF (Variance Inflation Factor) maiores do que três valores (VIF = 1.12; 3, e 3) 21 .

\section{DISCUSSÃo}

Os resultados revelaram que as atividades que têm a maior probabilidade de os brasileiros se envolverem são atividades que priorizam a sociabilidade, e que estão relacionadas com os jogos da seleção e com a procura de informações sobre o evento.

Ao comparar as variáveis em estudo em função do sexo, verificou-se que os homens são mais fanáticos pela copa e tem uma maior probabilidade de se envolverem em atividades relacionadas com o evento. Possivelmente, isto pode estar relacionado com a própria socialização e expectativas sociais dos papéis de género, pois os homens tendem a se socializar mais cedo com o futebol, por ser um desporto "tipicamente" masculino. Outras investigações também apontam para um maior envolvimento dos homens com o futebol, indicando que os homens brasileiros são torcedores mais fanáticos do que as mulheres brasileiras (Wachelke et al., 2008).

${ }^{21}$ Segundo O’brien (2007), o valor de VIF não deve ser maior do que 10. 

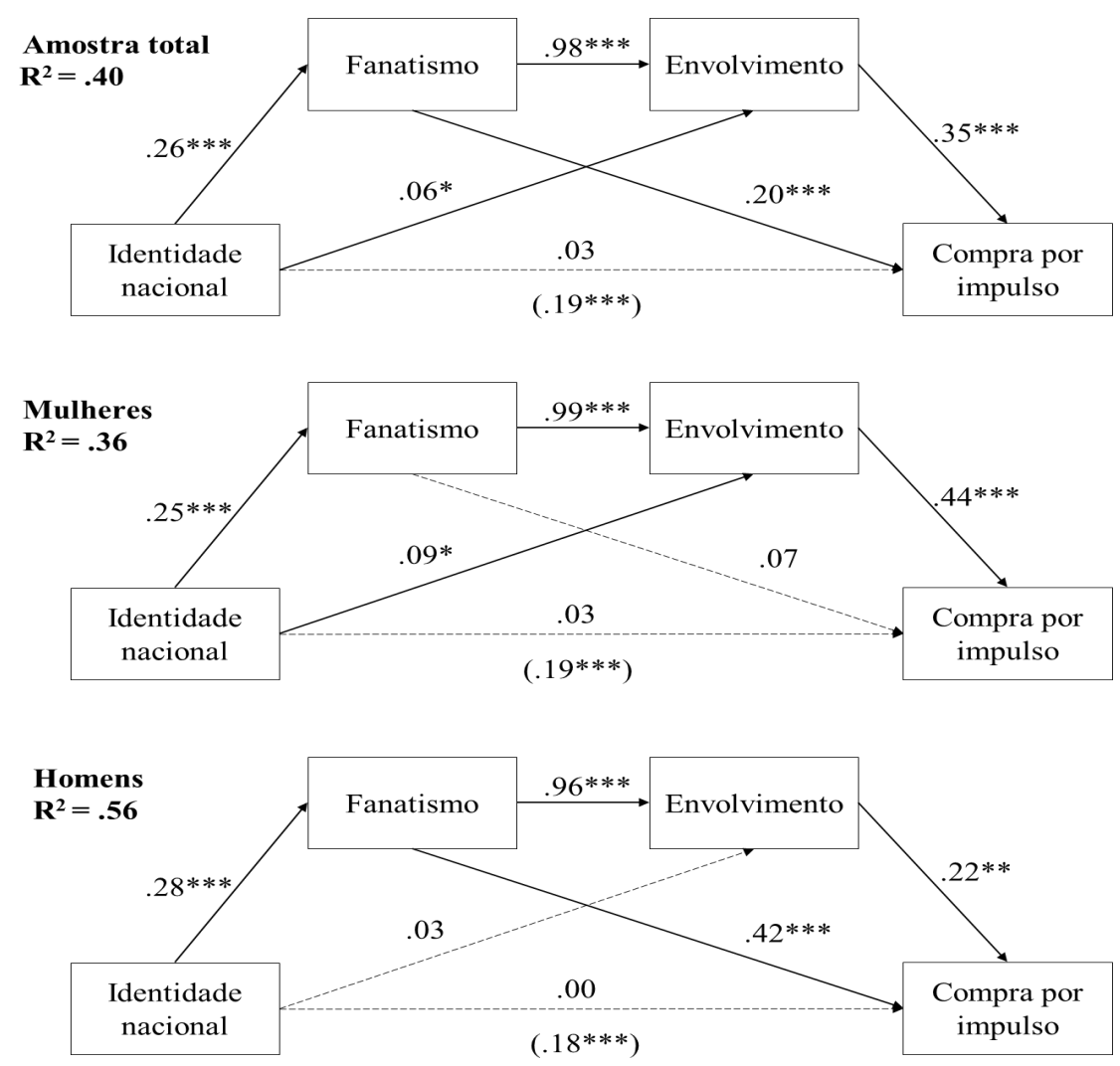

Figura 1. Relação entre a identidade nacional e a compra por impulso de acessórios de torcida mediado pelo fanatismo e envolvimento com a Copa. ${ }^{*} p<.05{ }^{* *} p<.01$; ${ }^{* * *} p<.001$

Entretanto, relativamente às emoções, não se verificou os resultados encontrados por Lins e Borsa (2014), que indicaram que os homens vivenciavam mais emoções positivas e menos emoções negativas em comparação com as mulheres quando pensavam na Copa do mundo. Cabe ressaltar que o estudo realizado por estes autores ocorreu quando a Copa foi sediada no Brasil, em 2014. É possível que estas diferenças na intensidade das emoções vivenciadas entre os sexos tenham sido maiores, pelo fato de o evento ter sido realizado no mesmo país dos participantes da pesquisa, tornando a identidade nacional mais saliente.

Importa destacar que as médias obtidas nas emoções, no fanatismo e no envolvimento foram baixas ou moderadas. É possível que os brasileiros não estivessem tão envolvidos com a Copa do mundo em 2018, devido à crise política que o país estava a vivenciar durante a realização do evento. Uma sondagem realizada pelo Instituto Paraná Pesquisas (2018), no mês que antecedia a abertura da Copa, revelou que cerca de $78 \%$ dos brasileiros estavam mais interessados em notícias sobre a Operação LavaJato $^{22}$ do que sobre a Copa do mundo da Rússia. Os resultados também corroboraram resultados anteriores, que mostraram a relação entre a identidade nacional e emoções positivas e negativas vivenciadas ao pensar na Copa (Lins \& Borsa, 2014).

Adicionalmente, a análise de mediação sequencial permitiu perceber melhor a relação entre a identidade nacional e a compra por impulso de acessórios de torcida, revelando que o Fanatismo pela Copa e o Envolvimento com o evento mediavam em sequência esta relação. Estes resultados evidenciam como a identidade nacional brasileira está relacionada com o futebol, ou seja, com o fanatismo pelo maior evento que representa este desporto: a Copa do mundo FIFA. 0 modelo aprofunda como a identidade social influencia o comportamento de compra impulsiva, englobando tanto variáveis atitudinais (identidade nacional e o fanatismo), como intenções comportamentais (envolvimento com a copa e a compra por impulso).

As pesquisas sobre a compra por impulso costumam considerar a identidade social apenas como uma variável antecedente, não examinando a relação entre estas duas variáveis, ou seja, não investigam o processo que há entre elas. Este estudo avança neste sentido, visto que se verificou que não há uma relação direta entre a identidade nacional e a compra por impulso de acessórios de torcida, mas que esta

${ }^{22}$ A Operação Lava Jato é um conjunto de investigações realizadas pela Polícia Federal do Brasil, com o objetivo de averiguar um esquema de corrupção e de lavagem de dinheiro, que envolvem políticos e empresários de grandes empresas brasileiras. 
relação é mediada por variáveis relacionadas ao evento (fanatismo e envolvimento). Entretanto, não estamos a nos referir a uma competição qualquer, mas a um evento que é parte da identidade brasileira. Assim, talvez não seria possível replicar este modelo em países onde o futebol não desempenha esse papel identitário, mesmo que o país estivesse a competir na Copa do mundo. Portanto, seria interessante confirmar esta hipótese em pesquisas futuras.

Para além disto, este processo parece ser moderado pelo sexo, visto que este modelo explica muito mais a compra por impulso dos homens, em comparação com as mulheres. Esta moderação não surpreende, visto que homens e mulheres fazem julgamentos e sentem as emoções com intensidades distintas quando pensam na Copa (Lins \& Borsa, 2014), e que os homens são mais fanáticos por futebol (Wachelke et al., 2008). Desse modo, é compreensível que a variância explicada do modelo aplicado ao sexo masculino tenha sido consideravelmente maior.

Por fim, este estudo revelou um resultado inesperado, nomeadamente na adaptação da escala de compra por impulso de Rook e Fisher (1995). Na escala original, o item "Eu planejo cuidadosamente a maioria dos produtos que eu compro" precisa ser invertido, pois se correlaciona negativamente com os demais itens, visto que quanto mais se planeiam as compras, menos impulsivo a pessoa tende a ser. Entretanto, ao adaptar para um produto ("Eu planejo cuidadosamente a maioria dos "acessórios de torcida" que eu compro"), este item passou a se correlacionar positivamente com os demais itens, não necessitando mais invertê-lo.

Tal resultado nos leva a refletir que, ao que parece, quando falamos de um comportamento de compra mais geral, o planeamento das compras tende a reduzir a compra impulsiva, mas quando se refere a um comportamento de compra relacionado a um produto específico, o planeamento prévio passa a estar relacionado com a compra por impulso. De facto, apesar de parecer uma contradição, Stern (1962) diz que a compra impulsiva também pode ser planeada. Isto ocorre quando o consumidor já tomou a decisão de compra, mas está apenas esperando um bom momento para poder efetuar a compra (por exemplo, quando há descontos).

Portanto, é possível que o envolvimento prévio do indivíduo com o produto (isto é, planear cuidadosamente a sua compra), possa estar relacionado com a impulsividade da compra. De facto, quando se trata de um produto específico, o cenário fica mais concreto, aproximando mais o consumidor da realidade do contexto de compra. Assim, sugere-se para estudos futuros verificar para quais tipos de produtos o "pensar cuidadosamente" poderia estar relacionado positivamente com a compra por impulso.

Os resultados também nos levam a refletir sobre a adaptação da escala de Rock e Fisher (1995) para um produto específico, no sentido de avaliar melhor o comportamento de compra por impulso. Visto que a compra por impulso pode ser realizada para os mais diversos tipos de produtos, parece que quando a escala está adaptada para um produto específico conseguimos obter variâncias explicadas maiores, do que quando a escala diz respeito a um comportamento geral.

Esta diferença entre "contexto geral versus específico", remete-nos para o que ocorre nos estudos sobre as atitudes, que mostram que as atitudes medidas para contextos mais específicos tendem a predizer melhor o comportamento do que atitudes mais gerais (Fishbein \& Ajzen, 1975). Neste sentido, quanto mais específico for o comportamento, mais forte será a relação entre a atitude-ação.

Portanto, fazendo um paralelo, quando se especifica um produto, melhor será a possibilidade de se identificarem variáveis determinantes no comportamento de compra por impulso deste produto, do que quando se avalia uma tendência mais geral para comprar impulsivamente. Assim, para estudos futuros, sugere-se aplicar a escala de compra por impulso de Rook e Fisher (1995), que avalia uma tendência mais geral, e adaptá-la para diversos tipos de produtos, utilizando as mesmas variáveis independentes, para que se possa identificar se estas variáveis estão relacionadas com uma tendência mais geral, ou em função do produto a ser comprado.

\section{CONCLUSÃO}

Este estudo identificou variáveis que influenciaram os brasileiros a comprar impulsivamente acessórios de torcida para apoiar o seu país na Copa do mundo FIFA 2018. Especificamente, explorou a relação entre a identidade social e o comportamento de compra por impulso. Apesar da estreita relação entre estas duas variáveis, ainda há poucos estudos existentes que examinam este processo. Portanto, compreender esta relação é relevante para as investigações da psicologia social aplicada aos estudos do consumo. Adicionalmente, também se constatou que o processo de compra pode ser diferente em função do sexo.

A principal limitação deste estudo é que o modelo testado é muito específico para um determinado contexto (durante a realização de um evento que acontece a cada quatro anos), e que também está intrinsecamente relacionado a cultura de um país (Brasil). Assim, este modelo talvez possa ser verificado apenas durante a realização da Copa do mundo, sendo, portanto, um "modelo temporário". Desse modo, 
estudos futuros poderiam ser realizados no intuito de saber se esse modelo se aplica, tanto em um período fora da Copa do mundo, como em outros países onde o futebol tem tradição e relevância social.

Como sugestão, um país e uma ocasião propícia para testar esse modelo seria Portugal, durante o próximo Campeonato Europeu de Futebol (EURO 2021) ${ }^{23}$. Para além do futebol ser um desporto tradicional no país, Portugal venceu a última edição da EURO, em 2016, e também venceu a Liga das nações da UEFA, em 2019, gerando uma elevada expectativa dos portugueses sobre o desempenho do país na competição, criando um cenário propício para a verificação do modelo.

Outra limitação do estudo foi considerar todos os produtos para apoiar o país na competição unicamente como "acessórios de torcida". Produtos diferentes (e.g., camisas, bolas, cachecóis, produtos de decoração) podem desencadear reações diversas em função do sexo. Portanto, para estudos futuros sugere-se adaptar a escala de compra por impulso para produtos específicos, em vez de uma categoria única incluindo todos os tipos de produtos. Adicionalmente, seria interessante pesquisar que tipos de produtos homens e mulheres compram para apoiar o seu país durante a Copa do mundo.

Ainda sobre a escolha de produtos para estudos que procurem identificar a relação entre a identidade social e a compra por impulso, é imprescindível que o produto a ser escolhido para o estudo tenha de fato uma relação com a identidade do grupo em questão. Se o produto não "fizer sentido" para o grupo que está salientado no estudo, esta relação também não existirá. Isto é, o produto precisa simbolizar e representar de certa forma a identidade social.

Apesar de terem sido variáveis utilizadas no estudo, as emoções (positivas e negativas) não foram incluídas no modelo testado. Segundo Iyer e colaboradores (2020), os estados emocionais são um fator importante que levam as pessoas a comprarem por impulso, o que pode ser considerado tanto como uma variável antecedente, mas principalmente como uma variável mediadora.

Estes autores sugerem a necessidade de compreender melhor este papel mediador das emoções, como também apontam para a necessidade de encontrar mais evidências empíricas para revelem quais emoções são mais proeminentes (Iyer et al., 2020). Deste modo, as emoções poderiam mediar a relação entre a identidade nacional e a compra por impulso. Recomenda-se para pesquisas futuras que esta hipótese seja testada.

Assim como este estudo, as pesquisas encontradas que investigaram a relação entre a identidade social e a compra impulsiva utilizaram predominantemente métodos correlacionais. Por isso, também se recomenda aplicar outros métodos para examinar esta relação, nomeadamente, métodos experimentais.

Por fim, importa também ressaltar que este estudo se enquadra numa série de outros estudos sobre identidade nacional que têm sido realizados enquanto importantes acontecimentos sociais estão a decorrer (e.g., megaeventos esportivos, como a Copa e as Olimpíadas; e as eleições presidenciais). São nestas ocasiões, afinal, que o sentimento de nacionalismo está mais acentuado, tornando-se um bom momento para a realização de estudos que objetivam investigar o papel desempenhado pela identidade social.

\section{REFERÊNCIAS}

Aquino, S. D., Natividade, J. C., \& Lins, S. L. B. (2020). Validity evidences of the Buying Impulsiveness Scale in the Brazilian context. PSICO-USF, 25(1), 15-25. https://doi.org/10.1590/141382712020250102

Chung, E., Beverland, M.B., Farrelly, F., \& Quester, P. (2008). Exploring consumer fanaticism: Extraordinary devotion in the consumption context. Advances in Consumer Research, 35, 333- 340.

Dittmar, H., Beattie, J., \& Friese, S. (1995). Gender identity and material symbols: objects and decision considerations in impulse purchases. Journal of Economic Psychology, 16(3), 491-511. https://doi.org/10.1016/0167-4870(95)00023-h

Fenton-O'Creevy, M., \& Furnham, A. (2019). Money attitudes, personality and chronic impulse buying. Applied Psychology, 0 (0), 1-16. https://doi.org/10.1111/apps.12215

Fishbein, M., \& Ajzen, I. (1975). Belief, attitude, intention, and behaviour: An introduction to theory and research. Addison-Wesley.

Fisher, R. J., \& Wakefield, K. (1998). Factors leading to group identification: A field study of winners and losers. Psychology \& Marketing, 15(1), 23-40. https://doi.org/10.1002/(SICI)15206793(199801)15:1<23::AID-MAR3>3.0.C0;2-P

Flight, R., Rountree, M., \& Beatty, S. (2012). Feeling the urge: affect in impulsive and compulsive buying. Journal of Marketing Theory and Practice, 20(4), 453-465. https://doi.org/10.2753/MTP1069-6679200407.

${ }^{23} 0$ campeonato estava previsto para ser realizado em 2020, mas foi adiado para 2021 devido à Pandemia de COVID-19. Esta será a 16 edição da competição, que será realizada em doze cidades de diferentes países europeus. 
Guéguen, N., Martin, A., \& Stefan, J. (2017). Holding your flag: The effects of exposure to a regional symbol on people's behavior. European Journal of Social Psychology, 47, 539-552. https://doi.org/10.1002/ejsp.2239

Hayes, A. F. (2017). Introduction to mediation, moderation, and conditional process analysis: A regressionbased approach. Guilford Publications.

Heere, B., Walker, M., Gibson, H., Thapa, B., Geldenhuys, S., \& Coetzee, W. (2013). The power of sport to unite a nation: the social value of the 2010 FIFA World Cup in South Africa. European Sport Management Quarterly, 13(4), 450-471. https://doi.org/10.1080/16184742.2013.809136

Instituto Paraná Pesquisas (2018). Pesquisa Brasil Online - Panorama Político Brasileiro atual. Retirado de: http://www.paranapesquisas.com.br/wp-content/uploads/2018/05/Lava-Jato-X-Copa-doMundo1.pdf

Iyer, G. R., Blut, M., Xiao, S. H., \& Grewal, D. (2020). Impulse buying: a meta-analytic review. Journal of the Academy of Marketing Science, 48, 384-404. https://doi.org/10.1007/s11747-019-00670-

Kwon, H. H., \& Armstrong, K. L. (2002). Factors influencing impulse buying of sport team licensed merchandise. Sport Marketing Quarterly, 11(3), 151-163.

Kwon, Y., \& Kwak. D.H. (2014). Revisiting the team identification-value- purchase relationship in the team-licensed merchandise consumption context: A multidimensional consumer value approach. Sport Marketing Quarterly, 23(2), 100-114.

Lage, C., Aversa, J., Diogo, C., Lins, S., \& Borsa, J. (2014). Alegria, vergonha e decepção: A percepção dos brasileiros da Copa do mundo. Comunicação apresentada na 9a Mostra Regional de Práticas em Psicologia, Rio de Janeiro. https://doi.org/10.13140/RG.2.2.18011.92969

Lima, M.E.O., \& Santos, E.V. (2016). Representações sociais do Brasil e identidade nacional. In M. E. 0. Lima, A. R. R. Torres \& E. M. Techio (Eds.), Identidade nacional e representações sociais do Brasil: Abordagens integrativas (pp. 79-102). BR: Scortecci.

Lins, S., \& Borsa, J. (2014). Identidade social, saúde mental e avaliação dos impactos da Copa do mundo FIFA 2014. Psicologia e Saber Social, https://doi.org/10.12957/psi.saber.soc.2014.12208

O’brien, R. M. (2007). A caution regarding rules of thumb for variance inflation factors. Quality \& Quantity, 41(5), 673-690. https://doi.org/10.1007/s11135-006-9018-6

Penfold, T. (2019). National identity and sporting mega-events in Brazil. Sport in Society, 22(3), 384-398. https://doi.org/10.1080/17430437.2018.1490266

Rook, D. W., \& Fisher, R. J. (1995). Normative influences on impulsive buying behavior. Journal of Consumer Research, 22(3), 305-313. https://doi.org/10.1086/209452

Santini, F., Ladeira, W., Vieira, V., Araújo, C., Sampaio, C. (2018). Antecedents and consequences of impulse buying: a meta-analytic study. RAUSP Management Journal, 54 (2), 2531-0488. https://doi.org/10.1108/rausp-07-2018-0037

Sharma, P., Sivakumaran, B., \& Marshall, R. (2010). Impulse buying and variety seeking: A trait-correlates perspective. Journal of Business Research, 63(3), 276-283. https://doi.org/10.1016/j.jbusres.2009.03.013.

Stern, H. (1962). The significance of impulse buying today. Journal of Marketing, 26, 59-62. https://doi.org/10.2307/1248439

Sullivan, G. (2009). Germany during the 2006 World Cup: The role of television in creating a national narrative of pride and "party patriotism". In E. Castelló, A. Dhoest, \& H. O'Donnell (Eds.). The Nation on Screen, Discourses of the National in Global Television (pp. 235 - 252). Cambridge.

Thorne, S., \& Bruner, G.C. (2006). An exploratory investigation of the characteristics of consumer fanaticism. Qualitative Market Research: An International Journal, 9 (1), 51-72. https://doi.org/10.1108/13522750610640558

Wachelke, J. (2008). Brazilian fans' social representations on soccer. International Journal of Sport Science, 4(13), 1-19. https://doi.org/10.5232/ricyde2008.01301

Wachelke, J., De Andrade, A., Tavares, L., \& Neves, J. (2008). Mensuração da identificação com times de futebol: evidências de validade fatorial e consistência interna de duas escalas. Arquivos Brasileiros de Psicologia, 60(1), 96-111.

Xiao, S. H., \& Nicholson, M. (2013). A multidisciplinary cognitive behavioural framework of impulse buying: A systematic review of the literature. International Journal of Management Reviews, 15(3), 333-356. https://doi.org/10.1111/j.1468-2370.2012.00345.x 
Historial do artigo

Recebido 07/2019

Aceite $\quad 05 / 2020$

Publicado 08/2020 
Revista PSICOLOGIA, 2020, Vol. 34 (1), doi: 10.17575/psicologia.v34i1.1681

Atas do X Simpósio Nacional de Investigação em Psicologia

\title{
Educação positiva: A escola como uma instituição promotora de bem- estar
}

\author{
Anita Figueira ${ }^{24 i} \&$ Margarida Pocinho ${ }^{\mathrm{i}}$ \\ i Universidade da Madeira
}

\begin{abstract}
Resumo: Este estudo tem como objetivo analisar a Educação Positiva, como uma possível alternativa pedagógica ao sistema educativo convencional. Devido às elevadas taxas de perturbações mentais entre os jovens em todo o mundo, a escola surge como um espaço privilegiado que oferece uma extraordinária oportunidade para aceder a um grande número de crianças e jovens, e combater esse fenómeno. A Educação Positiva integra diretrizes cientificamente validadas da Psicologia Positiva, e tem o propósito de promover o bem-estar e o sucesso académico dos alunos. Após a apresentação concetual da Educação Positiva, este estudo trás ao de cima diversas pesquisas atuais, por meio de uma Revisão Sistemática da Literatura efetuada na Biblioteca do Conhecimento Online (B-on). As investigações analisadas indicam que, através a aplicação de programas de intervenção baseados em evidências científicas que promovem o bem-estar na comunidade educativa, a escola pode de facto ser uma instituição positiva promotora de saúde mental.
\end{abstract}

\section{Palavras-chave: Educação positiva, Psicologia positiva, Bem-estar, Saúde mental.}

Positive Education: The school as a place of well-being: This study aims to analyze Positive Education as a possible pedagogical alternative to the conventional educational system. Due to the high rates of mental disorders among young people around the world, the school emerges as a privileged space offering an extraordinary opportunity to reach out to a large number of children and young people and to combat this phenomenon. Positive Education integrates scientifically validated guidelines from Positive Psychology and aims to promote students' well-being and academic success. After the conceptual presentation of Positive Education, this study demonstrates several current researches, through a Systematic Review of Literature done at the Online Knowledge Library (B-on). The investigations analyzed indicate that through the application of program-based intervention with scientific evidence that promote well-being in the educational community, the school can in fact be a positive institution that promotes mental health.

Keywords: Positive education, Positive psychology, Wellbeing, Mental.

A prevalência de perturbações mentais e de problemas de comportamento em crianças e jovens tem crescido consideravelmente nos últimos anos. Cerca de um em cada cinco alunos é afetado, revelando um real impacto no desempenho escolar para além de diversas consequências individuais, familiares e sociais (Ordem dos Psicólogos Portugueses, 2015).

De acordo com Seligman, Ernest, Gilham, Reivich e Linkins (2009), no final do ensino secundário cerca de $20 \%$ dos jovens experienciam um episódio de depressão clínica. A Organização Mundial da Saúde revela, também, estimativas chocantes acerca das taxas de prevalência de suicídio a nível mundial. Mais especificamente, as estatísticas indicam que o suicídio foi a segunda principal causa de morte de cidadãos na faixa etária entre os 15 e 29 anos de idade, no ano de 2015 (World Health Organization, 2017).

Na comunidade científica cada vez mais se procura compreender de que forma é possível combater e prevenir esta problemática, que é crescente nos dias de hoje. Investigadores como Seligman e colaboradores (2009) afirmam que, para combater este fenómeno, é necessário ensinar aos jovens habilidades e estratégias que aumentem a resiliência, as emoções positivas, o significado de vida e o bemestar, sem retirar espaço ao currículo.

\footnotetext{
${ }^{24}$ Morada para correspondência: Anita Figueira, Universidade da Madeira. Departamento de Humanidades- Caminho da Achada 10, Funchal, Portugal. E-mail: anita.figueira14@gmail.com. Esta investigação foi desenvolvida no âmbito da Dissertação de Mestrado em Psicologia da Educação.
} 


\section{Da Psicologia Positiva à Educação Positiva}

Devido ao papel crucial que a educação tem na sociedade atual, as instituições educacionais passaram a ser estudadas pela Psicologia Positiva no âmbito de se tornarem locais privilegiados para a promoção do bem-estar, tanto na comunidade escolar como na sociedade em geral (Cintra \& Guerra, 2017).

A Educação Positiva foi definida pela primeira vez por Seligman e os seus colaboradores (2009), como "a educação tanto para as habilidades tradicionais como para a felicidade". 0 termo surgiu no início do ano 2008, durante um encontro entre Seligman e os membros da equipa de Geelong Grammar School (GGS) (Norrish, 2015, citado por Cintra \& Guerra, 2017). A referida escola australiana foi a primeira a implementar os princípios da Psicologia Positiva em toda a instituição de ensino. Mais especificamente, através do aconselhamento com diferentes especialistas mundiais em psicologia positiva, e com base no modelo multidimensional PERMA de Florescimento desenvolvido por Seligman (2012, citado por White, 2016), a GGS desenvolveu o seu Modelo de Educação Positiva (Figuras 1 e 2) para complementar a aprendizagem tradicional. A partir do primeiro treino em grande escala do pessoal do GGS, progressivamente a equipa foi expandindo e criaram o Instituto de Educação Positiva da Geelong Grammar School. Atualmente, já treinaram mais de 10 mil educadores de mais de 600 escolas e organizações de diferentes países da Ásia, Europa e Médio Oriente (Bott et al., 2017).

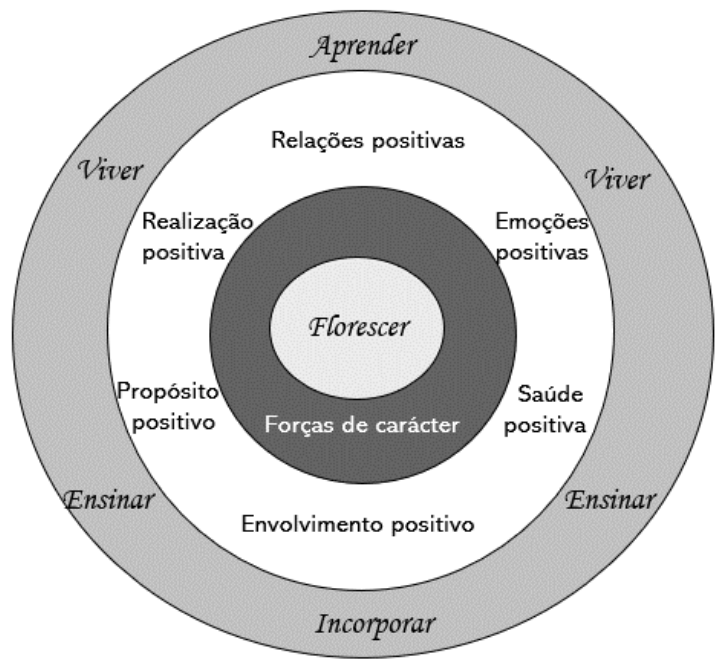

Figura 1. Modelo de Educação Positiva da Geelong Grammar School, adaptado de Hoare, Bott e Robison (2017).

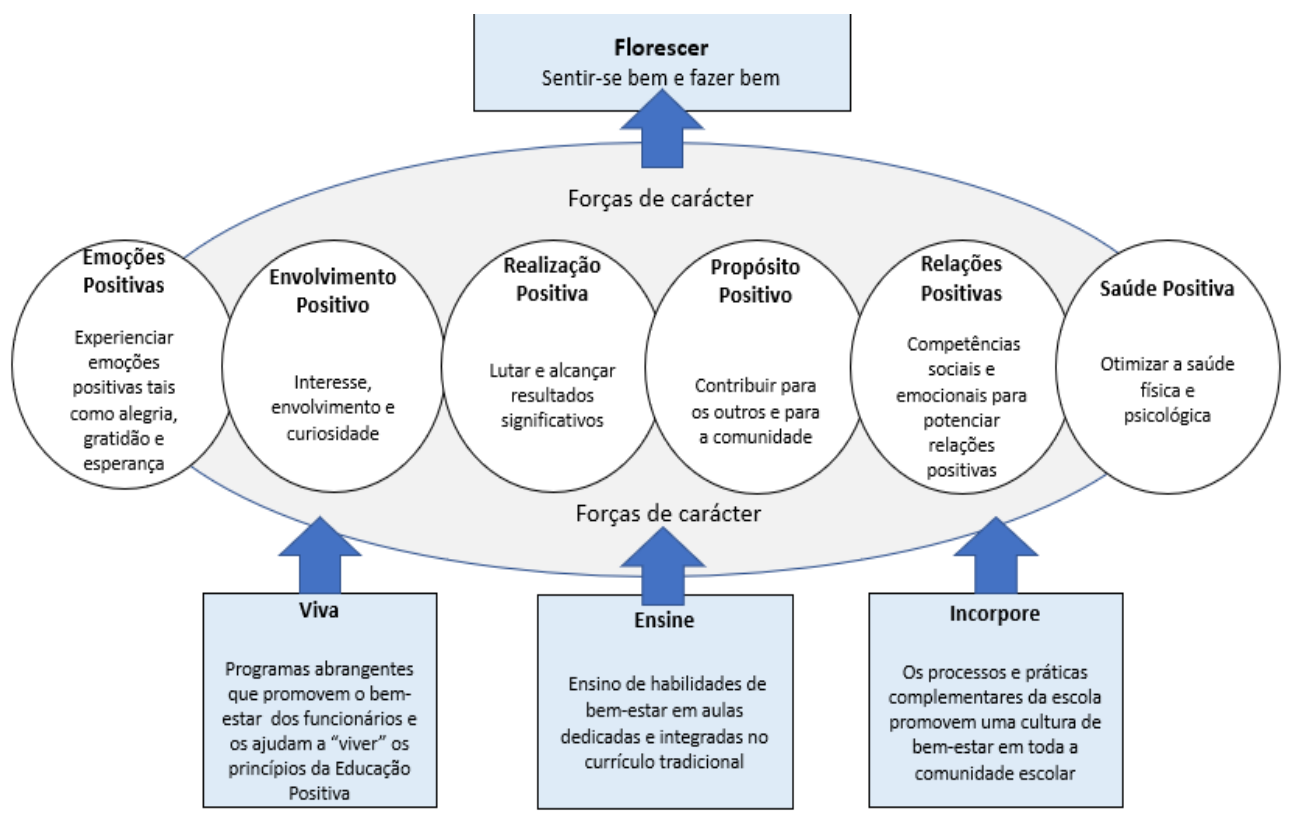

Figura 2. Esquema com os pressupostos do Modelo de Educação Positiva, adaptado de Norrish, Williams, O'Connor e Robinson (2013). 
Neste âmbito, são dadas várias oportunidades, desde cursos a workshops, para que os funcionários e os pais dos alunos consigam "aprender" os princípios da Educação Positiva, sendo encorajados a "viver" esses princípios através da modelação de comportamentos apropriados nas suas interações com os outros e com os alunos. Na escola, procura-se que os alunos possam viver esses princípios, através da promoção e ensino de competências nos alunos (Bott et al., 2017; Seligman \& Adler, 2018).

Seligman e Adler (2018) participaram na redação de um documento da Global Hapiness Policy Report relatando a disseminação da Educação Positiva em todo o mundo, e evidenciando alguns casos práticos de escolas na China, Índia, Emirados Árabes Unidos, Israel, Austrália, México, Peru, Estados Unidos e Inglaterra em que foram implementados e avaliados os programas de Educação Positiva. Os autores demonstraram diversas evidências que a Educação Positiva aumenta, de facto, os objetivos tradicionais das escolas (alfabetização e ciência, etc.), bem como um aumento significativo no bem-estar nos alunos e agentes educativos.

\section{MÉTODO}

Devido à dispersão de modelos pedagógicos em todo o mundo, e à necessidade emergente de avaliar e adaptar os sistemas educativos escolares (Pinto, Garcia, \& Letichevsky, 2006), procurámos investigar durante o período de junho a setembro do ano de 2018 quatro pedagogias conhecidas mundialmente: a Pedagogia de Waldorf, o Método Montessori, a Escola da Ponte e a Educação Positiva ${ }^{25}$. Sob uma perspetiva científica de carácter psicológico e psicopedagógico, procurou-se responder à questão "quais são os fatores psicológicos e psicopedagógicos que estão subjacentes a cada método pedagógico em estudo".

Como objetivo geral, procurou-se reunir um conhecimento científico mais profundo acerca dos múltiplos fatores psicológicos (de ordem cognitiva, afetiva, motivacional e desenvolvimental) mediadores do processo de ensino-aprendizagem das pedagogias, de forma a contribuir para um delineamento mais integrativo do ponto de vista psicopedagógico os sistemas educativos escolares.

Como método de investigação, foi utilizada a revisão sistemática da literatura que é, de acordo com Sousa e Ribeiro (2009), "uma revisão planeada da literatura científica, que usa métodos sistemáticos para identificar, selecionar e avaliar criticamente estudos relevantes sobre uma questão claramente formulada" (p. 241) permitindo assim reduzir possíveis erros que poderiam surgir numa revisão não planeada da literatura. Como principal referência deste método, foram utilizadas as recomendações do PRISMA - Principais Itens para Relatar Revisões Sistemáticas e Meta-análises (Preferred Reporting Items for Systematic reviews and Meta-Analyses) (Moher, Liberati, Tetzlaff, Altman \& The PRISMA Group, 2009). O PRISMA consiste num fluxograma de quatro etapas (figura 3) que permite expor os procedimentos e os resultados da investigação.

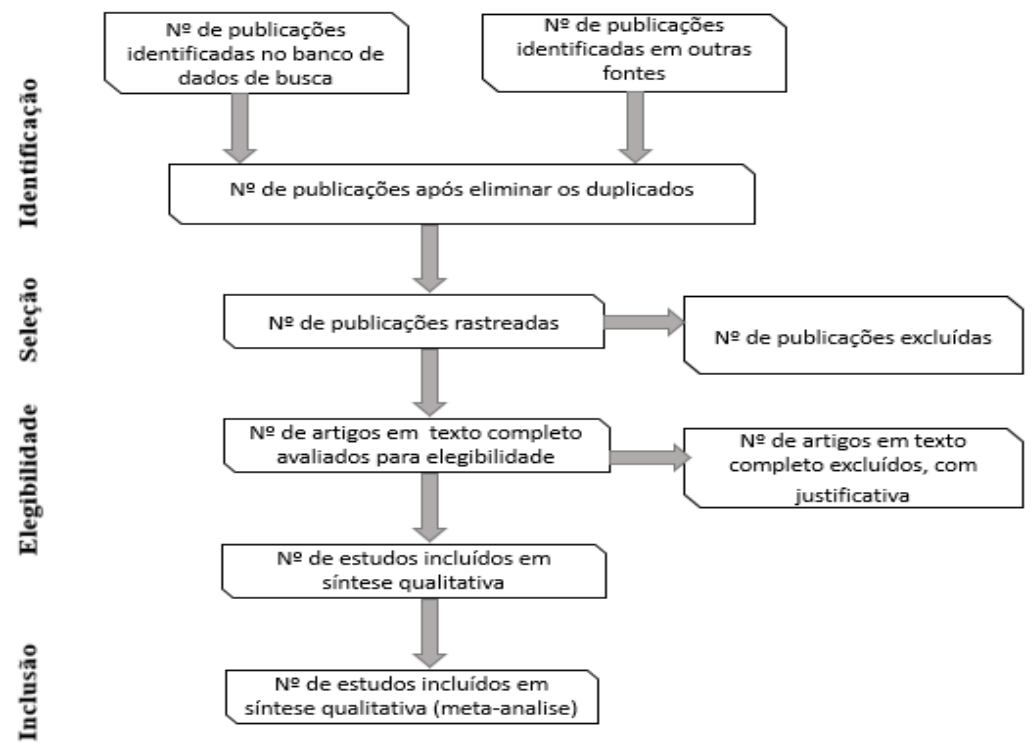

Figura 3. Fluxograma de quatro etapas- PRISMA, adaptado de Moher, Liberati, Tetzlaff, Altman e The PRISMA Group (2009).

${ }^{25}$ Este artigo é parte da dissertação de mestrado em Psicologia da Educação, sob orientação de Margarida Pocinho. 


\section{Etapas da RSL}

Em primeiro lugar, foi definido como fonte de pesquisa de dados, a Biblioteca de Conhecimento Online (Bon). Atualmente a B-on é reconhecida como uma grande referência no acesso à informação científica internacional (Active Media, 2018) pois permite o acesso livre a publicações científicas de diferentes plataformas online de bases de dados como a ProQuest, Eric, JSTOR, ResearchGate, SciELO e Elsevier.

De seguida, foram definidos os critérios de inclusão e exclusão (ver Tabela 1), os termos de pesquisa (Psicologia* AND Método Montessori; Psicologia* AND Pedagogia de Waldorf; Psicologia* AND Escola da Ponte; Psicologia* AND Educação Positiva), as opções de pesquisa, os idiomas (português, inglês e espanhol) e a data das publicações (2000-2018).

Tabela 1. Critérios de inclusão e exclusão

\begin{tabular}{|c|c|}
\hline Critérios de inclusão & Critérios de exclusão \\
\hline $\begin{array}{l}\text { Estudos Científicos, Dissertações de Mestrado e } \\
\text { Doutoramento, Revistas Científicas, em que os termos de } \\
\text { pesquisa selecionados estejam presentes no título e/ou } \\
\text { nos termos do assunto e/ou no resumo }\end{array}$ & $\begin{array}{l}\text { Livros e notícias sem revisão de pares mesmo que } \\
\text { os termos de pesquisa estejam presentes no título } \\
\text { e/ou nos termos do assunto e/ou no resumo }\end{array}$ \\
\hline Período de publicação entre 2000 e 2018 & Publicações cujo a data anteceda 2000 \\
\hline $\begin{array}{l}\text { Estudos empíricos ou teóricos com metodologia científica } \\
\text { válida (revisão cega de pares) }\end{array}$ & Publicações que sejam pagas para aceder \\
\hline $\begin{array}{l}\text { Estudos Científicos, Dissertações de Mestrado e } \\
\text { Doutoramento, Revistas Científicas que sejam em } \\
\text { português, inglês e espanhol }\end{array}$ & $\begin{array}{l}\text { Publicações que não sejam em português, inglês e } \\
\text { espanhol }\end{array}$ \\
\hline
\end{tabular}

A pesquisa eletrónica realizou-se no período de junho a julho de 2018, por meio da B-On. É de realçar que, apesar da investigação ter tido como foco quatro pedagogias, neste artigo somente serão apresentados os resultados da Educação Positiva.

Na Tabela 2, encontra-se descrito o número de publicações encontradas, bem como o número de publicações excluídas e incluídas durante a pesquisa.

Tabela 2. Pesquisa de termos cruzados- no de publicações excluídas e incluídas

\begin{tabular}{|c|c|c|c|}
\hline Pesquisa de termos cruzados & № de publicações & Excluídas & Incluídas \\
\hline 10 & 110 & $\begin{array}{c}39 \text { (duplicados } \\
\text { automaticamente) }\end{array}$ & 71 \\
\hline $2^{\underline{o}}$ & 71 & $\begin{array}{l}12 \text { (duplicados } \\
\text { manualmente) }\end{array}$ & 59 \\
\hline $3^{\circ}$ & 59 & $\begin{array}{c}40 \text { (aplicação de } \\
\text { critérios de inclusão e } \\
\text { exclusão) }\end{array}$ & 19 \\
\hline
\end{tabular}

De seguida, foi efetuada uma análise manual cuidadosa no texto completo de cada publicação, tendo em conta os critérios de inclusão, exclusão e a pertinência do conteúdo (objetivo, descrição da pedagogia abordada, estudos desenvolvidos e os fatores psicológicos e psicopedagógicos inerentes) das publicações. Na Tabela 3, encontra-se descrito o número de publicações que foram excluídas e incluídas após essa análise. 
Tabela 3. № de publicações excluídas e incluídas após uma análise manual das publicações

\begin{tabular}{ccc}
\hline Análise manual do texto completo & Excluídos & Incluídos \\
\hline 19 & 13 & 6 \\
\hline
\end{tabular}

Mais especificamente, foram identificadas 13 publicações que somente abordavam como assunto principal a Psicologia Positiva ao invés da Educação Positiva. Assim, as publicações com elegibilidade permaneceram na amostra e as restantes foram excluídas, mediante justificação. A amostra final reuniu um total de seis publicações (ver Tabela 4).

Tabela 4. Amostra final das publicações relacionadas com a Educação Positiva

\begin{tabular}{cc}
\hline Pedagogia & Amostra \\
\hline Educação Positiva & 6 \\
\hline
\end{tabular}

\section{RESULTADOS E DISCUSSÃO}

Após uma análise qualitativa da amostra, em que foi dada especial atenção às principais ideias do texto, objetivos e conclusões das publicações da amostra (ver Tabela 5), foram identificados os principais fatores psicológicos que emergiram com maior frequência nas publicações relacionadas com a Educação Positiva (ver Tabela 6).

Estes resultados podem ser explicados pelo facto da Educação Positiva integrar diretrizes cientificamente validadas da Psicologia Positiva, desde aplicações teóricas e práticas de pesquisas em áreas como o otimismo, resiliência, autorregulação, emoções positivas e inteligência emocional, com o objetivo de promover o sucesso escolar e o bem-estar dos alunos (Barrett, Bradsha \& Lewis-Palmer, 2008).

0 "bem-estar" é um dos fatores psicológicos que surgiu em todas as publicações, estando descrito de forma geral como sendo o objetivo principal da Educação Positiva. Seligman e colaboradores (2009) afirmam que uma das diretrizes da Educação Positiva é o desenvolvimento de programas de intervenção baseados em evidências científicas que promovem o bem-estar dos alunos. A sua equipa dedicou cerca de 15 anos a investigar, através de métodos rigorosos, se o bem-estar poderia ser ensinado aos estudantes através de dois programas diferentes para escolas, o Programa de Resiliência Penn (PRP) e o Currículo de Psicologia Positiva Strath Haven. Relativamente ao PRP, o principal objetivo do currículo é aumentar a capacidade dos alunos em lidar com os fatores de stress, promover o otimismo, ensinando os alunos a pensar de forma mais realista e flexível, e ensinar a assertividade, brainstorming criativo, a tomada de decisão, o relaxamento e várias outras capacidades de resolução de problemas. Neste artigo, os autores identificam diversas descobertas realizadas por meio de diferentes investigações sobre a aplicação do PRP, em comparação com grupos de controlo, nomeadamente: a redução e prevenção dos sintomas de depressão; a redução e prevenção da ansiedade; a possível redução de problemas de comportamento entre adolescentes, entre outros. De uma forma geral, Seligman e os seus colaboradores (2009) afirmam que as diversas pesquisas realizadas indicam que o PRP produz de forma positiva e credível uma melhoria no bem-estar dos alunos.

Outro fator psicológico que emergiu em todas as publicações foram as "emoções positivas". Diversos autores da nossa amostra destacaram o papel das emoções positivas no processo de aprendizagem e a sua implementação na Educação Positiva. Bozkurt (2014) enfatiza que a neuropsicologia explica atualmente que relação entre as emoções positivas e a aprendizagem está assente na forma como a informação é processada no cérebro Este resultado vai ao encontro da investigação científica na área. No estudo de Mega e colaboradores (2014, citado por Bzuneck, 2018), os autores indicaram que as emoções positivas experienciadas no contexto de sala de aula influenciam, de modo direto, a autorregulação, os fatores motivacionais de autoeficácia e as metas de realização dos alunos, fatores essenciais para o bom desempenho dos mesmos.

0 "engagement" é outro fator psicológico que apareceu em quase todas as publicações, estando normalmente correlacionado com outras variáveis. Ramachandram (2016) referencia vários estudos que indicam que o engagement dos alunos está positivamente relacionado com o suporte do professor. 0 autor enfatiza o papel do professor na Educação Positiva como figura que suporta e encoraja os alunos, trazendo benefícios para os mesmos (mais confiança) e para o processo de aprendizagem (maior performace académica). 
Tabela 5. Objetivos e conclusões das publicações relacionadas com a Educação Positiva

\begin{tabular}{lll}
\hline Autor e ano & \multicolumn{1}{c}{ Título } & \multicolumn{1}{c}{ Principais objetivos e conclusões } \\
\hline Artigo 3 & A Educação positiva & “ 0 presente estudo teve como principal objetivo explorar os \\
Gonçalves (2013) & na promoção do & impactos da Educação Positiva na promoção do Bem-estar \\
& bem-estar subjetivo & Subjetivo em 64 crianças, com 9 e 10 anos, do $4 .^{\text {o ano de }}$ \\
& em crianças do 4. o & escolaridade(...) As crianças submetidas ao programa de \\
& ano de escolaridade & intervenção, quando comparadas às do grupo de controlo, \\
& apresentam maior satisfação ao nível da dimensão Self, Família e \\
& Afetividade Positiva e menor satisfação no que respeita à Não \\
& Violência, vivenciando concomitantemente menor Afeto Negativo, \\
& possivelmente devido aos cuidados recebidos no programa e ao \\
& afastamento de situações adversas trabalhadas no decorrer das \\
& sessões “ (p.4).
\end{tabular}

Artigo 9

Bozkurt (2014)

Artigo 10

Ramachandram

(2016)
New horizons in

education: Positive

education and

emerging leadership

roles of counsellors

Positive education

and higher achievers

role of positive

psychology

Positive education:

Artigo 11

Seligman, Ernst,

Gillham, Reivich

\& Linkins (2009)

Artigo 12

Williams (2011)
Positive psychology

and classroom

interventions
Pathways to positive education at geelong
"The article proposes a system model where meso and micro level indicators of positive psychology/education interacts within an eco system where the role of school counselors as change agents becomes crucial. Mindsets and other potential barriers for the counselors are discussed along with resolution strategies" (p.452)

“(...) The delivery of Positive Education Programs in schools can equip both students and teachers with the skills and confidence to better manage their emotion, develop healthy thinking styles, form a positive self-identity, and foster heealthy relationships. Positive Education provides a new educational paradigm which prepares young people to thrive despite the complex life challenges they face in contemporary times" (p.848)

“(...) There is substantial evidence from well controlled studies that skills that increase resilience, positive emotion, engagement and meaning can be taught to schoolchildren. We present the story of teaching these skills to an entire school-Geelong Grammar School-in Australia, and we speculate that positive education will form the basis of a 'new prosperity', a politics that values both wealth and well-being" (p.293). grammar school
"Since 2005 Geelong Grammar School has been developing and implementing Positive Education - Positive Psychology applied to all aspects of school life. Appreciative Inquiry is one important way through which the school is developing this at an organisational level to ensure that Positive Education becomes an embedded organisational capability" (p.8). 
Artigo 19

Kristjánsson

(2012)
Positive psychology

and positive

education: Old wine

in new bottles?
“(...) In this critical review, I scrutinize positive education as a potential theory in educational psychology. Special attention is given to conceptual controversies and suggested educational interventions. Positive psychologists have yet to explore in detail the school as a positive institution. (...)"(p.86).

Tabela 6. Fatores Psicológicos subjacentes à Educação Positiva

\begin{tabular}{l}
\hline Fatores psicológicos \\
\hline Bem-estar \\
Emoções positivas \\
Resiliência \\
Identificação das próprias forças e fraquezas
\end{tabular}

Felicidade

Engagement

Criatividade

Relações positivas e de confiança

Autores
Gonçalves (2013)
Bozkurt, (2014)

Ramachandram, (2016)

Estigma, Ernst, Gillham, Reivich \& Lenis (2009)

Williams (2011)

Kristjánsson (2012)

Bozkurt, (2014)

Ramachandram, (2016)

Estigma, Ernst, Gillham, Reivich \& Lenis (2009)

Williams (2011)

Kristjánsson (2012)

Gonçalves (2013)

Bozkurt, (2014)

Ramachandram, (2016)

Estigma, Ernst, Gillham, Reivich \& Lenis (2009)

Kristjánsson (2012)

Ramachandram, (2016)

Estigma, Ernst, Gillham, Reivich \& Lenis (2009)

Williams (2011)

Kristjánsson (2012)

Gonçalves (2013)

Bozkurt, (2014)

Estigma, Ernst, Gillham, Reivich \& Lenis (2009)

Kristjánsson (2012)

Bozkurt, (2014)

Ramachandram, (2016)

Estigma, Ernst, Gillham, Reivich \& Lenis (2009)

Williams (2011)

A "felicidade" é um fator psicológico que também emergiu em diferentes artigos. Diversos especialistas na área da Educação e investigadores das mais reconhecidas universidades do Mundo têm 
vindo a revelar que os alunos com maiores índices de felicidade desenvolvem níveis superiores de atenção, pensamento criativo e envolvimento na comunidade, comparativamente a outros alunos que têm níveis mais baixos de felicidade (Espiner, \& Guild 2010, citados por Ramachandram, 2016). Os resultados do estudo desenvolvido por Gonçalves (2013), sobre a aplicação de um programa de promoção de bemestar subjetivo, indicaram maiores níveis de felicidade nas crianças sujeitas ao programa. Mais precisamente, o autor identificou um aumento ao nível das dimensões Self (i.e. autoestima, bom-humor, capacidade de relacionar-se, entre outros), Família (i.e. ambiente saudável, em harmonia, com relacionamentos satisfatórios, entre outros) e Afetividade Positiva (i.e. interesse, entusiasmo, inspiração, determinação, entre outros), e uma visível redução das dimensões Não Violência (i.e. comportamentos agressivos) e Afetividade Negativa (i.e. angústia, descontrolo, culpa, irritabilidade, nervosismo, entre outros), comparativamente ao grupo de controlo. Apesar das limitações existentes no estudo, o autor refere que estes resultados podem advir das aprendizagens que as crianças adquiriram ao longo do programa e do afastamento de situações adversas trabalhadas no decorrer das sessões.

No que concerne ao fator "identificação das forças e fraquezas", diversos autores descreveram em diferentes artigos como é que o mesmo pode ser implementado na Educação Positiva. Desta forma, autores como Seligman e seus colaboradores (2009) descrevem-no como uma ferramenta da Psicologia Positiva aplicada ao currículo "Signature Strengths". Noutro artigo, Williams (2011) faz referência à experiência obtida através da aplicação da Psicologia Positiva e Educação Positiva na escola australiana Geelong Grammar School (GGS), enaltecendo a abordagem utilizada baseada no foco e na promoção de pontos fortes dos indivíduos (alunos e staff) e do sistema escolar, o que possibilita a implementação de uma cultura positiva em todo o contexto escolar.

Para além destes fatores, os fatores de âmbito contextual também são relevantes para a implementação da Educação Positiva. Bozkurt (2014) propõe, no fim do seu artigo, um modelo de Educação Positiva assente na interação entre os elementos dos ecossistemas e os níveis meso e micro de Educação Positiva, visto que, segundo o autor, a eficiência e a eficácia da educação positiva não estão livres da influência do contexto ambiental.

\section{CONCLUSÃO}

0 método de investigação utilizado na investigação permitiu identificar e interpretar evidências de pesquisas disponíveis relevantes que conduziram a uma maior compreensão dos múltiplos fatores de ordem psicológica e psicopedagógica que estão subjacentes à Educação Positiva.

Neste âmbito, a Educação Positiva revela ser uma abordagem psicopedagógica que se destaca pela evidência científica nas áreas do bem-estar (psicológico, físico e intelectual), potenciando o "florescimento" e a aprendizagem dos alunos. Desta forma, através de um clima escolar positivo, o processo de aprendizagem é percecionado como um processo positivo e cooperativo, em que tanto os agentes educativos como os alunos aprendem e aplicam os princípios da Educação Positiva. Os fatores disseminados pela Educação Positiva (bem-estar, felicidade, resiliência, etc) são suportados, também, por outros autores como fatores essenciais para a promoção da aprendizagem. Pois, como Verdasca (2016) explicita, a interligação entre bem-estar e aprendizagem tem por base a conceção de que o bem-estar transporta consigo a motivação, felicidade e a tranquilidade. Tudo fatores que conjugam em si múltiplas dimensões (cognitiva, motivacional, socio-emocional), naturalmente potenciadoras e facilitadoras de aprendizagem. Por conseguinte, podemos referir que a aprendizagem é central para o bem-estar e o bemestar é fator determinante para a aprendizagem.

Diversas investigações da amostra indicam que através da aplicação de programas de intervenção baseados em evidências científicas que promovem o bem-estar na comunidade educativa, a escola pode de facto ser uma instituição positiva promotora de saúde mental para todos.

\section{REFERÊNCIAS}

Active Media. (2018). Biblioteca do conhecimento online. https://www.b-on.pt/.

Barrett, S., Bradshaw, C., \& Lewis-Palmer, T. (2008). Maryland statewide PBIS initiative: Systems, evaluation, and next steps. Journal of Positive Behavior Interventions, 10(2), 105-114. https://doi.org/10.1177/109830070731254

Bott, D., Escamilia, H., Kaufman, S., Kern, M., Krekel, C., Schlicht-Schmälzle, R., Seldon, A., Seligman, M., \& White, M. (2017). The State of Positive Education. World Government Summit. Retirado de https://www.worldgovernmentsummit.org/observer/reports/detail/the-state-of-positiveeducation

Bozkurt, T. (2014). New Horizons in Education: Positive Education and Emerging Leadership Roles of Counselors. Procedia Social and Behavioral Sciences, 140, 452-461. https: // doi.org / 10.1016 / j.sbspro.2014.04.452 
Bzuneck, J. (2018). Emoções acadêmicas, autorregulação e seu impacto sobre motivação e aprendizagem. Educação Temática Digital, 20(4), 1059-1075. https://doi.org/10.20396/etd.v20i4.8649692

Cintra, C. L., Guerra, V. M. (2017). Educação Positiva: A aplicação da Psicologia Positiva a instituições educacionais. Psicologia Escolar e Educacional, 21(3), 505-514. https://doi.org/10.1590/217535392017021311191

Gonçalves, S. C. (2013). A Educação Positiva na Promoção do Bem-estar Subjetivo em Crianças do 4.ํo ano de Escolaridade [Dissertação de Mestrado, Universidade do Algarve].

Hoare, E., Bott, D., \& Robinson, J. (2017). Learn it, live it, teach it, embed it: Implementing a whole school approach to foster positive mental health and wellbeing through positive education. International Journal of Wellbeing, 7(3), 56-71. https://doi.org/10.5502/ijw.v7i3.645

Kristjánsson, K. (2012). Positive psychology and positive education: Old wine in new bottles? Educational psychologist, 47(2), 86-105. https://doi.org/10.1080/00461520.2011.610678

Moher, D., Liberati, A., Tetzlaff, J., Altman, D. G., \& The PRISMA Group (2009). Preferred reporting items for systematic reviews and meta-analyses: The PRISMA Statement. https: // www.ncbi.nlm.nih.gov / pmc / articles / PMC2707599/

Norrish, J. M., Williams, P., O'Connor, M., \& Robinson, J. (2013) Na applied framework for Positive Education. International Journal of Wellbeing, 3(2), 147-161.

Ordem dos Psicólogos Portugueses (2015). A realidade dos Serviços de Psicologia da Educação Públicos e Privados. OPP.

Pinto, F., Garcia, V., \& Letichevsky, A. (2006). Pesquisa nacional qualidade na educação: A escola pública na opinião dos pais. Ensaio: Avaliação e Políticas Públicas em Educação, 14(53), 527-542. http://www.scielo.br/pdf/ensaio/v14n53/a08v1453.pdf

Ramachandram, V. (2016). Positive education and higher achievers role of positive psychology. Indian Journal of Health and Wellbeing, 7(8), 848.

Seligman, M., Ernst, R.M., Gillham, J., Reivich, K., \& Linkins, M. (2009). Positive education: Positive psychology and classroom interventions. Oxford Review of Education, 35(3), 293-311. https://doi.org/10.1080/03054980902934563

Seligman, M., \& Adler, A. (2018). Positive education. In The Global Happiness Council. Global Happiness Policy Report 2018 (pp. 53-73). https: // s3.amazonaws.com / ghc-2018 / GlobalHappinessPolicyReport2018.pdf

Sousa, M. S., Ribeiro, A. L. P. (2009). Revisão sistemática e meta-análise de estudos de diagnóstico e prognóstico: um Tutorial. Arquivos Brasileiros de Cardiologia, 92 (3), 241-25. http://www.scielo.br/scielo.php?pid=S0066-782X2009000300013\&script=sci_abstract\&tlng=pt

White, M. (2016). Why won't it stick? Positive Psychology and Positive Education. Psychology of WellBeing. 6(2), 1-16. https://doi.org/10.1186/s13612-016-0039-1

Williams, P. (2011). Pathways to positive education at Geelong Grammar School. Integrating positive psychology and appreciative inquiry. AI Practitioner, 13(2), 8-13. Retirado de https://www.hb.edu/uploaded/Innovation_Summit/Geelong_Grammar_School_-_P_Williams.pdf

World Health Organization. (2017). Depression and Other Common Mental Disorders: Global Health Estimates. https://apps.who.int/iris/bitstream/handle/10665/254610/WHO-MSD-MER-2017.2eng.pdf?sequence.

Historial do artigo

Recebido $\quad 07 / 2019$

Aceite $\quad 03 / 2020$

Publicado $\quad 08 / 2020$ 
Revista PSICOLOGIA, 2020, Vol. 34 (1), doi: 10.17575/psicologia.v34i1.1681.

Atas do X Simpósio Nacional de Investigação em Psicologia

\title{
Psychometric study of the short form of the Creative Personality Scale
}

\author{
Margarida Pocinho26i,ii, Soraia Garcêsi,ii, Saul Neves de Jesusii,iii, João Viseuii,iii \& Juan José \\ Miguel-Tobaliv \\ iUniversity of Madeira - Research Center for Regional and Local Studies (UMa-CIERL) \\ ii Research Centre for Tourism, Sustainability and Well-being (CinTurs) of University of Algarve \\ iii Faculty of Human and Social Sciences, University of Algarve \\ ${ }^{i v}$ Complutense University of Madrid
}

\begin{abstract}
Creativity is a complex phenomenon, being considered an important skill for personal and professional success. The current research aimed to study the psychometric properties of the short form of the Creative Personality Scale (CPS) in a Portuguese sample. An exploratory factor analysis (EFA) was performed with a sample of 1503 participants. A new solution of 9 items and one-factor was obtained. Reliability results showed a value of .86 and positive and significant correlations between all items were found. Also, a confirmatory factor analysis (CFA) was conducted with a sample of 380 subjects. This analysis showed an overall adequate fit. Finally, convergent validity was also tested using the average variance extracted (AVE) coefficient. The theoretical and practical implications of these results are discussed, as well as are presented the limitations of this study and suggestions for future research.
\end{abstract}

Keywords: Confirmatory factor analysis; Creative personality scale short-form; Creativity; Exploratory factor analysis.

Estudo psicométrico da versão reduzida da escala de personalidade criativa: A criatividade é um fenómeno psicológico complexo, sendo considerada uma competência essencial para o sucesso pessoal e profissional. 0 objetivo deste estudo foi analisar as caraterísticas psicométricas da versão reduzida da Escala de Personalidade Criativa (EPC), numa amostra composta por indivíduos portugueses. Foi realizada uma análise fatorial exploratória (AFE) com uma amostra de 1503 participantes, tendo sido identificada uma estrutura unidimensional composta por 9 itens. Foi obtida uma consistência interna de .86 e correlações positivas e significativas entre todos os itens. De seguida, efetuou-se uma análise fatorial confirmatória (AFC), com uma amostra de 380 sujeitos, onde se obteve um ajustamento adequado. Por fim, foi testada a validade convergente através do coeficiente average variance extracted (AVE). São discutidas as implicações teórico-práticas destes resultados, bem como são referidas as principais limitações deste estudo e são apresentadas sugestões para futuras investigações.

Palavras-chave: Análise fatorial confirmatória; Análise fatorial exploratória; Criatividade; Escala da personalidade criativa - versão reduzida.

Creativity can be defined as a cognitive process that has two core characteristics: novelty and usefulness. Those who involve themselves in a creative behavior tend to see problems with an open mind, leading to the development of new and different ideas (Azanedo, Fernández-Abascal, \& Barraca, 2014; 0'Connor, Gardiner, \& Watson, 2016). Linked to personality and cognitive factors, creativity is associated with one's ability to generate new products, reflections, and/or inventions that are seen by specialists as having great significance in science, society, and/or technological contexts (Leutner, Yearsley, Codreanu, Borenstein, \& Ahmetoglu, 2017; Ozdas \& Batdi, 2017). Abilities such as critical thinking and problemsolving skills are considered crucial in professional settings (Chiu, Hsu, Lin, Chen, \& Liu, 2017). Stakeholders are highlighting the importance of creativity and innovation to solve the problems that modern societies are facing (Vidal, 2005). Creative thinking is a vital factor for success in many fields, such as schools and organizations (O'Connor et al., 2016). Knowledge acquisition is no longer the single goal. The ability to use knowledge and transform it needs creative skills, thus these skills are one of the most crucial abilities that education should promote (Jónsdóttir, 2017). Creativity characteristics, such as autonomy, energy, or independence of thought are usually part of the creative personality profile (Wang, Chen, Zhang, \& Deng, 2017). The identification of a creative personality is complex (Krumm, Lemos, \&

${ }^{26}$ Correspondence address: Soraia Garcês, Campus Universitário da Penteada, Gabinete 1.75 (1ํopiso)- CIERL, 9020-105 Funchal. E-mail: soraiagarces@gmail.com 
Richaud, 2018), but there are some common traits between creative people, e.g., cognitive skills, such as, keen observation, ability to avoid perceptual sets; to see things in new ways, striving to express truth not evident to others, independence in expressions of opinion, willingness to work diligently for the sake of new knowledge, high intellectual abilities and good memory, ability to hold in mind multiple ideas and to compare them (Mynbayeva, Vishnevskaya, \& Sadvakassova, 2016, p. 408).

It is possible to find three different types of creative individuals. The first are problem solvers, who try to find solutions for a problem using creativity, for example engineers or scientists. The second group are artists, who develop art pieces which can be physical products, such as paintings or processes like a dance. The third type, and last, is composed by individuals who implement creativity as a lifestyle, using it when working, at home, and every other place (Vidal, 2005).

As a complex mental function linked to a variety of cognitive factors, creativity varies from individual to individual (Bendetowicz, Urbanski, Aichelburg, Levy, \& Volle, 2017). Thus, its' assessment is an ongoing issue (Forthmann et al., 2017). The self-assessment of a person's own skills is a powerful motivator and an important subject for personal and professional development. When an individual believes that he /she is good at what they do, they will continue doing it and will follow, for example, a career where they can use those skills. Those who perceive themselves as creative are more prone to choose activities where they can express their creativity. Individuals who perceive themselves as not creative or with little creative abilities will avoid this kind of activities (Kemmelmeier \& Walton, 2016). Throughout the years' creativity researchers have developed instruments to identify individuals with high and low creativity levels (Chiu, 2014). Some of these instruments are the California Personality Inventory (Helson, 1965), What Kind of Person Are You? (Torrance \& Katena, 1970), Adjective Check List (Gough \& Heilbrun, 1983), or the (Wechsler, 2006). In Portugal, creative personality assessment instruments are sparse. The Thinking and Creating Styles Scale (Wechsler, 2006) was adapted for a Portuguese sample (see Garcês, 2011; Garcês, Pocinho, Wechsler, \& Jesus, 2014; Nogueira, Almeida, Pocinho, Garcês, \& Wechsler, 2016), since then an effort to develop a Portuguese creative personality scale was made (Jesus et al., 2011; Garcês, 2013; Garcês et al., 2015). Identifying levels of creativity in individuals can have a major impact on the development of one's own skills and allow self-improvement. It can also be an asset for professional development, since nowadays creative individuals are sought for their ability to think 'outside-of-the-box' and bringing new ideas that can help an organization to thrive. The main goal of this research was to study the psychometric properties of the short-form of the Creative Personality Scale (CPS) in a sample of Portuguese individuals, since no short-form of this measure exists, to the best of our knowledge, in Portugal.

\section{Creative Personality Scale}

The Creative Personality Scale aims to evaluate peoples' creative characteristics (Jesus et al., 2011). Built from a pool of creative personality features and analyzed by renowned experts, a measure of 30 items was developed (Jesus et al., 2011). A higher score indicates more creative personality characteristics. Following this first attempt, several studies with this instrument were conducted. Godinho (2012) studied creativity as having a protective effect in inmates, regarding depression, anxiety, and stress. Reliability in this study was.89. Matos (2012) aimed to contribute to the validation of the CPS by applying it to a mental disability population. Results showed a reliability of .95 $(M=110.29 ; S D=20.47)$. Saramago (2012) used the creative personality as a mechanism to promote well-being, also with inmate participants. A reliability value of .89 . $(M=121.67$; SD $=15.72)$ was achieved. Vieira (2012) while comparing students' creativity and motivation in regular and alternative curriculums found a mean of 116.61(17.03) for the first group (regular curriculum) and a mean of 111.01 (SD = 19.61) for the second group (i.e., alternative curriculum). Also, reliability for the regular and alternative curricula was, respectively,.93 and .94. Globally, in this research, the internal consistency achieved a score of .94. A validation study that encompassed all data from these previous studies plus 414 university students, a total sample of 784 subjects, was conducted (Garcês et al, 2015). Reliability was .92 ( $M=117.93 ; S D=$ 15.72) (Garcês et al, 2015). The one-dimensionality of the scale was clear through the analysis of the scree plot and the explained variance was $31.18 \%$ (Garcês et al, 2015). The confirmatory analysis indicated the goodness of fit of the scale structure after the inclusion of modification indices (MI), with results of .041 for the Root Mean Square Residual (RMR); .92 for the Goodness of Fit Index (GFI); .91 for the Comparative Fit Index (CFI); and .05 for the Root Mean Square Error of Approximation (RMSEA). This study suggested the possibility of reducing the number of items, namely because this scale presented one single factor with several items and a short-form scale could be more useful to assess the characteristics of the creative personality (Cook, Kallen, \& Amtmann, 2009; Marsh, Hau, Balla, \& Grayson, 2010). Therefore, the current research aimed to study the psychometric characteristics of the short-form version of the Creative Personality Scale. Since this is the first effort to provide a short-form for this measure, this 
paper presents results from two factor analyses, exploratory and confirmatory. Convergent validity was also tested. Results are discussed focusing on the potential of this short-form to evaluate the creative personality in a Portuguese sample.

\section{METHOD}

\section{Participants}

Two samples were collected. An exploratory factor analysis (EFA) and a confirmatory factor analysis (CFA) were performed.

For the EFA, participants were selected from past studies (Borralha, 2018; Garcês, 2013; Garcês et al., 2015) where the Creative Personality Scale (30-item version) was used (Garcês et al., 2015). A random sample composed by 1503 individuals (58.4\% females) with ages ranging from 10 to 70 years old $(M=31 ; S D=13)$ was selected. For the CFA, another random sample was selected, this sample was composed by 380 respondents $(71.2 \%$ females) with ages ranging from 18 to 73 years old $(M=29 ; S D=$ 11).

\section{Measurements}

The Creative Personality Scale is a self-assessment measure of an individual's creative characteristics. Responses are given on a Likert format and vary from Completely Disagree (1) to Completely Agree (5). Total scores are obtained through the sum of the items that compose this instrument. As already reported in original validation study, a one-factor structure with 30 items was obtained, filling a gap in the research about creativity in the European Portuguese context, since there was no record, to our knowledge, of previous measures on creative personality (Garcês et al., 2015). The Creative Personality Scale presented an internal consistency of .92, item-total correlation values were above .30, and the total variance explained was 31.18\% (Garcês et al., 2015). The goodness-of-fit indices were also adequate after the addition of modification indices (MI), Root Mean Square Residual (RMR) =.041; Goodness of Fit Index $(\mathrm{GFI})=.92$; Comparative Fit Index $(\mathrm{CFI})=.91$; and Root Mean Square Error of Approximation $(\mathrm{RMSEA})=$ .05 .

\section{Statistical analysis procedures \\ Exploratory factor analysis}

Two EFA were performed with SPSS software version 24 . The first aimed to analyze the 30-item version and reduce it. The second tested the short-form of the scale previously obtained. A principal component analysis was used to extract only one factor, since previous studies on this measure registered a onefactor solution (Garcês, 2013; Garcês et al., 2015). Values for the Kaiser-Meyer-Olkin (KMO) test were observed to the continue EFA, values equal to or higher than .60 were desired (Pestana \& Gajeiro, 2008). Factor loadings were suppressed for a cut-off of .60. Matsunaga (2010) argued that a cut-off of .40 is marginally acceptable, higher cut-off values, e.g., .60 or .70 , would be more reliable ${ }^{27}$. For both EFA's the chosen cut-off value was .60. Explained variance and the scree plot graphic were also analyzed. Reliability was calculated through the Cronbach's Alpha coefficient.

\section{Confirmatory factor analysis}

Structural equation modeling (SEM) was used to perform the CFA, which was conducted with the Analysis of Moment Structures (AMOS) software version 22. The fit of the model was assessed by the following indices and cut-off values: Normed Chi-squared $\left(\chi^{2} / \mathrm{df}\right)<2$ with a non-significant $p$-value; RMR $\leq .05$; GFI > .90; AGFI > .90; CFI > .90; and RMSEA $\leq .05$ (Byrne, 2010; Pilati \& Laros, 2007; Schumacker \& Lomax, 2004; Ulman 2007). The MI were also observed for model improvement. These indices give an expected value of how the Chi-squared $\left(\chi^{2}\right)$ can decrease if they are added to a model (Schumacker \& Lomax, 2004).

Convergent validity was also calculated. This type of validity emerges when a given concept, in this case creative personality, explains at least half of the variance of its indicators (i.e., items) (Bagozzi \& Yi, 1988). To assess convergent validity the Average Variance Extracted (AVE) coefficient was used, the values of this coefficient should exceed .50 to prove the existence of convergent validity (Fornell \& Larcker,1981; Sharma, 1996). In addition to the Cronbach's alpha coefficient, another reliability measure, Composite Reliability (CR) coefficient, was used. CR values should exceed the threshold of .70 (Bagozzi \& Yi, 1988).

${ }^{27}$ According to the literature, loadings higher than: a) .71 are excellent; b) .63 are very good; c) .55 are good; (d) .45 are sufficient; and e) .32 are low (Tabachnick \& Fidell, 2007). 


\section{RESULTS}

\section{Exploratory factor analysis}

KMO test results showed a value of .96, considered very good, which allowed the continuation of the EFA. The explained variance with one factor was $31.16 \%$, a value similar to the $31.18 \%$ of the original study (Garcês et al., 2015). Factor loadings were above .60 and led to a new set of 12 items (items 7, 8, 9, 13, 14, $15,18,22,27,28,29$, and 30). These items were used in the second EFA. In this analysis, the KMO registered a value of .94 , a result that allowed the analysis to continue. The explained variance for one factor solution was $44.06 \%$, a higher value compared to the initial version of the scale. The scree plot (Figure 1) also indicated the existence of a one-factor solution. Item 22 did not load on the factor and so a solution of 11 items was the selected structure. Table 1 shows the factor loadings for the new solution of 11 items.

Table 1. Factor Loadings and Communalities for the Creative Personality Scale 11-item Solution

\begin{tabular}{lcc}
\hline Item & Loadings & $h^{2}$ \\
\hline $\begin{array}{l}\text { 7. Aprecio novas ideias. (I appreciate new ideas). } \\
\text { 8. Gosto de questionar e dar sugestões. (I like to question and give }\end{array}$ & .64 & .42 \\
$\begin{array}{l}\text { suggestions). } \\
\text { 9. Tenho abertura para receber novas ideias. (I am open to new ideas). }\end{array}$ & .66 & .45 \\
$\begin{array}{l}\text { 13. Descubro problemas no meio envolvente e ocorrem-me ideias para } \\
\text { a sua resolução. (I discover problems in the environment and ideas } \\
\text { come up for me to solve them). }\end{array}$ & .64 & .44 \\
$\begin{array}{l}\text { 14. Quando falho, não desisto e continuo a tentar novas soluçóes. } \\
\text { (When I fail, I do not give up and I continue to try new solutions). }\end{array}$ & .70 \\
$\begin{array}{l}\text { 15. Agrada-me resolver problemas de forma não habitual. (I like to } \\
\text { solve problems in an unusual way). }\end{array}$ & .72 & .48 \\
$\begin{array}{l}\text { 18. Aprecio atividades que possibilitem ter muitas ideias. (I appreciate } \\
\text { activities that allow me to have many ideas). }\end{array}$ & .74 & .52 \\
$\begin{array}{l}\text { 27. Adoro aperfeiçoar as minhas ideias até que fiquem bem definidas. } \\
\text { (I love perfecting my ideas until they are well defined). }\end{array}$ & .66 & .55 \\
28. Apesar de errar, creio e prezo o valor das minhas ações. (Although & .61 & .44 \\
$\begin{array}{l}\text { I make mistakes, I believe and appreciate the value of my actions). } \\
\text { 29. Consigo encontrar várias soluções para o mesmo problema. (I can } \\
\text { find several solutions to the same problem). }\end{array}$ & .71 & .37 \\
30. Tenho facilidade em encontrar a beleza das coisas. (I find it easy to \\
discover the beauty of things).
\end{tabular}




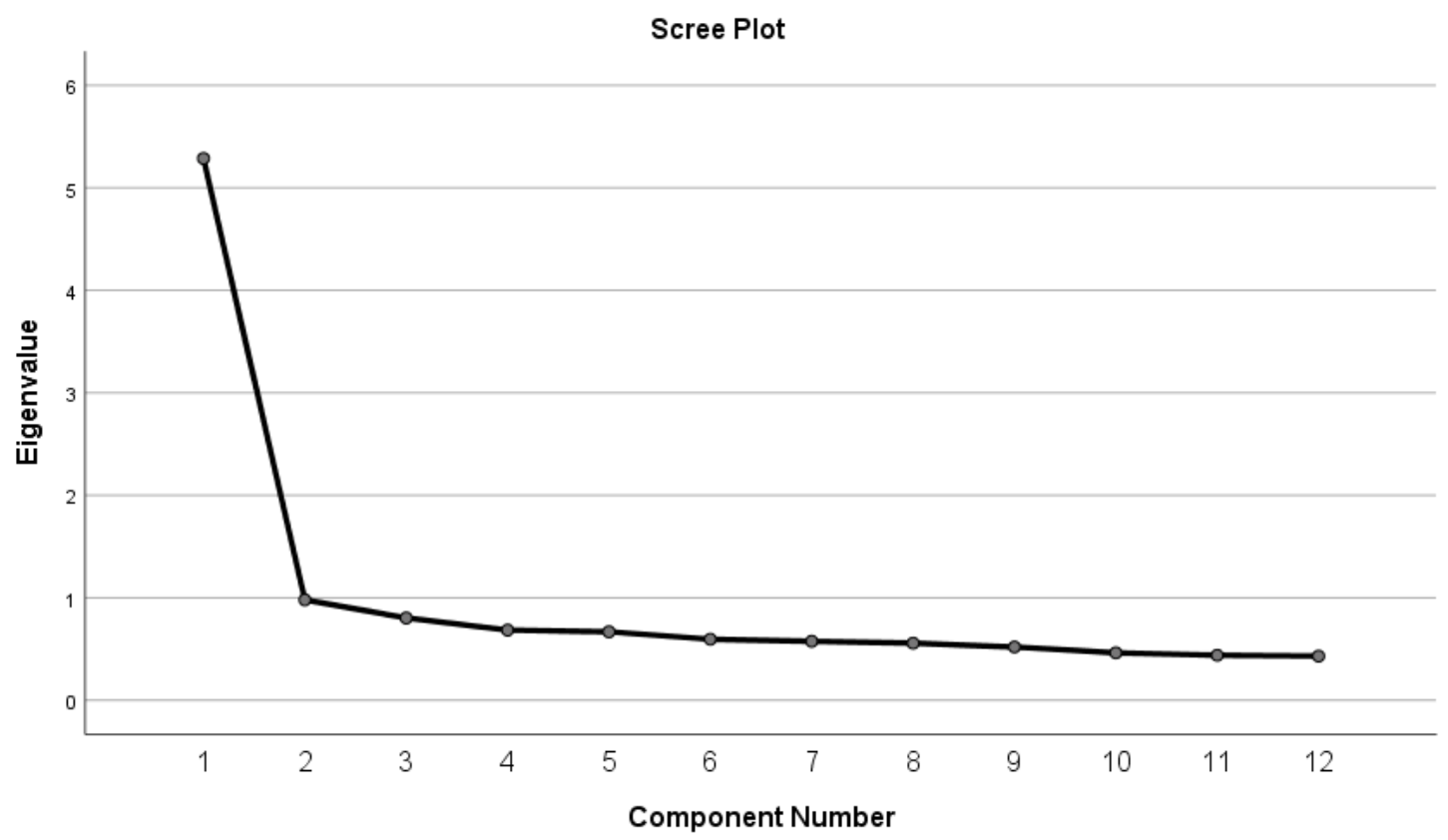

Figure 1. Scree plot for the Creative Personality Scale short form.

Reliability analysis, through the Cronbach's Alpha coefficient, showed a result of .88 (Pestana \& Gajeiro, 2008). Item-total correlation values presented a strong association with values above .50. Moreover, the reliability analysis indicated that the elimination of these items was not necessary. The scale's mean result was $44.32(S D=6.14)$. All 11 items were positive and significantly correlated for a $p$ value below 01 .

\section{Confirmatory factor analysis}

The initial one-factor model with 11 items presented the following fit: $\chi^{2} / \mathrm{df}=3.46, p=.00$; RMR $=.03$; GFI $=.93 ; \mathrm{AGFI}=.89 ; \mathrm{CFI}=.92$; and RMSEA $=.08$. This model did not display an adequate fit, namely regarding the AGFI value. Modification indices were included in the model to improve the fit. After their inclusion, the model achieved the following results: $\chi^{2} / \mathrm{df}=1.38, p=.07$; RMR = .02; GFI = .98; AGFI = .96; $\mathrm{CFI}=.99$; and RMSEA $=.03$. AVE results for this 11 -item structure were .36 , below cut-off value of .50 and CR was .85, above the threshold of .70. This led to an in-depth item analysis and item 9 and 11 were eliminated, since they presented loadings below .50 (Marôco, 2014). An analysis of the 9-item structure (Figure 2) was performed. With the addition of the modification indices, the model presented the following fit results: $\chi^{2} / \mathrm{df}=1.15, p=.291$; RMR $=.01 ; \mathrm{GFI}=.99 ; \mathrm{AGFI}=.97 ; \mathrm{CFI}=.99$; and RMSEA = 02 . AVE results (.37) were again below the cut-off value defined by the literature (i.e., 50). This may suggest that the items selected for the short-form version of this instrument are not the most suitable to explain the creative personality construct. Regarding the CR coefficient, the obtained values were above the threshold defined, .70, having achieved a value of .84. According to Bagozzi and Yi (1988), this result can be classified as very good. Reliability through Cronbach's Alpha coefficient for the 9-item scale was .86 and the explained variance was $48.08 \%$. 


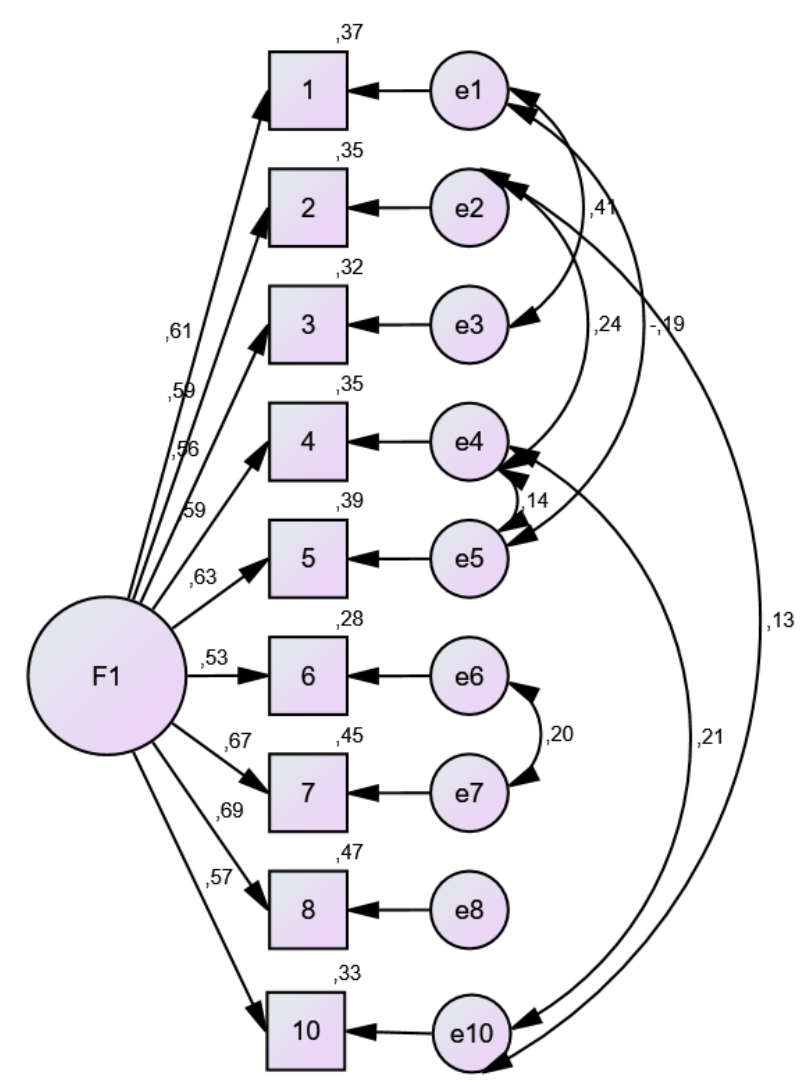

Figure 2. One-factor model with 9 items and modification indices.

\section{DISCUSSION}

This study performed an EFA and CFA for the short form of the Creative Personality Scale developed in Portugal (Garcês et al., 2015). Firstly, the analysis showed that an 11-item solution instead of the initial 30 items had adequate psychometric values. A strong correlation between items was observed and reliability values were classified as very good. This reduced solution showed a slightly lower reliability than the studies of Garcês and colleagues (2015), Godinho (2012), Matos (2012), and Saramago (2012). However, it still possesses a high-reliability score, which led the authors to believe that this short version holds an adequate internal consistency. The explained variance of $44.06 \%$ provided additional evidence for the reduction of the number of items, since these results indicate that the short form of this scale explains a great amount of the creative personality construct. The CFA also found an adequate fit. The addition of the MI allowed the improvement of the model and was justified by the high correlation values between the items, which was expected by the amount of shared variance between them. It is important to note that even before the addition of the MI some of the fit indices presented acceptable values. The 11-item solution presented a solid factor structure, a situation that indicated that it was not necessary to add more items. Yet, further item analysis showed that the items 9 and 11 had loadings below the threshold of .50 (Marôco, 2014). After testing for a 9-item solution, the overall fit of the model improved, and it explained $48.08 \%$ of the variance. However, the AVE value obtained was below the cut-off defined by the literature (.50). Nevertheless, Fornell and Larcker (1981) argued that in situations where AVE < .50 , the value of CR must be considered. This occurs because the AVE is a conservative indicator. After the assessment of the CR value, it was found that the obtained result may be classified as very good (Bagozzi \& Yi, 1988). Similar situations were reported in past studies, e.g., Lam (2012). This 9-item arrangement showed a better overall fit than the initial 30-item structure. This version of the Creative Personality Scale possesses one advantage compared to the long form, the answering time is lower which facilitates its application in organizational domains, giving an overall perspective of a person's creative characteristics (Garcês et al., 2015). However, in the future, it is important to further study the concurrent and discriminant validity of this measure. An analysis that was beyond the scope of the current research. It will also be important to develop cross-cultural studies to observe how well this measure fits in different cultures. The Creative Personality Scale short form provides an efficient instrument for the Portuguese context. It can also be an important instrument for career and vocational orientation, allowing the identification of personal and professional needs for the improvement of creative skills. This instrument 
can be an important tool to measure peoples' levels of creativity and a significant step in the development of creative thinking skills through creative problem techniques. Creativity is a very important variable nowadays and being able to identify those who express high or low levels of it can be determinant to thrive in modern society. Creativity is a characteristic that allows individuals to deal with uncertainty and complexity (Vidal, 2005). Life needs more than logical or convergent thinking (Kaufman, 2018). But for this, it is crucial that individuals perceived themselves as creative agents in the creative problem-solving process. The development of adequate instruments, which allow the identification of a creative personality, can then be a powerful tool to ignite people's creative abilities.

\section{REFERENCES}

Azanedo, C., Fernández-Abascal, E., \& Barraca, J. (2014). Character strengths in Spain: Validation of the Values in Action Inventory of Strengths (VIA-IS) in a Spanish sample. Clínica y Salud, 25, 123-130. http://dx.doi.org/10.1016/j.clysa.2014.06.002

Bagozzi, R., \& Yi, Y. (1988). On the evaluation of structural equation models. Journal of the Academy of Marketing Science, 16(1), 74-94. https://doi.org/10.1007/BF02723327

Bendetowicz, D., Urbanski, M., Aichelburg, C., Levy, R., \& Volle, E. (2017). Brain morphometry predicts individual creative potential and the ability to combine remote ideas. Cortex, 86, 216-229. https://doi.org/10.1016/j.cortex.2016.10.021

Borralha, S. (2018). Satisfaction of hotel professionals: Study of the relationships with personal and organizational variables [Unpublished doctoral dissertation, University of Algarve].

Byrne, B. (2010). Structural equation modeling with AMOS: Basic concepts, applications, and programming ( $2^{\text {nd }}$ ed.). Routledge.

Chiu, F. (2014). The effects of exercising self-control on creativity. Thinking Skills and Creativity, 14, $20-31$. https://doi.org/10.1016/j.tsc.2014.06.003

Chiu, F., Hsu, C., Lin, Y., Chen, H., \& Liu, C. (2017). Effects of the interaction between exercising self-control and PhoPhiKat on divergent and convergent thinking. Thinking Skills and Creativity, 24, 286-298. https://doi.org/10.1016/j.tsc.2017.05.001

Cook, K., Kallen, M., \& Amtmann, D. (2009). Having a fit: Impact of number of items and distribution of data on traditional criteria for assessing IRT's unidimensionality assumption. Quality of Life Research, 18, 447-460. https://doi.org/10.1007/s11136-009-9464-4

Forthmann, B., Holling, H., Zandi, N., Gerwig, A., Celik, P., Storme, M., \& Lubart, T. (2017). Missing creativity: The effect of cognitive workload on rater (dis-) agreement in subjective divergentthinking scores. Thinking Skills and Creativity, 23, 129-139. https://doi.org/10.1016/j.tsc.2016.12.005

Fornell, C., \& Larcker, D. (1981). Evaluating structural equation models with unobservable variables and measurement error. Journal of Marketing Research, 18, 39-50. https:/doi.org/10.2307/3151312

Garcês, S. (2011). Escala de estilos de pensar e criar: Adaptação e validação à população portuguesa. [Master thesis, University of https://digituma.uma.pt/bitstream/10400.13/431/1/MestradoSoraiaGarc\%C3\%AAs.pdf

Garcês, S. (2013). A multidimensionalidade da criatividade: A pessoa, o processo, o produto e o ambiente criativo no ensino superior [Doctoral dissertation, University of Madeira). DigitUMa. https://digituma.uma.pt/bitstream/10400.13/546/1/DoutoramentoSoraiaGarc\%c3\%aas.pdf

Garcês, S., Pocinho, M., Jesus, S., Viseu, J., Imaginário, S., \& Wechsler, S. (2015). Estudo de validação da Escala de Personalidade Criativa. Revista Iberoamericana de Diagnóstico y Evaluación - e Avaliação Psicológica, 40(2), 17-24. https://www.aidep.org/sites/default/files/articles/R40/Art2.pdf

Garcês, S., Pocinho, M., Wechsler, S., \& Jesus, S. (2014). Estilos de Pensar e Criar na Região Autónoma da Madeira. Revista Iberoamericana de Diagnóstico e Evaluación Psicológica, 38(2), 55-68. https://www.aidep.org/sites/default/files/articles/R38/Art3.pdf

Godinho, P. (2012). 0 Efeito Protetor da Criatividade face à Depressão, à Ansiedade e ao Stresse em Reclusos de Estabelecimentos Prisionais do Algarve [Unpublished Master's thesis, University of Algarve].

Gough, H. C., \& Heilbrun, A. B. (1983). The Adjective Check List manual (2nd ed.). Consulting Psychologists Press.

Helson, R. (1965). Childhood interest clusters related to creativity in women. Journal of Consulting and Clinical Psychology, 29, 352-361. https://doi.org/10.1037/h0022401

Jesus, S. N., Morais, F., Pocinho, M., Imaginário, S., Duarte, J., Matos, ... Sousa, F. (2011). Escala da Personalidade Criativa: Estudo preliminar para a sua construção. In A. S. Ferreira, A. Verhaeghe, D. R. Silva, L. S. Almeida, R. Lima, \& S. Fraga (Eds.) Actas do VIII Congresso Iberoamericano de 
Avaliação/evaluación Psicológica e XV Conferência Internacional de Avaliação Psicológica: Formas e Contextos. Universidade de Lisboa.

Jónsdóttir, S. (2017). Narratives of creativity: How eight teachers on four school levels integrate creativity into teaching and learning. Thinking Skills and Creativity, 24, 127-139. https://doi.org/10.1016/j.tsc.2017.02.008

Kaufman, S. (2018). My quest to understand human intelligence. In R. J. Sternberg (Ed.), The nature of human intelligence (pp. 215-229). Cambridge University Press.

Kemmelmeier, M., \& Walton, A. (2016). Creativity in men and women: Threat, other-interest, and self$\begin{array}{lllll}\text { assessment. } \quad \text { Creativity } & \text { Research }\end{array}$ https://doi.org/10.1080/10400419.2016.1125266

Krumm, G., Lemos, V., \& Richaud, M. (2018). Personality and creativity: A study in Spanish-Speaking children. International Journal of Psychological Research, 11(1), 33-41. https://doi.org/10.21500/20112084.2867

Lam, L. (2012). Impact of competitiveness on salespeople's commitment and performance. Journal of Business Research, 65, 1328-1334. https/doi.org/10.1016/j.busres.2011.10.026

Leutner, F., Yearsley, A., Codreanu, S., Borenstein, Y., \& Ahmetoglu, G. (2017). From Likert scales to images: Validating a novel creativity measure with image-based response scales. Personality and Individual Differences, 106, 36-40. https://doi.org/10.1016/j.paid.2016.10.007

Marôco, J. (2014). Análise de Equações Estruturais: Fundamentos teóricos, software e aplicações. ReportNumber Editora.

Marsh, H., Hau, K., Balla, J., \& Grayson, D. (2010). Is more ever too much: The number of indicators per factor in confirmatory factor analysis. Multivariate Behavioral Research, 22, 181-220. https://doi.org/10.1207/s15327906mbr3302_1

Matos, N. (2012). Avaliação da criatividade na deficiência mental [[Unpublished Master's thesis, University of Algarve.]

Matsunaga, M. (2010). How to factor-analyze your data right: Do's, don'ts, and how-to's. International Journal of Psychological Research, 3(1), 97-110. https://doi.org/10.21500/20112084.854

Mynbayeva, A., Vishnevskaya, A., \& Sadvakassova, Z. (2016). Experimental study of developing creativity of university students. Procedia - Social and Behavioral Sciences, 217, 407-413. https://doi.org/10.1016/j.sbspro.2016.02.113

Nogueira, S., Almeida, L., Garcês, S., Pocinho, M., \& Wechsler, S. (2016). The Style Troika Model (STM): A structural model of the thinking and creating styles scale. Journal of Creative Behavior, 50(4), 333346. https://doi.org/10.1002/jocb.81

O'Connor, P., Gardiner E., \& Watson, C. (2016). Learning to relax versus learning to ideate: Relaxationfocused creativity training benefits introverts more than extraverts. Thinking Skills and Creativity, 21, 97-108. https://doi.org/10.1016/j.tsc.2016.05.008

Ozdas, F., \& Batdi, V. (2017). A thematic-based meta analytic study regarding the effect of creativity on academic success and learning retention. Journal of Education and Training Studies, 5(3), 53-61. https://doi.org/10.11114/jets.v5i3.2043

Pestana, M., \& Gajeiro, J. (2008). Análise de dados para ciências sociais: A complementaridade do SPSS. Edições Sílabo.

Pilati, R., \& Laros, J. (2007). Modelos de equações estruturais em psicologia: Conceitos e aplicações. Psicologia: Teoria e Pesquisa, 23, 205-116. https://doi.org/10.1590/S0102-37722007000200011

Saramago, T. (2012). Criatividade e bem-estar na população reclusa. [Unpublished Master's thesis, University of Algarve].

Schumacker, R., \& Lomax, R. (2004). A beginner's guide to structural equation modeling. Lawrence Erlbaum Associates.

Sharma, S. (1996). Applied multivariate techniques. John Wiley and Sons Inc.

Tabachnick, B., \& Fidell, L. (2007). Using multivariate statistics. Pearson Education.

Torrance, E. P., \& Khatena, J. (1970). What kind of person are you? The Gifted Child Quarterly, 14, 71-75. https://doi.org/10.1177/001698627001400201

Ulman, J. (2007). Structural equation modeling. In B. Tabachnick, \& L. Findell (Eds.), Using Multivariate Statistics (pp. 676-780). Pearson Education.

Vidal, R. (2005). Creativity for operational researchers. Investigação Operacional, 25, 1-24. http://www.scielo.mec.pt/pdf/iop/v25n1/v25n1a01.pdf

Vieira, M. (2012). Criatividade e motivação em crianças e jovens: Um estudo comparativo [Unpublished Master's thesis, University of Algarve]. 
Wang, M., Chen, W., Zhang, C., \& Deng, X. (2017). Personality types and scholarly creativity in undergraduate students: The mediating roles of creative styles. Personality and Individual Differences, 105, 170-174. https://doi.org/10.1016/j.paid.2016.09.050

Wechsler, S. (2006). Estilos de Pensar e Criar. LAMP/PUC.

Historial do artigo

Recebido $\quad 07 / 2019$

Aceite $\quad 03 / 2020$

Publicado $\quad 08 / 2020$ 
Revista PSICOLOGIA, 2020, Vol. 34 (1), doi: 10.17575/psicologia.v34i1.1681.

Atas do X Simpósio Nacional de Investigação em Psicologia

\title{
Perfis emocionais de alunos do 1.o ciclo e a sua relação com a perceção de competência e a motivação em matemática
}

\author{
Vera Monteiro ${ }^{28 i}$, Natalie Nóbrega Santos ${ }^{i}$, Lourdes Matai, Cristina Sanches ${ }^{i} \&$ Marta $^{2}$ \\ Gomesii \\ ${ }^{i}$ Centro de Investigação em Educação, ISPA - Instituto Universitário \\ ii ISPA - Instituto Universitário
}

\begin{abstract}
Resumo: Este estudo utilizou a análise de clusters para identificar perfis emocionais dos alunos face à matemática e a sua relação com a perceção de competência e com a motivação. Participaram 356 alunos do 3.ํ e 4..$^{\circ}$ anos do 1.ำ ciclo. A recolha de dados realizou-se através do Questionário de Emoções de Realização, da Escala de Perceção de Competência em Matemática e do Questionário de Autorregulação Académica. Foram identificados três perfis emocionais diferenciados: positivo (níveis altos de prazer e baixos de ansiedade e aborrecimento); negativo (níveis altos de aborrecimento, médios de ansiedade e baixos de prazer) e moderado (níveis moderadamente altos de ansiedade e médios de aborrecimento e prazer). Os alunos do perfil positivo revelaram uma perceção mais elevada de competência para a matemática e valores mais elevados de motivação autónoma. Os resultados evidenciam a importância de se considerarem perfis emocionais para a compreensão dos processos de aprendizagem.
\end{abstract}

Palavras-chave: Emoções de realização académica; Matemática; Motivação académica; Perceção de competência.

Emotional profiles of elementary students and its relationship with their self-perception of competence and motivation in mathematics: This study used cluster analysis to identify students' emotional profiles towards mathematics and their relationship with motivation and perceived competence. Participants were 356 elementary students from the 3rd and 4th grades. Data was collected through the Portuguese version of the Achievement Emotions Questionnaire - Elementary School, Perceived Competence in Math Scale, and the Academic Self-Regulation Questionnaire. Three distinct emotional profiles were identified: positive (high levels of enjoyment and low levels of anxiety and boredom); negative (high levels of boredom, average levels of anxiety and low levels of enjoyment); and moderate (moderate-high levels of anxiety and moderate levels of boredom and enjoyment). Students of the positive profile presented higher levels of perceived competence as well as higher levels of intrinsic motivation than students from the other profiles. The results highlight the importance of considering the emotional profiles to understand learning processes.

Keywords: Achievement emotions; Mathematics; Academic motivation, Self-perception of competence.

As emoções desempenham um papel muito relevante na aprendizagem da matemática uma vez que estas influenciam a escolha de estratégias cognitivas, a tomada de decisão e a motivação dos alunos na realização das atividades (Kim \& Pekrun, 2014). A teoria de controlo-valor das emoções de realização académica (CVT) (Pekrun, 2006) sugere ainda que as emoções podem ser causadas por vários fatores individuais, nomeadamente as avaliações cognitivas de controlo e de valor relativamente às tarefas e resultados. A presente investigação foi conduzida para aprofundar o estudo das relações entre estas variáveis utilizando, para isso, uma abordagem centrada na pessoa.

Emoções de realização académica, perceção de competência e motivação para a aprendizagem As emoções são vistas como constructos psicológicos multidimensionais que compreendem processos afetivos, motivacionais, expressivos e fisiológicos que ajudam os indivíduos a adaptarem-se aos desafios e acontecimentos que ocorrem nas suas vidas. As emoções de realização académica estão ligadas diretamente às atividades ou aos resultados de aprendizagem (Pekrun, 2006).

\footnotetext{
${ }^{28}$ Morada para correspondência: Vera Monteiro, Rua Jardim do Tabaco 34, 1149-041, Lisboa. E-mail: veram@ispa.pt. Este trabalho foi financiado pela FCT-Fundação para a Ciência e a Tecnologia (PTDC/MHC-CED/1680/2014, UID/CED/04853/2016).
} 
Segundo a CVT (Pekrun, 2007), as emoções podem ser organizadas de acordo com a sua valência (positivas/ negativas) e grau de ativação (ativação/desativação). Podemos, então, classificar as emoções em: positivas de ativação (e.g., prazer); positivas de desativação (e.g., alívio); negativas de ativação (e.g., ansiedade) e negativas de desativação (e.g., aborrecimento). Os estudos têm demonstrado que, quando os alunos apresentam emoções positivas de ativação apresentam melhor desempenho académico, utilizam estratégias cognitivas mais profundas e estão mais motivados (Goetz, Frenzel, Pekrun, \& Hall, 2006; Pekrun, 2006).

As abordagens mais recentes sobre as emoções têm optado por identificar perfis emocionais que resultam da conjugação de diferentes emoções (abordagem centrada na pessoa) (Ganotice, Datu, \& King, 2016; Jarrell, Harley, \& LaJoie, 2016; Jarrell, Harley, LaJoie, \& Naismith, 2017; Raccanello, Rob, \& Burro, 2018; Siemer, Mauss, \& Gross, 2007). Esta abordagem coloca o indivíduo no foco da análise, permitindo compreender como os constructos psicológicos se manifestam nele e facilitando a identificação de perfis emocionais através da intensidade assumida por cada emoção (Jarrell et al., 2016). No estudo de Raccanello et al. (2018), os autores identificaram a existência de três perfis emocionais. Os alunos "felizes" foram caracterizados principalmente por níveis altos de prazer e baixos de aborrecimento; os alunos "descontraídos" que sentiam principalmente tranquilidade e algum prazer; e os estudantes "deprimidos", em que a tristeza era predominante, mas também a ansiedade e o prazer eram bastante relevantes. É de salientar que a maioria dos alunos pertencia aos dois perfis em que prevaleciam as emoções positivas.

Considerando a relação entre perceção de competência e emoções, esta pode ser explicada à luz da CVT (Pekrun \& Perry, 2014) que defende que as avaliações cognitivas que fazemos se relacionam com as experiências emocionais vividas nessas situações. Níveis elevados de perceção de competência e controlo podem levar a elevadas expectativas relativamente aos resultados, e a um controlo mais adaptativo da ação do sujeito numa determinada situação o que, por sua vez, conduzirá a experiências emocionais positivas (Goetz, Cronjaeger, Frenzel, Lüdtke, \& Hall, 2010).

Se as avaliações cognitivas que os estudantes fazem das situações a realizar e dos resultados que irão obter são importantes para estimular as emoções, estas últimas são relevantes para a predição da sua motivação académica (Dresel \& Hall, 2013). A motivação refere-se, de acordo com Ryan e Deci (2000), aos processos que levam um individuo a iniciar uma ação, a controlá-la e a mantê-la numa determinada direção. Estes autores consideram que a motivação pode diferenciar-se em motivação intrínseca, quando um individuo deseja realizar uma atividade pelo prazer e interesse inerentes a essa atividade, e motivação extrínseca, quando um sujeito realiza uma ação para obter algo ou para evitar uma situação desagradável.

Embora a motivação possa ser dicotomizada como intrínseca e extrínseca, a teoria da autodeterminação (Deci \& Ryan, 1985, 2012, 2016; Ryan \& Deci, 2017) especifica quatro tipos de motivação extrínseca que variam em termos do grau em que os comportamentos foram internalizados e, portanto, autodeterminados. A internalização pode ser entendida em termos de um continuum de autonomia, desde o comportamento menos autónomo (regulação externa) até o mais autónomo (integrado). Na regulação externa, o aluno faz a atividade para satisfazer um pedido externo ou para receber uma recompensa imposta externamente. Na regulação introjetada, o aluno aceita a razão pela qual tem de fazer a ação, mas não a aceita completamente. A ação é controlada ou coagida por contingências externas. Na regulação identificada, o aluno faz a ação porque acredita na sua importância pessoal. A regulação integrada é a mais autónoma, em que o estudante considera a razão para fazer a ação compatível com seus próprios valores e necessidades. Os estudos têm demonstrado que a motivação intrínseca e formas mais autónomas de motivação extrínseca (integrada e identificada) têm efeitos positivos no processo de aprendizagem, enquanto que formas de motivação mais controladas (externa e introjetada) têm efeitos menos positivos (principalmente a longo-prazo) no processo de aprendizagem (Lemos \& Veríssimo, 2014).

No que toca à relação entre emoções e motivação para a aprendizagem, os autores são unânimes em afirmar que as emoções se relacionam com a motivação dos sujeitos (Kim \& Pekrun, 2014; Pekrun, 2006, 2007). Os seus trabalhos mostraram que as emoções positivas de ativação (e.g., prazer) promovem a motivação intrínseca e extrínseca, e as emoções negativas de desativação (e.g., aborrecimento) inibem qualquer das orientações motivacionais. Por seu turno, as emoções positivas de desativação (e.g., alívio) e as emoções negativas de ativação (e.g., ansiedade) parecem ter um papel mais complexo na orientação motivacional dos estudantes. Refere Pekrun (2006) que a ansiedade pode induzir quer a motivação intrínseca, quer a motivação extrínseca de forma a evitar o insucesso. No entanto, Pekrun (2013) afirma que esta relação é bidirecional existindo relações recíprocas entre estes dois processos afetivos.

0 estudo realizado por Ganotice et al. (2016) identificou quatro perfis emocionais: a) vergonha adaptativa; b) mal-adaptativo; c) moderado; e d) adaptativo. Neste último perfil, os adolescentes 
apresentavam níveis mais elevados de emoções positivas e baixos níveis de emoções negativas. 0 perfil da vergonha adaptativa foi o mais representativo da amostra, em que os sujeitos tinham altos níveis de emoções positivas e baixos níveis de emoções negativas, exceto na vergonha que era um pouco mais alta que nos outros grupos. Os autores constataram que os alunos destes dois perfis apresentavam orientações motivacionais autónomas e controladas mais elevadas que os colegas dos restantes clusters, sugerindo que isto acontece porque os alunos que experienciam mais frequentemente emoções positivas (ou seja, prazer) tendem a ser mais autorregulados (Ganotice et al., 2016). 0 perfil moderado caracterizou-se por níveis moderados de emoções académicas positivas e negativas, tendo apresentado níveis baixos de motivação autónoma e controlada. 0 grupo "mal adaptativo" apresentou níveis baixos de emoções positivas e altos de emoções negativas, tendo evidenciado os níveis mais baixos de motivação controlada. 0 grupo moderado apresentou níveis relativamente baixos de motivação autónoma e controlada, enquanto o grupo não-adaptativo revelou níveis mais baixos de motivação controlada.

Tendo como referência uma abordagem centrada na pessoa, na presente investigação tivemos como objetivos identificar os perfis de emoções de realização académica de alunos do 3.ํㅜ e 4.ำ anos de escolaridade e analisar a sua relação com a sua perceção de competência e a sua motivação académica no contexto específico da matemática.

\section{MÉTODO}

Participaram 356 alunos do terceiro (64.6\%) e quarto ano (35.4\%) do 1. ciclo do ensino básico, com idades compreendidas entre os 7 e 12 anos $(M=8.81, D P=0.8)$, sendo $51.4 \%$ do sexo masculino. A sua participação foi voluntária (após a obtenção de autorização escrita da direção da escola e encarregados de educação) e foi assegurada a confidencialidade dos dados.

A versão portuguesa do Questionário de Emoções de Realização para alunos do Ensino Elementar (Sanches, Monteiro, Mata, Santos, \& Gomes, 2020) foi utilizada para avaliar as emoções de prazer, ansiedade e aborrecimento em três contextos das aulas de matemática. A perceção de competência em matemática foi avaliada com Escala de Perceção de Competência em Matemática (Santos, Mata, Monteiro, Sanches, \& Gomes, 2019). A motivação foi avaliada através do Questionário de Autorregulação Académico (Ryan \& Connell, 1989), adaptado à população portuguesa por Gomes, Monteiro, Mata, Peixoto, Santos e Sanches (2019). Este questionário mede a motivação intrínseca, assim como três tipos de regulação da motivação extrínseca: externa, introjetada e identificada.

Para identificar grupos homogéneos de estudantes com diferentes perfis de emoções de realização, foi realizada uma análise de clusters. Foi utilizado o método hierárquico de Ward para comparar o número de clusters possíveis, usando a distância euclidiana quadrada como medida de dissemelhança entre sujeitos. 0 número de clusters foi definido com base na teoria e na percentagem de variância explicada por cada uma das emoções de realização académica incluídas na análise. Uma vez identificado o número de clusters, foi utilizado o método não hierárquico K-means para ajustar a solução preliminar (Marôco, 2014).

\section{RESULTADOS}

Uma solução com três clusters foi identificada, os quais explicam 70.6\% do prazer, 50.2\% da ansiedade e $75.2 \%$ do aborrecimento, com valores superiores aos 50\% sugeridos em estudos similares (e.g., Hayenga \& Corpus, 2010). Esta solução mostrou-se estável e foi replicada usando duas metades da amostra selecionadas aleatoriamente $(\kappa=.83)$ e por ano de escolaridade $(\kappa=.81)$. 


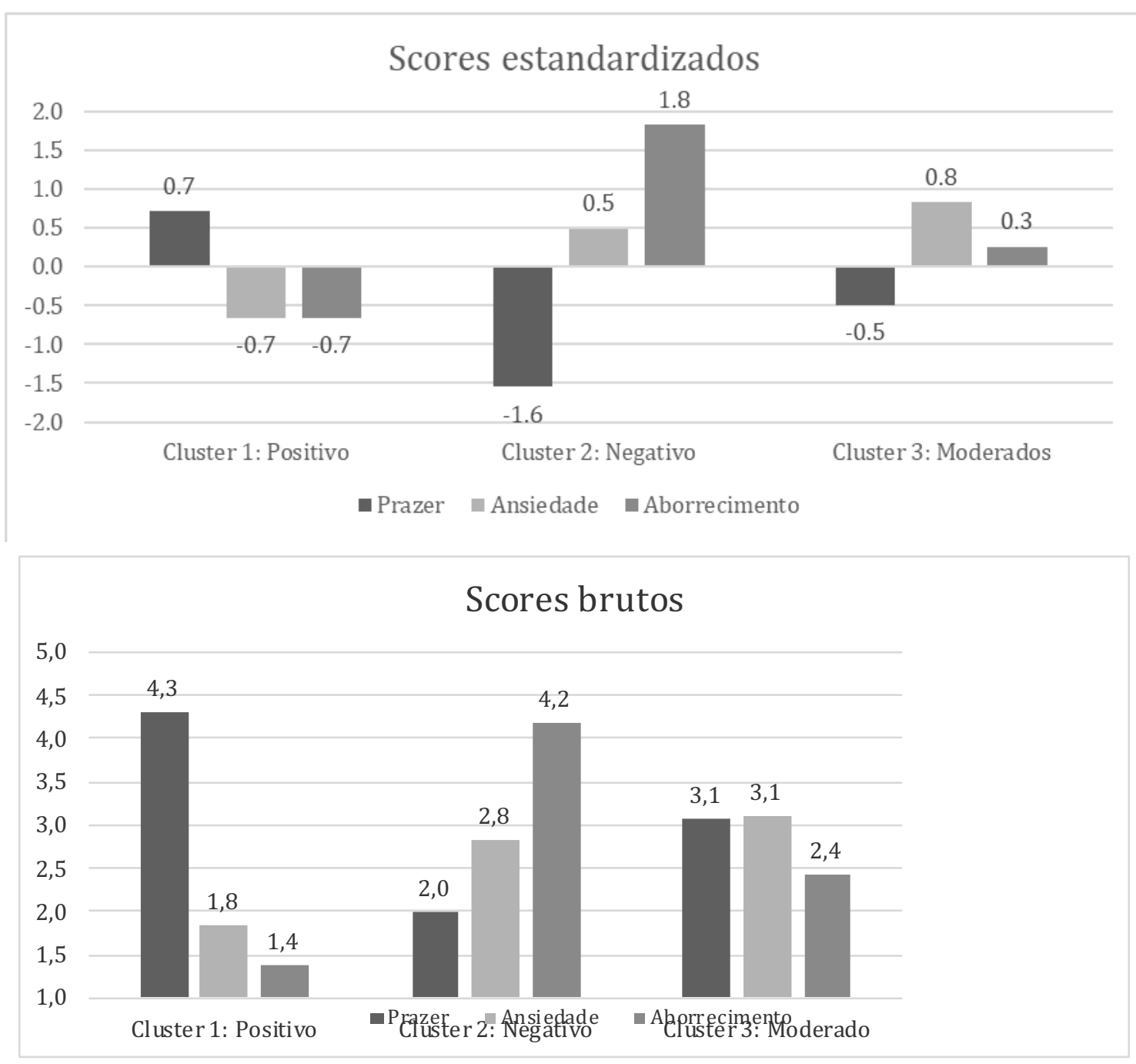

Figura 1. Perfis de emoções de realização académica em relação à matemática.

Na Figura 1, são representados os valores médios (tanto o score bruto da escala como o score estandardizado) obtidos pelos alunos nos três clusters. No primeiro cluster, encontramos alunos com um perfil emocional positivo, com valores de prazer acima da média e valores baixos de aborrecimento e ansiedade. A maioria dos alunos apresenta este perfil (ver Tabela 1). No segundo cluster, encontramos alunos com um perfil emocional negativo e com valores de ansiedade ligeiramente superiores à média e valores particularmente altos de aborrecimento, enquanto os valores de prazer se encontram muito abaixo da média. Este é o grupo com menor representatividade na nossa amostra. No último cluster, encontramos alunos com um perfil emocional moderado: níveis apenas ligeiramente inferiores à média, bem como de prazer, valores moderadamente altos de ansiedade e valores médios de aborrecimento. A distribuição dos alunos por cada perfil é semelhante tanto no 3. como no 4. ano, existindo apenas pequenas diferenças de sexo, com o perfil moderado a apresentar uma maior proporção de raparigas e o perfil positivo a apresentar uma maior proporção de rapazes (ver Tabela 1).

Tabela 1. Distribuição dos alunos por cluster, em função do sexo e do ano escolar

\begin{tabular}{lcccccccccc}
\hline & \multicolumn{2}{c}{ Positivo } & \multicolumn{2}{c}{ Negativo } & \multicolumn{3}{c}{ Moderado } & \multicolumn{3}{c}{ Associação entre variáveis } \\
& & $\boldsymbol{f}$ & $\mathbf{\%}$ & $\boldsymbol{f}$ & $\mathbf{\%}$ & $\boldsymbol{f}$ & $\mathbf{\%}$ & $\boldsymbol{X}^{2}$ & $\boldsymbol{p}$ & $\boldsymbol{V}$ \\
\hline Sexo & Masculino & 108 & 59.0 & 36 & 14.2 & 49 & 26.8 & 6.02 & .049 & .13 \\
& Feminino & 80 & 46.8 & 26 & 15.2 & 65 & 38.0 & & & \\
Ano escolar & $3 .$. ano & 129 & 56.3 & 34 & 14.8 & 66 & 28.8 & 3.95 & .139 & .10 \\
& $4 .$. ano & 59 & 46.8 & 18 & 14.3 & 49 & 38.9 & & & \\
Total & & 188 & 53.1 & 52 & 14.87 & 114 & 32.2 & & & \\
\hline
\end{tabular}


A análise de variância identificou diferenças significativas de tamanho elevado, entre os perfis ao nível da perceção de competência. 0 grupo com perfil positivo apresentou níveis significativamente mais elevados do que o grupo com perfil negativo $(p<.001)$.

No que se refere à motivação, os resultados da análise multivariada da variância indicam que existem diferenças elevadas entre os grupos $\left(F(8,656)=25.94\right.$, Pillai's $\left.V=.48, p<.001, \eta_{\mathrm{p}}{ }^{2}=.24\right)$, mais especificamente na regulação identificada e na motivação intrínseca (Tabela 2). 0 grupo de alunos com perfil emocional negativo apresenta valores mais baixos em ambas variáveis, comparativamente aos alunos do perfil moderado e positivo. Por sua vez, os alunos com perfil moderado apresentam níveis mais baixos em ambas variáveis que os seus colegas com perfil positivo $(p<.001)$.

Tabela 2. Distribuição dos alunos por cluster, em função do sexo e do ano escolar

\begin{tabular}{lccccccccc}
\hline Variável & \multicolumn{2}{c}{ Positivo } & \multicolumn{2}{c}{ Negativo } & \multicolumn{2}{c}{ Moderado } & \multicolumn{2}{c}{ Diferenças entre clusters } \\
& $\boldsymbol{M}$ & $\boldsymbol{D P}$ & $\boldsymbol{M}$ & $\boldsymbol{D P}$ & $\boldsymbol{M}$ & $\boldsymbol{D P}$ & $\boldsymbol{F}$ & $\boldsymbol{p}$ & $\boldsymbol{\mu}_{\mathbf{p}}^{2}$ \\
\hline Perceção de competência & 4.00 & 0.61 & 2.97 & 0.77 & 3.08 & 0.57 & 103.02 & $<.001$ & .369 \\
R Externa & 3.22 & 1.19 & 3.38 & 0.82 & 3.52 & 0.97 & 2.75 & .065 & .016 \\
R Introjetada & 3.06 & 1.08 & 2.85 & 0.87 & 3.04 & 0.89 & 0.85 & .427 & .005 \\
R Identificada & 4.48 & 0.53 & 3.60 & 0.80 & 4.15 & 0.54 & 45.68 & $<.001$ & .217 \\
M Intrínseca & 4.23 & 0.69 & 2.32 & 0.89 & 3.38 & 0.75 & 137.94 & $<.001$ & .455 \\
\hline
\end{tabular}

\section{DISCUSSÃo}

Neste estudo tivemos como principais objetivos identificar os perfis emocionais de alunos do $3^{\circ}$ e $4^{\circ}$ anos de escolaridade, usando uma abordagem centrada na pessoa e analisar a sua relação com a perceção de competência e com a motivação académica no domínio específico da matemática. Foram identificados três perfis emocionais: um positivo, um negativo e um moderado, dando-nos a entender que as emoções destes alunos em relação à matemática apresentam padrões complexos. Estes resultados são consistentes com os encontrados por Jarrell et al. $(2016,2017)$ e Raccanello et al. (2018). Salienta-se que a maioria dos alunos pertencia ao perfil positivo, o que pode ser indicador do bem-estar geral dos participantes envolvidos.

À semelhança do estudo de Raccanello et al. (2018), e em consonância com os princípios subjacentes à CVT (Pekrun \& Stephens, 2010), verificámos que a perceção de competência diferia consoante o perfil emocional dos estudantes. Os alunos do perfil positivo das emoções revelaram uma perceção de competência superior à dos seus colegas dos restantes perfis. De acordo com a CVT, o prazer de aprender depende da combinação de várias avaliações que o aluno faz, nomeadamente a sua perceção de competência, o valor atribuído à tarefa e os conteúdos a aprender. Verificámos no perfil positivo que estes alunos valorizam mais a matemática do que os seus colegas, sentem-se mais competentes e sentem de forma mais intensa o prazer na realização dessas tarefas. Pelo contrário, os alunos com perfil negativo consideram-se menos competentes a matemática e atribuem-lhe menos valor, provocando a presença de um misto de emoções negativas. Revelaram, por um lado, ansiedade porque antecipam o seu fracasso, mas especialmente aborrecimento porque as atividades são percebidas como demasiado exigentes para as suas competências (Pekrun et al., 2010).

0 perfil emocional, por sua vez, mostrou ter uma função relevante na promoção e/ou inibição da motivação intrínseca para a aprendizagem (Ganotice et al., 2016; Kim \& Pekrun, 2014). Á semelhança dos resultados dos estudos anteriores dos autores, na presente investigação os alunos com um perfil emocional positivo foram os que apresentaram uma motivação mais autónoma, mais intrínseca. Logo, a ativação de emoções predominantemente positivas, como o prazer, pode ter levado estes alunos a interessarem-se mais pelo estudo da matemática, a investirem mais nesta disciplina, conduzindo a comportamentos mais autodeterminados (Pekrun et al., 2002). Por outro lado, no perfil negativo em que o aborrecimento é a emoção vivida de forma mais intensa, quer a motivação intrínseca, quer a motivação por regulação identificada, foram minadas, apresentando estes alunos valores mais baixos que os colegas. Estes resultados são preocupantes e são consistentes com os de Pekrun (2006) e Pekrun et al. (2002). 0 facto de estes alunos sentirem pouco prazer pela matemática, e considerarem aquelas tarefas aborrecidas pode levá-los a investir e a esforçar-se pouco nas atividades, prejudicando a sua motivação. Já as crianças que sentem, moderadamente, ansiedade e prazer pelas realizações em matemática, mas que as consideram um pouco aborrecidas (perfil moderado), apresentaram mais motivação autónoma e controlada que os colegas do perfil negativo. Isto sugere que os alunos do perfil moderado, ao atribuírem 
mais valor à matemática que os seus colegas do perfil negativo, tentam de alguma forma esforçar-se e envolver-se nas atividades de matemática para não terem insucesso (Pekrun, 2006).

\section{CONCLUSÃO}

O presente estudo fornece evidências empíricas de que as experiências emocionais dos alunos em relação à matemática são complexas, reforçando os resultados de estudos anteriores (Ganotice et al., 2016; Jarrell et al., 2016, 2017), pelo que se salienta a importância de analisar as relações entre as emoções académicas e outras variáveis afetivas adotando uma abordagem centrada na pessoa. Acrescenta-se que, na presente investigação, foram analisadas apenas três emoções, pelo que será importante, em futuros estudos, incluir outras emoções (e.g. orgulho, vergonha e raiva) de forma a diversificar os perfis emocionais. Por outro lado, de modo a ter uma visão mais profunda das emoções dos alunos, seria importante complementar os dados obtidos com informação sobre o desempenho dos alunos e a perceção dos professores face ao seu envolvimento. Os resultados desta pesquisa realçam a necessidade de uma maior preocupação, por parte dos professores, em relação às avaliações cognitivas que os alunos fazem das tarefas e resultados em matemática, pois estas parecem estar muito relacionadas com o estado emocional e com as motivações na aprendizagem da matemática. A criação de um clima positivo de sala de aula pode facilitar o desenvolvimento de emoções positivas e, consequentemente, a promoção de uma motivação mais intrínseca (Ganotice et al., 2016).

\section{REFERÊNCIAS}

Deci, E. L., \& Ryan, R. M. (1985). Intrinsic motivation and self-determination in human behavior. Plenum. https://doi.org/10.1007/978-1-4899-2271-7

Deci, E. L., \& Ryan, R. M. (2012). Motivation, personality, and development within embedded social contexts: An overview of self-determination theory. In R. M. Ryan (Ed.), Oxford handbook of human motivation (pp. 85-107). Oxford University Press. https://doi.org/10.1093/oxfordhb/9780195399820.001.0001

Deci, E. L., \& Ryan, R. M. (2016). Optimizing students' motivation in the era of testing and pressure: A selfdetermination theory perspective. In J. Wang, C. W. Liu, \& R. M. Ryan (Eds.), Building autonomous learners: Research and practical perspectives using self-determination theory (pp. 9-29). Springer.

Dresel, M., \& Hall, N. C. (2013). Motivation. In N. C. Hall \& T. Goetz (Eds.), Emotion, Motivation, and Selfregulation: A Handbook for Teachers (pp. 57-122). Emerald.

Ganotice, F., Datu, J., \& King, R. (2016). Which emotional profiles exhibit the best learning outcomes? A person-centered analysis of students' academic emotions. School Psychology International, 37(5), 498-518. https://doi.org/10.1177/0143034316660147

Goetz, T., Cronjaeger, H., Frenzel, A. C., Lüdtke, O., \& Hall, N. C. (2010). Academic self-concept and emotion relations: domain specificity and age effects. Contemporary Educational Psychology, 35, 44-58. https://doi.org/10.1016/j.cedpsych.2009.10.001.

Goetz, T., Frenzel, A. C., Pekrun, R., \& Hall, N. C. (2006). The domain specificity of academic emotional experiences. The Journal of Experimental Education, 75(1), 5-29. https://doi.org/10.3200/JEXE.75.1.5-29

Gomes, M., Monteiro, V., Mata, L., Peixoto, F., Santos, N., \& Sanches, C. (2019). The Academic SelfRegulation Questionnaire: A study with Portuguese elementary school children. Psicologia: Reflexão e Crítica, 32, 8. https://doi.org/10.1186/s41155-019-0124-5

Hayenga, A. O., \& Corpus, J. H. (2010). Profiles of intrinsic and extrinsic motivations: A person-centered approach to motivation and achievement in middle school. Motivation and Emotion, 34(4), 371383. https://doi.org/10.1007/s11031-010-9181-x.

Jarrell, A., Harley, J. M., \& LaJoie, S. (2016). The link between achievement emotions, appraisals, and task performance: Pedagogical considerations for emotions in CBLEs. Journal of Computers in Education, 3(3), 289-307. https://doi.org/10.1007/s40692-016-0064-3.

Jarrell, A., Harley, J. M., LaJoie, S., \& Naismith, L. (2017). Success, failure and emotions: Examining the relationship between performance feedback and emotions in diagnostic reasoning. Educational Technology Research and Development, 65(5), 1263-1284. https://doi.org/10.1007/s11423-0179521-6.

Kim, C., \& Pekrun, R. (2014). Emotions and motivation in learning and performance. In J. Spector, M. Merrill, J. Elen, \& M. Bishop (Eds.), Handbook of Research on Educational Communications and Technology (pp. 65-75). Springer. https://doi.org/10.1007/978-1-4614-3185-5_6

Lemos, M. S., \& Veríssimo, L. (2014). The relationships between intrinsic motivation, extrinsic motivation, and achievement, along elementary school. Procedia-Social and Behavioral Sciences, 112, 930-938. https://doi.org/10.1016/j.sbspro.2014.01.1251 
Marôco, J. (2014). Análise estatística com o SPSS. Pêro Pinheiro: ReportNumber.

Pekrun, R. (2006). The Control-Value Theory of Achievement Emotions: Assumptions, Corollaries, and Implications for Educational Research and Practice. Educational Psychology Review, 18, 315-341. https://doi.org/10.1007/s10648-006-9029-9

Pekrun, R. (2007). Emotions in students' scholastic development. In R. Perry, \& J. Smart (Eds.), The scholarship of teaching and learning in higher education: An evidence- based perspective (pp. 553610). Springer.

Pekrun, R. (2013). Emotion, motivation and self-regulation: Common principles and future directions. In N. Hall \& T. Goetz (Eds.), Emotion, Motivation and Self-Regulation: A Handbook for Teachers (pp. 168-187). Emerald Group Publishing Limited.

Pekrun, R., \& Perry, R. (2014). Control-Value Theory of achievement emotions. In R. Pekrun \& L. Linnenbrink-Garcia (Eds.), International Handbook of Emotions in Education (pp. 120-141). Routledge.

Pekrun, R., \& Stephens, E. J. (2010). Achievement emotions: A control-value approach. Social and Personality Psychology Compass, 4(4), 238-255. https://doi.org/10.1111/j.17519004.2010.00259.x.

Pekrun, R., Goetz, T., Daniels, L. M., Stupnisky, R. H., \& Perry, R. P. (2010). Boredom in achievement settings: Control-value antecedentsand performance consequences of a neglected emotion. Journal of Educational Psychology, 102,531-549. https://doi.org/10.1037/a0019243

Pekrun, R., Goetz, T., Titz, W., \& Perry, R. P. (2002). Academic Emotions in Students' Self-Regulated Learning and Achievement: A Program of Qualitative and Quantitative Research. Educational Psychologist, 37(2), 91-105. https://doi.org/10.1207/S15326985EP3702_4

Raccanello, D., Rob, H., \& Burro, R. (2018). Salience of primary and secondary school students' achievement emotions and perceived antecedents: Interviews on literacy and mathematics domains. Learning and Individual Differences, 65, 65-79. https://doi.org/10.1016/j.lindif.2018.05.015

Ryan, R. M., \& Connell, J. P. (1989). Perceived locus of causality and internalization: Examining reasons for acting in two domains. Journal of Personality and Social Psychology, 57, 749-761. https://doi.org/10.1037/0022-3514.57.5.749

Ryan, R. M., \& Deci, E. L. (2000). Intrinsic and extrinsic motivations: Classic definitions and new directions. Contemporary Educational Psychology, 25, 54-67. https://doi.org/10.1006/ceps.1999.1020

Ryan, R. M., \& Deci, E. L. (2017). Self-determination theory: Basic psychological needs in motivation, development, and wellness. Guilford Publishing.

Sanches, C., Monteiro, V., Mata, L., Santos, N., \& Gomes, M. (2020). Psychometric properties of the Portuguese version of the Achievement Emotions Questionnaire for Elementary School. Análise Psicológica, 38(1), 127-139. http://dx.doi.org/10.14417/ap.1671

Santos, N., Mata, L., Monteiro, V., Sanches, C., \& Gomes, M. (2019). Perceção de competência e desempenho na matemática em alunos do 1. ciclo do ensino básico. Revista Portuguesa de Educação, 32(1),108124. https://doi.org/10.21814/rpe.14264

Siemer, M., Mauss, I., \& Gross, J. J. (2007). Same situation-different emotions: How appraisals shape our emotions. Emotion, 7, 592-600. https://doi.org/10.1037/1528-3542.7.3.592

Historial do artigo

Recebido $\quad 07 / 2019$

Aceite $\quad 03 / 2020$

Publicado $\quad 08 / 2020$ 
Revista PSICOLOGIA, 2020, Vol. 34 (1), doi: 10.17575/psicologia.v34i1.1681.

Atas do X Simpósio Nacional de Investigação em Psicologia

\title{
Impacto da inteligência emocional no engagement e no burnout dos professores: Diferenças de sexo
}

\author{
Natalie Nóbrega Santos ${ }^{29 i}$, Claúdia Andradeii \& Glória Franco ${ }^{\mathrm{ii}}$ \\ ${ }^{i}$ Centro de Investigação em Educação, ISPA - Instituto Universitário \\ ii Universidade da Madeira
}

\begin{abstract}
Resumo: Este estudo analisa, através de equações estruturais, a relação entre a inteligência emocional, o engagement e o burnout dos professores de ensino básico e secundário, as diferenças de sexo nessa relação e as diferenças de sexo nas médias latentes. Participaram 250 professores $(70.4 \%$ do sexo feminino) pertencentes ao $2^{\circ}$ (26.4\%) e 3ํㅜ (57.2\%) Ciclos e Secundário (16.4\%) da Região Autónoma da Madeira. Os resultados indicam que a inteligência emocional tem um efeito direto significativo nos níveis de burnout dos professores e um efeito indireto através do engagement. A relação entre as variáveis é semelhante para professores de ambos sexos e os professores do sexo feminino apresentam níveis mais altos de inteligência emocional. Conclui-se que os professores com maiores níveis de inteligência emocional envolvem-se mais no trabalho e utilizam todas as suas capacidades físicas, cognitivas e emocionais, enquanto desempenham as suas funções, estando mais protegidos dos sintomas de burnout.
\end{abstract}

\section{Palavras-chave: Inteligência emocional; Burnout; Professor; Engagement; Emoções.}

Emotional intelligence impact on engagement and teacher burnout: gender differences: This study analyzes, through structural equations, the relationship between emotional intelligence, engagement and burnout of elementary and secondary school teachers, the gender differences in this relationship and the gender differences in latent means. Two hundred and fifty teachers $(70.4 \%$ female) belonging to the 2 nd (26.4\%) and 3rd (57.2\%) cycles of elementary schools and secondary schools $(16.4 \%)$ of the Autonomous Region of Madeira participated. The results indicate that emotional intelligence has a significant direct effect on teacher burnout levels and an indirect effect through engagement. The relation between variables is similar for teachers of both sexes and female teachers have higher levels of emotional intelligence. It is concluded that teachers with higher levels of emotional intelligence are more involved in the work and use all their physical, cognitive and emotional capacities, while performing their functions, being more protected from the symptoms of burnout.

\section{Keywords: Emotional intelligence; Burnout; Teacher; Engagement; Emotions.}

A inteligência emocional (IE) é definida por Mayer e Salovey (1990) como a capacidade de perceber, avaliar e expressar corretamente as emoções, de utilizar sentimentos para facilitar a compreensão de si ou do outro e a capacidade de controlar as próprias emoções para promover o crescimento emocional e intelectual. Estudos recentes sinalizam a IE como uma variável preditora dos níveis de engagement nos professores (Almazrouei, Dahalan, \& Faiz, 2015; Andrade, Santos \& Franco, 2016; Castillo, FernándezBerrocal, \& Brackett, 2013; Pena, Rey, \& Extremera, 2012; Sun, Chen, \& Jiang, 2017), e que pode ajudar o professor a reduzir o seu burnout e envolver-se noutras atividades da escola para além das funções formais que exerce (Pena \& Extremera, 2012; Rey, Extremera, \& Pena, 2016; para uma revisão ver Mérida-López \& Extremera, 2017).

Schaufeli, Salanova, González-Romá e Bakker (2002) concetualizaram o engagement como um estado afetivo-cognitivo positivo, persistente e abrangente, que se caracteriza pelo vigor (nível de energia e resistência durante as atividades profissionais), pela dedicação (entusiasmo no trabalho) e pela absorção (capacidade de concentração do profissional na sua tarefa). A teoria clássica de Maslach e Leiter (1997) considera que, inicialmente, a pessoa sente-se segura e envolvida no seu trabalho e que o burnout surge quando esse envolvimento diminui devido a circunstâncias desfavoráveis, como por exemplo, no caso dos professores, comportamentos de indisciplina dos alunos, pressões de tempo ou excesso de

29 Morada para correspondência: Natalie Nóbrega Santos, Rua Jardim do Tabaco 34, 1149-041, Lisboa. E-mail: natalie_nobrega_santos@hotmail.com. Este trabalho foi financiado pela FCT - Fundação para a ciência e a Tecnologia (UID/CED/04853/2016). 
tarefas (Correia, Gomes, \& Moreira, 2010). A investigação sugere que os professores com níveis elevados de engagement são menos propensos a sofrer de burnout (Pena \& Extremera, 2012; Silva, 2016).

O burnout é um desconforto caracterizado por três sintomas: exaustão emocional (recursos emocionais dos profissionais são "drenados" e o cansaço se desenvolve); a perda do sentido de realização profissional (desenvolvem-se crenças de que seus esforços são irrelevantes); e a despersonalização (os profissionais apresentam uma atitude menos positiva nas suas relações interpessoais e no seu local de trabalho) (Grayson \& Alvarez, 2008; Maslach \& Jackson, 1986). Em Portugal, os estudos sinalizam que $27.2 \%$ de professores apresentam sintomas de burnout, e 48\% estão em risco de evoluírem para esse quadro (Gomes et al., 2006; Marques, Lima, \& Lopes da Silva, 2005; Santos-Rita, Patrão, \& Sampaio, 2010; Varela et al., 2018).

O burnout tem sido associado a efeitos negativos relacionados com o desempenho e com as relações familiares e sociais, assim como com o desenvolvimento de sintomatologia ansiosa e depressiva (Gouveia, 2010; Shin, Noh, Jang, Park, \& Lee, 2013; Szigeti, Balázs, Bikfalvi, \& Urbán, 2017), com práticas educativas menos efetivas (Patrão \& Santos-Rita, 2011), com a motivação e envolvimento dos seus estudantes nas atividades escolares (López \& Álvarez, 2019; Shen et al., 2015) e com o seu desempenho académico (Balkis, Duru, Bulus, \& Duru, 2011; Herman, Hickmon-Rosa, \& Reinke, 2018). Igualmente, os professores com burnout apresentam uma diminuição da participação nas atividades relativas ao ensino e menor preocupação em satisfazer as necessidades dos alunos (Pas, Bradshaw, Heshfeldt, \& Leaf, 2010; Philip \& Schupbach, 2010). As consequências negativas do burnout têm levado investigadores a estudar as variáveis que possam protegê-los de níveis elevados de exaustão emocional e despersonalização, assim como, ajudar a aumentar os níveis de realização profissional. Entre as variáveis mais estudadas pela literatura está a inteligência emocional e o engagement.

Com base nestas ideias, o nosso primeiro objetivo é avaliar se a IE favorece altos níveis de engagement e se pode ser um fator protetor contra a manifestação do burnout, mediado parcialmente pelo engagement.

Outro aspeto relevante a estudar refere-se às influências de sexo. A literatura refere resultados inconsistentes relativamente às diferenças de sexo, tanto no burnout (Purvanova \& Muros, 2010; Araújo, 2011; Cogaltay, Karadag, \& Anar, 2016; Grayson \& Alvarez, 2008; Santos, 2011; Silva \& Astorga, 2012; Kroupis et al., 2017; Ozan, 2009; Szigeti et al., 2017), como na IE (Karakus, 2013; Pena et al., 2012; Shehzad \& Mahmood, 2013). Relativamente ao engagement, os estudos encontrados com docentes não encontram diferenças significativas nas suas dimensões (Esteves, 2013; Silva \& Astorga, 2012). Por outro lado, apesar de existir vários estudos que investiguem a relação entre burnout ou engagement e a IE, existem poucas informações sobre as diferenças de sexo nessa relação. Apenas foi identificado um estudo com professores, de Karakus (2013), cujos resultados indicaram que a IE mostra efeitos negativos no burnout tanto nos professores como nas professoras, porém, para os homens o efeito da IE no burnout dependia da idade, dos níveis de stress e da ansiedade. Assim, o nosso segundo objetivo é avaliar se existem diferenças de sexo na relação entre inteligência, engagement e burnout e determinar se existem diferenças de sexo nas médias latentes destas variáveis.

\section{MÉTODO}

\section{Participantes}

0 estudo foi desenvolvido na Região Autónoma da Madeira, com a participação de 250 professores pertencentes ao 2ำ (26.4\%) e 3ำ (57.2\%) Ciclos do Ensino Básico e do Ensino Secundário (16.4\%), de 4 escolas públicas, $70.4 \%$ do sexo feminino e $29.6 \%$ do masculino. Os participantes apresentam entre 20 e 63 anos de idade $(M=41.67 ; D P=7.35)$, e um tempo de serviço entre 3 e 40 anos $(M=17.04, D P=7.47)$.

\section{Instrumentos}

Foi utilizado o Trait Meta-Mood Scale (TMMS-24) de Mayer e Salovey (1990, versão portuguesa de Queirós, Fernández-Berrocal, Extremera, Carral, \& Queirós, 2005), que avalia, através de 24 itens de escala tipo Likert de cinco pontos, a atenção às emoções (grau com que as pessoas acreditam prestar atenção aos seus sentimentos), a clareza de sentimentos (forma como as pessoas acreditam perceber as suas emoções) e a reparação do estado emocional (capacidade para interromper os estados emocionais negativos e prolongar os positivos). A consistência interna de cada uma das escalas foi adequada ( $\alpha$ $=.88, .84$, e .87 , respetivamente).

Também foi utilizada a versão reduzida do Utrech Work Engagement Scale (UWES-9) (MarquesPinto, 2000), constituída por 9 itens de escala tipo Likert de seis pontos, que avalia o vigor, a dedicação e a absorção que neste estudo apresentaram níveis adequados de consistência interna $(\alpha=.87,81$, e .70, respetivamente). 
Para avaliar o burnout foi utilizado o Inventário de Burnout de Maslach (MBI, Maslach \& Jackson, 1986; traduzido por Marques-Pinto, 2000), que avalia a exaustão emocional, despersonalização e a realização profissional, através de 22 itens de escala tipo Likert de seis pontos. Neste estudo, as escalas de exaustão emocional e realização profissional apresentam bons valores de fiabilidade $(\alpha=.85$ e .80 , respetivamente), enquanto a escala de despersonalização apresenta valores apenas minimamente aceitáveis $(\alpha=.62)$ semelhantes aos de outras investigações (Castillo et al., 2013; Kroupis et al., 2017; Santos, 2011; Szigeti et al., 2017).

\section{Procedimento}

Após o consentimento informado dos conselhos executivos das escolas e dos professores, os questionários foram entregues a cada docente para o seu preenchimento. Os instrumentos foram devolvidos em envelope fechado, fornecido pelos investigadores, de forma a proteger a confidencialidade das respostas.

Foram calculados os scores de cada uma das escalas do MBI, do UWES, e do TMMS. Após a eliminação de uma observação que apresentou valores outlier, foi estimado um modelo explicativo da influência da IE sobre o burnout, mediado pelo engagement através de equações estruturais, com o método de estimação Máxima Verosimilhança. Para tal, foi utilizado o software IBM® SPSS® AMOS (versão 25.0). A qualidade do ajustamento do modelo foi calculada de acordo com os seguintes índices de qualidade e respetivos valores de referência: $X^{2} / d f \leq 2-3$; CFI e TLI $\geq .95$; e RMSEA $\leq .06$ (Hair, Black, Babin, \& Anderson, 2014). Para refinar o modelo, recorreu-se aos índices de modificação como descrito por Marôco (2014).

A invariância de sexo do modelo explicativo foi avaliada por comparação do modelo não constrito com sucessivos modelos, onde foram fixados os pesos fatoriais e estruturais, interceptos e covariâncias em função do sexo. A significância estatística das diferenças dos modelos foi feita através da monitorização das mudanças significativas nos valores de qui-quadrado $\left(\Delta X^{2}\right)$, após a restrição de cada um dos valores. Valores significativos de $\Delta X^{2}$ indicam que existe variância do modelo em função do sexo, sinalizando a relação entre as variáveis como diferente para cada um dos grupos (Marôco, 2014).

Finalmente, foram comparadas as diferenças nas médias dos fatores latentes IE, engagement e burnout em função do sexo, utilizando modelos MIMIC (Multiple Imputation and Multiple Causes, Marôco, 2014), fixando os coeficientes de regressão no valor zero, e comparando a diferença na qualidade de ajustamento do modelo restrito e do modelo não restrito.

\section{RESULTADOS}

A média da amostra nas dimensões atenção, clareza e reparação emocional encontra-se nos níveis de IE considerados adequados (Queiros et al., 2005; Mayer \& Salovey, 1990); nas dimensões exaustão emocional, despersonalização e realização profissional (ver Tabela 1) encontram-se dentro dos níveis médios de burnout descritos por Marques-Pinto (2000); e nas dimensões de vigor, dedicação e absorção encontra-se entre os valores considerados de alto engagement (Schaufeli \& Bakker, 2003). As variáveis apresentam valores baixos de assimetria $(S k)$ e curtose $(K u)$ que indicam que não existem desvios graves do pressuposto de normalidade (Marôco, 2014).

As análises preliminares (ver Tabela 1), indicam que existem diferenças significativas entre professores do sexo masculino e feminino apenas nas escalas de despersonalização e de atenção às emoções, com as professoras apresentando níveis mais baixos de despersonalização e mais altos de atenção às emoções. Não foram observadas associações significativas entre a idade e o tempo de serviço e as dimensões avaliadas em nenhum dos sexos, pelo que estas variáveis não foram incluídas nas análises seguintes. 
Tabela 1. Análise descritiva das variáveis manifestas

\begin{tabular}{|c|c|c|c|c|c|c|c|c|c|c|c|c|c|}
\hline \multirow[t]{2}{*}{ Variáveis } & \multirow[t]{2}{*}{ Dimensões } & \multirow[b]{2}{*}{ Min. } & \multirow[b]{2}{*}{ Max. } & \multicolumn{3}{|c|}{ Amostra Total } & \multirow[b]{2}{*}{$K u$} & \multicolumn{2}{|c|}{ Feminino } & \multicolumn{2}{|c|}{ Masculino } & \multirow[t]{2}{*}{$t$} & \multirow[t]{2}{*}{$p$} \\
\hline & & & & $M$ & $D P$ & $S k$ & & $M$ & $D P$ & $M$ & $D P$ & & \\
\hline \multirow[t]{3}{*}{ Inteligência emocional } & Atenção & 8 & 39 & 29.96 & 5.64 & -0.78 & 0.78 & 30.58 & 5.26 & 28.45 & 6.26 & 2.74 & .006 \\
\hline & Clareza & 13 & 40 & 30.49 & 4.93 & -0.47 & 0.28 & 30.81 & 5.24 & 29.74 & 4.01 & 1.74 & $.084^{\mathrm{a}}$ \\
\hline & Reparação & 17 & 40 & 32.36 & 5.08 & -0.30 & -0.56 & 32.73 & 4.84 & 31.45 & 5.55 & 1.82 & .070 \\
\hline \multirow[t]{3}{*}{ Engagement } & Vigor & 1.33 & 6 & 4.77 & 1.03 & -1.10 & 1.03 & 14.28 & 3.09 & 14.41 & 3.08 & -0.31 & .758 \\
\hline & Dedicação & 1.33 & 6 & 4.84 & 1.05 & -1.17 & 0.91 & 14.57 & 3.18 & 14.47 & 3.07 & 0.23 & .815 \\
\hline & Absorção & 1.67 & 6 & 4.85 & 0.90 & -1.21 & 1.65 & 14.60 & 2.88 & 14.47 & 2.23 & 0.35 & .729 \\
\hline \multirow[t]{3}{*}{ Burnout } & Exaustão & 0 & 51 & 17.56 & 10.82 & 0.51 & -0.27 & 17.36 & 10.66 & 18.05 & 11.25 & -0.46 & .644 \\
\hline & Despersonalização & 0 & 22 & 4.18 & 4.50 & 1.33 & 1.56 & 3.78 & 4.29 & 5.15 & 4.89 & -2.20 & .028 \\
\hline & Realização & 10 & 48 & 35.89 & 7.89 & -0.75 & 0.13 & 35.77 & 8.24 & 36.16 & 7.01 & -0.36 & .722 \\
\hline \multirow[t]{2}{*}{ Socioprofissionais } & Idade & 20 & 63 & 41.71 & 7.34 & 0.20 & -0.14 & 41.66 & 7.05 & 41.84 & 8.05 & -0.17 & .863 \\
\hline & Tempo de serviço & 3 & 40 & 17.07 & 7.47 & 0.52 & -0.30 & 17.28 & 7.28 & 16.58 & 7.93 & 0.6 & .500 \\
\hline
\end{tabular}

Nota. $\mathrm{N}$ = 249. A = igualdade das variâncias não assumida, valores de significância corrigidos 
Foi avaliado um modelo explicativo da influência da IE sobre o burnout, mediado pelo engagement. 0 modelo revelou uma qualidade de ajustamento sofrível (ver Tabela 2). Os índices de modificação sugerem a correlação entre os erros da escala de exaustão emocional e da escala de despersonalização, sendo coerente com a teoria, pois existem modelos teóricos nos quais estas duas dimensões são combinadas num fator devido às elevadas correlações entre eles (Szigeti et al., 2017). Uma vez correlacionados os erros destas escalas, o modelo apresenta valores adequados (ver Tabela 2). 0 modelo final é apresentado na Figura 1. Todas as escalas contribuem significativamente no seu fator $(p<.001)$. No entanto, as escalas de atenção às emoções $(\lambda=.41)$, da IE e despersonalização $(\lambda=.32)$ do burnout, apresentam pesos fatoriais relativamente baixos $(\lambda<.50)$ mas minimamente aceitáveis $(\lambda>.30)$, de acordo com sugestões de Hair et al. (2014).

Tabela 2. Índices de Ajustamento do Modelo Explicativo do Burnout

\begin{tabular}{lcccccc}
\hline Modelos & $\boldsymbol{X}^{2}$ & $\boldsymbol{d f}$ & $\boldsymbol{X}^{2} / \boldsymbol{d} \boldsymbol{f}$ & $\boldsymbol{C F I}$ & $\boldsymbol{T L I}$ & RMSEA [90\% IC] \\
\hline Modelo Explicativo & 68.15 & 24 & 2.84 & .949 & .923 & $.086[.062, .111]$ \\
Modelo Corrigido & 46.57 & 22 & 2.02 & .973 & .957 & $.064[.037, .091]$ \\
\hline
\end{tabular}

Nota. IC = Intervalo de Confiança

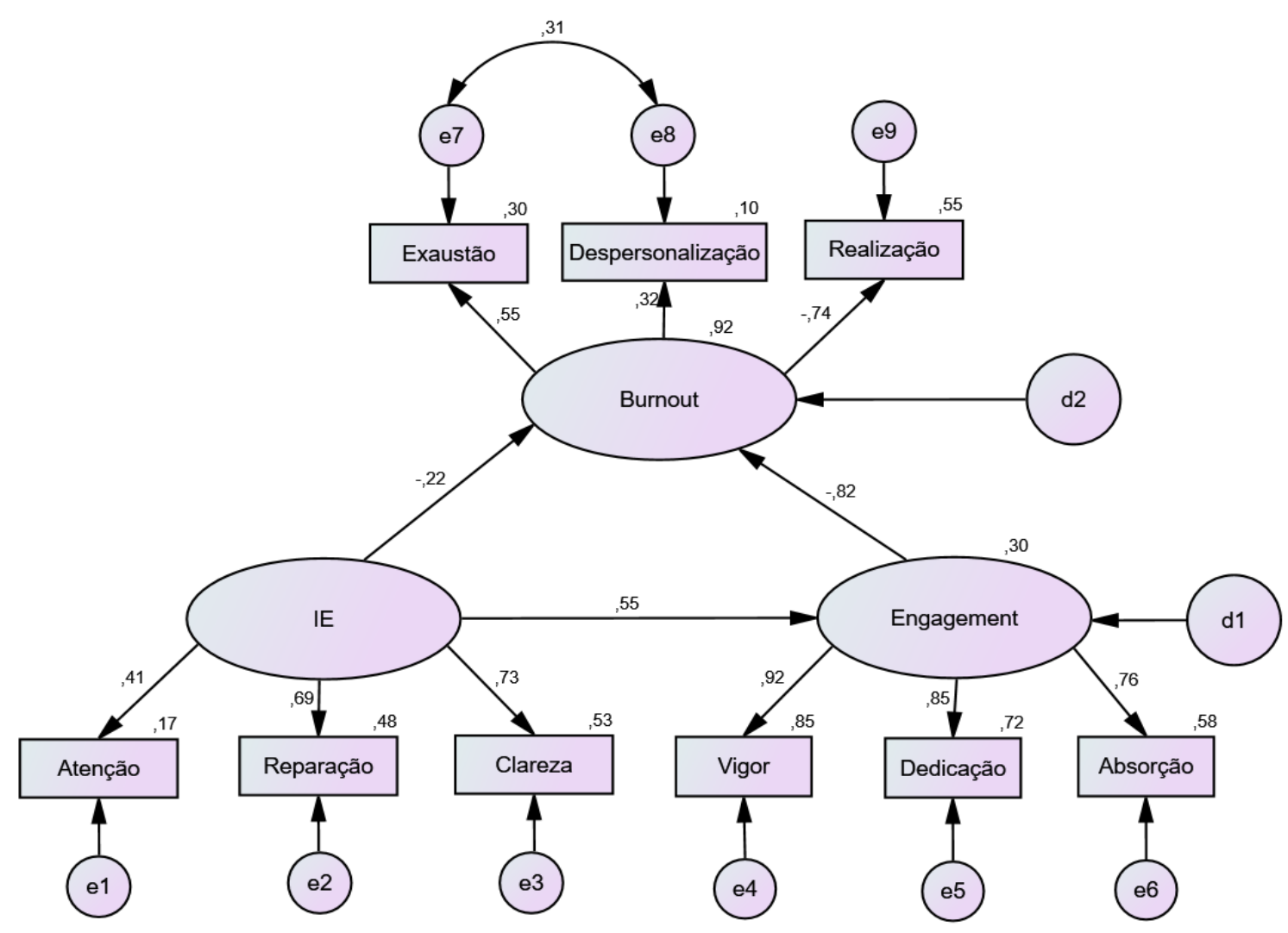

Figura 1. Modelo explicativo do burnout para uma amostra de 249 professores.

Todas as trajetórias são significativas, e explicam $92 \%$ do burnout. 0 efeito direto do engagement sobre o burnout é forte, $\beta=-.82, b=-7.13, p<.001$. O efeito direto da IE sobre o burnout é mais fraco, $\beta=-$ $.22, b=-0.57 p=.009$, mas acrescenta um efeito indireto significativo através do engagement, $\beta=-.45, b=$ $-1.15, p=.001$. 0 efeito da IE sobre o engagement, também é significativo, $\beta=.48, b=0.55, p=.001$, explicando $30 \%$ da sua variabilidade. Assim, maiores níveis de IE estão associados a maiores níveis de engagement e menores níveis de burnout. 
Os valores não significativos de $\Delta \mathrm{X}^{2}$ indicam que o modelo é invariante em função do sexo e adequado para explicar os dados dos professores dos dois sexos (ver Tabela 3).

Tabela 3. Estatística Descritiva dos Itens da Versão Portuguesa da DIDS

\begin{tabular}{lcccc}
\hline Invariância de sexo & $\boldsymbol{X}^{2}$ & $\boldsymbol{d} \boldsymbol{f}$ & $\boldsymbol{\Delta X}^{2}$ & $\boldsymbol{p}$ \\
\hline Com parâmetros livres & 75.93 & 46 & & .656 \\
Com pesos fatoriais fixos & 80.08 & 52 & 4.15 & .067 \\
Com interceptos fixos & 96.05 & 61 & 15.97 & .901 \\
Com pesos estruturais fixos & 96.63 & 64 & 0.58 & .848 \\
Com covariâncias fixas & 96.67 & 65 & 0.04 & \\
\hline
\end{tabular}

As estimativas de regressão estandardizadas, obtidas nos modelos MIMIC utilizados para comparar as médias latentes entre professores do sexo feminino e masculino, mostram efeitos significativos moderados do sexo na IE, $\beta=-.14, b=-.75, p=.018$, com os professores do sexo masculino apresentando um pior desempenho. 0 modelo restrito apresenta uma qualidade significativamente pior, $\Delta X^{2}(1)=5.131$, $p=.026$, confirmando a existência de diferenças significativas de sexo na IE. O teste de diferença de Quiquadrados indicam que não existem diferenças significativas entre os professores e as professoras no engagement, $\Delta X^{2}(1)=2.643, p=.104$, ou no burnout, $\Delta X^{2}(1)=0.474, p=.491$, pelo que se pode concluir que os professores do sexo masculino apresentam níveis semelhantes de engagement e burnout aos do sexo feminino.

\section{DISCUSSÃO}

Os nossos resultados indicam que a IE se apresenta como recurso pessoal para perceber, assimilar e regular os estados de ânimo negativos que podem favorecer o desenvolvimento de sentimentos de vigor, dedicação e absorção no trabalho dos professores e diminuir a sensação de exaustão emocional, despersonalização e de falta de realização profissional. Desta forma, desenvolver a capacidade para gerir as emoções e filtrar estímulos stressantes é cada vez mais importante no dia-a-dia de todos os envolvidos no contexto educativo.

O nosso modelo de equações estruturais indica que as relações entre os diferentes constructos avaliados são semelhantes para ambos os sexos, apenas existem diferenças de sexo para a IE. Segundo Petrides, Furnham, e Martin (2004), a IE é percebida sistematicamente como um atributo feminino, pelo que a perceção de superioridade das mulheres em relação aos homens, pode estar relacionada com o facto de que as mulheres demonstrarem maior competência social. Muller-Lissner (2001) afirma que até que persista uma educação diferenciada entre os sexos, dificilmente se comprovará a ideia de que o sexo feminino lida e manifesta melhor, ou de uma forma inata, as emoções. De qualquer forma, as diferenças encontradas ao nível da IE são bastante pequenas e não se refletem nos níveis de burnout, há semelhança dos resultados de Kroupis et al. (2017), Ozan (2009), e Szigeti et al. (2017). Outros estudos portugueses relatam maiores níveis de realização profissional (Araújo, 2011; Santos, 2011), baixos níveis de exaustão emocional (Santos, 2011) e altos níveis de despersonalização (Silva \& Astorga, 2012) no professor do sexo masculino. Contudo, estes estudos não avaliam os níveis de burnout integrando os sintomas de exaustão emocional, despersonalização e falta de realização profissional, como no nosso estudo.

A comparação de médias latentes realizada neste estudo, oferece evidências estatísticas mais robustas das diferenças entre grupos, uma vez que é contabilizado o erro de medida dos instrumentos utilizados (Vandenberg \& Lance, 2000). Nas análises preliminares do presente estudo, foram identificados maiores níveis de despersonalização nos professores do sexo masculino, como os resultados de Silva e Astorga (2012), mas esta escala apresenta limitações do ponto de vista da consistência interna, limitando a sua fiabilidade.

Os nossos resultados realçam a importância que a IE tem para os professores na manutenção do engagement no trabalho docente, e na proteção contra os sintomas de burnout. Considerando que vivemos num momento de mudanças educativas, importa refletir sobre a importância de incluir, de uma forma explícita, a aprendizagem de competências emocionais na formação docente. Estas competências não são só relevantes para o próprio bem-estar, como também para o desenvolvimento da IE dos alunos. Fernández-Berrocal e Extremera (2002), indicam que, na atualidade, o professor tem de ser capaz de ensinar a aritmética do coração e a gramática das relações sociais. Estes aspetos serão essenciais para assegurar os programas educativos, melhorar os resultados dos alunos e promover a sustentabilidade e a continuidade das aprendizagens. 
Este estudo apresenta algumas limitações, pois o caracter transversal e correlacional dos dados não permite determinar efeitos de causalidade entre as variáveis, pelo que são necessários estudos longitudinais de, pelo menos, um ano de intervalo (Maricutoiu, Sulea, \& Iancu, 2017), que permitam compreendem melhor a relação causal entre IE, engagement e burnout.

A amostra deste estudo não permitiu validar a invariância de sexo nos instrumentos utilizados, de forma a assegurar que os constructos avaliados são os mesmos para ambos os sexos (Marôco, 2014). Apesar de existirem estudos internacionais que comprovam a invariância de sexo do MBI, do UWES e do TMMS, não foram encontrados estudos para a população portuguesa. Neste sentido, são necessários mais estudos que permitam confirmar a validade destas escalas para comparar diferenças de sexo.

\section{REFERÊNCIAS}

Almazrouei, S. A. S., Dahalan, N., \& Faiz, M. H. (2015). The Impact of Emotional Intelligence on Employee Engagement. International Journal of Management and Commerce Innovations, 3(1), 376-387. https://doi.org/10.1177/026142940702300203

Andrade, C., Franco, M. G., \& Santos, N. N. (2016). Inteligência emocional e engagement em professores do ensino básico e secundário da Ilha da Madeira. Revista de estudios e investigación en psicología y educación, 3(2), 376-387. https://doi.org/10.17979/reipe.2016.3.2.1810

Araújo, A. M. (2011). Interacção das variáveis sócio-demográficas com o stress e o burnout em professores do 2. ${ }^{\mathrm{o}}$ e 3. ${ }^{\circ}$ ciclos de ensino secundário. EFDesportes.com, 16, 161. Retirado de https://www.efdeportes.com/efd161/burnout-em-professores-de-ensino-secundario.htm

Balkis, M., Duru, E., Bulus, M., \& Duru, S. (2011). The prevalence of burnout among prospective teachers, it's relation with demographic variables and academic achievement. Pamukkale University Journal of Education, 29, 151-165.

Castillo, R., Fernández-Berrocal, P., \& Brackett, M. A. (2013). Enhancing Teacher Effectiveness in Spain: A Pilot Study of The RULER Approach to Social and Emotional Learning. Journal of Education and Training Studies, 1(2). https://doi.org/10.11114/jets.v1i2.203

Cogaltay, N., Karadag, E., \& Anar, S. (2016). The factor affecting teachers' burnout: a meta-analytical study on the effect of gender and marital status. Hacettepe University Journal of Education, 32(3), 695707. https://doi.org/10.16986/HUJE.2016018517

Correia, T., Gomes, A. R., \& Moreira, S. (2010). Stress ocupacional em professores do ensino básico: um estudo sobre as diferenças pessoais e profissionais. No VII Simpósio Nacional de Investigação em Psicologia (pp. 1477-1493). Universidade do Minho. Retirado de http://hdl.handle.net/1822/10502

Esteves, A. M. A. (2013). O Burnout e o Engagement - Estudo numa amostra de professores do ensino superior público português [Dissertação de mestrado, Instituto Politécnico do Porto]. Retirado de http://hdl.handle.net/10400.22/5064

Fernández-Berrocal, P., \& Extremera, N. (2002). La inteligencia emocional como una habilidad esencial en la escuela. Revista Iberoamericana de Educación, 29,1-6.

Gomes, R. A., Silva, M. J., Mourisco, S., Silva, S., Mota, A., \& Montenegro, N. (2006). Problemas e desafios no exercício da actividade docente: um estudo sobre o estresse, burnout, saúde física e satisfação profissional em professores do 3 o ciclo e ensino secundário. Revista Portuguesa de Educação, 19(1), 67-93. Retirado de http://www.scielo.mec.pt/scielo.php?script=sci_arttext\&pid=S087191872006000100004

Gouveia, C. J. (2010). Burnout, ansiedade e depressão nos professores. [Dissertação de mestrado, $\begin{array}{llll}\text { Universidade } & \text { Le } & \text { Retirado } & \text { de }\end{array}$ http://repositorio.ul.pt/bitstream/10451/2742/3/ulfp037556_tm_tese.pdf.

Grayson, J. \& Alvarez, H. (2008). School climate factors relating to teacher burnout: a mediator mode. Teaching and Teacher Education, 23, 1349-1363. https://doi.org/10.1016/j.tate.2007.06.005

Hair, J. F., Black, W. C., Babin, B. J., \& Anderson, R. E. (2014). Multivariate data analysis. Pearson custom library. Pearson Education Limited. https://doi.org/10.1038/259433b0

Herman, K. C., Hickmon-Rosa, J., \& Reinke, W. M. (2018). Empirically derived profiles of teacher stress, burnout, self-efficacy, and coping and associated student outcomes. Journal of positive behavior interventions, 20, 90-100. https://doi.org/10.1177/1098300717732066

Karakus, M. (2013). Emotional intelligence and negative feelings: A gender specific moderated mediation model. Educational Studies, 39(1), 68-82. https://doi.org/10.1080/03055698.2012.671514

Kroupis, I., Kourtessis, T., Derri, V., Tzetzis, G., Kouli, O., \& Mavrommatis, G. (2017). Job satisfaction and burnout among Greek P.E. teachers. A comparison of educational sectors, level and gender. Cultura_Ciencia_Deporte, 12(34), 5-14. https://doi.org/10.12800/ccd.v12i34.827 
López, J. C., \& Álvarez, E. F. (2019). Relatioship between students' motivation and engagement in physical education and teacher's burnout. Sportis - Cientific Journal of School Sport Physical Education and Psychomotricity, 5, 101-117. https://doi.org/10.17979/sportis.2019.5.1.3634

Maricuțoiu, L. P., Sulea, C., \& Iancu, A. (2017). Work engagement or burnout: Which comes first? A metaanalysis of longitudinal evidence. Burnout Research, 5(May), 35-43. https://doi.org/10.1016/j.burn.2017.05.001

Marôco, J. (2014). Análise de equações estruturais. Fundamentos teóricos, software, \& aplicações. ReportNumber.

Marques-Pinto, A. (2000). Burnout profissional em professores portugueses: representações sociais, incidência e preditores. [Dissertação de Doutoramento, Universidade de Lisboa]. Retirado de http://hdl.handle.net/10451/11328

Marques-Pinto, A., Lima, M.L., \& da Silva, A. (2005) Como lidam os professores com o stress profissional? Proformar, 7. Retirado de http://proformar.pt/revista/edicao_7/copingProfsF.pdf

Maslach, C. \& Jackson, S. (1986). Maslach burnout inventory manual (2. ${ }^{\mathrm{a}}$ Ed.). Consulting Psychologist Press.

Maslach, C., \& Leiter, M. P. (1997). The truth about burnout. Jossey-Bass.

Mayer, J., \& Salovey, P. (1990). Emotional intelligence. Imagination, cognition and personality, 9(3), 185211. Retirado de http//www.unh.edu/emotional_ intelligence/EIAssets/EmotionalIntelligenceProper/EI1990\%20Emotional\%20intelligence.pdf

Mérida-López, S., \& Extremera, N. (2017). Emotional intelligence and teacher burnout: A systematic review. International Journal of Educational Research, 85, 121-130. https://doi.org/10.1016/J.IJER.2017.07.006

Muller-Lissiner, A. (2001). A Inteligência Emocional na Criança - Como estimulá-la no seu filho. Colecção Biblioteca Pergaminho.

Ozan, M. B. (2009). A study on primary school teacher burnout levels: The Northern Cyprus case. Education, 129(4), 692-703.

Pas, E., Bradshaw, C., Hershfeldt, P., \& Leaf, P. (2010). A multilevel exploration of the influence of teacher efficacy and burnout on response to student problem behavior and school-based service use. School Psychology Quarterly, 25(1), 13-27. https://doi.org/10.1037/a0018576

Patrão, I., \& Santos-Rita, J. (2011). Práticas educativas em professores portugueses: A influência das preocupações profissionais, condições organizacionais e reconhecimento. Nas Actas Do $12^{\circ}$ Colóquio de Psicologia e Educação (pp. 1460-1472). ISPA - Instituto Universitário. Retirado de http://hdl.handle.net/10400.12/1617

Pena, M., \& Extremera, N. (2012). Inteligencia emocional percibida en el profesorado de primaria y su relación con los niveles de burnout e ilusión por el trabajo (Engagement). Revista de Educacion, 359, 604-627. https://doi.org/10.4438/1988-592X-RE-2011-359-109

Pena, M., Rey, L., \& Extremera, N. (2012). Life Satisfaction and Engagement in Elementary and Primary Educators Differences in Emotional Intelligence. Revista de Psicodidáctica, 17(2), 341-358. https://doi.org/10.1387/Rev.Psicodidact.1220

Petrides, K. V., Furnham, A., \& Martin, G. N. (2004). Estimates of emotional and psychometric intelligence: evidence for gender-based stereotypes. The Journal of Social Psychology, 144(2), 149-162. https://doi.org/10.3200/SOCP.144.2.149-162

Philip, A., \& Schupbach, H. (2010). Longitudinal effects of emotional labour on emotional exhaustion and dedication of teachers. Journal of Occupational Health Psychology, 15, 494-504. https://doi.org/10.1037/a0021046

Purvanova, R. K., \& Muros, J. P. (2010). Gender differences in burnout: A meta-analysis. Journal of Vocational Behavior, 77(2), 168-185. https://doi.org/10.1016/J.JVB.2010.04.006

Queirós, M. M., Fernández-Berrocal, P., Extremera, N. Carral, J. M. C. e Queirós, P. S. (2005). Validação e fiabilidade da versão portuguesa modificada da Trait Meta-Mood Scale. Psicologia, Educação e Cultura, 9(1) 199-216.

Rey, L., Extremera, N., \& Pena, M. (2016). Emotional competence relating to perceived stress and burnout in Spanish teachers: a mediator model. PeerJ, 4, e2087. https://doi.org/10.7717/peerj.2087

Santos, S. F. (2011). Burnout e diferenças de género em Professores [Dissertação de mestrado, Universidade Lusófona de Humanidades e Tecnologias, Faculdade de Psicologia].

Santos-Rita, J., Patrão, I., \& Sampaio, D. (2010). Burnout, stress profissional e ajustamento emocional em professores portugueses do ensino básico e secundário. Nas Actas Do VII Simpósio Nacional de Investigação em Psicologia (pp. 1151-1161). Universidade do Minho. Retirado de http://repositorio.ispa.pt/bitstream/10400.12/1531/1/SNIP 2010 1151- 1161.pdf 
Schaufeli, W., \& Bakker, A. (2003). UWES - Utrecht Work Engagement Scale Preliminary Manual. Occupational Health Psychology Unit Utrecht University.

Schaufeli, W., Salanova, M., González-Romá, V., \& Bakker, A. (2002). The measurement of engagement and burnout: a two sample confirmatory factor analytic approach. Journal of Happiness Studies, 3, 7192. https://doi.org/10.1023/A:1015630930326.

Shehzad, S., \& Mahmood, N. (2013). Gender differences in emotional intelligence of university teachers. Pakistan Journal of Social and Clinical Psychology, 11(1), 16-21. Retirado de https://pdfs.semanticscholar.org/fb9b/ 336a585ae91025ebb963f7f8c41022043143.pdf.

Shen, B., McCaughtry, N., Martin, J., Garn, A., Kulik, N., \& Fahlman, M. (2015). The relationship between teacher burnout and student motivation. British Journal of Educational Psychology, 85, 519-532. https://doi.org/10.1111/bjep.12089

Shin, H., Noh, H., Jang, Y., Park, Y. M., \& Lee, S. M. (2013). A longitudinal examination of the relationship between teacher burnout and depression. Journal of Employment Counseling, 50, 124-137. https://doi.org/10.1002/j.2161-1920.2013.00031.x

Silva, A. P. (2016). Burnout, engagement, e perceção das exigências do trabalho: Comparação entre professores do ensino público e privado [Dissertação de mestrado, Instituto de Contabilidade e Administração do Porto]. Retirado de http://hdl.handle.net/10400.22/8709

Silva, M. \& Astorga, M. (2012). Engagement e Burnout em Professores Portugueses. International Journal of Developmental and Educational Psychology INFAD, 4(1), 63-72. Retirado de http://hdl.handle.net/10662/3040

Sun, P., Chen, J. J., \& Jiang, H. (2017). Coping humor as a mediator between emotional intelligence and job satisfaction. A study on Chinese Primary School Teachers. Journal of Personnel Psychology, 16, 155159. https://doi.org/10.1027/1866-5888/a000185

Szigeti, R., Balázs, N., Bikfalvi, R., \& Urbán, R. (2017). Burnout and depressive symptoms in teachers: Factor structure and construct validity of the Maslach Burnout inventory-educators survey among elementary and secondary school teachers in Hungary. Stress and Health, 33(5), 530-539. https://doi.org/10.1002/smi.2737

Vandenberg, R. J., \& Lance, C. E. (2000). A review and synthesis of the measurement invariance literature: Suggestions, practices, and recommendations for organizational research. Organizational Research Methods, 3, 4-70. https://doi.org/10.1177/109442810031002

Varela, R. C., della Santa, R., Silveira, H., Matos, C., Rolo, D., Areosa, J., \& Leher, R. (2018). Inquérito nacional sobre as condições de vida e trabalho (INCVTE) na educação em Portugal (Relatório). Jornal da FENPROF. Retirado https://www.fenprof.pt/Download/FENPROF/M_Html/Mid_332/Anexos/JF_INCVTE_20182.pdf 
Revista PSICOLOGIA, 2020, Vol. 34 (1), doi: 10.17575/psicologia.v34i1.1681.

Atas do X Simpósio Nacional de Investigação em Psicologia

\title{
Escala de comportamento pro-ambiental: Estudo exploratória de validação
}

\author{
Luciana Maria Ferreira $30 \mathrm{i}$ \\ i Universidade da Madeira
}

\begin{abstract}
Resumo: As alterações ambientais globais resultantes do rápido processo de urbanização, industrialização e mudanças no padrão de consumo das populações, têm impulsionado as comunidades científica e política a procurar respostas para o desenvolvimento sustentável. 0 acordo científico sobre o aquecimento global e padrões de consumo mundial, realçam a necessidade de se compreender com maior profundidade os mecanismos de comportamento e atitudes ambientais da população. Este estudo pretende analisar as relações entre o comportamento pró-ambiental através da Escala de Comportamento Ambiental (ECA) de Ferreira, Agrela, Garcês e Pocinho (Submetido), o sexo, a idade e o nível educacional. Trata-se de um estudo quantitativo correlacional, que utiliza uma amostra de conveniência. Os dados foram recolhidos através das redes sociais e correio eletrónico, através de uma amostra de 300 sujeitos. Os resultados serão discutidos e explorados posteriormente.
\end{abstract}

\section{Palavras-chave: Psicologia do ambiente; Atitudes pró-ambientais; Comportamento ambiental.}

Pro-environmental behaviour scale: Exploratory validation study: Global environmental changes resulting from the rapid process of urbanization, industrialization and changes in population consumption patterns have driven scientific and political communities to seek answers to sustainable development. The scientific agreement on global warming and world consumption patterns underlines the need to better understand the mechanisms of behaviour and environmental attitudes of the population. This study aims to analyse the relationships between pro-environmental behaviour through the Ferreira, Agrela, Garcês e Pocinho, Environmental Behaviour Scale (ECA), educational level, age and gender. This is a correlational quantitative study using a random sample. Data were collected through social networks and email, through a sample of 300 subjects. The results will be discussed and explored later.

Keywords: Environmental Psychology; Pro-environmental attitudes; Environmental behaviour.

Considerando o contexto da União Europeia, assiste-se, nas últimas décadas, a um conjunto de preocupações e respostas no que concerne ao meio ambiente, à sustentabilidade e bem-estar humano, que se fazem notar, de alguma forma, ao nível internacional (NASA, 2019; Chang, 2017; IPSS, 2014).

Nas últimas décadas do século XX, o estudo sobre a matéria ambiental foi negligenciado em Portugal, mas a partir da primeira década do novo século a narrativa ambiental tem começado a ganhar importância (Guimarães \& Amorim, 2016).

Em 2015, foi criada uma Rede Portuguesa de História Ambiental que estabelece um marco identitário desta disciplina em relação ao ambiente e sua relação com a sociedade, a cultura e a economia. Esta rede vem consolidar o conhecimento sobre toda a dinâmica que se dirige a questões ambientas, nos seus fenómenos naturais e da biodiversidade CITCEM (Transdisciplinary Research Centre Culture, 2019).

Segundo Guimarães e Amorim (2016), existe uma grande escassez de material científico, em Portugal, em relação às alterações climáticas. Os trabalhos realizados circunscreviam-se à geografia ou ao espaço físico-biológico, onde se desenvolvem as relações humanas que se traduzem em relações económicas. É com Orlando Ribeiro que a história ambiental se manifesta, através do destaque que este confere ao papel do homem na introdução de novas plantas e espécies animais, na alteração da paisagem e nas alterações de povoamento (Ribeiro, 1993). Outro contributo foi o de Feio (1983), em finais dos anos 40, onde descreve as alterações climáticas, seca, no Baixo Alentejo e Algarve. Outro marco, nesta matéria, deve-se às questões ambientais associadas ao desenvolvimento da indústria num estado que havia falhado nos seus esforços de modernização, e que se posicionou na periferia da economia mundial. Nos finais dos anos 80, é publicado um estudo em que se mostra o impacto de conflitos industriais e a

30 Morada para correspondência: Luciana M. Ferreira, Universidade da Madeira, Funchal. E-mail: lucianaferreira_998@hotmail.com 
poluição dos rios e da poluição mineira. 0 principal esforço para o reconhecimento dos problemas de poluição atmosférica e das águas associados à industrialização em Portugal, bem como das iniciativas dos governos para limitar o seu impacto ambiental, foi autoria de Ana Cardoso Matos (Guimarães \& Amorim, 2016).

Um novo ciclo emerge a partir da primeira década no novo século. Exemplos são os estudos de James W. Moore sobre a cultura do açúcar na Ilha da Madeira, e expansão da primeira economia-mundo e, posteriormente, os dois textos prolíferos para a narrativa ambiental portuguesa (Guimarães \& Amorim, 2016).

Em 2010, surge a dissertação de Maria Cristina Joana de Melo (2010) que explora a criação do ordenamento jurídico liberal sobre as florestas e as águas. Segundo Neto (2013), a História Ecológica ou Meio Ambiente inicia terreno na literatura do meio académico.

Em Portugal, pela primeira vez, desenvolve-se investigação histórica em relação ao clima, sendo que um dos resultados deste trabalho é a elevação da temperatura nos últimos 400 anos (Santos, 2015). Por sua vez, a identificação de fenómenos climatéricos extremos e das vulnerabilidades da costa portuguesa, o esforço de relacionar o comportamento da mortalidade com as mudanças climatéricas (Guimarães \& Amorim, 2016). 0 trabalho continua a ser realizado sendo imperioso o seu contributo no entendimento da urgência de construir uma sociedade mais resiliente e sustentável. Não obstante, na Região Autónoma da Madeira, os trabalhos realizados nesta matéria são escassos. Encontrou-se apenas uma referência a um estudo longitudinal de 5 anos, intitulado "Programas de Monitorização Ambiental da ETRS da Meias Serra" de 2005, onde foram estudadas as condições meteorológicas, qualidade do ar, poluentes atmosféricos ao nível do solo, águas superficiais e subterrâneas e comportamentos dos aterros, ruído, fauna e flora, saúde pública, e com especial enfoque os aspetos psicossociais. Neste último programa, desenvolvido por Branco et al. (2005), verificou-se que os fatores que poderão afetar a perceção de risco ambiental são a "familiaridade, medo do desconhecido, proximidade no espaço e no tempo" (p. 244). No entanto, quando inseridas, numa base de dados - A Biblioteca do Conhecimento Online (https://www.b-on.pt/, 2019) -, as palavras chave "ambiente" e "Ilha da Madeira", no ano de 2019, não surgiram quaisquer resultados. Parece-nos fundamental estudar estas questões, sendo que as vulnerabilidades de uma ilha acrescem em toda a urgência na matéria. Dentro desta contextualização, a Psicologia é solicitada a realizar o seu papel na compreensão das características diferenciadoras dos indivíduos, nos seus processos cognitivos e motivacionais, que promovem o comportamento próambiental.

\section{Comportamentos e consciência pró-ambientais}

A proteção do ambiente, e especificamente a ameaça às alterações climáticas, são um dos maiores desafios enfrentados pela nossa sociedade. De modo a endereçar os problemas ambientais, é fundamental compreender o comportamento pró-ambiental na população, assim como os fatores que o determina.

0 crescimento económico e o aumento do padrão de vida em muitos países foram seguidos de um crescimento no consumo, o que, por sua vez, leva à deterioração ambiental. Muitos estudos indicam que a deterioração ambiental está relacionada com o aumento das atividades económicas e ao aumento da procura de energia e recursos naturais (Levitus, et al., 2017). No entanto, também foi demonstrado que, embora muitos dos países implementem tecnologias amigas do ambiente como resultado de sociedades orientadas para o consumo e padrões de consumo insustentáveis a nível mundial, as fontes de poluição ambiental mudaram dos processos de produção para os de consumo, o que causou adicionalmente um agravamento da situação (Vasiljevic-Shikaleska, Trpovski, \& Gjozinska, 2018). Atualmente, a maioria dos problemas ambientais surge não apenas das atividades económicas, mas também da falta de legislação, instrumentos e regulamentos económicos e predominantemente da falta de conscientização ambiental e participação do público no comportamento pró-ambiental para a proteção ambiental (VasiljevicShikaleska, Trpovski, \& Gjozinska, 2018).

A consciência ambiental é uma definição muito genérica que se refere ao conhecimento sobre ambiente e atitude, valores e capacidades necessárias para resolver problemas relacionados ao meio ambiente. Pode ser definida como a capacidade de um indivíduo compreender a relação existente entre as atividades humanas, o status atual da qualidade ambiental (Liu, Vedlitz, \& Shi, 2014) e a vontade do indivíduo em participar atividades ambientais. Além disso, a consciencialização ambiental é assumida como um pré-requisito importante da proteção ambiental, e é considerada uma etapa inicial que leva à capacidade de manter um comportamento de cidadania responsável e uma ferramenta poderosa para promover a política ambiental (Vasiljevic-Shikaleska, Trpovski, \& Gjozinska, 2018).

o comportamento pró-ambiental, por outro lado, pode ser definido como um complexo de atividades realizadas para a proteção do meio ambiente em termos de mudança de comportamento e 
hábitos de consumo dos consumidores, de modo a contribuir para a conservação de energia e materiais, proteção da biodiversidade e gestão ecológica de resíduos (Čater \& Serafimova, 2019).

Determinar o que as pessoas sabem sobre o meio ambiente e as mudanças climáticas, como se sentem sobre ele e quais ações realizam, são essenciais para estabelecer a sustentabilidade da sociedade (Čater \& Serafimova, 2019). Verificou-se, por exemplo, que a preocupação ambiental pode ser um dos principais fatores no processo de tomada de decisão dos consumidores no ato de compra (Čater \& Serafimova, 2019).

Os resíduos pós-adquiridos são outra preocupação ambiental de grande importância. Uma das estratégias mais bem-sucedidas para lidar com os resíduos pós-adquiridos é a reciclagem que reduz o uso de matérias-primas, a poluição da água e do ar e o desperdício (Banerjee, 2015). Como um todo, a proteção ambiental depende não apenas dos programas implementados pelos órgãos reguladores, mas também das escolhas diárias feitas pelos consumidores em termos de como eles se comportam em relação ao meio ambiente, em relação ao que consomem ou ao que desejam deixar de consumir.

\section{Considerações sociodemográficas e comportamento pró-ambiental}

A extensa revisão da literatura mostrou que o comportamento pró-ambiental do consumidor é grandemente influenciado por um conjunto diversificado de fatores que, geralmente, podem ser agrupados em fatores sociodemográficos, sendo os mais influentes a idade, sexo, nível de escolaridade (Benedek \& Takácsné György, 2013) e estado civil (Boztepe, 2012).

Muitos dos estudos sugerem que a idade é um importante preditor de comportamento próambiental. Enquanto alguns concluíram que os idosos estão mais preocupados com o meio ambiente do que os mais jovens (Shen \& Saijo, 2008), outros descobriram que os mais jovens demonstram uma maior consciência de obrigação com o meio ambiente (Wiernik et al., 2013). No entanto, a relação entre idade e comportamento ambiental depende do comportamento específico em estudo (Charles \& Piazza, 2009). No que diz respeito à variável sexo, as mulheres praticam um estilo de vida mais ecológico, dedicando mais atenção a comportamentos pró-ambientais, como reciclagem, compra de produtos orgânicos e redução do uso de automóveis (Xiao \& McCright, 2014). Em relação à influência da formação educacional, observou-se que, em geral, existe uma relação positiva entre nível de educação e conscientização ambiental, o que implica que pessoas mais instruídas tendem a se preocupar mais com o meio ambiente e mais dispostas a contribuir em relação aos comportamentos pró-ambientais (Xiao \& McCright, 2014). Tendo em mente o exposto, estudar os atitudes e comportamentos pró-ambientais dos consumidores e os fatores que os afetam é fundamental para mitigar as mudanças climáticas e permitir salvar o ambiente e o bem-estar geral.

Pelo acima exposto consideramos as seguintes hipóteses:

Hipótese 1: 0 sexo feminino apresenta maior comportamento pró- ambiental.

Hipótese 2: Indivíduos com mais idade apresentam maior comportamento pró-ambiental.

Hipótese 3: 0 nível de educação superior relaciona-se com maior comportamento pró-ambiental. ambiental.

Hipótese 4: A consciência ambiental está positivamente relacionada com comportamento pró-

\section{MÉTODO}

\section{Participantes}

A amostra deste estudo é constituída por 300 indivíduos, maioritariamente portugueses, da Região Autónoma da Madeira. Dos participantes, 75\% (225) são do sexo feminino, 24.7\% (74) é do sexo masculino e $0.3 \%$ (1) insere-se na categoria "outro". As idades oscilaram entre os 15 e os 67 anos, situando-se a média das idades em $34.8(D P=11.9)$.

Em relação ao nível de escolaridade, $0.7 \%$ (2) possuem o 4o ano de escolaridade; $1.3 \%$ (4) o $6^{\circ}$ ano de escolaridade; $7.7 \%$ (23) o 9o ano de escolaridade; $30.3 \%$ (91) o $12^{\circ}$ ano de escolaridade; $44 \%$ (132) a licenciatura; $14 \%$ (42) o mestrado; e, $2 \%$ (6) o doutoramento

\section{Instrumentos}

Foi utilizada a Escala de Comportamento Ambiental (ECA) de Ferreira, Agrela, Garcês e Pocinho (submetido), que avalia o comportamento pró-ambiental, consciência e os transportes ecológicos através de 9 itens, numa escala Likert, com 5 pontos (1 - não concordo e 5 - concordo muito). Através do teste $K M O$ e Bartlett, a adequação da amostra mostrou um valor razoável de $.76(p<.001)$, o que permite realizar a análise fatorial. Com recurso à análise fatorial com rotação Varimax, os itens da ECA repartemse por 3 fatores, que que representam $66 \%$ do total da variância explicada. 0 fator 1 ( 5 itens), apresenta 
um $\alpha$ de .81. No que se refere ao fator 2, (2 itens), apresenta um $\alpha$ de .56. 0 fator 3, ( 2 itens), indica um $\alpha$ de 68 (ver Tabela 1).

Tabela 1. Resultado da análise fatorial da escala ECA

\begin{tabular}{lcc}
\hline Fatores & Itens da escala & $\alpha$ de \\
Cronbach
\end{tabular}

Fator 1 - Comportamento pró- ambiental

Fator 2 - Consciência ambiental

Fator 3 -Transportes ecológicos

\footnotetext{
"Em geral, penso/preocupo-me com as questões

ambientais"

"Faço separação do lixo"

“Evito a utilização de plástico na minha rotina

$$
\text { diária" }
$$

“Nas minhas compras seleciono produtos que

tenham embalagens reutilizáveis ou recicláveis"

"Encorajo amigos a reciclar"
}

.81

“Considero relevante conservar o ambiente

através de reservas naturais"

"Considero relevante proteger a biodiversidade"

"Utilizo transportes públicos"

.56

"Poupo combustível deslocando-me a pé ou de

bicicleta"

\section{Procedimento}

Para a realização do instrumento foram elaborados 20 itens, relacionados com o comportamento ambiental, consciência ambiental e variáveis pessoais. Numa primeira fase, foi efetuada uma análise preliminar com o intuito de verificar a adequabilidade do questionário, sendo que para tal foram recolhidas as respostas de 20 indivíduos, onde foram avaliados o tempo de preenchimento e a opinião dos participantes nas questões apresentadas. Os resultados, em relação à confiabilidade dos itens, conduziram à eliminação de 11 itens. Deste modo, optou-se por manter 9 dos itens iniciais, com valores de alfa de Cronbach aceitáveis. A média das pontuações nos itens correspondeu a 3.5, oscilando entre 2.3 e 4.7 .

O questionário com as informações relativas ao objetivo do estudo, foi distribuído através de redes sociais e correio eletrónico. No total foram recolhidas 300 respostas, tendo sido consideradas todas válidas.

\section{RESULTADOS}

0 tratamento dos dados foi realizado através do IMB SPSS (versão 25). De acordo com a Tabela 2, não foram encontradas diferenças estatisticamente significativas relativamente ao sexo, ao nível de $5 \%$ ( $p=$ 0.596).

Tabela 2. Diferenças de sexo consoante os fatores

\begin{tabular}{lccc}
\hline Teste $t$ & $\boldsymbol{p}$ & $\boldsymbol{t}$ & $\mathbf{d f}$ \\
& & & \\
\hline Comportamento pró-ambiental & .173 & .226 & 297 \\
Consciência ambiental & .643 & -.881 & 297 \\
Transportes ecológicos & .862 & .560 & 297 \\
\hline
\end{tabular}


Os dados da amostra indicam que existem diferenças significativas entre os indivíduos mais novos (idade inferior a 35 anos) e os mais velhos (idade superior a 35 anos). Os mais velhos apresentam valores inferiores nos transportes ecológicos, $t(298)=4.761, p<.001$ (ver Tabela 3).

Tabela 3. Diferenças de idade consoante os fatores

\begin{tabular}{lccc}
\hline Test t & $\boldsymbol{p}$ & $\boldsymbol{t}$ & $\mathbf{d f}$ \\
& & & \\
\hline Comportamento pró-ambiental & .429 & -.6 .723 & 298 \\
Consciência ambiental & .185 & -.969 & 298 \\
Transportes ecológicos & .000 & 4.761 & 298 \\
\hline
\end{tabular}

Verificaram-se diferenças no nível de educação através da Anova, com $F(2,297)=4298(p=.014)$ e o comportamento pró-ambiental. 0 teste pos-hoc de Bonferroni, mostra as comparações de escolaridade por Método Pairwaise (ver Tabela 4). As diferenças médias foram reportadas entre o nível superior de educação e o nível médio, sendo que os detentores de um grau superior apresentam diferenças superiores em $1.3(p<.01)$.

Tabela 4. Nível de escolaridade

\begin{tabular}{lccccc}
\hline Comparações & $\begin{array}{c}\text { (I) Nível de } \\
\text { Variável dependente }\end{array}$ & $\begin{array}{c}\text { (J) Nível de } \\
\text { escolaridade }\end{array}$ & $\begin{array}{c}\text { Diferença } \\
\text { média (I-J) }\end{array}$ & DP & Sig. \\
\hline Comportamento pró-ambiental & Superior & < Médio & $.1 .3^{*}$ & .44 & .01 \\
\hline
\end{tabular}

Nota. * A diferença média é significativa no nível .05.

Como se pode verificar na Tabela 5, a variável comportamento pró-ambiental tende a apresentar uma correlação significativa com a consciência ambiental, ao nível de $.01(r=.278)$. No que refere à variável consciência ambiental, esta tende a correlacionar-se com a variável os transportes ecológicos, ao nível de .05 ( $r=.125)$.

Tabela 5. Matriz de correlações das variáveis do estudo

\begin{tabular}{lccc}
\hline Correlações & $\begin{array}{c}\text { Comportamento } \\
\text { pró-ambiental }\end{array}$ & $\begin{array}{c}\text { Consciência } \\
\text { ambiental }\end{array}$ & $\begin{array}{c}\text { Transportes } \\
\text { ecológicos }\end{array}$ \\
\hline Comportamento pró-ambiental & 1 & 1 & \\
Consciência ambiental & $.278^{* *}$ & $.125^{*}$ & 1 \\
Transportes ecológicos & -.015 & \\
\hline
\end{tabular}

Nota. * A correlação é significativa no nível .05.; ** A correlação é significativa no nível 01.

\section{DISCUSSÃO}

A presente investigação foi concretizada com recurso à internet com o propósito de atingir um elevado número de participantes, resultando numa amostra de 300 participantes. 0 objetivo foi compreender os comportamentos pró-ambientais, como também as suas relações com o sexo, idade e nível de educação, através da criação de uma escala.

Em relação à hipótese "o sexo feminino apresenta maior comportamento ambiental" esta não se confirmou. Os dados não mostraram diferenças significativas em relação ao género e ao comportamento pró-ambiental (Lindner Radons, 2016).

No que concerne à hipótese "quanto maior a idade maior o comportamento pró-ambiental", os dados da amostra tendem a não confirmar a hipótese. São os indivíduos mais novos que utilizam mais os transportes ecológicos. Estes dados vão ao encontro do estudo de Garlet (2019).

Em relação à hipótese "o nível de educação mais elevado relaciona-se com um maior comportamento ambiental”, verificou-se, na amostra, uma correlação positiva e significativa, encontrando algum suporte. Os indivíduos com habilitações literárias mais elevadas apresentam moderadamente comportamentos mais favoráveis para com o ambiente (Xiao \& McCright, 2014). 
Nesta amostra existe uma tendência para que a consciência ambiental esteja positivamente e significativamente relacionada com comportamento pró-ambiental, sendo que existe alguma evidência que suporta a hipótese Nsiku-Lutete (2017) e Zhao et al. (2014).

\section{CONCLUSÃO}

Este estudo permitiu analisar, através da amostra, o comportamento pró-ambiental, a consciência e o uso de transportes ecológicos em relação às variáveis sexo, idade e educação. Os dados obtidos suportaram a literatura entre a variável consciência e comportamento pró-ambiental.

A amostra indica que existe alguma tendência para que o nível elevado de educação se correlacione positivamente com o comportamento pró-ambiental. Neste contexto, foram os indivíduos mais novos que apontaram uma média significativa superior na utilização dos transportes ecológicos, em comparação com os mais velhos. No entanto, não foram encontradas diferenças entre os sexos.

Este estudo apresenta limitações ao nível da simetria em relação às variáveis sexo, idade e educação. Tratou-se de um estudo exploratória para a criação de uma escala, aferindo valores razoáveis no teste $K M O$ e Bartlett de $.70(p<.001)$. No entanto, consideramos que é necessário efetuar algumas melhorias psicométricas no mesmo. Por outro lado, deparou-se com algumas fragilidades na construção dos itens, sendo que se pretende melhorar a sua construção em estudos futuros.

\section{REFERÊNCIAS}

Banerjee, R. (2015). Importance of Recycling. International Journal of Innovative Research in Electrical, Electronics, Instrumentation and Control Engineering, 3, 2321-5526.

Benedek, A., \& Takácsné György, K. (2013). A study of the factors influencing the environmental consciousness of consumers. Annals of the polish association of agricultural and agribusiness economists, 15(5), 15-19.

Boztepe, A. (2012). Green Marketing and Its Impact on Consumer Buying Behavior. European Journal of Economic and Political Studies, 2(12), 61-64.

Branco, A. A. (2005). Programas de monitorização ambiental da ETRS da Meia Serra. Valor Ambiente.

Čater, B., \& Serafimova, J. (2019). The Influence of Socio-Demographic Characteristics on Environmental Concern and Ecologically Conscious Consumer Behaviour among Macedonian Consumers. Economic and Business Review for Central and South-Eastern Europe, 21(2), 213-342. https://doi.org/10.15458/ebr.84

Chang, W. W. (2017). World trade and the environment: Issues and policies: World trade and the environment. Pacific Economic Review, 22(3), 435-479. https://doi.org/10.1111/14680106.12100

Charles, S. T., \& Piazza, J. R. (2009). Age Differences in Affective Well-Being: Context Matters. Social and Personality Psychology Compass, 3(5), 711-724. https://doi.org/10.1111/j.17519004.2009.00202.x

CITCEM (2017). Transformações Ambientais-CITCEM. https://citcem.org/linhas-deinvestigacao/transformacoes-ambientais

Feio, M. (1983). Le Bas Alentejo et l'Algarve (1949). Centro de Ecologia Aplicada da Universidade de Évora, INIC.

Garlet, V., Favarin, R. R., Beuron, T. A., Madruga, L. R. da R. G., Terra, C. C. de F., \& Borges, G. da R. (2019). Correlações entre Comportamentos e Competências: a busca de uma Universidade verde. Gestão.Org, 17(1), 73-87. https://doi.org/10.21714/1679-18272019v17Ed.p73-87

Guimarães, P. E., \& Amorim, I. (2016). A História Ambiental em Portugal: A emergência de um novo campo historiográfico. AREAS Revista Internacional de Ciencias Sociales, 35, 47-58.

IPSS, I. P. (2014). Climate Change 2014: Synthesis report. Contribution of working groups I, II and III to the fifth assessment report of the intergovernmental panel on climate change. IPCC.

Levitus, S., \& Antonov, J. I. (2017). Climate Data Record (CDR) Program Climate Algorithm Theoretical Basis Document (C-ATBD) Ocean Heat Content CDR [Data set]. NOAA National Centers for Environmental Information. https://doi.org/10.7289/V53F4MVP

Lindner Radons, D. F. (2016). Geração e gênero como moderadores no comportamento de compra próambiental. Pensamiento \& Gestión, 41, 148-173. https://doi.org/10.14482/pege.41.9707

Liu, X., Vedlitz, A., \& Shi, L. (2014). Examining the Determinants of Public Environmental Concern: Evidence from National Public Surveys. Environmental Science \& Policy, 39, 77-94. https://doi.org/10.1016/j.envsci.2014.02.006

NASA. (2019). Global Climate Change. Obtido de NASA: https://climate.nasa.gov/evidence/

Neto, M. (2013). Problemática do Saber Histórico - Guia de estudo. Palimage.

Ribeiro, O. (1993). Portugal. O Mediterrâneo e o Atlântico (1945). Edições Sá da Costa. 
Santos, J. e. (2015). New insights into the reconstructed tempera- ture in Portugal over the last 400 years. Climate of the Past, 11, 825-834. https://doi.org/10.5194/cp-11-825-2015

Shen, J., \& Saijo, T. (2008). Reexamining the relations between socio-demographic characteristics and individual environmental concern: Evidence from Shanghai data. Journal of Environment Psychology, 28(1), 42-50. https://doi.org/10.1016/j.jenvp.2007.10.003

Vasiljevic-Shikaleska, A., Trpovski, G., \& Gjozinska, B. (2018). Environmental Awareness And Of ProEnvironmental Consumer Behavior. Journal Of Sustainable Development, 8(20), 4-17.

Xiao, C., \& McCright, A. (2014). A Test of the Biographical Availability Argument for Gender Differences in Environmental Behaviors. Environment and Behavior, 46(2), 241-263. https://doi.org/10.1177/0013916512453991

Wiernik, B., Ones, D., \& Dilchert, S. (2013). Age and environmental sustainability: A meta-analysis. Journal of Managerial Psychology, 28(7/8), 826-856. https://doi.org/10.1108/JMP-07-2013-0221

Historial do artigo

Recebido $\quad 07 / 2019$

Aceite $\quad 03 / 2020$

Publicado $\quad 08 / 2020$ 
Revista PSICOLOGIA, 2020, Vol. 34 (1), doi: 10.17575/psicologia.v34i1.1681.

Atas do X Simpósio Nacional de Investigação em Psicologia

\title{
Possibilidades e desafios inclusivos educacionais: A realidade de Instituições de Ensino Superior Moçambicanas
}

\author{
Evelyn M. Fortes dos Santos ${ }^{31 i}$, Dayse Neri de Souza ${ }^{\mathrm{i}, \mathrm{ii}}$, Paula Vagos ${ }^{\mathrm{iii}}$ \& Bianca Gerente Biv $^{\mathrm{i}}$ \\ iCIDTFF, Universidade de Aveiro \\ ii Centro Universitário Adventista de São Paulo - UNASP-EC \\ iii Instituto de Desenvolvimento Humano Portucalense, Universidade Portucalense \\ iv Faculdade de Gestão de Turismo e Informática da Universidade Católica de Moçambique
}

\begin{abstract}
Resumo: A busca pela promoção de conceções e práticas inclusivas é determinante para a constatação da equidade de oportunidades, acesso à informação, garantia dos direitos e educação das pessoas com Necessidades Educativas Especiais. Independentemente do contexto geográfico ou cultural, compreendese que algumas estratégias de apoio são transversais, tais como as acessibilidades culturais/atitudinais, físicas/estruturais, materiais/digitais, as políticas educativas e o suporte familiar para o ajustamento destes estudantes, na trajetória de acesso, ingresso e permanência no Ensino Superior. Contemplando esta realidade, o presente estudo, de natureza qualitativa, objetivou percecionar, através do recurso de um inquérito online, as vivências de possibilidades e desafios inclusivos, descritos por 10 colaboradores de Instituições de Ensino Superior Moçambicanas, públicas e privadas. Os dados foram tratados com apoio do software webQDA. Concluiu-se que, apesar das necessidades verificadas, muitas estratégias já têm sido implementadas e, apesar dos desafios, há inclusão.
\end{abstract}

\section{Palavras-chave: Inclusão; Ensino superior; Necessidades educativas especiais; Moçambique.}

Inclusive educational possibilities and challenges: the reality of Mozambican Higher Education Institutions: The search for promotion of inclusive conceptions and practices is crucial for the realization of equity of opportunity, access to information, guarantee of rights and education of people with Special Educational Needs. Regardless of geographical or cultural context, it is understood that some support strategies are transversal, such as cultural/attitudinal, physical/structural and material/digital accessibilities; educational policies and family support for the adjustment of these students in the path of access, admission and stay in Higher Education. Contemplating this reality, this qualitative study aimed to perceive, through an online survey, the experiences of inclusive possibilities and challenges, described by 10 representatives of Mozambican Higher Education Institutions, public and private. Data were processed with the support of webQDA software. It was concluded that, despite the verified needs, many strategies have already been implemented and despite the challenges, there is inclusion

Keywords: Inclusion; Higher education; Special educational needs; Mozambique.

O conceito de inclusão propõe um olhar atento às necessidades específicas das pessoas, a partir das suas peculiaridades e particularidades, no contexto em que estão inseridas, no país em que se encontram e na Instituição de Ensino Superior (IES) que as recebe, "a fim de torná-la acolhedora para todas as pessoas, especialmente aquelas em desvantagem por suas deficiências" (Nhemachena et al., 2012, p. 74) . Tendo em consideração este panorama de significâncias, tem-se verificado que as práticas inclusivas modificam estruturas, suportes materiais e digitais, currículos e até mesmo acessibilidades culturais/atitudinais, de forma a promover a equidade de acesso à informação, educação e a igualdade de oportunidades (Santos et al., 2015). Contudo, a realidade dos desafios inclusivos é mais pronunciada nos países em desenvolvimento, nos quais se verifica um apoio ainda limitado, falta de formação docente e atitudes negativas em relação às pessoas com deficiência (Nhemachena et al., 2012), de entre outros fatores. No entanto, esta prática se ressignifica de acordo com um determinado contexto, seja familiar, regional, nacional, escolar ou universitário, com as necessidades que são encontradas, as potencialidades que são conhecidas e com as especificidades de cada pessoa (Santos et al., 2017).

\footnotetext{
31 Morada para correspondência: Evelyn dos Santos, Universidade de Aveiro - Departamento de Educação e Psicologia, 3810-193, Aveiro Portugal. E-mail: evelynsantos@ua.pt. Este trabalho foi financiado por fundos nacionais através da FCT, I.P., no âmbito do projeto UID/CED/00194/2019, com a referência SFRH/BD/124543/2016.
} 
A Convenção das Nações Unidas (ONU), preconiza a salvaguarda dos direitos humanos das pessoas com deficiência como um dever da sociedade, visando assegurar o respeito pela integridade, dignidade e liberdade individual das pessoas com deficiência ou incapacidade. Moçambique, um país marcado pela exclusão social e pobreza, "realça as dificuldades enfrentadas em pleno século XXI, no que se refere à disponibilidade e acesso de recursos básicos, como educação, saúde, emprego, proteção social" (Maleane \& Suaiden, 2010). Entretanto, é signatário das convenções e acordos da ONU acima mencionados. 0 governo baseia a inclusão na sua política nacional, salientando que deve haver educação para todos sem discriminação (Nhemachena et al., 2012).

\section{MÉTODO}

Este estudo, de cariz qualitativo, debruça-se na prerrogativa de proporcionar uma maior aproximação a um novo contexto, objetivando percecionar a realidade de possibilidades e desafios inclusivos, descritos por colaboradores de Instituições de Ensino Superior (IES) moçambicanas, indagados por meio de um inquérito online ${ }^{32}(\mathrm{IO})$, favorecendo a recolha de dados à distância. Estas opções fazem-nos buscar descrições detalhadas de situações, pessoas e interações, averiguando o que faz sentido na realidade e como faz sentido para os sujeitos investigados (Amado, 2013).

A elaboração do IO foi ancorada pela bibliografia científica, sendo composto por questões fechadas, abertas e escala de likert, centrando-se na caracterização pessoal, profissional e sociodemográfica e nas principais problemáticas inclusivas. As questões do IO foram sujeitas a uma validação interna, junto das orientadoras científicas do estudo, e a uma validação externa, junto de dois colegas investigadores.

Para a constituição da recolha de dados procedeu-se com a criação de uma base de contactos das IES. Os dados foram obtidos no site do Ministério da Educação Moçambicano, e através de outras redes interligadas com o Ensino Superior em Moçambique. A recolha de dados seguiu-se com o envio do link do IO, por e-mail, aos 90 contactos conseguidos, junto da apresentação do estudo, consentimento livre e esclarecido e termos éticos, assegurando o anonimato e a utilização dos dados para fins únicos desta investigação.

Dos noventa contactos levantados ${ }^{33}$, foram recebidas 10 respostas. 0 tratamento dos dados do Inquérito Online foi abarcado pela técnica de análise de conteúdo, com apoio do software de análise qualitativa webQDA e a análise estatística descritiva teve apoio do pacote estatístico SPSS, versão $26^{34}$.

\section{Contexto e participantes}

Moçambique é um país com cerca de 29,67 milhões de pessoas, situado no sudeste do Continente Africano, em processo de reconstrução pós-guerras, com uma história cercada por lutas, sofrimento e desigualdades. 0 povo moçambicano é marcado pela pobreza, pela discrepância de oportunidades entre os diferentes géneros (Nhemachena et al., 2012), pela falta de acessibilidades estruturais, digitais, materiais (Joanguete, 2011) e de equidade das pessoas com NEE. Entretanto, as práticas inclusivas no Ensino Superior tornaram-se uma questão atual nos debates sobre o continente africano, abraçando a filosofia Ubuntu ${ }^{35}$, para a redução das desigualdades, também no campo da inclusão (Shanyanana, 2016).

Os dados Error! Reference source not found. apresentam o perfil sociodemográfico dos participantes e enquadram a realidade das problemáticas inerentes à trajetória inclusiva vivenciada em IES moçambicanas.

\footnotetext{
320 Inquérito Online utilizado é composto, tal como descrito na Figura 1, por questões fechadas, abertas e escala de likert. No entanto, contemplando os objetivos do presente estudo, ressalta-se que as análises realizadas neste documento se centraram somente nos dados provenientes das questões fechadas, que correspondiam a caracterização dos participantes, bem como das questões abertas.

33 Ressalta-se que os contactos das IES moçambicanas não foram facilmente encontrados. A busca foi realizada, num primeiro momento, nos sites e, posteriormente, em redes sociais como o Facebook. Acredita-se que algumas IES não tenham respondido pela falta de informação do contacto atualizado nas páginas institucionais. Reitera-se ainda que dos 90 e-mail enviados, cerca de 30 retornaram como "não enviados".

${ }^{34}$ Apesar da estruturação do instrumento (perguntas fechas e escala de likert), as respostas foram analisadas estritamente numa perspetiva qualitativa.

${ }^{35}$ Ubuntu: conceito africano que significa acolhimento, respeito, entreajuda, partilha, comunidade, cuidado, confiança e generosidade "eu sou porque tu és. Eu só posso ser pessoa através das outras pessoas". (Nelson Mandela)
} 
Tabela 1. Caracterização dos participantes

\begin{tabular}{|c|c|c|}
\hline CARACTERIZAÇÃO & $\mathbf{N}$ & $\%$ \\
\hline \multicolumn{3}{|l|}{ Sexo } \\
\hline Masculino & 7 & 70,0 \\
\hline Feminino & 3 & 30,0 \\
\hline Total & 10 & 100,0 \\
\hline \multicolumn{3}{|l|}{ Idade } \\
\hline $20-29$ & 1 & 10,0 \\
\hline $30-39$ & 6 & 60,0 \\
\hline $40-49$ & 1 & 10,0 \\
\hline $50-59$ & 2 & 20,0 \\
\hline Total & 10 & 100,0 \\
\hline \multicolumn{3}{|l|}{ Função que exerce na IES } \\
\hline Diretor da Instituição de Ensino Superior & 4 & 40,0 \\
\hline Documentalista & 1 & 10,0 \\
\hline Docente & 2 & 20,0 \\
\hline Diretor de Departamento & 3 & 30,0 \\
\hline Total & 10 & 100,0 \\
\hline \multicolumn{3}{|l|}{ Há quantos anos exerce a função } \\
\hline Menos de 1 ano & 1 & 10,0 \\
\hline de 1 a 5 anos & 4 & 40,0 \\
\hline de 5 a 10 anos & 5 & 50,0 \\
\hline Total & 10 & 100,0 \\
\hline \multicolumn{3}{|c|}{ Qual o regime da sua Instituição de Ensino Superior? } \\
\hline Pública & 2 & 20,0 \\
\hline Privada & 8 & 80,0 \\
\hline Total & 10 & 100,0 \\
\hline \multicolumn{3}{|l|}{ A sua IES situa-se em que parte do país? } \\
\hline Norte & 3 & 30,0 \\
\hline Sul & 4 & 40,0 \\
\hline Centro & 3 & 30,0 \\
\hline Total & 10 & 100,0 \\
\hline
\end{tabular}

As IES participantes representam as províncias de Niassa, Sofala, Maputo, Cabo Delgado, Inhambane e Nampula, de entre as onze que compõe Moçambique (ver Error! Reference source not found.1), entre públicas e privadas. Na sua maioria (60\%), os colaboradores respondentes possuem experiência no âmbito da inclusão, visto que estão vivendo uma época de maior atenção às ações afirmativas para os grupos minoritários, e as temáticas das acessibilidades e da equidade constituem este debate, pois a reflexão em prol da inclusão possibilita "um conjunto de reestruturações tanto no âmbito da formação como da educação dos alunos, o que poderá permitir trazer algumas alterações que devem pressupor uma escola inclusiva e para todos" (Terenciano \& Wamusse, 2018, p. 72). 


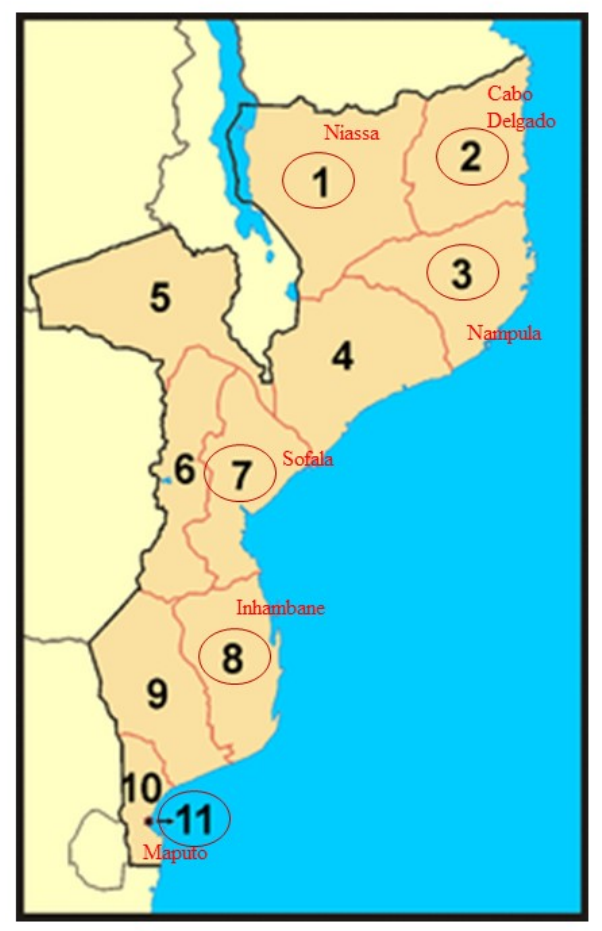

Figura 1. Regiões das IES participantes (Fonte: Google imagens, com modificações da autora).

\section{RESULTADOS E DISCUSSÃO}

A partir dos objetivos traçados para o estudo, os dados provenientes das questões abertas foram elencados em duas grandes dimensões de análise: i) possibilidades inclusivas e ii) desafios inclusivos das IES. A Error! Reference source not found.2, permite retratar estas duas dimensões, bem como descrever as categorias e subcategorias que emergiram após a análise dos dados. Amado (2013, p. 313) revela que "o primeiro grande objetivo da análise de conteúdo é o de organizar os conteúdos de um conjunto de mensagens num sistema de categorias que traduzem as ideias-chave veiculadas pela documentação em análise".

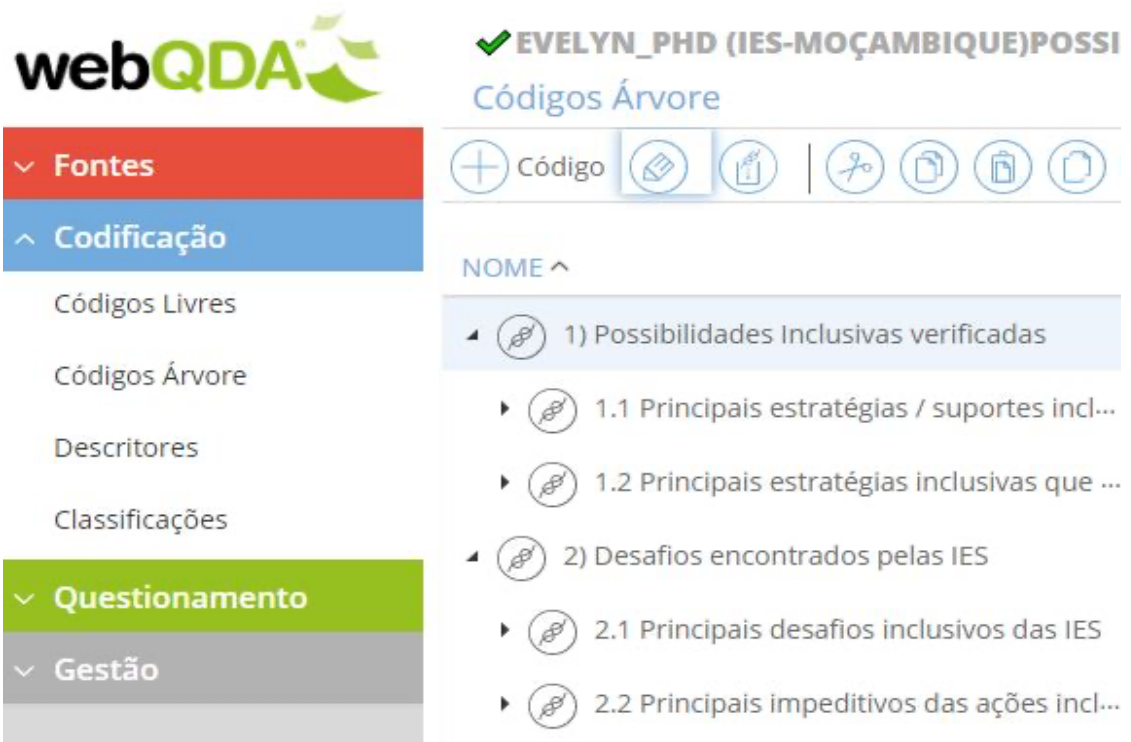

Figura 2. Árvore de categorias do estudo, Fonte: webQDA.

Os resultados serão apresentados por meio de tabelas que irão contemplar o número de referências/unidades de texto a cada uma das categorias e subcategorias descritas, realçando as mais 
referenciadas. Os elementos de destaque serão exemplificados com excertos dos discursos dos participantes, a partir de uma identificação própria, garantindo o anonimato dos sujeitos.

Os dados da Tabela 2 revelam a falta de qualificação dos profissionais em IES, visto que nenhum participante possuía formação especializada até o momento do estudo. Estes dados vieram reiterar a preocupação de investigadores moçambicanos (Nhemachena et al., 2012; Terenciano \& Wamusse, 2018) ao se depararem com a realidade da falta de preparo docente. Nesta perspetiva, Terenciano \& Wamusse (2018) afirmam que é necessário ajustar-se às necessidades, investindo na formação dos professores, de forma a que possam desenvolver cada vez mais as suas competências para a atuação nos espaços educacionais inclusivos, pois, segundo os autores, os professores com maior informação e formação possuem mais zelo no trabalho com os estudantes com NEE.

Tabela 2. Caracterização das experiências inclusivas

\begin{tabular}{lcc}
\hline \multicolumn{1}{c}{ EXPERIÊNCIAS INCLUSIVAS } & N & $\%$ \\
\hline Possui experiência no âmbito da Inclusão de estudantes com NEE? & 60,0 \\
Sim & 6 & 40,0 \\
Não & 4 & 100,0 \\
Total & 10 & 100,0 \\
Possui formação especializada na área das NEE e/ou inclusão? & 10 & \\
Não & 10 & \\
\hline
\end{tabular}

\section{Dimensão de análise 1 - Possibilidades inclusivas}

De entre a dimensão "Possibilidades Inclusivas", buscou-se conhecer os suportes que eram verificados pelas IES participantes, bem como elencar as estratégias que poderiam ser implementadas, de acordo com a realidade local. Os resultados apresentados na Error! Reference source not found., permitiram a visualização das possibilidades verificadas. De entre a categoria principais "estratégias/suportes inclusivos", com 33 referências, estão o "suporte familiar" (16 referências), seja no âmbito da valorização, motivação, incentivo para iniciar e dar continuidade aos estudos ou numa dimensão de encorajamento, motivação, apoio e incentivo das capacidades pessoais e sociais. Segundo os participantes:

...acima de tudo, estudantes com NEE precisam ser encorajadas para fazer o que devem fazer e isso parte de onde ele tem passado mais tempo, que é no leito familiar (I4).

Cada vez mais tem sido discutida e evidenciada a importância da relação entre família e escola para o ajustamento e o sucesso académicos dos estudantes. Contudo, essa realidade ainda é pouco referenciada no âmbito do Ensino Superior, mas os resultados nos levam a crer que esta participação pode ser, de igual modo, fundamental, como nos demais ciclos de ensino (Santos et al., 2017).

Na perspetiva de um participante, as políticas nacionais têm favorecido o suporte inclusivo. No entanto, não parecem colmatar as demandas de necessidades apresentadas. Desta forma, de entre as principais estratégias inclusivas que poderiam ser implementadas, a referência às "políticas nacionais para a inclusão" volta a ser visualizada a partir de 6 unidades de texto. "As políticas de educação inclusiva" constituem um exemplo do modo como as tendências e os traços da sociedade criam condições específicas de funcionamento dos sistemas de educação" (Terenciano \& Wamusse, 2018, p. 73). 
Tabela 3. Apresentação dos resultados da Dimensão de análise 1

\begin{tabular}{|c|c|}
\hline DIMENSÃO 1: POSSIBILIDADES INCLUSIVAS & Refs \\
\hline \multicolumn{2}{|l|}{ Subcategorias: } \\
\hline Categoria 1.1 Principais estratégias / suportes inclusivos verificados & 33 \\
\hline Formação da comunidade académica & 2 \\
\hline Acessibilidade cultural/atitudinal & 3 \\
\hline Acessibilidade física/estrutural & 5 \\
\hline Acessibilidade digital/material & 2 \\
\hline Recursos financeiros/ financiamento & 1 \\
\hline Acessibilidade académica / curricular & 2 \\
\hline Suporte familiar para os estudantes & 16 \\
\hline Políticas nacionais & 1 \\
\hline \multicolumn{2}{|l|}{ Categoria 1.2 Principais estratégias inclusivas que poderiam ser } \\
\hline implementadas & 18 \\
\hline Acessibilidade Física/estrutural & 4 \\
\hline Acessibilidade Cultural/Atitudinal & 4 \\
\hline Acessibilidade Digital/Material & 2 \\
\hline Acessibilidade académica / curricular & 1 \\
\hline Formação para os professores / comunidade académica & 1 \\
\hline Políticas nacionais para a inclusão & 6 \\
\hline Total & 51 \\
\hline
\end{tabular}

\section{Dimensão de análise 2 - Desafios inclusivos das IES}

Os resultados apresentados na Error! Reference source not found., evidenciam a dimensão de análise "Desafios inclusivos das IES" e revelam, de entre as suas categorias, os principais desafios inclusivos encontrados, bem como os fatores que são considerados impeditivos para as ações inclusivas.

Os dados apontam, mais uma vez, para a importância do "suporte familiar" e a "formação da comunidade académica", bem como a "falta de atenção e/ou iniciativas formativas por parte das IES". Neste âmbito, Terenciano e Wamusse (2018) afirmam que "é preciso que os professores, corpo técnico, e os demais entes que compõem o corpo educativo, sejam formados realmente” (p. 80). Esta atenção, informação e formação não deve ser simplesmente centrada nos docentes, importante, mas não só. A comunidade educativa precisa, como um todo, estar informada, consciencializada e formada, pois a perspetiva da inclusão em países em desenvolvimento, como é o caso de Moçambique, por vezes torna-se árdua e desafiadora em relação a equidade de direitos e oportunidades das pessoas com Necessidades Especiais, em todos os ciclos de ensino (Nhemachena et al., 2012).

Com pouco ou nenhuma informação aos familiares, estes por vezes sentem-se inseguros e acabam apresentando dificuldades em apoiar e incentivar a transição dos estudantes com NEE para o Ensino Superior (Santos et al., 2017). Ainda neste domínio, evidencia-se o impacto da NEE na vida familiar, de entre os mitos, os receios e a exclusão vivenciada que, por vezes, são o reflexo desta falta de ajustamento. Na perspetiva do participante I3:

O reconhecimento de pessoas NEE é um assunto delicado e mal visto no seio da sociedade moçambicana, com forte impacto na vida de pessoas com NEE e das suas famílias.

Entre os principais impeditivos das ações inclusivas nas IES estão a "relutância com a inovação" (3 referências) e a "falta de políticas nacionais" (3 referências) visto que:

A instituição aqui é a menor do mundo. As pessoas ainda não estão prontas para inovações. Vivem num "mesmismo" de antigamente (I9). 
Tabela 4. Apresentação dos resultados da Dimensão de análise 2

\begin{tabular}{lc}
\hline DIMENSÃO 1: POSSIBILIDADES INCLUSIVAS & Refs \\
\hline Subcategorias: & 23 \\
Categoria 2.1 Principais desafios inclusivos das IES & 4 \\
Acessibilidade Física/Estrutural & 5 \\
Formação da comunidade académica & 4 \\
Acessibilidade/Digital Material & 1 \\
Acessibilidade Cultural/Atitudinal & 2 \\
Acessibilidade académica / curricular & 1 \\
Recursos financeiros/ financiamento & 6 \\
Falta / pouco suporte familiar & 3 \\
Categoria 2.2 Principais impeditivos das ações inclusivas nas IES & 7 \\
Falta de vontade da IES & 1 \\
Relutância com a inovação & 3 \\
Falta de políticas nacionais & 3 \\
Total & 30 \\
\hline
\end{tabular}

No âmbito das "políticas nacionais", verifica-se que, por vezes, tornam-se "impeditivas" ou não são percebidas. Contudo, são de suma importância para que se "assegurem as oportunidades, mas também que essas possam garantir o suporte financeiro, essencial para os estudantes com NEE que necessitam, pois financeiramente desprotegidos por um conjunto de circunstâncias, por vezes não poderão finalizar o seu percurso académico" (Santos, 2014, p. 71).

\section{CONCLUSÃO}

A partir das presentes (in)conclusões, tencionou-se dar a conhecer um pouco da realidade vivenciada por colaboradores de IES, de forma a descrever as condições que as IES moçambicanas apresentam para que os estudantes possam apropriar-se do que lhes é oferecido, buscando um espaço para reflexão e conhecimento das possibilidades e dos desafios, mas que, acima de tudo, consagre e reverta essas informações em práticas e espaços de equidade. Verifica-se a realidade da inclusão no Ensino Superior como algo acessível, apesar dos desafios, por isso é importante que os estudantes com NEE, as suas famílias e toda a comunidade académica, perceba as possibilidades e conheça os suportes em prol da inclusão no Ensino Superior moçambicano.

No âmbito das 51 referências que adentraram a dimensão "Possibilidades Inclusivas", os resultados evidenciaram que, de entre as principais estratégias/suportes inclusivos, estão o "suporte familiar" e as "acessibilidades físicas/estruturais". A partir do "suporte familiar", os participantes revelaram que as famílias demonstram iniciativas de valorização e estímulo em acreditar que os estudantes com NEE podem estudar, formar-se, ter um emprego e serem pessoas realizadas, desenvolvendo as suas habilidades e competências. Na realidade das "acessibilidades físicas/estruturais" as adaptações centram-se principalmente nos acessos, visto que a autonomia do estudante e a liberdade de ir e vir é um dos principais direitos que estes jovens tem e/ou buscam ter.

De entre as 18 referências sobre as principais estratégias inclusivas que poderiam ser implementadas, são destacadas 6 unidades de texto reafirmando a importância do investimento nas "políticas nacionais de apoio à inclusão", visto que na perspetiva dos colaboradores as políticas educativas são as que conduzem o destino da educação de um país, criando condições para potenciar a inclusão e a permanência de estudantes com NEE no Ensino Superior.

No que toca às 30 referências integradas na dimensão "Desafios inclusivos das IES", os "principais desafios" centraram-se na promoção da "formação da comunidade académica" para trabalhar com os estudantes com NEE. Por um lado, entre a limitação advinda da formação inicial, por outro, devido a falta de recursos humanos qualificados para a formação contínua nesta área, junto da descrição de "falta / pouco suporte familiar". Segundo os participantes, um dos "impeditivos de ações inclusivas na IES" é a 
"relutância com a inovação", que é corroborado com a perceção de que as "políticas educativas do país podem ser potenciadoras" para a inclusão no Ensino Superior. Contudo, tornam-se por vezes "impeditivas", no sentido de que não investem recursos necessários e não garantem as condições básicas de acesso e permanência de estudantes com NEE no Ensino Superior.

Centrados nestas (in)conclusões, acreditamos que os colaboradores das IES moçambicanas, a partir de suas vivências, proporcionam a oportunidade da comunidade académica vir a refletir sobre a inclusão, na sua amplitude, como uma envolvência educacional, social e ambiental. Desta forma, passamos a olhar a realidade do outro numa forma mais abrangente, e conseguimos enxergar algumas das barreiras ambientais que impedem a participação plena de pessoas com NEE na sociedade. A partir deste estudo, verificou-se que tanto as possibilidades quanto os desafios possuem dimensões muito similares aos que encontramos atualmente em Portugal e que, de entre necessidades verificadas, muitas estratégias já têm sido implementadas e, apesar dos desafios, há inclusão.

\section{REFERÊNCIAS}

Amado, J. (2013). Manual de investigação qualitativa em educação. Imprensa da Universidade de Coimbra. Joanguete, C. (2011). Política pública moçambicana sobre a inclusao digital. Redmarka: Revista Digital de Marketing Aplicado, 3(7), 61-82. http://dialnet.unirioja.es/servlet/articulo?codigo=4126640

Maleane, S. O. T., \& Suaiden, E. J. (2010). Inclusão, exclusão social e pobreza em Moçambique em pleno século XXI. Inclusão Social, 4(1), 67-75. http://revista.ibict.br/inclusao/article/view/1645

Nhemachena, L., Kusangaya, S., \& Gwitira, I. (2012). An Evaluation of Inclusive Education of Students with Visual Impairment in Schools and University in Beira, Mozambique. Journal of the American Academy of Special Education Professionals, 76-98. https://eric.ed.gov/?id=EJ1135515

Santos, E. (2014). Ingresso e permanência de estudantes com NEE no ensino superior: um estudo qualitativo [Dissertação de mestrado, Universidade de Aveiro]. http://hdl.handle.net/10773/14358

Santos, E., Gonçalves, M., Ramos, I., Castro, L., \& Lomeo, R. (2015). Inclusão no Ensino Superior: Perceções dos estudantes com Necessidades Educativas Especiais sobre o ingresso à universidade. Revista Portuguesa de Educação, 28(2), 251. https://doi.org/10.21814/rpe.7741

Santos, E., Vagos, P., \& Neri de Souza, D. (2017). Inclusão de estudantes com NEE no ensino superior português: reflexões teóricas dos principais suportes e fragilidades. In E. T. D. M Dias, L. M. V. Kim, \& M. M. Baptista (Ed.), Polifonias Psicológicas e educacionais (pp. 181-205). Paco editorial.

Shanyanana, R. N. (2016). Reconceptualizing Ubuntu as inclusion in African higher education. Towards Equalization of Voice, 4(4), 104-120. https://www.researchgate.net/publication/325429712

Terenciano, F., \& Wamusse, B. G. (2018). A percepção dos professores primários sobre a educação inclusiva na cidade de Pemba-Moçambique. Revista Educação, 13(1), 69-83. http://revistas.ung.br/index.php/educacao/article/view/3131 
Revista PSICOLOGIA, 2020, Vol. 34 (1), doi: 10.17575/psicologia.v34i1.1681.

Atas do X Simpósio Nacional de Investigação em Psicologia

\title{
Implantação do programa de orientação profissional para estudantes universitários: Relato de experiência de intervenção em contexto de estágio
}

\author{
Maria Cristina Pinto Gattai $36 i$ \\ iFaculdade de Ciências Humanas e da Saúde, Curso de Psicologia, Pontifícia Universidade de São Paulo - \\ PUCSP, São Paulo, Brasil
}

\begin{abstract}
Resumo: Este artigo tem como objetivo relatar a experiência de implantação de um programa de intervenção em orientação profissional em contexto de estágio para o Núcleo de Psicologia Organizacional, Supervisão de Estágio, oferecido aos alunos do último ano do curso de psicologia da Pontifícia Universidade Católica de São Paulo. O serviço, denominado Delineamento Individual de Carreira (DICA), tem por objetivo ajudar o indivíduo a obter, organizar e utilizar informações objetivas a respeito de si e do mundo do trabalho, ampliando seu índice de empregabilidade, através de três pilares de atendimento: autoconhecimento (identificando as potencialidades, fragilidades, valores, interesses e habilidades, através da avaliação psicológica e outras ferramentas), trilhas de carreira (promovendo a reflexão sobre perspetivas para o futuro e traçando estratégias para seu atingimento) e preparação para processo seletivo (através de ações como elaboração de currículo e simulações de entrevistas e dinâmicas de grupo). 0 serviço iniciou em abril de 2018 tendo atendido 14 orientandos de um total de 73 alunos inscritos. As principais demandas foram a busca pelo processo seletivo; autoconhecimento e trilhas de carreira. As sessões de orientação tinham duração de uma hora e variaram de um a cinco encontros semanais. De forma geral todos os orientandos ficaram satisfeitos com o serviço oferecido. Para os dez orientandos que buscavam uma inserção no mercado de trabalho, a maioria afirmou sentir-se mais seguro para participar de processos seletivos sendo que seis deles (60\%) conquistaram seu primeiro estágio remunerado atribuindo o sucesso obtido ao programa de $\mathrm{OP}$, principalmente por terem maior segurança nas entrevistas e nas dinâmicas de grupo alegando que algumas situações lembravam muito as proporcionadas pelo DICA.
\end{abstract}

Palavras-chave: Orientação profissional; Carreira; Avaliação psicológica; Autoconhecimento.

Implementation of the professional guidance program for university students: Report of intervention experience in the context of internship: This article aims to report the experience of implementing an intervention program in professional guidance in the context of internship for the Organizational Psychology Center, Internship Supervision, offered to students of the last year of the course of psychology of the Pontifical Catholic University of São Paulo. The service, called Individual Career Design (DICA), aims to help the individual obtain, organize and use objective information about themselves and the world of work, expanding their employability index, through three pillars of care: selfknowledge (identifying potentialities, weaknesses, values, interests and skills, through psychological evaluation and other tools), career trails (promoting reflection on perspectives for the future and plotting strategies for its achievement) and preparation for the selection process (through actions such as curriculum elaboration and simulations of interviews and group dynamics). The service began in April 2018 having attended 14 guidance from a total of 73 students enrolled. The main demands were the search for the selection process; self-knowledge and career trails. The orientation sessions lasted one hour and ranged from one to five meetings per week. In general, all the guidance was satisfied with the service offered. For the ten guidance that sought an insertion in the labor market, the majority said they felt safer to participate in selection processes and six of them (60\%) they conquered their first paid internship by attributing the success obtained to the PO program mainly because they had greater security in interviews and group dynamics, claiming that some situations reminded a lot of those provided by DICA.

Keywords: Career orientation; Career; Psychological assessment; Self knowledge.

${ }^{36}$ Morada para correspondência: Maria C. P. Gattai, Rua Copacabana 457, aptoo 51B, 02461-000, São Paulo, Brasil. E-mail: crisgattai@uol.com.br 
Escolher uma profissão e decidir em qual área se especializar, nem sempre é uma tarefa fácil de ser superada sem o apoio de um processo de Orientação Vocacional e/ou Profissional.

Orientação Vocacional (OV) subtende que já existe uma vocação no orientando que precisa ser descoberta por intermédio de um orientador. Orientação Profissional (OP), considera o homem com maior autonomia para fazer suas opções profissionais (Moura, 2011).

A OV foi, por muito tempo, uma prática diretiva; o orientador, com base em ferramentas psicométricas, realizava diagnósticos e prognósticos indicando as profissões mais adequadas ao perfil do orientando. Mas, esse tipo de orientação começou a modificar-se com a publicação dos trabalhos de vários autores, como os de Carl Rogers, com a abordagem não diretiva nas práticas terapêuticas (Rogers, 1974); Donald Super, para o qual a escolha profissional é um processo que ocorre ao longo da vida, com escolhas e recolhas (Super, 1980); John Holland, cujos interesses profissionais são reflexos da personalidade do indivíduo determinando a direção da escolha profissional (Holland, 1977); Rudolf Bohoslavsky, com o método clínico de Orientação compreendendo o indivíduo como sujeito de escolhas (Lehman, Silva, Ribeiro \& Uvaldo, 2011).

Para Levenfus (2002), a OV informa o orientando sobre as profissões e mercado de trabalho, e a OP também assessora o orientando a conhecer suas características pessoais, familiares e sociais, visando o matching de suas afinidades com as peculiaridades de determinada profissão.

A OP orienta na escolha de uma profissão para que seja exercida com êxito e satisfação pessoal (Pieron, 1993). Auxilia na descoberta dos conhecimentos, interesses, motivações, habilidades e a identificar quais competências profissionais precisam desenvolver ou aprimorar para conferir maior qualidade de vida e felicidade em determinada profissão.

Entre os contextos em que pode ser conduzida, destaca-se o educacional voltado para o estudante universitário, focalizando uma ou mais profissões com as quais seu perfil mais se identifica, auxiliando-o na compreensão de suas limitações e na promoção de reajustes necessários (Rocha, 2002) visando o aprimoramento ou desenvolvimento de certas competências esperadas para a profissão.

Em OP, há diferentes abordagens psicológicas, como também grande diversidade de possibilidades interventivas, tanto nos referenciais metodológicos (método estatístico ou clínico), quanto nas etapas, quantidade e duração das seções além das ferramentas utilizadas.

No método estatístico, o orientando é considerado sem condições de decidir sobre sua carreira cabendo ao orientador o papel de investigar suas aptidões, habilidades e interesses objetivando identificar a carreira mais adequada ao seu perfil através de testes psicométricos.

No método clínico, o orientador assume o papel de esclarecer e informar as habilidades e motivações do orientando, instrumentalizando-o para uma decisão mais consciente, pela via do autoconhecimento e da articulação entre o universo subjetivo do orientando e os aspectos do mundo do trabalho (Sparta et al., 2006).

Não há consenso quanto às etapas de um processo de OP. Para Soares (1993), elas devem desenvolver-se em três etapas: autoconhecimento; conhecimento das profissões; escolha propriamente dita da profissão. Para Parsons (2005), também deve pautar-se em três etapas: análise das características do indivíduo (compreensão de si mesmo, suas aptidões, capacidades, interesses, ambições, recursos e limites); análise das características das ocupações (conhecimento dos requisitos e condições de sucesso, vantagens e desvantagens, remuneração, oportunidades e as perspetivas nos diferentes tipos de trabalho); cruzamento das informações. Para Lima (2007), o processo deve ter duas etapas. A primeira engloba os interesses, aptidões e características de personalidade do orientando que facilitam o autoconhecimento; a segunda se relaciona com o conhecimento do mercado de trabalho e com as oportunidades profissionais. Ao finalizar as duas etapas, o orientando estará apto a fazer sua escolha profissional.

De certa forma, a OP deve pautar-se na promoção do autoconhecimento, no fornecimento de informações sobre a profissão, conhecimento do mercado trabalho e no processo de tomada de decisão pelo orientando de forma mais madura e consciente. Apesar de não mencionada na literatura, é importante acrescentar a etapa de preparação do orientando para os processos seletivos para ingresso no mercado de trabalho.

Não há consenso em OP sobre a quantidade de sessões e sua duração. Müller (1998, citado por Moura, 2014) sugere quinze sessões (ver Tabela 1). 
Tabela 1. Sessões de OP segundo Müller

\begin{tabular}{ll}
\hline Sessões & Conteúdo \\
\hline Sessões 1 a 3 & Motivo da consulta, enquadre, contrato, primeiro diagnóstico. \\
Sessões 4 a 6 & Ajuste do primeiro diagnóstico; incorporação de técnicas auxiliares; prognóstico \\
Sessões 7 a 9 & Esclarecimento operativo acerca dos pontos de urgência detectados \\
Sessões 10 a 12 & Técnicas informativas \\
Sessões 12 a 15 & Continuação do esclarecimento, síntese e conclusões \\
\hline
\end{tabular}

Fonte: Adaptado de Müller (1988, p.34, citado por Moura, 2014, p. 51)

Barbosa (2012) sugere oito sessões, com duas horas de duração cada. Melo-Silva e Jacquemin (2001) indicam nove de duas horas cada.

A literatura também não indica consenso nas ferramentas utilizadas em $O P$, já que são determinadas em função das necessidades do orientando e dos objetivos a serem alcançados.

Quanto ao orientador, o seu papel não deve focar somente em informações a respeito das carreiras profissionais, mas sim estimular o autoconhecimento, identificar as competências e as motivações do orientando conduzindo-o a uma escolha mais consciente e madura considerando as várias profissões e as demandas do mercado de trabalho e prepará-lo para o enfrentamento mais assertivo nos processos seletivos para o ingresso na carreira.

\section{MÉTODO}

O estudo é descritivo, tipo relato de experiência, desenvolvido no contexto da disciplina Estágio Supervisionado em Psicologia Organizacional, como um novo campo de estágio para os alunos do último ano de graduação (nono e décimo períodos) do curso de Psicologia da PUC-SP, campus Monte Alegre que, diferentemente dos oito primeiros semestres do curso, tem a característica de ser anual e não semestral.

Participaram como orientadores os nove alunos do grupo de supervisão, orientado pela professora idealizadora do projeto.

A proposta interventiva se enquadra na modalidade clínica, no contexto educacional, com foco em estudantes universitários, utilizando-se de ferramentas como entrevistas, escuta qualificada, testes, reflexões sobre si mesmo, informações sobre o mercado de trabalho, influências na escolha da profissão, exploração sobre as especialidades oferecidas pelo curso superior escolhido e a diferença entre eles, o processo de tomada de decisão, o medo de uma decisão errada, habilidades, motivações e valores pessoais coerentes com a escolha profissional.

\section{Relato da experiência}

O serviço surgiu da parceria realizada entre o Núcleo de Psicologia Organizacional (NPO) e a Coordenadora Geral de Estágios (CGE), que efetua a gestão académico-administrativa da atividade de estágio na universidade e favorece o acesso dos estudantes no mercado de trabalho, para atender a demanda de alunos de diversos cursos da Pontifícia Universidade Católica de São Paulo (PUC-SP) com dúvidas sobre a escolha feita pelo curso de graduação ou em qual área de formação se especializar; com dificuldades de inserção no mercado de trabalho; dificuldades na elaboração de currículo; dificuldades em participar de forma mais assertiva das etapas de entrevista e de dinâmicas de grupo nos processos seletivos que se candidatavam; e dificuldades em serem inseridos no mercado de trabalho como estagiários.

O serviço foi estruturado, após revisão da literatura sobre o tema com o grupo de alunos do NPO. Para a divulgação do serviço, firmou-se parceria com a Agência de Publicidade da instituição para a criação da uma página do Facebook, e a divulgação do serviço no site institucional da universidade.

O programa, denominado Delineamento Individual de Carreira (DICA), foi organizado em até cinco sessões semanais com uma hora de duração cada, com etapas definidas de acordo com os objetivos/pilares escolhidos pelos orientandos:

- Autoconhecimento: identificar potencialidades, fragilidades, valores, interesses e habilidades, utilizando ferraments como entrevista, testes e exercícios psicológicos;

- Trilhas de Carreira: proporcionar a reflexão sobre perspectivas para o futuro e, traçar estratégias para seu atingimento através de ferramentas como análise do mercado de trabalho, de recursos humanos, estruturas organizacionais; entrevista com profissionais da área; 
- Processo Seletivo: preparar o orientando para participar de processos seletivos através da elaboração de currículo, simulações de entrevistas e dinâmicas de grupo.

Os atendimentos tiveram início em abril de 2018 após seu lançamento nas redes sociais da PUC-SP. 0 interessado encaminhava uma mensagem via inbox/messenger do Facebook. Uma mensagem automática era disparada solicitando o preenchimento de uma ficha de inscrição com a escolha do horario disponibilizado pelos orientadores. As sessões eram sigilosas, e sem caráter psicoterapêutico. Caso surgisse esse tipo de demanda, o orientando era instruído a buscar o serviço oferecido pela clínica escola oferecido pelo curso de psicologia da PUC-SP.

Quanto aos objetivos e ferramentas utilizadas nas sessões para os três pilares, tem-se :

- 1á sessão: com fins de diagnóstico sendo utilizadas entrevista semiestruturada buscando informações como: o curso escolhido pelo orientando; motivações para a escolha do curso; área de especialização que pretende seguir; conhecimento do mercado de trabalho; histórico profissional, expectativas em relação ao atendimento no DICA, agenda de atendimentos.

- 2a a 4⿳亠丷a sessões: ampliar o autoconhecimento e identificar planos para o futuro. Ferramentas: testes psicológicos; inventários; exercícios para autoconhecimento; entrevista semiestruturada; pesquisa sobre profissões de interesse; elaboração de currículo; entrevista com profissionais da área; dramatização de entrevistas de seleção e dinâmicas de grupo; reflexão sobre dúvidas e medos quanto ao processo de tomada de decisão; exercício de análise do critério de escolha.

- 5a sessão: análise sobre o processo de tomada de decisão; devolutiva e avaliação do programa de OP proporcionado.

\section{RESULTADOS}

Dos 73 inscritos no DICA, 17 (23.28\%) desistiram por incompatibilidade de horários e 42 (58.90\%) aguardaram em fila de espera para serem atendidos em 2019. Dos 14 (19.18\%) atendidos, 13 estudavam no campus Monte Alegre e 1 no campus Consolação (ver Tabela 2).

Tabela 2. Atendimentos em 2018

\begin{tabular}{lc}
\hline Descrição & Quantidade de alunos \\
\hline Busca por atendimento & 73 \\
Desistências & 17 \\
Pessoas atendidas & 14 \\
\hline
\end{tabular}

Apesar de apenas 73 alunos terem feito inscrição no DICA, foram feitas 126 curtidas de alunos na página do Facebook, revelando a importância do serviço oferecido pela NPO.

Os 14 orientandos atendidos eram provenientes de vários cursos sendo a maioria de Publicidade e Propaganda, seguido dos cursos de Direito e Administração (ver Tabela 3).

Tabela 3. Curso de origem

\begin{tabular}{lcc}
\hline Curso & Qtde de alunos & \% \\
\hline Publicidade e Propaganda & 04 & 28.57 \\
Direito & 03 & 21.43 \\
Administração & 03 & 21.43 \\
Engenharia de Produção & 02 & 14.29 \\
Letras & 01 & 7.14 \\
Artes do Corpo & 01 & 7.14 \\
Total & 14 & 100.00 \\
\hline
\end{tabular}

Os 14 orientandos eram bolsistas e concluíram o programa de OP; 7 alunos (50.00\%) optou pelo pilar processo seletivo; 4 (28.57\%) por autoconhecimento; 2 (14.29\%) pelos pilares autoconhecimento e processo seletivo; e 1 (7.14\%) por trilhas de carreira (ver Tabela 4). 
Tabela 4. Atendimentos por pilar

\begin{tabular}{lc}
\hline Pilares de atendimento & Quantidade de alunos \\
\hline Processo Seletivo & 7 \\
Autoconhecimento & 4 \\
Autoconhecimento e Processo Seletivo & 2 \\
Trilhas de Carreira & 1 \\
Total & 14 \\
\hline
\end{tabular}

A maioria dos orientandos realizou o programa em três ou quatro sessões alegando não possuírem disponibilidade de horário devido as obrigações do curso (seminários, TCCs, provas) necessitando do tempo para se prepararem (ver Tabela 5).

Tabela 5. Sessões por pilar

\begin{tabular}{lcccccc}
\hline Pilares de atendimento & \multicolumn{3}{c}{ Quantidade de Sessões } & \multicolumn{2}{c}{ Total } \\
\cline { 2 - 6 } & $\mathbf{1}$ & $\mathbf{2}$ & $\mathbf{3}$ & $\mathbf{4}$ & $\mathbf{5}$ & \\
\hline Processo Seletivo & 1 & - & 2 & 3 & 1 & 7 \\
Autoconhecimento & 2 & - & 1 & - & 1 & 4 \\
Autoconhecimento e Processo Seletivo & - & - & 1 & 1 & - & 2 \\
Trilhas de Carreira & - & 1 & - & - & - & 1 \\
Total & 3 & 1 & 4 & 4 & 2 & 14 \\
\hline
\end{tabular}

Fonte: elaborado pela autora

Baseando-se nos instrumentos utilizados e nas entrevistas conduzidas pelos orientadores, a maioria dos orientandos manteve ou acrescentou um novo interesse a partir das profissões escolhidas. Somente um aluno afirmou ter feito a escolha errada, pois o curso Artes do Corpo não possui muitas oportunidades de carreira, decidindo que faria outro curso como Comunicação ou Jornalismo.

Entre os que buscaram processo seletivo (10 alunos), a maioria (90\%) afirmou sentir-se mais seguro para participar de processos seletivos; $6(60 \%)$ conquistaram seu primeiro estágio graças à participação no DICA, por adquirirem maior segurança nas entrevistas e nas dinâmicas de grupo: "algumas situações dos processos seletivos que participei lembram as atividades vivenciadas no programa de OP" (conforme informações do sujeito - sic).

Afirmaram sentirem mais seguros diante da escolha do curso realizada, ou em descobrir um foco de maior interesse em uma área de atuação, que não haviam considerado antes. Mostraram-se satisfeitos com o programa de OP e com a forma conduzida, fazendo descobertas sobre si mesmos e aproveitando o que foi trabalhado e refletido com as ferramentas utilizadas. Fizeram elogios em relação às atividades desenvolvidas principalmente no pilar processo seletivo, pela característica mais dinâmica e lúdica das atividades propostas de simulações.

No último encontro, a devolutiva contou com a técnica Linha do Tempo, relembrando todas as atividades propostas ao orientando, sessão por sessão. 0 orientador fez o fechamento com as principais conclusões e decisões tomadas entregando um relatório.

\section{DISCUSSÃO}

A partir da prática de estágio supervisionado, foi possível alcançar os objetivos esperados com esse novo campo de estágio, gerando aquisição de habilidades por parte dos alunos contribuindo para sua formação profissional e atuação no campo da OP.

Constatou-se a importância da atuação do profissional de Psicologia em OP, não somente para o ensino médio, mas também para os estudantes universitários que tem dúvidas em relação a qual área de especialização a seguir e que não tem a oportunidade de compartilhar suas angustias e conflitos com alguém mais preparado seja pela formação; ou pela escuta qualificada ou por considerar a individualidade e o protagonismo de cada sujeito na tomada de decisão sobre a carreira a ser seguida.

A implantação de um programa de OP, em instituição de ensino superior, se faz necessária para que os alunos possam ter apoio emocional e suporte teórico para uma tomada de decisão mais madura e 
consciente, frente à escolha profissional e orientações assertivas em como enfrentar processos seletivos com segurança.

O campo de estágio forneceu aos alunos embasamento teórico e prático, promovendo o esclarecimento sobre a atuação do psicólogo na OP em diversas possibilidades e oportunidades interventivas; que a OP não segue um modelo único, mas um modelo singular voltado aos objetivos de cada orientando que também é singular.

\section{Limitações do estudo e perspetivas futuras}

Os resultados indicam que se trata de um serviço de importância para a universidade e para os orientandos que se sentiram mais confiantes em suas opções de curso, ou em participar de processos seletivos após a passagem pelo DICA.

Como fatores limitantes tem-se:

- número reduzido de estagiários orientadores (apenas 9 alunos por supervisor/ano);

- campo de estágio ligado à idealizadora do projeto, quando o NPO conta com três supervisores;

- pouca disponibilidade de tempo para os estagiários se dedicarem ao DICA, devido a carga horária elevada da matriz curricular para o último ano do curso.

Como perspectivas futuras, espera-se a continuidade da oferta do serviço enquanto campo de estágio, e que seja estendido a todos os alunos do NPO (em torno de 32 alunos) e a realização novos estudos e pesquisas para validação do modelo interventivo proposto, com levantamento dos dados recolhidos, com as orientações realizadas e a publicação de artigos sobre uma área privilegiada de intervenção do psicólogo.

\section{REFERÊNCIAS}

Alonso, W. C. \& Melo-Silva, L. L. (2013). Avaliação de uma Intervenção em Orientação Profissional na Perspectiva de Ex-Estagiários. Psicologia: Ciência e Profissão, 33 (1), 84-99.

Anastasi, A. \& Urbina, S. (2000). Testagem psicológica. Artes Médicas.

Bandura, A. (1977). Self-efficacy: The exercise of control. W.H. Freeman and Company.

Barbosa, A. \& Lamas, K. (2012). A orientação profissional como atividade transversal ao currículo escolar. Estudos de Psicologia, 17(3), 461-468.

Bock, A. M. B.; Furtado, O. \& Teixeira, M. de L. T. (2009). Psicologias: uma introdução ao estudo de psicologia. Editora Saraiva.

Bock, A.M.B, Amaral, C. M. M., Silva, F.F., Calejon, L.M.C., Andrade, L.Q., Uvaldo, M.C.C., . ..Nascimento, R.S.G.F. (1995). A Escolha Profissional em Questão. Casa do Psicólogo.

Bock, S. D. (2002). Orientação profissional - A abordagem sócio-histórica. Cortez.

Bock, S. D. (2006). Orientação Profissional: a abordagem sócio-histórica. Cortez.

Colognese, S. C. (2000). O adolescente e a escolha profissional. Interações: Estudos e Pesquisa em Psicologia. São Paulo, 5(9), 111-125.

Ferreira, W. B.(2006). Inclusão x exclusão no Brasil: reflexões sobre a formação docente dez anos após Salamanca. In D. Rodrigues (org.) Inclusão e educação: doze olhares sobre a educação inclusiva. Summus.

Gil, A. C. (2006). Métodos e técnicas de pesquisa social (5a ed.). Atlas.

Holland, J. L. (1997). Making vocational choices: a theory of vocation personalities and work environments (3a . ed.). Psychological Assessment Resources.

Hoyt, K. B. (2005). Career education as a federal legislative effort. In K. B. Hoyt (Ed.), Career education: History and future (pp. 3-74). National Career Development Association.

Lehman, Y.P., Silva, F.F., Ribeiro, M.A. \& Uvaldo, M.C.C. (2011). Segunda demanda chave para a orientação profissional: como ajudar o indivíduo a entender os determinantes de sua escolha e poder escolher? Enfoque psicodinâmico. In M.A. Ribeiro \& L.L. Melo-Silva (Orgs.), Compêndio de orientação profissional e de carreira: perspectivas históricas e enfoques teóricos clássicos e modernos (pp.111-134). Vetor.

Leitão, L. M. \& Miguel, J. P. (2004) Avaliação dos interesses. In L.M. Leitão (Org.). Avaliação psicológica em orientação escolar e profissional (pp. 179-262). Quarteto.

Lent, R.; Hacckett, G., \& Brown, S. D. (2004). Una perspectiva Social Cognitiva de la transición entre la escuela y el trabajo. Laboratorio de Evaluación Psicológica y Educativa - Evaluar, 4, 1-22.

Levenfus, R.S.e Cols. (1997). Psicodinâmica da escolha profissional. Artes Médicas.

Levenfus, R. S.; \& Soares, D. H. P.(2010). Orientação Vocacional Ocupacional. ARTMED.

Levenfus, R. S.(2002). Orientação vocacional ocupacional: novos achados teóricos, técnicos e instrumentais para a clínica, a escola e a empresa. ARTMED. 
Lima, M. T. (2007). Orientação Profissional. Princípios Teóricos, Práticas e Textos para Psicólogos e Educadores. -. São Paulo: Vetor.

Magalhães, S, M. O.(2014). Matriz de habilidades e interesses profissionais (2ae ed). Casa do Psicólogo.

Marconi, M. A. \& Lakatos, E. M. (2005). Fundamentos da metodologia científica (6 ${ }^{\mathrm{a}}$ ed). Atlas.

Mattiazzi, B. (1997). A natureza dos interesses e a orientação vocacional (3ª̀. ed.). Vozes.

Melo-Silva, L. L. (2011). Intervenção e Avaliação em Orientação Profissional e de Carreira. In M. A. Ribeiro \& L. L. Melo-Silva (Orgs.), Compêndio de orientação profissional e de carreira: Enfoques contemporâneos e modelos de intervenção (Vol. 2). Vetor.

Melo-Silva, L. L. \& Jacquemin, A. (2001). Intervenção em Orientação Profissional: Avaliando resultados e processos. Vetor.

Moura, C. (2011). Orientação profissional: sob o enfoque da análise do comportamento (3a ed). Editora Alínea.

Moura, M. L. (2014). Contribuições para a orientação profissional na estratégia clínica a partir da psicoterapia breve psicanalítica. [Dissertação de Mestrado, Instituto de Psicologia da Universidade de São Paulo].

Nauta, M.M. (2010). The development, Evolution, and status of Holland's theory of vocational personalities: reflections and future directions for counseling psychology. Journal of Counselin Psychology, 57(1), 11-22.

Neiva, K. M. C. (2007). Processos de escolha e orientação profissional. Vetor.

Pasqualini, J. C.; Garbulho, N. F.\& Schut, T. (2004). Working with kids: early professional guidance as a contribution to child education. Revista Brasileira de Orientação Profissional, 5(1), 71-85.

Parsons, F. (2005). Choosing a vocation. Boston: Houghton Mifflin. (Original publicado em 1909).

Pieron, H. (1993). Dicionário de Psicologia (8ª ed.). Globo.

Primi, R.; Muniz, M.; Mansão, C.M. \& Nunes, M.F.O. (2010). SDS - Questionário de busca autodirigida: manual técnico da versão brasileira. Casa do Psicólogo.

Primi, R., Moggi, M. A., \& Casellato, E. O. (2004) Estudo correlacional do inventário de busca auto-dirigida (self-directed search) com o IFP. Psicologia Escolar e Educacional, 8(1), 47-54.

Rocha, M. L. (2002). Contexto do adolescente -. In S.H. Koller (Org.). Adolescência e psicologia: concepções, práticas e reflexões críticas (pp. 25-32). Conselho Federal de Psicologia.

Rogers, C. R. (1974). A terapia centrada no paciente. Moraes Editores.

Savickas, M. L.(2005). The theory and practice of career cosntruction. In S. D. Brown, S.D. \& Lent, R. W. Carrer development and conseling: putting theory and reserach to work (pp.42-70). John Wiley \& Sons.

Schein, E. H. (1996). Identidade profissional: como usar suas inclinações e suas opções de trabalho. Nobel.

Scorsolini-Comin, F.; Nedel, A. Z. \& Santos, M. A. (2011). Temos nosso próprio tempo: grupo de orientação das escolhas profissionais com alunos do ensino médio. Vínculo, 8(1), 2-9.

Selig, G. \& Valore, L. (2010). Imagens da aposentadoria no discurso de pré-aposentados: subsídios para a orientação profissional. Cadernos De Psicologia Social Do Trabalho, 13(1), 73-87.

Silva, L. L. M. \& Jacquemin, A. (2001). Intervenção em Orientação Vocacional/Profissional. Vetor.

Soares, D. H. P. (Org.) (1993). Pensando e vivendo a orientação profissional. Summus.

Spaccaquerch, E, M. E. \& Fortim, I.(2009) Orientação profissional: passo a passo. Paulus.

Sparta, M.; Bardagi, M. P. \& Teixeira, M. A. (2006). Modelos e Instrumentos de Avaliação em Orientação Profissional: Perspectiva Histórica e Situação no Brasil. Revista Brasileira de Orientação Profissional, 7(2), 19-32.

Super, D. E. \& Bohn, M. J. Jr. (1980). Psicologia Ocupaciona. Atlas.

Vieira, V. S.; Bezerra, J. V. A. \& Fernandes, A. C. C. (2013, 04-06 Julho). Orientação vocacional e profissional. In Anais IX Congresso de Iniciação Científica do IFRN (pp. 2063 - 2067). Ministério da Educação. Instituto Federal de Educação Ciência e Tecnologia - Pró Reitoria de Pesquisa e Inovação.

Werlang, B. S. G.; Villemor-Amaral, A. E. \& Nascimento, R. S. G. F. (2010). Avaliação psicológica, testes e possibilidades de uso. In Conselho federal de Psicologia (Ed.), Avaliação psicológica: diretrizes na regulamentação da profissão (pp.101-128). Conselho Federal de Psicologia.

Yin, R. K. (2005). Estudo de caso: planejamento e métodos (3ª ed). Bookman.

Historial do artigo

Recebido 29/07/2019

Aceite 23/03/2020

Publicado 08/2020 
Revista PSICOLOGIA, 2020, Vol. 34 (1), doi: 10.17575/psicologia.v34i1.1681.

Atas do X Simpósio Nacional de Investigação em Psicologia

\title{
Uso de estratégias de aprendizagem em cursos oferecidos à distância
}

\author{
Mayra Antonelli-Ponti37i, Raissa Barbara Nunes Moraes Andradeii, Fabiana Maris \\ Versutii, Fabiana Roberta Scarpino Franco ${ }^{\mathrm{i}}$ \& Cristina Costa-Lobo ${ }^{i i i}$ \\ i Universidade de São Paulo \\ ii Centro Universitário UNIFAFIBE \\ iii Instituto de Estudos Superiores de Fafe
}

\begin{abstract}
Resumo: Foram analisadas estratégias de aprendizagem (EA) utilizadas por estudantes de um curso de curta duração, na modalidade educação à distância $(\mathrm{EaD})$. A amostra $(n=44)$ Brasileira, com idade média $35.6(D P=11.5)$, respondeu à Escala de Estratégias de Aprendizagem (EEA) com opções de resposta de 0 a 10. A EEA tem evidências de validade verificadas por meio de análise fatorial exploratória, que resultou em uma estrutura de quatro fatores ( $\alpha$ Cronbach entre 0.68 e 0.90 ). Os participantes recorreram a diversas EA e o fizeram em diferentes níveis. As Estratégias Cognitivas $(M=8.1 ; D P=2.7)$ foram as mais utilizadas, seguidas por Estratégias de Controle da Emoção $(M=7.9 ; D P=2.3)$, Estratégias Autorregulatórias $(M=7.9 ; D P=2.6)$ e por Estratégias de Ajuda Interpessoal $(M=3.6 ; D P=3.6)$. Participantes mais novos e com experiência em EaD pontuaram mais num item da categoria de Estratégias de Controle da Emoção, num item da categoria de Estratégias Autorregulatórias e maior uso de Estratégias de Ajuda Interpessoal. Os resultados apoiam o delineamento de cursos de difusão, realizados na modalidade à distância no âmbito de ensino superior.
\end{abstract}

Palavras-chave: Curso online; Educação a distância; Estratégias de aprendizagem; Ensino superior.

Use of learning strategies in distance learning courses: $\mathrm{W}$ We analyzed learning strategies used by students of a short duration course in distance education. The sample $(n=44)$, Brazilian, mean age of 35.6 ( $\mathrm{SD}=11.5$ ), answered to the Learning Strategies Scale (LSS) with response options from zero to 10. The LSS has validity verified through exploratory factor analysis, which results in a four-factor structure $(\alpha$ Cronbach between 0.68 and 0.90 ). Participants used a variety of learning strategies and did it in different levels. Cognitive Strategies $(M=8.1, S D=2.7)$ were the most used, followed by Emotion Control Strategies $(M=7.9, \mathrm{SD}=2.3)$, Self-Regulatory Strategies $(M=7.9 ; S D=2.6)$ and the Interpersonal Assistance Strategies $(M=3.6, S D=3.6)$. Younger participants with distance education experience scored more in one item in the Emotion Control Strategies category, in an item in the category of Self-regulatory Strategies and greater use of interpersonal assistance strategies. The results will aid in the design of distance education courses carried out in the distance modality in higher education.

\section{Keywords: Online course; Distance education; Learning strategies; Higher education.}

A distância física entre professores e estudantes, e a comunicação com o uso de mídias que caracterizam o Ensino a Distância (EaD), desafiam as instituições exigindo investimentos em tecnologia para a mediação e, ao mesmo, tempo mudança na cultura dos professores e estudantes que têm como parâmetro preponderante o modelo pedagógico presencial (Antonelli-Ponti, Valenti, Díaz, Picoli, \& Versuti, 2018; Beluce \& Oliveira, 2018; Paixão, Linhares \& Cerveró, 2019; Valenti, Antonelli-Ponti, Picoli, Díaz, \& Versuti, 2019). Aos estudantes EaD são atribuídas responsabilidades diferentes das requeridas pelo ensino presencial (Martins, Zerbini \& Medina, 2019). 0 papel dos estudantes no seu próprio resultado e aprendizagem é enfatizado, colaborando para que eles evidenciem competências como disciplina e autonomia (Badia \& Monereo, 2010; Oliveira, Boruchovitch \& Santos, 2009).

Vale destacar a escassez de pesquisas no Brasil, dedicadas a conhecer as diversas variáveis externas e internas ao estudante, que favorecem a promoção da aprendizagem na modalidade EaD, especialmente há necessidade de estudos que explorem como ocorre o processo da aprendizagem, gerando resultados que possam ampliar as possibilidades de sucesso acadêmico de um número maior de participantes, bem como minimizar falhas neste processo, de modo a propiciar ganhos qualitativos na

${ }^{37}$ Morada para correspondência: Mayra Antonelli-Ponti, Universidade de São Paulo, Brasil. E-mail: antonelli.may@alumni.usp.br. Este projeto foi financiado pela Coordenação de Aperfeiçoamento de Pessoal de nível superior (CAPES). 
oferta e na formação profissional dos estudantes (Abbad, Zerbini \& Souza, 2010; Martins \& Zerbini, 2014a; Saldanha, 2016).

Martins e Zerbini (2014), realizaram um estudo de revisão da produção de conhecimentos em EaD no Brasil e destacaram variáveis externas ao estudante relacionadas à qualidade dos cursos ofertados nesta modalidade, tais como preparação adequada de recursos humanos e tecnológicos; oferecimento ao estudante de possibilidades de acompanhamento adequado do curso, tutoria e avaliação, de forma a garantir a qualidade durante o desenvolvimento do curso.

A EaD manuseia diversos processos de interação que possibilitam a autoaprendizagem e a interaprendizagem concomitantes (Rosa \& Cecílio, 2010), ao mesmo tempo que promovem o uso de Estratégias de Aprendizagem (EA) em diversas frequências, a depender da fase em que o estudante se encontra no curso (Marini \& Boruchovitch, 2014). Estas EA podem envolver processos cognitivos e comportamentais, os quais interferem sobre os processos de escolha, processamento, retenção e recuperação de informações, sendo usadas, em geral, no contato do aprendiz com os conteúdos disciplinares (Portilho \& Dreher, 2012). Outras estratégias envolvem aspectos da motivação para aprender, a autogestão de esforços, a automonitorização do progresso, e até mesmo controle da ansiedade frente a determinadas situações que estariam dissipando a atenção do aprendiz (Warr \& Allan, 1998; Zerbini \& Abbad, 2008). O estudo de Versuti, Andrade e Zerbini (2020) faz uma classificação em relação às estratégias de aprendizagem, dividindo-as em quatro categorias:

1. Estratégias de Controle da Emoção: domínio da ansiedade e prudência na desatenção causada por sentimentos de ansiedade;

2. Estratégias Autorregulatórias: permitem que os discentes planifiquem, controlem e avaliem o processo de aprendizagem;

3. Estratégias Cognitivas: onde o discente utiliza a repetição, organização e elaboração, auxiliandoos na retenção e a utilização de novos conhecimentos;

4. Estratégias de Ajuda Interpessoal: solicitação de ajuda de terceiros, objetivando o auxílio de outras pessoas como colegas ou tutores.

A segunda e a quarta categoria, apesar de estarem separadas, fazem parte das estratégias comportamentais.

No ambiente virtual, as EA configuram-se em competências e habilidades indispensáveis para a convivência e permanência na sociedade-rede (Badia \& Monereo, 2010). Os ambientes virtuais têm potencial para promover o desenvolvimento de competências para uma aprendizagem mais independente e flexível, considerando tempo, espaço e ritmo do aprendiz, por meio de um conjunto de recursos didáticos adaptados a tecnologias e múltiplas mídias (Antonelli-Ponti et al., 2018; Paixão et al., 2019; Valenti et al., 2019). As EA consideram as peculiaridades do indivíduo, e são consideradas favoráveis ao processo de aprendizagem bem-sucedida, tendo ainda potencial para extrapolar tais benefícios para outros âmbitos da vida (Oliveira et al., 2009).

Ao elaborar um curso EaD deve levar-se em conta o formato de curso. Existem diferenças, em particular, no uso de EA em relação ao formato e tempo de duração e dos cursos (Versuti et al, 2020). 0 perfil do público também deverá guiar as escolhas de materiais didáticos, mídias e até linguagem mais adequada (Beluce \& Oliveira, 2018; Valenti et al., 2019). Desta forma, o objetivo do presente projeto foi avaliar o uso das estratégias de aprendizagem de estudantes de um curso de ciências de curta duração na modalidade EaD. Esta avaliação poderá servir como delineamento para novos cursos, além de colaborar na ação tanto dos professores formuladores de cursos à distância, quanto dos tutores que acompanham os estudantes durante o processo.

\section{MÉTODO}

\section{Instrumento}

A Tabela 1 apresenta um resumo dos dados do instrumento aplicado no presente estudo, após passar por análises exploratórias para verificação de evidências de validade do mesmo. 0 instrumento de estratégias de aprendizagem foi proposto por Martins e Zerbini (2014), que em seu estudo tiveram como objetivo a coleta de dados de estratégias de aprendizagem em cenários educacionais de nível superior à distância. Observa-se, a partir da Tabela 1, que os índices de confiabilidade variaram de .77 a .90 , indicando que a escala apresenta consistência interna. As cargas fatoriais, por sua vez, variaram de .33 a .76, o que indica que a escala é válida e contempla itens representativos do fator. 
Tabela 1. Instrumento utilizado na pesquisa após validação estatística

\begin{tabular}{lccccc}
\hline Instrumento & Escalas obtidas & № de & Alfa & \multicolumn{2}{c}{ Pesos fatoriais } \\
itens & & Mínimo & Máximo \\
\hline \multirow{3}{*}{ Estratégias de } & Estratégias Cognitivas & 15 & 0.90 & 0.33 & 0.72 \\
Aprendizagem & Controle da Emoção & 4 & 0.77 & 0.46 & 0.76 \\
& Estratégias Autorregulatórias & 7 & 0.86 & -0.50 & -0.69 \\
& Busca de ajuda interpessoal & 3 & 0.68 & 0.45 & 0.69 \\
\hline
\end{tabular}

Fonte: Martins e Zerbini (2014).

\section{Curso-alvo}

O curso Genética do Comportamento Humano: conceitos, crenças e consequências, de modalidade exclusivamente online, é o primeiro curso de uma proposta de divulgação científica e extensão universitária nesta modalidade com temas em Psicobiologia. 0 curso faz parte do projeto intitulado Psicobio em Rede (PBR) o qual preconiza a disponibilização de cursos completos para população interessada no tema (Antonelli-Ponti et al, 2018).

0 objetivo do curso é ampliar o acesso ao conhecimento sobre o tema, como forma de diminuir possíveis preconceitos associados a conceções erróneos e não científicos sobre o desenvolvimento dos comportamentos humanos. Para isso, o curso contou com videoaulas, materiais de leitura, participação em fóruns, supervisão de dúvidas e atividades avaliativas. A oferta dos materiais foi dividida em seis módulos, um por semana, e a carga horária total foi de 30 horas.

\section{Amostra}

A amostra foi composta por 44 estudantes EaD, com idade média 35.6 anos (DP=11.5), predominantemente mulheres (68.2\%), com Ensino Superior completo (77.3\%). A maioria (81.8\%) dos participantes já estudou em modalidade EaD anteriormente.

\section{Procedimento}

O projeto de pesquisa foi submetido ao Comitê de Ética da Faculdade de Filosofia, Ciências e Letras de Ribeirão Preto da Universidade de São Paulo. Após a aprovação (CAAE 44415715.5.0000.5407), a Escala de Estratégias de Aprendizagem foi disponibilizada digitalmente por meio da ferramenta Google forms aos participantes concluintes da última semana do curso-alvo. Os participantes escolheram participar voluntariamente da pesquisa e foram informados acerca dos aspectos da mesma pelo Termo de Consentimento Livre e Esclarecido garantindo os aspectos éticos da coleta de dados.

\section{Análise de dados}

Os dados foram analisados descritivamente. A diferença entre os grupos acerca de cada item foi testada por meio do teste de hipóteses Wilcoxon Mann-Whitney para amostras independentes, o qual foi escolhido após a violação dos pressupostos de normalidade, apresentando nos testes de Kolmogorov-Smirnov e Shapiro-Wilk valor de $p>.05$. Todas as análises foram conduzidas com a utilização do software estatístico SPSS.

\section{RESULTADOS}

Calcularam-se as médias por item e por categoria de Estratégias de Aprendizagem (EA) A categoria EA Ajuda Interpessoal $(M=3.6 ; D P=3.6)$, com três itens, foram as menos utilizadas (ver Tabela 2). 
Tabela 2. Média e desvio padrão dos itens da categoria “Ajuda Interpessoal” da Escala de Estratégias de Aprendizagem

\begin{tabular}{lcc}
\hline Categoria & Itens & Média (DP) \\
\hline & Busquei auxílio do tutor para esclarecer minhas dúvidas & $3.4(3.9)$ \\
Estratégias de Ajuda & sobre o conteúdo. & $3.6(3.4)$ \\
Interpessoal & Busquei auxílio de colegas nos fóruns para esclarecer & minhas dúvidas. \\
& Troquei mensagens com os colegas para esclarecer & $3.7(3.5)$ \\
\hline
\end{tabular}

As EA Controle da Emoção, categoria formada por quatro itens demonstrou alta utilização pelos estudantes $(M=7.9 ; D P=2.3)$ (ver Tabela 3$)$.

Tabela 3. Média e desvio padrão dos itens da categoria “Controle da Emoção" da Escala de Estratégias de Aprendizagem

\begin{tabular}{lcc}
\hline Categoria & Itens & Média (DP) \\
\hline $\begin{array}{l}\text { Estratégias de controle da } \\
\text { emoção }\end{array}$ & Mantive a calma quando tive dificuldades. & $8.5(1.6)$ \\
& Repeti a mim mesmo, quando me senti ansioso, que & $8.0(2.9)$ \\
& tudo sairia bem ao final do curso. & $7.3(2.4)$ \\
& Mantive a calma com a possibilidade de ter um & $7.8(1.9)$ \\
& rendimento abaixo do esperado. & \\
\hline
\end{tabular}

As EA Autorregulatórias $(M=7.9 ; D P=2.6)$, com seis itens, tiveram alta utilização, semelhante às EA Controle da Emoção (ver Tabela 4).

Tabela 4. Média e desvio padrão dos itens da categoria "Autorregulatórias" da Escala de Estratégias de Aprendizagem

\begin{tabular}{|c|c|c|}
\hline Categoria & Itens & Média $(D P)$ \\
\hline \multirow{12}{*}{ Estratégias autorregulatórias } & Esforcei-me mais quando percebi que estava perdendo & $8.6(1.7)$ \\
\hline & a concentração. & \\
\hline & Forcei-me a manter a atenção nos estudos quando me & $7.8(2.6)$ \\
\hline & senti desinteressado & \\
\hline & Esforcei-me mais quando percebi que estava perdendo & $7.9(2.5)$ \\
\hline & o interesse no assunto. & \\
\hline & Elaborei perguntas para testar minha compreensão & $6.4(3.4)$ \\
\hline & sobre os conteúdos do curso. & \\
\hline & Revisei a matéria para verificar o quanto eu dominava o & $8.0(2.5)$ \\
\hline & conteúdo & \\
\hline & Esforcei-me para verificar minha compreensão sobre o & $8.5(1.8)$ \\
\hline & que estava sendo ensinado. & \\
\hline
\end{tabular}



Tabela 5)

As EA Cognitivas $(M=8.1 ; D P=2.7)$, com 11 itens, foram as mais utilizadas por esta amostra (ver

Tabela 5. Média e desvio padrão dos itens da categoria "Cognitivas" da Escala de Estratégias de Aprendizagem

\begin{tabular}{|c|c|c|}
\hline Categoria & Itens & Média $(D P)$ \\
\hline & $\begin{array}{l}\text { Busquei outras fontes de pesquisas, fora da internet, relacionadas ao } \\
\text { curso para me ajudar a aprender. }\end{array}$ & $9.1(1.6)$ \\
\hline & $\begin{array}{l}\text { Busquei sites relacionados ao conteúdo do curso para me ajudar a } \\
\text { aprender. }\end{array}$ & $8.4(1.9)$ \\
\hline & $\begin{array}{l}\text { Realizei os exercícios práticos propostos ao longo do curso para me } \\
\text { ajudar a aprender. }\end{array}$ & $9.0(1.6)$ \\
\hline & Fiz anotações sobre o conteúdo do curso. & $6.9(3.4)$ \\
\hline & Fiz resumos do conteúdo do curso. & $7.3(2.8)$ \\
\hline \multirow[t]{6}{*}{ Estratégias cognitivas } & Fiz esquemas do conteúdo do curso como método para aprender. & $8.4(1.8)$ \\
\hline & Refleti sobre as implicações que os conteúdos aprendidos poderiam ter. & $8.3(2.8)$ \\
\hline & $\begin{array}{l}\text { Busquei desenvolver uma ideia global sobre como os conteúdos do curso } \\
\text { se relacionavam entre si. }\end{array}$ & $7.3(3.1)$ \\
\hline & Associei os conteúdos do curso aos meus conhecimentos anteriores. & $6.1(3.9)$ \\
\hline & $\begin{array}{l}\text { Diferenciei, ao analisar os conteúdos do curso, os aspectos mais } \\
\text { importantes dos menos importantes. }\end{array}$ & $9.0(1.5)$ \\
\hline & $\begin{array}{l}\text { Identifiquei situações diárias em que eu pudesse aplicar os conteúdos do } \\
\text { curso. }\end{array}$ & $8.8(1.5)$ \\
\hline
\end{tabular}

0 grupo de participantes mais novos, 18 a 34 anos, apresentou média estatisticamente significativa $(U=162.0 ; W=.415 .0 ; p<.05)$ maior $(M=9.1 ; D P=0.9)$ do que os participantes mais velhos, 35 a 62 anos, $(\mathrm{M}=7.9 ; \mathrm{DP}=1.9)$ no item "Mantive a calma quando tive dificuldades".

Ter experiência anterior com EaD também teve diferença estatisticamente significativa, sempre com médias mais altas nos itens "Mantive a calma diante dos erros que cometi ao realizar atividades do curso", "Elaborei perguntas para testar minha compreensão sobre os conteúdos do curso", "Busquei auxílio de colegas nos fóruns para esclarecer minhas dúvidas" e "Troquei mensagens com os colegas para esclarecer dúvidas sobre o conteúdo do curso" $(M=8.1, D P=1.7 ; M=6.9, D P=3.3 ; M=4.2, D P=3.5 ; M=4.2$, $D P=3.5$, respectivamente) do que os participantes sem experiência anterior com $\operatorname{EaD}(M=6.5, D P=2.1$; $M=4.3, D P=3.3 ; M=1.3, D P=2.0 ; M=1.4, D P=2.3)$. As estatísticas do teste de hipóteses foram: $U=78.0$, $W=114.0, p<0.05 ; U=72.0, W=108.0, p<.05 ; U=67.0, W=103.0, p<.05 ; U=58.0, W=94.0, p<.05$.

\section{DISCUSSÃo}

Este estudo investigou quais Estratégias de Aprendizagem (EA) são mais frequentemente aplicadas por estudantes de um curso de difusão EaD. Todas as categorias de EA foram utilizadas pelos participantes, em maior ou menor intensidade. As EA Cognitivas foram as mais utilizadas por esta amostra, participante de um curso de curta duração, corroborando com os achados de Versuti et al (2020). As EA Autorregulatórias e de Controle da Emoção, como categoria, apresentaram médias iguais demonstrando que estas EA foram utilizadas com frequência similar. As EA de Ajuda Interpessoal foram as menos utilizadas.

Em todas as etapas do processo de aprendizagem as EA Cognitivas são requeridas. É por meio desta categoria de estratégias que os discentes são capazes de executar e reorganizar suas ideias, objetivando a compreensão do conteúdo (Portilho \& Dreher, 2012). Portanto, era esperado que esta categoria de itens fosse tão pontuada. O curso usado como ambiente para esta pesquisa continha proposições de exercícios e atividades que são indispensáveis para a sequência do processo, algumas destas proposições estavam contempladas nos itens da Escala de Estratégias de Aprendizagem como 
parte da categoria EA Cognitivas, a saber: o item "Busquei outras fontes de pesquisas, fora da internet, relacionadas ao curso para me ajudar a aprender" com média bastante alta se alinha com os materiais em forma de links externos ao site do curso, e que eram disponibilizados aos estudantes; o item "Realizei os exercícios práticos propostos ao longo do curso para me ajudar a aprender" também com média alta, é explicado pela obrigatoriedade na execução de exercícios para obter pontuação e progredir no curso.

Estudo realizado por Marini e Boruchovitch (2014), sobre as EA Cognitivas utilizadas por estudantes brasileiros do ensino superior, revelou que nos primeiros semestres os estudantes fizeram grande uso das estratégias cognitivas, mas ao adquirirem conhecimentos sobre o conteúdo esta utilização foi diminuída. Relacionando os resultados acima mencionados com os dados do presente estudo, podemos dizer que as EA variam conforme compreensão do estudante em relação ao tema, ou ainda, em relação ao comprometimento, visto que nos primeiros semestres da graduação é necessário um esforço maior para se inserir no contexto, depois esses hábitos podem enfraquecer. No caso da presente pesquisa, os estudantes estavam participando de um curso de difusão, o qual não envolvia comprometimento académico decisivo, nem mesmo financeiro, e o único prejuízo por baixo desempenho ou por não finalizar o curso seria a não obtenção do certificado.

As características do curso também podem explicar o grande controle emocional averiguado pelos resultados da categoria EA Controle da Emoção. 0 baixo desempenho dos participantes em um curso de difusão não teria consequências impactantes em suas vidas quanto acontece em um curso de graduação, por exemplo. A motivação e as atitudes sobre o processo de ensino e aprendizagem são desiguais para ambientes específicos de aprendizagem e práticas de instrução (Oliveira et al., 2009). Se considerarmos somente o aspeto relacionado à finalização do curso, a alta frequência na utilização das EA Autorregulatórias seria surpreendente. Este resultado, no entanto, revela o interesse dos estudantes no tema do curso.

A comunicação interpessoal é imensamente relevante no EaD, sendo uma variável que interfere na produtividade, na comunicação e na cooperação entre os discentes e tutores (Zerbini \& Abbad, 2008). A solicitação de ajuda interpessoal, que poderia ser caracterizada como uma forma de interaprendizagem (Rosa \& Cecílio, 2010) por meio de EA desta categoria, foi pouco realizada pelos discentes no presente estudo, bem como ocorreu em ambiente de formação superior (Marini \& Boruchovitch, 2014).

Demonstrou-se que os estudantes não têm o hábito de informar ao tutor sobre suas dificuldades, impedindo o docente de compreender as necessidades dos discentes (Beluce \& Oliveira, 2018). Os estudantes que utilizaram das estratégias interpessoais, no entanto, parecem ter usado em alta frequência, visto que, apesar de as médias serem baixas, a pontuação máxima foi 10. Esta situação propõe a hipótese que os participantes se sintam hesitantes para buscar ajuda de seus pares e tutores, ou podemos conjecturar que os estudantes busquem o entendimento dos conteúdos por si próprios, a autoaprendizagem (Rosa \& Cecílio, 2010), empregando para isso, possivelmente, as estratégias cognitivas.

A experiência anterior com EaD demonstrou uma variável importante no uso de EA. Isso é corroborado por Martins, Zerbini e Medina (2019), que apontam que a familiaridade com o ambiente virtual de aprendizagem pode proporcionar um uso mais frequente de EA que, por sua vez, podem aumentar a retenção de conteúdo por parte dos aprendizes. É possível que os estudantes mais novos, que demonstraram mais calma diante de dificuldades, também tenham maior familiaridade com ferramentas comuns a EaD, conferindo a este grupo maior segurança para contornar situações desfavoráveis num ambiente virtual de aprendizagem.

Os estudantes da presente amostra obtiveram ao menos a nota sete (7.0), que foi a nota mínima para chegar até o último módulo. Estudos futuros pretendem relacionar as EA com o desempenho médio de cada estudante, como forma de averiguar a correlação entre o uso de EA e realização educacional.

Promover a utilização de EA tem potencial para estimulação da aprendizagem e de superação de bloqueios individuais e sociais (Badia \& Monereo, 2010). É possível que as estratégias possam ser adquiridas por meio treinamento, com a intenção de agregar a qualidade da aprendizagem em uma atividade ou ambiente específico (Warr \& Allan, 1998; Zerbini \& Abbad, 2008). Para futuros cursos, poderá ser aventada a possibilidade de que docentes e tutores façam o curso anteriormente e apliquem as EA como forma de compreensão sobre a experiência dos estudantes (Beluce \& Oliveira, 2018). Com base nesta vivência e no perfil dos estudantes (Valenti et al., 2019), os tutores instruirão a turma sobre o uso de EA, inclusive informando aos discentes sobre as vantagens de sua utilização.

Por fim, os dados evidenciaram que a produção do conhecimento acerca das EA fomentariam a proposição de interações em EaD, adequadas às demandas dos estudantes, de modo a estimular a criação de sistemas tutoriais eficazes para apoiar a formação do estudante em diversos momentos do processo de ensino e aprendizagem. Tal conhecimento pode suprir lacunas teórico-metodológicas destacadas neste 
estudo e contribuir para o avanço da área (Abbad, Zerbini \& Souza, 2010; Martins \& Zerbini, 2014a; Saldanha, 2016).

\section{REFERÊNCIAS}

Abbad, G.; Zerbini, T., \& Souza, D. B. L. (2010). Panorama das pesquisas em Educação a Distância no Brasil. Estudos de Psicologia (UFRN), 15, 291-298. https://doi.org/10.1590/S1413-294X2010000300009

Antonelli-Ponti, M., Valenti, L., Díaz, D. A. C., de Picoli, R. M. D. M., \& Versuti, F. M. (2018). Divulgação científica em psicobiologia: educação a distância como estratégia para a promoção da extensão universitária. Expressa Extensão, 23(3), 183-197. http://10.15210/EE.V23I3.13668

Badia, A., \& Monereo, C. (2010). Ensino e aprendizagem de estratégias de aprendizagem em ambientes virtuais. In C. Coll \& C. Monereo, Psicologia da educação virtual: aprender e ensinar com as tecnologias da informação e da comunicação (pp. 311-328). Artmed.

Marini, J.S, \& Boruchovitch, E. (2014). Estratégias de aprendizagem de estudantes brasileiros do ensino superior: Considerações sobre adaptação, sucesso acadêmico e aprendizagem autorregulada. Revista E-Psi, 4(1), 102-126.

Martins, L. B., \& Zerbini, T. (2014a). Educação a distância em instituições de ensino superior: uma revisão de pesquisas. Revista Psicologia: Organizações e Trabalho, 14(3), 271-282.

Martins, L. B., \& Zerbini, T. (2014b). Escala de estratégias de aprendizagem: Evidências de validade em contexto universitário híbrido. Psico-USF, 19, 317-328. https://doi.org/10.1590/141382712014019002007

Martins, L. B., Zerbini, T., \& Medina, F. J. (2019). Impact of Online Training on Behavioral Transfer and Job Performance in a Large Organization. Revista de Psicologia del Trabajo y de Las Organizaciones, 35(1), 27-37. https://doi.org/10.5093/jwop2019a4

Versuti, F. B., Andrade, R. B. N. M., \& Zerbini, T. (2020) Estratégias de Aprendizagem em Cursos Ofertados à Distância: Diferença entre Cursos de Licenciatura e Extensão. Psicologia: Teoria e Pesquisa, 36, e3631. https://dx.doi.org/10.1590/0102.3772e3631

Oliveira, K. L., Boruchovitch, E.,\& Santos, A. A. A. (2009). Estratégias de Aprendizagem e desempenho acadêmico: evidências de validade. Psicologia: teoria e pesquisa, 25(4), 531-536. https://doi.org/10.1590/S0102-37722009000400008.

Paixão, P. B. S., Linhares, R. N., \& Cerveró, A. C. (2019). Modelo teórico-aplicativo de alfabetização informacional em cursos a distância. Ciência da Informação, 48(1), 181-200.

Portilho, E. M. L., \& Dreher, S. A. S. (2012). Categorias metacognitivas como subsídio à prática pedagógica. Educação e Pesquisa, São Paulo, 38(1), 181-196. https://doi.org/10.1590/S151797022011005000009.

Rosa, R., \& Cecílio, S. (2010). Educação e o uso pedagógico das tecnologias da informação e comunicação: a produção do conhecimento em análise. Educação em foco. Juiz de Fora, 15(1), 107-126.

Saldanha, L. C. D (2016). Meta-análise da pesquisa em educação a distância. Arte Factum - Revista de Estudos em Linguagem e Tecnologia, 12(1), 1-14.

Valenti, L., Antonelli-Ponti, M., de Picoli, R. M. D. M., Díaz, D. A. C., \& Versuti, F. M (2019). Treinamento para docência no ensino superior: descobrindo a diversidade discente. In M. Flores, Atas do Congresso Internacional sobre Avaliação no Ensino Superior (pp. 1-8). Universidade do Minho, Instituto de Educação Centro de Investigação em Estudos da Criança.

Warr, P., \& Allan, C. (1998). Learning strategies and occupational training. Internacional Review of Industrial and Organizational Psychology, 13, 83-121.

Zerbini, T., \& Abbad, G. (2008). Estratégias de aprendizagem em curso a distância: validação de uma escala. Psico-USF, 13(2), 177-187. https://doi.org/10.1590/S1413-82712008000200005.

Historial do artigo

Recebido $\quad 07 / 2019$

Aceite $\quad 04 / 2020$

Publicado $\quad 08 / 2020$ 
Revista PSICOLOGIA, 2020, Vol. 34 (1), doi: 10.17575/psicologia.v34i1.1681.

Atas do X Simpósio Nacional de Investigação em Psicologia

\title{
LightOnYou: Mindfulness, yoga and meditation for elementary school students
}

\author{
Filipa Catarina Coelho ${ }^{38 i}$, Saúl Neves de Jesus ${ }^{i} \&$ Rafaela Maia $^{\mathrm{i}}$ \\ i Universidade do Algarve, Faro, Portugal
}

\begin{abstract}
The educational context is a context in which the psychologist is fundamental due to the needs that the school presents. The role of the school psychologist is to respond to problems, promote and prevent the mental health of the school community. The role of the psychologist in this context is varied, and it is important to create dynamics that will improve the quality of service provision and the success of students attending school. The dynamics created for this specific project include interventions based on mindfulness. The mindfulness approaches (MBIs) include several interventions such as meditation and yoga. These approaches are implemented to improve the physical and emotional conditions of patients (Wahbeh, Elsas, \& Oken (2008). This work aimed to introduce a study that integrates the multimodal psychoeducational intervention based on mindfulness, yoga, and meditation in a school context, involving the participation of 32 students aged between 10 and 16 years. The analysis of comparison between groups through the Mann-Whitney test demonstrated the differences found in the pre-test and post-test of the intervention carried out by the group LightOnYou.

The multimodal techniques adopted constitute an adequate response (low cost) to the identified needs of the schools involved as well as an adequate way to promote the development of emotional and social skills.
\end{abstract}

Keywords: Educational Psychology; Mindfulness; Yoga; Meditation.

LightOnYou: Mindfulness, yoga e meditação para estudantes do ensino básico: 0 contexto educacional, é um contexto no qual o psicólogo é fundamental devido às necessidades que a escola apresenta. 0 papel do psicólogo escolar passa pela resposta a problemas, promoção e prevenção da saúde mental da comunidade escolar. 0 papel do psicólogo neste contexto é variado, sendo importante para que sejam criadas dinâmicas que permitam melhorar a qualidade na prestação de serviços e o sucesso escolar dos alunos que o frequentam. A dinâmica criada para este projeto específico passa pelas intervenções baseadas no mindfulness. As abordagens mente-corpo (MBIs) incluem diversas intervenções, como meditação e yoga. Estas abordagens são implementadas de forma a melhorar as condições físicas e emocionais dos pacientes (Wahbeh, Elsas, \& Oken (2008). O presente trabalho pretendeu introduzir um estudo que integra a intervenção multimodal psicoeducacional, baseada na Mindfulness, yoga e meditação em contexto escolar, envolvendo a participação de 32 estudantes com idades entre 10 e 16 anos. A análise de comparação entre grupos através do teste Mann-Whitney demonstrou as diferenças encontradas no pré-teste e pós-teste da intervenção efetuada pelo grupo LightOnYou. As técnicas multimodais adotadas constituem uma resposta adequada (baixo custo) às necessidades identificadas das escolas envolvidas, bem como uma forma adequada de promoção do desenvolvimento de competências emocionais e sociais.

\section{Palavras-chave: Psicologia Educacional; Mindfulness; Yoga; Meditação.}

The educational context is a context in which the psychologist is fundamental due to the needs that the school presents. The role of the school psychologist is to respond to problems, promote and prevent the mental health of the school community. It is in this context that children present several problems that negatively influence school success. The psychological intervention in a school context must include strategies that help in emotional management; in the decrease of aggressive behaviors; improvement of school performance; well-being, among others... There are strategies that promote various skills, enhancing the development of students in the socio-emotional, school, and behavioral fields (Ordem dos Psicólogos Portugueses, 2018). School psychologists intervene from different levels, including universal

${ }^{38}$ Correspondence address: Filipa Catarina Coelho, Departamento Psicologia e Ciências da Educação, Campus Gambelas 8005-139 Faro, Portugal. E-mail: phdlightonyou@gmail.com 
prevention (promotion of optimal development of the general population); selective prevention (for populations at risk, with the aim of remediation); indicated prevention (for populations with serious and/or permanent problems, having long-term interventions) (Ordem dos Psicólogos Portugueses, 2018). The role of the psychologist in this context is varied, and it is important to create dynamics that will improve the quality of service provision and the school success of the students who attend it. The dynamics created for this specific project include interventions based on mindfulness.

The mindfulness approaches (MBIs) include several interventions such as meditation and yoga. These approaches are implemented to improve the physical and emotional conditions of patients (Wahbeh, Elsas, \& Oken (2008). MBIs are positive due to their low cost, easy to apply intervention and effective results (Tatton-Ramos, Simões, Niquice, Bizarro, \& Russell, 2016). In the school context, MBIs are tools implemented with the objective of promoting emotional regulation, self-esteem, development of social skills and attention, promotion of well-being (physical and psychological) and prevention of depressive and anxious symptoms, reduction of stress, increased memory capacity, resilience, anxiety in the face of tests, greater happiness, improvement of school performance (Tatton-Ramos, Simões, Niquice, Bizarro, \& Russell, 2016; Crescentini, Capurso, Furlan, \& Fabbro, 2016; Bazzano, Anderson, Hylton, \& Gustat, 2018; Cheng, 2016; Vascouto, Sbissa, \& Takase, 2013). Thus, these tools are described as mindfulness, meditation, and yoga techniques, respectively. Mindfulness is considered as a state of full attention, where the individual is concentrated in the present moment, intentionally and without judgment (Vandenberghe, \& Sousa, 2006). Mindfulness has four fundamental criteria: the fact that it is not elaborate, without judgement, centered here and now and where our thoughts, feelings and sensations arise when we are concentrated, recognizing and accepting them (Tatton-Ramos, Simões and Niquice, 2016). In turn, meditation is considered a technique with the objective of promoting selfknowledge, health, well-being, and balance. It is a practice of easy access and understanding (Vascouto, Sbissa \& Takase, 2013). A meditation-based awareness practice, according to Cheng (2016), has positive results in memory capacity, inhibitory control, and resilience. Finally, we have yoga, considered a meditation practice that requires greater mental strength and has the objective of personal transformation and promotion of physical, emotional, and mental well-being. This practice promotes the development of various brain functions (psychological, cognitive, motor, affective and social) (Ribeiro, Fernandes, Carvalhal, Costa and Maciel, 2019). However, like all approaches, MBIs also have limitations. Regarding MBIs applied in a school context, these limitations include the cost of hiring an external professional if there is no qualified professional in mindfulness within the school, heterogeneous results (Sanger \& Dorjee, 2015; Bazzano, Anderson, Hylton, \& Gustat, 2018).

Thus, this study integrates a multimodal psychoeducational intervention based on mindfulness, meditation, and yoga in a school context. One of the objectives of this study is to demonstrate the use of these techniques as a (low-cost) response to school intervention needs. The objective of this study was also due to the difficulties of interpersonal relationships, aggressive behaviors, and indifference towards the school as these were questions raised by teachers and the School Psychology and Guidance Service where the intervention took place. It is also added that few studies have been carried out in Portugal using MBIs, however, none has adopted multimodal techniques.

\section{METHOD}

\section{Participants}

The study involved the participation of 32 students aged between 10 and 16 years, of both genders, attending the 2nd (5th and 6th grade) and 3rd Cycle (7th to 9th grade) of Canedo school (Group 1) and Couto-Mineiro de Pejão school (Group 2), located on Santa Maria da Feira and Castelo de Paiva city's respectively, Aveiro district, Portugal.

\section{Measurements}

Subjective Happiness Scale (Pais-Ribeiro, 2012). The SHS presents 4 items, 2 (2 and 3), to compare with their peers and 2 (1 and 4), which present a description of happiness and unhappiness. It contains two conflicting statements that express the level of happiness or lack of it. The total score is the average of the average responses on 4 items. The higher the score, the higher the happiness.

Brief Resilient Coping Scale (Pais-Ribeiro, \& Morais, 2010). BRCS presents 4 items to evaluate the ability to handle stress in an adaptive way. Those with a score between 4 and 20 years deal with stress in an adaptive way. Those with a score below 13 have low resilience, those over 17 have strong resilience. 
Behavioral Health Measure (Pais-Ribeiro, 2001). BHM is a questionnaire that assesses overall mental health, including psychopathological symptoms, well-being, and functioning. The results of any selected scale average the values of its items. The higher the total, the higher the level of mental health.

Idate - Anxiety-Trait (Fioravanti, Santos, Maissonette, Cruz and Landeira-Fernandez, 2006). IDATE has two scales that assess anxiety status (IDATE-E) or trait (IDATE-T). However, only the anxiety-trace scale was used. This consists of 20 items and the participant receives an instruction that should answer as "usually feels" according to a Likert 4-point scale.

Test Anxiety Inventory (TAI) (Taylor, \& Deane, 2002). TAI contains 20 items divided into 3 subscales, 4 items for the total anxiety scale (TAI-T), 8 items for the anxiety worry scale (TAI-P), 8 items for the anxiety-emotionality scale (TAI-E). The higher the score, the lower the anxiety on the tests.

D2 - Attention Test (Brickenkamp, \& Zilmer, 1998). The d2 attention test allows the evaluation of the subjects' selective attention and concentration capacity. It measures information processing speed, accuracy and qualitative aspects related to performance. Through d2's quotation the user has access to six results: Total of Characters processed - indicator of speed of execution, productivity capacity and subject motivation; Total of Hits - indicator of accuracy and efficiency; Total of Effectiveness - indicator of attention control and the relationship between speed and thoroughness in the task; Concentration Index indicator of concentration capacity; Variability Index - indicator of consistency in the execution of the task percentage of Errors - indicator of thoroughness and quality of performance.

\section{Procedure}

A psycho-educational intervention was implemented in the adherent schools, developed by the researcher, called LightOnYou. The intervention was based on Mindfulness, Yoga and Meditation Techniques practices, operated in 12 sessions (lasting about one hour each), distributed between January and June 2019. The sessions were integrated in an extracurricular activity. Four groups were created, most of them heterogeneous. The groups were composed of students from the 2nd and 3rd cycles, with the weekly sessions; all trainees were informed of the objectives and the voluntary nature of the group's participation, as well as the subsequent use of the data collected through informed consent, respecting the ethical and deontological principles inherent to the development of an investigation.

The tools were applied in the school context, in the psychology office in two moments (January and June, respectively). The completion of the questionnaires took between 30 and 45 minutes. A sociodemographic questionnaire was constructed for the present study, which allows to characterize the sample in its sociodemographic variables, considering, in particular, the age of the participants, gender, year of schooling attended, time dedicated to homework, time dedicated to the study, whether it likes to go to school, how it considers its health, the degree of satisfaction with its appearance, whether it receives the support of other people, questions about sleep, emotions and violence.

Session planning. The planning involved dynamic activities of short duration (Table 1). These activities include psycho-educational practices based on mindfulness, yoga, and meditation, providing and stimulating the management of emotions, self-knowledge, increased self-esteem, self-control and the development of personal and interpersonal conflict resolution skills, as well as memorization and concentration techniques. At the end of each session students were recommended to apply the practices learned through self-training (at home, during class or in another extra-group context) (Table 1).

\section{Data analyses}

The data were entered in the Statistical Package for the Social Sciences (SPSS), version 24. This program was used to perform a mean comparison analysis between pre-test and post-test, using the MannWhitney Test. 
Table 1. Planning of the interventional exercises with psychoeducational strategies based on yoga, meditation, and mindfulness

\section{Some session theme's}

Breathing exercises in the beginning and the end of each session such as pranayama, diaphragm breathing or focus on breathing: contemplative breathing, just observing, without changing the rhythm of the breath, letting it naturally, without take effort to inhale or exhale;

Interpersonal relationship management exercises and reinforcing self-esteem exercise's - stimulation of gratitude, compliments, identify positive characteristics from themselves and their colleagues, positive affirmations;

Emotional management exercises: identify, accept, and observe all type of emoticons (observing without judgment the body, bodily sensations, possible emotions, and thoughts that arise);

Attention and concentration exercises (with olfactory, tactile, and gustatory sensations training); attention, concentration, and relaxation exercise's (from a focus) such as Dharana (example: construction and painting of mandalas);

All body sensitize with Yoga exercises such as Ásanas;

Meditation exercise (with creative visualization); Dhyana (Guided meditation, Mantras, felling Smells, flavors and texture and "thoughts hunt");

Study visit to "Porto City Garden" with yoga and meditation exercises in contact with nature.

\section{RESULTS}

The results of the analysis performed by the Mann-Whitney test show that there are significant differences between the pre-test and post-test in terms of time spent on homework, time spent on the study, the under-run of the behavioral health measure, and the D2 attention test in terms of total processed characters, total hits, task effectiveness, concentration index, and variability index. We also point out that the post-test has a greater global mental health, processed characteristics, and total hits in the attention test D2 (Table 2).

Table 2. Comparison analysis of means between groups using the Mann-Whitney Test

\begin{tabular}{|c|c|c|c|c|c|}
\hline & Moment & $\overline{\mathbf{N}}$ & $\begin{array}{l}\text { Mean } \\
\text { Rank }\end{array}$ & $\begin{array}{c}\text { Sum of } \\
\text { Ranks }\end{array}$ & $\bar{U}$ \\
\hline \multirow[t]{3}{*}{ You like to go to school? } & Pre-test & 32 & 28.41 & 909.00 & \multirow[t]{3}{*}{381,00} \\
\hline & Pos-test & 31 & 35,71 & 1107,00 & \\
\hline & Total & 63 & & & \\
\hline \multirow[t]{3}{*}{ Sleep } & Pre-test & 32 & 32.38 & 1036.00 & \multirow[t]{3}{*}{508.00} \\
\hline & Pos-test & 32 & 32.63 & 1044.00 & \\
\hline & Total & 64 & & & \\
\hline \multirow[t]{3}{*}{ Stress Symptoms } & Pre-test & 32 & 34.67 & 1109.50 & \multirow[t]{3}{*}{442.50} \\
\hline & Pos-test & 32 & 30.33 & 970.50 & \\
\hline & Total & 64 & & & \\
\hline \multirow[t]{3}{*}{ Depressive Symptoms } & Pre-test & 32 & 32.19 & 1030.00 & \multirow[t]{3}{*}{490.00} \\
\hline & Pos-test & 31 & 31.81 & 986.00 & \\
\hline & Total & 63 & & & \\
\hline \multirow[t]{2}{*}{ Targeting aggressive behavior at school } & Pre-test & 32 & 33.06 & 1058.00 & \multirow[t]{2}{*}{494.00} \\
\hline & Pos-test & 32 & 31.94 & 1022.00 & \\
\hline
\end{tabular}




\begin{tabular}{|c|c|c|c|c|c|}
\hline & Total & 64 & & & \\
\hline \multirow[t]{3}{*}{ Scale of subjective happiness } & Pre-test & 32 & 33.92 & 1085.50 & \multirow{3}{*}{402.50} \\
\hline & Pos-test & 30 & 28.92 & 867.50 & \\
\hline & Total & 62 & & & \\
\hline \multirow[t]{3}{*}{ Resilient coping brief scale } & Pre-test & 32 & 30.28 & 969.00 & \multirow[t]{3}{*}{441.00} \\
\hline & Pos-test & 32 & 34.72 & 1111.00 & \\
\hline & Total & 64 & & & \\
\hline \multirow[t]{3}{*}{ Global Mental Health } & Pre-test & 32 & 24.52 & 784.50 & \multirow[t]{3}{*}{$256.50^{*}$} \\
\hline & Pos-test & 26 & 35.63 & 926.50 & \\
\hline & Total & 58 & & & \\
\hline \multirow[t]{3}{*}{ Test Anxiety Inventory } & Pre-test & 32 & 35.33 & 1130.50 & \multirow{3}{*}{389.50} \\
\hline & Pos-test & 31 & 28.56 & 885.50 & \\
\hline & Total & 63 & & & \\
\hline \multirow[t]{3}{*}{ D2 TC - total of characters processed } & Pre-test & 32 & 22.53 & 721.00 & \multirow[t]{3}{*}{$193.00^{* * *}$} \\
\hline & Pos-test & 32 & 42.47 & 1359.00 & \\
\hline & Total & 64 & & & \\
\hline \multirow[t]{3}{*}{ D2 TA - total hits } & Pre-test & 32 & 22.75 & 728.00 & \multirow[t]{3}{*}{$200.00^{* * *}$} \\
\hline & Pos-test & 32 & 42.25 & 1352.00 & \\
\hline & Total & 64 & & & \\
\hline \multirow{3}{*}{$\begin{array}{l}\text { D2 efficiency - indicator to control the } \\
\text { attention and the relation between speed } \\
\text { and meticulousness of the task }\end{array}$} & Pre-test & 32 & 42.38 & 1356.00 & \multirow[t]{3}{*}{$196.00^{* * *}$} \\
\hline & Pos-test & 32 & 22.63 & 724.00 & \\
\hline & Total & 64 & & & \\
\hline \multirow[t]{3}{*}{ D2 - IC - concentration index } & Pre-test & 32 & 44.38 & 1420.00 & \multirow[t]{3}{*}{$132.00^{* * *}$} \\
\hline & Pos-test & 32 & 20.63 & 660.00 & \\
\hline & Total & 64 & & & \\
\hline \multirow[t]{3}{*}{ D2 - IV - variability index } & Pre-test & 32 & 44.61 & 1427.50 & \multirow[t]{3}{*}{$124.500^{* * *}$} \\
\hline & Pos-test & 32 & 20.39 & 652.50 & \\
\hline & Total & 64 & & & \\
\hline \multirow[t]{3}{*}{ D2 E\% - percentage of errors } & Pre-test & 32 & 36.80 & 1177.50 & \multirow[t]{3}{*}{374.500} \\
\hline & Pos-test & 32 & 28.20 & 902.50 & \\
\hline & Total & 64 & & & \\
\hline
\end{tabular}

Note: ${ }^{* * *} p<.001 ;{ }^{* *} p<.01{ }^{*} p<.05$

\section{DISCUSSION}

To our knowledge, this study is the first to access the benefits/improvement perceived by students of a multimodal intervention based on Mindfulness, Yoga and Meditation. The mainly emotional gains of this study are congruent with the results of other studies in which MBIs and/or Yoga techniques are integrated (separately or together), demonstrating that students benefit from the psychosocial and emotional domains based on these interventions (Bazzano et al., 2018; Crescentini et al., 2016; FerreiraVorkapic et al., 2015; Franke, Huebner, \& Hills, 2017).

This study corroborates previous studies indicating the benefits of yoga, mindfulness, and meditation. As demonstrated in the results, these practices have provided students with several benefits, such as improved global mental health, processed characters, and total hits on the D2 attention test. According to previous studies, attention shows positive results in adolescents suffering from stress, test anxiety, eating disorders and Asperger's Syndrome (Cheng, 2016). Like mindfulness, meditation also has benefits such as: increased levels of relaxation, concentration and focus, decreased anxiety (Vascouto, Sbissa, Takase, 2013). In addition, yoga practice also has benefits reflected in previous studies, namely the reduction of risk factors for diseases such as anxiety, depression, and stress (Ribeiro, Fernandes, Carvalhal, Costa and Maciel, 2019).

The subjective results of improved school performance are in line with other studies showing that relaxation and mindfulness practices are positively correlated with academic performance and classroom climate (López-gonzález, Amutio, Oriol, \& Bisquerra, 2016), which can also be explained by the benefit associated with neurological development (Mak, 2018). 
In short, the main conclusion of this study is that the multimodal techniques adopted constitute an adequate (low-cost) response to the specific needs identified by the adherent schools (by teachers and by the Psychology and School Guidance Service), as they demonstrate a good adequacy to the development of emotional and social skills. In addition, the adopted techniques are indicated as pleasant, showing good acceptability, congruent with the general conclusions (Zenner et al., 2014). Finally, we highlight the selfselection of the participants given the voluntary nature of the group's participation, which may attract participants who may feel more affinity with the theme, leading to a bias in the results. Furthermore, the lack of a control group, as well as other measures, makes it impossible to compare results between and within the group. Further research should overcome the limitations: increase the number of the sample as well as diversify data collection. In addition, classroom intervention with some teacher integration seems appropriate.

\section{REFERENCES}

Bazzano, A. N., Anderson, C. E., Hylton, C., \& Gustat, J. (2018). Effect of mindfulness and yoga on quality of life for elementary school students and teachers: results of a randomized controlled school-based study. Psychology Research and Behavior Management, 11, 81-89. https://doi.org/10.2147/PRBM.S157503

Brickenkamp, R., \& Zilmer, E. (1998). The D2 Test of Attention. Hogrefe \& Huber Publishers.

Cheng, F. K. (2016). Is meditation conducive to mental well-being for adolescents? An integrative review for mental health nursing. International Journal of Africa Nursing Sciences, 4, 7-19. https://doi.org/10.1016/j.ijans.2016.01.001

Crescentini, C., Capurso, V., Furlan, S., \& Fabbro, F. (2016). Mindfulness-Oriented Meditation for Primary School Children : Effects on Attention and Psychological Well-Being. Frontiers in Psychology, 7, 112. https://doi.org/10.3389/fpsyg.2016.00805

Fiovaranti, A.C.M., Santos, L. F., Maissonette, S., Cruz, A. P. M., \& Landeira-Fernandez, J. (2006). Avaliação da estrutura fatorial da escala de ansiedade-traço do idate. Avaliação Psicológica, 5(2), 217-224.

Ferreira-Vorkapic, C., Feitoza, J. M., Marchioro, M., Simões, J., Kozasa, E., \& Telles, S. (2015). Are there benefits from teaching yoga at schools? A systematic review of randomized control trials of yogabased interventions. Evidence-Based Complementary and Alternative Medicine, 2015, 1-17. https://doi.org/10.1155/2015/345835

Franke, K. B., Huebner, E. S., \& Hills, K. J. (2017). Cross-sectional and prospective associations between positive emotions and general life satisfaction in adolescents. Journal of Happiness Studies, 18, 1075-1093. https://doi.org/10.1007/s10902-016-9763-8

López-gonzález, L., Amutio, A., Oriol, X., \& Bisquerra, R. (2016). Habits related to relaxation and mindfulness of high school students : Influence on classroom climate and academic performance. Revista de Psicodidatica, 21(1), 121-138. https://doi.org/10.1387/RevPsicodidact.13866

Mak, C. (2018). Efficacy of mindfulness-based interventions for attention and executive function in children and adolescents: A systematic review. Mindfulness, 9, 59-78. https://doi.org/10.1007/s12671-017-0770-6

Ordem dos Psicólogos Portugueses (Maio, 2018). A necessidade e o papel dos psicólogos no contexto educativo.

http://recursos.ordemdospsicologos.pt/files/artigos/parecer_sobre_a_necessidade_e_a_imp_dos_p sis_no_ctxt_educ.pdf

Pais-Ribeiro, J.L. (2001). Mental health inventory: Um estudo de adaptação à população portuguesa. Psicologia, Saúde \& Doenças, 2 (1), 77-99.

Pais-Ribeiro, J.L. \& Morais, R. (2010). Adaptação portuguesa da escala breve de coping resiliente. Psicologia, Saúde \& Doenças, 11(1), 5-13.

Pais-Ribeiro, J.L. (2012). Validação transcultural da escala de felicidade subjetiva de Lyubomirsky e Lepper. Psicologia, Saúde \& Doenças, 13(2), 157-168.

Ribeiro, A. A., Fernandes, C. T., Carvalhal, M. I. M. M., Costa, A. S., \& Maciel, C. M. L. A. (2019). A prática do yoga na escola: Elementos preliminares e perceções de estudantes do ensino técnico integrado ao nível médio. Profiscientia, 12, 89-102.

Sanger, K. L., \& Dorjee, D. (2015). Mindfulness training for adolescents : A neurodevelopmental perspective on investigating modifications in attention and emotion regulation using event-related brain potentials. Cognitive, Affective, \& Behavioral Neuroscience, 15, 696-711. https://doi.org/10.3758/s13415-015-0354-7

Tatton-Ramos, T. P., Simões, R. A. G., Niquice, F. L. A., Bizarro, L., \& Russell, T. A. (2016). Mindfulness em ambientes escolares: adaptações e protocolos emergentes. Temas em Psicologia, 24, 1375-1388. https://doi.org/10.9788/TP2016.4-10 
Taylor, J., Deane, F. P. (2002). Development of a short form of the test anxiety inventory (TAI). The Journal of General Psychology, 129, 127-136. https://doi.org/10.1080/00221300209603133

Vandenberghe, L., \& Sousa, A. C. A. (2006). Mindfulness nas terapias cognitivas e comportamentais. Revista Brasileira de Terapias Cognitivas, 2(1), 35-44.

Vascouto, H. D., Sbissa, P., Takase, E. (2013). Utilização da meditação no contexto escolar: Uma análise da literatura. Revista Caminhos, 4(6), 87-106.

Wahbeh, H., Elsas, S-M., Oken, B. S. (2008). Mind-body interventions: Applications in neurology. Neurology, 70, 2321-2328. https://doi.org/10.1212/01.wnl.0000314667.16386.5e

Zenner, C., Herrnleben-kurz, S., \& Walach, H. (2014). Mindfulness-based interventions in schools - a systematic review and meta-analysis. Frontiers in psychology, 5. https://doi.org/10.3389/fpsyg.2014.00603

$\begin{array}{lr}\text { Historial do } & \text { artigo } \\ \text { Recebido } & 07 / 2019 \\ \text { Aceite } & 05 / 2020 \\ \text { Publicado } & 08 / 2020\end{array}$


Revista PSICOLOGIA, 2020, Vol. 34 (1), doi: 10.17575/psicologia.v34i1.1681.

Atas do X Simpósio Nacional de Investigação em Psicologia

\title{
Un estudio basado en la teoría del autogobierno mental y las barreras de la creatividad en estudiantes de Derecho: Implicaciones
}

\author{
Verónica López-Fernández ${ }^{39}$ i, Cristian Camilo Arias-Castroii, Sara Margarida \\ Fernandes ${ }^{\mathrm{iii} i \mathrm{iv}}$, Cristina Costa-Lobo ${ }^{\mathrm{iv}, \mathrm{v}, \mathrm{vi}}$ \& Enrique Vázquez-Justo ${ }^{\mathrm{iii}, \mathrm{v}}$ \\ i Universidad Internacional de La Rioja (UNIR) \\ ${ }^{i i}$ Universidad Cooperativa de Colombia \\ iii IJP- Portucalense Institute for Legal Research and INPP- Portucalense Institute for Human Development, \\ Universidade Portucalense Infante D. Henrique \\ iv INPP- Portucalense Institute for Human Development, Universidade Portucalense Infante D. Henrique \\ ${ }^{\checkmark}$ Brain and Behavior Institute, Universidade Fernando Pessoa \\ vi Cátedra UNESCO de Juventude, Educação e Sociedade, Universidade Católica de Brasília
}

\begin{abstract}
Resumen: La psicología aporta una analogía sobre el funcionamiento de un gobierno, para adaptarlo al autogobierno individual. Los grupos están formados por individuos, en particular los contextos educativos, sociales y políticos. Por ello, es importante avanzar en estudios que reflejen el autogobierno mental de un individuo para mejorar el rendimiento grupal. El objetivo de este estudio es conocer el estilo de pensamiento predominante de un grupo de estudiantes de Derecho colombianos y conocer su relación con las barreras creativas. Los resultados muestran predominio del estilo ejecutivo y que el judicial correlaciona con las barreras creativas. Se discuten las repercusiones al respecto.
\end{abstract}

Palabras clave: Autogobierno mental; Creatividad; Barreras; Estilo de pensamento predominante.

A study based on the theory of mental self-regulation and the barriers of creativity in law students: implications: Psychology provides an analogy about the functioning of regulation to adapt it to self-regulation. The groups are made up of individuals, in particular educational, social and political contexts. Therefore, it is important to move forward in studies that reflect the mental self-government of an individual to improve group performance. The objective of this study is to know the predominant thinking style of a group of Colombian law students and to know its relationship with creative barriers. The results show a predominance of the executive style and that the legal correlates with creative barriers. The repercussions in this regard are discussed.

Keywords: Mental self-regulation; Creativity; Barriers; Predominant thinking style.

La psicología ha tomado prestada una analogía sobre las funciones del gobierno y su estructura, con el fin de aplicarla al gobierno mental de una persona. Así, Sternberg y Lubart (1997) crearon la teoría del autogobierno mental, que trata de explicar y describir las diferentes maneras que tienen las personas a la hora de utilizar las habilidades intelectuales (Gutierrez-Braojos, Salmeron-Vilchez, Martin-Romera \& Salmerón, 2013). Dentro de su teoría, se describen diferentes estilos de pensamiento, y una de sus dimensiones que más ha trascendido es la que hace referencia a las funciones de los estilos descritos. De esta forma, tendríamos el estilo legislativo, judicial y ejecutivo (Gutierrez-Braojos et al., 2013). Ahora bien, para entender mejor los estilos y sus respectivas funciones, primero debemos entender qué supone un estilo de pensamiento. Según autores como Zhang y Sternberg (2005) los estilos de pensamiento se refieren al modo concreto e individual predominante en un sujeto a la hora de formar ideas o juicios sobre las cosas o situaciones.

Ahora bien, el estilo legislativo está relacionado con procesos de creación, la planificación de respuestas y puede tratarse de sujetos con compromiso sobre la tarea y manifiestan además cómo hacerla (Sternberg \& Grigorenko, 1995); el estilo ejecutivo, es propio de sujetos a los que les resulta fácil acomodar su estilo de trabajo al seguimiento de normas y reglas, aunque en ocasiones pueda modificarlas (González-Pienda, Roces, Bernardo \& García, 2002). La función judicial es propia de sujetos que disfrutan

\footnotetext{
39 Dirección de envio: Verónica López Fernández, Universidad Internacional de La Rioja (UNIR). Avenida de la Paz, 137, 26004, Logroño (La Rioja). España. Phone: (+34) 941210 211. E-mail: veronica.lopez@unir.net
} 
de analizar fenómenos, tomar posturas críticas, así, podría decirse que este tipo de sujeto tiene una tendencia a evaluar a los demás.

Diferentes estudios han analizado la relación de los diferentes estilos de pensamiento con otras habilidades, como por ejemplo la creatividad en contextos universitarios (Martínez y Brufau, 2010; Sternberg, Castejón y Bermejo, 1999)

La creatividad es un constructo complejo de definir y abordar. Algunas definiciones, como la de Plucker, Beghetto y Dow (2004) proponen que la creatividad alude a la interacción de multitud de aptitudes, procesos y por supuesto, contexto, que da como resultado un producto perceptible de manera individual o grupal, útil y novedoso dentro de un contexto social determinado.

La complementaridad de los benefícios que la creatividad permite tanto personal, como socialmente, es clara, aunque todavía hay falta de concordancia en la definición de creatividad, así como de la forma en cómo se puede medir y desarrollar (Costa-Lobo et al., 2018). Sin embargo, podría afirmarse actualmente que la creatividad es considerada una competencia fundamental para la subsistencia del proprio individuo, que necesita ser desarrollada y explorada en sus diferentes dimensiones, así como los procesos que están envueltos (Garcês, 2014).

No obstante, esta falta de unanimidad total a la hora de definir el constructo, contrasta con la homogeneidad del discurso sobre la importancia de la creatividad. Así, por ejemplo la Alianza para las competencias del siglo XXI (P21), que hace alusión a las competencias y habilidades que serán fundamentales para el éxito en el siglo XXI, aluden a la creatividad como una de ellas (P21, 2007, 2013).

Estudios han demostrado resultados positivos de programas de intervención, enfatizando la noción de un potencial creativo inherente a todos los individuos y susceptible de ser desarrollada de forma sistemática (Runco, 2014; Sastre-Riba y Pascual-Sufrate, 2013). En este sentido, existe una diversidad de estrategias y de programas de entrenamiento ante las competencias de resolución creativa de problemas.

Es primordial que la supervisión de la intervención psicológica en la creatividad sea capaz de acompañar los cambios constantes del conocimiento y de los contextos (López-Fernández y LlamasSalguero, 2018), debiendo por ello tornarse ella misma, objetivo de transformación. En este contexto, merece la pena perguntar: ¿qué tipo de tendencias se verifican en la intervención psicológica, en particular en los contextos educativos, y que modelos han sido estudiados sobre la criatividad? El constructo de la creatividad ha venido a dar un gran giro a la Psicologia de la educación, precisamente por su transversalidad (Hernández Arteaga, Alvarado Pérez y Luna, 2015). Además de las perspetivas bastante tradicionales sobre los benefícios de la creatividad, educadores y psicólogos de diversas especialidades considerarán notorias las contribuciones de la creatividad en áreas tan distintas como el liderazgo en el trabajo, el éxito vocacional y en la vida adulta, el funcionamento psicológico saludable, o coping, el crecimiento emocional, el mantenimiento de relaciones amorosas saludables y tratamientos terapeuticos más eficazes. Los psicólogos educacionales y escolares, han venido a revelar preocupaciones en lo que concierne a la problemática de la violencia juvenil, para ello, solicitaron que fuese explorado el papel que la creatividad puede desempeñar en la reducción de la violencia y en la promoción de la resolución de conflitos (Kreidler, 2001).

Las características psicológicas de los individuos creativos varían entre sí, considerando las competencias que estas presentan como la perseverancia, la autonomía, la asunción de riesgos, aptitudes sociales, confianza (Navarro, 2008).

Algunos estudios han evidenciado que existe relación entre los diferentes estilos y la creatividad y se ha visto que el estilo legislativo y judicial, especialmente el primero de ellos se relacionan con el pensamiento divergente o creativo (Gutierrez-Braojos et al., 2013). El contexto de educación superior, requiere que las instituciones, ya sean universitarias o de gestión de recursos, aboguen por la generación de ideas nuevas, para que los miembros de las comunidades sean profesionales con espíritu crítico, transformador y reflexivo (Gutierrez-Braojos et al., 2013). Sin embargo, muchas veces los contextos educativos y no educativos, locales y globales, no son favorecedores a la hora de formar a personas creativas, sino que más bien proporcionan barreras a la capacidad creativa de una persona.

Costa-Lobo et al. (2018) enfatizan la pertinencia de desarrollar estrategias de intervención en contexto educativo, fundamentalmente centradas en la preocupación en considerar el significado y la comprensión; alternar entre aprendizaje de competencias y tareas complejas e innovadoras; proporcionar contextos de aprendizaje, desafiar actitudes y creencias así como competencias y conocimiento, e impedir la redundancia y la repetición.

En este sentido, dado que muchas de las personas que ocupan puestos directamente relacionados con la gestión de recursos a través de funciones adscritas a gobiernos locales y globales, tienen una formación académica relacionada con la titulación de Derecho, es fundamental siguiendo a Aljojo (2017) lograr el ajuste del estilo de pensamiento al contexto académico, que previamente implica conocer los 
estilos a desarrollar en función de la especialidad disciplinar y las funciones que se le atribuyen. Este hecho resulta de gran interés, pues generalmente el Derecho no se relaciona con la creatividad y sin embargo, debido a sus funciones, han de resolver diferentes problemáticas que requqrirían un pensamiento divergente.

En base a ello, este estudio pretende conocer los estilos preferentes de un grupo de alumnos que cursan derecho en Colombia, y analizar si estos estilos se relacionan o no con las barreras para impulsar la creatividad.

\section{MÉTODO}

\section{Hipótesis}

Se espera que las barreras para la creatividad correlacionen positivamente con el estilo judicial y ejecutivo, y de forma negativa con el estilo legislativo.

\section{Muestra}

La muestra estuvo compuesta por 30 estudiantes de Derecho de la Universidad Cooperativa de Colombia, con una media de edad de 21,57 años y desviación típica de 2,46. Por género, del total, 8 fueron hombres y 22 mujeres. La muestra estudiaba Derecho en diferentes cursos o semestres. En la Tabla 1 puede verse dicha distribución.

Tabla 1. Descripción del semestre de estudio de la muestra

\begin{tabular}{lcccc}
\hline & Frecuencia & Porcentaje & Porcentaje válido & Porcentaje acumulado \\
\hline Semestre & 1 & 3.3 & 3.3 & 3.3 \\
Cuarto & 4 & 13.3 & 13.3 & 16.7 \\
Décimo & 16 & 53.3 & 53.3 & 70.0 \\
Noveno & 5 & 16.7 & 16.7 & 86.7 \\
Octavo & 1 & 3.3 & 3.3 & 90.0 \\
Primero & 2 & 6.7 & 6.7 & 96.7 \\
Quinto & 1 & 3.3 & 3.3 & 100.0 \\
Tercero & 30 & 100.0 & 100.0 & \\
Total & 30.0 & & \\
\hline
\end{tabular}

\section{Instrumentos}

Para recoger los datos de la muestra se emplearon los siguientes instrumentos:

Inventario de barreras del pensamiento creativo y la acción innovadora (basado en Lorna y adaptado por Talavera et al., 2015). Consiste en 22 ítems que muestran afirmaciones y el sujeto ha de valorar en qué medida se siente de acuerdo con cada una, en una escala de 1 a 6 , siendo la más próxima a 6 el mayor desacuerdo (por lo que, a mayor puntuación, mayores barreras). Este instrumento ha sido utilizado en investigaciones semejantes a la presente en otros estudios (como por ejemplo, Rábanos y Torres, 2012).

Cuestionario Sternberg-Wagner para la evaluación de los estilos de pensamiento (en Talavera et al., 2015). Consta de 24 afirmaciones, 8 de cada uno de los tres estilos, y cada sujeto ha de marcar el grado en que cada situación describe el grado de adecuación de cada ítem (del 1 al 7, siendo 7 totalmente). Estudios como el de Hoffmann, Uriel, Da Lama y Liporace (2019) han informado de que la consistencia interna de los factores extraídos al analizar las propiedades psicométricas del instrumento alcanzó valores apropiados en contextos latinoamericanos.

\section{Procedimiento}

El presente estudio es descriptivo y correlacional, con una metodología de aplicación de formularios a través de Google Drive. Los datos fueron recogidos en una muestra de estudiantes de Derecho matriculados en diferentes semestres en Colombia. Su aplicación fue individual. Los participantes contestaron a las preguntas de manera voluntaria, asimismo, dieron aprobación de la toma de datos en un consentimiento informado virtual. 


\section{Análisis de datos}

Los datos fueron analizados en el software estadístico para MAC SPSS 24, en el cual fueron aplicados análisis descriptivos y de correlación de Pearson.

\section{RESULTADOS}

Analizados los resultados de la muestra del estudio, consistente en 30 sujetos estudiantes de Derecho, se encontró que el perfil de estilo de pensamiento con mayor puntuación en dicha muestra fue el estilo ejecutivo, seguido del legislativo y por último el judicial, tal y como se puede apreciar en la Tabla 2.

Tabla 2. Estadísticos descriptivos de los estilos de pensamiento y las barreras creativas

\begin{tabular}{lccccc}
\hline & $\boldsymbol{N}$ & Mínimo & Máximo & Media & Desv. estándar \\
\hline Estilo Ejecutivo & 30 & 3.88 & 7.00 & 5.5750 & 0.72412 \\
Estilo Legislativo & 30 & 3.13 & 7.00 & 5.2750 & 1.01200 \\
Estilo Judicial & 30 & 3.00 & 7.00 & 5.0625 & 1.04105 \\
Barreras Creativas & 30 & 45 & 216 & 112.43 & 50.430 \\
N válido (por lista) & 30 & & & & \\
\hline
\end{tabular}

En dicho cuadro, además, respecto a las barreras creativas, se evidencia que las barreras del grupo son de 112,43, que podría considerarse como una puntuación media en las barreras que se muestran ante la creatividad.

En relación con el análisis de correlación efectuado, solo se ha encontrado que el estilo judicial correlaciona significativamente con las barreras creativas y de forma directa, como puede verse en la Tabla 3.

Tabla 3. Análisis de Correlación Entre los Estilos de Pensamiento y las Barreras Creativas

\begin{tabular}{|c|c|c|c|c|c|}
\hline & & $\begin{array}{c}\text { Estilo } \\
\text { Ejecutivo }\end{array}$ & $\begin{array}{c}\text { Estilo } \\
\text { Legislativo }\end{array}$ & $\begin{array}{c}\text { Estilo } \\
\text { Judicial }\end{array}$ & $\begin{array}{l}\text { Barreras } \\
\text { Creativas }\end{array}$ \\
\hline \multirow{3}{*}{ Estilo Ejecutivo } & Correlación de Pearson & 1 & $0.380^{*}$ & ,338 & 0.017 \\
\hline & Sig. (bilateral) & & 0.038 & 0.068 & 0.927 \\
\hline & $N$ & 30 & 30 & 30 & 30 \\
\hline \multirow{3}{*}{$\begin{array}{l}\text { Estilo } \\
\text { Legislativo }\end{array}$} & Correlación de Pearson & $0.380^{*}$ & 1 & $0.631^{* *}$ & 0.255 \\
\hline & Sig. (bilateral) & 0.038 & & 0.000 & 0.174 \\
\hline & $N$ & 30 & 30 & 30 & 30 \\
\hline \multirow{3}{*}{ Estilo Judicial } & Correlación de Pearson & 0.338 & $0.631^{* *}$ & 1 & $0.471^{* *}$ \\
\hline & Sig. (bilateral) & 0.068 & 0.000 & & 0.009 \\
\hline & $N$ & 30 & 30 & 30 & 30 \\
\hline \multirow{3}{*}{$\begin{array}{l}\text { Barreras } \\
\text { Creativas }\end{array}$} & Correlación de Pearson & 0.017 & 0.255 & $0.471^{* *}$ & 1 \\
\hline & Sig. (bilateral) & 0.927 & 0.174 & 0.009 & \\
\hline & $N$ & 30 & 30 & 30 & 30 \\
\hline
\end{tabular}

Nota. $^{*}$ La correlación es significativa en el nivel 0.05 (bilateral); ${ }^{* *}$ La correlación es significativa en el nivel 0.01 (bilateral); Obsérvese que se ha empleado el estadístico de Pearson con un tamaño muestral de 30. En este sentido, se ha encontrado que existe una relación estadísticamente significativa entre las dos variables de interés del estudio: uno de los estilos de pensamiento y las barreras creativas. Sin embargo, a efectos prácticos cabe resaltar que, dado el valor $r$ obtenido, si se calcula el $R^{2}$ obtendremos un porcentaje más realista de lo que constituye la variancia explicada de una de ellas por parte de la otra, que en este caso sería del $22.18 \%$. Esto es importante porque según Palmer, Jiménez y Montaño (2001) la significatividad estadística está influenciada por el tamaño muestral utilizado, de forma que si se va incrementando, es más fácil encontrar una relación significativa ya que disminuye el valor criterio de referencia, por lo que conviene tomar en consideración, para efectos prácticos más realistas, del valor $R^{2}$. 


\section{DISCUSIÓN}

Estudios previos realizados con diferentes perfiles profesionales muestran un predominio de estilo de pensamiento diferente. Por ejemplo, Aguilar y Morales (2007) encontraron en un grupo de 100 docentes universitarios, que el perfil de pensamiento de mayor frecuencia fue el estilo judicial, seguido del ejecutivo y finalmente el legislativo. Por otra parte, otro estudio con universitarios (112 estudiantes de magisterio) evidenció que el estilo de pensamiento de los participantes, mostraba una presencia alta en el estilo legislativo de mayor número de personas que en el ejecutivo y el judicial (Talavera et al., 2015). En base a los resultados del presente estudio y de estudios previos expuestos, parece ser que la distribución del estilo de pensamiento predominante es diferente en función del grupo ante el que nos encontramos, y sus características idiosincráticas.

Por ejemplo, en el caso de la muestra de este estudio, el estilo predominante que es el ejecutivo, que se caracterizaría más por seguir las reglas, trabajar con problemas estructurados y previamente planeados, tal y como apuntan Aguilar y Morales (2007).

Respecto a las barreras creativas la puntuación obtenida puede considerarse como una puntuación media en las barreras que se muestran ante la creatividad. Habitualmente este tipo de barreras hacen alusión a la confianza de uno mismo, la conformidad, capacidad de abstracción, análisis, ejecución de tareas y barreras físicas (Talavera et al., 2015). En este estudio no se ha analizado pormenorizadamente el tipo de barreras, aunque sí el cómputo total de barreras, y en esa línea general, estudios previos han encontrado mayores barreras en sus muestras (como es el caso de 184,02 de media en el estudio de Talavera et al., 2015).

En cuanto al análisis correlacional efectuado, sólo se ha encontrado una correlación estadísticamente significativa y positiva entre el estilo judicial y las barreras creativas, en concreto de $.471(\mathrm{p}=.009)$ de intensidad media. Considerado además el valor $\mathrm{R}^{2}$, que aporta información práctica y complementaria (Palmer, Jiménez y Montaño, 2001), se puede decir que ambas variables comparten varianza del $22.18 \%$. Esto quiere decir que las personas con este estilo de pensamiento predominante suelen llevar a cabo actividades de juicio y de tipo evaluativo de aquellas alternativas existentes para poder solventar un problema (Aguilar \& Morales, 2007) y que este estilo de pensamiento se relaciona con mayores barreras a la hora de ser creativo (recordemos que la relación es directa y positiva). Por otra parte, no se han encontrado relaciones significativas entre las barreras y los otros dos estilos, aunque previamente otros autores habían encontrado relación entre el estilo legislativo y la creatividad, esperándose en este estudio una relación inversa entre estilo legislativo y las barreras a la creatividad. Esto es, a mayor puntuación en barreras, menos puntuación en estilo legislativo. Sin embargo, en esta línea, tal y como señalan Gutierrez-Braojos et al. (2013), Almansa Martínez y López Martínez, tampoco encontraron relación entre el estilo legislativo y el pensamiento divergente.

Al contrario, por último, se esperaba relación estadísticamente significativa y positiva entre el estilo ejecutivo y las barreras y no se ha demostrado. En esta línea, un estudio previo encontró con estudiantes de enfermería relación entre creatividad y dicho estilo, de forma significativa y positiva (Gutierrez-Braojos et al., 2013).

Una interpretación que se presenta como adecuada mediante estos hallazgos es que la creatividad es muy compleja y no han de tenerse en cuenta solo aspectos cognitivos, sino además emocionales, actitudinales, metacognitivos (Barron, 2007).

\section{CONCLUSIONES}

Para concluir este trabajo, es de relevancia recordar la complejidad del constructo de creatividad, por lo que estos resultados deben tomarse con precaución, dado que no recoge todas las dimensiones que componen la creatividad.

El hecho de que en un grupo se aprecie un predominio de un estilo de pensamiento sobre otros, no implica algo negativo en sí mismo, ya que los tres estilos son formas de trabajar con los recursos que el individuo tiene.

Lo importante de este tipo de estudios, radica en que es importante que en un grupo todos los estilos se hallen representados, para que en los diferentes momentos en los que nos encontremos, podamos emplear unos estilos en lugar de otros, apoyados por personas que muestran esas preferencias. De esta forma, el proceso de solución de problemas puede efectuarse de una manera más eficiente y eficaz si empleamos los recursos más apropiados en cada momento.

Además, sería muy importante y recomendable que en grupos de trabajo, se pueda analizar previamente qué barrera pueden inhibir la creatividad de los individuos para rebatirlas y ampliar la perspectiva de los problemas ante los que nos encontremos o la gestión de los recursos de una forma eficiente. 
No cabe duda de que incluir la creatividad como solución de problemas y optimización de recursos en los gobiernos locales y globales, y por supuesto en los autogobiernos personales es la base del cambio y la productividad.

El presente trabajo, presenta una cotización para la United Nations Educational, Scientific and Cultural Organization (UNESCO), que presenta como principal objetivo el desarrollo de herramientas educacionales que buscan ayudar a los individuos en lo que se refiere al acceso a la educación de calidad. Este estudio sigue la línea de trabajos de la Cátedra 812, dedicada a los pilares de la Juventud, Educación y Sociedad, habiendo publicado ya estudios previos en el ámbito de la creatividad (Costa-lobo et al., 2018).

La Declaración de Incheon 2030, establece una nueva visión para la educación, refiriendo que en 2030, sea posible garantizar que todos los estudiantes adquieran conocimientos y habilidades necesarios para promover la sostenibilidad del desarrollo, incluyendo, entre otros, ciudadanía global. La agenda $20 / 30$, es por ello, un plan de acción para las personas y para el planeta, que busca fortalecer la paz universal.

Se requieren más estudios en esta línea que arrojen más luz sobre este tema y que además superen las limitaciones del presente trabajo. Fundamentalmente las mejoras habrían de centrarse en aumentar el tamaño muestral, acceder a la muestra de forma aleatoria, incluir alumnado de diferentes titulaciones y explorar la capacidad creativa, las barreras creativas y el estilo de pensamiento con diferentes instrumentos que puedan ayudar a captar cada uno de los tres constructos que se pretenden evaluar. Además, sería relevante, tras estos estudios, llevar a cabo programas de intervención ajustados a las necesidades de la muestra para posteriormente volver a evaluar a los sujetos y explorar si éstos han sido o no efectivos y si el alumnado en realidad ha logrado romper con las barreras más creativas de su entorno. Sin duda, es un campo apasionante que requiere ser explorado de forma rigurosa.

\section{REFERENCIAS}

Aguilar, M., \& Morales, M. (2007). Estilos de pensamiento, tipos de liderazgo y estilos educativos en docentes universitarios. Revista de Psicología [Psychology Journal], 9(1), 81-91

Aljojo, N. (2017). Differences in styles of thinking "in light of Sternberg's theory": A case study of different educational levels in Saudi Arabia. Journal of Technology and Science Education, 7(3), 333-346. http://dx.doi.org/10.3926/jotse.291

Barron, F. (2007). Enhancement and the Fulfillment of Potential. In M. A. Runco (Ed.), Creativity. theories and themes: research, development, and practice (pp. 319-374). Academic Press.

Costa-Lobo, C., Monteiro, F., Stoltz, T., Vázquéz-Justo, E., Carvalho, T., \& Cabrera, J. (2018). Development of creative potential: what has literature said in the last ten years? In L. Gómez Chova, A. López Martínez, I. Candel Torres (Eds.), ICERI18 Proceedings (pp.7008-7013). Sevilla, Spain. ISBN: 97884-09-05948-5.

Garcês, S. F. (2014). A Multidimensionalidade da criatividade: A pessoa, o processo, o produto e o ambiente criativo no ensino superior [The Multidimensionality of Creativity: The Person, Process, Product, and Creative Environment in Higher Education] [Dissertação de Mestrado, Universidade da Madeira]. Retrieved from http://digituma.uma.pt/bitstream/10400.13/546/1/DoutoramentoSoraiaGarcês.pdf\%0Ahttp://h dl.handle.net/10400.13/546

González-Pienda, J. A., Roces, C., Bernardo, A. B., \& García, M. (2002). Estilos de aprendizaje y estilos de pensamiento. Manual de Psicología de la Educación [Educational Psychology Handbook]. Ediciones Pirámide.

Grigorenko, E. L., \& Sternberg, R. J. (1995). Thinking styles. In D. H. Saklofske \& M. Zeidner (Eds.). International handbook of personality and intelligence (pp. 205-229). Plenum Press.

Gutierrez-Braojos, C., Salmeron-Vilchez, P., Martin-Romera, A., \& Salmerón, H. (2013). Efectos directos e indirectos entre estilos de pensamiento, estrategias metacognitivas y creatividad en estudiantes universitarios. Anales de psicología [Annals of Psychology], 29(1), 159-170. http://dx.doi.org/10.6018/analesps.29.1.124651

Hernández Arteaga, I., Alvarado Pérez, J. C., \& Luna, S. M. (2015). Creatividad e innovación: competencias genéricas o transversales en la formación profesional. Revista Virtual Universidad Católica del Norte [Virtual Journal North Catholic University], 1(44), 135-151.

Hoffmann, A. F., Uriel, F., Da Lama, R. F., \& Liporace, M. F. (2019). Inventario de Estilos de Pensamiento: Propiedades psicométricas en universitarios de Buenos Aires. Interacciones, [Interactions] 5(2), 165. http://dx.doi.org/10.24016/2019.v5n2.165

Kreidler, W. J. (2001). La resolución creativa de conflictos: manual de actividades. Fundación para el Bienestar Humano SURGIR. Recuperado de 
https://www.oei.es/historico/noticias/spip.php?article273\&debut_5ultimasOEI=55 el 4 de Noviembre de 2019

López-Fernández, V., \& Llamas-Salguero, F. (2018). Neuropsicología del proceso creativo. Un enfoque educativo. Revista Complutense de Educación, [Complutense Education ] 29(1), 113. http://dx.doi.org/10.5209/RCED.52103

Martínez, O. L., \& Brufau, R. M. (2010). Estilos de pensamiento y creatividad. Anales de Psicología[Annals of Psychology], 26(2), 254-258.

Navarro, J. (2008). Mejora de la creatividad en el aula de primaria [Improvement of creativity in the primary classroom] [Tesis Doctoral, Universidad de Murcia]. Recuperado de https://www.tdx.cat/bitstream/handle/10803/11009/NavarroLozano.pdf;jsessionid=471EDE01 097B864FA33CFA4B988F0DDF?sequence=1 el 4 de Noviembre de 2019.

Palmer, A., Jiménez, R., \& Montaño, J. J. (2001). Tutorial sobre el coeficiente de correlación lineal de Pearson en Internet. Psicología. com [Psychology.com], 5(1), 1-19.

Plucker, J. A., Beghetto, R. A., \& Dow, G. (2004). Why isn't creativity more important to educational psychologists? Potential, pitfalls, and future directions in creativity research. Educational Psychologist, 39, 83-96. http://dx.doi.org/10.1207/s15326985ep3902_1

P21. (2007). The Intellectual and Policy Foundations of the 21st Century Skills Framework. Washington DC, Partnership for 21st Century http://route21.p21.org/images/stories/epapers/skills_foundations_final.pdf (consultado el 20 de septiembre de 2019)

P21(2013). Reimagining Citizenship for the 21st Century: A Call to Action for Policymakers and Educators. Washington DC, Partnership for 21st Century Skills.www.p21.org/storage/documents/Reimagining_Citizenship_for_21st_Century_webversion.p df (consultado el 20 de septiembre de 2019)

Rábanos, N. L., \& Torres, P. A. (2012). Efectos de un programa para desarrollar las habilidades creativas. Electronic Journal of research in educational Psychology, 10(3), 1139-1158.

Runco, M. A. (2014). Creativity: Theories and themes: Research, development, and practice (2nd ed.). Elsevier Academic Press.

Sastre-Riba, S., \& Pascual-Sufrate, M. T. (2013). Alta capacidad intelectual, resolución de problemas y creatividad. Revista de neurología [Journal of neurology], 56(1), 67-76. http://dx.doi.org/10.33588/rn.56S01.2013025

Sternberg, R. J., Castejón, J. L., \& Bermejo, M. R. (1999). Estilo intelectual y rendimiento académico. Revista de investigación educativa [Educational research journal], 17(1), 33-46.

Sternberg, R., Lubart, T. (1997) La creatividad en una cultura conformista. Un desafío a las masas [Creativity in a conformist culture. A challenge to the masses]. Paidós.

Talavera, M., Hurtado, A., Cantó, J., \& Martín, D. (2015). Valoración de la creatividad grupal y barreras del pensamiento creativo en universitarios. Journal of Learning Styles, 8(15), 70-90.

UNESCO. 2014. Education for All Global Monitoring Report 2013/14. Teaching and Learning: Achieving quality for all - Gender Summary. Retrieved from: http://unesdoc.unesco.org/images/0022/002266/226662E.pdf

Zhang, L., \& Sternberg, R. (2005). A threefold model of intellectual styles. Educational Psychology Review, 17(1), 1-53. http://dx.doi.org/10.1007/s10648-005-1635-4

Historial do artigo

Recebido $\quad 07 / 2019$

Aceite $\quad 03 / 2020$

Publicado 08/2020 
Revista PSICOLOGIA, 2020, Vol. 34 (1), doi: 10.17575/psicologia.v34i1.1681.

Atas do X Simpósio Nacional de Investigação em Psicologia

\title{
"Job Shadowing: Ready, Set... GO!" - Uma intervenção no 3o ciclo do Ensino Básico
}

\author{
Márcia C. G. Rodrigues ${ }^{40 i}$ \& Ester do Carmo M. Câmara ${ }^{\mathrm{i}}$ \\ ${ }^{i}$ Escola Básica do $2^{\circ} 3 \stackrel{0}{ }$ Ciclos do Caniço, Madeira, Portugal
}

\begin{abstract}
Resumo: 0 programa "Job Shadowing: Ready, Set... GO!" pretende apoiar a tomada de decisão dos alunos durante o $3^{\text {o }}$ ciclo, relativamente ao futuro escolar/profissional. Pretende-se: Verificar a confiança para a tomada de decisão; a influência das expetativas parentais nesta decisão e a perceção dos filhos sobre os estilos educativos parentais. Tem a duração de 3 anos e iniciou-se no ano letivo 2017/2018, com 86 alunos do $7^{\circ}$ ano. A avaliação foi realizada com o recurso a pré-teste (7음 ano) e pós-teste ( $9^{\circ}$ ano), relativamente a cada um dos objectivos referidos. Os resultados salientam que a maioria dos alunos revelam confiança para a tomada de decisão. Os pais manifestaram expetativas altas quanto ao futuro dos filhos, e estes sentem que têm espaço necessário para esta decisão. Os dois anos seguintes visam proporcionar aos alunos uma experiência de Job Shadowing, exploração de interesses, competências e valores.
\end{abstract}

Palavras-chave: Orientação escolar e profissional; Percurso académico; Job shadowing; Autoconhecimento.

"Job Shadowing: Ready, Set... GO!" - An intervention for 3rd. cycle of basic education: The "Job Shadowing: Ready, Set... GO!" program aims to support students' decision-making during the 3rd cycle regarding their school or career future. It is intended to: verify the confidence degree for decision making; the influence of parental expectations on this decision and the children's perception of parenting styles. This project has a duration of 3 years. It started in the 2017/2018 school year, with a sample of 867 th students. The evaluation was performed using pre-test (7th year) and post-test (9th year) for each of the above objectives. The results highlight that most of the students show a secure degree of confidence to make their decision. Parents expressed high expectations about their children's future and they feel their parents gave them the necessary space for this decision. The next two years aim to provide students with a job shadowing experience, exploration of interests, skills and values.

Keywords: School and professional guidance; Academic pathway; Job shadowing; Self-knowledge.

No final do 9o ano, os jovens enfrentam a sua primeira tomada de decisão, obrigando-os à reflexão acerca do seu projeto de vida. A preparação para este momento deve iniciar-se o mais cedo possível, fomentando o autoconhecimento, a exploração e ponderação da decisão (Abreu, 1996), desenvolvendo a Perspetiva Temporal de Futuro (PTF) (Carvalho, 2007). Esta engloba caraterísticas como a motivação e a cognição e refere-se à criação de objetivos e projetos de futuro (Carvalho, 2012/2015; Silva, Carvalho, \& Pocinho, 2010).

A orientação vocacional em contexto escolar foi aprofundada por Jesse Davis (Aubrey, 1977, citado por Ribeiro \& Uvaldo, 2007) e possibilita a autodescoberta e a exploração de alternativas em momentos cruciais de tomada de decisão (Carvalho, 2007). Ocorre em comunhão com o contexto, privilegiando uma abordagem ecológica e contextual, considerando a família, escola, pares, comunidade e serviços de apoio especializado (Bronfenbrenner, 1977).

O conhecimento experimental assume um papel central, pois, quando associado a currículos académicos, os efeitos a longo prazo são notórios (Arrigton, 2000; Kazis, 2016).

o Job Shadowing permite aos estudantes acompanhar o quotidiano de um trabalhador de uma área de interesse, durante determinado tempo (Arrington, 2000; Service, Dalgic, \& Thornton, 2017), observando caraterísticas essenciais para a profissão (Blake \& Stalberg, 2009) e aprendendo competências transversais: comunicação, trabalho em equipa e pensamento crítico (Arrington, 2000).

${ }^{40}$ Morada para correspondência: Márcia C. G. Rodrigues, Rua João Batista de Sá, 9125-017-Caniço, Santa Cruz, Caniço. E-mail: marciarmarques@hotmail.com 
Em Portugal, este procedimento tem vindo a conquistar a sua posição no domínio da Orientação Vocacional. Os programas "Orientação para a Carreira (POC)" (Silva, Carvalho \& Pocinho, 2010) e "\# Tagga o teu futuro: Desafios sem Barreiras" (EDP, 2016), constituíram a base deste projeto.

A tomada de decisão é influenciada por diferentes contextos do meio relacional do indivíduo. Saliente-se a inegável influência da escola, nomeadamente do docente através da relação afetiva que estabelece com os alunos (Carvalho, 2007).

O Serviço de Psicologia e Orientação possibilita a mobilização de recursos individuais, ajudando na exploração de interesses e no desenvolvimento pessoal para a carreira (Carvalho, 2007; Faria, Königstedt, \& Taveira, 2011).

A família, especificamente os pais, têm um papel essencial nas decisões de carreira, sendo privilegiada a sua inserção na intervenção (Faria, 2008).

Por fim, os pares, pois a partir da opinião destes, o jovem constrói uma imagem pessoal, que influencia as suas relações (Carvalho, 2007).

\section{MÉTODO}

\section{Justificação da Ação}

Explorar desde cedo as opções e promover o autoconhecimento está na base da construção deste projetopiloto, com a duração de 3 anos, correspondentes ao 3o ciclo do Ensino Básico. 0 presente artigo reporta os resultados obtidos no primeiro ano de implementação, o ano letivo 2017/2018.

\section{Objetivos e Hipóteses da Investigação}

Objetivos gerais. a) Promover a maturidade vocacional e a perspetiva temporal de futuro; b) Desenvolver competências vocacionais e de promoção do sucesso académico;

Objetivos específicos. a) Verificar a confiança para a tomada de decisão; b) Verificar a influência das expetativas parentais na tomada de decisão; c) Adquirir a perceção dos filhos sobre os estilos educativos parentais; d) Proporcionar experiências reais do exercício da profissão de interesse; e) Promover a exploração da oferta formativa; f) Avaliar interesses, aptidões e valores profissionais.

\section{Participantes}

A amostra é de conveniência, selecionada mediante os seguintes critérios: 1) Matrícula efetuada no estabelecimento de ensino onde foi aplicado o projeto; 2) Frequência do 70 ano; 3) Não abrangência por outros projetos. Emergiram 4 turmas de $7^{\circ}$ ano, que perfaz um total de 86 alunos.

Tabela 1. Caraterização da amostra segundo o sexo, idade, naturalidade e histórico de reprovação

\begin{tabular}{lc}
\hline Sexo: & $\%$ \\
Masculino & 51.20 \\
Feminino & 48.80 \\
Idade: & 60.70 \\
$\mathbf{1 3}$ & 25.00 \\
$\mathbf{1 4}$ & 8.30 \\
$\mathbf{1 5}$ & 1.20 \\
$\mathbf{1 6}$ & 86.90 \\
Naturalidade: & 8.30 \\
Funchal & 2.40 \\
Venezuela & 1.20 \\
Inglaterra & \\
Porto & 82.10 \\
Histórico de reprovação: & 14.30 \\
Reprovou algum ano & Não reprovou nenhum ano \\
\hline
\end{tabular}


De acordo com a Tabela $1,51.20 \%$ dos alunos são do sexo masculino e $48.80 \%$ do sexo feminino, a maioria com 13 anos de idade (60.70\%). Quanto à naturalidade, $86.90 \%$ é natural do Funchal, seguindose Venezuela (8.30\%), Inglaterra (2.40\%) e Porto (1.20\%). Relativamente ao histórico de reprovação, $82.10 \%$ dos alunos nunca reprovou (ver Tabela 1 ).

\section{Recolha de dados}

Será contínua e multicontextual, com o recurso a pré-teste e pós-teste para avaliar a maturidade para a tomada de decisão. 0 Questionário Pré-teste (Calado, 2009) de elevada consistência interna (coeficientes alfa de .93 e .95., no pré e pós-teste), compõem-se de 22 itens, respondidos mediante uma escala tipo Likert de 5 pontos (1: Nada Confiante; 5: Muito Confiante).

As perceções dos filhos sobre os estilos educativos parentais foram avaliadas mediante a Escala de Estilos Educativos Parentais (Barros de Oliveira, 1994). Avalia a perceção dos filhos relativamente ao estilo educativo dos pais, mediante dois fatores: Exigência e Responsividade. É composta por 19 itens, respondidos através de uma escala likert de 5 pontos (1: "Totalmente em desacordo"; 5: "totalmente de acordo"). Possui boa consistência interna e níveis altos de fidelidade.

Para avaliar as expectativas parentais face ao desempenho dos filhos, utilizou-se o questionário "0 percurso escolar do meu filho" (Lopes, 2010). É composto por 6 questões, 3 de resposta aberta e 3 de resposta múltipla.

Com uma periodicidade anual, os alunos responderão a um questionário de satisfação, construído com base na revisão da literatura sobre o tema. Composto por 5 itens, um de resposta aberta e quatro de resposta múltipla.

\section{Procedimento}

Construção do programa. Após a revisão teórica, foram tidas em conta as principais linhas e tarefas orientadoras da intervenção. No primeiro ano, foram abordadas as temáticas da autoestima, autoeficácia, autoconhecimento e importância da tomada de decisão informada e consciente. No segundo ano, serão abordados o planeamento de carreira, barreiras percecionadas, preparação para a experiência de Job Shadowing, e avaliação póstuma da mesma. 0 terceiro ano dedicar-se-á à avaliação de competências, interesses, valores e caraterísticas de personalidade e à exploração da oferta formativa.

Formação aos diretores de turma. Efetuaram-se várias reuniões com os diretores de turma, indicando linhas orientadoras da intervenção, fornecendo material e estratégias de divulgação dos conteúdos. 0 programa iniciou-se no $2^{\mathrm{o}}$ período, com uma reunião com os encarregados de educação, para adquirir a autorização da participação dos educandos e informá-los do projeto. Este foi dinamizado nas aulas de Formação Pessoal e Social, semanalmente (45 minutos). 0 tratamento de dados foi realizado com o programa Statistical Package for Social Sciences (SPSS), versão 25, com um nível de confiança de 95\%.

\section{RESULTADOS E DISCUSSÃO}

\section{Confiança para a tomada de decisão}

Aquando do tratamento do questionário de pré-teste, procedeu-se a uma análise de conteúdo para facilitar a reprodução dos resultados. Agruparam-se as questões em função de: "Confiança para a tomada de decisão" (ver Figura 1) e "Dificuldades Percecionadas" (ver Figuras 2 e 3). 


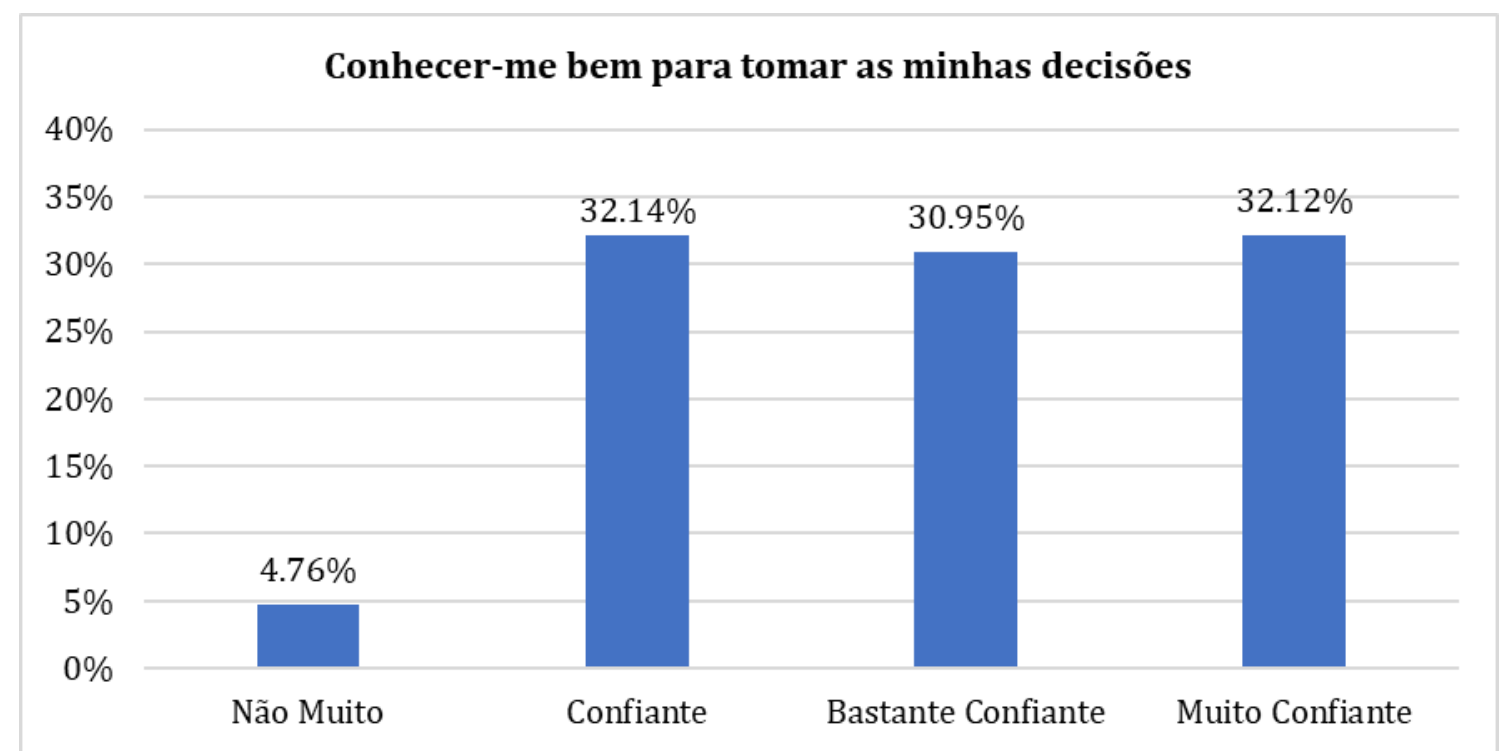

Figura 1. Autoconhecimento e tomada de decisão (Questionário de pré-teste)

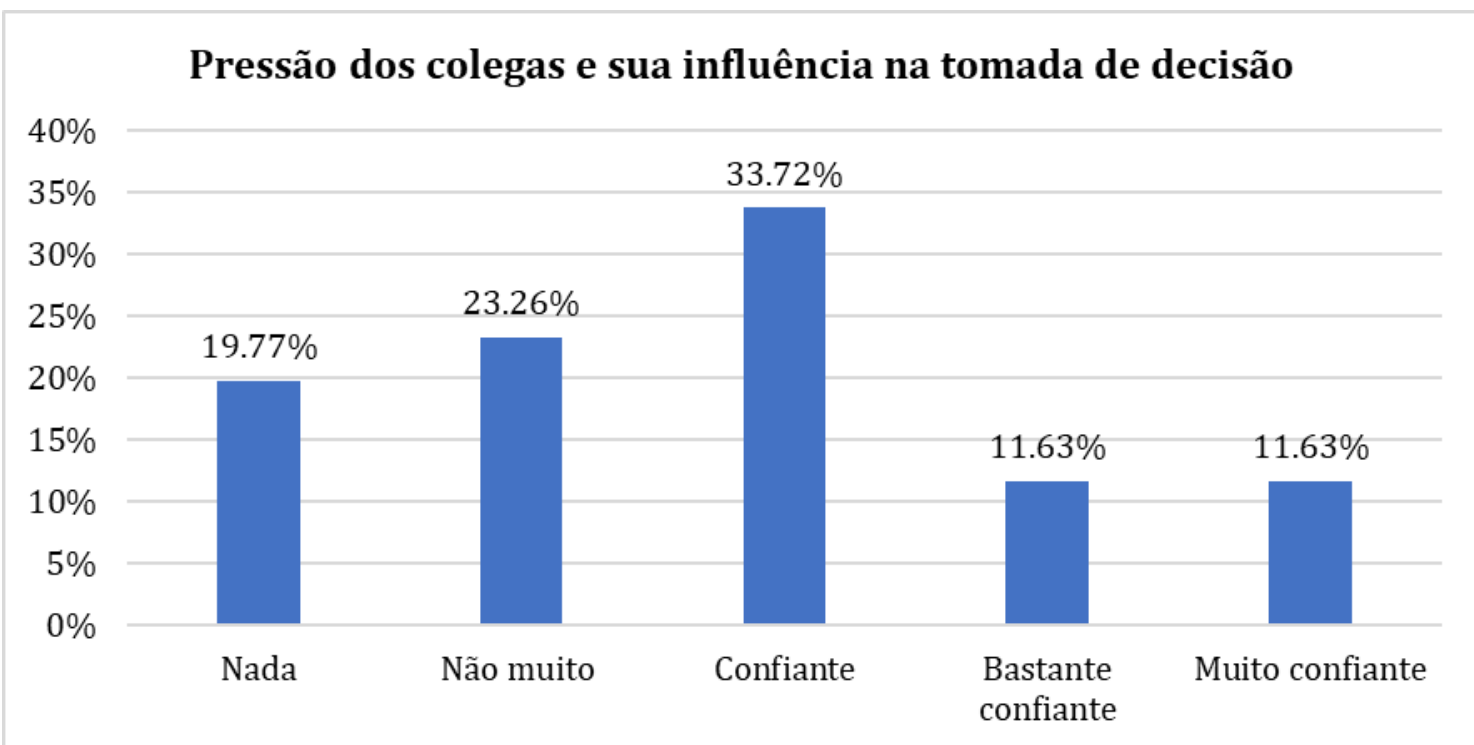

Figura 2. Influência dos pares na tomada de decisão (Questionário de pré-teste)

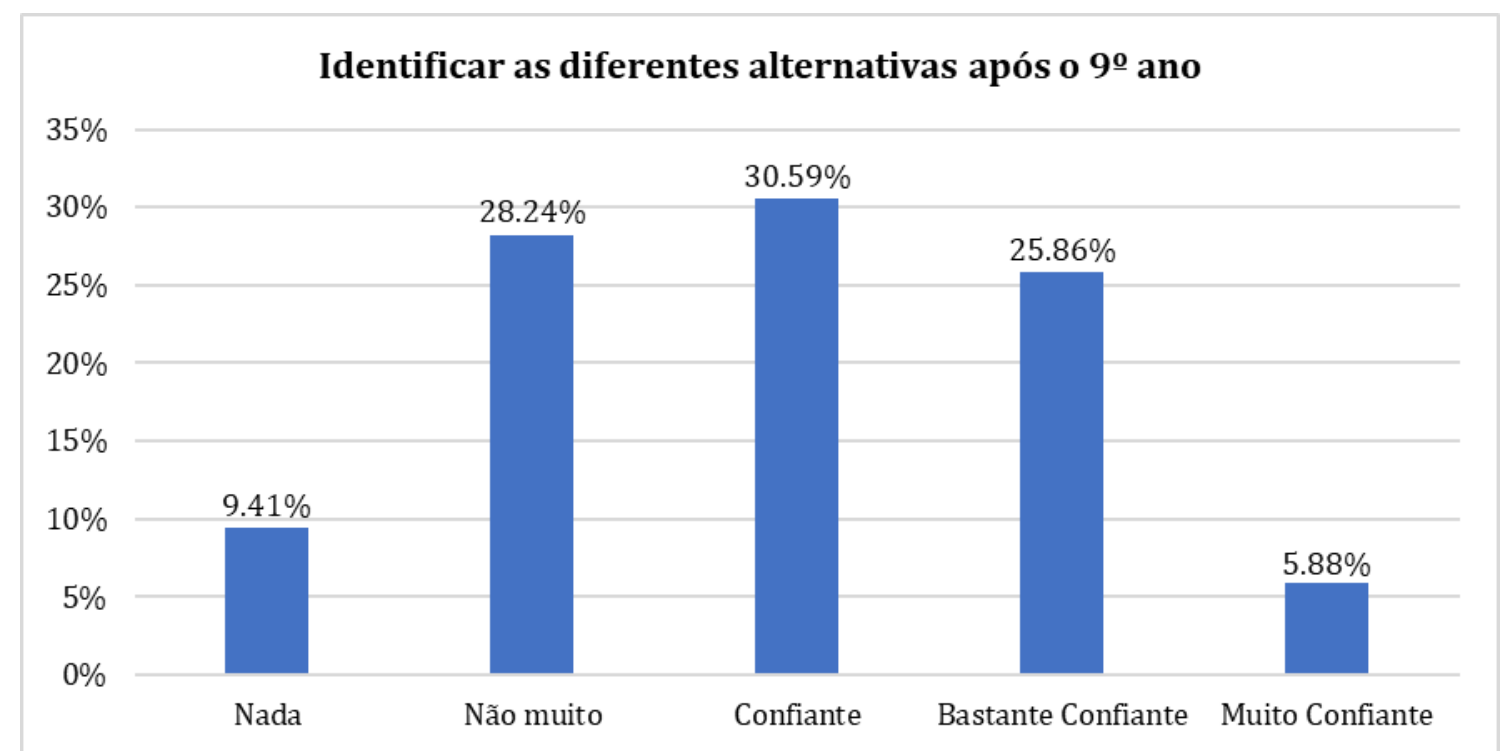


Figura 3. Identificação de alternativas após o 9a ano (Questionário de pré-teste)

Relativamente à Figura 1,63.09\% dos alunos sente-se confiante para a tomada de decisão. As figuras 2 e 3 salientam que $43.03 \%$ dos alunos manifesta inseguranças relativas à pressão interpares (ver Figura 2), e $37.65 \%$ sente dificuldades relativamente à identificação das alternativas após o 9o ano (ver Figura 3).

Conforme Fouad e colaboradores (2016), as dificuldades percecionadas ao tomar uma decisão prendem-se com o vasto leque de opções, a inadequação destas às preferências pessoais e a falta de preparação para este momento. A informação que os estudantes adquirem pode ser percecionada como insuficiente. Desenvolver a consciência de carreira e proporcionar experiências de exploração são duas vias que permitem formar planos realísticos (Arrington, 2000; Krieshok, Black, \& McKay, 2009).

Um dos fatores associados à variação do grau de confiança para a tomada de decisão é o sexo do aluno. Procedeu-se a esta análise quanto à confiança para a tomada de decisão (ver Figura 4) e dificuldades percecionadas (ver Figuras 5 e 6).

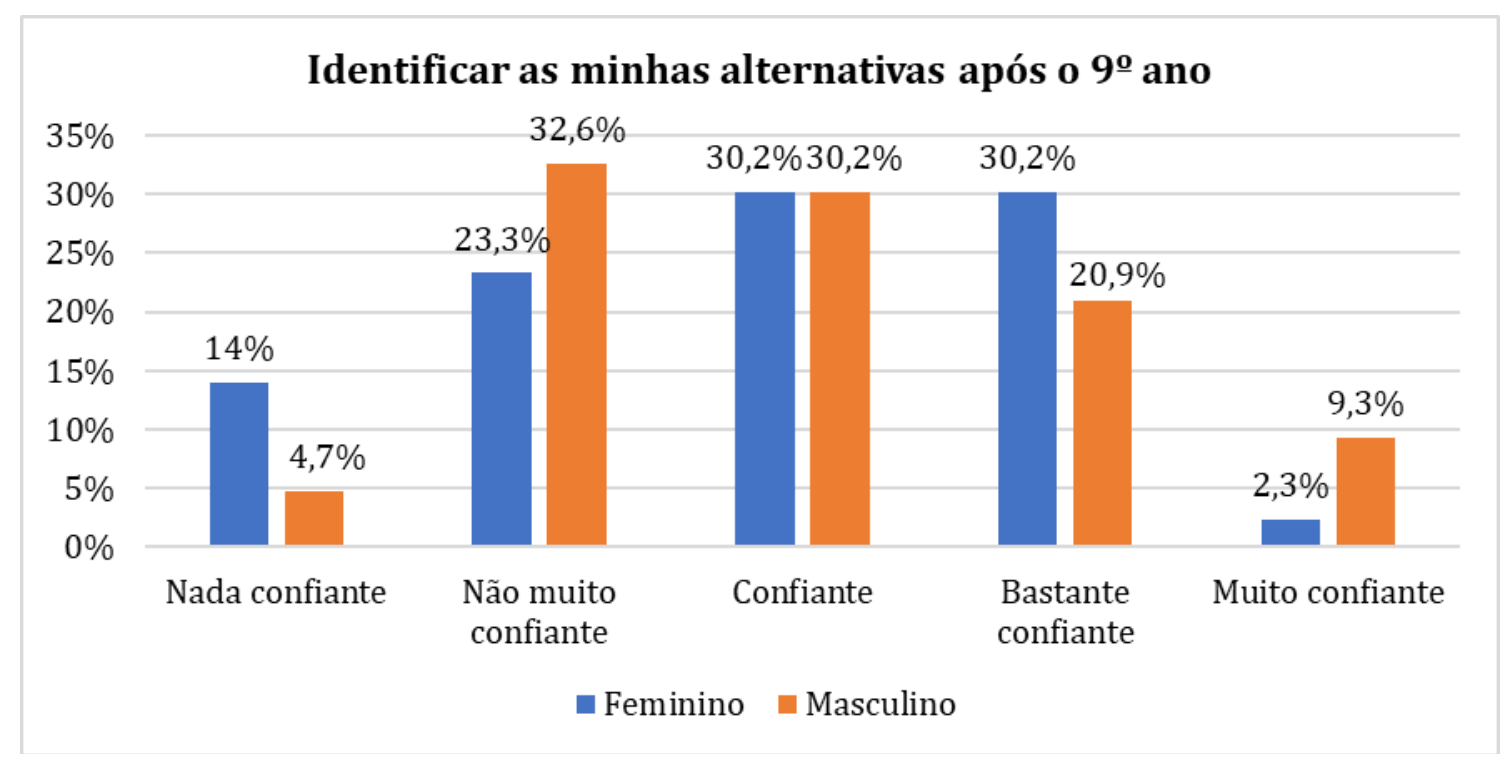

Figura 4. Confiança para a tomada de decisão e sexo

Os rapazes demonstraram mais confiança na tomada de decisão, ao pontuar nas categorias associadas a graus de confiança positiva. Este resultado vai ao encontro de Pocinho, Correia, Carvalho e Silva (2010), em que os rapazes apresentaram níveis de confiança para a tomada de decisão superiores. Pelo contrário, Figueira (2012) refere que as meninas apresentam maior capacidade de refletir sobre o desenvolvimento da carreira, conferindo-lhes maior confiança para a tomada de decisão.

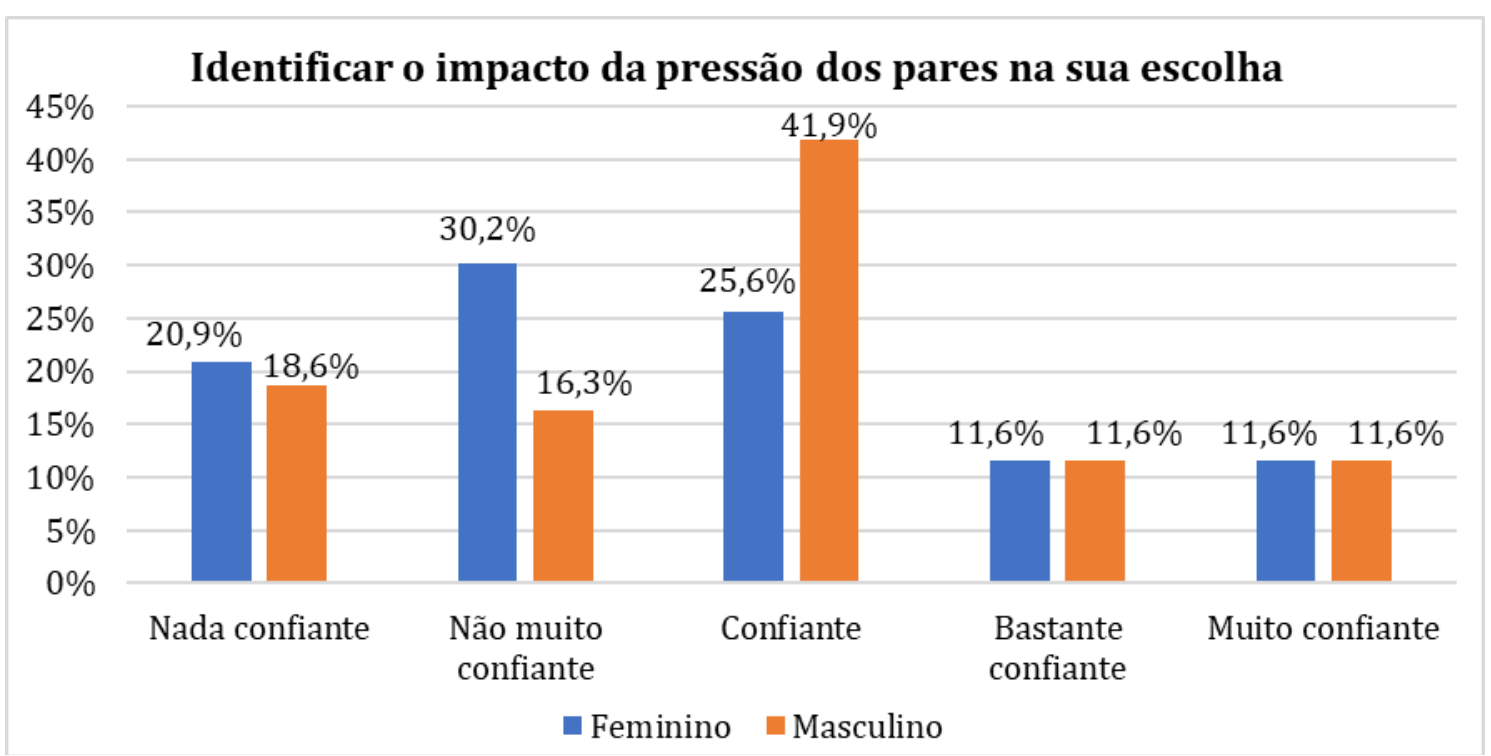

Figura 5. Identificar as alternativas após o 9oano e sexo 
A figura demonstra uma homogeneidade no sexo, referente à capacidade de identificar as alternativas após o 9o ano. Procedeu-se à união das categorias "Nada confiante" e "Não muito confiante", verificando-se que os rapazes $(39,30 \%)$ mostram menos confiança que as raparigas $(35,3 \%)$. Estes resultados corroboram os de Jorge (2011), em que as raparigas apresentam maior capacidade de planeamento e procura de informação.

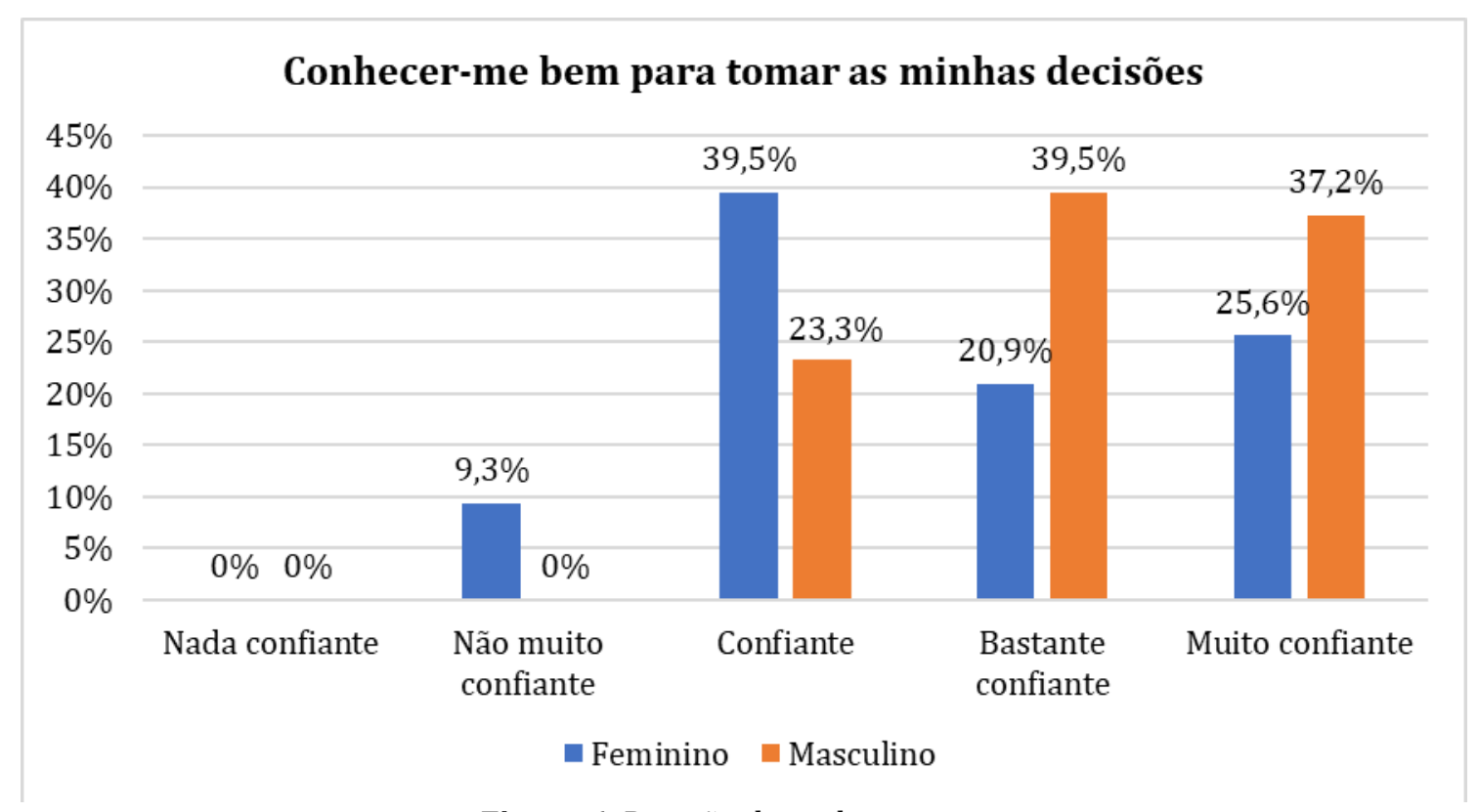

Figura 6. Pressão dos colegas e sexo

No geral, as raparigas apresentam uma maior dificuldade em identificar a pressão dos pares na tomada de decisão, com valores superiores em "Nada confiante" $(20,90 \%)$ e "Não muito confiante" $(30,20 \%)$. Isto salienta que as raparigas podem demonstrar maior preocupação em identificar as barreiras ao seu percurso. Em Fernandes (2018), as raparigas também apresentaram maiores níveis de preocupação relativamente à tomada de decisão do que os rapazes, contrastando com Pocinho e colaboradores (2010) que referem que os rapazes revelam menos prontidão e têm maior acesso a informações, passíveis de influência por terceiros.

\section{Expetativas parentais e sua influência nas escolhas profissionais dos filhos}

As figuras 7 e 8 referem-se às expetativas parentais quanto ao prosseguimento dos estudos para o ensino superior e/ou entrada no mercado de trabalho, após o $12^{\circ}$ ano de escolaridade (ver Figura 7) e área privilegiada na universidade (ver Figura 8).

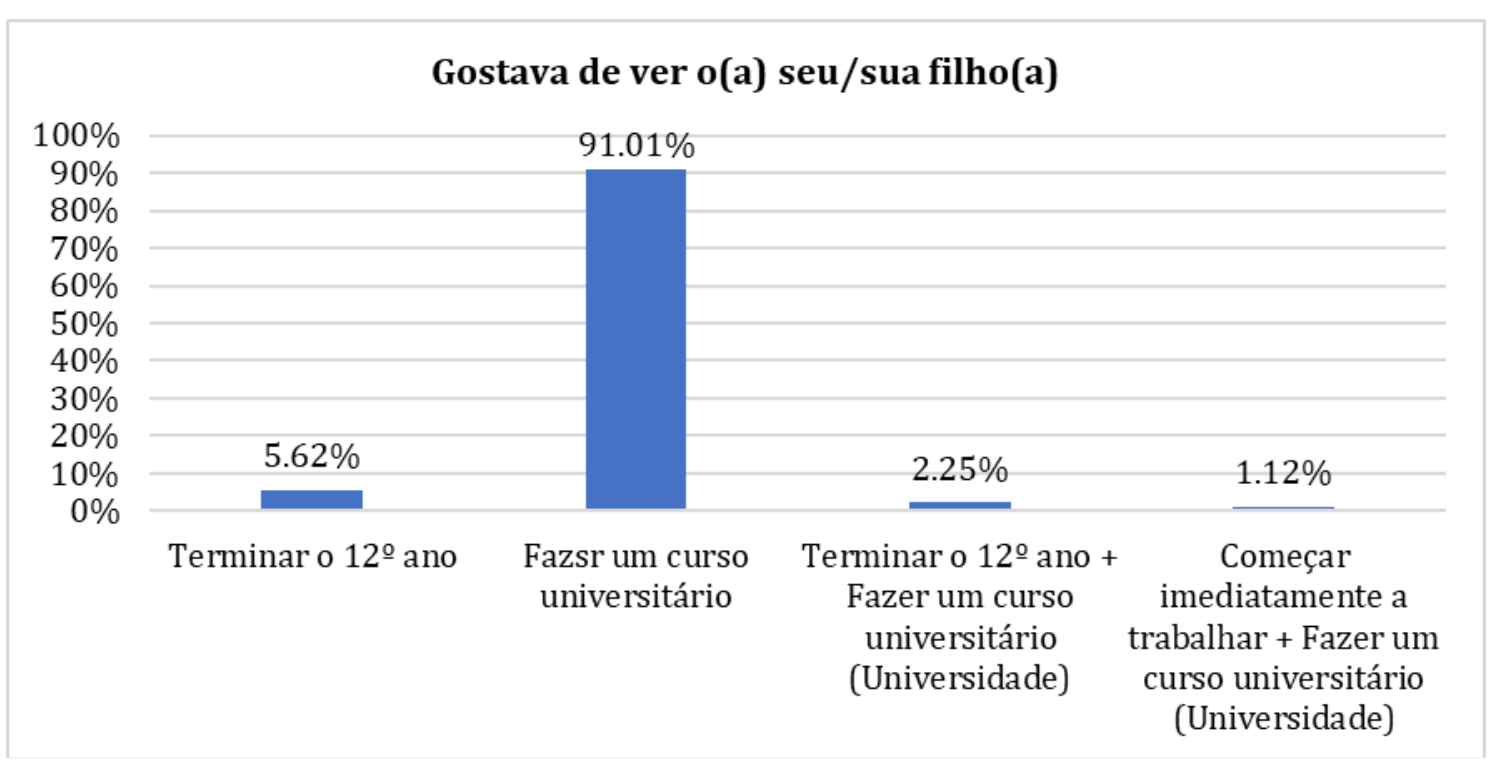

Figura 7. Expetativas parentais sobre a tomada de decisão 


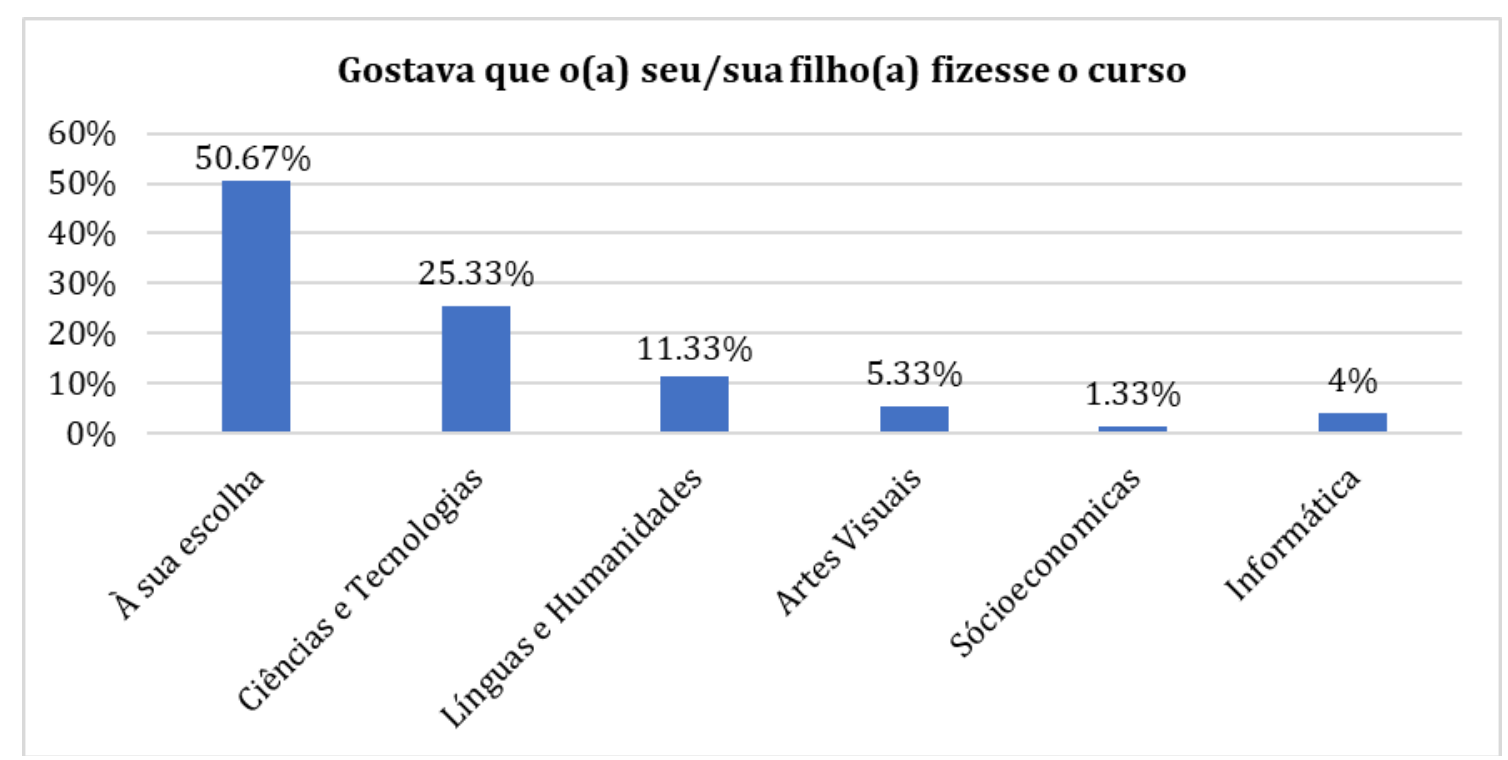

Figura 8. Expetativas parentais sobre o curso escolhido pelos filhos.

Verifica-se que $91.01 \%$ dos pais gostava de ver os filhos na universidade (ver Figura 7), numa área que os faça felizes e realizados (45.00\%) (ver Figura 8). Estes resultados corroboram Lopes (2010), onde a maioria dos pais manifesta o desejo de que os filhos frequentem o ensino superior (80\%), numa área à sua escolha (60\%). Assim, os pais exercem influência no desenvolvimento vocacional dos filhos, que tendem a escolher profissões que correspondam às suas expectativas (Helwig, 1998; Watson \& McMahon, 2005, citados por Araújo, 2009).

Para perceber o papel dos pais nas diferentes turmas, procedeu-se à análise das médias relativamente à expetativa parental do futuro escolar e/ou profissional dos filhos (ver Figura 9) e área correspondente (ver Figura 10).

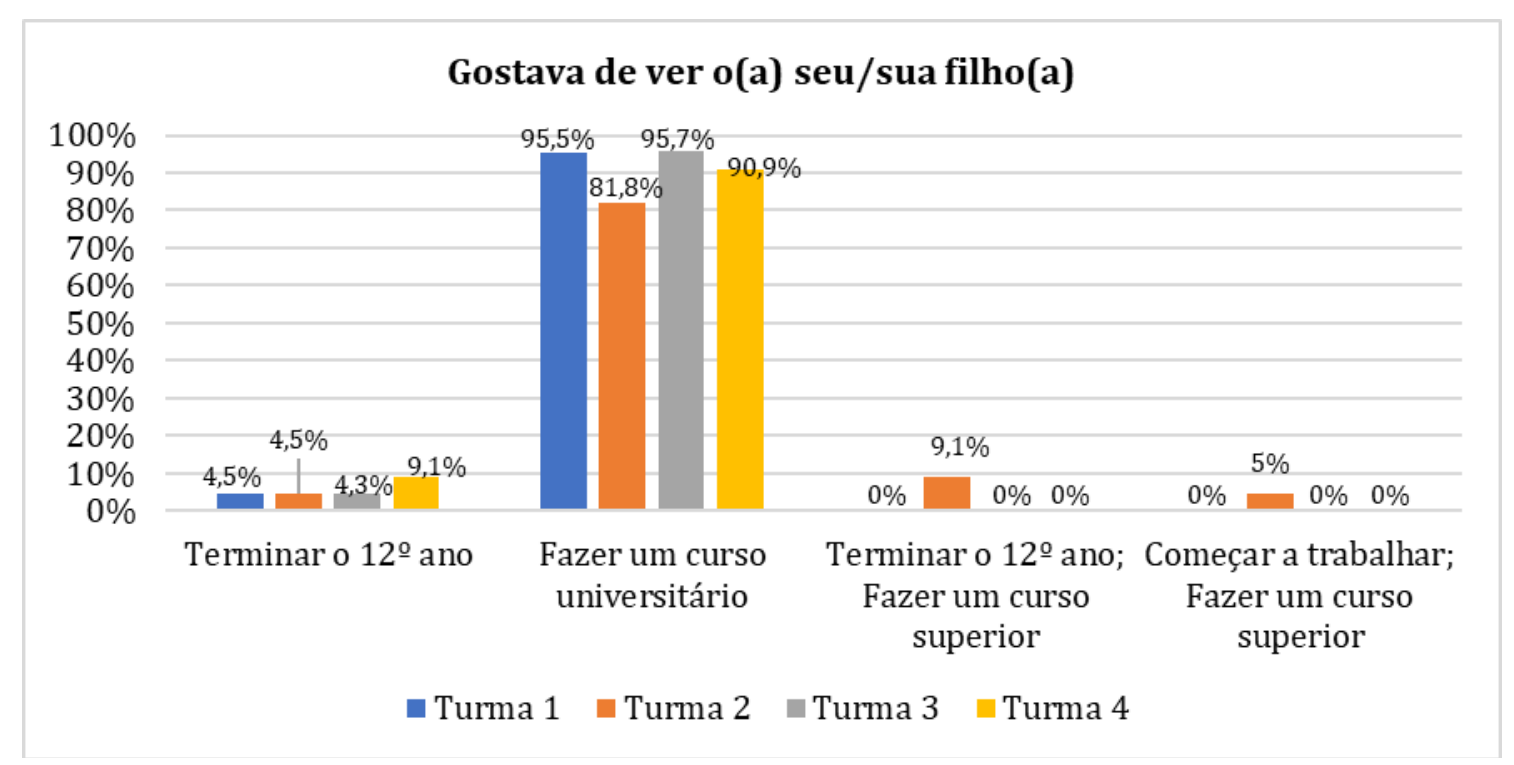

Figura 9. Expetativas parentais nas diferentes turmas. 


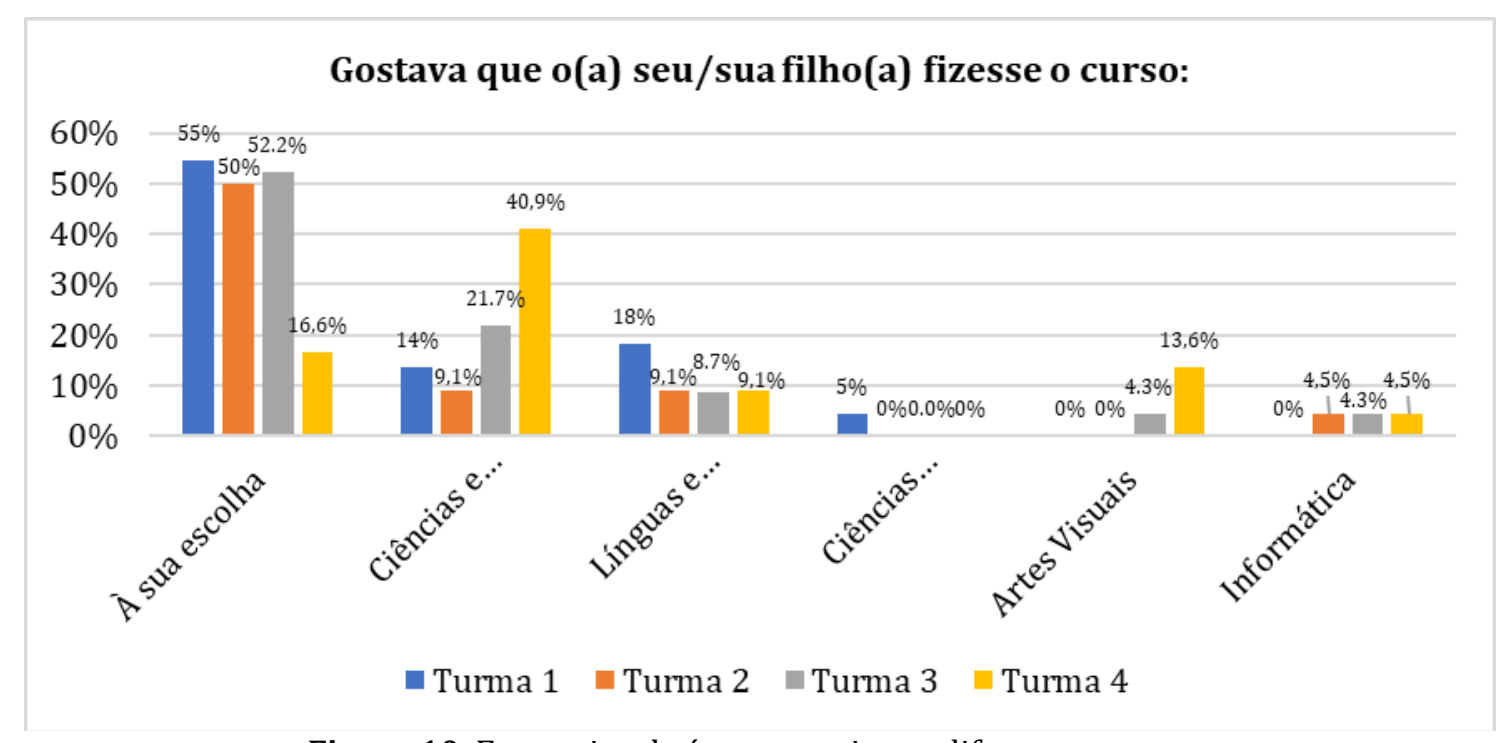

Figura 10. Expetativa de área a seguir nas diferentes turmas.

É evidente a expetativa dos pais das quatro turmas em que os filhos frequentem o ensino superior, especialmente turma 2 (ver Figura 9).

$\mathrm{Na}$ figura 10, os pais das turmas 1, 2 e 3 salientam que o curso a escolher para o ensino secundário seja realizado numa área à sua escolha. Na turma 4, a maioria dos pais gostava que os filhos prosseguissem o curso de Ciências e Tecnologias (40,9\%).

Desta forma, é evidente o impacto fulcral da família no processo de exploração de carreira, funcionando como orientadora e facilitadora (Faria, 2008; Pinto \& Soares, 2001).

\section{Estilos Educativos Parentais - Perspetiva dos filhos}

As figuras 11, 12 e 13 referem-se às expetativas dos filhos, referente à: 1) Exigência, ao nível da compreensão/rigor na educação (ver Figura 11); 2) Supervisão, relativamente ao amor (ver Figura 12) e ao respeito pelas suas ideias (ver Figuras 13).

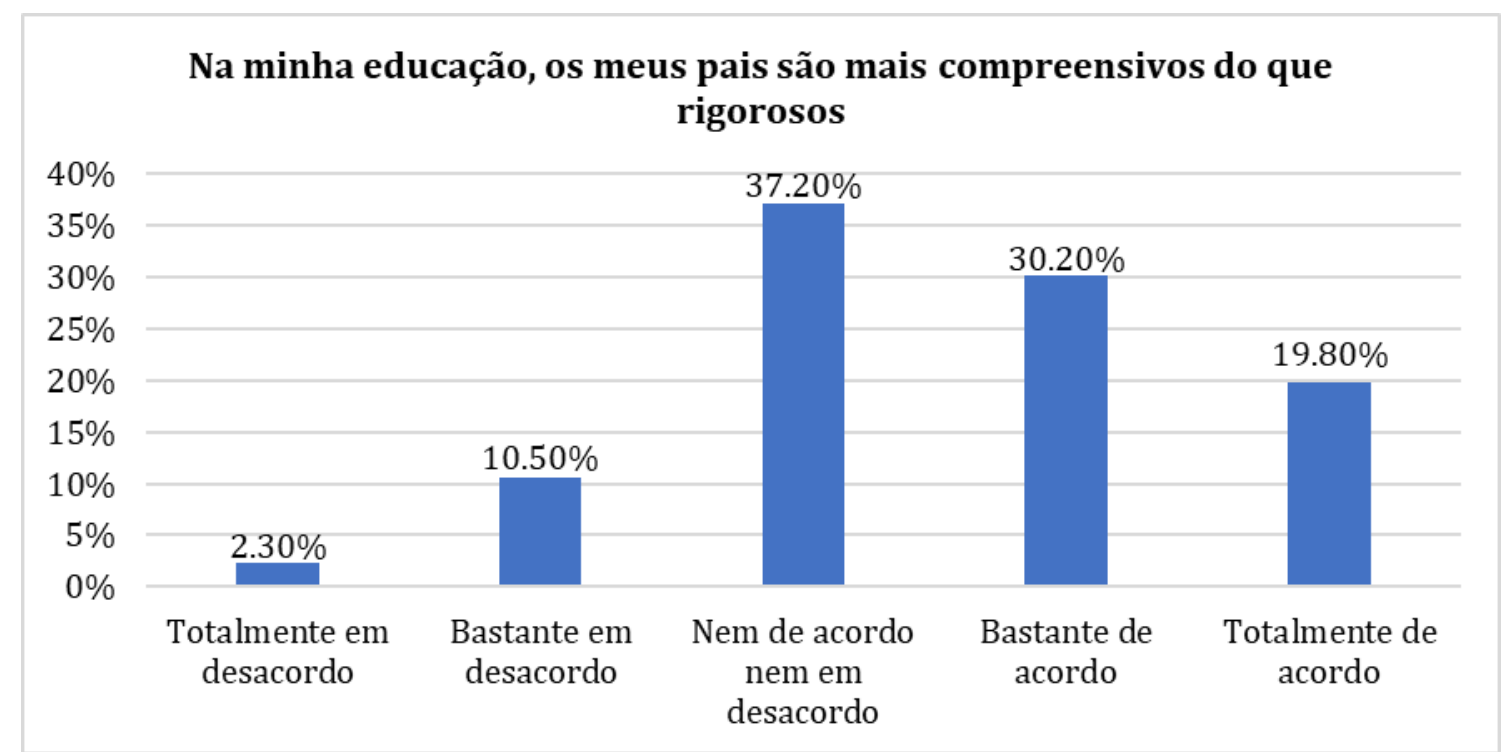

Figura 11. Expetativas dos filhos sobre a compreensão e rigor na educação. 


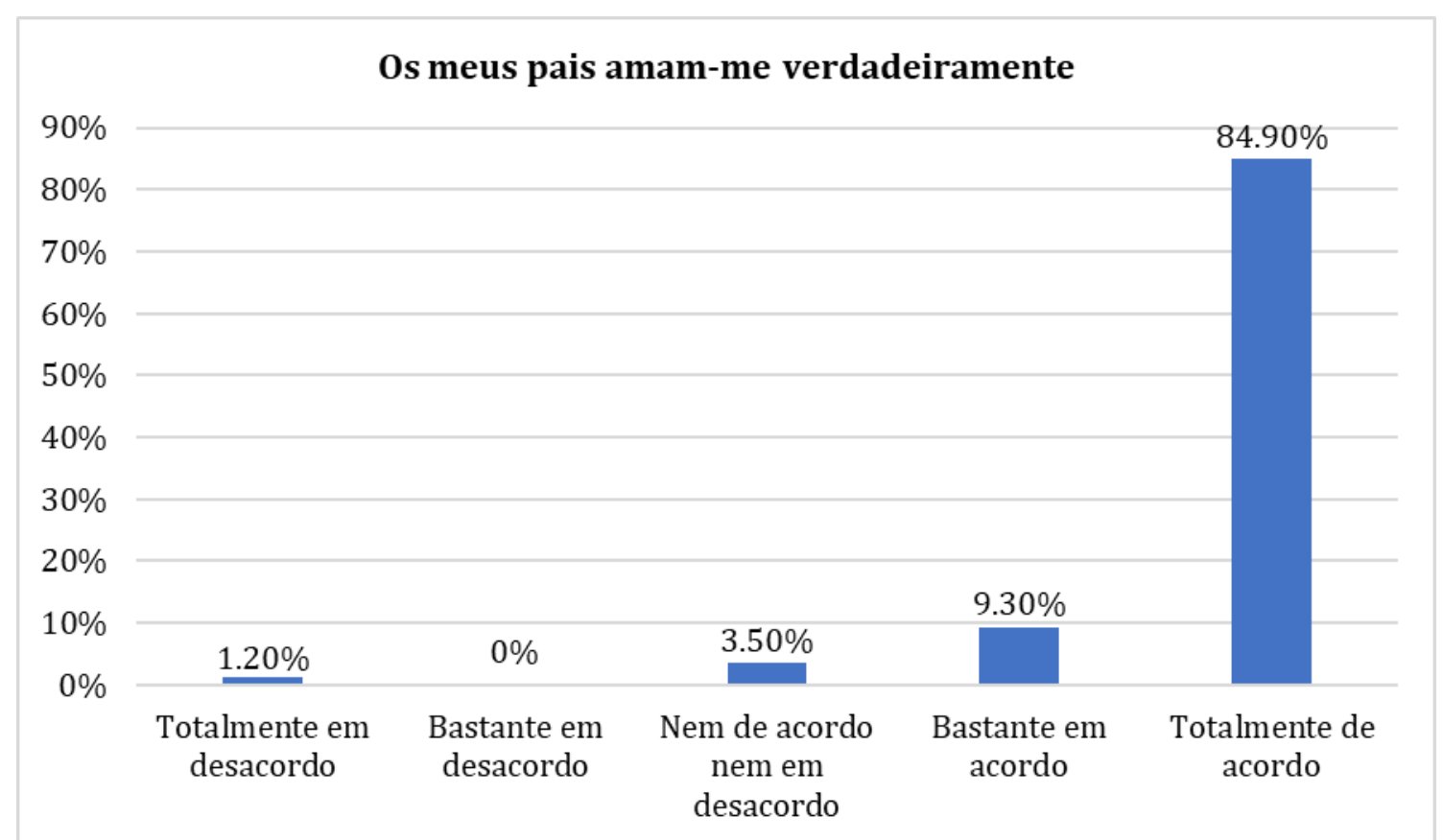

Figura 12. Expetativas dos filhos sobre o amor na educação.

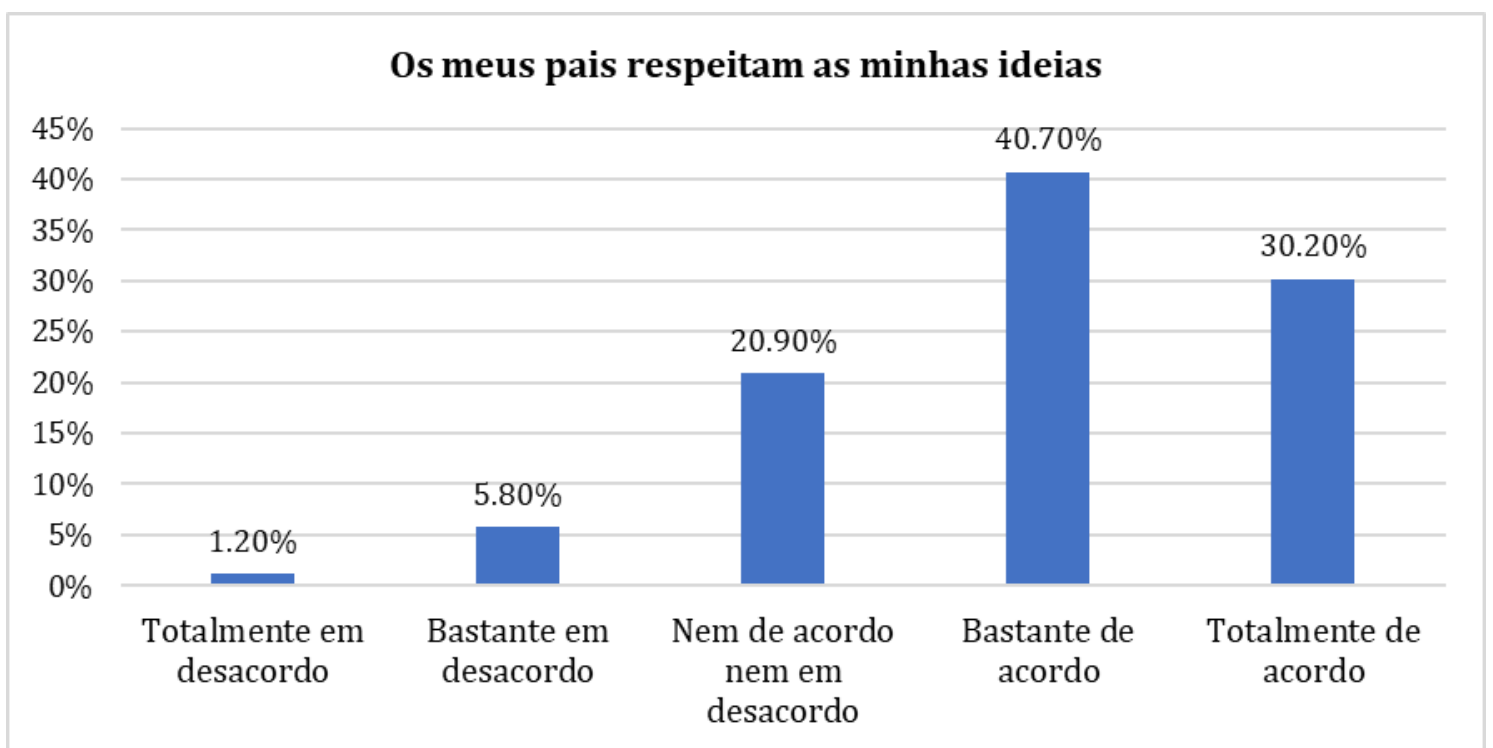

Figura 13. Expetativas dos filhos sobre o respeito dos pais pelas suas ideias.

As figuras anteriores mostram que os jovens sentem o apoio dos familiares para a prossecução dos planos futuros, sendo que $50 \%$ salientam que os pais são mais compreensivos do que rigorosos na educação (ver Figura 11). Quanto à supervisão, 94.20\% denotam que os pais os amam verdadeiramente (ver Figura 12) e $70.90 \%$ sente que estes respeitam as suas ideias (ver Figura 13). 0 suporte familiar surge como a alavanca para que os jovens se sintam seguros na tomada de decisão. Também Ducharne, Cruz, Marinho e Grande (2006), no estudo de atualização da Escala de Estilos Educativos Parentais, verificaram $45.40 \%$ dos adolescentes perceciona a atitude dos pais altamente responsiva e supervisora.

\section{Grau de satisfação com a intervenção}

Para adquirir o impacto do programa nas diferentes turmas, procedeu-se à análise do aconselhamento do programa aos amigos (ver Figura 14), motivos para tal (ver Figura 15) e grau de satisfação com o programa (ver Figura 16). 


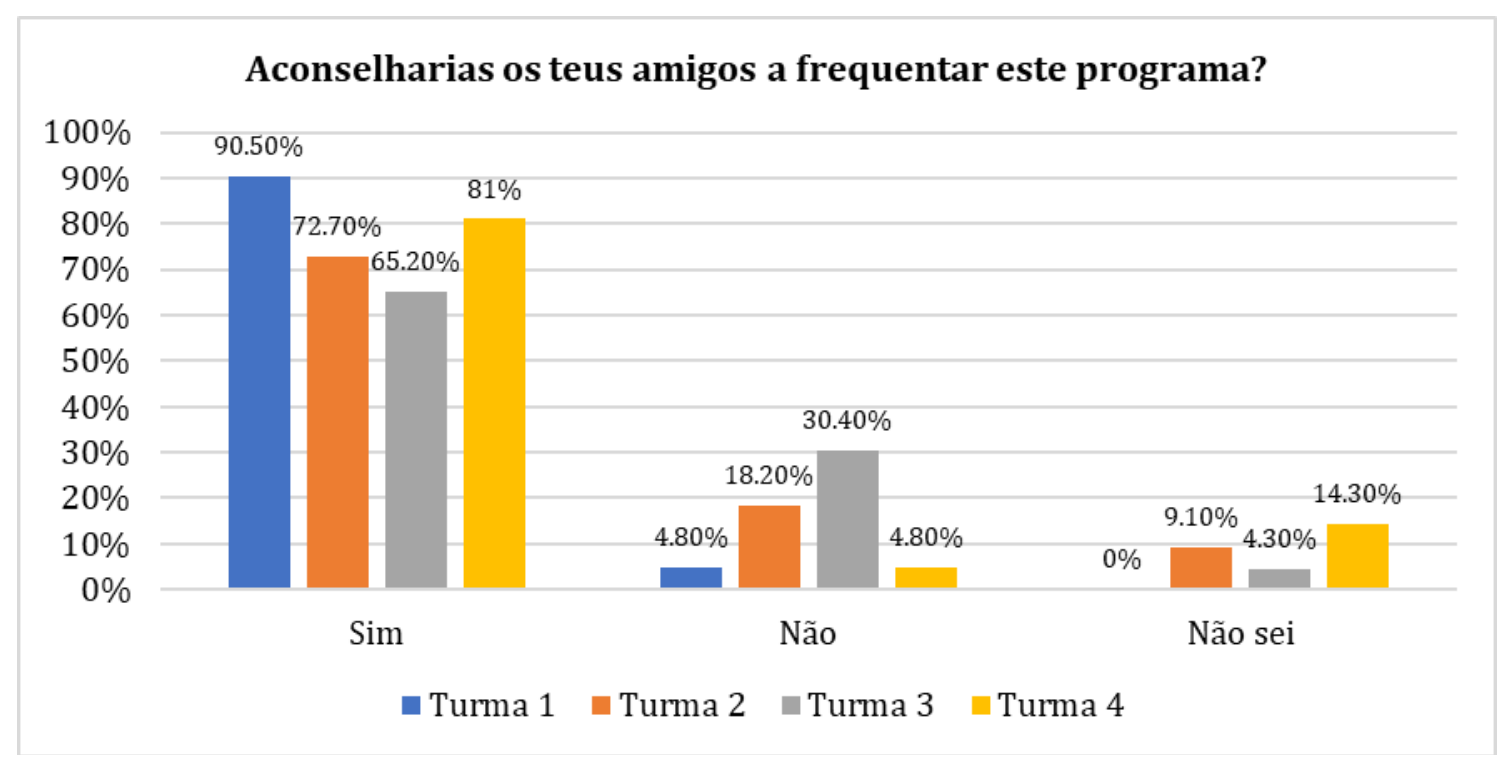

Figura 14. Aconselhamento do programa nas diferentes turmas.

Todas as turmas aconselhariam o programa aos amigos. A turma 1 é a que mais se salientou nesta questão. Pelo contrário, é na turma 3 que surgem as opiniões mais negativas, sendo que foi nesta turma que se registou um maior número de casos que não aconselharia o programa.

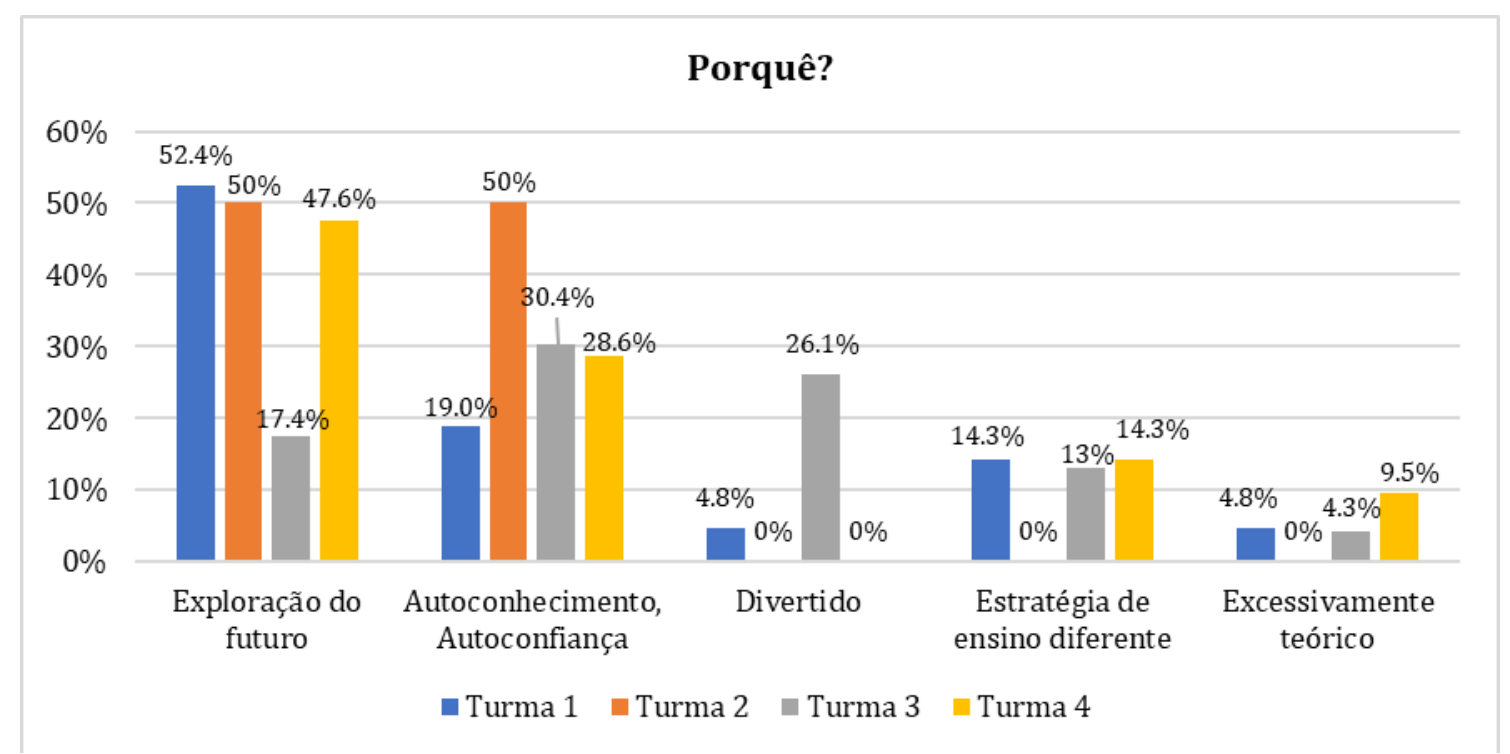

Figura 15. Motivos para aconselhamento do programa nas diferentes turmas.

Todas as turmas aconselhariam o programa pela oportunidade de exploração do futuro, autoconhecimento e autoconfiança. Os estudantes aproveitam estes programas para adquirir mais conhecimentos sobre os seus pontos fortes/fracos, denotando a sua importância no desenvolvimento vocacional (English, 2018). 


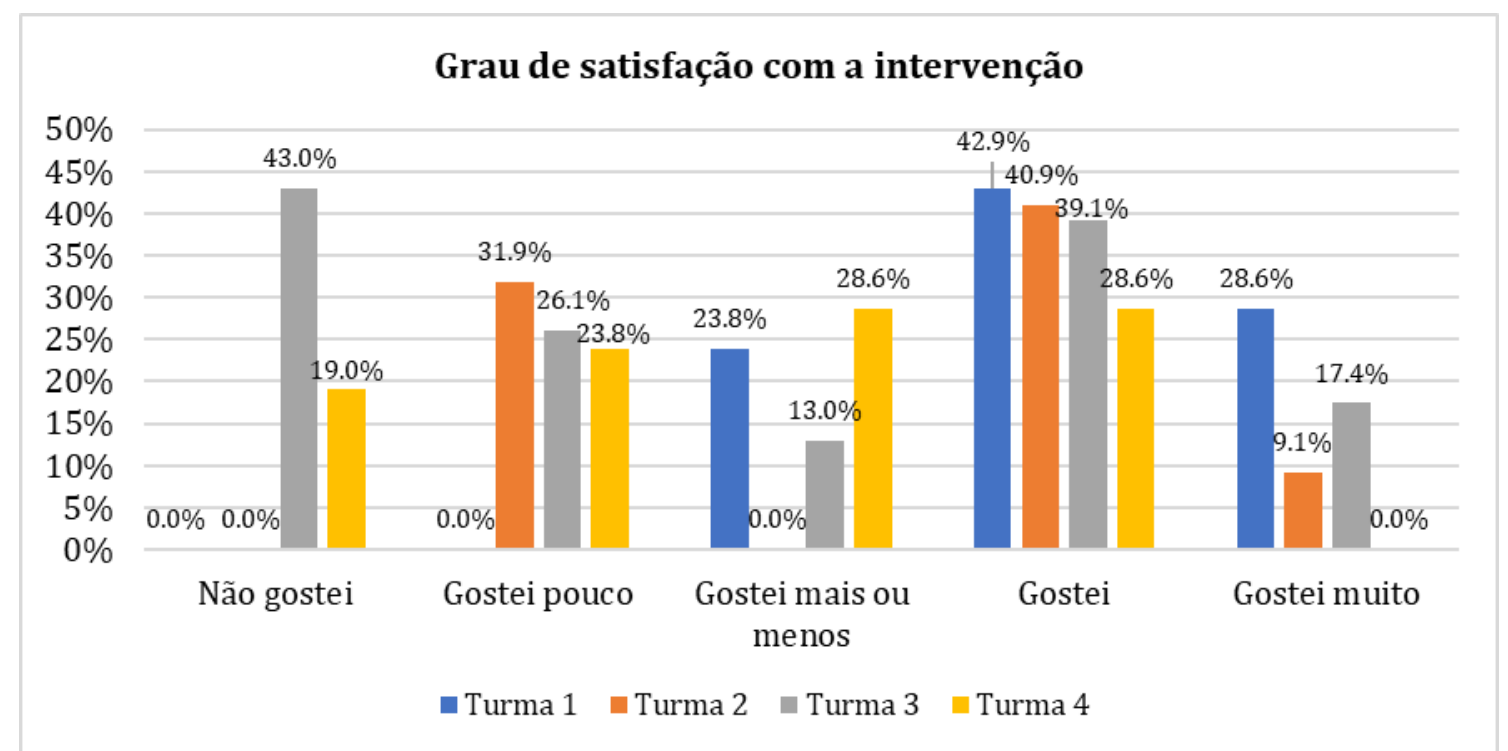

Figura 16. Grau de satisfação com a intervenção nas diferentes turmas.

Todas as turmas gostaram do primeiro ano da intervenção. Porém, é na turma 4 que se revelam os resultados mais negativos, salientando a necessidade de adotar estratégias distintas perante diferentes públicos. Qualquer impacto de uma intervenção é sempre positivo, porque ao se identificarem ou não com determinadas atividades, estratégias, estão a criar a sua própria identidade (English, 2018).

\section{CONCLUSÃO}

É notório o potencial destas intervenções no desenvolvimento da maturidade vocacional, permitindo o maior ganho de informações, o aumento da autoestima, autorrealização e valorização pessoal (Ornelas, 2017). Seguidamente, salienta-se a pertinência do trabalho paralelo com os pais, dotando-os de ferramentas que lhes permitam ser um pilar seguro na tomada de decisão. A oportunidade de realizar uma experiência laboral permitirá aos alunos uma maior consciencialização para o mercado de trabalho (Silva, Carvalho, \& Pocinho, 2010), e para a existência de uma multiplicidade de competências para além das técnicas (socioemocionais, pessoais e relacionais) (Silva, Carvalho, \& Pocinho, 2010; Figueira, 2012). Preparar os jovens para os desafios vindouros é um processo preventivamente planeado, antecipado e aplicado.

\section{REFERÊNCIAS}

Abreu, M. V. (1996). Pais, professores e psicólogos. Contributos para o desenvolvimento de uma prática relacional nas escolas. Coimbra Editora.

Araújo, A. (2009). Antecedentes, Dinâmica e Consequentes do Desenvolvimento Vocacional na Infância [Tese de Doutoramento, Universidade do Minho].

Arrington, K. (2000). Middle grades career planning programs. Journal of Career Development, 27(2), 103109. https://doi.org/10.1177/089484530002700204

Barros de Oliveira, J. H. (1994). Psicologia da Educação Familiar. Almedina.

Blake, K., \& Stalberg, E. (2009). Me and my shadow: observation, documentation, and analysis of serials and electronic resources workflow. Serials Review, 35(4), 242-252. https://doi.org/10.1016/j.serrev.2009.08.018

Bronfenbrenner, U. (1977). Toward an experimental ecology of human development. American Psychologist, 32(7), 513-531. https://doi.org/10.1037/0003-066X.32.7.513.

Calado, I. S. (2009). Um programa de planeamento de carreira para estudantes do 9o ano de escolaridade [Dissertação de mestrado não publicada, Universidade de Lisboa].

Carvalho, R. G. (2007). Perspetiva temporal de futuro em contexto educativo: Um estudo com estudantes do 9o ano de uma escola rural da Madeira [Dissertação de Mestrado não publicada, Universidade Aberta].

Carvalho, R. G. (2012). A personalidade na compreensão do percurso escolar na adolescência [Tese de Doutoramento não publicada, Universidade de Lisboa].

Carvalho, R. G. (2015). Quantitative and qualitative assessment of adolescents' future time perspective. Paidéia, 25(61), 163-172. https://doi.org/ 10.1590/1982-43272561201504. 
Ducharne, M. A. B., Cruz, O., Marinho, S., \& Grande, C. (2006). Questionário de Estilos Educativos Parentais (QEEP). Psicologia e Educação, 5(1), 63-75.

EDP (2016). \#Tagga o teu futuro: Desafio sem barreiras. Disponível em https://taggaoteufuturo.edp.pt/sem-barreiras.

English, N. (2018). Job shadowing as a mechanism for college and career readiness [Tese de doutoramento, School of Education, University of Pittsburgh].

Faria, L. C. (2008). A eficácia da consulta psicológica vocacional de jovens: Estudo do impacto de uma intervenção. [Tese de doutoramento não publicada, Universidade do Minho].

Faria, L., Königstedt, M., \& Taveira, M. D. C. (2011). Avaliação da eficácia das intervenções vocacionais. Psicologia, Educação e Cultura, 15(1), 107-123.

Fernandes, E. M. M. (2018). Construção da carreira no ensino secundário: Estudo exploratório com psicólogos e estudantes [Dissertação de Mestrado não publicada, Universidade da Madeira].

Figueira, D. R. F. (2012). Desenvolvimento vocacional no 2o ciclo do Ensino Básico: A experiência da implementação de um programa numa escola rural da Madeira [Dissertação de mestrado não publicada, Universidade da Madeira].

Fouad, N. A., Ghosh, A., Chang, W., Figueiredo, C., \& Bachhuber, T. (2016). Career exploration among college students. Journal of College Student Development, 57(4), pp. 460 - 464. https://doi.org/ https://doi.org/10.1353/csd.2016.0047.

Jorge, L. (2011). Construção e Validação de uma Escala de Avaliação da Consciência de Carreira para a Infância. [Dissertação de Mestrado não publicada, Universidade da Madeira].

Kazis, R. (2016). MDRC Research on career pathways. Disponível em: http://doingwhatmatters.cccco.edu/portals/6/docs/sw/Career_Pathways_2016_Issue_Brief.pdf.

Krieshok, T. S., Black, M. D., \& McKay, R. A. (2009). Career decision-making: The limits of rationality and the abundance of non-conscious processes. Journal of Vocational Behavior, 75, 275-290. https://doi.org/10.1016/j.jvb.2009.04.006.

Lankard, B. (1995). Family role in career development. Disponível em: https://files.eric.ed.gov/fulltext/ED389878.pdf

Lopes, A. R. (2010). Projectos vocacionais, crenças de auto-eficácia e expectativas parentais em estudantes do $7^{\circ}$ ano de escolaridade em situação de sucesso/insucesso escolar [Dissertação de Mestrado não Publicada, Faculdade de Psicologia da Universidade de Lisboa].

Ornelas, M. M. A. (2017). A Perspetiva Temporal de Futuro na Construção de Carreira de Adolescentes de Meios Socioeconómicos Desfavorecidos [Dissertação de mestrado não publicada, Universidade da Madeira].

Pocinho, M. D., Correia, A., Carvalho, R. G., \& Silva, C. (2010). Influência do sexo, da família e dos serviços de psicologia e orientação na tomada de decisão de carreira. Revista Brasileira de Orientação Profissional, 11(2), 201-212. Disponível em http://pepsic.bvs-psi.org.br/rbop

Rebelo-Pinto, H. R., \& Soares, M. C. (2000). Influência parental no desenvolvimento vocacional. Relatório de investigação. Instituto de Orientação Profissional. https://doi.org/10.21631/rpp36_111

Ribeiro, M. A., \& Uvaldo, M. C. C. (2007). Frank Parsons: Trajetória do pioneiro da orientação vocacional, profissional e de carreira. Revista Brasileira de Orientação Profssional, 8(1), 19-31. Disponível em: http://pepsic.bvsalud.org/scielo.php?script=sci_arttext\&pid=S1679-33902007000100003

Service, B., Dalgic, G. E., Thornton, K. (2017). Benefits of a shadowing/mentoring intervention for New Zealand school principals. Professional Development in Education, 44(4), 507-520. https://doi.org/10.1080/19415257.2017.1378705.

Silva, C. S, Carvalho, R. G., \& Pocinho, M. (2010). Ligação entre vivências profissionais e actividades académicas. In A. Estrela et al. (Eds.) A escola e o mundo do trabalho: actas do XVII Colóquio da Association Francophone Internationale pour la Recherche Scientifique en Educacion (pp. 16281635). EDUCA/Secção Portuguesa da AFIRSE. https://doi.org/0.13140/RG.2.1.2883.2806.

Swanson, J. L. (1999). Stability and change in vocational interest. In M. L. Savickas \& A. R. Spokane (Eds.), Vocational interests: Meaning, measurement, and counselling use (pp.135-158). David-Black.

Historial do artigo

Recebido $\quad 07 / 2018$

Aceite $\quad 04 / 2020$

Publicado 08/2020 
Revista PSICOLOGIA, 2020, Vol. 34 (1), doi: 10.17575/psicologia.v34i1.1681.

Atas do X Simpósio Nacional de Investigação em Psicologia

\title{
Cross-hemispheric transcranial Direct Current Stimulation over the parietal cortex impairs task switching performance
}

\author{
Jorge Leite 41 ii,i,iii, Óscar F. Gonçalves ${ }^{\mathrm{i}}$, Soraia Silvai, Diogo Branco ${ }^{\mathrm{i}}$, Patrícia Coelho $^{\mathrm{i}}$ \& \\ Sandra Carvalho ${ }^{\mathrm{i}, \mathrm{iii}}$ \\ ${ }^{i}$ Neurotherapeutics and Experimental Psychopathology Group, Psychological Neuroscience Laboratory, \\ CIPsi, School of Psychology, University of Minho, Braga, Portugal. \\ ii University Portucalense, Portucalense Institute for Human Development - INPP, Oporto, Portugal. \\ iii Department of Physical Medicine and Rehabilitation, Spaulding. Neuromodulation Center, Spaulding \\ Rehabilitation Hospital, Harvard Medical School, Boston, MA, United.
}

\begin{abstract}
Several studies suggested the involvement of the parietal cortex in task switching, however it remains unclear if cross-hemispheric transcranial Direct Current Stimulation (tDCS) over the parietal cortex is able to neuromodulate task-shifting performance. Seventeen volunteers participated in this study. Participants were randomly assigned to receive: Right Anodal - Left Cathodal (RA-LC), Sham and Left Anodal - Right Cathodal (LA - RC) tDCS, in three sessions, with an intersession interval of at least 72h. Pairwise Bonferroni corrected analysis showed that RA-LC significantly increased switch cost performance, when comparing to sham. The present results suggest that activity shift towards the right hemisphere actually impaired performance. This is consistent with the role of the left parietal cortex on endogenous preparation and adjustment of goal directed behaviors, and as such, the inhibition of that specific area resulted in a detrimental effect.
\end{abstract}

Keywords: Task switching; Parietal cortex; tDCS; Switch cost; Cognitive flexibility.

Estimulação por corrente contínua bi-hemisférica sobre o córtex parietal influencia o desempenho numa tarefa de task switching: Vários estudos sugerem o envolvimento do córtex parietal em task switching. No entanto, os efeitos da estimulação transcraniana transcraniana de corrente contínua (ETCC) sobre o córtex parietal no desempenho de tarefas de mudança não está claro. Dezessete voluntários participaram neste estudo. Os participantes foram aleatorizados para receber: ETCC/ánodo direito - cátodo esquerdo (AD-CE), Sham e ETCC/ánodo esquerdo - cátodo direito, em três sessões, com intervalo inter-sessão mínimo de 72h. Comparações de Bonferroni mostraram que a ETCC/AD-CE aumentou significativamente o desempenho no custo da mudança, quando comparado ao sham. Estes resultados sugerem que a mudança de atividade para o hemisfério direito prejudicou o desempenho. Este dado é consistente com o papel do córtex parietal esquerdo na preparação endógena e no ajuste de comportamentos direcionados por objetivos.

Palavras-chave: Task switching; Córtex parietal; ETCC; Custo de mudança; Flexibilidade cognitiva.

Task switching (TS) refers to the ability to respond to unexpected demands and rapidly shifting attention between tasks (Chan, Koval, Johnston, \& Everling, 2017). This ability to change the attentional focus and type of response is a subcomponent of a broader concept known as "cognitive flexibility" (Leber, TurkBrowne, \& Chun, 2008). Neuroimaging data suggests the involvement of a fronto-parietal network, that is sometimes left-lateralized (Brass \& Von Cramon, 2002; Braver, Reynolds, \& Donaldson, 2003; Dove, Pollmann, Schubert, Wiggins, \& Von Cramon, 2000; Liston, Matalon, Hare, Davidson, \& Casey, 2006; Sohn, Ursu, Anderson, Stenger, \& Carter, 2000; Worringer, Langner, Koch, Eickhoff, Eickhoff, \& Binkofski, 2019). A previous study tested the effects of dorsolateral prefrontal (DLPFC) cortex transcranial direct current stimulation (tDCS) on task switching. $1 \mathrm{~mA}$ tDCS over the left DLPCF or over the left primary motor area was able to increase set shifting performance (Leite, Carvalho, Fregni \& Gonçalves, 2011). In a later study

\footnotetext{
${ }^{41}$ Correspondence address: Jorge Leite, Rua Dr. António Bernardino de Almeida, 541,4200-072 Porto E-mail: jorgel@upt.pt. SC and OFG are members of CIPsi, supported by the Portuguese Foundation for Science and Technology and the Portuguese Ministry of Science, Technology and Higher Education through national funds and co-financed by FEDER through COMPETE2020 under the PT2020 Partnership Agreement (POCI-01-0145-FEDER-007653).SC, JL, and OFG were funded by the Portuguese Foundation for Science and Technology and COMPETE through the grants: P2020-PTDC/MHC-PCN/3950/2014; PTDC/PSI-ESP/30280/2017 and PTDC/PSI-ESP/29701/2017). SC was also funded by the individual grant IF/00091/2015
} 
cross-hemispheric tDCS over PFC was shown to be able to modulate task-switching ability in two different cognitive flexibility tasks (Leite, Carvalho, Fregni, Boggio, \& Gonçalves, 2013). These findings have been corroborated by other research groups (Morales-Quezada, Leite, Carvalho, Castillo-Saavedra, Cosmo, \& Fregni, 2016). However, it remains unclear if cross-hemispheric tDCS over the parietal cortex is able to neuromodulate shifting task-shifting performance.

Transcranial Direct Current Stimulation (tDCS) is a safe, painless non-invasive brain stimulation technique that uses at least two electrodes (one positive - cathode, and one negative - anode) to apply a small electrical current in the scalp (usually between 1and $2 \mathrm{~mA}$ ). The effects of the stimulation are dependent on several aspects of the stimulation, such as: current density, duration of stimulation, and type of electrodes montage (direction of the electrical current). Thus, the stimulation applied to a specific area of the scalp can neuromodulate the underlying brain area, by inducing changes in the membrane potential of the neurons. Cathodal tDCS is thought to induce hyperpolarization and anodal tDCS depolarization of neuronal membrane of the motor cortex (Nitsche \& Paulus, 2000). The effects of tDCS on cognitive tasks, and specifically in tasks that involves shifting abilities are promising, especially when applied over the PFC. In this pilot study we tested the effects of single session cross-hemispheric tDCS versus uni-hemispheric tDCS over the parietal cortex on task switching performance in healthy volunteers.

\section{METHOD}

\section{Participants}

A total of 17 college students who volunteered (age: $21.65 \pm 4.42,14$ females) participated in this pilot study. All participants were healthy, right-handed, as assessed by the Edinburgh Handedness Inventory (EHI $\geq 80$ ), with normal or corrected-to-normal visual acuity and without present or past history of neurological or psychiatric disorders. All participants gave their written informed consent prior to their inclusion in the study and the study was performed in accordance with the declaration of Helsinki.

\section{Design}

This pilot study used a within-subject design. The participation in the study consisted of 3 sessions, with an intersession interval of at least $72 \mathrm{~h}$. Each session had 5 moments 1) Pre-task assessment; 2) Introduction to the task with feedback; 3) tDCS without task (3 minutes); 4) Task Switching combined with tDCS; 5) Post-task assessment (Figure 1). In the first session, participants were asked to answer the following questionnaires: EHI to assess laterality or dominance of the right or left hand for everyday activities, and the Beck Depression Inventory (BDI) to assess mood (participants rating above 9 were excluded). Both the Visual Analogue Scale (VAS) and The Positive and Negative Affect Schedule (PANAS) were administrated prior and after task performance in each session. The objective of these assessments was to evaluate possible adverse effects due to the stimulation. The VAS consisted of a total of 10 questions regarding possible symptoms such as discomfort, tiredness, itching, pain, mood, tingling, burn sensation, migraine, sleepiness, or other. Participants had to rate in a scale from 1 (very little), 5 (normal) to 10 (too much) (intervals included) and follow a visual face depicting a smile face (from 1 to 5) or sad face (from 6 to 10). Participants were randomized for the following tDCS conditions: 1. Right Anodal Left Cathodal (RA-LC); 2. Sham; and 3. Left Anodal - Right Cathodal (LA - RC). Therefore, the order in which each participant received each tDCS condition was completely random.

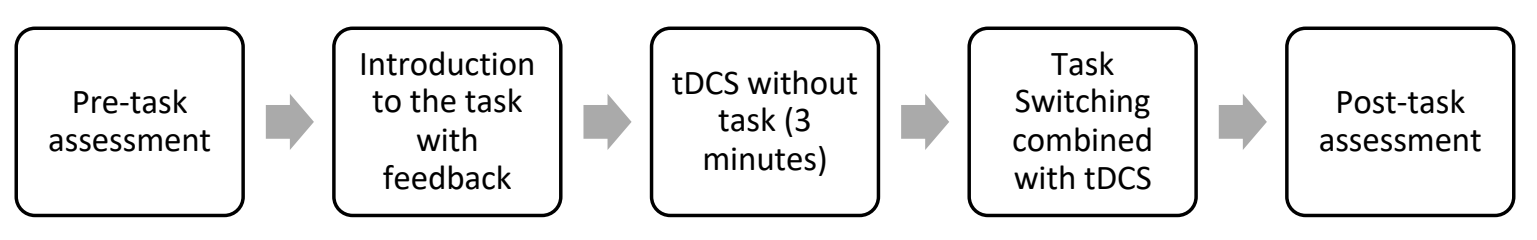

Figure 1. Schematic Representation of the procedure for each experimental session.

\section{Main task}

Task Switching. In this task, participants were presented with a cue that lasted $500 \mathrm{~ms}$, prior to the appearance of the target. The target consisted of a number inside a colored circle (either red or green) that was presented for 2500 ms. If the color presented was red, participants had to press the "Z" key for odd or the "M" key for even. If the color of the circle was red, participants should follow the magnitude 
rule (classifying if the number presented was either greater or not than five) by pressing "Z" key if the number presented was $>5$ and " $M$ " key if the number presented was $<5$, using the left or right index respectively. After a key press from participants, the target was replaced by another fixator for $500 \mathrm{~ms}$. If two consecutive trials were of the same color, there was no rule change (parity or magnitude), however if in two consecutive trials there was a change in color, there was a rule change, and that constituted repeat and switch trials, respectively.

There were a total of 160 trials, in 80 of those trials there was no rule change - repeat trials, while for the remainder 80 , there was a rule switch.

Transcranial Direct Current Stimulation. tDCS was applied using 35/35 $\mathrm{cm}^{2}$ saline-soaked electrode sponges, using an Eldith DC Stimulator Plus (Neuroconn, Germany). The experiment had a within-subject design, in which all participants were randomized to receive 3 tDCS conditions in a counterbalanced manner: 2 active (Right Anodal - Left Cathodal (RA-LC) and Left Anodal - Right Cathodal (LA - RC) over the parietal cortex) and one sham. There was at least a $6 \mathrm{~cm}$ distance between the anode and the cathode. It was applied a current density of $0.057 \mathrm{~mA} / \mathrm{cm}^{2}$ for a total duration of $30 \mathrm{~min}$, and 15-s ramp up and 15$s$ ramp down for the active condition. The sham tDCS was applied following procedure of active tDCS, expect for the duration of the stimulation that was $15 \mathrm{~s}$.

\section{Data Analysis}

For the primary outcome, switch cost indexes for response time (RT) were calculated subtracting the mean RT of responses to switch condition from the mean RT of responses to non-switch ones, for correct trials only. Switch costs for accuracy were calculated subtracting the accuracy from responses to switch condition from responses to non-switch ones.

Two one-way repeated measures ANOVAs with three levels (RA-LC; RC-LA and sham tDCS) for switch cost accuracy and response time were calculated.

T-tests were used to evaluate differences in symptoms from pre to postDCS as assessed by a 10point Visual Analogue Scales (VAS).

\section{RESULTS}

\section{tDCS effect on Response Time of the Switch Cost}

There were significant differences in terms of switch costs $F(2,28)=4,01, p<.05$ dependent on stimulation. Bonferroni pairwise comparisons showed that the RT of the Switch Cost increased significantly $(M=102.84, S D=18.24)$ for the RA-LC condition, when compared with the sham condition $(M=49.44, S D=17.84)(p=.03, d=2.96)$ (Figure 2).
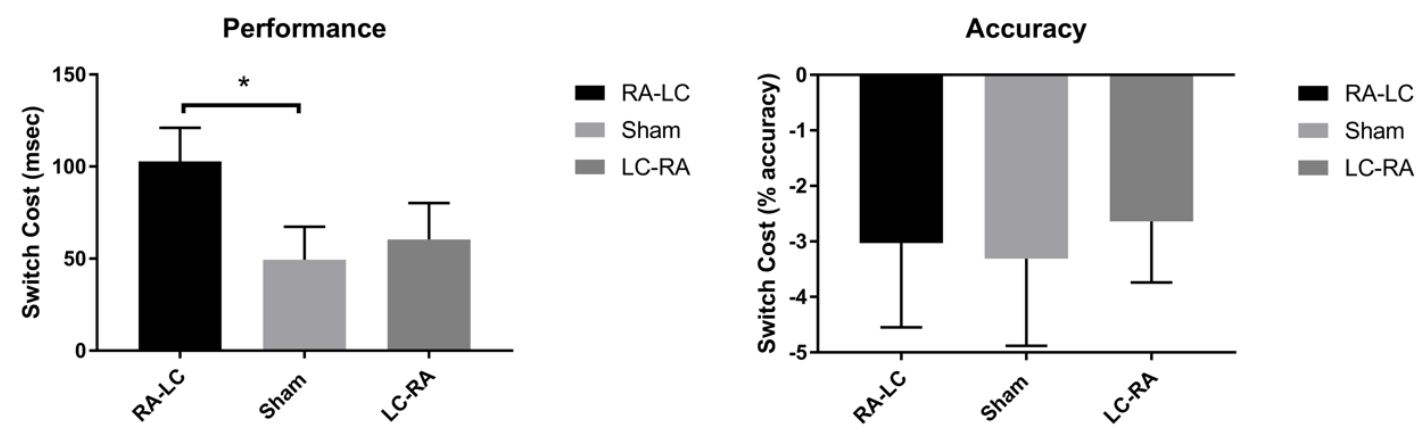

Figure 2. transcranial direct current stimulation (tDCS) effect on response time (msec) of the switch cost and on accuracy (RA-LC = Right Anodal - Left Cathodal tDCS , LA-RC = Left Anodal - Right Cathodal tDCS)

\section{tDCS effect on Accuracy of the Switch Cost}

No significant differences were found between conditions $F(2,28)=0.1, p>.05 .05$ (figure 2).

\section{Self- Report Measures}

Visual Analogue Scale (VAS). Participants reported a significant increase in discomfort, $t(16)=-3,32, p=$ $.004, d=1.04$, and headache, $t(16)=-3.86, p=.001, d=1.02$, following RA-LC stimulation; a significant increase in discomfort, $t(16)=-4.02, p<.001, d=1.09$, and itching, $t(16)=-4.61, p<.001, d=1.11$, after LA-RC stimulation, and a significant increase in headache, $t(16)=-3.22, p=.005, d=0.72$, after the sham condition. However, as can been seen from the following table, these symptoms were mild, not reaching, on average 4 points in a 10-point scale (Table 1 ). 
Table 1. Results of Visual Analogue Scale (VAS) pre and post of the three stimulation conditions

\begin{tabular}{|c|c|c|c|}
\hline \multicolumn{4}{|c|}{ RA-LC } \\
\hline & Prestimulation & Poststimulation & \\
\hline & $(n=17)$ & $(n=17)$ & \\
\hline & Mean (SD) & Mean (SD) & $t(16)$ \\
\hline Discomfort & $1.76(1.52)$ & $3.82(2.24)$ & $-3.32^{*}$ \\
\hline Headache & $1.24(0.97)$ & 3.94 (2.99) & $-3.86^{*}$ \\
\hline \multicolumn{4}{|c|}{ LA-RC } \\
\hline & Prestimulation & Poststimulation & \\
\hline & $(n=17)$ & $(\mathrm{n}=17)$ & \\
\hline & Mean (SD) & Mean (SD) & $t(16)$ \\
\hline Discomfort & $1.53(1.00)$ & $3.94(2.54)$ & $-4.02^{*}$ \\
\hline Itchiness & $1.12(0.33)$ & $3.82(2.58)$ & $-4.61^{* *}$ \\
\hline \multicolumn{4}{|c|}{ Sham } \\
\hline & Prestimulation & Poststimulation & \\
\hline & $(\mathrm{n}=17)$ & $(\mathrm{n}=17)$ & \\
\hline & Mean (SD) & Mean (SD) & $t(16)$ \\
\hline Headache & $1.29(0.59)$ & $2.88(2.45)$ & $-3.22 *$ \\
\hline
\end{tabular}

Note. ${ }^{*} p<.005 ;{ }^{* *} p<.001$

\section{DISCUSSION}

In this pilot study we tested the effects of single session cross-hemispheric tDCS over the parietal cortex on task switching performance in healthy volunteers. Using a within subjects design we tested three different tDCS conditions, 2 active (RA-LC; RC-LA) and a sham condition.

Results show that RA-LC tDCS, over the parietal cortex, increased switch cost in terms of response time when comparing to sham tDCS. This is partially consistent with a previous study in which RA-LC over the prefrontal cortex impaired task switching performance in a vowel/consonant task (Leite et al., 2013). These results highlight the critical interdependence of the PFC hemispheres in task switching performing, suggesting the existence of different roles for each one. This seems to be not the case for the parietal cortex, at least by using a cross-hemispheric tDCS montage, namely because in the present study, there were no effects of parietal cortex stimulation in terms of switching cost accuracy.

Despite the fact that task switching is dependent on an extensive network that included the PFC and the Parietal cortex, it is not surprising to consider that specific regions may be also responsible for specific functions that underlie the general cognitive ability. For instance, Ravizza and Carter (2008) suggested that rule switching is more dependent on the prefrontal cortex, while perceptual shifting is more dependent on the parietal cortex. Studies targeting other cognitive functions also suggested that PFC and parietal cortex may have different functions that underlie the same cognitive ability. For instance, Pergolizzi and Chua (2016) showed that tDCS over the PPC, but not over the PFC was able to decrease false memory recognition and bias in item and source memory. However, both regions are thought to be involved in the same memory processes.

However, if rule switching would be a task that was more dependent on the PFC, one would expect no effect on task performance of parietal tDCS, or a similar, detrimental effect, regardless of polarity. In the present results, only shifting the activity towards the right hemisphere produced a detrimental effect on task performance.

Our results are consistent with the assumption that task switching may be a more left- lateralized function, especially during endogenous preparation for a response (Sohn et al., 2000). If we look at the neurophysiological effect of tDCS, the current direction will produce a distinct effect in the neural tissue underlying the electrode. Under the anode, the current flows inward, making the neurons more prone to depolarize, while under the cathode, the current flows outward, making neurons more prone to hyperpolarize (Jackson et al., 2016). Following this assumption, in the RA-LC tDCS condition, there is a hemispheric shift towards the right hemisphere, which may have interfered with the endogenous preparation required to successfully perform the switch. Surprisingly, a shift of activity towards the left hemisphere did not produce any significant results in terms of task performance. 
This study is not without limitations. First of all, it is not possible to fully understand the role of the parietal cortex in task switching, as there were no other regions to be stimulated. Additionally, the task was based on a rule change and as such, perceptual changes could not be adequately tested.

The present results are a clear indicator that although most of the studies using tDCS have been targeting the PFC, it is also possible to neuromodulate this specific function using other cortical targets, such as the parietal cortex. Future studies should further explore the role of the PFC and parietal cortex on cognitive flexibility, as well as changing the degree of rule and perceptual changes required to perform the task, thus recruiting more the PFC or the parietal cortex respectively. Also, it would be important for future studies to target the PFC and parietal cortex simultaneously, in order to assess the impact of the combined neuromodulatory effects on task performance.

In sum, these results highlight the importance of studying the role of the parietal cortex in terms of task switching, especially because activity shift towards the right hemisphere (i.e., RA-LC) actually impaired performance. These results are consistent with the role of the left parietal cortex on endogenous preparation and adjustment of goal directed behaviors, and as such, the inhibition of that specific area resulted in a detrimental effect.

\section{REFERENCES}

Brass, M., \& Von Cramon, D. Y. (2002). The role of the frontal cortex in task preparation. Cerebral cortex, 12(9), 908-914. https://doi.org/10.1093/cercor/12.9.908

Braver, T. S., Reynolds, J. R., \& Donaldson, D. I. (2003). Neural mechanisms of transient and sustained cognitive control during task switching. Neuron, 39(4), 713-726. https://doi.org/10.1016/S08966273(03)00466-5

Chan, J. L., Koval, M. J., Johnston, K., \& Everling, S. (2017). Neural correlates for task switching in the macaque superior colliculus. Journal of neurophysiology, 118(4), 21562170. https://doi.org/10.1152/jn.00139.2017

Dove, A., Pollmann, S., Schubert, T., Wiggins, C. J., \& Von Cramon, D. Y. (2000). Prefrontal cortex activation in task switching: an event-related fMRI study. Cognitive brain research, 9(1), 103-109. https://doi.org/10.1016/S0926-6410(99)00029-4

Jackson, M. P., Rahman, A., Lafon, B., Kronberg, G., Ling, D., Parra, L. C., \& Bikson, M. (2016). Animal models of transcranial direct current stimulation: methods and mechanisms. Clinical Neurophysiology, 127(11), 3425-3454. https://doi.org/10.1016/j.clinph.2016.08.016

Leber, A. B., Turk-Browne, N. B., \& Chun, M. M. (2008). Neural predictors of moment-to-moment fluctuations in cognitive flexibility. Proceedings of the National Academy of Sciences, 105(36), 13592-13597. https://doi.org/10.1073/pnas.0805423105

Leite, J., Carvalho, S., Fregni, F., Boggio, P. S., \& Gonçalves, Ó. F. (2013). The effects of cross-hemispheric dorsolateral prefrontal cortex transcranial direct current stimulation (tDCS) on task switching. Brain stimulation, 6(4), 660-667. https://doi.org/10.1016/j.brs.2012.10.006

Leite, J., Carvalho, S., Fregni, F., \& Gonçalves, O. F. (2011). Task-specific effects of tDCS-induced cortical excitability changes on cognitive and motor sequence set shifting performance. PloS one, 6(9), e24140. https://doi.org/10.1371/journal.pone.0024140

Liston, C., Matalon, S., Hare, T. A., Davidson, M. C., \& Casey, B. J. (2006). Anterior cingulate and posterior parietal cortices are sensitive to dissociable forms of conflict in a task-switching paradigm. Neuron, 50(4), 643-653. https://doi.org/10.1016/j.neuron.2006.04.015

Morales-Quezada, L., Leite, J., Carvalho, S., Castillo-Saavedra, L., Cosmo, C., \& Fregni, F. (2016). Behavioral effects of transcranial pulsed current stimulation (tPCS): Speed-accuracy tradeoff in attention switching task. Neuroscience research, 109, 48-53. https://doi.org/10.1016/j.neures.2016.01.009

Nitsche, M. A., \& Paulus, W. (2000). Excitability changes induced in the human motor cortex by weak transcranial direct current stimulation. The Journal of physiology, 527(3), 633-639. https://doi.org/10.1111/j.1469-7793.2000.t01-1-00633.x

Pergolizzi, D., \& Chua, E. F. (2016). Transcranial direct current stimulation over the parietal cortex alters bias in item and source memory tasks. Brain and cognition, 108, 56-65. https://doi.org/10.1016/j.bandc.2016.06.009

Ravizza, S. M., \& Carter, C. S. (2008). Shifting set about task switching: Behavioral and neural evidence for distinct forms of cognitive flexibility. Neuropsychologia, 46(12), 2924-2935. https://doi.org/10.1016/j.neuropsychologia.2008.06.006

Sohn, M. H., Ursu, S., Anderson, J. R., Stenger, V. A., \& Carter, C. S. (2000). The role of prefrontal cortex and posterior parietal cortex in task switching. Proceedings of the National Academy of Sciences, 97(24), 13448-13453. https://doi.org/10.1073/pnas.240460497 
Worringer, B., Langner, R., Koch, I., Eickhoff, S. B., Eickhoff, C. R., \& Binkofski, F. C. (2019). Common and distinct neural correlates of dual-tasking and task-switching: a meta-analytic review and a neurocognitive processing model of human multitasking. Brain Structure and Function, 1-25. https://doi.org/10.1007/s00429-019-01870-

Historial do artigo

Recebido $\quad 07 / 2019$

Aceite $\quad 11 / 2019$

Publicado $\quad 08 / 2020$ 
Revista PSICOLOGIA, 2020, Vol. 34 (1), doi: 10.17575/psicologia.v34i1.1681.

Atas do X Simpósio Nacional de Investigação em Psicologia

\title{
Psychology of Tourism: Exploring tourists' wellbeing and creative personalities
}

\author{
Soraia Garcês ${ }^{42 i, i i}$, Margarida Pocinhoi,ii, Saul Neves de Jesusii,iii \& Ester Câmara ${ }^{\mathrm{i}}$ \\ ${ }^{i}$ Research Center for Regional and Local Studies (UMa-CIERL), University of Madeira \\ ii Researcher at the Research Centre for Tourism, Sustainability and Well-being (CinTurs), University of \\ Algarve \\ iii Faculty of Human and Social Sciences, University of Algarve
}

\begin{abstract}
Psychology of Tourism" is emerging as a new sub-field both in Psychology and in Tourism studies. Tourism is related to the movement of people and Psychology as the science that studies human behaviour has in tourism great potential for research and intervention. Thus, this paper gives one of the first attempts of research in "Psychology of Tourism" in Portugal, by exploring tourists' wellbeing and creative personalities. It participated 857 tourists with Portuguese nationality and the Creative Personality Scale-short Form and the Tourism Wellbeing Scale were the used instruments. Results showed significant differences between Portuguese who travelled within Portugal (domestic) and those who went abroad (international), being the latter the ones that reported more wellbeing and creativity. Conclusions showed that those who ventured out of the country express more wellbeing and creativity but more research in this field is needed.
\end{abstract}

\section{Keywords: Creativity; Domestic tourist; International tourist; Psychology of tourism; Wellbeing.}

Psicologia do Turismo: explorando o bem-estar e a personalidade criativa dos turistas: A "Psicologia do Turismo" é um novo subcampo de estudo nas áreas da Psicologia e do Turismo que está a surgir. 0 Turismo relaciona-se com o movimento de pessoas e a Psicologia como a ciência que estuda o comportamento humano tem no Turismo um grande potencial de investigação e de intervenção. Este estudo oferece uma primeira tentativa na investigação sobre "Psicologia do Turismo" em Portugal, através da análise exploratória do bem-estar e da personalidade criativa dos turistas. Participaram 857 turistas de nacionalidade Portuguesa, aos quais se aplicou a Escala da Personalidade Criativa- versão reduzida e a Escala do Bem-Estar do Turista. Encontraram-se diferenças significativas entre os turistas que viajaram dentro do país (doméstico) e aqueles que viajaram para fora de Portugal (internacional), tendo estes últimos reportado maior bem-estar e criatividade. Conclusões indicam que os turistas que viajam para fora de Portugal expressam maior bem-estar e criatividade, no entanto é necessária mais investigação neste campo.

Palavras-chave: Bem-Estar; Criatividade; Turista doméstico; Turista internacional; Psicologia do turismo.

"Psychology of Tourism" is an emergent field that brings psychological principles to better understand the tourists, the host communities and the workers/stakeholders of the tourism industry (Garcês, Pocinho, \& Jesus, 2019). Psychology has a long history of practice and research and it is identified as being "the scientific study of the mind and behaviour" (McLeod, 2019). Tourism, on the other hand is seen as a "(...) social, cultural and economic phenomenon related to the movement of people to places outside their usual place of residence" (Department of Economic and Social Affairs - Statistical Division [DESA], 2010, p.1). As Garcês et al., (2019) acknowledged both fields study, or have as main subjects, people, therefore, there is a clear relationship between both fields. Prior studies have also pointed out connections between the two fields. As an example, Dann (1981) wrote about tourists' motivations. Plog (1987) developed a typology of tourists based on personality characteristics. Further along Pearce and Stringer (1991) reviewed and analysed psychological contributions to the field of tourism, offering ideas for future studies including topics such as tourists' behaviours. Crotts and van Raaij $(1994$, p.1) applied the idea of economic psychology to tourism and stated that Psychology is of crucial importance for the development of economic theory, the practice of marketing, and research on travel and tourism. One

${ }^{42}$ Correspondence address: Soraia Garcês, Campus Universitário da Penteada, gabinete 1.75 (1ํopiso)- CIERL, 9020-105 Funchal. E-mail: soraiagarces@gmail.com. Special thanks to ARDITI - Agência Regional para o Desenvolvimento da Investigação Tecnologia e Inovação through the support provided under the Project M1420-09-5369-FSE-000001 - Post Doctoral Research Grant of the first author. 
should take into account the results of research on human behaviour, because people react upon the economic conditions as they perceive them rather than objectively defined conditions.

Pearce and Packer (2012), in a review, proposed that tourism researchers can gain from the growth of psychology theories and empathized topics such as motivation, attitudes, satisfaction, memory, and personal development, for example. Simková (2014) acknowledged that ideas from environmental and social psychology are used to help to better comprehend tourists namely their behaviours, attitudes and motivations. However, as the author also noted marketing is still seen as the most crucial issue in tourism. As tourism is growing immensely (Chen, King, \& Lee, 2018) the idea of "Psychology of Tourism" is crucial to help to understand and to develop new strategies that can support both fields towards a place of innovation with impact in society. Such efforts are already being seen through the use of principles from Positive Psychology and its application in understanding tourism phenomenon'. In this regard, Filep (2016) presented the idea of positive tourism, a new field that aims to study human flourishing in tourism with an emphasis in tourists, local communities and/or tourism workers. Defined as the "study of hedonic and eudaimonic human well-being and conditions (or various circumstances) for flourishing as they relate to individual tourists, members of host communities and tourism workers in diverse sectors of the tourism industry" (Filep, Laing, \& Csikszentmihalyi, 2017, p. 10), positive tourism is, in itself, one of the first theoretical attempts in merging Psychology and Tourism as a scientific sub-field of studies, to the authors best knowledge. Thus, Positive Psychology comes as an asset to the idea of "Psychology of Tourism" since it is considered the science of wellbeing (Seligman, 2010). Positive Psychology has a broad scope and a multidisciplinary view that allies' researches from multiple fields (Titus, 2017), hence its endeavours can clearly extend to tourism settings. In a systematic literature review, linking Positive Psychology and Tourism (Garcês, Pocinho, Jesus, \& Rieber, 2018), results showed that tourism is seen as a way to promote wellbeing for host communities and tourists. Also, in the same study, it was found that wellbeing can be understood as an opportunity for businesses innovation and that it is deeply related to nature. Despite being a new idea, wellbeing is being approached by tourism businesses as a unique opportunity for new tourism experiences (Smith \& Diekmann, 2017). In this sense as lifestyles are changing, the European Travel Commission (2016) questioned how people will balance life between their consumer endeavours and leisure time and if it is still possible to find new paths for happiness. However, as mentioned by Uysal, Sirgy, Woo and Kim (2016) experiences through tourism can help increase life satisfaction and as the ETC (2016, p.79) stated "we see that consumers today are happy to accept that understanding a foreign culture is complex (...) being in a foreign country presents an opportunity for novelty: being able to learn something that could not be learned anywhere else." This idea of looking for something novel is aligned not only with the current needs and desires of tourists but also with one of Peterson and Seligman (2004) character strengths, namely creativity. Creativity has, usually, two accepted characteristics: the idea of usefulness and uniqueness (Bacon, 2005). In a systematic literature review that highlighted creativity in tourism it was found that "creativity is explored, mainly, as a background for tourism development through organizational, structural and product innovation where workers and future ones are understood as important for its growth as is the place and its culture" (Garcês, Pocinho, \& Jesus, 2018b, p.8). Also, Pappalepore, Maitland and Smith (2010) in a study about tourism experiences in creative urban settings found out that these experiences were influenced by people's creativity. Passafaro et al. (2015) has acknowledged that people seek experiences that are aligned with who they are. So, being creativity and wellbeing two very powerful variables for feeling well and searching for new outlooks in life, including travelling, it is considered by the authors two major constructs to be explored. Therefore, with this exploratory study, the aim is to analyze tourists' wellbeing and creative personalities and make a first effort in exploring Portuguese tourists' personal characteristics through Positive Psychology concepts.

\section{METHOD}

\section{Participants}

A total of 857 tourists with Portuguese nationality participated in this study. Women partake $68.9 \%$ $(n=589)$ of the sample, while $31.1 \%(n=266)$ of men took part in the study. The minimum age was 17 years old and the maximum 76 years, with a Mean $(M)$ of 29, and a Standard Deviation (SD) of 11.89. The participants were distributed in two groups: a) domestic tourism (Portuguese tourists who in their last tourism experience travelled within Portugal) and b) international tourism (Portuguese tourists who in their last tourism experience travelled outside of Portugal). The domestic tourists acknowledged for $54.9 \%(n=450)$ and the international tourists made for $45.1 \%(n=369)$ of the sample. 


\section{Measurements}

Two instruments were used. The first one was the Creative Personality Scale-short Form - EPC (Pocinho, Garcês, Jesus, Viseu, \& Tobal, 2019). This instrument measures creative personality characteristics and is composed of 11 items. It is a one factor scale, and reliability of the original study was .877. In the present research, internal consistency was .841. The second instrument used was the Tourism Wellbeing Scale EBT (Garcês, Pocinho, \& Jesus, 2018a, 2020). This measure was built to evaluate tourists' wellbeing through a eudemonic view, based on Positive Psychology principles. The first version had 30 items (Garcês et al., 2018a) but later on, it was reduced to eight items after exploratory and confirmatory factor analyses (Garcês et al., 2020). This reduction allowed to have a more concise measure and, hopefully, avoid social desirability and boredom that could skew results. Operationally, EBT measures eight variables namely: a) positive emotions; b) engagement; c) relationships; d) meaning; e) accomplishment; f) creativity; e) optimism; and e) spirituality. Overall it also measures wellbeing through its one-factor dimension which is the sum of all eight items. The reliability of the original study was .874 (Garcês et al., 2020). The current research internal consistency of this measure was .848. In a complement, sociodemographic data makes too part of this measure. It also includes an open-ended question that asks for the place of the last tourism experience people undertook and which is the place people should consider when answering the wellbeing related items.

\section{Procedure}

Data was collected through social media sharing of the instruments and by convenience in social groups. After data collection two groups were formed: a) domestic tourists and b) international tourists. As the United Nations World Tourism Organization (2017) stated domestic tourism is related to the activities undertaken by visitors in their own countries, therefore in this study domestic tourists were considered to be tourists with Portuguese nationality who travelled within Portugal, including the islands of Madeira, Porto Santo and Azores. International tourists were understood as Portuguese who travelled abroad to foreign countries. Statistical analysis undertook an exploratory emphasis. Therefore, it was used Pearson correlation to look for significant relationships between variables and t-Student tests to compare means among the selected groups. Normality was assumed through the central limit theorem since the sample is n>30 (Burdenski, 2000; Pestana \& Gajeiro, 2008).

\section{RESULTS}

In an overall analysis, the results from the creativity measure showed a $M=44.55$, with a $S D$ of 5.51 . According to the scoring provided by the authors of the instrument, average creativity runs from 44 to 47 points (Pocinho et al., 2019), thus these results correspond to average results within the creativity measure. In the tourism wellbeing scale, results showed $M=40.29$ and a $S D$ of 9.39. In the validation study, average wellbeing runs from 39 to 44 points (Garcês et al., 2020), meaning that the current study also reports average wellbeing scores.

Since normality was assumed, the relationship between the main variables of this study was analysed through the Pearson correlation. Table 1 shows its results and as it is possible to observe, there is a positive and significant correlation between creativity and wellbeing.

Table 1. Correlation between creativity and wellbeing in tourism

\begin{tabular}{llcc}
\hline & & Creativity & Wellbeing \\
\hline Creativity & Pearson Correlation & 1 & $.32^{* *}$ \\
& $p$ & & .000 \\
Wellbeing & Pearson Correlation & $.32^{* *}$ & 1 \\
& $p$ & .000 & \\
\hline
\end{tabular}

$\overline{* *} p<.01$

A comparative analysis between groups was performed with the use of the $t$-Student independent test. For the creativity variable, homogeneity was assumed following the non-significant values of the Levene Test, $F=2.39, p=.122$. A significant difference between domestic tourists $(M=44.00, S D=5.20)$ and international tourists $(M=45.15, S D=5.71), t(817)=-3.005, p=.003$, was found, where international tourists scored higher than domestic ones.

For the wellbeing variable, homogeneity was again assumed following the non-significant values of the Levene Test, $F=.545, p=.46$. As occurred with the previous analysis, a significant difference between domestic tourists $(M=39.12, S D=9.46)$ and international tourists $(M=42.07, S D=8.33), t(817)=-4.675$, $p=.000$, was found with international tourists presenting higher wellbeing than domestic tourists. 
An in-depth analysis for the wellbeing sub-variables was performed, considering the eight individuals variables that this measure also evaluates. Table 2 shows the results for the performed $t$-test, Means $(M)$ and Standard-Deviations $(S D)$.

Table 2. $t$-test for wellbeing sub-variables

\begin{tabular}{|c|c|c|c|c|c|}
\hline Variables & Type of Tourist & $M$ & $S D$ & $t$ & $p$ \\
\hline \multirow[t]{2}{*}{ Optimism } & Domestic & 5.31 & 1.56 & -1.47 & .141 \\
\hline & International & 5.46 & 1.40 & & \\
\hline \multirow[t]{2}{*}{ Meaning } & Domestic & 4.88 & 1.61 & -3.50 & $.001^{* *}$ \\
\hline & International & 5.27 & 1.49 & & \\
\hline \multirow[t]{2}{*}{ Positive Emotions } & Domestic & 6.23 & 1.21 & -2.15 & $.032 *$ \\
\hline & International & 6.39 & .94 & & \\
\hline \multirow[t]{2}{*}{ Creativity } & Domestic & 5.44 & 1.64 & -6.57 & $.000^{* *}$ \\
\hline & International & 6.10 & 1.26 & & \\
\hline \multirow[t]{2}{*}{ Engagement } & Domestic & 4.38 & 1.90 & -3.17 & $.002^{* *}$ \\
\hline & International & 4.80 & 1.81 & & \\
\hline \multirow[t]{2}{*}{ Accomplishment } & Domestic & 3.96 & 2.02 & -7.07 & $.000^{* *}$ \\
\hline & International & 4.91 & 1.84 & & \\
\hline \multirow[t]{2}{*}{ Spirituality } & Domestic & 3.92 & 1.90 & -.93 & .351 \\
\hline & International & 4.05 & 1.91 & & \\
\hline \multirow[t]{2}{*}{ Positive Relationships } & Domestic & 5.13 & 1.69 & -1.53 & .126 \\
\hline & International & 5.30 & 1.60 & & \\
\hline
\end{tabular}

*. $p<.05 ;{ }^{* *} . p<.001$

Through the analysis of the results from Table 2, it can be observed that there are significant differences between domestic and international tourists for the variables: meaning, positive emotions, creativity, engagement and accomplishment, being international tourists those who scored higher in all sub-variables.

\section{DISCUSSION}

The study performed one of the very first attempts in exploring wellbeing and creative personalities of Portuguese tourists with psychological tools to the authors best knowledge. Results showed that within this sample creativity is significantly and positively related to wellbeing in tourism experiences. As a character strength from Positive Psychology (Peterson \& Seligman, 2004) which is the science of wellbeing (Seligman, 2010), these results come as an empirical statement that indeed both variables are connected. Thus, tourism experiences can gain a lot if stakeholders bet in creative activities as a source to promote wellbeing in tourists, particularly for those who perceived themselves as having a more creative personality.

Another important result from this study is the fact that international tourists (Portuguese who went abroad in their last tourism experience) showed higher scores both for creativity and wellbeing when compared to domestic tourists (Portuguese who travelled within Portugal in their last tourism experience). Also, in an analysis of the eight sub-variables of the tourism wellbeing scale, significant results were obtained for meaning, positive emotions, creativity, engagement and accomplishment, being the international tourists those who scored higher. It is, therefore, hypothesized that Portuguese who travelled abroad saw their experiences as more meaningful, with more positive emotions and creativity, engage more with the community, and perceive their tourism experience more as an accomplishment than Portuguese who travelled within Portugal in their last tourism experience. As ETC (2016) acknowledged being outside of the country of residence is a novel opportunity that allows to learn things that were not possible in the home country. Therefore, the results can be seen as showing that going away from home for a certain period of time can be a source of wellbeing and a transformative experience. As Kirillova, Lehto and Cai (2017, p. 509) stated "when placed in a conducive environment with beautiful scenery, novel cultural context, and the potential for meaningful connections with others, 'peak' experiences can initiate transformative meaning-making in a tourist". 
As in any study, the current one, presents some limitations. There is a lack of studies in this field and particularly in understanding tourists' wellbeing and creative personalities, thus comparing results was not achievable. Also, the instruments used are both self-reports and very new in the field which may be considered not desirable. Likewise, being an attempt at exploring a new sub-field of studies the analysis herein lacks robustness to completely explain such complex variables as are creativity and wellbeing. Future research is needed to fulfil these limitations and to continue to increase the field of "Psychology of Tourism". Thus, it is suggested to continue to study both variables, but also to study them in another context and/or culture, through international cross-cultural studies. Similarly, more in -depth research with sophisticated statistical analysis is important to further allow to understand tourists and what they want and desire, and thus promoting new strategies to develop the field and increase people wellbeing when travelling outside and inside of their country of residence.

\section{REFERENCES}

Bacon, S. (2005). Positive Psychology's Two Cultures. Review of General Psychology, 9(2), 181-192. https://doi.org/10.1037/1089-2680.9.2.181

Burdenski, T. (2000). Evaluating, Univariate, Bivariate, and Multivariate Normality Using Graphical and Statistical Procedures. Multiple Linear Regression Viewpoints, 26 (2), 15-28. https://pdfs.semanticscholar.org/1eb7/c37e392419eb3a6246236f8e6fad6cf55df6.pdf

Chen, Y., King, B., \& Lee, H. (2018). Experiencing the destination brand: Behavioral intentions of arts festival tourists. Journal of Destination Marketing, 10, 61-67. https://doi.org/10.1016/j.jdmm.2018.06.004

Crotts, J., \& van Raaij, W. (1994). Economic Psychology of Travel and Tourism. The Haworth Press, Inc.

Dann, G. (1981). Tourist motivation an appraisal. Annals of Tourism Research, 8(2), 187-219. https://doi.org/10.1016/0160-7383(81)90082-7

Department of Economic and Social Affairs - Statistics Division (2010). International Recommendations for Tourism $\quad$ Statistics United Nations https://unstats.un.org/unsd/publication/seriesm/seriesm_83rev1e.pdf

European Travel Commission (2016). Lifestyle trends \& Tourism: How changing consumer behavior impacts travel to Europe. European Travel Commission. https://etccorporate.org/uploads/reports/Trend-5-Lifestyle-Trends-Tourism-No-escape-2016.pdf

Filep, S. (2016). Tourism and positive psychology critique: Too emotional? Annals of Tourism Research, 59, 113-115. https://doi.org/10.1016/j.annals.2016.04.004

Filep, S., Laing, J., \& Csikszentmihalyi, M. (2017). What is positive tourism? Why do we need it? In S. Filep, J. Laing, \& M. Csikszentmihalyi (Eds.), Positive Tourism (pp. 2-16). Routledge.

Garcês, S., Pocinho, M., \& Jesus, S. (2018a). Development of the Tourism Wellbeing Scale. Revista de Divulgação Científica-AICA, 10, 55-63. https://en.calameo.com/read/002167773470718f240a0

Garcês, S., Pocinho, M., \& Jesus, S. (2018b). Review of Optimism, Creativity and Spirituality in Tourism Research. Tourism and Hospitality Management, 24(1), 1-11. https://doi.org/10.20867/thm.24.1.6

Garcês, S., Pocinho, M., \& Jesus, S. (2020). Psychometric analysis of the Tourism Wellbeing Scale (TWS): a multidisciplinary approach. Anais da Academia Brasileira de Ciências, 92(1), e20190232. https://doi.org/10.1590/0001-3765202020190232

Garcês, S., Pocinho, M., \& Jesus, S. (2019). Psychological wellbeing as a creative resource for businesses in the tourism industry - a multidisciplinary view. In S. Teixeira, \& J. Ferreira (Eds), Multilevel Approach to Competitiveness in the global tourism Industry (pp.98-119), IGI Global. https://doi.org/10.4018/978-1-7998-0365-2.ch007

Garcês, S., Pocinho, M., Jesus, S., \& Rieber, M. (2018). Positive psychology and tourism: a systematic literature review. Tourism \& Management Studies, 14(3), 41-51. http://tmstudies.net/index.php/ectms/article/view/1048/pdf_105

Kirillova, K., Lehto, X., \& Cai L. (2017). What triggers transformative tourism experiences? Tourism Recreation Research, 42(4), 498-511. https://doi.org/10.1080/02508281.2017.1342349

McLeod, S. (2019). What is Psychology? https://www.simplypsychology.org/whatispsychology.html

Pappalepore, I., Maitland, R., \& Smith, A. (2010). Exploring urban creativity: Visitor Experiences of Spitalfields, London. Tourism, Culture \& Communication, 10, 217-230. https://doi.org/10.3727/109830410X12910355180946

Pearce, P., \& Packer, J. (2013). Minds On the Move: New links from Psychology to Tourism. Annals of Tourism Research, 40, 386-411. https://doi.org/10.1016/j.annals.2012.10.002

Pearce, P., \& Stringer, P. (1991). Psychology and tourism. Annals of Tourism Research, 18(1), 136-154. https://doi.org/10.1016/0160-7383(91)90044-C 
Pestana, M. H., \& Gageiro, J. N. (2008). Análise de dados para ciências sociais: A complementaridade do SPSS. Edições Sílabo.

Peterson, C., \& Seligman, M. E. P. (2004). Character strengths and virtues: A handbook and classification. American Psychological Association.

Plog, S. (1987). Understanding psychographics in tourism research. John Wiley \& Sons.

Pocinho, M., Garcês, S., Jesus, S., Viseu, J., \& Tobal, J. (2019). Psychometric study of the short-form of the creative personality scale[ Manuscript submitted for publication]. CIERL, University of Madeira.

Seligman, M. (2010, October 7). Flourish: Positive psychology and positive interventions. http://tannerlectures.utah.edu/_documents/a-to-z/s/Seligman_10.pdf

Simková, E. (2014). Psychology and its application in tourism. Procedia - Social and Behavioral Sciences, 114, 317-321. https://doi.org/10.1016/j.sbspro.2013.12.704

Smith, M., \& Diekmann, A. (2017). Tourism and wellbeing. Annals of Tourism Research, 66, 1-13. http://dx.doi.org/10.1016/j.annals.2017.05.006

Titus, C. S. (2017) Aquinas, Seligman, and positive psychology: A Christian approach to the use of the virtues in psychology. The Journal of Positive Psychology, 12(5), 447-458. https://doi.org/10.1080/17439760.2016.1228005

United Nations World Tourism Organization (2016, July). UNWTO Tourism Highlights - 2106 edition. http://www.e-unwto.org/doi/pdf/10.18111/9789284418145

Uysal, M., Siry, M., Woo, E., \& Kim, H. (2016). Quality of life (QOL) and well-being research in tourism. Tourism Management, 53, 244-261, http://dx.doi.org/10.1016/j.tourman.2015.07.013

Historial do artigo

Recebido $\quad 07 / 2019$

Aceite $\quad 03 / 2020$

Publicado $\quad 08 / 2020$ 
Revista PSICOLOGIA, 2020, Vol. 34 (1), doi: 10.17575/psicologia.v34i1.1681.

Atas do X Simpósio Nacional de Investigação em Psicologia

\title{
Thinking the future and developing skills for the workplace: A project with college students
}

\author{
Filipa Oliveira ${ }^{43 i}$, Carla Vale Lucasi, Luísa Soares $^{1} \&$ Ricardo Martins $^{\text {ii }}$ \\ i Universidade da Madeira, Madeira, Portugal \\ ii Académica da Madeira, Madeira, Portugal
}

\begin{abstract}
Today's workplace imposes new challenges for college students. Psychologists that work in Higher Education play an important role as facilitators on the transition from university to work, helping students to think and design the future and careers. Under this scope, the project named "Invest in yourself: win competitive advantage in the transition to the workplace" was implemented. This project was co-financed by the Nacional Agency of Erasmus +, resulting from the partnership between the Psychological Counselling Service and the Student's Association of the University of Madeira. It aimed to promote career adaptability and the development of soft skills among College students, through different activities. Thus, over 20 months, college students had several opportunities to promote their critical reflexivity about the future and today's workplace and to design a career more aligned with personal interests, goals, and values, which constitutes a competitive advantage in the transition to the workplace.
\end{abstract}

Keywords: College students; Transition to work, Career adaptability, Soft-skills.

Pensar o futuro e desenvolver competências para o mercado de trabalho: Um projeto no contexto universitário: 0 mundo de trabalho impõe múltiplos desafios aos estudantes. Os psicólogos do Ensino Superior desempenham um papel importante enquanto facilitadores do processo de transição para o mercado de trabalho, auxiliando a pensar e a desenhar o futuro/carreira. Neste âmbito, foi desenvolvido o Projeto "Investe em ti: ganha vantagem competitiva na transição para o mercado de trabalho", cofinanciado pela Agência Nacional Erasmust, resultado da parceria entre o Serviço de Psicologia e a Associação de Estudantes da Universidade da Madeira. Este projeto visou promover a adaptabilidade de carreira e o desenvolvimento de soft-skills em estudantes universitários, através de diferentes iniciativas. Ao longo de 20 meses, foram criadas oportunidades para os estudantes exercitarem o seu pensamento crítico, ganharem uma maior consciencialização acerca dos requisitos do mercado de trabalho, bem como desenharem um futuro/carreira alinhado com interesses, valores e objetivos pessoais, elementos essenciais para uma transição bem-sucedida para o mundo de trabalho.

Palavras-chave: Estudantes universitários; Transição para o trabalho; Adaptabilidade de carreira, Softskills.

Do you usually think about the future and prepare yourself for it? Do you often make decisions and take responsibility for your actions? Do you look continuously for opportunities to develop as a person? Do you try your best, adopting a problem-solving posture when facing problems and obstacles?

These questions encapsulate the meaning of career adaptability, a key element to win a competitive advantage in the labor market (Savickas, 1997).

Career adaptability is the "readiness to cope with the predictable tasks of preparing for and participating in the work role and with the unpredictable adjustments prompted by changes in work and working conditions" (Savickas, 1997). It comprises four psychosocial resources/self-regulation strengths or capacities, as concern - looking ahead and prepare for the future; control - take charge of the future by being responsible and conscientious in making career-related decisions; curiosity - exploring the environment and thinking about the self in many possible situations, scenarios, and roles; and confidence - having self-efficacy to undertake necessary activities to implement a life design (Koen, Klehe, \& Van Vianen, 2012; Savickas \& Porfeli, 2012).

${ }^{43}$ Correspondence address: Carla Vale Lucas, Serviço de Psicologia da Universidade da Madeira, Colégio dos Jesuítas, Colégio dos Jesuítas Rua dos Ferreiros, 9000-039 Funchal - Portugal; email: carla.lucas@staff.uma.pt 
Career adaptability is related with self-efficacy in finding a job (Gamboa, Paixão, \& Palma, 2014; Guan et al., 2013), career success and well-being among individuals (Koen, Klehe, \& Van Vianen, 2012), to name some of its benefits.

Today's world is far different from the world of previous generations. It is extremely dynamic, uncertain, complex, and highly competitive. Transitions from education to work have become more prolonged and increasingly unpredictable (Lehmann, 2004) and the notion of lifetime employment has been replaced by lifetime employability. Consequently, planning for the future can be done by individuals with some anxiety, which may lead to the postponement of some important career decisions (Campbell \& Ungar, 2004; Krumboltz, 1992).

All this entails new challenges for young people, regardless of social context, gender or race (Lehmann, 2004), and for European Union, which set as their top priorities increasing employability and tackling high rates of youth unemployment.

In this regard, universities can play an important role. They can help to engage a change in the mindset of college students, which is extremely necessary to meet today's work demands, enabling them to become critical and reflective citizens (Coelho et al., 2014). They can also promote student career adaptability to manage the transition to professional contexts (Monteiro \& Almeida, 2015), helping students to build their lives and career projects, so that they are more adaptable and aligned with whom they want to become, to better contribute for society, and to maintain employable. Furthermore, besides investing in increasing student's human capital, by developing their technical-professional skills, universities should open avenues for the development of other personal and social skills, such as the ones identified in the "Future of Jobs Report" (Centre for the New Economy and Society, 2018) of the World Economic Forum (i.e. critical thinking; resilience, flexibility; complex problem solving; emotional intelligence; leadership; among others).

Within these goals, psychologists working in higher education, besides promoting psychological well-being and academic success, are key agents that can help students (re)define their vision of future, assume responsibility and authorship of their life/career project, foster a proactive attitude, promote career adaptability and self-efficacy in relation to life/career project (Oliveira, Soares, \& Lucas, 2016). Activities such as counseling, psychotherapy, pedagogical support, tutoring, seminars, workshops, psychoeducational activities, among others, are means to achieve those purposes.

Under this scope, we present the first insights of a project aimed at college students, named "Invest in yourself: win competitive advantage in the transition to the workplace".

\section{METHOD}

The project "Invest in yourself: win competitive advantage in the transition to the workplace" was carried out over twenty months and was divided into two phases, beginning in February $2018^{44}$.

It resulted from the collaborative effort between the Psychological Counselling Service of the University of Madeira, that works towards promoting psychological wellbeing and personal development of college students and the Students Association of the University of Madeira, an academic structure that represents students. It also occurred the collaboration of other entities, namely the Employment Service of the Institution that aims to support the incorporation or reintegration of unemployed youth and adults into the labor market.

The project main's goal was to help college student's win a competitive advantage in the transition and adaptation to professional life, by promoting career adaptability and the development of soft-skills, essential for the workplace. In more detail, it aimed to encourage the development of an open and curious mind about the future; encourage a more proactive attitude, in the context of active and responsible citizenship; promote collaborative work and problem-solving skills; promote self-knowledge and the development of psychological resources (i.e. self-efficacy) to successfully deal with the tasks inherent to the transition/adaptation to work.

\section{Participants and procedure}

This project mobilized several students from the University of Madeira, enrolled in different levels of academic education, from areas such as management, business studies, psychology, biochemistry, physical education/sports, engineering, and educational sciences.

44 This project was co-financed by the National Agency of Erasmus+ (reference number 2017-3-PT02-KA347-004736), being also under the program called Universitas - a program that offers several actions and initiatives to promote the adaptation of students to college life and to help prepare for the workplace. 
At least 152 students (68\% women, and $29 \%$ men) attended the formal initiatives, $72 \%$ of the college students ranged between 18 and 24 years old, $11 \%$ between $25-20$ and $16 \%$ having more than 30 years old.

Activities were publicized to the Academia through posters/flyers, shared in public spaces, official website, and networks, by SMS and with the help of college students.

Formal activities had restrictions on the number of participants, to create smaller groups, where discussion and reflection could turn more feasible. To participate in the formal activities' students had to enroll by filling a form, available online and in some common spaces, with the help of other peers, coresponsible for publicizing the initiatives. Although it was recommended enrolling in all the sessions of an activity, students could choose the sessions that best fit their needs and/or availability. This was also a way to allow and promote self-determination among participants.

\section{Intervention Plan}

According to Savickas et al. (2009), initiatives should seek to adopt a dynamic approach. Therefore, formal and non-formal activities were implemented with different characteristics, formats and time duration (i.e. Open days and distribution of psycho-educative materials; Skills Training/workshops such as "Think the future. Build yours", "Communicate better at the workplace", "Job Interview", "Key skills for work"; Discussion Forum "Thinking on the future and acting on the present"; Tertulia "Among peers"; Peddy Paper "Skills Camp"). Table 1 shows an overview of the activities and aims. A portfolio of the activities can be found online ${ }^{45}$.

The common principles of the activities were to create opportunities for students to exercise critical and reflexive thinking about future and work, promote self-knowledge, help to design a life project and define personal and career goals, develop psychological resources like the ones described in career adaptability, and prompt the development of soft-skills.

Formal activities, like the skills training/workshops, used active methodologies. They usually started with ice-breaking activities, to create a secure environment where participants could feel comfortable to share ideas. Group dynamics and individual exercises were used to help further promote reflection and self-knowledge. Questions were presented and answered by participants and by the facilitator to increase reflection. With the same purpose, games and metaphors were used to better enable reflexivity about key skills. The discussion focused both on emotions and ideas, gathered from personal experience, reading and research (Osterman \& Kottkamp, 1993). During sessions, participants received a booklet with exercises to be done in and out of session. Each session usually finished with a challenge to be done at home, aimed at facilitating the transference of the abilities learned to everyday life.

The activities plan was set considering reflections made while working with college students (Oliveira et al., 2016). It was also designed based on Life design theory (Savickas et al., 2009), and the concepts of career adaptability and self-efficacy in the transition to the workplace, that can be developed in individuals (Savickas \& Porfeli, 2012). They were also grounded in the principles of positive psychology (Seligman \& Csikszentmihalyi, 2000) and Banduras' social cognitive theory.

Activities were designed and implemented mostly by the psychologists from the Psychological Counselling Service, that provided information and resources to facilitate individuals' personal inquiry and professional growth (Osterman \& Kottkamp, 1993). Other activities had collaborations from the psychologist of the Employment Service of the University, professors, professionals from different domains, and local and national political decision-makers. Students were also a key piece in all the initiatives, both in the backstage (sharing and advertising the initiatives) and in the main role, sharing ideas, thoughts, and training personal and social skills. They were also responsible for organizing some of the non-formal activities held, in a peer-to-peer intervention, following the benefits of mentoring by peers (Coelho et al., 2014).

${ }^{45}$ Portfolio of activities can be found at https://academica.uma.pt/?page_id=10643 
Table 1. Activities and aims

\begin{tabular}{|c|c|c|c|c|}
\hline Type & Name & Facilitators & Duration & Main characteristics and aims \\
\hline $\begin{array}{l}\text { Non-formal } \\
\text { Different } \\
\text { activities }\end{array}$ & $\begin{array}{l}\text { Open days held under } \\
\text { the Event "Employability } \\
\text { Forum" organized by the } \\
\text { University of Madeira. }\end{array}$ & $\begin{array}{l}2 \text { psychologists of the } \\
\text { Psychological } \\
\text { Counselling Service }\end{array}$ & $\begin{array}{l}\text { Two full days in each } \\
\text { cycle ( } 7 \text { hours per } \\
\text { day) } \\
\text { Total of } 28 \text { hours }\end{array}$ & $\begin{array}{l}\text { Presentation of Project goals' and activities; increase awareness about valued skills in } \\
\text { today's workplace and how to develop them and promote critical thinking about the } \\
\text { importance of designing a future/career with meaning. } \\
\text { Over } 2 \text { days (in each cycle), we posed questions to students about what it means } \\
\text { words such as "future", "present", and "success". Students were invited to share, with } \\
\text { the academic community, their thoughts, by posting them on a mural. Students were } \\
\text { also invited to participate in some games (i.e. electric maze, tumble...), used as } \\
\text { metaphors for today's workplace. }\end{array}$ \\
\hline $\begin{array}{l}\text { Formal } \\
\text { Skills } \\
\text { training (1) }\end{array}$ & $\begin{array}{l}\text { "Pensa o futuro. Constrói } \\
\text { o teu." ("Think the } \\
\text { future. Build yours") }\end{array}$ & $\begin{array}{l}2 \text { Psychologists of the } \\
\text { Psychological } \\
\text { Counselling Service }\end{array}$ & $\begin{array}{l}\text { Total of } 9 \text { hours, } \\
\text { distributed in } \\
\left.3 \text { sessions ( } 1^{\text {st }} \text { cycle }\right)\end{array}$ & $\begin{array}{l}\text { Increase awareness about work requirements and the importance of career } \\
\text { adaptability to face a highly competitive environment; promote the development of } \\
\text { key skills and psychological resources, facilitators of the transition to the workplace; } \\
\text { promote self-knowledge, authorship, and responsibility for the construction of a life } \\
\text { project; discuss self-efficacy beliefs and the importance of developing it, over time. }\end{array}$ \\
\hline $\begin{array}{l}\text { Formal } \\
\text { Skills } \\
\text { training (2) }\end{array}$ & $\begin{array}{l}\text { "Comunicar bem no } \\
\text { Mercado de trabalho" } \\
\text { (Communicate better at } \\
\text { the workplace) }\end{array}$ & $\begin{array}{l}2 \text { psychologists of the } \\
\text { Psychological } \\
\text { Counselling Service }\end{array}$ & $\begin{array}{l}6 \text { hours, distributed } \\
\text { in } 2 \text { sessions }\left(1^{\text {st }}\right. \\
\text { cycle })\end{array}$ & $\begin{array}{l}\text { Promote reflection about the importance of communication and the barriers that } \\
\text { come in between, with consequences in professional and non-professional } \\
\text { relationships; increase self-knowledge about communication style and put on } \\
\text { practice assertiveness abilities. }\end{array}$ \\
\hline $\begin{array}{l}\text { Formal } \\
\text { Skills } \\
\text { training } \\
(3 / 4)\end{array}$ & $\begin{array}{l}\text { "A Entrevista de } \\
\text { emprego" ("Job } \\
\text { Interview") }\end{array}$ & $\begin{array}{l}2 \text { psychologists } \\
\text { (Psychological } \\
\text { Counselling Service } \\
\text { and Employability } \\
\text { Service) }\end{array}$ & $\begin{array}{l}3 \text { hours }(1 \text { session })- \\
1^{\text {st }} \text { cycle } \\
6 \text { hours }(2 \text { sessions })- \\
2^{\text {nd }} \text { cycle }\end{array}$ & $\begin{array}{l}\text { Raise awareness about skills valued by employees, the role of non-verbal } \\
\text { communication, and common mistakes on interviews; increase self-knowledge, } \\
\text { essential while answering questions on an interview; role-playing interview scenarios }\end{array}$ \\
\hline $\begin{array}{l}\text { Formal } \\
\text { Skills } \\
\text { training (5) }\end{array}$ & $\begin{array}{l}\text { "Competências chave } \\
\text { para o Mercado de } \\
\text { trabalho" (Key skills for } \\
\text { the work market) }\end{array}$ & $\begin{array}{l}2 \text { psychologists of the } \\
\text { Psychological } \\
\text { Counselling Service }\end{array}$ & $\begin{array}{l}6 \text { hours (distributed } \\
\text { in } 3 \text { sessions) }\end{array}$ & $\begin{array}{l}\text { Raise awareness about today's work market requirements and about the role that } \\
\text { motivation, proactivity, problem-solving skills, resilience, and assertiveness plays in } \\
\text { today's world; develop essential skills to better face the challenges of work transition, } \\
\text { as career adaptability; }\end{array}$ \\
\hline $\begin{array}{l}\text { Non-formal } \\
\text { Tertulia }\end{array}$ & $\begin{array}{l}\text { Sunset "Entre Pares" } \\
\text { (Among peers) }\end{array}$ & $\begin{array}{l}\text { College students } \\
\text { (peer to peer) }\end{array}$ & 2 hours & $\begin{array}{l}\text { Share experiences/perceptions by peers about the future, work market and other key } \\
\text { elements to win competitive advantage at the workplace. }\end{array}$ \\
\hline $\begin{array}{l}\text { Formal } \\
\text { Forum }\end{array}$ & $\begin{array}{l}\text { "Pensar no Futuro, Agir } \\
\text { no Presente" (Thinking } \\
\text { on the future and acting } \\
\text { on the present) }\end{array}$ & $\begin{array}{l}\text { Professionals from } \\
\text { different areas, } \\
\text { political decision- } \\
\text { makers, college } \\
\text { students, } \\
\text { psychologists of the } \\
\text { Psychological } \\
\text { Counselling Service }\end{array}$ & $\begin{array}{l}2 \text { days (total of 10,5 } \\
\text { hours) }\end{array}$ & $\begin{array}{l}\text { Promote the dialogue between students and political decision-makers about } \\
\text { employability, today's work market, problems that youth face and solutions for it. } \\
\text { The first part of the forum was kept for formal communications. Later, students were } \\
\text { organized in groups, randomly selected, creating discussion panels. They worked } \\
\text { towards identifying problems and obstacles to employability, developing a proposal } \\
\text { with solutions and presenting them to political decision-makers. }\end{array}$ \\
\hline
\end{tabular}


Non-formal "Skills camp: Assume o

Peddy paper comando" (Skills Camp:

Take charge)

Non-formal Distribution of Psychoeducative materials
College students

with the supervision

of psychologists)

Psychologists of the

Psychological

Counselling Service
2 hours (to be held in

September)

During the academic year
Promote the development of skills such as proactivity, collaborative work, creative problem solving.

Different articles and materials were made available online to increase reflection about the future and career:

Articles: "A importância de pensar o futuro e a carreira" (In English: The importance of thinking the future and career) and "Como se posiciona face ao futuro e à vida" (In

English: How do you position yourself in the future and in life) published in the

Students Association Magazine, named "Et al." (issuu 85 and 86); Booklets: "Como quero que seja o meu futuro?" (In English: How I want my future to be); "Invista em si: competências chave na procura de trabalho (In English: Invest in yourself: key skills

while searching for a job): A responsabilidade de ser feliz é sua (in english: The 


\section{Qualitative and quantitative evaluation of the initiatives}

The evaluation of formal activities was made by inviting students to fill a questionnaire with closed and open-ended questions. The closed questions were aimed to evaluate the event and to self-evaluate their engagement. The open-ended questions aimed to collect perceived strengths and weaknesses of the initiative and to put participants identifying one or more significant event ('event" meaning something said or done in session).

\section{RESULTS AND DISCUSSION}

Table 2 shows indicators of the project's preliminary results, namely the number of participants and the data collected from the evaluation questionnaires.

Over 20 months, the project involved 6 formal initiatives, 4 non-formal initiatives and approximately 72 hours of direct intervention, reaching 152 students on the formal activities. More students were involved in the non-formal activities, although not accounted in the numbers here presented.

Overall, in the formal activities held, participants perceived the value of the initiatives as being very good (77\%), showing also a good perceived engagement with the activities held ( $94 \%$ identifying themselves as engaged or very engaged), which are good indicators of the learning experience. Studies show that students who are sufficiently engaged with learning course material and perceive the value of a course as high will have an overall positive learning experience (Floyd, Harrington, \& Santiago, 2009).

On the qualitative evaluation of the initiatives, students identified as strengths, to name some: the group dynamic; the interactivity that enable to create a secure place for sharing opinions and actively participating; the practical exercises and examples of real-life situations, making it possible to develop a reflexive thinking; and the organization of the materials and activities. Some students further categorized the event as a "good time".

These elements seem to be good indicators of the learning process since learning is more effective when people become personally engaged, and when it takes place as a collaborative activity (Osterman and Kottkamp, 1993). Indeed, having fun while promoting a critical reflexibility about life, the future and the work environment, posing questions and using different types of methodologies to further instigate the reflection, allowed to create an environment more prone to learning, where students assumed the main role, as authors, and feel comfortable to share ideas/thoughts with peers and the facilitators.

Students' answers about relevant events in session allow us to see in more detail how the session met students' needs to learn, another important element for fostering engagement and contributing for the learning process (Osterman \& Kottkamp, 1993). Examples of student's quotes are: “...it led me to understand that sometimes risking is needed and thus confidence and self-knowledge are essential for takeoff"; "...it allowed me to think on abilities that I have and how I can empower them" (Skills training 1); "... I need to do introspection about who I am and how I see myself in 5 years" (Skills training 4); "... it led me to acknowledge the need to change my behavior, being more proactive in some occasions (...) not allowing fear of making mistakes guide my way" (Skills training 5). Overall, the answers given emphasized the importance of self-knowledge, assuming authorship and responsibility for designing and implementing a life project, not letting it only at the demand of luck, destiny, and others, as stated by Oliveira et al. (2016).

Participants identified as weaknesses of the initiatives, the duration of the sessions (too long or too short) and the time of the academic year in which they were held. As so, the collected inputs led us, to adjust the duration of some sessions, to better fit student's needs. However, choosing the adequate time to offer some activities will always pose as a challenge since, year by year, the academic schedule gets shorten and students' academic demands get higher. Thus, besides trying to adjust the duration of the activities to fit student's academic schedule, we need to continue working on adding more weight to students' decisional balance, offering more reasons for them to choose to empower their skills to better face work market. To make this happen, peers and professors play a crucial role as disseminators of the initiatives as well as their relevance to personal and professional development. 
Table 2. Overview of some indicators of projects' results (number of participants and evaluation questionnaire)

\begin{tabular}{|c|c|c|c|c|c|}
\hline & \multicolumn{3}{|c|}{ Participants } & \multicolumn{2}{|l|}{ Evaluation Questionnaire } \\
\hline & Total (n) & Gender (n) & $\operatorname{Age}(n)$ & Quantitative evaluation & Perceived involvement \\
\hline $\begin{array}{l}\text { Skills training 1 "Pensa o futuro. } \\
\text { Constrói o teu." ("Think the future. } \\
\text { Build yours") }\end{array}$ & 16 & $\begin{array}{l}\text { Women: } 11(69 \%) \\
\text { Men: } 5(31 \%)\end{array}$ & $\begin{array}{l}18 \text { - } 24 \text { years: } 14 \\
25 \text { - } 30 \text { years: } 2\end{array}$ & $\begin{array}{l}\text { Very good: } 70 \% \\
\text { Good: } 30 \%\end{array}$ & $\begin{array}{l}\text { Very involved: } 30 \% \\
\text { Involved: } 70 \%\end{array}$ \\
\hline $\begin{array}{l}\text { Skills training } 2 \text { "Comunicar bem no } \\
\text { Mercado de trabalho" ("Communicate } \\
\text { better at the workplace") }\end{array}$ & 17 & $\begin{array}{l}\text { Women: } 12(71 \%) \\
\text { Men: } 5(29 \%)\end{array}$ & $\begin{array}{l}18-24 \text { years: } 11 \\
25-30 \text { years: } 4 \\
+30 \text { years: } 2\end{array}$ & $\begin{array}{l}\text { Very good: } 67 \% \\
\text { Good: } 33 \%\end{array}$ & $\begin{array}{l}\text { Very involved: } 11 \% \\
\text { Involved: } 78 \% \\
\text { Not very involved: } 11 \%\end{array}$ \\
\hline Skills training 3/4 "Entrevista de & 16 & $\begin{array}{l}\text { Women: } 11(69 \%) \\
\text { Men: } 5(31 \%)\end{array}$ & $\begin{array}{l}18 \text { - } 24 \text { years: } 13 \\
25-30 \text { years: } 1 \\
+30 \text { years: } 2\end{array}$ & $\begin{array}{l}\text { Very good: } 82 \% \\
\text { Good: } 18 \%\end{array}$ & $\begin{array}{l}\text { Very involved: } 27 \% \\
\text { Involved: } 73 \%\end{array}$ \\
\hline emprego" ("Job Interview") & 24 & $\begin{array}{l}\text { Women: } 21(88 \%) \\
\text { Men: } 3(12 \%)\end{array}$ & $\begin{array}{l}18 \text { - } 24 \text { years: } 19 \\
25 \text { - } 30 \text { years: } 2 \\
+30 \text { years }: 3\end{array}$ & $\begin{array}{l}\text { Very good: } 78 \% \\
\text { Good: } 22 \%\end{array}$ & $\begin{array}{l}\text { Very involved: } 39 \% \\
\text { Involved: } 55 \% \\
\text { Neutral: } 6 \%\end{array}$ \\
\hline $\begin{array}{l}\text { Skills training } 5 \text { "Competências chave } \\
\text { para o Mercado de trabalho" ("Key } \\
\text { skills for work") }\end{array}$ & 21 & $\begin{array}{l}\text { Women: } 14(67 \%) \\
\text { Men: } 7 \text { (33\%) }\end{array}$ & $\begin{array}{l}18-24 \text { years: } 19 \\
+30 \text { years: } 2\end{array}$ & $\begin{array}{l}\text { Very good: } 88 \% \\
\text { Good: } 12 \%\end{array}$ & $\begin{array}{l}\text { Very involved: } 32 \% \\
\text { Involved: } 55 \% \\
\text { Neutral } 13 \%\end{array}$ \\
\hline $\begin{array}{l}\text { Forum "Pensar no Futuro, Agir no } \\
\text { Presente" ("Thinking on the future and } \\
\text { acting on the present") }\end{array}$ & 58 & $\begin{array}{l}\text { Women: } 34(59 \%) \\
\text { Men: } 19(33 \%)\end{array}$ & $\begin{array}{l}<18 \text { years: } 1 \\
18-24 \text { years: } 34 \\
25-30 \text { years: } 8 \\
+30 \text { years: } 15\end{array}$ & - & - \\
\hline Formal ativities - total values & 152 & $\begin{array}{l}\text { Women:103 (68\%) } \\
\text { Men: } 44(29 \%)\end{array}$ & $\begin{array}{l}<18: 1(1 \%) \\
\text { 18 - } 24 \text { years: } 110(72 \%) \\
25-30 \text { years: } 17(11 \%) \\
+30 \text { years: } 24(16 \%)\end{array}$ & $\begin{array}{l}\text { Very good: } 77 \% \\
\text { Good: } 23 \%\end{array}$ & $\begin{array}{l}\text { Very involved: } 28 \% \\
\text { Involved: } 66 \% \\
\text { Neutral: } 4 \% \\
\text { Not very involved: } 2 \%\end{array}$ \\
\hline
\end{tabular}




\section{LIMITATIONS}

The project lacks a more detail assessment of its efficacy, namely measuring the impact on student's career adaptability, self-efficacy in the transition to work, and student's engagement. We also recognize that it would be preferable to work with the same group over specific skills training, allowing time for changes to happen, and gathering data before and after the interventions.

\section{CONCLUSION}

The project "Invest in yourself: win competitive advantage in the transition to the workplace" represents the effort of psychologists, student's, and people from different domains, and entities from the local and national community, to empower college students to become more successful on the transition to workplace and to maintain employable over time (Maree, 2010; Savickas, 2012).

Therefore, as a university, we cannot only work on promoting student's human capital (technical and professional skills). We also cannot give solutions to students, nor they would be applicable in an everchanging environment. We can only aspire to work towards increasing awareness about today's workplace requisites, to foster student's self-knowledge, helping them to design a life/career project, prompting critical reflexivity, promoting the development of key psychological resources, and helping them to turn more flexible and adjustable.

An adjustable individual is someone who reveals great concern about his/her vocational future, takes more control over his future, shows great curiosity by exploring its many possible selves and future sceneries and opportunities, and exhibit confidence in achieving his goals (Savickas \& Porfeli, 2012), thus someone with a key characteristic that can enable him/her to win competitive advantage in the multiple transitions required in today's workplace.

As so, our quest as psychologists working in a Higher Education should be to create avenues for reflection and opportunities for growth in students, spreading the idea that future success (both in life and career) depends upon the quality of the investment that is done on the present.

\section{REFERENCES}

Campbell, C., \& Ungar, M. (2004). Constructing a Life That Works: Part 1, Blending Postmodern Family Therapy and Career Counseling. The Career Development Quarterly, 53(1), 16-27. https://doi.org/10.1002/j.2161-0045.2004.tb00652.x

Centre for the New Economy and Society. (2018). The Future of Jobs Report [Insight Report]. World Economic Forum.

Coelho, C., Faria, C., Oliveira, F., Vale Lucas, C., Vasconcelos, S., \& Soares, L. (2014). Pilot project for peer tutoring: a case study in a Portuguese university. Journal of Applied Research in Higher Education, 6(2), 314-324. https://doi.org/10.1108/JARHE-09-2013-0038

Gamboa, V., Paixão, O., \& Palma, A. I. (2014). Career adaptability and self-efficacy in school-work transition: the role of the perceived employability: a study with Higher Education students. Revista Portuguesa de Pedagogia, 48(2), 133-156. https://doi.org/10.14195/1647-8614_48-2_7

Guan, Y., Deng, H., Sun, J., Wang, Y., Cai, Z., Ye, L., Fu, R., Wang, Y., Zhang, S., \& Li, Y. (2013). Career adaptability, job search self-efficacy and outcomes: A three-wave investigation among Chinese university graduates. Journal of Vocational Behavior, 83(3), 561-570. https://doi.org/10.1016/j.jvb.2013.09.003

Koen, J., Klehe, U.-C., \& Van Vianen, A. E. M. (2012). Training career adaptability to facilitate a successful school-to-work transition. Journal of Vocational Behavior, 81(3), 395-408. https://doi.org/10.1016/j.jvb.2012.10.003

Krumboltz, J. D. (1992). The wisdom of indecision. Journal of Vocational Behavior, 41(3), 239-244. https://doi.org/10.1016/0001-8791(92)90025-U

Lehmann, W. (2004). "For Some Reason, I Get a Little Scared": Structure, Agency, and Risk in SchoolWork Transitions. Journal of Youth Studies, 7(4), 379-396. https://doi.org/10.1080/1367626042000315185

Maree, J. G. (2010). Brief Overview of the Advancement of Postmodern Approaches to Career Counseling. Journal of Psychology in Africa, 20(3), 361-367. https://doi.org/10.1080/14330237.2010.10820387

Monteiro, S., \& Almeida, L. S. (2015). The relation of career adaptability to work experience, extracurricular activities, and work transition in Portuguese graduate students. Journal of Vocational Behavior, 91, 106-112. https://doi.org/10.1016/j.jvb.2015.09.006

Oliveira, F., Soares, L., \& Lucas, C. V. (2016). Life and career behaviour: Some considerations about the need to assist college students to change their mind set and behaviours toward life construction. RUMOS, Revista Científica Da Universidade Do Mindelo, 3(1), 109-122. 
Osterman, K., \& Kottkamp, R. (1993). Rethinking Professional Development. In Reflective Practice for Educators - Improving Schooling Through Professional Development (pp. 18-42). CORWIN PRESS, INC.

S. Floyd, K., Harrington, S., \& Santiago, J. (2009). The Effect of Engagement and Perceived Course Value on Deep and Surface Learning Strategies. Informing Science: The International Journal of an Emerging Transdiscipline, 12, 181-190. https://doi.org/10.28945/435

Savickas, M. L. (1997). Career Adaptability: An Integrative Construct for Life-Span, Life-Space Theory. The Career Development Quarterly, 45(3), 247-259. https://doi.org/10.1002/j.21610045.1997.tb00469.x

Savickas, M. L. (2012). Life Design: A Paradigm for Career Intervention in the 21st Century. Journal of Counseling \& Development, 90(1), 13-19. https://doi.org/10.1111/j.1556-6676.2012.00002.x

Savickas, M. L., Nota, L., Rossier, J., Dauwalder, J.-P., Duarte, M. E., Guichard, J., Soresi, S., Van Esbroeck, R., \& van Vianen, A. E. M. (2009). Life designing: A paradigm for career construction in the 21st century. Journal of Vocational Behavior, 75(3), 239-250. https://doi.org/10.1016/j.jvb.2009.04.004

Savickas, M. L., \& Porfeli, E. J. (2012). Career Adapt-Abilities Scale: Construction, reliability, and measurement equivalence across 13 countries. Journal of Vocational Behavior, 80(3), 661-673. https://doi.org/10.1016/j.jvb.2012.01.011

Seligman, M. E. P., \& Csikszentmihalyi, M. (2000). Positive psychology: An introduction. American Psychologist, 55, 5-14.

Historial do artigo

Recebido $\quad 07 / 2019$

Aceite $\quad 01 / 2010$

Publicado 08/2020 
Revista PSICOLOGIA, 2020, Vol. 34 (1), doi: 10.17575/psicologia.v34i1.1681.

\title{
Significado e empatia: Efeitos de uma intervenção baseada em Teatro Playback - resultados preliminares
}

\author{
Nuno Amarante ${ }^{46 i}$, António-José Gonzalezi, Paulo Martinsii, Maria João Gouveia ${ }^{\mathrm{i}}$ \& \\ Susana Lavado iii \\ ${ }^{i}$ AppsyCI - Applied Psychology Research Center Capabilities and Inclusion, ISPA - Instituto Universitário, \\ Lisboa, Portugal \\ ii FMH-UL - Laboratório de Psicologia do Desporto, Faculdade de Motricidade Humana, Universidade de \\ Lisboa, Lisboa, Portugal \\ iii Nova SBE - Nova School of Business and Economics, Lisboa, Portugal
}

\begin{abstract}
Resumo: 0 Teatro Playback (TP) é um tipo de teatro em que são representadas, através do improviso, histórias pessoais partilhadas pela audiência, sendo cocriados, através da escuta empática e da expressão artística, novos significados para as experiências dos narradores. Uma vez que o significado é um dos constituintes do bem-estar, pretende-se investigar se uma intervenção de TP com 12 sessões tem efeito na experiência de significado e empatia dos participantes. Utilizou-se um delineamento experimental com medidas de pré-pós-teste e distribuição aleatória dos participantes no grupo experimental e controlo. Não foi possível concluir, estatisticamente, que a intervenção tenha tido efeito nos participantes. Discutem-se implicações teóricas sobre a ligação do TP aos construtos de empatia e significado. Complementar a análise quantitativa com uma qualitativa dos resultados da intervenção poderá trazer novas pistas para a utilização do TP como promotor do bem-estar.
\end{abstract}

Palavras-chave: Teatro playback; Bem-estar; Significado; Empatia.

Meaning and Empathy: effects of an intervention based on Playback Theatre - preliminary results: Playback Theater (PT) is a type of theater in which personal stories shared by the audience are represented through improvisation, and where new meanings for the narrators' experiences are cocreated through empathic listening and artistic expression. Since meaning is one of the components of well-being, the goal is to investigate whether a 12 session PT intervention has an effect on participants' levels of meaning and empathy. A randomized controlled trial was conducted but, statistically, it could not be concluded that the intervention had an effect on the participants. Important theoretical contributions are made linking PT to the constructs of empathy and meaning. Complementing the quantitative analysis of the results with a qualitative one may provide new clues to the use of TP as a well-being promotion intervention.

\section{Keywords: Playback theatre; Well-being; Meaning; Empathy.}

O Teatro Playback (TP) é um formato teatral de improviso, sem guião, que consiste numa cocriação entre os atores e a audiência, e acontece tipicamente em contextos comunitários com audiências reduzidas (de até 50 pessoas), tendo como objetivo a transformação social (Salas, 1993). São solicitadas aos membros da audiência histórias pessoais narradas na primeira pessoa, que são depois devolvidas por uma equipa de atores, em palco, na forma de representação artística com recurso à expressão corporal e vocal, panos, música e luz. 0 TP não tem agenda pré-definida, não se sabe o que vai acontecer em cada performance, mas é estruturado por rituais bem definidos (Fox, 1999). Jonathan Fox (2013), um dos fundadores do TP, alega ainda que este tipo de teatro não é terapia, mas é terapêutico e pode atingir muitos dos objetivos da psicoterapia. Existe, de facto, alguma investigação empírica que parece apontar neste sentido. Na Tabela 1 encontram-se listadas todas as publicações relativas a estudos experimentais e quasi-experimentais sobre os efeitos do Teatro Playback, sendo que em todos os casos se encontram resultados que parecem indicar efeitos terapêuticos. Destaca-se o número reduzido de estudos, apenas 5 , e somente 1 ter delineamento com grupo de placebo.

\footnotetext{
${ }^{46}$ Morada para correspondência: Nuno Amarante, ISPA - Instituto Universitário, Rua Jardim do Tabaco, 34, 1149-041. E-mail: nuno.a.g.amarante@gmail.com
} 
Tabela 1. Estudos experimentais e quasi-experimentais sobre os efeitos do Teatro Playback.

\begin{tabular}{|c|c|c|c|c|c|c|c|}
\hline Autor & Local & $\mathbf{N}$ & Contexto & População & Delineamento & Intervenção* & Principais resultados \\
\hline $\begin{array}{l}\text { Bornmann } \\
\text { et al. } \\
\text { (2011) }\end{array}$ & EUA & 24 & Escola & $\begin{array}{c}\text { Crianças; } \\
\text { Adolescentes }\end{array}$ & $\begin{array}{c}\text { Pré e pós teste } \\
\text { com grupo } \\
\text { placebo }\end{array}$ & $\begin{array}{l}\text { Assistir a } 1 \\
\text { sessão de } 1 \\
\text { dia }\end{array}$ & $\begin{array}{c}\text { Redução de níveis de } \\
\text { tolerância com a } \\
\text { agressão; não se } \\
\text { alteraram níveis de } \\
\text { empatia }\end{array}$ \\
\hline $\begin{array}{l}\text { Moran et } \\
\text { al. (2011) }\end{array}$ & EUA & 19 & $\begin{array}{l}\text { Instituição } \\
\text { de Saúde } \\
\text { Mental }\end{array}$ & $\begin{array}{c}\text { Adultos c/ } \\
\text { doença } \\
\text { mental grave }\end{array}$ & $\begin{array}{l}\text { Qualitativo } \\
\text { (entrevistas) e } \\
\text { pré e pós teste }\end{array}$ & $\begin{array}{c}\text { Treino de } 10 \\
\text { semanas / } 90 \\
\text { min. }\end{array}$ & $\begin{array}{l}\text { Aumento de autoestima, } \\
\text { autoconhecimento e } \\
\text { empatia; TP potencia } \\
\text { recovery de pessoas com } \\
\text { doença mental grave }\end{array}$ \\
\hline $\begin{array}{l}\text { Cheung et } \\
\text { al. (2016) }\end{array}$ & $\begin{array}{l}\text { Hong } \\
\text { Kong }\end{array}$ & 8 & $\begin{array}{c}\text { Escola } \\
\text { Secundária }\end{array}$ & Adolescentes & $\begin{array}{c}\text { Qualitativo } \\
\text { (Interpretative } \\
\text { Phenomenological } \\
\text { Analysis) }\end{array}$ & $\begin{array}{c}\text { Treino de } 30 \\
\text { horas }\end{array}$ & $\begin{array}{l}\text { Treino em TP é um meio } \\
\text { para atingir, desenvolver } \\
\text { e compreender empatia }\end{array}$ \\
\hline $\begin{array}{l}\text { Smigelsky } \\
\text { et al. } \\
(2016)\end{array}$ & USA & 10 & $\begin{array}{l}\text { Associação } \\
\text { de ex- } \\
\text { reclusos }\end{array}$ & $\begin{array}{l}\text { Polícias e ex- } \\
\text { reclusos }\end{array}$ & $\begin{array}{c}\text { Pré e pós teste } \\
\text { sem controlo }\end{array}$ & $\begin{array}{c}\text { Treino de } 2 \\
\text { meses / } 20 \\
\text { sessões de } 2 \mathrm{~h}\end{array}$ & $\begin{array}{c}\text { Aumento da capacidade } \\
\text { de dar significado a } \\
\text { eventos stressantes de } \\
\text { vida; aumento de atitudes } \\
\text { positivas entre os dois } \\
\text { grupos (polícias e ex- } \\
\text { reclusos) }\end{array}$ \\
\hline $\begin{array}{l}\text { Yotis et al. } \\
\text { (2017) }\end{array}$ & Grécia & NA & $\begin{array}{c}\text { Instituições } \\
\text { de Saúde } \\
\text { Mental }\end{array}$ & Adultos & $\begin{array}{c}\text { Qualitativo } \\
\text { (estudo de caso) }\end{array}$ & Assistir & $\begin{array}{l}\text { TP pode ser usado para } \\
\text { reduzir o estigma social e } \\
\text { pessoal para com pessoas } \\
\text { com perturbações } \\
\text { mentais }\end{array}$ \\
\hline $\begin{array}{l}\text { Chung et } \\
\text { al. (2018) }\end{array}$ & EUA & 18 & Instituições & Idosos & $\begin{array}{c}\text { Pré e pós teste } \\
\text { sem controlo }\end{array}$ & $\begin{array}{c}\text { Treino de } 6 \\
\text { semanas / } 90 \\
\text { min. }\end{array}$ & $\begin{array}{l}\text { Melhoria do bem-estar } \\
\text { emocional; sem } \\
\text { alterações nas funções } \\
\text { cognitivas }\end{array}$ \\
\hline
\end{tabular}

*Intervenção: Treino - intervenção consistiu em formação em TP sendo que os participantes dos estudos representaram em palco as histórias uns dos outros; Assistir - intervenção consistiu em ser membro da audiência de TP com uma equipa de atores formados em TP que representaram as histórias dos participantes

Dentro da investigação em psicologia do bem-estar, tem havido um crescente interesse na dimensão significado e a literatura sobre TP parece indicar efeitos cuja descrição é semelhante à da operacionalização de significado, ou seja, do ponto de vista teórico poderá haver uma ligação entre significado e TP. 0 significado é um constituinte do bem-estar, e diversos autores argumentam que é uma variável fundamental para conseguir obter uma mensuração compreensiva do bem-estar mental (Huta, 2013). A investigação sobre a psicologia do bem-estar tem sugerido que todos os conteúdos estudados nesta área podem ser enquadrados em dois grandes campos - hedonia e eudemonia -, e que, para estudar o bem-estar, é importante considerar constructos de ambos (Huta, 2016). Huta e Waterman (2014) realizaram uma revisão sistemática da literatura, na qual argumentam que a investigação sobre bemestar é algo confusa porque diferentes autores têm estudado e comparado conceitos destes dois campos 
(eudemonia e hedonia), utilizando diferentes modos de os definir, o que leva a uma aparente falta de consistência nesta área de investigação (Huta, 2016). Concluem que existem quatro categorias dentro das quais se consideram e estudam os respetivos constructos do bem-estar: orientações (aquilo que se procura na vida), comportamentos (atividades que se escolhe), experiências (emoções e sensações subjetivas) e funcionamento (competências estáveis e hábitos). No presente estudo, o significado é enquadrado enquanto experiência, mais associada à orientação eudemónica (procura de excelência e autorrealização) do que à hedónica (procura de prazer e conforto) (Huta, 2017; Huta \& Waterman, 2014). 0 significado pode ser operacionalizado através de 3 facetas. A primeira é a coerência e consiste em sentir as atividades e experiências de vida como sendo coerentes entre si, seguindo princípios consistentes, e conseguir que sejam partilhadas de forma a que constituam uma narrativa com sentido (Huta, 2017), sendo ainda possível ver onde encaixam em relação a outros aspetos da vida no geral (Martela \& Steger, 2016). Ou seja, a coerência poderá ser entendida como um mapa onde é possível observar como as atividades e experiências de vida se posicionam umas em relação às outras. A segunda faceta é a significância, e consiste em sentir que as experiências e atividades são preciosas dentro do contexto do quadro de valores pessoais (Huta, 2017) e que valem a pena ser partilhadas (Martela \& Steger, 2016). Se a coerência for ilustrada como sendo um mapa, a significância poderá ser o tesouro que esse mapa permite encontrar, portanto será aquilo que dá valor ao mapa. Por fim, a terceira faceta é o propósito ou implicações alargadas, e consiste em considerar que há ligações, implicações e relevância naquilo que se faz, em relação a outros aspetos da vida ou mesmo do mundo em geral (Huta, 2017). Se a coerência for o mapa, a significância o tesouro, o propósito poderá ser as considerações sobre as consequências alargadas de usar o mapa para encontrar o tesouro: quem e como irá afetar? Onde? Porquê? Para quê, então, procurá-lo?

Diferentes autores referem diferentes formas de promover o significado. No entanto, é reduzida a investigação empírica sobre eficácia de intervenções que promovam o significado e, apesar do TP poder ser enquadrado teoricamente como uma intervenção de promoção do significado, não existe nenhuma investigação sobre esta sua potencial aplicação. Jo Salas (1983), outra das fundadoras do TP, argumenta que "as histórias são fragmentos das vidas dos narradores, caóticos e apenas meio-compreendidos, sendo que a equipa de artistas recebe a narração, condensa alguns aspetos, expande outros, e sem discussão, apresenta uma peça teatral onde é possível o narrador da história testemunhar a sua experiência de forma mais cristalizada, clara e coerente" (p. 18). Rowe (2005) tenta ilustrar o processo do TP referindo que o "passado é irrecuperável e apenas pode ser-lhe dado significado (signified no original) através da mediação complexa entre memória e representação" (p. 2), i.e., os atores e narradores, ao co-construir a história, são mediadores do passado, das narrações que emergem. Nesta sequência, argumentamos que o TP poderá ser enquadrado teoricamente como uma intervenção de promoção de significado visto que, na literatura do bem-estar, algumas das estratégias de promoção de significado envolvem: "focar nas experiências pessoais através do uso intencional de metáforas ou narrativas, (...) sendo necessário, tentar de alguma forma, ligar os pontos" (Huta, 2015, p. 168) e "ajudar os indivíduos a reavaliar e reintegrar experiências anteriores, da sua vida, no seu contexto e circunstâncias atuais (...) procurando tecer uma narrativa de vida coerente com um tema predominante e central" (Shin e Steger, 2014, p. 96). Existe uma proposta teórica, fundamentada por um estudo de caso, que propõe a integração de TP com Psicoterapia Narrativa de Michael White (Barak, 2013). Barak argumenta que o TP pode ser visto como um processo de reescrever e refinar a história do narrador. No entanto, este autor refere que a sua proposta consiste numa integração de algumas ideias do TP, mas não se trata de TP. Apesar da Psicoterapia Narrativa e TP apresentarem semelhanças, existem importantes diferenças: em TP o ênfase é colocado na criação artística e não depende de objetivos terapêuticos sendo que, caso dependesse, poderia levar a uma perda do seu valor estético e teatral (Barak, 2013).

Um dos pressupostos do TP, e parte integrante do treino de ator de TP, é a escuta ativa e empática. Existe já alguma investigação sobre o impacto do TP na empatia, mas apenas 2 estudos (Bornmann \& Crossman, 2011; Cheung Ng \& Graydon, 2016) são experimentais (ver Tabela 1). A empatia é um constructo multidimensional definido como "a capacidade de entender e partilhar o estado emocional de outra pessoa" (Pechorro, Jesus, Kahn, Gonçalves, \& Barroso, 2018, p. 157). Hannah Fox (2007), outra das fundadoras do TP, afirma que "em TP a cura é trazida pela empatia, os membros da audiência, ao ouvir a história do narrador, e ao vê-la ser interpretada em palco, emocionam-se porque se lembram de uma parte das suas próprias histórias, subitamente sentem-se menos isolados, e experienciam interligação (interconnectedness no original) humana" (p. 99). Bornmann e Crossman (2011), num estudo que procurou avaliar o efeito do TP na empatia da audiência, argumentam que "os atores procuram nas suas memórias por identificação e empatia enquanto ao mesmo tempo preservam alguma distância para assegurar uma representação ética e estética da história, sendo que a audiência observa esta troca empática e, através da expressão artística, internaliza empatia pelo narrador" (p. 166). 
Como vimos, o TP pode ser enquadrado teoricamente como uma intervenção promotora de significado. Por outro lado, um aspeto central do TP é a empatia. Já existe alguma evidência sobre o impacto do TP na empatia, mas tanto quanto é do nosso conhecimento, nenhum estudo, até ao momento procurou analisar o seu papel no significado. Assim, este ensaio aleatório controlado tem como objetivo estudar o efeito do TP no aumento da experiência de significado e empatia da audiência.

\section{MÉTODO}

\section{Participantes}

Foi realizada uma sessão de apresentação aberta divulgada nas redes sociais, com inscrição online necessária, sendo intitulado o projeto como "Grupo de Desenvolvimento Pessoal através do Teatro Playback". Inscreveram-se 90 pessoas, das quais compareceram 64. Após apresentação do estudo foi solicitado aos participantes que, se concordassem em participar, preenchessem o consentimento informado e o questionário de pré-teste em papel. Inscreveram-se no estudo 48 participantes que foram divididos aleatoriamente entre o grupo experimental e de controlo (com recurso à ferramenta online random.org).

\section{Instrumentos}

Para medir o significado foi usada a Escala de Experiência de Significado (MES) de Huta e Ryan (2010). Este instrumento de 12 itens pretende medir o significado enquanto uma experiência subjetiva. É pedido aos participantes que avaliem numa escala de sete pontos (1=Não, de todo; 7=Sim, muito), como habitualmente se sentem em relação às suas atividades e experiências (e.g. "São algo que posso estimar"). Os resultados de cada participante são obtidos através da média das respostas aos itens, tendo a escala demonstrado bons níveis de consistência interna neste estudo no pré ( $\alpha=0.82)$ e pós-teste $(\alpha=0.98)$.

Para a empatia foi usada a Escala de Empatia Básica, versão breve adaptada (BES-A) de Jolliffe e Farrington (2006, versão portuguesa de Pechorro, Jesus, Kahn, Gonçalves e Barroso, 2018). Este instrumento mede as duas dimensões da empatia: afetiva (com 3 itens, e.g. "Depois de falar com um amigo que está triste geralmente também fico triste") e cognitiva (com 4 itens, e.g. "Geralmente costumo perceber quando as pessoas estão contentes"). É pedido aos participantes que classifiquem cada item numa escala de concordância de 5 pontos (Discordo totalmente a Concordo totalmente). Os resultados de cada participante são obtidos através do somatório das respostas aos itens, tendo a escala demonstrado igualmente níveis de consistência adequados no pré $(\alpha=0.74)$ e pós-teste $(\alpha=0.82)$.

\section{Procedimento}

O grupo experimental assistiu a 12 sessões de TP com duração de 2 horas, 1 vez por semana ao longo de 3 meses. Apenas 17 iniciaram efetivamente o grupo (i.e., estiveram presentes numa sessão) e 3 desistiram após 1 ou 2 sessões. Os restantes 14 participantes estiveram presentes em pelo menos 7, com frequência média de 9 sessões, correspondendo a uma taxa de retenção de $82 \%$. Cada sessão consistiu num aquecimento com recurso a dinâmicas de grupo (15 minutos), performance de TP (90 minutos) e uma fase de partilha livre (15 minutos). 0 grupo de controlo foi do tipo lista de espera, ficando a aguardar uma segunda edição da intervenção. Depois das 12 sessões, foi solicitado a ambos os grupos que preenchessem novamente o questionário para o pós-teste através de formulário online. Completaram 13 participantes do grupo experimental e 19 do grupo de controlo (54\% e 79\%).

\section{RESULTADOS}

Para analisar os resultados da intervenção, uma vez que não se verificaram os pressupostos para a aplicação do teste paramétrico (normalidade e homocedasticidade), recorreu-se ao teste não paramétrico para comparação de médias de amostras independentes de Wilcoxon-Mann-Whitney que indicou não haver diferenças na média entre grupos no pós-teste da variável Significado $(p=0.29)$ e Empatia $(p=$ 0.47). Na tabela 2, encontram-se as médias e desvios padrão das duas escalas separadas por grupo e por momento temporal. 
Tabela 2. Comparação das médias do grupo de controlo com os do grupo experimental, para as duas variáveis dependentes consideradas.

\begin{tabular}{lccccccccc}
\hline & \multicolumn{3}{c}{ Significado (MES) } & \multicolumn{3}{c}{ Empatia (BES) } \\
\cline { 2 - 9 } & \multicolumn{2}{c}{ Pré-teste } & \multicolumn{2}{c}{ Pós-teste } & \multicolumn{2}{c}{ Pré-teste } & \multicolumn{2}{c}{ Pós-teste } \\
& $M$ & $D P$ & $M$ & $D P$ & $M$ & $D P$ & $M$ & $D P$ \\
Grupo de controlo & 5.95 & 0.80 & 5.54 & 1.25 & 27.63 & 4.35 & 28.21 & 4.84 \\
Grupo experimental & 5.83 & 0.78 & 6.03 & 0.99 & 27.08 & 5.25 & 29.85 & 2.85 \\
\hline
\end{tabular}

Notas: score do significado calculado pela média e varia entre 1 e 7 ; score da empatia é calculado pelo somatório e varia entre 7 e 35 .

\section{DISCUSSÃO}

Apesar do TP poder ser enquadrado teoricamente como uma intervenção promotora de significado e existir alguma evidência sobre o efeito do TP na empatia, a partir deste estudo nada se pode afirmar sobre a potencial utilização do TP enquanto promotor destas variáveis, uma vez que não se encontraram efeitos significativos da intervenção nas duas escalas utilizadas. Relativamente às limitações metodológicas que poderão ter levado a não ser possível confirmar a hipótese inicial considera-se, por um lado, que o tamanho da amostra poderá não ter sido suficiente para conseguir detetar os efeitos de mudança ou o número de sessões não tenha sido suficiente para exercer esses efeitos. Outra possibilidade é estarmos perante o efeito de teto na medida em que o perfil das pessoas que se inscreveram é com inclinação para este tipo de atividades que poderão por si já ter um elevado nível de experiência de significado e empatia. De notar, através da Tabela 2, os scores elevados em ambas as variáveis no préteste: são relativamente próximos dos valores máximos das escalas. Assim sendo, será indicado repetir este estudo com uma amostra maior e participantes com perfis mais diversificados recorrendo a um grupo natural. De notar, também, que intervenções que promovam dimensões mais associadas à orientação eudemónica, como é o significado, podem não ser detetáveis logo após a intervenção, mas sim 3 meses depois em follow-up, havendo um "atraso" entre a intervenção e os benefícios (Huta \& Ryan, 2010), sendo por isso que se planeia fazer recolha de dados e nova comparação em follow-up para o presente estudo. Por fim, uma vez que o grupo de controlo é do tipo lista de espera, irá necessariamente ser feita uma segunda edição do grupo para estes participantes poderem ter a oportunidade de usufruírem dos potenciais benefícios que esta intervenção possa proporcionar, sendo que é intenção da equipa de investigação repetir a intervenção, não só para os participantes do grupo de controlo, como também abrir a novos participantes, na tentativa de alargar a amostra. Será recrutado um novo grupo de controlo natural (turmas de alunos universitários) para servir de comparação à segunda edição do grupo de TP.

Uma das participantes do grupo comentou no final de uma sessão já ter frequentado psicoterapia psicodramática, mas só com o grupo de TP é que sentiu ter resolvido um tema pessoal: deu um novo significado a um "monstro verde dos ciúmes" que por vezes a assombrava. Colocamos a hipótese de que a razão para a participante ter tido esta experiência subjetiva, prende-se com o facto de em TP ela ter visto não só o seu "monstro verde" ser representado simbolicamente em palco, bem como outros "monstros verdes" que apareceram, na mesma sessão, noutras histórias. É habitual em performances de TP haver um fio condutor que liga, de alguma forma, todas as histórias partilhadas na mesma performance, denominado de red thread (Chesner, 2002), o que pode ter conferido um novo nível de coerência e significância relativamente a esta temática desta participante. Na mesma sessão, foi possível observar uma história de outro participante que narrou como foi ele próprio infiel e causador do "monstro", e ainda uma história sobre poliamor onde não se concebe a existência do ciúme, o que pode ter trazido novos níveis de compreensão sobre este tema, das suas causas e diferentes perspetivas do mesmo. Este estudo sugere ser possível atingir novos níveis de significado e empatia através do TP, permitindo entender que não se está sozinho no sofrimento, e aceder a aspetos do mundo interior que a palavra e a cognição, por si só, não alcançam.

Em conclusão, o presente estudo traz contributos teóricos para a utilização do TP como intervenção para a promoção de significado e empatia. Complementar a análise quantitativa com uma qualitativa dos resultados da intervenção poderá trazer novas pistas para a utilização do TP como promotor do bem-estar. 


\section{REFERÊNCIAS}

Barak, A. (2013). Playback theatre and narrative therapy: introducing a new model. Dramatherapy, 35(2), 108-119. https://doi.org/10.1080/02630672.2013.821865

Bornmann, B. A., \& Crossman, A. M. (2011). Playback Theatre: Effects on students' views of aggression and empathy within a forensic context. Arts in Psychotherapy, 38(3), 164-168. https://doi.org/10.1016/j.aip.2011.04.004

Chesner, A. (2002). Playback Theater and Group Communication. Em A. Chesner (Ed.), Creative advances in groupwork (pp. 40-66). Jessica Kingsley Publishers.

Cheung Ng, H., \& Graydon, C. (2016). In search of empathy in Playback Theatre: a preliminary study. Person-Centered \& Experiential Psychotherapies, 15(2), 126-141. https://doi.org/10.1080/14779757.2016.1172252

Chung, K., Lee, E., Tan, J., Teo, D., Lee, C., Ee, S., ... Chee, C. (2018). Effects of Playback Theatre on cognitive function and quality of life in older adults in Singapore: A preliminary study. Australasian Journal on Ageing, 37(1), E33-E36. https://doi.org/10.1111/ajag.12498

Fox, H. (2007). Playback Theatre: Inciting dialogue and building community through personal story. The Drama Review: TDR, 51(4), 89-105. https://doi.org/10.1162/dram.2007.51.4.89

Fox, J. (1999). Introduction. Em J. Fox \& H. Dauber (Eds.), Gathering Voices: Essays on Playback Theatre (pp. 9-16). Tusitala Publishing.

Fox, J. (2013). The Therapy Question. Interplay, 18(2), 29-30. Obtido de https://www.iptn.info/uploads/iptn/201503/20150306_200603_3AG4S7xK1v_f.pdf

Huta, V. (2013). Pursuing eudaimonia versus hedonia: Distinctions, similarities, and relationships. Em A. S. Waterman (Ed.), The best within us: Positive psychology perspectives on eudaimonia. (pp. 139158). American Psychological Association. https://doi.org/10.1037/14092-008

Huta, V. (2015). The Complementary Roles of Eudaimonia and Hedonia and How They Can Be Pursued in Practice. Em S. Joseph (Ed.), Positive Psychology in Practice: Promoting Human Flourishing in Work, Health, Education, and Everyday Life: Second Edition (pp. 159-182). Wiley. https://doi.org/10.1002/9781118996874.ch10

Huta, V. (2016). Eudaimonia versus Hedonia: What Is the Difference? And Is It Real? International Journal of Existential Psychology and Psychotherapy, 7(2), 8. Obtido de http://journal.existentialpsychology.org/index.php/ExPsy/article/view/230

Huta, V. (2017). Meaning as a Subjective Experience. Journal of Constructivist Psychology, 30(1), 20-25. https://doi.org/10.1080/10720537.2015.1119088

Huta, V., \& Ryan, R. M. (2010). Pursuing Pleasure or Virtue: The Differential and Overlapping Well-Being Benefits of Hedonic and Eudaimonic Motives. Journal of Happiness Studies, 11(6), 735-762. https://doi.org/10.1007/s10902-009-9171-4

Huta, V., \& Waterman, A. S. (2014). Eudaimonia and Its Distinction from Hedonia: Developing a Classification and Terminology for Understanding Conceptual and Operational Definitions. Journal of Happiness Studies, 15(6), 1425-1456. https://doi.org/10.1007/s10902-013-9485-0

Jolliffe, D., \& Farrington, D. P. (2006). Development and validation of the Basic Empathy Scale. Journal of Adolescence, 29(4), 589-611. https://doi.org/10.1016/j.adolescence.2005.08.010

Martela, F., \& Steger, M. F. (2016). The three meanings of meaning in life: Distinguishing coherence, purpose, and significance. Journal of Positive Psychology, 11(5), 531-545. https://doi.org/10.1080/17439760.2015.1137623

Moran, G. S., \& Alon, U. (2011). Playback theatre and recovery in mental health: Preliminary evidence. Arts in Psychotherapy, 38(5), 318-324. https://doi.org/10.1016/j.aip.2011.09.002

Pechorro, P., Jesus, S., Kahn, R., Gonçalves, R., \& Barroso, R. (2018). A Versão Breve da Escala de Empatia Básica numa Amostra Escolar de Jovens Portugueses: Validade, Fiabilidade e Invariância. Revista $\begin{array}{llll}\text { Iberoamericana de Diagnóstico y } & \text { Evaluación, 49(4), 157-169. }\end{array}$ https://doi.org/10.21865/RIDEP49.4.13

Rowe, N. (2005). Personal stories in public places: An investigation into Playback Theatre. [Tese de Doutoramento não publicada, York University]. Obtido de http://www.playbacktheatre.org/playbacktheatre/wp-content/uploads/2010/04/RowePersonal-Stories-in-Public-Places.pdf

Salas, J. (1983). Culture and Community: Playback Theatre. The Drama Review: TDR, 27(2), 15-25. https://doi.org/10.2307/1145490

Salas, J. (1993). Improvising Real Life: Personal Story in Playback Theatre (20. a ed.). New York: Tusitala.

Shin, J. Y., \& Steger, M. (2014). Promoting Meaning and Purpose in Life. Em A. C. Parks \& S. M. Schueller (Eds.), The Wiley Blackwell Handbook of Positive Psychological Interventions (pp. 90-110). Chichester: Wiley-Blackwell. https://doi.org/10.1002/9781118315927.ch25 
Smigelsky, M. A., Neimeyer, R. A., Murphy, V., Brown, D., Brown, V., Berryhill, A., \& Knowlton, J. (2016). Performing the Peace: Using Playback Theatre in the Strengthening of Police-Community Relations. Progress in Community Health Partnerships: Research, Education, and Action, 10(4), 533539. https://doi.org/10.1353/cpr.2016.0061

Yotis, L., Theocharopoulos, C., Fragiadaki, C., \& Begioglou, D. (2017). Using playback theatre to address the stigma of mental disorders. Arts in Psychotherapy, 55, 80-84. https://doi.org/10.1016/j.aip.2017.04.009

Historial do artigo

Recebido $\quad 07 / 2019$

Aceite $\quad 03 / 2020$

Publicado $\quad 08 / 2020$ 
Revista PSICOLOGIA, 2020, Vol. 34 (1), doi: 10.17575/psicologia.v34i1.1681.

\title{
Cross-hemispheric tDCS over the temporal lobe affects the consolidation of previous learning in healthy volunteers
}

\author{
Sandra Carvalho ${ }^{47, i i}$, Óscar F. Gonçalves ${ }^{\mathrm{i}}$, Sofia Fariai, Patrícia Coelhoi, Diogo Branco ${ }^{\mathrm{i}}$ \& \\ Jorge Leite ${ }^{\mathrm{i} i, \mathrm{iii}}$ \\ ${ }^{i}$ Psychological Neuroscience Laboratory, CIPsi, School of Psychology, University of Minho, Braga, Portugal \\ ii Spaulding Neuromodulation Center Department of Physical Medicine \& Rehabilitation, Spaulding \\ Rehabilitation Hospital \& Massachusets General Hospital. Harvard medical School, Boston, USA. \\ iii University Portucalense, Portucalense Institute for Human Development - INPP, Porto, Portugal.
}

\begin{abstract}
Transcranial direct current stimulation (tDCS) over the temporal lobe improves memory performance. Little is known about the effects of tDCS when the memory trace is still unstable. In this study, we assess the effects of cross-hemispheric tDCS over the temporal lobe, during a verbal-memory interference task. Fifteen healthy volunteers learned two 12-word lists. After learning the first list, they learned the second one, while receiving tDCS. Then they were instructed to recall words from the lists in two moments: immediately after learning the second one and 20 minutes after the first recall. Results show that left anode/right cathode tDCS applied during the second list learning significantly decreased the percentage of words correctly recalled from the first list, for the immediate, as well as for the delayed recall, when comparing to sham. This study shows that cross-hemispheric tDCS over the temporal lobe modulates memory performance by interfering with previously learned memories.
\end{abstract}

\section{Keywords: Memory interference; Learning; tDCS; Temporal lobe.}

Estimulação bi-hemisférica por corrente contínua interfere com a consolidação de aprendizagens prévias em voluntários saudáveis: Estudos mostraram que a estimulação transcraniana por corrente continua (ETCC) aplicada sobre o lobo temporal parece aumentar o desempenho mnésico. Contudo, é desconhecido o efeito da ETCC quando a memória de traço é instável. Neste estudo, testamos os efeitos da ETCC bi-lateral sobre o lobo temporal durante uma tarefa de interferência na memória verbal. Quinze voluntários saudáveis, após aprenderem a primeira lista, aprenderam a segunda lista enquanto recebiam ETCC. Posteriormente, relembram as palavras das listas em dois momentos: a) após a aprendizagem da segunda lista; b) 20 minutos após a primeira vez que relembraram as palavras. Resultados mostraram que ETCC ânodo esquerdo/cátodo direito, aplicada durante a aprendizagem da segunda lista, reduziu significativamente a percentagem de palavras da primeira lista relembradas em ambos os momentos, quando comparado com o placebo. Este estudo mostra que a ETCC sobre o lobo temporal pode modular o desempenho mnésico, interferindo com memórias anteriormente aprendidas.

Palavras-chave: Interferência mnésica; Aprendizagem; ETCC; Lobo temporal.

Memory consolidation refers to the process by which short-term memories during a learning process become stable or fixed over time, and as such, available for a later retrieval (Nader \& Einarsson, 2010). Studies on memory consolidation performed on rats showed that when, both a concurrent learning (Duncan, 1949) and/or electroconvulsive shocks (Gordon \& Spear, 1973) were applied moments after a recently learned information, the retrieval of such information was impaired. These findings suggest that it is possible to interfere with learning processes, and with the consolidation of memories.

Memory formation and consolidation, or even retrieval have been conceptualized as depending on an interplay between the prefrontal and the hippocampus regions (Komorowski, Garcia, Wilson, Hattori, Howard, \& Eichenbaum, 2013; Preston \& Eichenbaum, 2013). However, neuroimaging data also suggests distinct recruitment of temporal lobe and the prefrontal areas depending on the stage of the memory consolidation process (Simons \& Spiers, 2003). For instance, Takashima and colleagues (2006), studying

\footnotetext{
47 Correspondence address: Sandra Carvalho, School of Psychology, University of Minho, Campus de Gualtar, 4710-057, Braga, Portugal. Email: sandrarc@psi.uminho.pt. SC and OFG are members of CIPsi, supported by the Portuguese Foundation for Science and Technology and the Portuguese Ministry of Science, Technology and Higher Education through national funds and co-financed by FEDER through COMPETE2020 under the PT2020 Partnership Agreement (POCI-01-0145-FEDER-007653). SC, JL and OFG were funded by the Portuguese Foundation for Science and Technology and COMPETE through the grants: P2020-PTDC/MHC-PCN/3950/2014; PTDC/PSIESP/30280/2017 and PTDC/PSI-ESP/29701/2017). SC was also funded by the individual grant IF/00091/2015.
} 
declarative memory consolidation, showed that the hippocampus is highly activated during the encoding and early consolidation processes; however, during long-term memory coding processes, the main activation seems to be over the prefrontal cortex. The involvement of different brain regions on memory processes is also a supported by lesions studies, in which patients with lesions to the medial temporal lobe exhibit deficits in long-term memory, despite the fact that their short-term memory remains intact (Baddeley and Warrington 1970; Milner, 1972).

Transcranial direct current stimulation (tDCS) is a non-invasive neuromodulation technique that uses controlled, constant and low electric current delivered to the scalp. It induces changes in cortical excitability, by depolarizing (anodal) or hyperpolarizing (cathodal) the membrane resting potential (Nitsche et al., 2008). tDCS effects vary depending on the current intensity administered, the electrodes size, the duration of the stimulation and the area that is being stimulated (Nitsche et al., 2008). Studies that assess the effects of tDCS over the prefrontal cortex on memory consolidation, support the idea that tDCS has a positive effect on consolidation, if applied during encoding and retrieval stages (Carvalho et al., 2015; Fregni et al., 2005; Javadi \& Walsh, 2012), or even during sleep (Marshall et al., 2004). As memory also depends on the medial temporal lobe, other studies have also shown that the application of anodal stimulation over the temporal lobe improves memory consolidation and reduces the number of false memories (Boggio et al., 2009a; Boggio et al, 2009b; Ross et al., 2010).

However, to the best of our knowledge, this is the first study probing the usefulness of using tDCS in conjugation with a concurrent word list, in order to decrease the number of correctly recalled words, from a previously learned word list. More specifically, the aim of this study is to use tDCS over the temporal lobe during the learning of a concurrent list of words, and to assess its impact on the immediate and delayed recall of a previous learned word list. The main hypothesis is that delivering bi-hemispheric tDCS over the temporal lobes during the learning of a second list of words (i.e., concurrent) will decrease the immediate and delayed recall performance on a previous learned list.

\section{METHOD}

\section{Participants}

The sample was composed by 15 healthy subjects, with ages between 18 and 22 years (all females; $M_{\text {age }}=$ 19.88, $S D_{\text {age }}=1.36$ ). Participants were all right-handed (Edinburgh Handedness Inventory, EHI $\geq 80$; Oldfield, 1971), had normal or corrected-to-normal vision and no history of psychiatric or neurologic disorders. The sample was recruited without any kind of monetary compensation. Participants were excluded if they were using any medication or psychotropic drugs at the time of the study, or if they had clinically relevant levels of depression as assessed by the Beck Depression Inventory (BDI-II; Beck et al., 1996).

\section{Experimental Procedure}

This experiment consisted of assessing the impact of active or sham tDCS in memory consolidation during an interfering memory task. There were three stimulation conditions, with one-week interval between them in order to prevent carryover effects. Session 1 included the written informed consent, a sociodemographic questionnaire, the laterality test (Oldfield, 1971), the eligibility questionnaire for tDCS, the BDI and the cognitive task; Session 2 included the cognitive task; and Session 3 included the BDI and the cognitive task. In every session, before and after the cognitive task, participants were screened about positive and negative affect using The Positive and Negative Affect Schedule (PANAS) and about possible levels of discomfort, fatigue, pain, itching, humor, tingling, burning, headache and sleepiness (among others) using a continuous Visual Analog Scale (VAS).

\section{Cognitive Task}

In total, there were six lists of words (two were randomly selected per session and counterbalanced across participants). Each list was comprised of 12 unrelated, neutral words. Each trial was presented for two seconds with an interstimulus interval (ISI) of one second. Participants were required to go through five learning blocks of the list of words (i.e., List A), and at the end of each block they had 60 seconds to recall the maximum number of words presented before.

After they learned the list with a 100\% accuracy, tDCS was delivered. After an initial 3-min period of tDCS, participants were instructed to learn a second list (i.e., list B), following a similar protocol to what has been done for the first list. Total duration of stimulation was $12 \mathrm{~min}$. Afterwards, participants were asked to recall all the words they remembered from the first list (List A), and then to recall those of the second list (List B). A second recall moment occurred 20 minutes later, when participants were again asked to remember the words from List A and B (Figure 1). 
a)

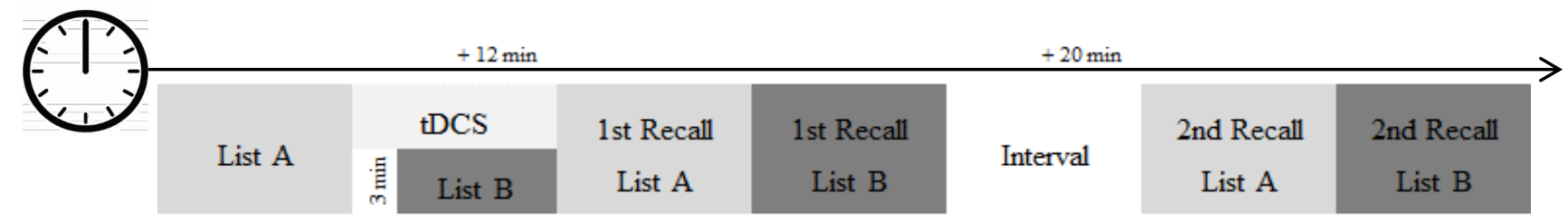

b)

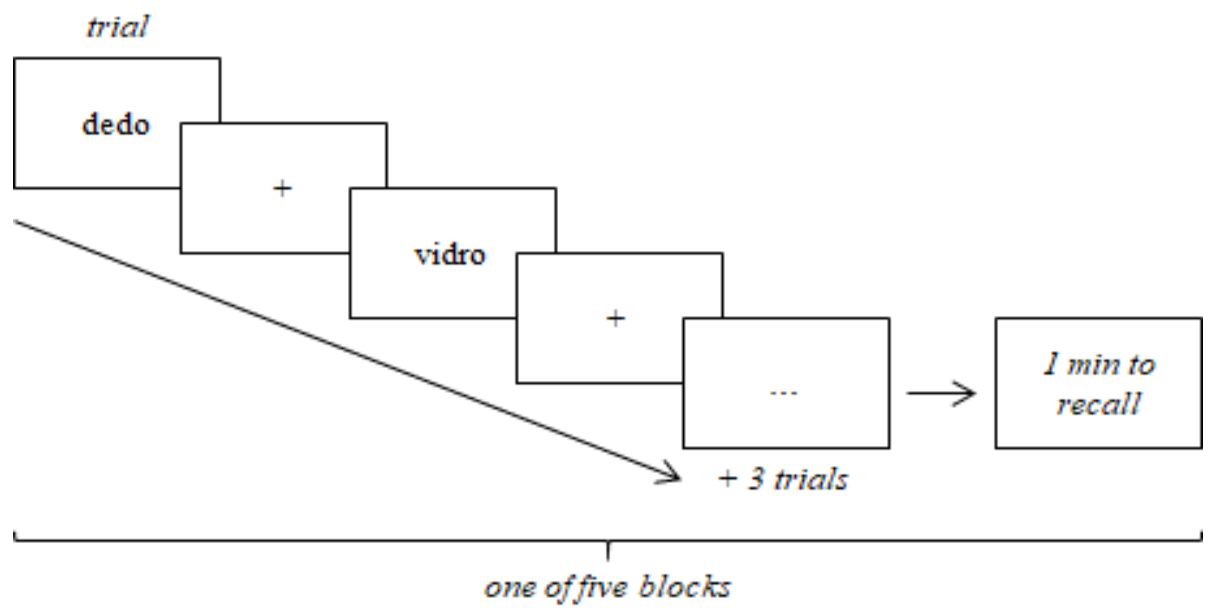

Figure 1. a) Schematic illustration of the experimental protocol; b) Lists learning procedure.

\section{Transcranial Direct Current Stimulation}

a)

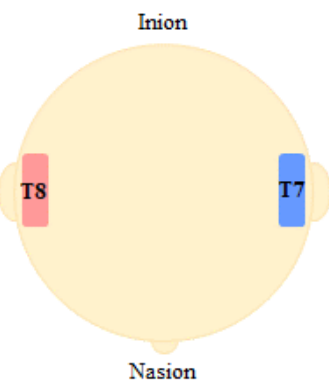

b)

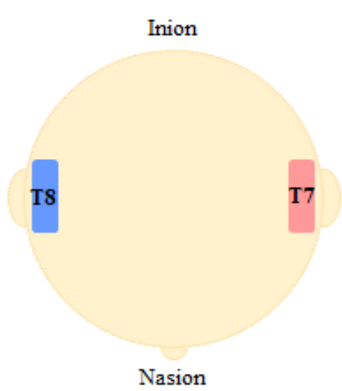

Figure 2. Schematic illustration of the experimental tDCS montages: a) electrode positioning for active and sham conditions with anode placed over the left temporal lobe (T7) and cathode over the right temporal lobe (T8); b) electrode positioning for the active condition with the anode placed in T8 and the cathode in T7.

Participants were counterbalanced to receive all of three tDCS conditions: cathode right/ anode left (LA-RC); anode right/cathode left (RA-LC); and sham tDCS. Electrodes were placed over left temporal lobe (T7) and right temporal lobe (T8) according to the $10-20$ system with at least six cm distance between them, following previous studies (Leite et al., 2013; Kekic et al., 2017) (Figure 2). The current was delivered to the scalp using a constant current stimulation, Magstim Eldith DC+-stimulator, by means of saline-soaked pair of surface sponge electrodes $(5 \times 7 \mathrm{~cm})$.

In active conditions (LA-RC; RA-LC), direct current of $2 \mathrm{~mA}$ was delivered for 720 seconds (12 minutes), with a 15 second ramp-up and ramp-down. To mimic the active condition, the sham group received stimulation for 45 seconds with the same ramp-up and down (with 15 seconds ramp-up and down). In total, the trial was comprised of three stimulation sessions. Sessions were performed with at least 72 hours between them, in order to prevent stimulation carryover effects. 


\section{Statistical Analysis}

To account for possible side effects of the stimulation, paired samples T-tests were run, using the scores reported in the VAS and PANAS. Mean percentage of words recalled from List A in both recall moments were calculated. Comparisons were made for the mean percentage of words correctly recalled in the three stimulation conditions, to evaluate the effect of stimulation in memory consolidation. Comparisons between groups (LA-RC, RA-LC, sham) regarding the percentage of words recalled from List A were assessed using one-way independent samples ANOVA. Post hoc comparisons with Fisher's Least Significant Difference (LSD) were also performed, when the main analysis revealed significant effects. All data analyses were performed using SPSS version 21 (IBM, United States).

\section{RESULTS}

\section{Differences in PANAS and VAS between pre and poststimulation}

There were no serious or adverse side effects reported due to the intervention (Table 1). Participants did not show significant changes in PANAS for any of the stimulation conditions. Regarding VAS, participants scored significantly higher on 'itch' in poststimulation for LA-RC, $t(14)=-2.26, p=.04$, and RA-LC conditions, $t(14)=-3.34, p=.005$; as well as on 'anthill' in LC-RA condition, $t(14)=-2.51, p=.03$. After the experimental protocol, participants reported significantly lower scores on 'affect' in LA-RC condition, $t(14)=2.56, p=.02$, and for 'fatigue' in sham condition. No significant changes were reported in any of the other categories evaluated on the VAS, $t(14)=2.55, p>.05$ (Table 1 ).

Table 1. Descriptive statistics from VAS between pre and poststimulation

\begin{tabular}{|c|c|c|c|}
\hline & \multicolumn{3}{|c|}{ LA - RC } \\
\hline & Prestimulation & Poststimulation & \\
\hline & $(n=15)$ & $(n=15)$ & \\
\hline & Mean (SD) & Mean (SD) & $t(14)$ \\
\hline Itch & $1.13(.52)$ & $1.67(.98)$ & $-2.26^{*}$ \\
\hline \multirow[t]{5}{*}{ Affect } & $4.67(1.45)$ & $4.40(1.30)$ & $2.26^{*}$ \\
\hline & \multicolumn{3}{|c|}{ LC - RA } \\
\hline & Prestimulation & Poststimulation & \\
\hline & $(n=15)$ & $(n=15)$ & \\
\hline & Mean (SD) & Mean (SD) & $t(14)$ \\
\hline Itch & $1.07(.26)$ & $3.20(2.57)$ & $-3.34^{*}$ \\
\hline \multirow[t]{5}{*}{ Affect } & $1.00(0)$ & $2.47(2.27)$ & -2.51 \\
\hline & \multicolumn{3}{|c|}{ Sham } \\
\hline & Prestimulation & Poststimulation & \\
\hline & $(n=15)$ & $(\mathrm{n}=15)$ & \\
\hline & Mean (SD) & Mean (SD) & $t(14)$ \\
\hline Fatigue & $3.87(1.92)$ & $3.27(1.94)$ & $2.55^{*}$ \\
\hline
\end{tabular}

Note. ${ }^{*} p<.05$

\section{Percentage of words recalled from List $A$ on the first and second recall's}

In the first recall, the ANOVA showed a main effect of stimulation on the percentage of words recalled from List A, $F(2,28)=3.58, p<.05$. Post-hoc analysis using paired sample $t$-tests with the Fisher's Least Significant Difference (LSD) showed that LA-RC $(M=29.03, S D=13.88)$ significantly decreased the percentage of words correctly recalled of list A when compared to sham $(M=39.44, S D=9.92, p=.018$, $d=0.43)$. No significant differences were found between LC-RA $(M=31.50, S D=10.93, p>.05)$ and the other conditions (Figure 2). 
A

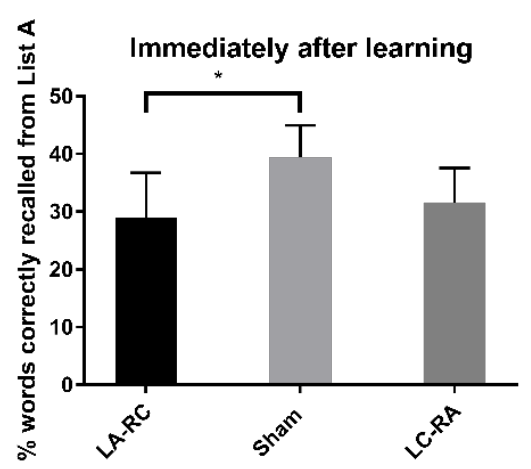

B

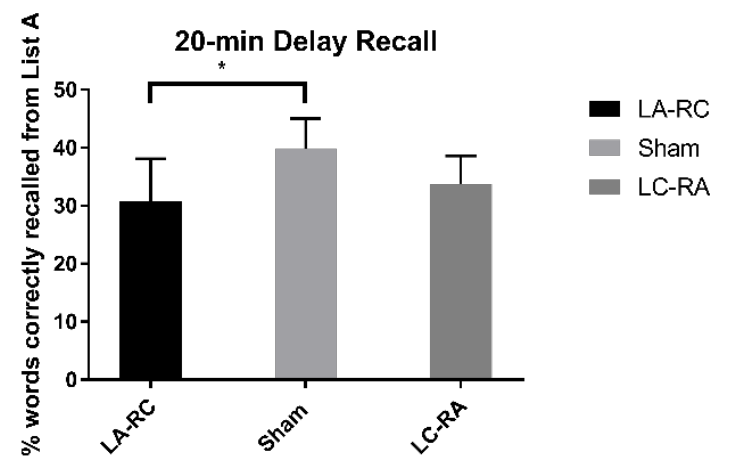

Figure 3. Mean amplitude of words recalled from List A across stimulation conditions for all the participants immediately (A) and 20-min delay (B) recall. Bars represent 95\% CI.

In the second recall, the ANOVA showed similar results to those reported in the first recall moment, $F(2,28)=3.69, p<.05$. LA-RC $(M=30.84, S D=13.15)$ significantly decreased the percentage of correctly recalled words when comparing to sham $(M=39.77, S D=9.59 ; p=.018, d=0.76)$. No significant differences were found when comparing LC-RA $(M=33.82, \mathrm{SD}=8.62)$ with LA-RC $(p>.05)$ and sham $(p>$ .05). Surprisingly there were no differences in terms of recall across conditions for List A and B (Figure 4).
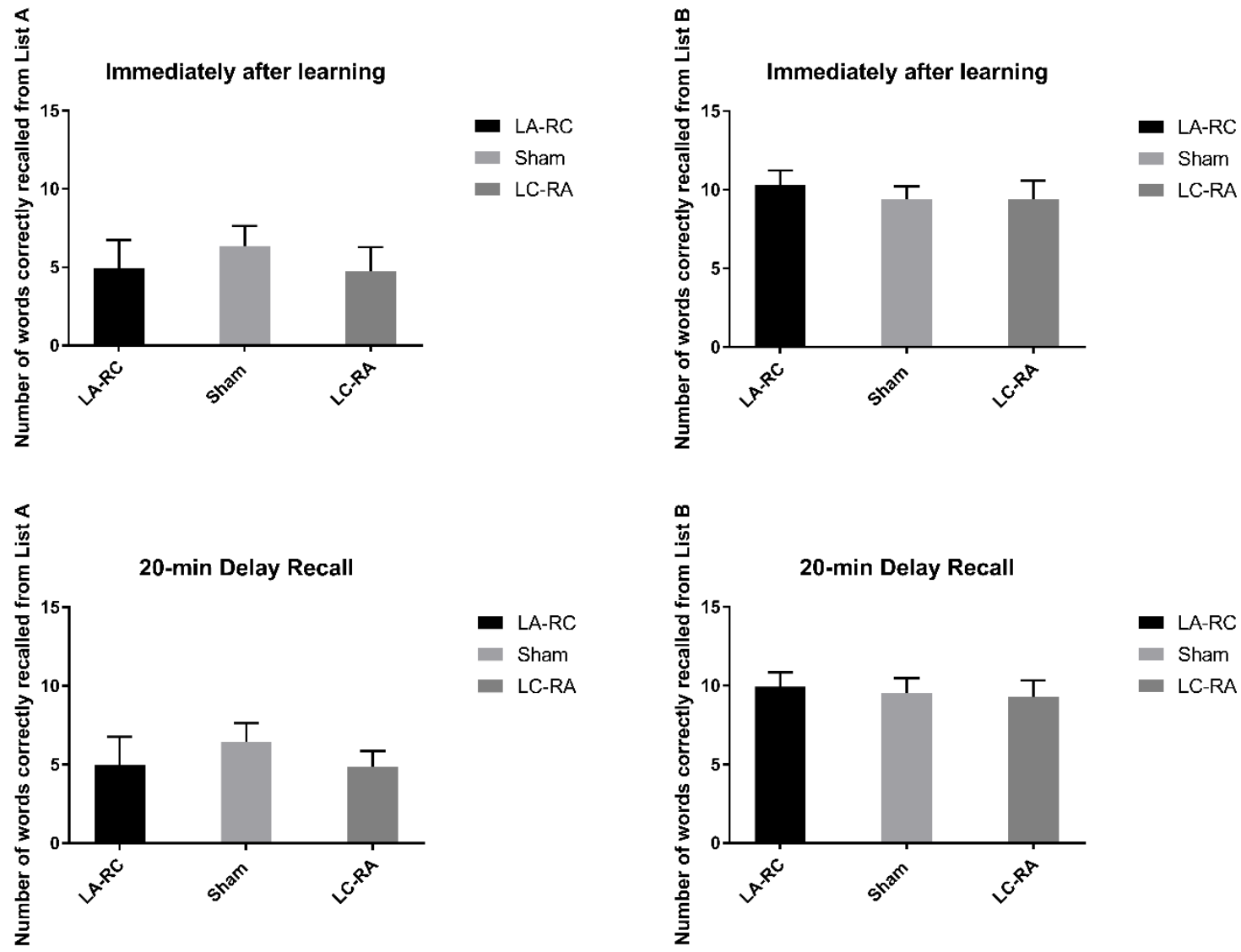

Figure 4. Total number of words correctly recalled from List A and B. Top figures represent immediate recall, while bottom figures represent the 20-min delayed recall. Bars represent standard error. 


\section{DISCUSSION}

In this study, we tested the effects of cross-hemispheric tDCS delivered to the temporal lobe during a concurrent word list learning and, consequently its effects on the consolidation of a previously learned word list.

By shifting the activity balance towards the left hemisphere (LA-RC), a lower percentage of words were recalled from LA in comparison with the percentage of words recalled in sham, suggesting the existence of an interference effect in the learning of the list of words, which persisted for at least for 20min. This effect seems to be unrelated to the direct effects of tDCS during List B learning, as there were no differences in terms of List B learning due to tDCS.

Lesions studies have suggested that better semantic memory is associated with smaller resection volumes and greater left temporal pole integrity (Skirrow et al., 2014). This highlights the role of the left temporal lobe on semantic memory and may explain some of the results. Moreover, the role of the medial temporal lobe in encoding and early memory consolidation processes has already been shown (Takashima et al., 2006). Therefore, by shifting the balance towards the left temporal lobe during the concurrent list learning, a retroactive interference process effect across lists was favored. Namely, the learning process of the second list retroactively interfered with the previously learned list, by decreasing the percentage of correctly recalled words. One possibility here is that LA-RC tDCS actually increased the recall of the second list, and the present results may be explained by changes at the recall level. However, this is highly unlikely, because there were no difference across tDCS conditions during the recall of list A and B. Furthermore, retroactive interference in memory is though do be due to an inhibitory failure and does not seem to be related to retrieval, but instead to encoding and storage processes (O'Reilly \& Rudy, 2001; Sutherland \& Rudy, 1989). In this sense, LA-RC tDCS may have increased this failure at the inhibitory level and thus related to a memory interference effect during memory encoding and storage process, rather than tDCS-induced changes at the recall level. Moreover, the lack of differences across the number of words correctly retrieved from List A and B, suggests that the combination of tDCS with the concurrent list, may have changed the complex configural structure that was created during List A consolidation. For instance, when someone is learning a list of words, words are coded alongside with multiple elements, such as cues, targets and context. These multiple elements are thought to protect that specific list learning, from interferences that may arise from other lists of words with different elements, as unique content would be created for all the lists (Humphreys et al., 1989). In theory, the goal of this complex configural structure would be to maintain the integrity of distinct memories. As there were no effects of tDCS on List B learning and, as such, it is possible that this tDCS-enhanced interference mechanism actually operated through interference at the complex configural structure of learning, thus resulting in a retroactive inhibition, which was able to impact a previous learned list. This is only a hypothesis, however this is the first time that tDCS is reported as being able of interfering with learning, and moreover, interfering with the process of memory consolidation, probably by changing the complex configural structure associated with memory. This claim gains further support at the 20-min delayed recall condition. Again, there were no differences in terms of List A or B recall, however LA-RC tDCS reduced the percentage of correctly evoked words from list A. Thus suggesting that this tDCS interference effect may actually translate to long-term memory retention.

It is noteworthy, that this study must be seen as exploratory and has several limitations. First, no other region was tested (such as Prefrontal Cortex) and future studies should compare the effects of tDCS on memory learning in different regions. Moreover, these effects should be tested on other types of memory, such as spatial, which are thought to more right lateralized. Furthermore, it will be important in future studies to test the effects of context on learning, in order to assess this hypothesis that tDCS may have interfered with this unique coding.

This study highlights that tDCS combined with word list learning may interfere with previous learning, potentially by a mechanism of retroactive inhibition that may actually change the complex configural structure of memories. Furthermore, this effect lasts at least for 20 min after tDCS, which suggests that there are transfer effects to long-term memory retention. The data from the present study, although exploratory, can be useful for probing the mechanism by which memories become consolidated, or even how memory consolidation can be altered. By studying such processes, it is possible to improve our understanding about how memories become stable over time, and especially how several types of memories (such as traumatic ones), can potentially be altered.

\section{REFERENCES}

Baddeley, a. D., Warrington, E., K. (1970). Amnesia and the distinction between long- and short-term memory. Journal of Verbal Learning and Verbal Behavior, 9(2), 176-189. https://doi.org/10.1016/S0022-5371(70)80048-2 
Beck, A. T., Steer, R. A., \& Brown, G. K. (1996). Manual for Beck Depression Inventory-II. Psychological Corporation.

Boggio, P. S., Fregni, F., Valasek, C., Ellwood, S., Chi, R., Gallate, J., ... Snyder, A. (2009a). Temporal lobe cortical electrical stimulation during the encoding and retrieval phase reduces false memories. PLoS ONE, 4(3). https://doi.org/10.1371/journal.pone.0004959

Boggio, P. S., Khoury, L. P., Martins, D. C. S., Martins, O. E. M. S., Macedo, E. C., Fregni, F. (2009b). Temporal cortex direct current stimulation enhances performance on a visual recognition memory task in Alzheimer disease. Journal of Neurology, Neurosurgery, and Psychiatry, 80(4), 444-447. https://doi.org/10.1136/jnnp.2007.141853

Carvalho, S., Boggio, P. S., Gonçalves, Ó. F., Vigário, A. R., Faria, M., Silva, S., ... Leite, J. (2015). Transcranial direct current stimulation based metaplasticity protocols in working memory. Brain stimulation, 8(2), 289-294. https://doi.org/10.1016/j.brs.2014.11.011

Duncan, C. P. (1949). The retroactive effect of electroconvulsive shock on learning. Journal of Comparative and Physiological Psychology, 42(1), 32-44. https://doi.org/10.1037/h0058173

Fernández, G., Weyerts, H., Schrader-Bolsche, M., Tendolkar, I., Smid, H., Tempelmann, C., ... Heinze, H. (1998). Successful Verbal Encoding into Episodic Memory Engages the Posterior Hippocampus: A Parametrically Analyzed Functional Magnetic Resonance Imaging Study. Journal of Neuroscience, 18(5), 1841-1847. Retrieved from PubMed database

Fregni, F., Boggio, P. S., Nitsche, M., Bermpohl, F., Antal, A., Feredoes, E., ... Pascual-Leone, A. (2005). Anodal transcranial direct current stimulation of prefrontal cortex enhances working memory. Experimental brain research, 166(1), 23-30. https://doi.org/10.1007/s00221-005-2334-6

Golby, A. J., Poldrack, R. A., Brewer, J. B., Spencer, D., Desmond, J. E., Aron, A. P., \& Gabrieli, D. E. (2001). Material-specific lateralization in the medial temporal lobe and prefrontal cortex during memory encoding. Brain, 124(9), 1841-1854. Retrieved from PubMed database

Gordon, W. C., \& Spear, N. E. (1973). Effect of reactivation of a previously acquired memory on the interaction between memories in the rat. Journal of Experimental Psychology, 99(3), 349-355. https://doi.org/10.1037/h0035301

Javadi, A. H., \& Walsh, V. (2012). Transcranial direct current stimulation (tDCS) of the left dorsolateral prefrontal cortex modulates declarative memory. Brain Stimulation, 5(3), 231-241. https://doi.org/10.1016/j.brs.2011.06.007

Komorowski, R.W., Garcia, C.G., Wilson, A., Hattori, S., Howard, M. W., Eichenbaum, H. (2013). Ventral hippocampal neurons are shaped by experience to represent behaviorally relevant contexts. Journal of Neuroscience, 33(18)8079-87. https://doi.org/10.1523/JNEUROSCI.5458-12.2013.

Kekic, M., McClelland, J., Bartholdy, S., Boysen, E., Musiat, P., Dalton, B., ... Schmidt, U. (2017). Singlesession transcranial direct current stimulation temporarily improves symptoms, mood, and selfregulatory control in bulimia nervosa: a randomised controlled trial. PloS one, 12(1), e0167606. https://doi.org/10.1371/journal.pone.0167606

Leite, J., Carvalho, S., Fregni, F., Boggio, P. S., \& Goncalves, O. F. (2013). The effects of cross-hemispheric dorsolateral prefrontal cortex transcranial direct current stimulation (tDCS) on task switching. Brain Stimul. 6, 660-667. https://doi.org/10.1016/j.brs.2012.10.006

Marshall, L., Molle, M., Hallschmid, M., \& Born, J. (2004). Transcranial direct current stimulation during sleep improves declarative memory. The Journal of Neuroscience, 24(44), 9985-9992. https://doi.org/10.1523/JNEUROSCI.2725-04.2004

Milner, B. (1972). Disorders of learning and memory after temporal lobe lesions in man. Clinical Neurosurgery, 19:421-46. https://doi.org/10.1093/neurosurgery/19.cn_suppl_1.421

Nader, K., \& Einarsson, E. O. (2010). Memory reconsolidation: an update. The Year in Cognitive Neuroscience, 1191, 27-41. https://doi.org/10.1111/j.1749-6632.2010.05443.x

Nitsche, M. A., Cohen, L. G., Wasserman, E. M., Priori, A., Lang, N., Antal, A., ... Pascual-Leone, A. (2008). Transcranial direct current stimulation: state of the art 2008. Brain Stimulation, 1(3), 206-223. https://doi.org/10.1016/j.brs.2008.06.004

O'Reilly, R. C., \& Rudy, J. W. (2001). Conjunctive representations in learning and memory: principles of cortical and hippocampal function. Psychological review, 108(2), 311. https://doi.org/10.1037/0033-295X.108.2.311

Oldfield, R. C. (1971). The assessment and analysis of handedness: the Edinburgh inventory. Neuropsychologia, 9(1), 97-113. https://doi.org/10.1016/0028-3932(71)90067-4

Preston, A. R., Eichenbaum, H. (2013). Interplay of hippocampus and prefrontal cortex in memory. Current Biology, 23(17), R764-R773. https://doi.org/10.1016/j.cub.2013.05.041 
Ross, L. A., McCoy, D., Wolk, D. A., Coslett, H. B., \& Olson, I. R. (2010). Improved proper name recall by electrical stimulation of the anterior temporal lobes. Neuropsychologia, 48(12), 3671-1674. https://doi.org/10.1016/j.neuropsychologia.2010.07.024

Simons, J. S., \& Spiers, H. J. (2003). Prefrontal and medial temporal lobe interactions in long term memory. Nature Reviews, 4(8), 637-648. https://doi.org/10.1038/nrn1178

Skirrow, C., Cross, J. H., Harrison, S., Cormack, F., Harkness, W., Coleman, R., ... Baldeweg, T. (2014). Temporal lobe surgery in childhood and neuroanatomical predictors of long-term declarative memory outcome. Brain, 138(1), 80-93. https://doi.org/10.1093/brain/awu313

Sutherland, R. J., \& Rudy, J. W. (1989). Configural association theory: The role of the hippocampal formation in learning, memory, and amnesia. Psychobiology, 17(2), 129-144. https://doi.org/10.3758/BF03337828

Takashima, A., Petersson, K. M., Rutters, F., Tendolkar, I., Jensen, O., Zwarts, M. J., ... Fernández, G. (2006). Declarative memory consolidation in humans: a prospective functional magnetic resonance imaging study. Proceedings of the National Academy of Sciences, 103(3), 756-761. https://doi.org/10.1073/pnas.0507774103

Historial do artigo

Recebido $\quad 07 / 2019$

Aceite $\quad 03 / 2020$

Publicado 08/2020 
Revista PSICOLOGIA, 2020, Vol. 34 (1), doi: 10.17575/psicologia.v34i1.1681.

\title{
Estudos preliminares das características psicométricas da versão curta da Escala Básica de Empatia em adultos
}

\author{
Clara Carvalho48i, António-José Gonzalezii, Nuno Amarante, Miguel Basto-Pereiraiii \& \\ Darrick Jolliffe $\mathrm{e}^{\mathrm{iv}}$ \\ i ISPA - Instituto Universitário de Ciências Psicológicas, Sociais e da Vida \\ ii APPsyCI - Applied Psychology Research Center Capabilities \& Inclusion, ISPA - Instituto Universitário \\ iii William James Center for Research, ISPA - Instituto Universitário \\ iv University of Greenwich
}

\begin{abstract}
Resumo: 0 estudo da empatia em Portugal tem-se focado na população adolescente, em relação à qual diversas evidências relevantes têm vindo a ser produzidas. Na falta de instrumentos validados para a população adulta portuguesa, é objetivo deste estudo apresentar os estudos psicométricos da versão breve da Basic Empathy Scale nesta população, incluindo as validades convergente, de construto, de grupos conhecidos e consistência interna. Para tal, foi usada uma amostra por conveniência $(N=257)$ que respondeu à escala de empatia, bem como a uma escala de Intensidade Afetiva. A análise fatorial mostrou que a versão breve da BES é constituída pelas 2 componentes sugeridas na literatura, cognitiva e afetiva. As mulheres mostraram resultados significativamente superiores, quer na escala total, quer nas subescalas. No geral, a versão reduzida da BES demonstrou propriedades psicométricas apropriadas para a população adulta portuguesa, nomeadamente no que se refere a fiabilidade, validade de construto, validade convergente e validade de grupos conhecidos.
\end{abstract}

Palavras-chave: Empatia; Versão Curta da Escala Básica de Empatia; População Adulta Portuguesa; Propriedades Psicométricas.

Preliminary study of the psychometric properties of the Short version of Basic Empathy Scale in adults: The study of empathy in Portugal has focused on the adolescent population, for which several relevant evidences have been produced. In the absence of validated instruments for the Portuguese adult population, this study aims to present the psychometric studies of the brief version of Basic Empathy Scale in this population, including the convergent validity, of construct, of known groups and internal consistency validity. For this, a convenience sample $(\mathrm{N}=257)$ was used, which responded to the Empathy Scale, as well as to Affective Intensity Scale. The factor analysis showed that the shortened version of BES is made up of the 2 components suggested in the literature, cognitive and affective. Women showed significantly higher results on both full scale and subscales. Overall, the shortened version of BES showed good psychometric qualities, notably as in regard to reliability, construct validity, convergent validity and known group validity.

Keywords: Empathy; Short Version of Basic Empathy Scale; Portuguese Adult Population; Psychometric Properties.

A empatia é uma forma básica de comunicação social que pode ocorrer em variados e diferentes contextos (Godinho, 2015), e com importantes implicações nas interações sociais, já que indivíduos com níveis mais altos de empatia sentem maior satisfação nas relações profissionais, de amizade, familiares e conjugais, e têm maior facilidade em evitar o conflito e a rutura (Davis \& Oathout, 1987; 1992; Davis, 1983; Ickes, 1997; Lon \& Andrews, 1990; Domingos, 2011; Góis \& Miguéis, 2012). A investigação tem consistentemente demonstrado que a empatia está negativamente associada a comportamentos agressivos e disfuncionais (Marshal \& Marshall, 2011; Jolliffe \& Farrington, 2004), e positivamente relacionada com o comportamento pró-social (Eisenberg \& Miller, 1987; Fengqin \& Zhaohui, 2016). A empatia é, por isso, um construto essencial para a conceção de programas de treino de competências empáticas (Van Berkhout \& Malouff, 2015) nos mais variados contextos. Assim sendo, afigura-se pertinente a existência de um instrumento efetivo de mensuração dos níveis de empatia para a população adulta portuguesa. Entre os investigadores portugueses, tem-se refletido sobre a importância deste

48 Morada para correspondência: António Gonzalez, ISPA, Rua Jardim do Tabaco, 34, 1140-041, Lisboa E-mail: psiantoniogonzalez@gmail.com 
construto no desenvolvimento do ser humano, sendo, no entanto, as investigações realizadas essencialmente focadas na área da adolescência (Anastácio, 2013; Matias, 2012; Pechorro, Ray, SalasWright, Marôco \& Gonçalves, 2015).

Percorrendo a literatura, notamos que os estudos nacionais e internacionais (Anastácio, 2013; Depraz, 2005; Domingos, 2011; Stein, 1917; Eisenberg \& Miller, 1987; Marshall, 2011; Rogers, 1992) reúnem evidências de que a compreensão da empatia pode dar um contributo importante e válido para a intelecção do funcionamento psíquico do indivíduo como sujeito ativo, tanto no seu desenvolvimento intrapsíquico, quanto no interpsíquico, enquanto ator social. Apesar das evidências que encontramos, revisões da literatura como a de Hall e Schwartz (2019), que analisaram cerca de 489 estudos editados entre 2001 e 2017, indicam-nos que existe uma grande variedade de definições do conceito de empatia. Estes autores defendem, ainda, que essa variedade pode apresentar-se como um obstáculo para o avanço qualitativo das investigações sobre este construto, uma vez que foram encontradas inconsistências, quer no conceito, quer na operacionalização dos instrumentos de mensuração. De acordo com Basto-Pereira e Farrington (2018), os investigadores têm-se deparado com a dificuldade em encontrar um instrumento que meça efetivamente a empatia e as suas dimensões, sendo que os instrumentos existentes apresentam limitações ligadas a propriedades psicométricas pobres, deficiências no conceito e na validade de construto e estabilidade temporal desconhecida.

Parece ser consensual que a empatia se baseia em duas dimensões: a cognitiva e a afetiva. Perante a problemática da grande variedade de dimensões existentes em diversos instrumentos, que desviam o foco para outros construtos que não a empatia, Jolliffe e Farrington (2006) construíram a Basic Empathy Scale (BES) para a população adolescente. A BES é composta por um conjunto de 20 itens que se dividem por duas subescalas: Empatia cognitiva, com 9 itens, que visa medir a capacidade de compreender as emoções de outra pessoa, e Empatia afetiva, com 11 itens, que procura medir a congruência emocional para com as emoções de outra pessoa (Jolliffe \& Farrington, 2006; Basto-Pereira \& Farrington, 2019; Godinho, 2015). Esta escala tem sido alvo de várias adaptações e validações para a população adolescente, na Europa e no resto do mundo, em países como China, Peru, Eslováquia e Coreia do Sul, entre outros, apresentando em todos eles boas propriedades psicométricas (Basto-Pereira \& Farrington, 2019). De salientar que apenas em França se adaptou a BES para a população adulta, num estudo de Carré (2013). Em Portugal, autores como Pechorro et al. (2015) e Pechorro, Ray, Raine, Marôco, e Gonçalves (2017), Matias (2012) e Anastácio, Vagos, Nobre-Lima, Rijo e Jolliffe (2016), adaptaram e validaram a BES para a população adolescente, encontrando igualmente boas propriedades psicométricas, inclusivamente a sua versão curta, adaptada e validada por Pechorro, Jesus, Kahn, Gonçalves, e Barroso (2018). Uma vez que não se encontram publicações sobre a validação desta escala para a população portuguesa adulta, é objetivo deste estudo explorar preliminarmente as propriedades psicométricas da Basic Empathy Scale (Jolliffe \& Farrington, 2006), na sua versão curta para esta população.

Primeiro, pretende-se testar a validade de constructo, avaliando se a estrutura desta versão da BES, nomeadamente a alocação dos itens por dimensões, é replicada no presente estudo, indo de acordo com o que é teoricamente esperado. Foi também avaliado o indicador de fiabilidade, consistência interna, dos itens em relação a cada uma das subescalas e total. Além destes indicadores, testou-se a validade de grupos conhecidos, que consiste em verificar se o instrumento é capaz de detetar diferenças entre dois grupos que são conhecidos por se diferenciarem no construto avaliado (Davidson, 2014). No âmbito desta forma de validade, no presente estudo procurou-se avaliar se, como indica a literatura, as dimensões medidas nesta versão da BES discriminam corretamente as diferenças entre os sexos indicadas na literatura (e.g., Albiero, Matricardi, Speltri \& Toso, 2009; Anastácio et al., 2016; D’Ambrosio, Olivier, Didon, \& Besche, 2009; Geng, Xia \& Qin, 2012; Jolliffe \& Farrington, 2006; Salas-Wright et al., 2012). Neste sentido, a existência destas diferenças indicará que o instrumento de avaliação é capaz de discriminar entre dois grupos que é sabido serem diferentes no conceito avaliado.

Pretende-se ainda demonstrar a validade convergente, correlacionando a BES-A com a Escala Breve de Intensidade Afetiva (EBIA; Prada, Rodrigues, \& Palma, 2009), esperando-se uma correlação positiva, nomeadamente com a dimensão afetiva, pois os indivíduos com elevada intensidade afetiva mostram-se mais ativos, sociáveis e reativos emocionalmente (Larsen \& Diener, 1987). Aparentemente, existe relação entre os níveis de intensidade afetiva e dimensões de estilo emocional (e.g., resposta, expressividade e orientação emocional) (Flett, Bator, \& Blankstein, 1988), o que constitui suporte para a validade convergente da BES. 


\section{MÉTODO}

\section{Participantes}

Neste estudo participaram, de forma voluntária, numa amostra recolhida por conveniência, 257 sujeitos de ambos os sexos. A amostra é maioritariamente constituída por sujeitos do sexo feminino (79.0\%). Os participantes têm, na sua maioria, idade compreendida entre os 40 e os 49 anos (32.0\%) e 168 indivíduos revelaram ter escolaridade ao nível do Ensino Superior (65.4\%), conforme se pode observar na tabela 1.

Tabela 1. Características sociodemográficas $(\mathrm{N}=257)$

\begin{tabular}{lcc}
\hline & Frequência & Percentagem \\
\hline Sexo & 203 & 79.0 \\
Feminino & 54 & 21.0 \\
Masculino & & \\
Escolaridade & 14 & 5.4 \\
$<10^{\circ}$ ano & 16 & 6.2 \\
$10^{\circ}-11^{\circ}$ ano & 59 & 23.0 \\
$12^{\circ}$ ano & 168 & 65.4 \\
Superior & & \\
Idade & 26 & 10.1 \\
$18-29$ & 76 & 29.6 \\
$30-39$ & 84 & 32.7 \\
$40-49$ & 53 & 20.6 \\
$50-59$ & 18 & 7.0 \\
$>69$ & & \\
\hline
\end{tabular}

\section{Instrumentos}

A Basic Empathy Scale foi desenvolvida por Jolliffe e Farrington (2006). Os itens na versão breve portuguesa foram traduzidos por Pechorro et al. (2018). Esta escala (BES - A) é composta por um conjunto de 7 itens que se dividem, tal como a versão longa de 20 itens, por duas subescalas: Empatia cognitiva com 4 itens (Itens 9, 10, 12,14) e Empatia afetiva com 3 itens (Itens 2, 5, 17). Esta escala pode ser cotada somando os 7 itens em escala de resposta de 5 pontos $(1=$ Totalmente falso e $5=$ Totalmente verdade). A BES-A permite obter os totais parciais, nomeadamente para a empatia cognitiva e afetiva, para além do cálculo da pontuação total, que pode variar entre os 7 (défice de empatia) e os 35 pontos (alto nível de empatia) (Carré et al., 2013; Godinho, 2015). Em estudos anteriores conduzidos na população portuguesa, em diferentes amostras populacionais, este instrumento tem apresentado consistentemente boas propriedades psicométricas (e.g., Pechorro et al., 2015; Pechorro et al., 2018), nomeadamente no que se refere à consistência interna, onde os valores encontrados têm sido consistentemente satisfatórios $(\alpha>.70)$.

A Escala Breve de Intensidade Afectiva (EBIA), adaptada e validada por Prada, Rodrigues, \& Palma (2009), é constituída por um conjunto de 20 itens, divididos por 3 dimensões, numa estrutura trifatorial: Intensidade Positiva (8 itens), Intensidade Negativa (6 itens) e Serenidade (6 itens). Os itens são definidos como afirmações que expressam reações emocionais a eventos típicos do quotidiano, sendo solicitado que os participantes indiquem a forma como habitualmente reagem a tais eventos, numa escala de resposta com seis pontos (1=Nunca e 6=Sempre). Quanto mais elevada a pontuação total, maior será o nível de intensidade afetiva do participante. Este instrumento de avaliação apresenta bons indicadores de validade e fiabilidade na população portuguesa (Prada et al., 2009). Nomeadamente no que se refere à consistência interna, tanto no estudo anteriormente referido $(\alpha=.82)$ como na presente amostra $(\alpha=.80)$, foram encontrados valores de consistência interna satisfatórias.

\section{Procedimento}

Após ter sido pedida a autorização aos autores para a utilização das escalas, iniciou-se a recolha dos dados através de um inquérito on-line, usando para o efeito o aplicativo do Google Forms. 0 inquérito foi 
disseminado através das redes sociais, nomeadamente o Facebook, Messenger e WhatsApp. Na folha de rosto do inquérito, procedeu-se ao esclarecimento sobre o objetivo do estudo, deram-se as instruções de resposta e pediu-se a leitura e aceitação do Consentimento Informado, frisando a participação voluntária, o anonimato e a confidencialidade no tratamento dos dados obtidos. Após a recolha, passaram-se os dados para uma folha de Excel através de uma ferramenta do próprio Google Forms, e posteriormente procedeu-se ao tratamento estatístico utilizando o software da IBM SPSS 25 (IBM Corp. Release, 2017).

\section{RESULTADOS}

A análise da estrutura relacional dos 7 itens da BES-A breve foi efetuada através do método da fatorização de eixo principal, utilizando a rotação Promax. Os fatores comuns retidos foram os que apresentaram um eigenvalue superior a 1 . A validade da análise fatorial foi feita através do KMO (0.724, razoável) e teste de Bartlett ( $p<.001$, significativo). A análise fatorial convergiu para uma solução com 2 fatores principais que explicam $54.11 \%$ da variância total. A saturação dos itens em cada uma das dimensões pode ser apreciada na Tabela 2.0 primeiro fator integra os itens relacionados com a dimensão cognitiva, enquanto os itens relacionados com a dimensão afetiva se encontram agrupados no segundo fator.

Tabela 2. Matriz de Fatores Principais e variância explicada

\begin{tabular}{lcc}
\hline & \multicolumn{2}{c}{ Fatores Principais } \\
\cline { 2 - 3 } & $\mathbf{1}$ & $\mathbf{2}$ \\
\hline BES_12cogn & $\mathbf{0 . 8 0 8}$ & 0.225 \\
BES_10cogn & $\mathbf{0 . 7 8 8}$ & 0.143 \\
BES_9cogn & $\mathbf{0 . 7 8 5}$ & 0.170 \\
BES_14cogn & $\mathbf{0 . 6 3 0}$ & 0.041 \\
BES_17afet & 0.117 & $\mathbf{0 . 8 8 2}$ \\
BES_5afet & 0.119 & $\mathbf{0 . 6 8 0}$ \\
BES_2afet & 0.164 & $\mathbf{0 . 4 9 0}$ \\
Variância explicada & 34.62 & 19.49 \\
\hline
\end{tabular}

Na Figura 1, podemos observar que os itens de cada dimensão se concentram próximos do eixo dessa mesma dimensão, os itens de cada subescala estão posicionados de acordo com a sua carga fatorial nos dois fatores extraídos após rotação, e claramente não estão correlacionados entre si, apontando para que cada um deles meça exatamente o fator ao qual pertence: empatia afetiva e empatia cognitiva.

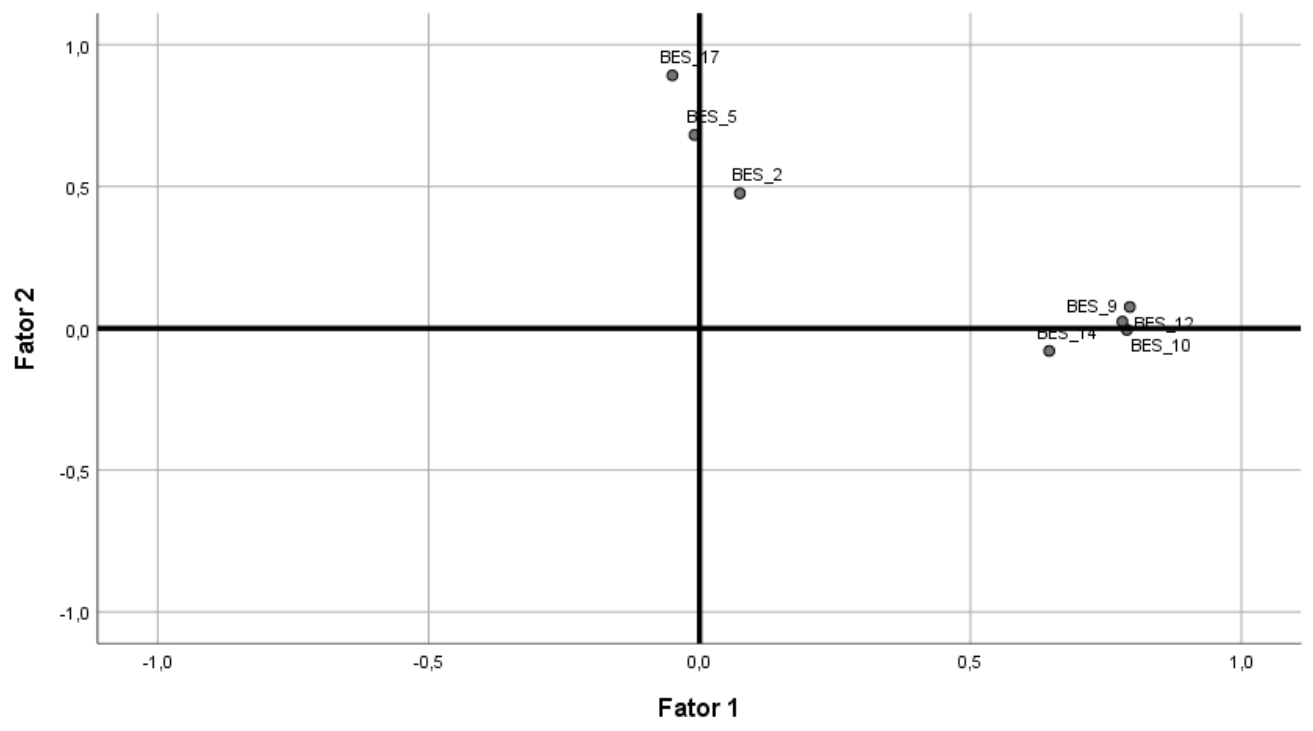

Figura 1. Agrupamento dos itens das duas subescalas. 
Os coeficientes de consistência interna, analisada com o coeficiente Alfa de Cronbach, são superiores a .70 para a escala total e as duas subescalas. A consistência global obtida foi de $\alpha=.72$. (ver Tabela 3).

Tabela 3. Consistência Interna das subescalas e escala total

\begin{tabular}{lc}
\hline Escala & Alfa de Cronbach \\
\hline Afetiva & 0.84 \\
Cognitiva & 0.72 \\
BES total & 0.72 \\
\hline
\end{tabular}

Os coeficientes de correlação entre as dimensões da BES são estatisticamente significativos e positivos. No que se refere à validade convergente (ver Tabela 4), as correlações da BES-Breve com a Escala Breve de Intensidade Afetiva (EBIA) são positivas e moderadas $(r>.30)$.

Tabela 4. Matriz de Correlações da BES Breve e Escala Breve de Intensidade Afetiva

\begin{tabular}{lccc}
\hline & BES Cognitiva & BES Total & Intensidade Afetiva \\
\hline BES Afetiva & $.159^{*}$ & $.76^{* *}$ & $.39^{* *}$ \\
BES Cognitiva & & $.76^{* *}$ & $.30^{* *}$ \\
BES Total & & & $.45^{* *}$ \\
\hline
\end{tabular}

Notas: ${ }^{*} p<.05 ;{ }^{* *} p<.01$

A comparação dos valores obtidos pelas amostras feminina e masculina indicam diferenças estatisticamente significativas $(p<.001)$, sendo que as mulheres obtêm valores significativamente mais elevados quer nas dimensões afetiva e cognitiva quer no valor global da BES breve (ver Tabela 5).

Tabela 5. Comparação por sexo

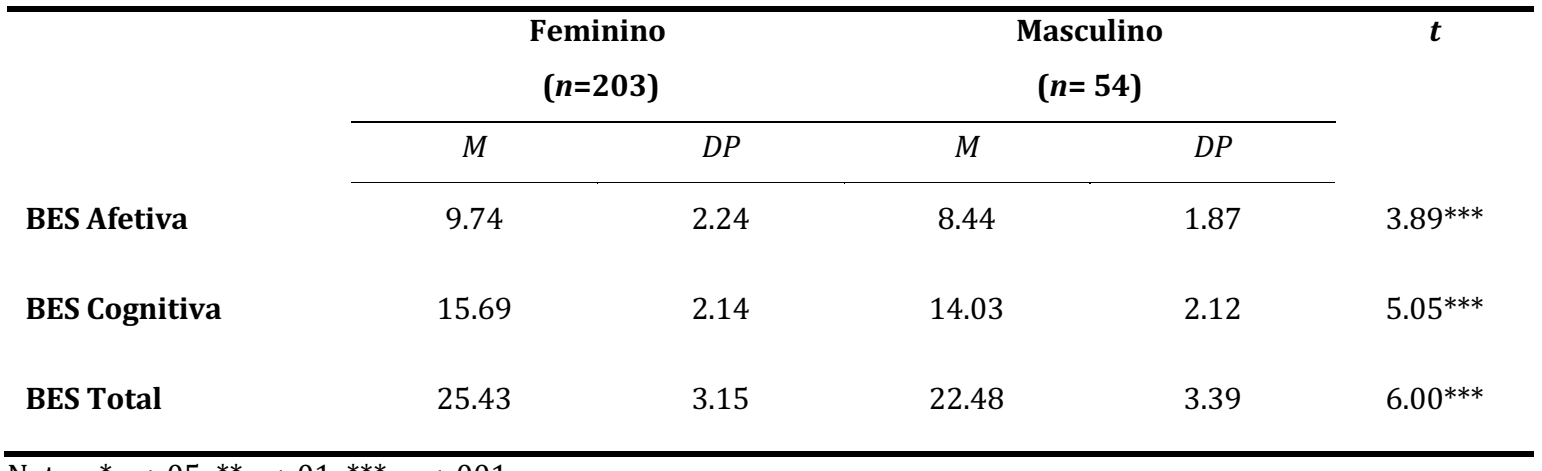

Notas: ${ }^{*} p<.05 ;{ }^{* *} p<.01 ;^{* * *} ; p<.001$

\section{CONCLUSÃO}

Dada a importância dos níveis de empatia nas relações humanas, torna-se relevante o estudo de medidas fidedignas para a sua avaliação e compreensão, e que permitam a sua utilização em vários contextos de intervenção (e.g., educacional, clínico, social, forense). O objetivo central deste estudo incidiu sobre a análise das propriedades psicométricas da Escala Básica de Empatia - versão breve (BES-A) - para a população Portuguesa adulta, e a apresentação dos resultados da consistência interna, validade convergente, validade de construto e validade de grupos conhecidos (e.g., sexo).

De modo geral, o modelo da BES-A apresentou um bom ajustamento aos dados e uma boa fiabilidade das subescalas e da escala total. Relativamente à análise do sexo, os resultados mostram que o sexo feminino, comparativamente ao masculino, apresenta um índice de empatia mais elevado tanto nas dimensões afetiva e cognitiva, como na totalidade da escala, confirmando o que era esperado. Como se previa, as correlações da BES-A com a EBIA foram positivas, moderadas e significativas. Assim, valores mais altos de empatia afetiva parecem estar associados aos valores mais altos de intensidade afetiva. A correlação entre as subescalas da BES-A é positiva e estatisticamente significativa, contudo baixa. Na verdade, em vários estudos anteriores (e.g., Pechorro et al., 2015; Sánchez-Pérez et al., 2014) as correlações apresentadas são inferiores a .40, uma vez que tanto a empatia afetiva como a cognitiva são 
dimensões com características independentes, mas que fazem parte de um mesmo conceito comum, a empatia. De facto, uma recente meta-análise na área das neurociências (Fan et al., 2014) sugere que estas duas formas de empatia correspondem a diferentes ativações neuronais. No entanto, a construção de uma versão breve da BES, constituída pelos itens que melhor caracterizam cada uma das subescalas, reforça ainda mais essas diferenças, resultando ainda em valores inferiores de correlação entre as duas subescalas, tal como foi verificado em estudos prévios (ver, por exemplo, Pechorro et al., 2018).

Tendo em conta os resultados globais, verificamos que os dados corroboram os estudos de Jolliffe e Farrington (2006) e de Pechorro, Ray, Salas-Wright, Marôco e Gonçalves (2015) quando referem que a BES pode ser utilizada com uma boa precisão, pelo que consideramos este instrumento útil e fidedigno na medição dos níveis de empatia. A BES-A breve demonstrou qualidades psicométricas satisfatórias, surgindo como uma medida adequada para o construto da empatia através das suas duas dimensões: empatia afetiva e empatia cognitiva. Estudos futuros deverão explorar a estabilidade temporal da BES breve na população adulta portuguesa, assim como explorar as propriedades desta escala em diferentes grupos populacionais. Por fim, a BES-A breve é também um instrumento de utilização rápida e simples, que não necessita de meios dispendiosos para a sua aplicação.

\section{REFERÊNCIAS}

Albiero, P., Matricardi, G., Speltri, D., \& Toso, D. (2009). The assessment of empathy in adolescence: A contribution to the Italian validation of the Basic Empathy Scale. Journal of Adolescence, 32, 393408. https://doi.org/10.1016/j.adolescence.2008.01.001

Anastácio, S. (2013). Estudo da relação entre a empatia e a vinculação aos pais e aos pares na adolescência [Dissertação de Mestrado, Universidade de https://estudogeral.sib.uc.pt/handle/10316/23982

Anastácio, S., Vagos, P., Nobre-Lima, L., Rijo, D., \& Jolliffe, D. (2016). The Portuguese version of the Basic Empathy Scale (BES): Dimensionality and measurement invariance in a community adolescent sample. European Journal of Developmental Psychology, 13, 614-623. https://doi.org/10.1080/17405629.2016.1167681

Basto-Pereira, M. \& Farrington, D.P. (2018, Outubro). The Basic Empathy Scale: Psychometric properties and contributions to the understanding of antisocial behaviour, [Artigo Apresentado]. Measuring Empathy and Behavioural Outcomes Conference, Greenwich, UK.

Carré, A., Stefaniak, N., D'Ambrosio, F., Bensalah, L., \& Besche-Richard, C. (2013). The Basic Empathy Scale in Adults (BES-A): Factor structure of a revised form. Psychological Assessment, 25(3), 679-691. https://doi.org/10.1037/a0032297

D’Ambrosio, F., Olivier, M., Didon, D., \& Besche, C. (2009). The Basic Empathy Scale: A French validation of a measure of empathy in youth. Personality and Individual Differences, 46(2), 160-165. https://doi.org/10.1016/j.paid.2008.09.020

Davidson M. (2014). Known-Groups Validity. In A. C. Michalos (Ed.) Encyclopedia of Quality of Life and Well-Being Research. Springer. https://doi.org/10.1007/978-94-007-0753-5_1581

Davis, M. H. (1983). Measuring individual differences in empathy: Evidence for a multidimensional approach. Journal of Personality and Social Psychology, 44, 113-126. https://doi:10.1037/00223514.44.1.113

Davis, M. H. (1983). The effects of dispositional empathy on emotional reactions and helping: a multidimensional approach. Journal of Personality, 51, 167-184. https://doi. org/10.1111/j.14676494.1983.tb00860.x

Davis, M. H. \& Oathout, H.A. (1987). Maintenance of satisfaction in romantic relationship: empathy and relational competence. Journal of Personality and Social Psychology, 53, 397-410. https://doi.org/10.1037/0022-3514.53.2.397

Davis, M. H. \& Oathout, H.A. (1992). The effect of dispositional empathy on romantic relationships behaviors: Heterosocial anxiety as a moderating influence. Journal of Personality and Social Psychology, 18, 76-83. https://doi.org/10.1177/0146167292181011

Domingos, M.E. (2011). A liderança sob a perspectiva do outro: dissertação sobre a empatia, o respeito, a cordialidade e os seus efeitos no desempenho profissional, pessoal e organizacional. [Tese de Mestrado, Universidade Católica Portuguesa]. https://repositorio.ucp.pt/handle/10400.14/8240

Depraz, N. (2005). A ética relacional: uma prática de ressonância interpessoal. Revista Do Departamento De Psicologia, 17(2), 19-34. https://doi.org/10.1590/S0104-80232005000200003

Fengqin, D. \& Zhaohui, L. (2016). Association between empathy and prosocial behavior: A systematic review and meta-analysis. Advances in Psychological Science, 24(8), 1159-1174. https://doi.org/10.3724/SP.J.1042.2016.01159

Flett, G. L., Blankstein, K. R., Pliner, P., \& Bator, C. (1988). Impression-management and self-deception 
components of appraised emotional experience. British Journal of Social Psychology, 27, 67-77.

Eisenberg, N., \& Miller, P. A. (1987). Empathy, sympathy and altruism: Empirical and conceptual links. In N. Eisenberg \& J. Strayer (Eds.), Empathy and its development (pp. 292-316). University Press. https://psycnet.apa.org/record/1987-98639-015

Fan, Y., Duncan, N.W., de Greck M., Northoff, G. (2011). Is there a core neural network in empathy? An fMRI based quantitative metaanalysis. Neuroscience \& Biobehavioral Reviews, 35, 903-911.

Flett, G. L., Bator, C., \& Blankstein, K. R. (1988). Affect intensity and components of emotional style. Personality and Individual Differences, 9, 185-186. https://doi.org/10.1016/0191-8869(89)901694

Geng, Y., Xia, D., \& Qin, B. (2012). The Basic Empathy Scale: A Chinese validation of a measure of empathy in adolescents. Child Psychiatry \& Human Development, 43(4), 499-510. https://doi.org/10.1007/s10578-011-0278-6

Godinho, T. (2015). Contributos para a compreensão do processo de empatia e do seu desenvolvimento [Tese de Doutoramento, Universidade de Évora, Escola de Ciências Sociais]. https://dspace.uevora.pt/rdpc/bitstream/10174/14535/11/Doutoramento\%20\%20T\%C3\%A2nia\%20Godinho.pdf

Góis, S. \& Migueis, M. (2012). Qualidade da interação Professor-Aluno no 1ํo Ciclo do Ensino Básico: Do diálogo experiencial à educação emocional. Em L. Mata, M. A. Martins, V. Monteiro, J. Morgado, F. Peixoto, A. C. Silva \& J. M. C. Silva (Eds.), Atas do 13.o colóquio de Psicologia e Educação (pp. 188201). ISPA - Instituto Universitário. http://repositorio.ispa.pt/handle/10400.12/5541

IBM Corp. (2017). IBM SPSS Statistics para Windows, Versão 25.0. IBM Corp.

Ickes, W., \& Simpson, J. A. (1997). Managing empathic accuracy in close relationships. In W. J. Ickes (Ed.), Empathic accuracy (pp. 218-250). The Guilford Press.

Jolliffe, D. \& Farrington, D.P. (2006). Development and validation of the Basic Empathy Scale. Journal of Adolescence, 29, 589-611. https://doi.org/10.1016/j.adolescence.2005.08.010

Hall, J., \& Schwartz, R. (2019) Empathy present and future, The Journal of Social Psychology, 159, 3, 225243. https://doi.org/10.1080/00224545.2018.1477442

Long, E. C., \& Andrews, D. W. (1990). Perspective taking as a predictor of marital adjustment. Journal of Personality and Social Psychology, 59(1), 126-131. http://dx.doi.org/10.1037/0022-3514.59.1.126

Matias, M. (2012). A Avaliação da Empatia na Adolescência: estudos de validação da versão portuguesa da "Basic Empathy Scale" [Dissertação de Mestrado, Universidade de Coimbra] https://eg.uc.pt/handle/10316/26137

Larsen, R. J., \& Diener, E. (1987). Affect Intensity as an individual difference characteristic: A review. Journal of Research in Personality, 21, 1-39. https://doi.org/10.1016/0092-6566(87)90023-7

Marshall, L. E. \& Marshall, W.L. (2011). Empathy and antisocial behavior. The Journal of Forensic Psychiatry \& Psychology, 22(5), 742 759, https://doi.org/10.1080/14789949.2011.617544

Pechorro, P., Ray, J., Salas-Wright, C., Marôco, J. \& Gonçalves, R. A. (2015). Adaptation of the Basic Empathy Scale among a Portuguese sample of incarcerated juvenile offenders. Psychology, Crime \& Law. 21(7), 699-714. https://doi.org/10.1080/1068316X.2015.1028546

Pechorro, P., Ray, J., Raine, A., Marôco, J., \& Gonçalves, R. (2017). The Reactive-Proactive Aggression Questionnaire: Validation among a Portuguese sample of incarcerated juvenile delinquents. Journal of Interpersonal Violence, 32, 1995-2017. https://doi.org/10.1177/0886260515590784

Pechorro, P., Jesus, N., Kahn R. E., Gonçalves, R. A., e Barroso, R. (2018). A Versão Breve da Escala de Empatia Básica numa Amostra Escolar de Jovens Portugueses: Validade, Fiabilidade e Invariância. Revista Iberoamericana de Diagnóstico y Evaluación - e Avaliação Psicológica, 49(4), 157-169. https://doi.org/10.21865/RIDEP49.4.13

Prada, M., Rodrigues, D., \& Palma, T. (2013). 8 ou 80! Adaptação e validação da Escala Breve de Intensidade Afectiva. Laboratório de Psicologia, 7, 85-99. https://doi.org/10.14417/lp.688

Rogers, C. (1992). Terapia centrada no cliente. Martins Fontes.

Salas-Wright, C., Olate, R., \& Vaughn, M. (2012). Assessing empathy in Salvadoran high-risk and ganginvolved adolescents and young adults: A Spanish validation of the Basic Empathy Scale. International Journal of Offender Therapy and Comparative Criminology, 57, 1393-1416. https://doi.org/10.1177/0306624X12455170

Sánchez-Pérez, N., Fuentes, L. J., Jolliffe, D., \& González-Salinas, C. (2014). Assessing children's empathy through a Spanish adaptation of the basic empathy scale: Parent's and child's report forms. Frontiers in Psychology, 5, 1438. https://doi.org/10.3389/fpsyg.2014.01438

Stein, E. (2004). El Problema de la Empatia. Trotta.

Van Berkhout, E. \& M Malouff, J. (2015). The Efficacy of Empathy Training: A Meta-Analysis of Randomized Controlled Trials. Journal of counseling psychology, 63 (1), 32-41. 
Versão curta da escala Básica da Empatia em Adultos

https://doi.org/10.1037/cou0000093

Historial do artigo

Recebido 31/07/2019

Aceite $\quad 10 / 06 / 2020$

Publicado 08/2020 


\title{
Que competências podem ser preditoras do desempenho em leitura? Um estudo longitudinal*
}

\author{
Inês Ferraz ${ }^{49 i}$ \\ ${ }^{i}$ Centro de Investigação em Estudos da Criança
}

\begin{abstract}
Resumo: Apesar do investimento na promoção das competências linguísticas na educação pré-escolar, o número de crianças com dificuldades na aprendizagem da leitura continua elevado. Tendo como suporte a teoria piagetiana, a investigação sobre a consciência fonológica e o conhecimento das letras, através dum estudo longitudinal do pré-escolar ao 2. ${ }^{\circ}$ ano de escolaridade, analisou-se o contributo das operações lógicas piagetianas, da consciência fonológica e do conhecimento das letras no desempenho em leitura, considerando a idade e habilitações dos pais, em 116 crianças da Madeira. No pré-escolar, as crianças mais velhas apresentam melhores resultados na seriação e classificação do que as mais novas. No 1. ano, a seriação mostrou ser preditor do reconhecimento de palavras. No 2.ำ ano, a consciência fonológica e o conhecimento das letras foram preditores da identificação de palavras. A seriação e a classificação foram os melhores preditores da compreensão da leitura. Crianças com pais mais habilitados apresentaram melhores resultados.
\end{abstract}

\section{Palavras-chave: Aprendizagem da leitura; Operações lógicas; Consciência fonológica; Conhecimento das} letras.

What skills can predict reading performance? A longitudinal study: Despite the investment in the promotion of language skills in pre-school education, the number of children with learning disabilities remains high. Based on Piagetian theory, research on phonological awareness and knowledge of letters through a longitudinal study, from preschool to 2nd grade, the contribution of Piagetian logical operations, phonological awareness and knowledge of letters in reading performance, considering the age and parental skills, in 116 children from Madeira. In preschool older children have better grading and grading results than younger children. In the 1st year, seriation proved to be a predictor of word recognition. In the 2nd year, phonological awareness and knowledge of letters were predictors of word identification. Seriation and classification were the best predictors of reading comprehension. Children with parents with higher education level had better results.

Keywords: Reading; Logical operations; Phonological awareness; Knowledge of letters.

A leitura é um processo linguístico e cognitivo. A nível linguístico, convoca o conhecimento lexical, o conhecimento morfossintático e a consciência fonológica. A nível cognitivo, convoca, além de processos cognitivos básicos, como os processos percetivos e léxicos envolvidos no conhecimento das letras e na identificação das palavras escritas, processos de ordem superior. Estes processos de ordem superior podem, por sua vez, ser classificados como: i) processos de integração, nos quais assumem especial importância a realização de inferências; ii) macroprocessos, com especial relevância para a identificação das ideias principais; iii) processos de elaboração, como as previsões ou a criação de imagens mentais; iv) processos metacognitivos, que permitem a identificação da perda de compreensão e a ativação de estratégias para a recuperar (Giasson, 1993).

A consciência fonológica e o conhecimento das letras têm sido identificados como os preditores mais potentes relativamente à aprendizagem da leitura, considerada na vertente descodificação. Este valor preditivo é registado quando a aprendizagem da leitura se processa em línguas de ortografia opaca, como é o caso do inglês (Carson, Boustead, \& Gillon, 2014), mas também quando se processa em línguas de ortografia transparente, como é o caso do português (Figueira \& Botelho, 2017; Reis, Faísca, Castro, \& Petersson, 2010). Todavia, o peso destes preditores decresce à medida que as crianças avançam na escolaridade (Reis, Faísca, Castro \& Petersson, 2010; Sim-Sim, Silva, \& Nunes, 2008).

\footnotetext{
${ }^{49}$ Morada de correspondência: Inês Ferraz, Centro de Investigação em Estudos da Criança. Universidade do Minho. Instituto de Educação. Campus de Gualtar, 4710-057, Braga, Portugal. E-mail: inesprferraz@gmail.com

*Este artigo retoma, em parte, o conteúdo do estudo: Ferraz, I., Viana, F., \& Pocinho, M. (2018). Operações lógicas, consciência fonológica e conhecimento das letras na educação pré-escolar. Caleidoscópio: Revista de Comunicação e Cultura, 16(1) 4-15. https://doi.org/10.4013/cld.2018.161.01
} 
A constatação da importância da consciência fonológica para a aprendizagem da leitura introduziu mudanças significativas na Educação Pré-Escolar. As Orientações Curriculares para a Educação PréEscolar, publicadas em 1997 (Ministério da Educação, 1997), apontavam para a importância da sua promoção em contexto de jardim de infância e a revisão das mesmas, em 2016 (Silva, Marques, Mata, \& Rosa, 2016), acrescenta propostas concretas, quer para a sua promoção, quer para a sua avaliação.

É no período sensório-motor que se começam a elaborar as estruturas que suportam as condições cognitivas que marcam o início do processo de formação do leitor, uma vez que é neste período que se dá o desenvolvimento do pensamento simbólico e representativo (Piaget, 1956). No período pré-operatório as relações passam a estabelecer-se sobre as representações (Piaget, 1964). Sendo a linguagem escrita uma representação da linguagem oral, esta relação torna-se crucial. Tendo em consideração as caraterísticas da cognição pré-operatória e operatória concreta, definidas por Piaget (1964), e as mudanças em termos dos esquemas cognitivos utilizados, a aquisição das operações lógicas (caraterísticas do período operatório concreto) pode ser considerada fundamental para a aprendizagem da leitura.

Numerosos estudos demonstram a existência de uma relação entre as Operações Lógicas seriação, inclusão de classe e classificação - e a capacidade de leitura (Sequeira, 1989). A maior ou menor facilidade em aprender a ler é predita pelo desempenho em tarefas cognitivas, como a classificação, a seriação e a conservação da quantidade (Clark, 1983; Hooper, Sipple, Goldman, \& Swinton, 1979; Lunzer, Dolan, \& Wilkinson, 1976; Weed \& Ryan, 1983; Webster \& Ammon, 1994). Existe uma elevada correlação entre seriação e a «prontidão» para aprender a ler (Scott, 1969). Este tipo de resultados conduziu à reconvocação da teoria piagetiana, e à necessidade de desenvolver um estudo em que, a par da consciência fonológica e do conhecimento das letras, fossem também avaliadas as operações lógicas de seriação, classificação e inclusão de classes, cujo domínio ocorre por volta dos 7 anos. Este marco, em termos de idade cronológica, não é coincidente com a idade legal de entrada no 1.ํ Ciclo do Ensino Básico.

Atendendo à inexistência de estudos que, a par do conhecimento de letras e da consciência fonológica, incluam também a consideração das operações lógicas piagetianas como potenciais preditores do desempenho em leitura, pretende-se com este estudo identificar o contributo relativo e independente destes três preditores. Pretende-se, também, verificar o seu potencial valor preditivo atendendo à variável idade, pelo que este contributo será analisado considerando dois grupos de alunos: i) os nascidos entre 1 de janeiro e 30 de junho (mais velhos); ii) os nascidos entre 1 de julho e 31 de dezembro do ano em que completam os 6 anos (mais novos).

Os dados mais recentes do Ministério da Educação (Vale, Monteiro, Fonseca, Monteiro, Rebelo, Marques, \& Ferreira, 2019), mostram que as habilitações escolares dos pais estão correlacionadas com o desempenho académico dos filhos (provas finais de 9.․ano), pelo que os resultados obtidos são também analisados sob este ponto de vista, no sentido de avaliar se estas correlações são detetadas precocemente no percurso académico. Dados semelhantes tinham sido já observados noutros estudos, de âmbito mais restrito, com as habilitações académicas dos pais a influenciarem positivamente o resultado escolar dos filhos (Rezende \& Candian, 2012; Ferraz, Pocinho, Pereira, \& Soares, 2012).

\section{MÉTODO}

\section{Participantes}

Foi efetuado um estudo longitudinal, desde o final do ano pré-escolar ao 2.. ano de escolaridade, com três momentos de avaliação: final do ano pré-escolar, final do 1.o ano e final do 2.o ano de escolaridade (ver Tabela 1). Foram respeitados os procedimentos éticos requeridos e obtidos os consentimentos informados dos encarregados de educação.

Participaram neste estudo 116 crianças de 6 grupos naturais que, no primeiro momento do estudo, frequentavam contextos de educação pré-escolar públicos na Região Autónoma da Madeira (Portugal). Os grupos são idênticos em termos de género, estatuto socioeconómico e de tempo de frequência de educação pré-escolar. 
Tabela 1. Distribuição da amostra de acordo com o género e a fase de avaliação

\begin{tabular}{lcccccc}
\hline & \multicolumn{2}{c}{ Pré-Escolar } & \multicolumn{2}{c}{ 1.o ano } & \multicolumn{2}{c}{ 2.o ano } \\
& $N$ & $\%$ & $N$ & $\%$ & $N$ & $\%$ \\
\cline { 2 - 7 } Masculino & 65 & 56 & 53 & 55.2 & 52 & 55.3 \\
Feminino & 51 & 44 & 43 & 44.8 & 42 & 44.7 \\
Total & 116 & 100 & 96 & 100 & 94 & 100 \\
\hline
\end{tabular}

\section{Instrumentos e procedimento}

No final do ano pré-escolar $(\mathrm{M} 1=$ Momento 1) foram aplicados, individualmente, os seguintes instrumentos de avaliação: i) PACF - Prova de Avaliação da Consciência Fonológica - Forma A (Ferraz, Pocinho \& Fernandes, 2018); ii) PCL - Prova de Conhecimento das Letras (criada para o efeito); iii) As Provas Piagetianas (Piaget \& Szeminska,1941; Piaget \& Inhelder, 1959, 1966). No 1.o ano (M2 = Momento 2), foram aplicados os seguintes instrumentos de avaliação: i) Teste de Avaliação da Fluência e da Precisão de Leitura «O Rei» (Carvalho, 2010); ii) TOL - Teste de Leitura de Palavras, que integra a Bateria de Avaliação da Leitura (Ribeiro, Viana, Santos, Cadime, Chaves-Sousa, Vale, \& Spinillo, 2014); iii) PRP Prova de Reconhecimento de Palavras (Viana \& Ribeiro, 2010). Os dois primeiros testes foram aplicados individualmente; o último foi aplicado coletivamente.

No 2.․ ano (M3 = Momento 3), os instrumentos utilizados foram: i) Teste de Avaliação da Fluência e da Precisão de Leitura «O Rei» (Carvalho, 2010); ii) PRP - Prova de Reconhecimento de Palavras (Viana \& Ribeiro, 2010); iii) ACL - Avaliação da Compreensão Leitora (Català, Català, Molina, \& Monclús, 2001, traduzido para Português Europeu (Mendonça, 2008). 0 primeiro teste foi aplicado individualmente; os dois últimos foram aplicados coletivamente.

\section{RESULTADOS}

Nas tabelas 2 e 3 são apresentadas a estatística descritiva e os resultados obtidos no Momento 1. No ano pré-escolar, tanto na Prova de Avaliação da Consciência Fonológica como na Prova de Conhecimento das Letras (PCL), as crianças mais velhas apresentam melhores desempenhos do que as mais novas (ver Tabela 2). Não se registam diferenças em função da idade das crianças tanto na Prova da Avaliação da Consciência Fonológica $(t(94)=.286 ; p=.685)$, como na Prova de Conhecimento das Letras $(U=$ $1433.50 ; p=.170$ ). Nas Provas Piagetianas, as crianças mais velhas apresentam resultados superiores aos das mais novas do mesmo ano de escolaridade (ver Tabela 3 ) tanto na Operação Lógica de seriação $(U=$ $1303.50, p=.016)$, como na classificação $(U=1333.00, p=.023)$.

Tabela 2. Estatística descritiva dos resultados obtidos no Momento 1 na PACF e na PCL

\begin{tabular}{lcccccc}
\hline & $\boldsymbol{M}$ & $\boldsymbol{D P}$ & Min. & Max. & Assimetria & Curtose \\
\hline PACF total & 49.81 & 11.73 & 18 & 72 & -.397 & -.307 \\
PCL total & 8.97 & 8.02 & 0 & 26 & .951 & -.388 \\
PACF & & & & & & \\
$\quad$ Mais velhos & 50.12 & 12.03 & 18 & 70 & -.585 & .187 \\
$\quad$ Mais novos & 49.50 & 11.53 & 25 & 72 & -.209 & -.742 \\
PCL & & & & & & -.812 \\
$\quad$ Mais velhos & 10.14 & 8.64 & 0 & 26 & .739 & .205 \\
$\quad$ Mais novos & 7.85 & 7.27 & 0 & 26 & 1.180 &
\end{tabular}

Nota . PACF = Prova de Avaliação da Consciência Fonológica; PCL = Prova de Conhecimento das Letras. 
Tabela 3. Resultados obtidos no Momento 1 nas Provas Piagetianas em função da idade

\begin{tabular}{lcccccc}
\hline & \multicolumn{2}{c}{ Seriação } & \multicolumn{2}{c}{ Classificação } & \multicolumn{2}{c}{ Inclusão } \\
Níveis de desempenho & $\boldsymbol{N}$ & $\mathbf{\%}$ & $\boldsymbol{N}$ & $\mathbf{\%}$ & $\boldsymbol{N}$ & $\mathbf{\%}$ \\
\hline 1 (ausência) & 64 & 55.2 & 1 & 0.9 & 82 & 70.7 \\
2 (intermédio) & 49 & 42.2 & 71 & 61.2 & 31 & 26.7 \\
3 (êxito) & 3 & 2.6 & 44 & 37.9 & 3 & 2.6 \\
& & & & & & \\
Mais velhas: & & & & & & \\
1 (ausência) & 25 & 43.9 & 1 & 1.8 & 40.2 \\
2 (intermédio) & 30 & 52.6 & 28 & 49.1 & 16 & 28.1 \\
3 (êxito) & 2 & 3.5 & 28 & 49.1 & 1 & 1.8 \\
& & & & & & \\
Mais novas: & & & & & & \\
1 (ausência) & 39 & 66.1 & 0 & 0 & 42 & 71.2 \\
2 (intermédio) & 19 & 32.2 & 43 & 72.9 & 15 & 25.4 \\
3 (êxito) & 1 & 1.7 & 16 & 27.1 & 2 & 3.4 \\
\hline
\end{tabular}

Na Tabela 4 é apresentada a estatística descritiva dos resultados obtidos nas provas de leitura aplicadas no Momento 2 (M2 - final do 1.ํaa ano de escolaridade). Quer na Prova de Reconhecimento de Palavras $(U=1008.50 ; p=.294)$, quer no Teste de Avaliação da Fluência e Precisão da Leitura «O Rei» ( $U$ $=1114.50 ; p=.786)$, quer ainda no Teste de Leitura de Palavras $(U=1126.00 ; p=.852)$ não se registam diferenças em função da idade.

Tabela 4. Estatística descritiva dos resultados do Momento 2 na PRP, no REI e no TLP por idade

\begin{tabular}{lcccccc}
\hline & $\boldsymbol{M}$ & $\boldsymbol{D P}$ & Min. & Max. & Assimetria & Curtose \\
\hline PRP: & 20.01 & 9.18 & 6 & 40 & .427 & -.895 \\
Mais velhos & 21.39 & 10.56 & 6 & 40 & .162 & -1.323 \\
Mais novos & 18.57 & 7.33 & 8 & 37 & .607 & -.369 \\
& & & & & \\
REI: & 58.27 & 51.98 & 6 & 278 & 1.639 & 3.207 \\
$\quad$ Mais velhos & 61.10 & 58.18 & 6 & 278 & 1.556 & 2.775 \\
$\quad$ Mais novos & 55.32 & 45.06 & 10 & 226 & 1.674 & 3.480 \\
& & & & & -124 & -1.217 \\
TLP: & 17.53 & 7.04 & 3 & 30 & -.217 & -1.359 \\
$\quad$ Mais velhos & 17.35 & 7.69 & 3 & 30 & .092 & -1.188 \\
Mais novos & 17.72 & 6.36 & 6 & 29 & & \\
\hline
\end{tabular}

Nota. PRP = Prova de Reconhecimento de Palavras; REI = Teste de Avaliação da Fluência e Precisão da Leitura «0 Rei»; TLP = Teste de Leitura de Palavras. Mais velhos = nascidos entre janeiro e junho; Mais novos = nascidos entre julho e dezembro.

A consciência fonológica, o conhecimento das letras e a operação lógica de seriação revelaram ser preditores dos resultados na Prova de Reconhecimento de Palavras. Já para o Teste de Avaliação da Fluência e da Precisão de Leitura «O Rei», os preditores foram a consciência fonológica e o conhecimento das letras. Os preditores da Prova de Leitura de Palavras foram a consciência fonológica e o conhecimento das letras. A consciência fonológica e o conhecimento das letras revelaram ser preditores do desempenho em todas as provas de leitura.

$\mathrm{Na}$ tabela 5 apresenta-se a estatística descrita dos resultados obtidos nas provas de leitura aplicadas Momento 3 (2.ํ ano de escolaridade). Quer na Prova de Reconhecimento de Palavras $(U=985.50 ; p=.375)$, quer no Teste de Avaliação da Fluência e Precisão da Leitura «O Rei» $(U=1039.00 ; p=$ 
.631), quer ainda no Teste de Avaliação da Compreensão Leitora ( $U=1038.50 ; p=.627$ ) não se registam diferenças em função da idade. Verifica-se que na PRP - Prova de Reconhecimento de Palavras -, os alunos mais novos apresentam resultados superiores aos dos mais velhos, tendência que se constata também no Teste de Avaliação da Fluência e Precisão da Leitura «0 Rei» e na ACL - Avaliação da Compreensão Leitora (ver Tabela 5).

Tabela 5. Estatística descritiva dos resultados do Momento 3 na PRP, no REI e na ACL por idade

\begin{tabular}{lcccccc}
\hline & $\boldsymbol{M}$ & $\boldsymbol{D P}$ & Min. & Max. & Assimetria & Curtose \\
\hline PRP & 27.99 & 9.13 & 9 & 40 & -.311 & -1.045 \\
Mais velhos & 27.00 & 9.79 & 9 & 40 & -.147 & -1.207 \\
Mais novos & 29.07 & 8.31 & 11 & 40 & -.469 & -.807 \\
& & & & & \\
REI & 160.96 & 69.48 & 6 & 281 & -.062 & -.627 \\
Mais velhos & 157.20 & 75.41 & 6 & 281 & -.005 & -.734 \\
Mais novos & 165.04 & 62.99 & 28 & 276 & -.082 & -.531 \\
& & & & & .273 & -.578 \\
ACL & 11.95 & 7.04 & 2 & 23 & .336 & -.595 \\
Mais velhos & 11.71 & 4.13 & 5 & 20 & .198 & -.545 \\
Mais novos & 12.22 & 4.59 & 2 & 23 & & \\
\hline
\end{tabular}

Nota. PRP = Prova de Reconhecimento de Palavras; REI = Teste de Avaliação da Fluência e Precisão da Leitura «0 Rei»; ACL = Avaliação da Compreensão Leitora; Mais velhos = nascidos entre janeiro e junho; Mais novos = nascidos entre julho e dezembro.

Os preditores dos resultados obtidos na Prova de Reconhecimento de Palavras, e no Teste de Avaliação da Fluência e da Precisão de Leitura «O Rei», foram a consciência fonológica e o conhecimento das letras. No teste de Avaliação da Compreensão Leitora, os preditores foram a operação lógica de seriação, a consciência fonológica e o conhecimento das letras. No teste de Avaliação da Compreensão Leitora, constatou-se que há uma tendência preditiva da operação lógica de classificação, embora não significativa do ponto de vista estatístico.

Analisando os desempenhos em função das habilitações académicas do pai e da mãe, no ano préescolar verifica-se que, na Prova de Conhecimento das Letras e na de Classificação, as crianças com pais possuidores de habilitações académicas mais elevadas apresentaram melhores resultados $\left(\chi^{2}=16.191, p\right.$ $=.003$ e $\chi^{2}=14.102, p=.007$, respetivamente). Quanto às habilitações académicas das mães, apenas se registaram diferenças significativas na Prova de Conhecimento das Letras $\left(\chi^{2}=15.588, p=.004\right)$ a favor das crianças cujas mães possuíam habilitações mais elevadas.

No 1.ำ ano, as crianças cujo pai possui habilitações mais elevadas têm melhores resultados em todas as provas: Prova de Reconhecimento de Palavras $\left(\chi^{2}=11.081, p=.026\right)$; Teste de Avaliação da Fluência e Precisão da Leitura «0 Rei» $\left(\chi^{2}=24.687, p=.000\right)$; Teste de Leitura de Palavras $\left(\chi^{2}=22.532, p\right.$ $=.000$ ). Procedendo à mesma análise para as habilitações académicas da mãe, verifica-se que estas apenas influenciam o desempenho dos filhos apenas no Teste de Avaliação da Fluência e Precisão da Leitura «0 Rei» $\left(\chi^{2}=15.880, p=.003\right)$ e no Teste de Leitura de Palavras $\left(\chi^{2}=12.933, p=.012\right)$.

No 2.․aำ as crianças cujo pai possui habilitações académicas superiores obtêm melhores resultados na Prova de Reconhecimento de Palavras $\left(\chi^{2}=10.370, p=.035\right)$ e no Teste de Avaliação da Fluência e Precisão da Leitura «O Rei» $\left(\chi^{2}=15.236, p=.004\right)$. Por seu turno, as habilitações académicas das mães influenciam os desempenhos dos filhos no Teste de Avaliação da Fluência e Precisão da Leitura «O Rei» $\left(\chi^{2}=13.648, p=.009\right)$ e no teste de Avaliação da Compreensão Leitora $\left(\chi^{2}=19.076, p=.001\right)$.

\section{DISCUSSÃo}

No primeiro momento de avaliação - ano pré-escolar - as crianças mais velhas apresentaram resultados significativamente mais elevados do que as mais novas nas provas de seriação e de classificação. De acordo com Piaget (1964) a aquisição das operações lógicas é uma caraterística do período operatório concreto, altura em que as crianças adquirem as competências necessárias para realizar ações mentais que evitem contradição e confusão entre transformações relevantes e irrelevantes, passando a ser capazes de realizar ajustamentos ou regulações operatórias. Estes resultados são semelhantes aos dos 
estudos de Clark (1983) e de Hooper, Sipple, Goldman e Swinton (1979), comprovando que a idade é um fator a ter em conta nas tarefas de seriação, de classificação e de inclusão de classes.

Relativamente aos resultados do Momento 2 (1.ํao ano escolaridade), a consciência fonológica, o conhecimento das letras e a Prova Piagetiana de seriação revelaram ser os melhores preditores dos resultados obtidos na Prova de Reconhecimento de Palavras. Estes resultados corroboram os obtidos por Bradley e Bryant (1991), nos quais o conhecimento das letras se correlacionava positivamente com o progresso inicial na aprendizagem da leitura. Corroboram ainda, embora parcialmente, os dados obtidos por Weed e Ryan (1983), nos quais se encontraram correlações significativas entre a capacidade de seriação e o desempenho em leitura. Por sua vez, a consciência fonológica e o conhecimento das letras revelaram-se preditores do desempenho no Teste de Avaliação da Fluência e Precisão da Leitura «O Rei» e no TLP - Teste de Leitura de Palavras, resultados que vão no mesmo sentido dos obtidos por Figueira e Botelho (2017).

No terceiro momento do estudo (2. .9 ano de escolaridade), a consciência fonológica e $o$ conhecimento das letras são preditores do desempenho na PRP - Prova de Reconhecimento de Palavras e no Teste de Avaliação da Fluência e Precisão da Leitura «0 Rei». Estes resultados são idênticos aos encontrados por Ribeiro (2012).

No que concerne à compreensão da leitura, avaliada através da ACL - Avaliação da Compreensão da Leitura, constatou-se que os resultados obtidos na PCL - Prova de Conhecimento de Letras e nas Provas de seriação e de classificação foram os melhores preditores. Estes resultados corroboram os de Lunzer, Dolan e Wilkinson (1976), nos quais o conhecimento das letras é preditor do desempenho na compreensão de textos. Vão, ainda, ao encontro dos resultados do estudo de Webster e Ammon (1994) que comprovaram que a capacidade de classificação se correlacionava significativamente com compreensão da leitura.

No que se refere à idade, no 1.음 ano não se verificaram diferenças estatisticamente significativas em nenhuma das provas de leitura, contrariando os dados obtidos noutros estudos (e.g., Bonin, Barry, Méot, \& Chalard, 2004). Também no 2.ำ ano, a variável idade não exerceu qualquer influência nos resultados obtidos nas provas de leitura. Estes resultados contrastam com os de Salles, Piccolo, Zamo e Toazza (2013), uma vez que estes comprovaram que a idade de aquisição da leitura tinha implicações no seu desempenho.

Em relação às habilitações dos pais, verificou-se que os filhos de pais com maiores habilitações apresentam melhores desempenhos nas provas de leitura, à semelhança do que aconteceu nos estudos de Rezende e Candian (2012) e de Ferraz, Pocinho, Pereira e Soares (2012).

Os dados obtidos neste estudo permitem verificar que os resultados nas provas piagetianas de avaliação das operações lógicas de seriação, classificação e inclusão de classes influenciam a leitura, na vertente da descodificação, e são fortemente preditoras da compreensão. Uma das limitações do estudo prende-se com a escassez da literatura no que diz respeito às Provas Piagetianas, o que dificultou a justificação e a comparação dos dados obtidos com outros estudos efetuados.

Nas Orientações Curriculares para a Educação Pré-Escolar (Silva, Marques, Mata, \& Rosa, 2016), a alusão às operações lógicas é apenas efetuada no domínio da matemática. Em face destes resultados, sugere-se que, em termos pedagógicos, seja dada uma maior atenção às propostas pedagógicas que apoiem a emergência destas operações.

\section{REFERÊNCIAS}

Bonin, P., Barry, C., Meot, C. \& Chalarda, M. (2004). The influence of age of acquisition in word reading and other tasks: a never-ending story? Journal of Memory and Language, 50, 456-476. https://doi.org/10.1016/j.jml.2004.02.001

Bradley, L., \& Bryant, P. (1991). Phonological skills before and after learning to read. In S. Brady, D. Shankweiler (Ed.), Phonological Processes in Literacy: A Tribute to Isabelle Y. Liberman (pp. 37-45). Lawrence Erlbaum.

Carson, K., Boustead, T., \& Gillon, G. (2014). Predicting reading outcomes in the classroom using a computer-based phonological awareness screening and monitoring assessment (Com-PASMA). International Journal of Speech-Language Pathology, 16(6), 552-561. https://doi.org/10.3109/17549507.2013.855261

Carvalho, A. (2010). Teste de Avaliação da Fluência e Precisão de Leitura - O Rei. Edipsico.

Català, G., Català, M., Molina, E., \& Monclús, R. (2001). Evaluación de la comprensión lectora. GRAÓ.

Clark, J. V. (1983). Development of seriation and its relation to the achievement of inferential transitivity. Journal of Research in Science Teaching, 20(8), 781-794. https://doi.org/10.1002/tea.3660200808 
Ferraz, I., Viana, F., \& Pocinho, M., (2018). Operações Lógicas, Consciência Fonológica e Conhecimento das Letras na Educação Pré-Escolar. Caleidoscópio: Revista de Comunicação e Cultura, 16(1) 4-15. https://doi.org/10.4013/cld.2018.161.01

Ferraz, I., Pocinho, M., \& Fernandes, T. (2018). PACF - Prova de Avaliação da Consciência Fonológica (Formas A e B). Universidade da Madeira. https://digituma.uma.pt/handle/10400.13/2017

Ferraz, I., Pocinho, M., Pereira, A., \& Soares, A. (2012). Phonological awareness training programme. Uschina foreign language, 2, 944-959. https://doi.org/10.17265/1539-8080/2012.02.005

Figueira, A., \& Botelho, A. (2017). Consciência fonológica e desempenho geral na leitura. Que relação? Estudo com alunos dos $2^{\circ}$ e $3^{\circ}$ anos de escolaridade. Práxis Educativa, 12(1), 48-63. https://doi.org/10.5212/PraxEduc.v.12i1.0003

Giasson, J. (1993). A compreensão na leitura. Edições Asa.

Hooper, F. H., Sipple, T., Goldman, J., \& Swinton, S. (1979). A cross-sectional investigation of children's classificatory abilities. Genetic Psychology Monographs, 99(1), 41-89.

Lunzer, E., Dolan, T., \& Wilkinson, J. (1976). The effectiveness of measures of operativity, language and short-term memory in the prediction of reading and mathematical understanding. British Journal of Education Psychology, 46, 295-305.

Mendonça, S. (2008). Provas de avaliação da compreensão leitora: estudos de validação. [Dissertação de Mestrado não publicada, Universidade do Minho].

Ministério da Educação (1997). Orientações Curriculares para a Educação Pré-Escolar. Ministério da Educação.

Vale, A., Monteiro, A., Fonseca, D., Monteiro, I., Rebelo, I., Marques, P., \& Ferreira, R. (2019). Processo de Avaliação Externa da Aprendizagem - Provas de Aferição, Provas Finais e Exames Nacionais 2018. Ministério da Educação. https://www.dge.mec.pt/sites/default/files/JNE/relatorio_anual_do_jne_2018_final_lv.pdf

Mota, M., \& Santos, A. (2009). 0 papel da consciência fonológica na leitura contextual medida pelo teste de Cloze. Estudos de Psicologia, 14(3), 207-212. https://doi.org/10.1590/S1413294X2009000300004

Piaget, J. (1956). Le langage et la pensée chez l'enfant (4ํㅡㄹ. ed.). Delachaux et Niestlé.

Piaget, J. (1964). Six études de psychologie. Denoël-Gonthier.

Piaget, J., \& Inhelder, B. (1959). La genèse des structures logiques élémentaires. Delachaux et Niestlé.

Piaget, J., \& Inhelder, B. (1966). La psychologie de l'enfant. Presses Universitaires

Piaget, J., \& Szeminska, A. (1941). La genèse du nombre chez l'enfant. Delachaux et Niestlé.

Reis, A., Faísca, L., Castro, S., \& Peterson, K. (2010). Preditores da leitura ao longo da escolaridade: alterações dinâmicas no papel da consciência fonológica e da nomeação rápida. In Atas do VII Simpósio Nacional de Investigação em Psicologia, Universidade do Minho (pp. 3117-3132). https://repositorio-aberto.up.pt/bitstream/10216/21499/2/85069.pdf

Rezende, W., \& Candian, J. (2012). A família, a escola e o desempenho dos alunos: notas de uma interação cambiante. In Anais do III Congresso Ibero-Americano de Política e Administração da Educação (pp. 1-19).

Ribeiro, I., Viana, F., Santos, S., Cadime, I., Chaves-Sousa, S., Vale, A. \& Spinillo, A., (2014). Bateria de Avaliação da Leitura. Cegoc.

Ribeiro, M. 2012. Trajetórias na aprendizagem da leitura e da escrita: um estudo longitudinal com alunos do 1. ciclo do ensino básico [Dissertação de Doutoramento em Psicologia, Universidade de Coimbra].

Salles, J., Piccolo, L., Zamo, R., \& Toazza, R. (2013). Normas de desempenho em tarefas de leitura de palavras/pseudopalavras isoladas para crianças de 1.ำ ano a 7.․ano. Estudos e Pesquisas em Psicologia, 13(2), 397-419.

Scott, R. (1969). Social class, race, seriating, and reading readiness: A study of their relationship at the kindergarten level. Journal Genetic Psychology, 115, 87-96.

Sequeira, F. (1989). Um estudo dos processos cognitivos determinantes do sucesso na aprendizagem da leitura. Sugestão para elaboração de novos programas escolares. In F. Sequeira; I. Sim-Sim (Ed.), Maturidade linguística e aprendizagem da leitura, (vol.1, pp. 79-98). Universidade do Minho.

Silva, L., Marques; L., Mata, L., \& Rosa, M. (2016). Orientações Curriculares para a Educação Pré-Escolar. Ministério da Educação - Direção-Geral da Educação. https://www.dge.mec.pt/sites/default/files/Noticias_Imagens/ocepe_abril2016.pdf

Sim-Sim, I., Silva, A. C., \& Nunes, C. (2008). Linguagem e comunicação no jardim-de infância. Direção Geral de Inovação e Desenvolvimento Curricular.

Viana, F. L., \& Ribeiro, I. S. (2010). P.R.P. - Prova de Reconhecimento da Palavras. Cegoc. 
Webster, L., \& Ammon, P. (1994). Linking written language to cognitive development: reading, writing, and concrete operations. Research in the Teaching of English, 28, 89-109. https://www.jstor.org/stable/40171326

Weed, K., \& Ryan, E. (1983). Alphabetical seriation as a reading readiness indicator. Journal of General Psychology, 109, 201-210. https://doi.org/10.1080/00221309.1983.10736086

Historial do artigo

Recebido $\quad 07 / 2019$

Aceite $\quad 03 / 2020$

Publicado $\quad 08 / 2020$ 
Revista PSICOLOGIA, 2020, Vol. 34 (1), doi: 10.17575/psicologia.v34i1.1681.

\title{
Espontaneidade como precursor da criatividade: Um estudo com estudantes universitários de Desporto e Psicologia
}

\author{
Paulo Martins ${ }^{50 i}$, António-José Gonzalez ${ }^{\mathrm{ii}}$, Margarida Limaiii, \& Hugo Duarte ${ }^{\mathrm{iv}}$ \\ ${ }^{i}$ Laboratório de Psicologia do Desporto, Faculdade de Motricidade Humana, Universidade de Lisboa, Lisboa, \\ Portugal \\ ii APPsyCI, Applied Psychology Research Center Capabilities \& Inclusion, ISPA - Instituto Universitário \\ iii Faculdade de Psicologia e de Ciências da Educação, Universidade de Coimbra, Coimbra, Portugal \\ ${ }^{i v}$ ISPA - Instituto Universitário, Lisboa, Portugal
}

\begin{abstract}
Resumo: O objetivo deste estudo é identificar as perceções de Espontaneidade e Criatividade em grupos distintos de estudantes universitários de Desporto e Psicologia. Participaram no estudo 225 indivíduos, entre os 18 e os 52 anos de idade, dos quais 133 são mulheres e 92 são homens. A espontaneidade foi medida pelo Revised Spontaneity Assessment Inventory (SAI-R) enquanto a criatividade foi mensurada pela Escala de Personalidade Criativa - Forma Reduzida (EPC- Forma Reduzida). Os resultados mostram que os estudantes de ciências do desporto têm níveis mais elevados de Espontaneidade do que os estudantes de psicologia. Os resultados revelaram também que não há diferenças entre os níveis de Criatividade para as variáveis sexo e tipo de curso. Encontraram-se também correlações positivas moderadas entre espontaneidade e criatividade. Estes resultados suportam a ideia de que em estudantes com experiências em que o corpo é utilizado como veículo do ato criativo, tais experiências fomentam dinâmicas de auto-percepção de espontaneidade mais elevadas no contexto de corporeidades diversas, como por exemplo modalidades desportivas. Por último, a espontaneidade revelou-se uma variável preditora da criatividade.
\end{abstract}

Palavras-chave: Criatividade; Espontaneidade; Desporto; Psicologia; Corpo.

Spontaneity as a precursor of creativity: a comparative study using sports and psychology students: This study aims to identify the expressions of spontaneity and creativity in two different groups, namely sports and psychology students. This study included 225 subjects, between the ages of 18 and 52, of which 133 were females and 92 males. Spontaneity was measured using the Revised Spontaneity Assessment Inventory (SAI-R), while creativity was measured using the Creative Personality Scale (CPS). The results showed that sport students have higher levels of Spontaneity than psychology students. Additionally, no differences were found between the levels of Creativity concerning the variables gender and type of study. Mild positive correlations were found between spontaneity and creativity. Such results lend support to the idea that, within a diverse group of sporting activities, students using their body as a vehicle for creative actions record a higher self-assessment of spontaneity. Lastly, , spontaneity was shown to be a predictive variable of creativity.

Keywords: Creativity; Spontaneity; Sport; Psychology; Body.

Na conceção moreniana (Abreu, 2002), o estado espontâneo é composto por sentimentos positivos, pautado por disponibilidade e autonomia intelectual e direcionado para a descoberta de novas experiências orientadas pela adequação. Este estado é anterior ao ato criativo propriamente dito, e é um facilitador positivo da saúde mental (Kipper \& Shemer, 2006).

A espontaneidade tem sido concetualizada como uma energia, mas também como uma competência, resultando que, no campo teórico, tenha sido postulada a existência de um estado mental experiencial específico correspondente à espontaneidade (Davelaar, Araujo, \& Kipper, 2008; Kipper, Green, \& Prorak, 2010; Kipper \& Shemer, 2006). Segundo Moreno (1975, p. 76) “(a espontaneidade) é o que impele o indivíduo para uma resposta adequada a uma nova situação, ou uma nova resposta a uma situação antiga. Assim a criatividade está relacionada ao ato em si, e a espontaneidade a uma prontidão para agir".

${ }^{50}$ Morada para correspondência: Paulo Martins, FMH - Faculdade de Motricidade Humana, 1499-002 Cruz Quebrada - Dafundo, Lisboa. Email: pmartins@fmh.ulisboa.pt 
No cânone original de Moreno, é colocada a hipótese de que a espontaneidade é um precursor da criatividade. Recentemente, Kipper (2006) sublinha que o estado criativo é definido como um estado de ativação interna caracterizado pela procura, geração e contemplação de ideias criativas, e que o significado de ato criativo pode ser visto como a operacionalização do trabalho sobre a ideia criativa. Assim, a elaboração do produto criativo num formato físico pode ser utilizada repetidamente para fomentar a espontaneidade, que se orientará depois para a criatividade psicodramática (Kipper, 2006; Kipper, Davelaar \& Herst, 2009; Kipper et al., 2010; Moreno, 1975). 0 desenvolvimento da espontaneidade é realizado através da interatividade, nomeadamente através da introdução de situações relacionais não esperadas pelo protagonista da dramatização, sendo uma oportunidade para comunicar e cooperar com outros indivíduos. Na essência, está em causa um conjunto de momentos, que vai da ativação da prontidão para agir, até à mudança (fisicalidade) propriamente dita (Kipper et al., 2010). 0 desporto é outro ambiente onde as experiências humanas se realizam numa lógica em torno do conceito de interatividade de cooperação com os outros. No desporto, a criatividade é também fundamental para encontrar respostas aos problemas impostos pela competição (Stavrou, Psychountaki, Georgiadis, Karteroliotis, \& Zervas, 2015).

Csikszentmihalyi (1996) propõe o conceito de fluxo (flow) como modo de alcançar a criatividade, aspecto que concorre para experiências ótimas com fortes implicações também no desempenho desportivo (Stavrou, et al., 2015). De acordo com o autor, há íntimas relações entre a espontaneidade e o fluxo, uma vez que ambos descrevem uma experiência ótima. Contudo divergem, no sentido em que o fluxo se refere aos sentimentos que a pessoa tem enquanto realiza a ação, e o estado espontâneo diz respeito a um estado experiencial presente antes do despoletar do ato criativo (Kipper, 2000; Kipper et al., 2009; Kipper et al., 2010). Acresce dizer que no desporto moderno, o corpo só é visto enquanto total quando estrutura e função são fundamentalmente iguais, embora regularmente entendidas como duas faces da mesma moeda. E, nesta ordem de ideias, o desenvolvimento do corpo pressupõe a sua progressiva socialização (Sérgio, 2012). Nesta medida, o dualismo cartesiano é questionado pelo rigor, a precisão e a matemática de um corpo total. A este propósito, Sérgio (1981, p.33) afirma que "o psiquismo radica na motricidade, e cumpre-se através dele para concluir o primado do ser sobre o pensar".

Mediante este paradigma, seria expectável que, no campo científico do desporto, proliferassem os trabalhos onde os efeitos psicológicos da espontaneidade fossem avaliados em atletas ou em sujeitos com prática regular de atividades desportivas. Todavia, e não obstante o reconhecimento que a espontaneidade pode assumir no desporto, na investigação ainda é insuficiente o conhecimento de base empírica, questionando em que medida a espontaneidade é precursora da criatividade. Recentemente, com o objetivo de fundir o papel orientador do ambiente com o papel ativo desempenhado pelas características individuais no desempenho desportivo, foram também desenvolvidos estudos sobre a personalidade criativa no desporto. Estes estudos permitiram compreender que as personalidades dos atletas mais criativos estão ligadas à emergência do pensamento situacional (Cavallera, Boari, Labbrozzi \& Del Bello, 2011). No entanto, a amostra é limitada a jovens com baixa prática desportiva e não foram consideradas outras características psicológicas que favorecem o estado criativo como, por exemplo, o fluxo ou a espontaneidade, nem feitas comparações com outros contextos que não o desportivo.

Noutro estudo, Stavrou et al., (2015), argumentam que o estado de fluxo permite uma maior espontaneidade para encontrar respostas criativas, mas não utilizam nenhum instrumento de avaliação desse construto específico. Nesta medida, o uso de outros instrumentos será útil para entender e interpretar a experiência competitiva desportiva. Assim, neste trabalho, procuramos contribuir para este tópico, através de um estudo exploratório, avaliando os níveis de espontaneidade e criatividade em estudantes de psicologia e de ciências do desporto. Este estudo tem por objetivo explorar as relações entre os níveis de espontaneidade e de criatividade em jovens estudantes do ensino superior, tendo em conta a maior probabilidade de, em função das exigências curriculares, o grupo de estudantes de desporto ter um maior número de horas de prática física/desportiva semanal do que os de psicologia.

\section{MÉTODO}

\section{Participantes}

Participaram no estudo 225 estudantes de duas faculdades de Lisboa, sendo 81 estudantes do primeiro ano de ciências do desporto e 144 estudantes do primeiro ano de ciências psicológicas, com idades compreendidas entre os 18 e os 52 anos e uma média de idades de 21.5 anos e desvio-padrão de 6.41 . Destes, 133 (59.1\%) são mulheres e 92 homens (40.9\%) e maioritariamente (88\%) vivem na Área Metropolitana de Lisboa. 


\section{Instrumentos}

Para medir a espontaneidade, foi utilizado o Revised Spontaneity Assessment Inventory (SAI-R), criado por Kipper e Shemer (2006), traduzido e validado para português por Gonzalez, Martins, Lima e Costa (submetido). 0 inventário é composto por 18 itens que descrevem vários sentimentos e pensamentos como "com energia", "desinibido/a" e "capaz de fazer qualquer coisa, dentro dos limites". 0 questionário é precedido pela questão inicial: "Com que intensidade é que você tem estes sentimentos ou pensamentos durante um dia típico?". A resposta é assinalada numa escala de tipo Likert de cinco pontos ordenada de 1 (muito fraca) a 5 (muito forte).

Para medir a criatividade, foi utilizada a Escala de Personalidade Criativa - Forma Reduzida (Garcês, Pocinho, Jesus, Viseu, \& Tobal, 2018) - que é a versão curta da Escala de Personalidade Criativa (Garcês, Pocinho, Jesus, Viseu, Imaginário, \& Muglia, 2015). 0 questionário é composto por 11 itens que correspondem a caraterísticas criativas individuais, como por exemplo "Tenho abertura para receber novas ideias" e "Agrada-me resolver problemas de forma não habitual.". As respostas são dadas numa escala de tipo likert de 5 pontos ordenada entre 1 (discordo totalmente) e 5 (concordo totalmente).

\section{Procedimento}

Os alunos foram contatados através dos professores da faculdade onde estudavam. No momento da aplicação foram explicados os objetivos do estudo, sublinhando que a sua participação era voluntária e que poderiam interromper a mesma caso não desejassem continuar. Foram igualmente garantidos o anonimato e a confidencialidade das respostas. Posto isto, os questionários foram administrados e o seu preenchimento tomou aproximadamente entre 10 a 15 minutos.

\section{Análise de dados}

Para analisar os dados foi usado o software SPSS 24.0. Depois de organizados os dados, procedemos à análise descritiva dos mesmos, calculando-se as médias e desvios-padrão, bem como as frequências absolutas e relativas. Assim, para decisão dos testes estatísticos a utilizar, testou-se a normalidade recorrendo ao teste Kolmogorov-Smirnov e a homogeneidade da variância recorrendo ao teste de Levene. Para avaliar a fiabilidade foi ainda calculada a consistência interna através do alfa de Cronbach.

Para a análise inferencial testaram-se as diferenças entre grupos, recorrendo aos testes $t$-student $\mathrm{e}$ as relações entre variáveis, através de testes de associação recorrendo ao coeficiente de correlação de Pearson. Utilizámos finalmente, a regressão linear simples para resumir a natureza da correlação e avaliar a sua expressão preditora, tendo como variável dependente a criatividade e como variável independente a espontaneidade. Para a verificação dos pressupostos para a utilização da regressão, avaliou-se a normalidade da distribuição dos resíduos através de histograma e análise de P-P Plot dos resíduos.

Assim sendo, apesar da não verificação do pressuposto, a distribuição é aproximadamente normal o que viabiliza a continuação da análise de regressão. Para todas as análises, o nível de significância foi fixado em $p \leq .05$, excetuando para o modelo de regressão linear simples que foi fixado em $p \leq .01$ (Marôco, 2011).

\section{RESULTADOS}

0 teste de Kolmogorov-Smirnov revelou a normalidade da distribuição da amostra, bem como da homogeneidade da variância para as variáveis sexo e faculdade de pertença. Adicionalmente, foi obtido um alfa de Cronbach de .92 para o inventário de espontaneidade, valor que pode ser considerado excelente para a fiabilidade do instrumento. Na escala de personalidade criativa foi obtido um alfa de Cronbach de .84, valor que pode ser considerado muito bom.

Quanto aos pressupostos para a utilização da regressão linear (ver Figura 1), quer o histograma (curva da área), quer o gráfico P-P Plot (sobreposição dos resíduos à linha diagonal) apresentam uma distribuição normal, o que viabiliza a continuação da análise de regressão. 

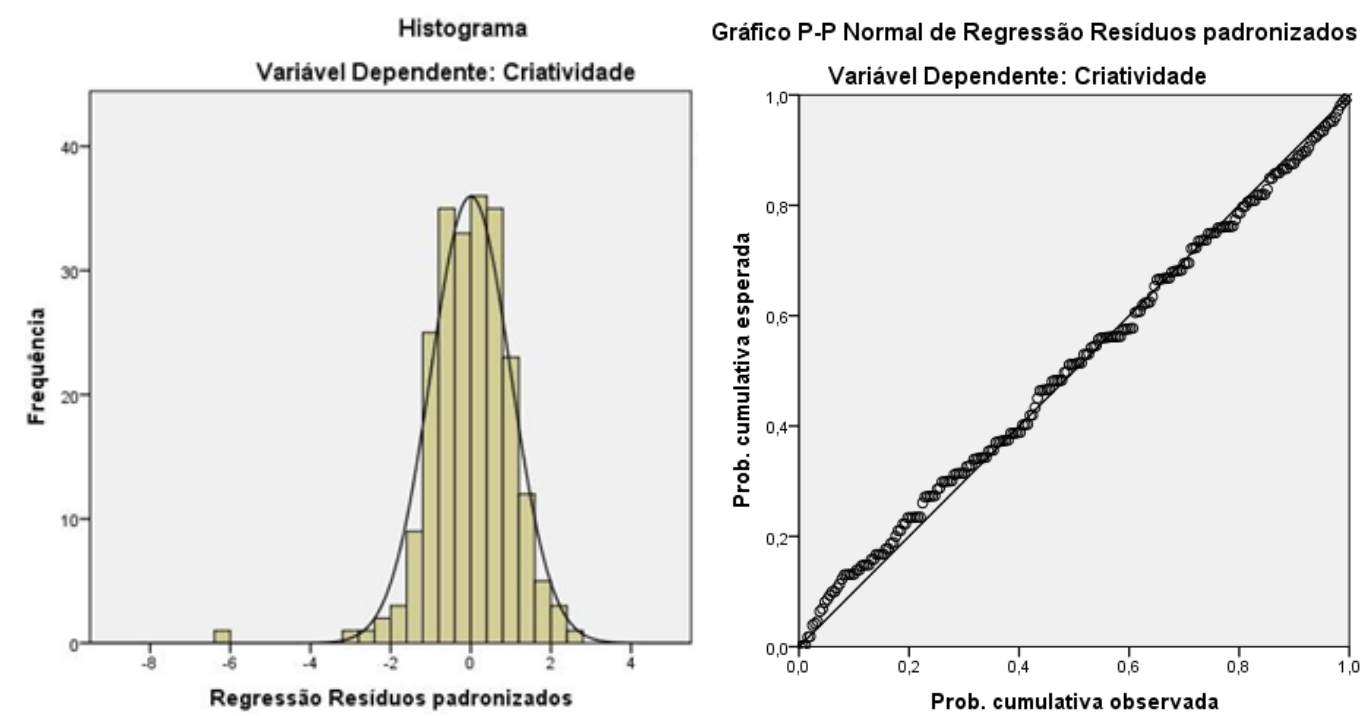

Figura 1. Validação dos pressupostos do modelo de regressão linear

Os resultados (ver Tabela 1) mostram que os homens têm níveis mais elevados de espontaneidade e que existem diferenças estatisticamente significativas quando consideramos a variável sexo. Os resultados mostram também que os homens têm níveis mais elevados de personalidade criativa, mas essas diferenças não são estatisticamente significativas quando consideramos a variável sexo.

0 mesmo procedimento de análise foi realizado para a variável universidade de pertença, isto é, foram realizadas comparações entre os níveis de espontaneidade e criatividade considerando esta variável. Os resultados mostram que os estudantes de ciências do desporto têm níveis de espontaneidade significativamente mais elevados do que os estudantes de ciências psicológicas. No caso da variável personalidade criativa, os estudantes de ciências do desporto apresentam níveis mais elevados, mas as diferenças encontradas não são significativas do ponto de vista estatístico.

Tabela 1. Comparação dos valores de Espontaneidade e Personalidade Criativa segundo as variáveis sexo e universidade de pertença (média, desvio padrão e significância de teste de diferenças de médias)

\begin{tabular}{lcccccc}
\hline & \multicolumn{3}{c}{ Sexo } & \multicolumn{2}{c}{ Universidade } \\
\cline { 2 - 7 } Dimensões: & Feminino & Masculino & & Psicologia & Desporto \\
& $(\mathrm{n}=133)$ & $(\mathrm{n}=92)$ & $p$ & $(\mathrm{n}=144)$ & $(\mathrm{n}=91)$ & $p$ \\
\cline { 2 - 8 } Espontaneidade & $3.24(.63)$ & $3.53(.52)$ & .001 & $3.26(.64)$ & $3.53(.50)$ & .002 \\
Personalidade criativa & $3.91(.51)$ & $4.03(.46)$ & .06 & $3.95(.51)$ & $3.96(.45)$ & .849 \\
\hline
\end{tabular}

Foram ainda realizadas comparações de médias para as variáveis espontaneidade e personalidade criativa, considerando o sexo para cada uma das universidades do estudo. Os resultados (ver Tabela 2) revelaram que, à semelhança dos resultados gerais, no caso dos estudantes de psicologia, os homens mostraram níveis de espontaneidade e personalidade criativa mais elevados do que as mulheres, mas essa diferença não é estatisticamente significativa. No caso dos estudantes de ciências do desporto, as diferenças estão também em linha com os resultados anteriores, sendo que neste caso as diferenças são significativas para a variável espontaneidade, mas não são significativas para a variável personalidade criativa. 
Tabela 2. Comparação dos valores de Espontaneidade segundo 'universidade de pertença x sexo' e Personalidade Criativa segundo as variáveis "universidade de pertença x sexo" (média, desvio padrão e significância de teste de diferenças de médias)

\begin{tabular}{|c|c|c|c|c|c|c|}
\hline \multirow[b]{3}{*}{ Dimensões: } & \multicolumn{6}{|c|}{ Universidade } \\
\hline & \multicolumn{2}{|c|}{ Psicologia } & \multicolumn{4}{|c|}{ Desporto } \\
\hline & Feminino & Masculino & $p$ & Feminino & Masculino & $p$ \\
\hline Espontaneidade & $3.23(.66)$ & $3.38(.56)$ & .243 & $3.28(.47)$ & $3.61(.48)$ & .008 \\
\hline Personalidade criativa & $3.92(.52)$ & $4.05(.50)$ & .196 & $3.81(.46)$ & $4.01(.44)$ & .076 \\
\hline
\end{tabular}

Os resultados mostraram que o coeficiente de correlação de Pearson indica uma relação positiva entre a espontaneidade e a personalidade criativa, $R=.396, F(223)=41.57, p=.001$, que a sua relação é linear e que o modelo é adequado. Por último, os resultados revelaram que a espontaneidade é preditora da personalidade criativa, $t=6.45, p=.001$, ou seja, o efeito da variável espontaneidade é significativo mostrando que quanto maior o nível de espontaneidade, maior é a expressão da personalidade criativa.

Complementarmente, estes resultados indicam que a espontaneidade é uma variável a considerar num eventual modelo explicativo do ato criativo pois, com base na estatística da correlação quadrada, $R^{2}$ $=.16$, a variância explicada é de $16 \%$.

\section{DISCUSSÃO E CONCLUSÃO}

O principal objetivo do presente estudo foi o de contribuir para o desenvolvimento da investigação sobre as ligações entre espontaneidade e criatividade, procurando compreender em que medida esta relação pode ser influenciada pela prática regular de atividades físicas organizadas, como por exemplo o desporto. 0 estudo revelou que os estudantes da licenciatura em ciências do desporto têm níveis significativamente mais elevados de espontaneidade do que os estudantes de ciências psicológicas, suportando a ideia de que a prática regular de atividades onde as expressões físico-motoras são estimuladas através da interatividade de cooperação com o outro podem facilitar a elevação de níveis de espontaneidade. Estes resultados permitem um revisitar das teorias de Moreno e seus seguidores (Kipper, 2006) que, ao discutir o Cânone da Espontaneidade / Criatividade se referem a despoletadores da criatividade não apenas internos / intelectuais, mas igualmente externos / físicos. Recentemente (Gonzalez, Martins, \& Lima, 2018) tem sido sublinhada a eficácia do psicodrama no aumento da espontaneidade, podendo colocar-se a hipótese de que os frequentes aquecimentos físicos presentes nesta prática clínica pode ser parte da explicação desse aumento da espontaneidade.

Os resultados deste estudo indicam, também, que não existem diferenças significativas nos níveis de auto-perceção de criatividade entre os estudantes de ciências do desporto e de psicologia. Nesta medida, os dados obtidos sobre as diferenças entre sexos (apenas significativos nos estudantes de desporto) estão em linha com os estudos anteriores, onde as diferenças ou não existem ou são apenas ligeiramente mais altos em homens do que em mulheres (Kipper, 2000; Kipper et al., 2009; Kipper et al., 2010).

Finalmente, os resultados da sustentam empiricamente a ideia de que a espontaneidade tem uma relação preditora da personalidade criativa, e que poderá ser um fator preponderante na determinação da criatividade, nomeadamente do ato criativo, o que parece ter vindo a ser sugerido na literatura (Stavrou et al., 2015).

Na perspetiva da continuidade deste estudo, podemos salientar que seria importante realizar estudos adicionais associados às medidas de espontaneidade e criatividade com outras medidas teoricamente associadas, como por exemplo flow, impulsividade e bem-estar. Tais estudos permitiriam verificar os efeitos da espontaneidade sobre outros construtos, por sua vez associados à saúde mental.

\section{REFERÊNCIAS}

Abreu, J. (2002). O Modelo do Psicodrama Moreniano. Quarteto.

Cavallera, G. M., Boari, G., Labbrozzi, D., \& Del Bello, E. D. (2011). Morningness- eveningness personality and creative thinking among young people who play recreational sport. Social Behavior and Personality: An International Journal, 39(4), 503-518. https://doi.org/10.2224/sbp.2011.39.4.503.

Csikszentmihalyi, M. (1996). Creativity, Flow and the Psychology of Discovery and Invention. Harper Collins Publications. 
Cukier, R. (2002). Palavras de Jacob Levy Moreno: vocabulário de citações do Psicodrama, da Psicoterapia de Grupo, do Sociodrama e da Sociometria. Ágora.

Davelaar, P., Araujo, F., \& Kipper, D. (2008). The Revised Spontaneity Assessment Inventory (SAI-R): Relationship to goal orientation, motivation, perceived self-efficacy, and self-esteem. Arts in Psychotherapy, 35(2), 117-128. https://doi.org/10.1016/j.aip.2008.01.003

Fox, J. (2002). O Essencial de Moreno: Textos sobre psicodrama, terapia de grupo e espontaneidade. Ágora.

Garcês, S., Pocinho, M., Jesus, S., Viseu, J., Imaginário, S., \& Muglia, W. (2015). Estudo de Validação da Escala de Personalidade Criativa. Revista Iberoamericana de Diagnóstico y Evaluación - e Avaliação Psicológica, 40(2), 17-24.

Garcês, S., Pocinho, M., Jesus, S., Viseu, J., \& Tobal, J. (2018). Exploratory and confirmatory analysis of the Creative Personality Scale short-form. Manuscrito submetido para publicação.

Gonzalez, A.-J., Martins, P., \& Lima M. (2018). Studying the efficacy of psychodrama with the Hermeneutic Single Case Efficacy Design: results from a longitudinal study. Frontiers in Psychology, 9. https://doi.org/10.3389/fpsyg.2018.01662

Gonzalez, A.-J., Martins, P., Lima, M., \& Costa, J. (submetido). Análise das qualidades psicométricas da versão portuguesa do Inventário de Avaliação da Espontaneidade (SAI-RP). Análise Psicológica.

Kipper, D. (2000). Spontaneity: Does the experience match the theory? The International Journal of Action Methods: Psychodrama, Skill Training and Role Playing, 33-47.

Kipper, D. (2006). The Canon of Spontaneity - Creativity Revisited: The Effect of Empirical Findings. Journal of Group Psychotherapy, Psychodrama and Sociometry, 59(3), 117-126. https://doi.org/10.3200/JGPP.59.3.117-126

Kipper, D., Davelaar, P., \& Herst, S. (2009). The relationship between spontaneity and inhibition. The arts in psychotherapy, 36(5), 329-334. https://doi.org/10.1016/j.aip.2009.09.005

Kipper, D., Green, D., \& Prorak, A. (2010). The relationship among spontaneity, impulsivity, and creativity. Journal of Creativity in Mental Health, 5(1), 39-53. https://doi.org/3953.10.1080/15401381003640866

Kipper, D., \& Shemer, H. (2006). The Revised Spontaneity Assessment Inventory (SAI-R): spontaneity, well-being, and stress. Journal of Group Psychotherapy, Psychodrama \& Sociometry, 59(3), 127-136.

Marôco, J. (2011). Análise Estatística com o SPSS statistics. Report Number.

Magalhães, E., Salgueira, A., Gonzalez, A.-J., Costa, J., Costa, M., Costa, P., \& Lima, M. (2014). NEO-FFI: Psychometric properties of a short personality inventory in a portuguese context. Psicologia, Reflexão e Crítica, 27(4), 642-657. http://dx.doi.org/10.1590/1678-7153.201427405.

Moeller, F., Barrat, E., Dougherty, D., Schmitz, J., \& Swann, A. (2001). Psychiatric aspects of impulsivity. $\begin{array}{llll}\text { American of Journal Psychiatry, } & \text { 158(11), }\end{array}$ https://doi.org/10.1176/appi.ajp.158.11.1783

Moreno, J. (1974). The creativity theory of personality: spontaneity, creativity, and human potentialities. In I. A. Greenberg (Ed.), Psychodrama theory and therapy (pp. 73-84). Behavioral publications.

Moreno, J. (1975). Psicodrama. Cultrix.

Moreno, J. (1983). Fundamentos do Psicodrama. Summus.

Moreno, J. (1999). Psicoterapia de Grupo e Psicodrama. Campinas. Livro Pleno.

Moreno, J. (2008). Quem sobreviverá? Fundamentos da sociometria, da psicoterapia de grupo e do psicodrama. Daimon.

Sérgio, M. (1981). Filosofia das atividades corporais. Compendium.

Sérgio, M. (2012). Crítica da razão desportiva. Instituto Piaget - Divisão documental

Stavrou, N. A., Psychountaki, M., Georgiadis, E., Karteroliotis, K., \& Zervas, Y. (2015). Flow theory - goal orientation theory: positive experience is related to athlete's goal orientation. Frontiers in Psychology, 6, 1499. https://doi.org/10.3389/fpsyg.2015.01499

Historial do artigo

Recebido $\quad 07 / 2019$

Aceite $\quad 05 / 2020$

Publicado 08/2020 